FELIPE LOPES DE SOUZA

BAYESIAN ESTIMATION OF DIRECTIONAL WAVE SPECTRUM USING VESSEL MOVEMENTS AND WAVE-PROBES 
FELIPE LOPES DE SOUZA

BAYESIAN ESTIMATION OF DIRECTIONAL WAVE SPECTRUM USING VESSEL MOVEMENTS AND WAVE-PROBES

Thesis presented to the Escola Politécnica Universidade de São Paulo, to obtain the degree of Doctor of Science. 
FELIPE LOPES DE SOUZA

\section{BAYESIAN ESTIMATION OF DIRECTIONAL WAVE SPECTRUM USING VESSEL MOVEMENTS AND WAVE-PROBES}

Thesis presented to the Escola Politécnica Universidade de São Paulo, to obtain the degree of Doctor of Science.

Research Area:

Mechanical Engineering

Control and Automation

Advisor: Prof. Dr. Eduardo Aoun Tannuri 
Autorizo a reprodução e divulgação total ou parcial deste trabalho, por qualquer meio convencional ou eletrônico, para fins de estudo e pesquisa, desde que citada a fonte.

Este exemplar foi revisado e corrigido em relação à versão original, sob responsabilidade única do autor e com a anuência de seu orientador.

São Paulo, de de

Assinatura do autor:

Assinatura do orientador:

Catalogação-na-publicação

de Souza, Felipe Lopes

BAYESIAN ESTIMATION OF DIRECTIONAL WAVE SPECTRUM USING

VESSEL MOVEMENTS AND WAVE-PROBES / F. L. de Souza - versão corr. -- São Paulo, 2019.

$254 \mathrm{p}$.

Tese (Doutorado) - Escola Politécnica da Universidade de São Paulo. Departamento de Engenharia Mecatrônica e de Sistemas Mecânicos.

1.Espectro Direcional 2.Estimação Bayesiana 3.Wave-Probe 4.Posicionamento Ótimo de Sensores I.Universidade de São Paulo. Escola Politécnica. Departamento de Engenharia Mecatrônica e de Sistemas Mecânicos II.t. 


\section{ACKNOWLEDGEMENT TO FINANCIAL SUPPORTS}

This study was financed in part by the Coordenação de Aperfeiçoamento de Pessoal de Nível Superior - Brasil (CAPES) - Finance Code 001. The research grant conceded by CAPES was indispensable for the development of this work.

The other resources for this research were provided by the Tanque de Provas Numérico da Universidade de São Paulo (TPN-USP) - Numerical Offshore Tank of the University of São Paulo, in a partnership with the Brazilian petroleum company Petrobras and the Norwegian petroleum company Equinor, previously called Statoil, and was financed inside the context of the project "Wave Measurements Aboard FPSO Unit in Peregrino Field". 


\begin{abstract}
The exploration of oil and natural gas in offshore fields has motivated advanced researches about the environmental forces in the oceans. The waves, in particular, have been measured using different techniques, as meteorological buoys, with recent works proposing motion-based estimations procedures using the vessel, or a floating facility, in analogy with the buoys, as a wave sensor. Even though this approach has a number of benefits, the vessels, as dynamic systems, have a cut-off frequency that degrades the estimation of high-frequency waves, which are important for non-linear drift effects predictions. In order to solve this problem, it is proposed the incorporation of wave-probes - gauges used to measure the wave elevation in a point - installed on the hull of the vessel, based on literature suggestions and simple analytical arguments, using the Bayesian statistics as the standing point of a more complete estimation algorithm. In order to incorporate the measurements of the wave-probes, an extended linear model is proposed, showing that only corrections for the vertical motions of the vessel are necessary. The ideal installation positions of the wave-probes are defined using as base the utility Bayesian optimal design of experiments, which is shown to guarantee an upper bound for other optimal criteria, with the "Elbow Criterion" defining the optimal number of sensors to be employed. Based on the previous solutions, other proposals are made: a heuristic to solve the optimal sensor placement problem and an optimal prior exploring the probabilistic nature of the algorithm. Finally, all the proposals are tested numerically and experimentally, with a vessel model in a towing tank, concluding that the addition of the wave-probes is able to improve not only the estimation of high-frequency waves, but also the estimation over a large range of frequencies. For unimodal seas with intermediate draft, the addition of just one waveprobe reaches approximately a $37 \%-55 \%$ improvement in the energy parameter estimations $-H_{S}$ and $T_{P}$; the addition of two or more probes reaches approximately a $62 \%-65 \%$ improvement in the same parameters estimations; the addition of four probes achieved the best cost benefit for mean direction estimation; and the addition of six probes is shown to be the recommendation for the best high-order directional estimation in the entire range of the spectrum.
\end{abstract}

Key-words: Directional Spectrum, Bayesian Estimation, Wave-Probe, Optimum Sensor Placement. 


\section{RESUMO}

A prospecção de óleo e gás natural em campos offshore tem motivado pesquisas avançadas sobre as forças ambientais em oceanos. As ondas, em particular, têm sido medidas através de diferentes técnicas, como boias meteorológicas, com trabalhos recentes propondo técnicas baseadas em movimento para que os navios, em analogia com as boias, possam ser usados como sensores de onda. Apesar desse método ter uma série de vantagens, os navios, como sistemas dinâmicos, têm uma frequência de corte que dificulta a estimação de ondas de altas frequências, que são importantes para a previsão de efeitos de deriva não-lineares. Para resolver esse problema, sugere-se a adição de wave-probes instalados no costado da embarcação, usando como justificativas sugestões da literatura e simples argumentos analíticos, com estatística Bayesiana como fundamentação para um algoritmo de estimação mais completo. Para que as medidas dos wave-probes possam ser incorporadas, um modelo linear estendido é proposto, mostrando que apenas correções para os movimentos verticais do navio são necessárias. A posição ideal de instalação dos wave-probes é definida usando como base o projeto ótimo de experimentos Bayesianos por utilidade, mostrando que o mesmo garante o limite superior de outros critérios de optimalidade, com o "critério cotovelo" definindo o número ótimo de sensores a serem usados. Com base nas soluções anteriores, outras propostas são feitas: uma heurística para resolver o problema de posicionamento ótimo dos sensores e uma priori ótima, explorando a natureza probabilística do algoritmo. Ao final, todas as propostas são testadas numericamente e experimentalmente, utilizando um modelo em escala em um tanque de provas, concluindo que a adição de wave-probes é capaz de melhorar não só a estimação de ondas em alta-frequência, mas também a estimação em uma ampla gama de frequências. Para mares unimodais, com calado intermediário, a adição de apenas um sensor alcançou uma melhoria de aproximadamente $37-55 \%$ na estimação dos parâmetros relacionados à energia - $H_{S}$ e $T_{P}$; a adição de dois ou mais sensores alcançou melhorias de $62-65 \%$ na estimação de tais parâmetros; a adição de quatro sensores alcançou o melhor custo benefício para estimação da direção média; e a adição de seis sensores se mostrou ideal para estimação de ordem elevada do espectro direcional de energia.

Palavras-chave: Espectro Direcional, Estimação Bayesiana, Wave-Probe, Posicionamento Ótimo de Sensores. 


\section{LIST OF FIGURES}

Figure 1 - Meteorological buoy (a), radar (b) and satellite imagery (c) .................19

Figure 2 - Example of microwave wave-probe................................................21

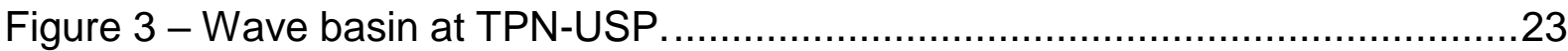

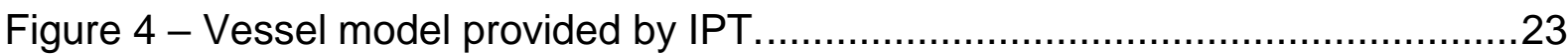

Figure 5 - Naval nomenclature for vessels and seas...........................................

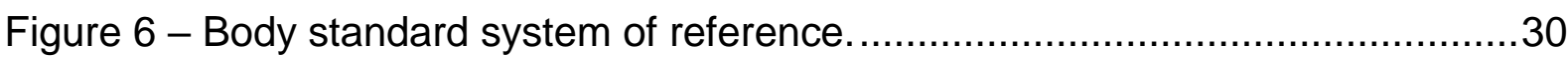

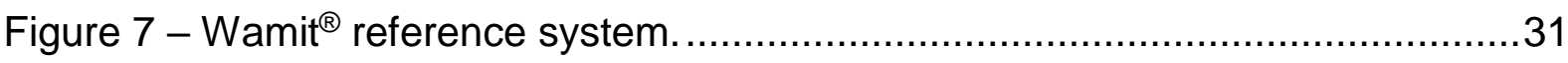

Figure 8 - Directional spectrum polar plot reference.......................................... 31

Figure 9 - Irregular sea, formed by the superposition of regular waves...................34

Figure 10 - Energy spectral density determined by harmonic decomposition............35

Figure 11 - Example of JONSWAP energy spectral density...................................38

Figure 12 - Example of cosine-squared directional spread function. .......................38

Figure 13 - Example of directional energy spectrum. ........................................38

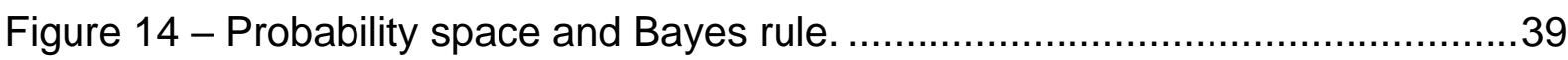

Figure 15 - Euclidian space clusters ............................................................ 46

Figure 16 - Least increment in inertia. ...................................................... 47

Figure 17 - Wrong solution with greater increment in inertia. ..............................48

Figure 18 - Example with five groups and four clusters, with high inertia. ................49

Figure 19 - Same example with five clusters, and considerably less inertia.............49

Figure 20 - Same example with six clusters, and slightly less inertia. ...................50

Figure 21 - Five clusters selected, based on the 'elbow' criterion of the example. ...50

Figure 22 - Low-frequency waves versus high-frequency waves. .........................57

Figure 23 - Wave-probe measurement affected by the surge movement.................58

Figure 24 - Wave-probe measurement affected by the sway movement..................58

Figure 25 - Wave-probe measurement affected by the heave movement. ...............58

Figure 26 - Wave-probe measurement affected by the roll movement. ...................59

Figure 27 - Wave-probe measurement affected by the pitch movement. .................59

Figure 28 - Wave-probe measurement affected by the yaw movement. .................59

Figure 29 - Experimental computation cost and polynomial fitting..........................66

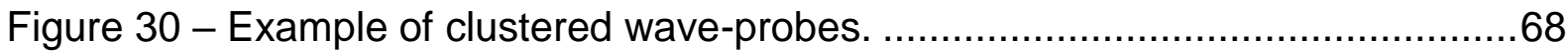

Figure 31 - Pre-processing before the optimization algorithm. .............................68

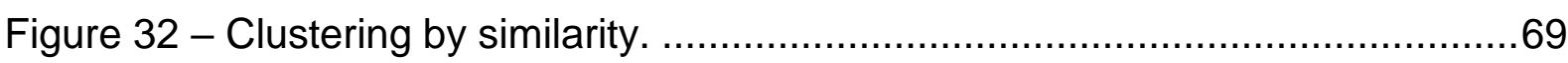


Figure 33 - Optimization algorithm...........................................................

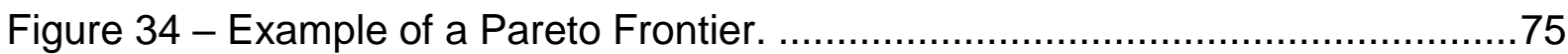

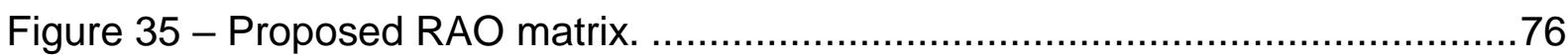

Figure 36 - Position of the wave-probes for the experiments. .............................. 85

Figure 37 - Parametric errors during verification with $40 \omega$ and $40 \beta \ldots \ldots \ldots \ldots \ldots \ldots . . .87$

Figure 38 - Parametric errors during verification with $40 \omega$ and $20 \beta \ldots \ldots \ldots \ldots \ldots \ldots . . .89$

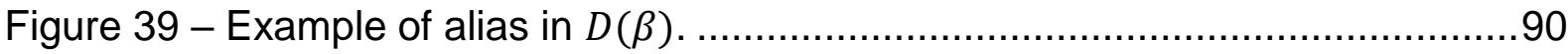

Figure 40 - Parametric errors during verification with $20 \omega$ and $20 \beta \ldots \ldots \ldots \ldots \ldots \ldots . . . .91$

Figure 41 - Parameter errors without removing bias...........................................92

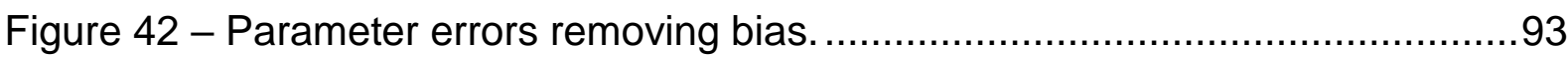

Figure 43 - Trace criterion versus different error metrics in noiseless estimation. ....95

Figure 44 - Regions with low trace have lower worst-case.................................101

Figure 45 - Chosen combination for detailed analysis. ......................................102

Figure 46 - Histogram of the combinations performing noiseless estimation..........102

Figure 47 - Trace criterion versus different error metrics in estimation with noise. .105

Figure 48 - Regions with low trace also have lower worst-case. .........................110

Figure 49 - Histogram of the combinations performing estimation with noise.........112

Figure 50 - Best trace criterion for different number of wave-probes....................114

Figure 51 - Best position of the wave-probes for different number of gauges. …...115

Figure 52 - Error metrics for different number of wave-probes. ..........................116

Figure 53 - Histogram of the errors for different number of wave-probes. ..............118

Figure 54 - One hundred positions grouped together in 24 clusters. ....................122

Figure 55 - Values obtained for the addition of one wave-probe. .........................122

Figure 56 - Values obtained for the addition of three wave-probes. .....................123

Figure 57 - Positions suggested by the algorithm for a single wave-probe.............123

Figure 58 - Suggested solutions for the three-wave-probe problem......................124

Figure 59 - Histogram in a particular sea vector row. The occurrence of zero values is greater than shown in the histogram, it was cropped for visualization purposes. ....126

Figure 60 - Comparison between the column of the priors.................................127

Figure 61 - Penalization of the conventional prior..........................................129

Figure 62 - Penalization of the maximum likelihood quadratic prior (MLQP) ..........129

Figure 63 - Trace criterion versus errors for different priors................................130

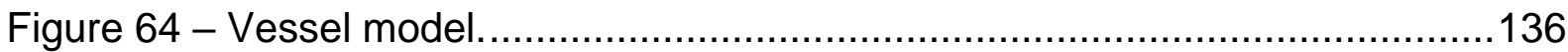

Figure 65 - Main dimensions of the real vessel. ............................................137 


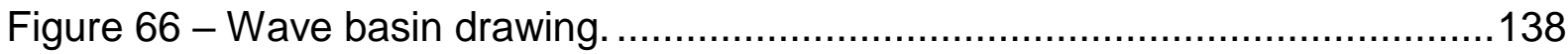

Figure 67 - Valid region due to physical constraints. ........................................138

Figure 68 - Reflective dummy and Qualisys ${ }^{\circledR}$ camera. ......................................139

Figure 69 - CAD drawing of the model test with the wave-probes.......................139

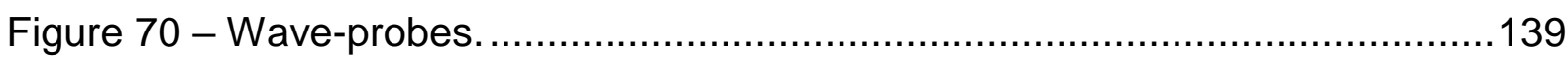

Figure 71 - Wave-probes dynamic response, in real scale.................................140

Figure 72 - Test with similar configuration. ..................................................140

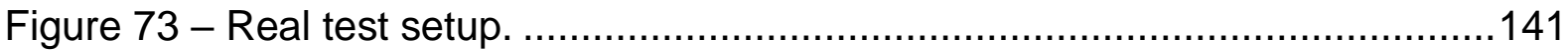

Figure 74 - 'Elbow' criterion applied to the clustering of the metocean data. ..........143

Figure 75 - Spectra density in each cluster.....................................................144

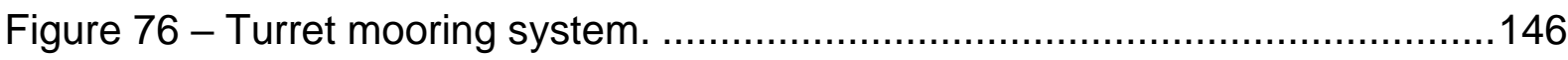

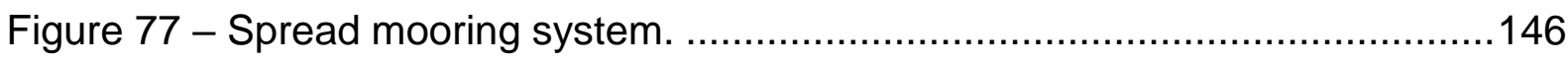

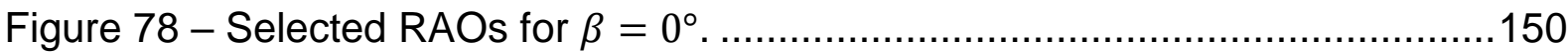

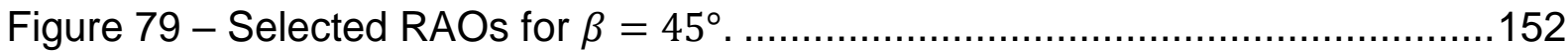

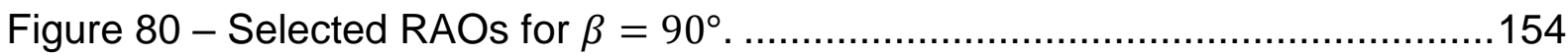

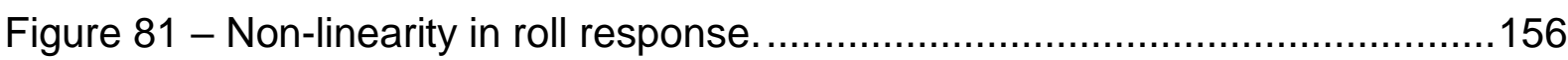

Figure 82 - Example of wave-probe response affected by the roll non-linearity. ....157

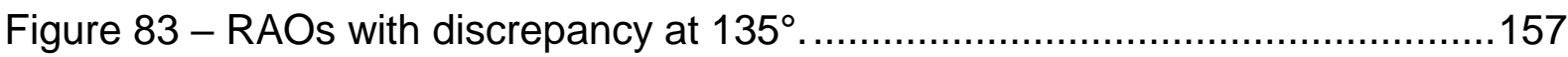

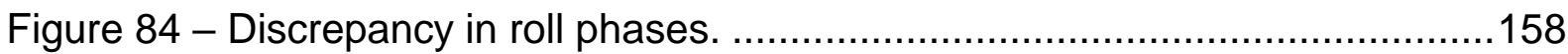

Figure 85 - Expanded linear model with roll phase shift. ....................................158

Figure 86 - Selected RAOs for the ballasted condition, $\beta=0^{\circ}$............................160

Figure 87 - Selected RAOs for the ballasted condition, $\beta=45^{\circ} \ldots \ldots \ldots \ldots \ldots \ldots \ldots \ldots \ldots . . . . . . . . . . .161$

Figure 88 - Selected RAOs for the ballasted condition, $\beta=90^{\circ} \ldots \ldots \ldots \ldots \ldots \ldots \ldots \ldots . . . . . . . . . . . . .163$

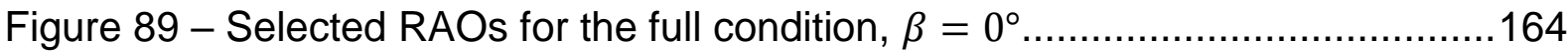

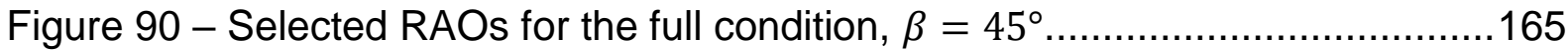

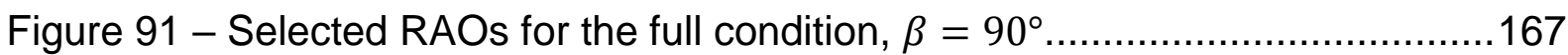

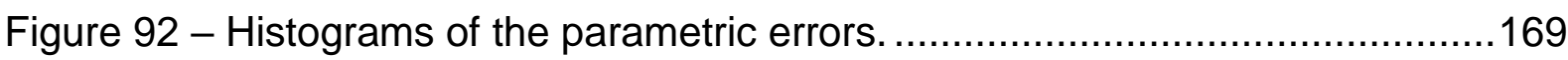

Figure 93 - Histogram of the directional spread function error.............................172

Figure 94 - Low TP wave, coming from $0^{\circ}$, estimated without wave-probes...........173

Figure 95 - Low TP wave, coming from $0^{\circ}$, estimated with 1 wave-probe. .............173

Figure 96 - Low TP wave, coming from $0^{\circ}$, estimated with 2 wave-probes.............174

Figure 97 - Low $T P$ wave, coming from $0^{\circ}$, estimated with 4 wave-probes..............174

Figure 98 - Low $T P$ wave, coming from $0^{\circ}$, estimated with 6 wave-probes.............174 
Figure 99 - Low TP wave, coming from $90^{\circ}$, estimated without wave-probes........175

Figure 100 - Low TP wave, coming from $90^{\circ}$, estimated with 2 wave-probes.........175

Figure 101 - Low TP wave, coming from $180^{\circ}$, estimated without wave-probes.....176

Figure 102 - Low TP wave, coming from $180^{\circ}$, estimated with 2 wave-probes.......176

Figure 103 - Low TP wave, coming from $45^{\circ}$, estimated without probes. ..............177

Figure 104 - Low TP wave, coming from $45^{\circ}$, estimated with 2 wave-probes........177

Figure 105 - Low TP wave, coming from $45^{\circ}$, estimated with 4 wave-probes........177

Figure 106 - Low TP wave, coming from $45^{\circ}$, estimated with 6 wave-probes.........178

Figure 107 - Low TP wave, coming from $135^{\circ}$, estimated without wave-probes.....178

Figure 108 - Low TP wave, coming from $135^{\circ}$, estimated with 2 wave-probes.......178

Figure 109 - Low TP wave, coming from $135^{\circ}$, estimated with 4 wave-probes.......179

Figure 110 - Low TP wave, coming from $135^{\circ}$, estimated with 6 wave-probes.......179

Figure 111 - Energy spectral density with 0 and 2 probes..............................179

Figure 112 - Medium TP wave, coming from $0^{\circ}$, estimated without wave-probes. .180

Figure 113 - Medium TP wave, coming from $0^{\circ}$, estimated with 2 wave-probes. ...181

Figure 114 - Medium TP wave, coming from $0^{\circ}$, estimated with 6 wave-probes. ...181

Figure 115 - Medium TP wave, at $135^{\circ}$, estimated without wave-probes. .............181

Figure 116 - Medium TP wave, at $135^{\circ}$, estimated with 2 wave-probes. ...............182

Figure 117 - Medium TP wave, at $135^{\circ}$, estimated with 6 wave-probes. ...............182

Figure 118 - High TP wave, coming from $135^{\circ}$, estimated without wave-probes....183

Figure 119 - High $T P$ wave, coming from $135^{\circ}$, estimated with 2 wave-probes......183

Figure 120 - High TP wave, coming from $135^{\circ}$, estimated with 6 wave-probes......183

Figure 121 - Histograms for the ballasted condition. ....................................184

Figure 122 - Ballasted condition, medium $T P$, without probes...........................187

Figure 123 - Ballasted condition, medium $T P$, with 2 probes............................187

Figure 124 - Ballasted condition, medium $T P$, with 6 probes..............................187

Figure 125 - Ballasted condition, high $T P$, without probes................................188

Figure 126 - Ballasted condition, high $T P$, with 2 probes............................... 188

Figure 127 - Ballasted condition, high $T P$, with 6 probes................................ 188

Figure 128 - Histograms for the full draft condition. ......................................189

Figure 129 - Full draft condition, low $T P$, without probes. ................................191

Figure 130 - Full draft condition, low $T P$, with 1 probe.................................... 192

Figure 131 - Full draft condition, medium TP, without probes............................192 
Figure 132 - Full draft condition, medium $T P$, with 1 probe. ...............................192

Figure 133 - Full draft condition, high $T P$, without probes...................................193

Figure 134 - Full draft condition, high $T P$, with 1 probe. ......................................193

Figure 135 - Histograms of the estimation of bimodal seas................................194

Figure 136 - Bow-Quartering, Low TP, without wave-probes. ……….................196

Figure 137 - Bow-Quartering, Low TP, with 2 wave-probes. ..............................196

Figure 138 - Bow-Quartering, Low TP, with 4 wave-probes. ................................196

Figure 139 - Bow-Quartering, Low TP, with 6 wave-probes. ...............................197

Figure 140 - Head-Beam, Low TP, without wave-probes. .................................197

Figure 141 - Head-Beam, Low TP, with 2 wave-probes. ...................................197

Figure 142 - Head-Beam, Low TP, with 4 wave-probes. ....................................198

Figure 143 - Head-Beam, Low TP, with 6 wave-probes. ...................................198

Figure 144 - Bow-Bow, Low TP, without wave-probes. ........................................198

Figure 145 - Bow-Bow, Low TP, with 2 wave-probes........................................199

Figure 146 - Bow-Bow, Low TP, with 4 wave-probes.......................................199

Figure 147 - Bow-Bow, Low TP, with 6 wave-probes. .......................................199

Figure 148 - Histograms comparing the conventional and the MLQP prior. ...........200

Figure 149 - Directional spectrum at $135^{\circ}$ estimated with conventional prior. ........204

Figure 150 - Directional spectrum at $135^{\circ}$ estimated with MLQP........................205

Figure 151 - Energy spectral density at $135^{\circ}$ for different priors...........................205

Figure 152 - Directional spectrum at $0^{\circ}$ estimated with conventional prior. ............205

Figure 153 - Directional spectrum at $0^{\circ}$ estimated with MLQP. ...........................206

Figure 154 - Energy spectral density at $0^{\circ}$ for different priors.............................206

Figure 155 - Histograms for the bimodal set comparing the priors.......................207

Figure 156 - Quartering-bow spectrum, conventional prior..................................209

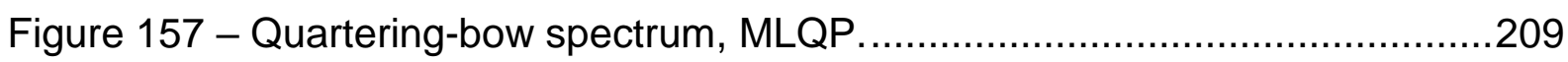

Figure 158 - Head-beam spectrum, conventional prior......................................210

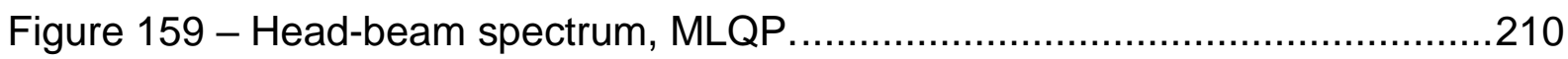

Figure 160 - General framework for Bayesian estimation.................................213

Figure 161 - The superposition of two potentials satisfies the boundary condition. 226

Figure 162 - Seas used in the sensibility analysis. ...........................................230

Figure 163 - Wave-probe measurement affected by the surge movement.............230

Figure 164 - Wave-probe measurement affected by the sway movement.............231 
Figure 165 - Wave-probe measurement affected by the heave movement. 231

Figure 166 - Wave-probe measurement affected by the roll movement. 231

Figure 167 - Wave-probe measurement affected by the pitch movement. 232

Figure 168 - Wave-probe measurement affected by the yaw movement. 232

Figure 169 - Vessel response in a beam sea. 233

Figure 170 - Wave-elevation in six different port positions. 233

Figure 171 - Wave-elevation in six different starboard positions. 234

Figure 172 - Amplification coefficient based on the wave-elevation on the port. ....234

Figure 173 - Maximum disturbance cause by each DoF. 235

Figure 174 - Vessel response in a head sea. 236

Figure 175 - Wave-elevation in six different port positions. 236

Figure 176 - Maximum disturbance cause by each DoF. 237

Figure 177 - Directional spread functions. 239

Figure 178 - Estimation with interior-point-convex algorithm 241

Figure 179 - Estimation with trust-region-reflective algorithm without initial guess. 242

Figure 180 - Estimation with trust-region-reflective, initial guess equals to zero.....243

Figure 181 - Different discretizations superimposed. 245

Figure 182 - Rediscretization using interpolation 245

Figure 183 - Original and rediscretized spectrum. .248

Figure 184 - Comparison of the spectral energy densities. 249

Figure 185 - Rediscretized and ideal spectrum. 249

Figure 186 - Rediscretized spectrum by simple interpolation. 250

Figure 187 - Spectral energy density of the interpolated spectrum. 250

Figure 188 - Interpolation of spectrum slightly translated. 251

Figure 189 - Comparison with the ideal spectrum. 251

Figure 190 - The amplification factor changes drastically. 251

Figure 191 - Rediscretization is robust against translations. 252

Figure 192 - Rediscretization still compatible with the ideal spectrum. .252 


\section{LIST OF TABLES}

Table 1 - Trace criterion for all possible 3DoF combination of a FPSO employed in a conventional Bayesian estimation, with periods ranging from $8 \mathrm{sec}$. to $30 \mathrm{sec}$., from the best to the worst. 61

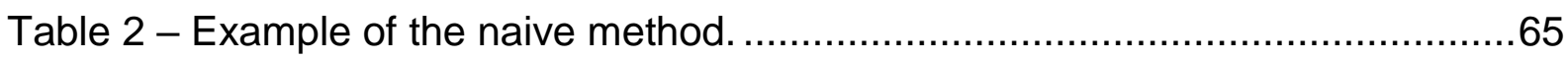

Table 3 - Position of the wave-probes in the model scale, in millimeters..................85

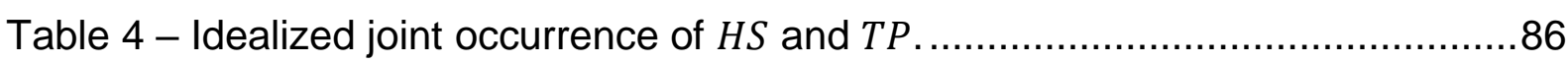

Table 5 - Comparison of the estimation errors for different noise magnitudes. ......105

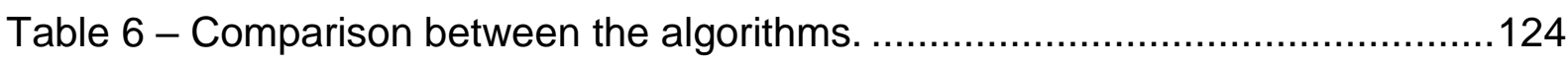

Table 7 - Proposed discretization to calculate the new prior..................................125

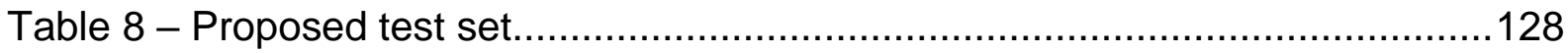

Table 9 - Inertia and draft properties of the vessel. ..........................................137

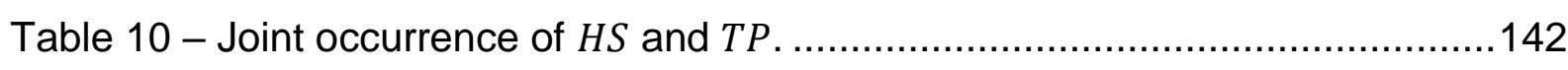

Table 11 - Clustered joint occurrence of $H S$ and TP ......................................144

Table 12 - Selected duplets: the averages of each cluster.................................145

Table 13 - Decenary and centenary waves. ……………...............................145

Table 14 - Extreme seas set of experiments. ..............................................147

Table 15 - Selected duplets for the multiple drafts set..........................................149 


\section{LIST OF SYMBOLS}

\section{In the context of Wave-Theory}

\begin{tabular}{ll}
$\varphi(x, y, z, t)$ & Scalar potential function \\
$\boldsymbol{v}(x, y, z, t)$ & Velocity vector \\
$\boldsymbol{g}$ & Gravity vector \\
$h$ & Depth \\
$\varphi_{j}$ & Radiation potential \\
$\varphi_{0}$ & Unperturbed potential \\
$\varphi_{7}$ & Scattering potential \\
$\varphi_{A}$ & Diffraction potential \\
$\xi_{j}$ & Complex amplitude of degree of freedom j \\
$\zeta$ & Free surface elevation \\
$\boldsymbol{I}_{\boldsymbol{A}}$ & Added mass matrix \\
$\boldsymbol{B}$ & Radiation damping matrix \\
$A$ & Wave amplitude \\
$\xi_{j}$ & Complex amplitude of degree of freedom j \\
$\boldsymbol{F}$ & Hydrodynamic force vector \\
$\boldsymbol{M}$ & Hydrodynamic moment vector \\
$\boldsymbol{C}$ & Restoration matrix \\
$\boldsymbol{R} \boldsymbol{A} \boldsymbol{O}$ & Response amplitude operator \\
$\beta$ & Incoming wave direction \\
$\omega$ & Wave angular frequency \\
$S(\omega)$ & Energy spectral density \\
$S(\omega, \beta)$ & Directional energy spectrum \\
$\Phi_{i_{j}}(\omega)$ & Cross spectra between degree of freedom i and $\mathrm{j}$ \\
$H_{S}$ & Significant height \\
$T_{P}$ & Peak period \\
$T_{1}$ & Mean centroid wave-period \\
$D(\omega, \beta)$ & Directional spread function \\
$\boldsymbol{S}$ & Spread factor \\
& \\
\hline
\end{tabular}

In the context of Bayesian Estimation

$\begin{array}{ll}P(A) & \text { Probability of event A } \\ P(A \mid B) & \text { Probability of A given B } \\ x & \text { State to be estimated } \\ y & \text { Measurement } \\ \mu & \text { Mean value } \\ \sigma^{2} & \text { Variance } \\ \boldsymbol{\Sigma} & \text { Covariance matrix } \\ \boldsymbol{\Gamma} & \text { Penalization matrix }\end{array}$


In the context of Bayesian Estimation of Wave Spectrum

$\boldsymbol{\Phi}_{m} \quad$ Cross-spectrum vector for angular frequency $\mathrm{m}$

$A_{m} \quad$ RAO matrix for angular frequency $\mathrm{m}$

$x_{m} \quad$ Directional spectrum vector for angular frequency $\mathrm{m}$

b Measurement vector

A RAO matrix

$\boldsymbol{x} \quad$ Directional spectrum vector

$\boldsymbol{H}_{\boldsymbol{i}} \quad$ Prior matrix i

$u_{i} \quad$ Hyperparameter $\mathrm{i}$

$J(x) \quad$ Estimation functional

In the context of Optimal Design of Experiments

$\boldsymbol{X} \quad$ Experiment matrix

a Parameter vector

$\boldsymbol{y} \quad$ Response vector

$\eta \quad$ Particular design of experiment

$U($.$) \quad Utility function$

W Weight matrix

In the context of Error Criteria Definition

$\mathrm{Err}_{S_{\omega}} \quad$ Energy spectral density error

$\operatorname{Err}_{D_{\beta}} \quad$ Directional spread function error

$\operatorname{Err}_{H_{S}} \quad$ Significant height error

$E r r_{T_{1}} \quad$ Mean centroid wave period error

$\operatorname{Err}_{T_{P}} \quad$ Peak period error, estimated using T1

$\mathrm{Err}_{S} \quad$ Spread factor error

$\operatorname{Err}_{S_{\omega \beta}} \quad$ Directional spectrum error

$\operatorname{Err}_{x} \quad$ Directional spectrum vector error 


\section{CONTENTS}

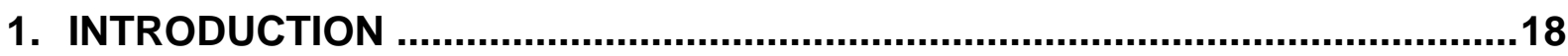

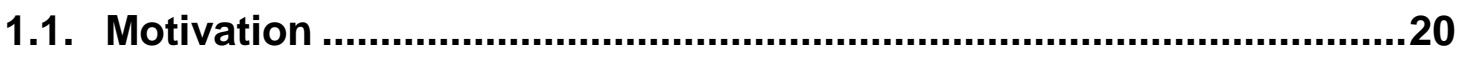

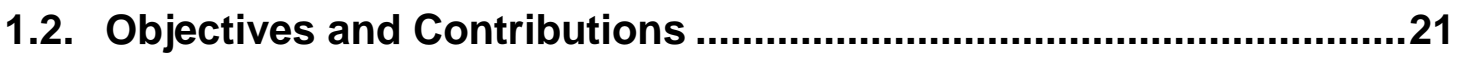

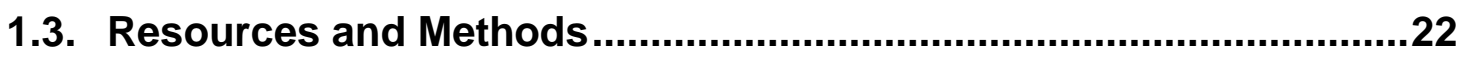

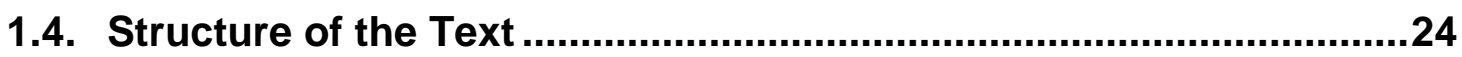

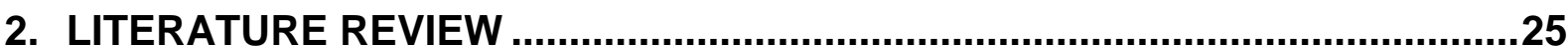

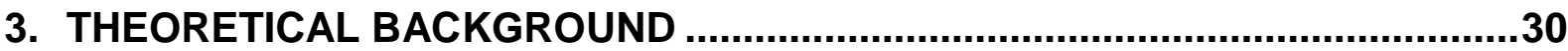

3.1. Frames of Reference and Nomenclature..........................................30

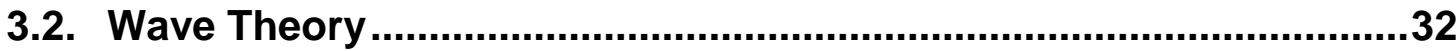

3.2.1. Linear response model ......................................................... 32

3.2.2. Irregular wave statistics and models........................................34

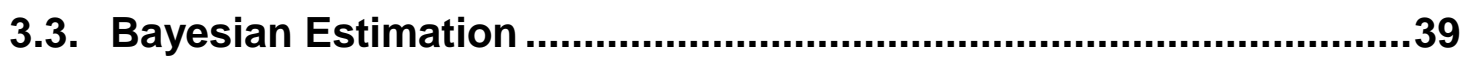

3.3.1. General Bayesian estimation ................................................39

3.3.2. Bayesian estimation applied to wave spectrum estimation........ 43

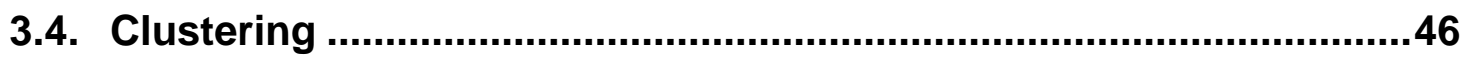

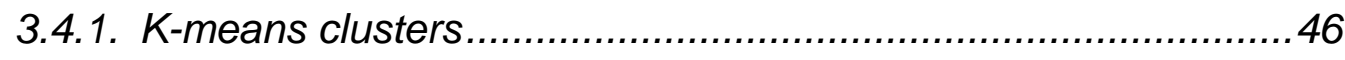

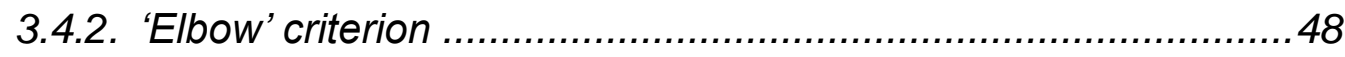

3.5. Optimal Design of Experiments ........................................................51

3.5.1. Optimal design of experiments general theory ..........................51

3.5.2. Bayesian optimal design of experiments ..................................52

3.5.3. Utility optimal design of experiments .......................................53

3.5.4. Optimal design of experiments applied to input estimation.........55

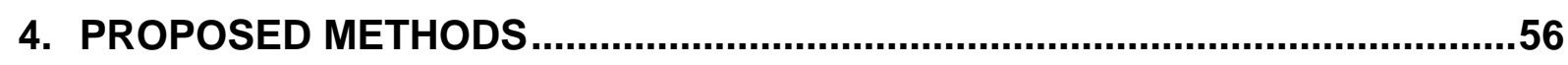

4.1. The Extended Linear Model............................................................56

4.2. Positioning and Selecting the Number of Probes .............................60

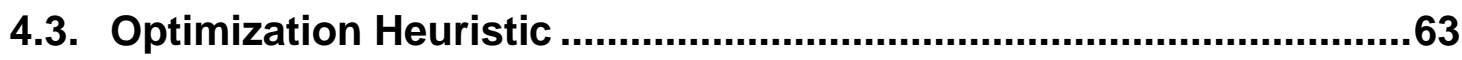


4.4. Optimization of Degrees of Freedom Usage by Frequency..............75

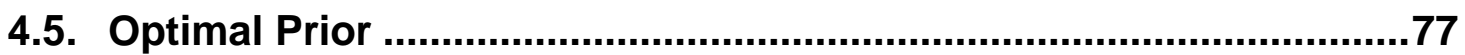

5. NUMERICAL VALIDATION.......................................................................... 82

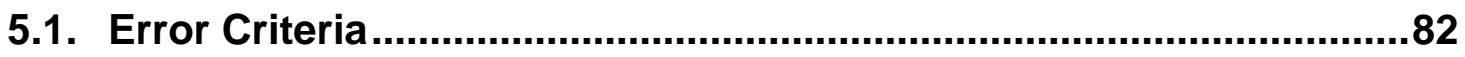

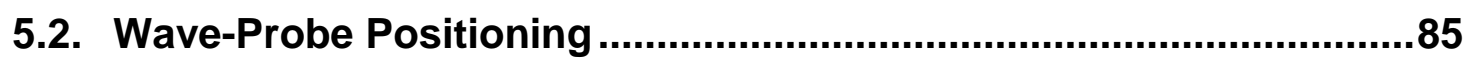

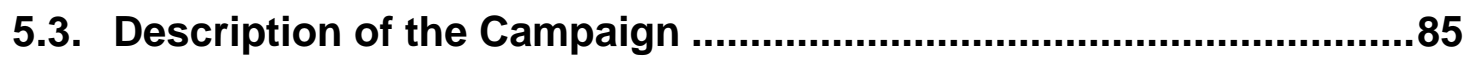

5.4. Verification of the Error Metrics.....................................................86

5.5. Validation of the Trace Criterion .....................................................95

5.5.1. Noiseless simulation ................................................. 95

5.5.2. Simulations with noise .................................................. 104

5.6. Study of the Usage of Different Number of Wave-Probes ..............114

5.7. Validation of the Heuristic ..............................................................122

5.8. Validation of the Optimal Prior.....................................................125

6. EXPERIMENTAL VALIDATION .............................................................136

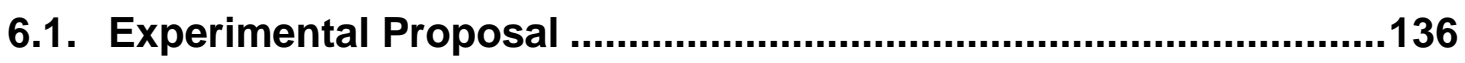

6.1.1. Experimental setup................................................ 136

6.1.2. Wave selection ............................................................. 141

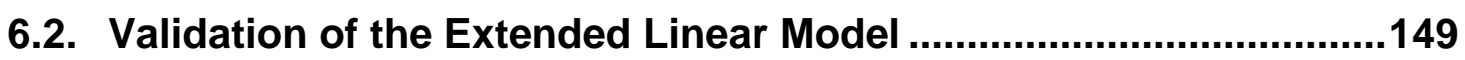

6.2.1. Validation of the extended linear model for intermediate draft. 149

6.2.2. Validation of the extended linear model for extreme draft .......159

6.3. Validation of the Usage of the Wave-Probes .................................168

6.3.1. Validation of the wave-probes usage for intermediate draft.... 168

6.3.2. Validation of the wave-probes usage for extreme draft .......... 184

6.3.3. Validation of the wave-probes usage for bimodal seas ........... 193

6.4. Validation of the Optimal Prior....................................................200

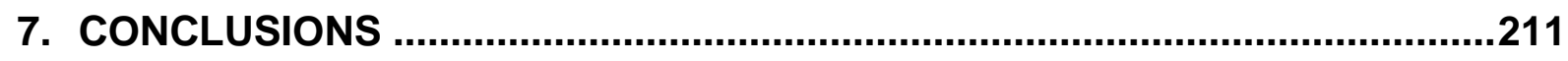


8. PROPOSALS FOR FUTURE RESEARCHES - AN UNIFIED FRAMEWORK FOR BAYESIAN ESTIMATION .213

REFERENCES

APPENDIX A - POTENTIAL THEORY OF SURFACE WAVES ….......................222

APPENDIX B - SUPERPOSITION OF VESSEL MOTIONS.................................230

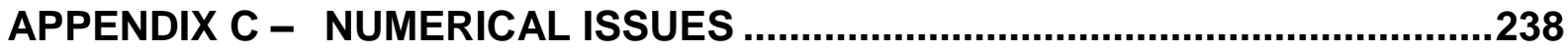




\section{INTRODUCTION}

The economical exploration of seas, mainly oil and natural gas in offshore fields, has motivated advanced studies and researches about oceanic systems and environmental forces acting on them.

In order to reduce operating costs, those researches are conducted aiming two main goals. The first one is the improvement of the systems design, acquiring statistical bases for scenario modelling, validating mathematical theories and validating experimental model tests in towing tanks. The second one is the amplification of safe operating windows, using real-time monitoring systems capable of predicting dynamic behaviors. The correct modelling of environmental forces plays a major role in both.

Oceanic systems - mostly moored offshore floating facilities - experiences forces from wind, current and waves, and a number of models were proposed to describe them properly, with different levels of complexity. The in loco measurement of those environmental actors, however, has always been a challenge.

The waves, in particular, have been measured using meteorological buoys, satellite imagery and radar reconstruction, but all of those solutions have problems, respectively: difficult maintenance and high rate of damage after extreme conditions; poor resolution around interesting regions; and high sensibility to installation errors with high maintenance and acquisition costs.

The meteorological buoys are floating bodies from which wave induced movements are taken, and are the most common method of wave monitoring. With the recordings of the movements, it is possible to recover the properties of the wave that excited the body and estimate the directional sea spectrum by the methods described in (NOAA, 1996). The inherent drawbacks of this method are: firstly, it is, usually, a moored system, which means that the depth of the region is a constraint, unless drifting buoys are used; secondly, this kind of system has a high rate of failures during stormy weather and extreme external load conditions, as the study of (SHIGEMURA, et al., 1988) shows, for a buoy system in Japan.

The satellite imagery method, on its turn, was conceived as a climate monitoring system, returning global statistics of the oceans in areas of thousands of kilometers squared. This kind of system may be useful to predict the main sea components acting 
on a floating facility inside its monitoring area, since this component varies slowly within the large region, but probably will not be able to provide accurate information about secondary components that are also important for the facility dynamic response.

Lastly, the radar method utilizes an on-board system to monitor the wave elevation pattern around the offshore installation. This approach presents clear advantages over the two previous methods; notwithstanding, the installation and maintenance of the antenna is a serious problem and can potentially degrade critically the measurements. Even though the method is able to reconstruct entirely the directional spread of the sea, other known problem with radars is their poor energy prediction capability, since it depends on the correct measurement of the waves amplitude, which is not done easily due to improper scaling, (TRAENKMANN, 2008).

Figure 1 illustrates those three methods.

Figure 1 - Meteorological buoy (a), radar (b) and satellite imagery (c).

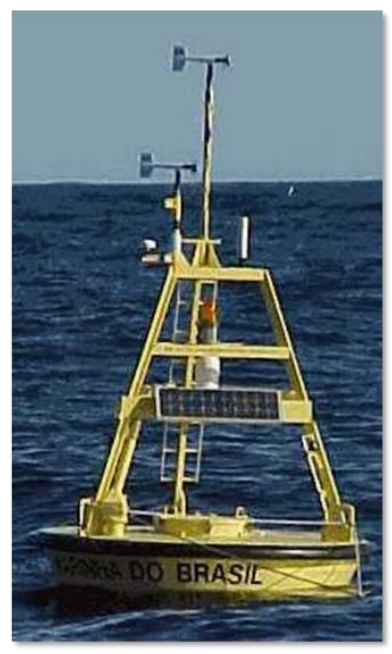

(a)

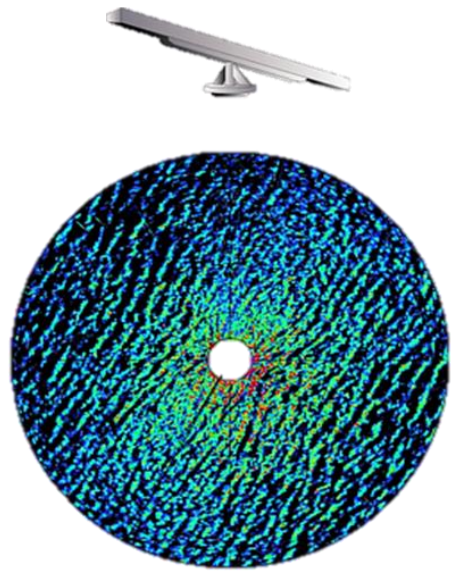

(b)
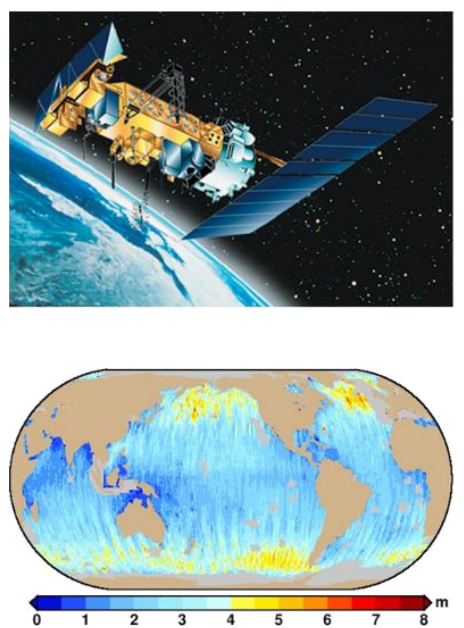

(c)

Source: (FURG, 2008) (a), (TRAENKMANN, 2008) (b), (NOAA, 2016) (c).

Recent works in the wave estimation area, however, were able to solve the problems presented using the oceanic system itself as a measurement buoy, which is called wave-buoy analogy. In this way, motion sensors already installed on the facilities, i.e., inertial units, can be used, reducing installation and maintenance costs; guaranteeing the maximum resolution in the area of interest - the vessel itself; and avoiding damage while monitoring extreme events.

Despite the improvements, the dynamical behavior of Very Large Crude Carriers, VLCCs, one of the most used vessels for floating offshore facilities, presents 
a low cut-off frequency, around eight seconds, making them unable to predict an important range of the wave spectrum, responsible for effects like drift forces. Therefore, the methods are still not satisfactory for this important kind of vessel.

This thesis proposes a possible solution to this drawback, using wave-probes gauges used to measure the wave elevation in a point - installed on the hull of the oceanic system as a complementary measuring equipment.

The approach is justified by the asymptotical behavior of waves encountering a free floating system after the cut-off frequency, in which the system starts to behave like a wall, reflecting the wave encountering it, i.e., amplifying the wave-elevation measurement. Hence, the proposal is able to incorporate high frequencies in the spectra estimation without incurring the problems presented so far.

\subsection{Motivation}

As already stated, the proposed solution improves the motion-based estimation method, allowing a better estimation of the high-frequency components of the wave spectrum, and, consequently, of the drift forces acting on the vessel.

The correct prediction of the drift forces is important for the mooring design of anchored systems, since they can induce both a constant force acting on the system, mean drift load, and a low-frequency harmonic component capable of inducing resonance, slow drift load. Furthermore, a real-time prediction of those forces can anticipate movements during high-risk operations.

Other systems that can benefit from better estimations are dynamic positioning systems (DP), which use global positioning systems (GPS) and thrusters commanded by a control law to guarantee a fixed vessel position; mainly in deep seas in which conventional mooring is not possible. It is known that a correct drift force prediction, used as a feed-forward compensator, is able to improve the DP control response, reducing the demanded power and increasing the safe operating window.

Finally, it is important to emphasize that the proposal is feasible, from an operational point of view, because today there are microwave gauges capable of performing the measurement from distance, i.e., without being physically on the hull and in contact with the water surface, (RS AQUA, 2016) for example, Figure 2. This is 
important in order to avoid disturb usual platform operations as off-loadings, which sometimes demands hull contact with other vessels, like tugboats.

Figure 2 - Example of microwave wave-probe.

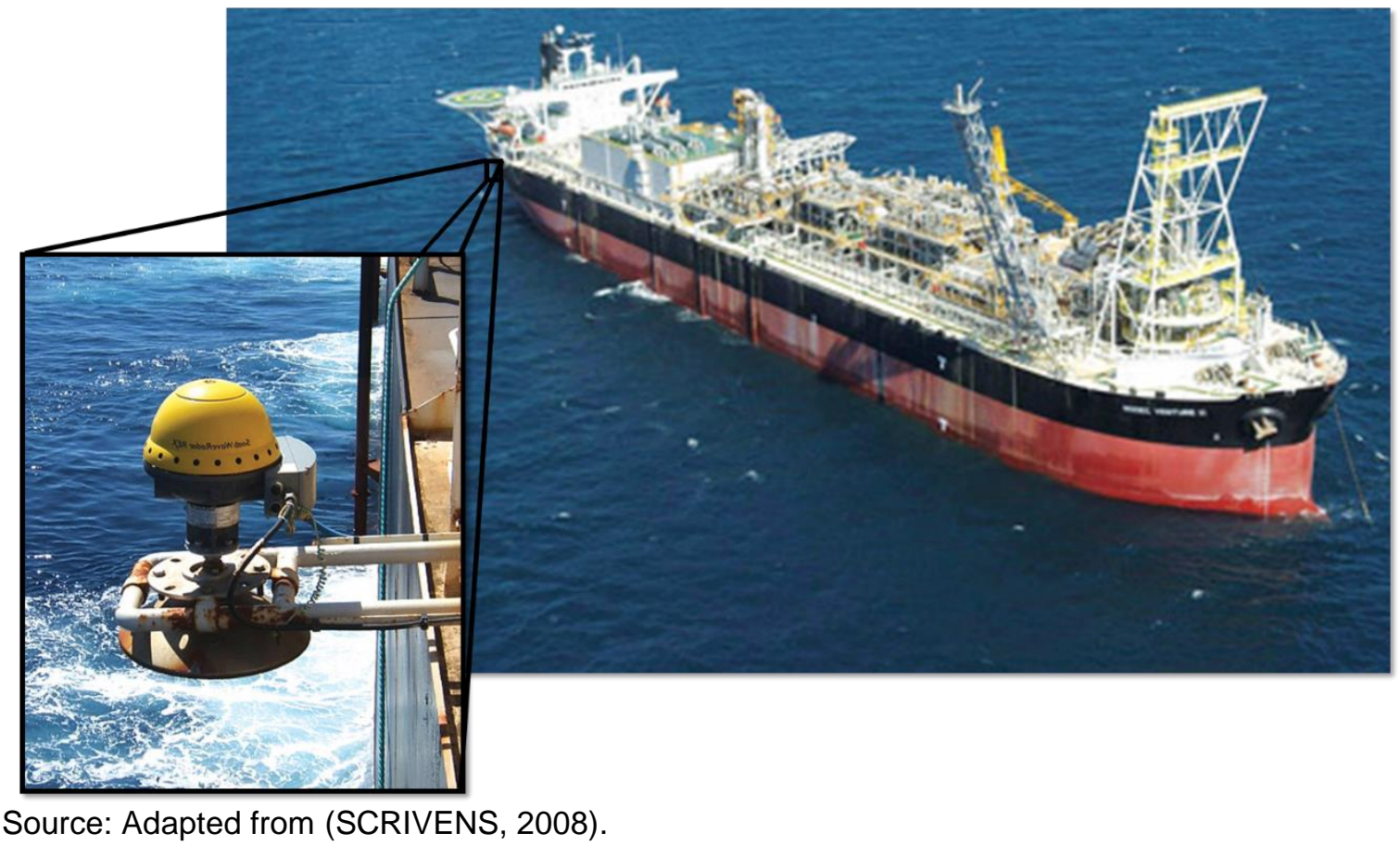

\subsection{Objectives and Contributions}

The main objective of this thesis is the study of the incorporation of wave-probe measurements in the motion-based wave estimation method. To accomplish this, the work:

1. Proposes a method to expand the wave-buoy analogy to incorporate waveprobes, using an estimation algorithm based on Bayesian statistics;

2. Proposes a method to select the best number and position of the waveprobes, which can also be applied to select the best vessel motions to be used in the estimation;

3. Validates all the propositions numerically and experimentally.

During the research, other possible improvements were identified and incorporated as new contributions:

1. Proposition of a new multi-objective optimization heuristic for optimal sensor placement, optimized for the particular problem;

2. Proposition of an optimal prior for a given set of sea states. 


\subsection{Resources and Methods}

This study was financed in part by the Coordenação de Aperfeiçoamento de Pessoal de Nível Superior - Brasil (CAPES) - Finance Code 001. The research grant conceded by CAPES was indispensable for the development of this work.

The other resources for this research were provided by the Tanque de Provas Numérico da Universidade de São Paulo (TPN-USP) - Numerical Offshore Tank of the University of São Paulo, in a partnership with the Brazilian petroleum company Petrobras and the Norwegian petroleum company Equinor, previously called Statoil, and was financed inside the context of the project "Wave Measurements Aboard FPSO Unit in Peregrino Field", coordinated by Prof. Alexandre Simos and by Prof. Eduardo Tannuri. The facilities are described by (TPN, 2016).

The vessel dynamic behavior and the wave-elevation on the hull were obtained using the wave interaction analysis software Wamit ${ }^{\circledR}$, running in the computational cluster structure provided by the laboratory. It uses the three-dimensional panel method to solve equations based on the potential theory. The equations and the theory underlining the computations are described by (WAMIT, 2015).

The numerical simulations, development of optimization strategies and postprocessing of the experiments were conducted using the scientific computing platform Matlab $^{\circledR}$, which contains toolbox for statistical analysis and numerical optimization essentials for this work. This simplified the development of the algorithms.

The experimental part was conducted in the TPN wave basin. The basin is capable of generating waves from its entire perimeter, and uses active wave absorption. The vessel movements in all degrees of freedom were measured by a high precision camera system monitoring reflective dummies on the hull. The basin is shown in Figure 3.

The vessel model was provided by the Instituto de Pesquisas Tecnológicas (IPT) - Technological Research Institute. It is a Floating Production Storage and Offloading (FPSO) vessel unit based on a moored Very Large Crude Carrier (VLCC), with a scale of 1:90. Figure 4 shows the unit. 
Lastly, the validation methods were based on different error criteria applied on the estimation of JONSWAP type spectrum with cosine-squared spread function, and are better explained in the sections Numerical Validation and Experimental Validation.

Figure 3 - Wave basin at TPN-USP.

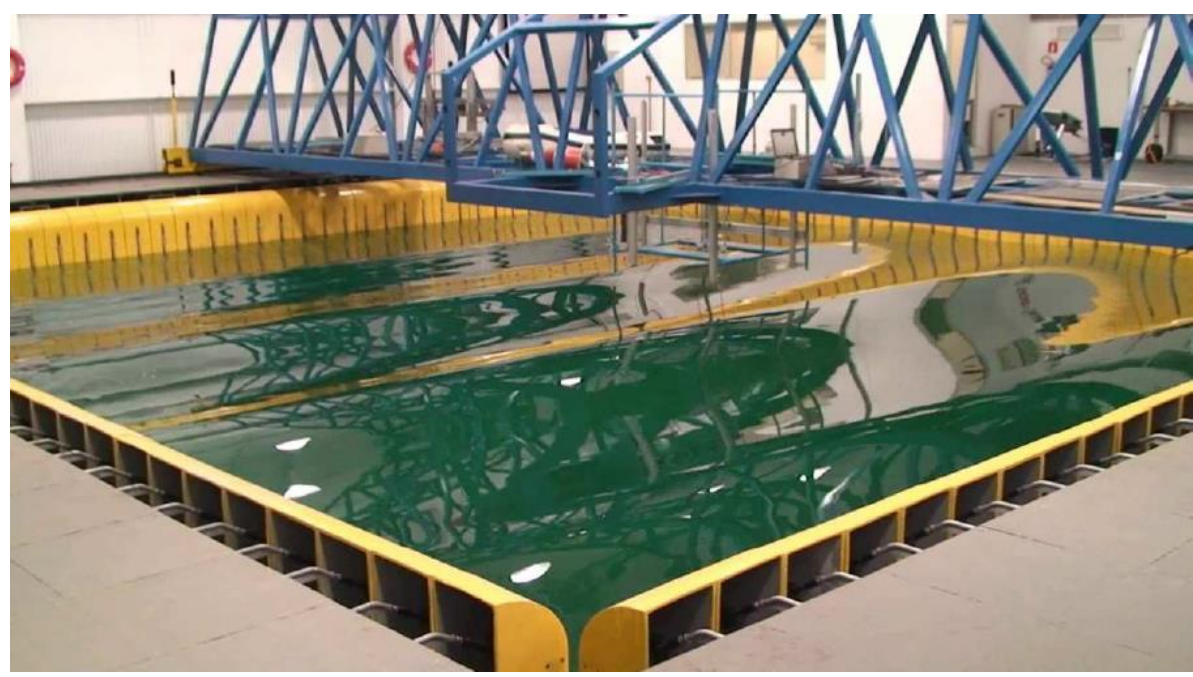

Source: (MELLO, 2012).

Figure 4 - Vessel model provided by IPT.

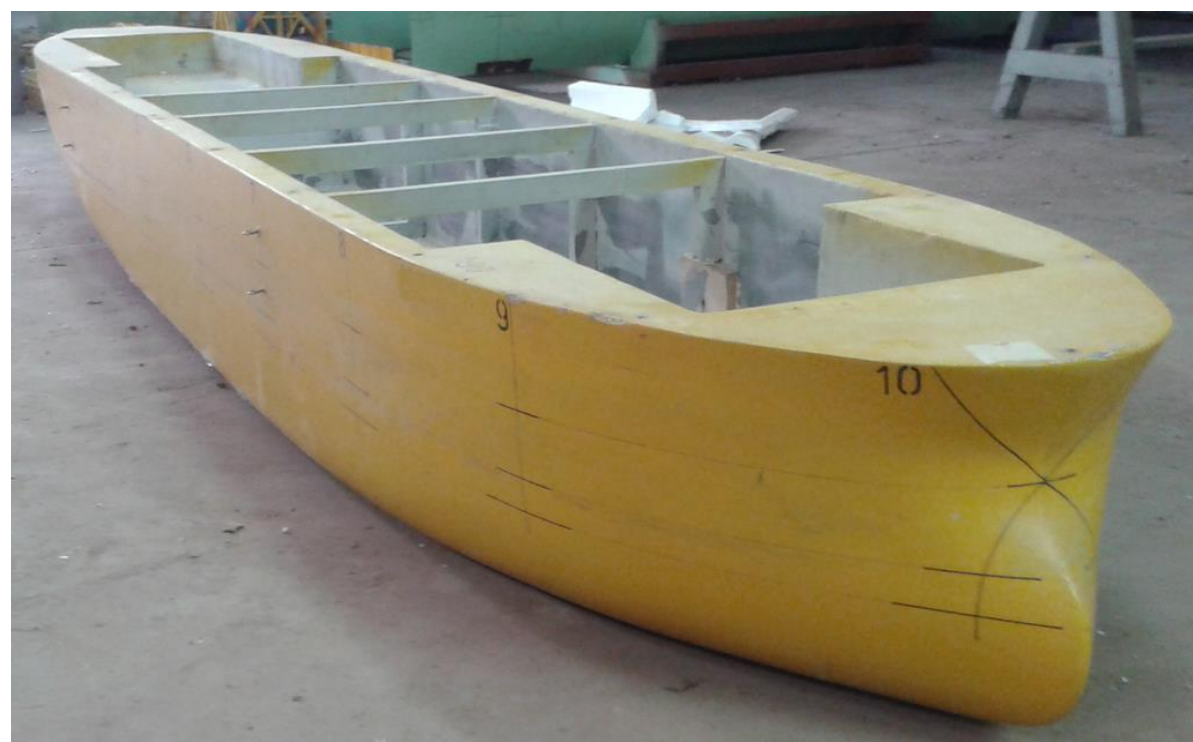

Source: Photographed by Pedro Mello. 


\subsection{Structure of the Text}

The next chapters start with a revision of the literature in the area, in the section Literature Review, providing information about the historical development and the current research and methods to estimate directional sea wave spectra. References about wave-probes application and second order wave effects as feed-forward compensators are also listed, to emphasize their importance.

The review is followed by the section Theoretical Background, which provides all the theory used in this work. It describes Wave Theory, and the statistical methods Bayesian Estimation, Clustering and Optimal Design of Experiments.

After explaining the base theory, the work follows to the section Proposed Methods, which contains the contributions of the text to the area, being the kernel of the thesis.

The methods proposed are then validated numerically and experimentally in the sections Numerical Validation and Experimental Validation. All the validating procedures are explained and justified, with benchmark comparisons in some cases.

Complementary information and deductions are presented in appendixes, guaranteeing a full treatment of the problem without compromising the flow of the work.

Finally, the thesis ends in the sections Conclusions and Proposals for Future Researches - An Unified Framework for Bayesian Estimation, summarizing the results of the thesis and providing insights for future researches in the area. 


\section{LITERATURE REVIEW}

Researches about experimental wave measurement have been conducted since the end of the second world war. The first attempts to measure waves in seas can be traced back to the works of Longuet-Higgins and his development of measurement buoys in 1946 (LONGUET-HIGGINS, 1946) apud (DARBYSHIRE, 1961). Even the use of on-board measurement systems had already been described in works from Tucker in 1956, (TUCKER, 1956) apud (DARBYSHIRE, 1961), in which ship-borne pressure transducer were employed.

Although those early works were only concerned about the overall spectral energy in the sea, the directional spectrum estimation was put into perspective around 1960, by works from (COTÉ, et al., 1960) apud (MITSUYASU \& MIZUNO, 1976); and (LONGUET-HIGGINS, et al., 1963) apud (MITSUYASU \& MIZUNO, 1976), who proposed the cosine-squared parametric form of the directional spread function, which is used until today.

A parametric description of the spectrum of a fully developed sea was proposed a few years later by Pierson and Moskowitz, based on statistical theory, in its classical paper from 1964, (PIERSON \& MOSKOWITZ, 1964); and in 1973, the JONSWAP parametric model was developed to model situations in which the wave spectrum had not yet reached the fully developed condition, (HASSELMAN, et al., 1973).

Even though different estimations methods were used, mainly the Discrete Time Fourier Transform method and the parametric method, after Longuet-Higgins, the first proposal of a high-resolution method, capable of predicting the entire directional spread function and not only the principal direction, was given by Capon in 1969, who used the maximum-likelihood method to estimate seismic waves, (CAPON, 1969).

The usage of vessel movements to improve the estimation capabilities is also found in pre-1980 works. Takekuma and Takahashi, in 1972, proposed the use of pitch movement measurements as an alternative to the ship-borne wave recorders, calling the new method "Reverse Operational Method" of sea spectrum estimation, although without considering the directional spectrum, (TAKEKUMA \& TAKAHASHI, 1972).

Pos-1980, estimation of the full directional spectrum became the standard practice. In the context of on-board measurements, (WEBSTER \& DILLINGHAM, 
1981) showed theoretically how it is possible to use a system of on-board sensors to estimate the directional spectra, as long as "the response amplitude operators are linear and have some directional dependence". In the context of estimation algorithms, mostly for an array of wave-probes mounted in vessels with low interference in the domain field, e.g., catamarans and swat ships, the Maximum Likelihood Method (MLM) was applied to the problem of directional sea spectrum estimation by (ISOBE, et al., 1984); the usage of the Maximum Entropy Principle was proposed by (KOBUNE \& HASHIMOTO, 1986); and the usage of Bayesian Estimation was proposed by (HASHIMOTO \& KOBUNE, 1988), which was said to be smoother than the MLM, mainly when there are more variables to estimate than equations relating it, and based on a general method proposed by Akaike some years earlier, (AKAIKE, 1980).

In 1987, the MLM was applied to the directional spectrum estimation by using only vessel movements in the work of Hirayama, (HIRAYAMA, 1987) apud (ISEKI \& OHTSU, 1999), which was expanded later by Iseki and co-authors in 1992, (ISEKI, et al., 1992). The Bayesian Estimation for this particular problem was introduced by the authors few years later, in the work (ISEKI \& OHTSU, 1999). It is important to notice that, up to this moment, a great effort was being made to solve the so called triplevalued function, which corrects the Doppler Effect that changes the frequency of a wave encountering a vessel with non-negligible forward speed.

In 2003, in the context of dynamic positioned vessels, (TANNURI, et al., 2003) set aside the triple-valued problem, as DP vessels are supposed to be stationary, and compared the Bayesian estimation against a more complex parametric estimation based on a bimodal spectrum proposed by (HOGBEN \& COBB, 1986), which is a superposition of a generalized JONSWAP energy distribution with the cosine-squared spread function; giving a slightly preference to parametric models after numerical and experimental tests. In the work, the authors also proposed a change in the motion base used to estimate the sea, using the sway movement instead of the roll movement, usually adopted due to the wave-buoy analogy, arguing that the roll motion is affected by viscous non-linear effects that would probably degrade its response calibration, while the sway motion has the same asymmetric behavior concerning the port and starboard incoming wave directions, which is indispensable for a good direction estimation. 
Nielsen started his investigation into the subject in 2005, in his $\mathrm{PhD}$ thesis (NIELSEN, 2005). In the work, he emphasized the limitation of motion-based methods because of the natural filtering properties of the dynamic system, and compared the different estimation methods, although not giving preference to any. In the same year, (PASCOAL, et al., 2005) put into perspective the non-linearity and non-convexity of the parametric estimation optimization problem, suggesting the use of global search procedures as the genetic algorithm to find the best solution. The problem was also discussed by (NIELSEN, 2006), who showed that improper first guesses for gradientbased algorithms could potentially reach extremely bad solutions in the parametric estimation. One possible solution was given by (PASCOAL \& SOARES, 2006), who suggested a mixed approach to solve the problem, i.e., starting with non-parametric estimation and use it as the first guess for a parametric fitting.

In 2007, a short full-scale experimental campaign was described by Simos, following the previous work of his research group with Tannuri, (SIMOS, et al., 2007). In the work, the motion-based estimation using an FPSO was compared against a wave-buoy measurement close to the location of the vessel. In this case, however, the data suggested that the Bayesian Estimation was more robust than the parametric model, demanding, at the same time, much less computational power.

In the following years, other comparisons and improvements in the Bayesian method were made, for example: (NIELSEN, 2007), (NIELSEN, 2008a), (SIMOS, et al., 2010) (NIELSEN, 2011), (ISEKI \& HIRAYAMA, 2012), (TANNURI, et al., 2012), (BISPO, et al., 2012), (NIELSEN, et al., 2013) and (ISEKI \& NIELSEN, 2015). In general, the improvements proposed in those papers are all included in the estimation presented in this thesis, and the comparisons are usually favorable to the Bayesian estimation against the parametric model, although Nielsen works do not suggest a clear preference. The results were also compared against estimations from wavebuoys, meteorological satellites and radar imagery, with good consistence.

Other investigation issues deserve more attention. For example, (NIELSEN, 2008b) suggests, in the context of parametric estimation, to incorporate measurements of the relative motion between the vessel and the water surface for improve the highfrequency estimation, which provides evidence for the use of wave-probes in a more broad sense. (STREDULINSKY \& THORNHILL, 2011) also propose a sensor fusion 
approach, but suggesting the motion-base estimation as a possible correction to radar measurements, since radars are known to have good direction and spread estimation but poor energy estimation capabilities.

The works from Lajic in 2010 also deserve attention, (LAJIC, 2010) and (LAJIC, et al., 2010). They suggest a new method for selecting the best estimation base called "Sensor Fusion Quality Test", using the online measurement of the cross residual estimation - the difference between the estimation of the significant sea height from two different degrees of freedom - to find the combination of vessel movements which has the best agreement among the measurements it contains. It is interesting to emphasize the effort from Lajic to find an automatic method to select the best base of movements to be used, based on the fault detection theory, although the drawback that the method only provides answer for the ongoing sea state, needing an extensive campaign to provide information for all the possible states. A similar problem is discussed in this thesis.

Finally, there are more recent works in the subject, but they do not provide more information for the exact problem discussed here, for example: (MOGSTER, 2015), which discusses the non-stationary problem; (SINKE, 2015), which discusses the motion-based and radar fusion; (NIELSEN, et al., 2015), which describes an interesting new technique using a mixed model-based and signal-based approach to enhance the waves prediction; and (MONTAZERI, et al., 2016a) and (MONTAREZI, et al., 2016b), which improve some aspects of the parametric estimation.

From the perspective of the DP systems, there are also papers that discussed wave-estimation, but they usually adopt methods that estimate the drift-forces in a more straightforward manner, which is shown in the next paragraphs.

Most of the literature indicates Pinkster as the pioneer in the usage of wave estimation in feedforward compensators, (PINKSTER, 1978). However, the effects of the drift-forces on the vessels, and its consequences as regarding DP systems, were described in earlier works: (REMERY \& HERMANS, 1971) apud (PINKSTER, 1978), (PINKSTER, 1976) apud (PINKSTER, 1978), (VAN OORTMERSSEN, 1976) apud (PINKSTER, 1978) and (FALTISEN \& LOKEN, 1978) apud (PINKSTER, 1978). 
In his work, Pinkster suggested a method to estimate the slow drift force by the direct integration of the relative motion measured in the hull of the vessel. He justified the feasibility of the method arguing that only a finite number of weighted sensors need to be used, as the water height varies slowly along the water-line. Despite that, a significant number of eight wave-probes were used, and only some forces could be estimated.

A more recent work from Aalbers, in which Pinkster is co-author, (AALBERS, et al., 2001), proposed a second method, using two wave-probes located symmetrically at the port and starboard, which are used to provide information about the direction of the incoming wave, and one wave-probe located at the bow of the ship, to acquire energy information. The second method is interesting for this work because it uses the drift force transfer functions to estimate the forces, justifying the directional spectrum approach. Despite that, Aalbers concluded that the second method is generally worse than the Pinkster method and only works for head seas, although he recognizes the need of further developments.

Lastly, a modern treatment of the wave feedforward usage in DP systems can be found in (HUGHES, et al., 2009) and (HUGHES, et al., 2010). Initially, they explain the reason why first order forces cannot be counteracted by the traditional DP systems, since they are forces that change direction faster than the usual propellers are able to; despite this, they result in zero-mean movements. Second order forces, however, which are described mostly by high-order terms in the Bernoulli's equation, are nonzero mean, which eventually results in a non-negligible movement, with a low frequency component that can easily be close to the DP system resonance; consequently, they must be taken in account, at least in the design phase. The works also use the Pinkster approach, with twenty wave-probes around the hull, showing how the method is still the standard practice in this subject.

The proposals of this works can be seen as an expansion of the idea of using relative motions, discussed originally by (NIELSEN, 2008b), to high-order nonparametric procedures for full directional spectra. They can also be seen as a combination between Bayesian estimation and the Pinkster method, aiming at improving both: increasing the estimation range of the Bayesian algorithm and demanding, at the same time, less wave-probes than the Pinkster method. 


\section{THEORETICAL BACKGROUND}

In this section, the theoretical concepts used in this work are presented.

\subsection{Frames of Reference and Nomenclature}

First, it is interesting to present the naval nomenclature used for vessels and waves encountering it, Figure 5 . Waves coming from $0^{\circ}$, for example, encounter the stern of the vessel first and the sea is called following sea.

Figure 5 - Naval nomenclature for vessels and seas.

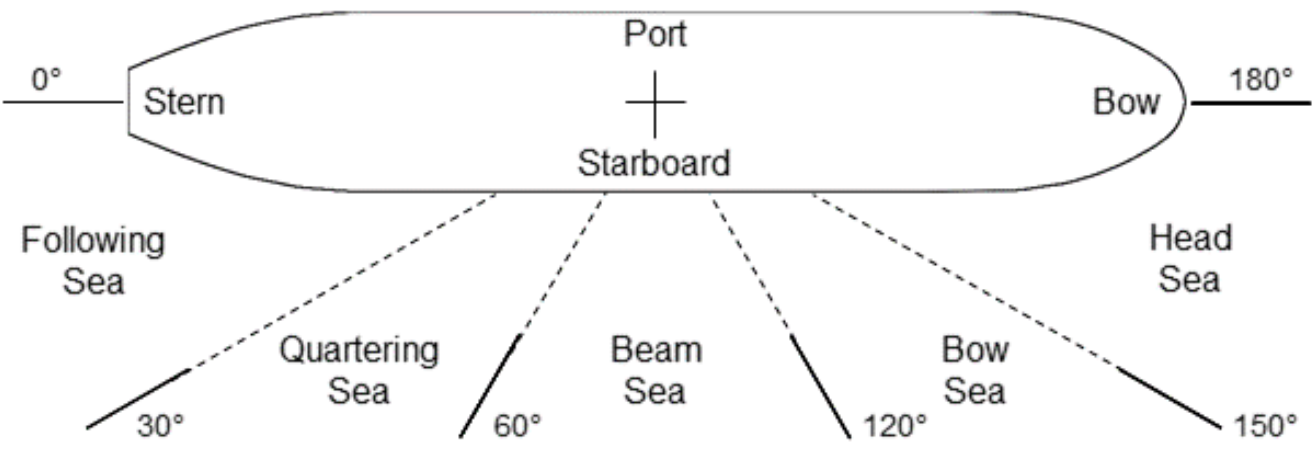

Source: Elaborated by the author, inspired by a similar figure from (NIELSEN, 2005).

The local coordinates are chosen to be equal to the unperturbed body system, also following the standard nomenclature, Figure 6 . Even though the direction of each coordinate can vary among the literature, the adopted convention is the same as the Wamit ${ }^{\circledR}$ software, mentioned in the section 1.3 Resources and Methods, simplifying the algorithms, Figure 7.

Figure 6 - Body standard system of reference.

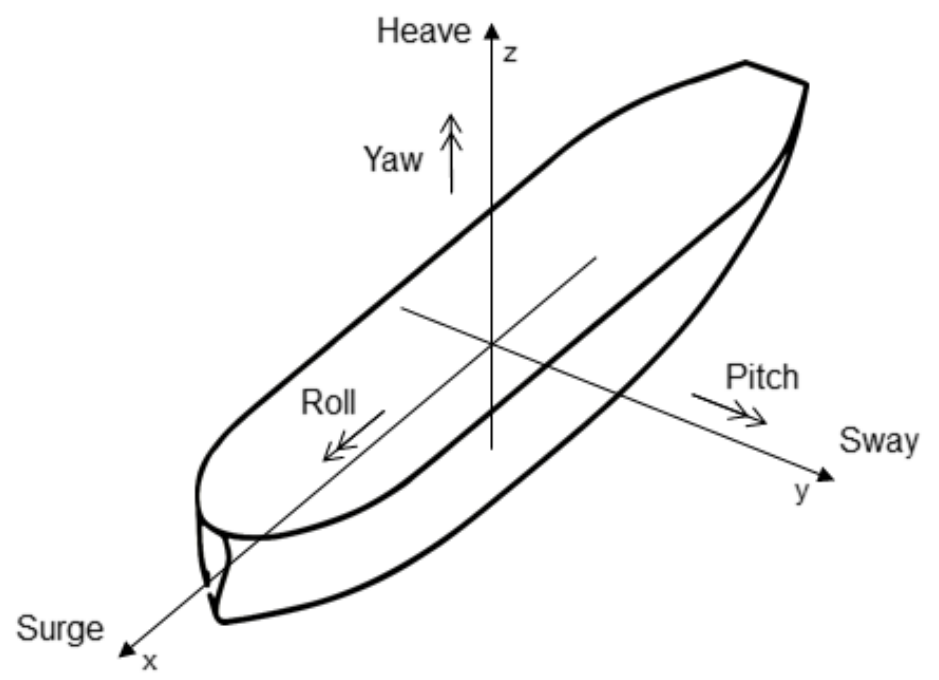

Source: Elaborated by the author. 
Figure $7-$ Wamit $^{\circledR}$ reference system.

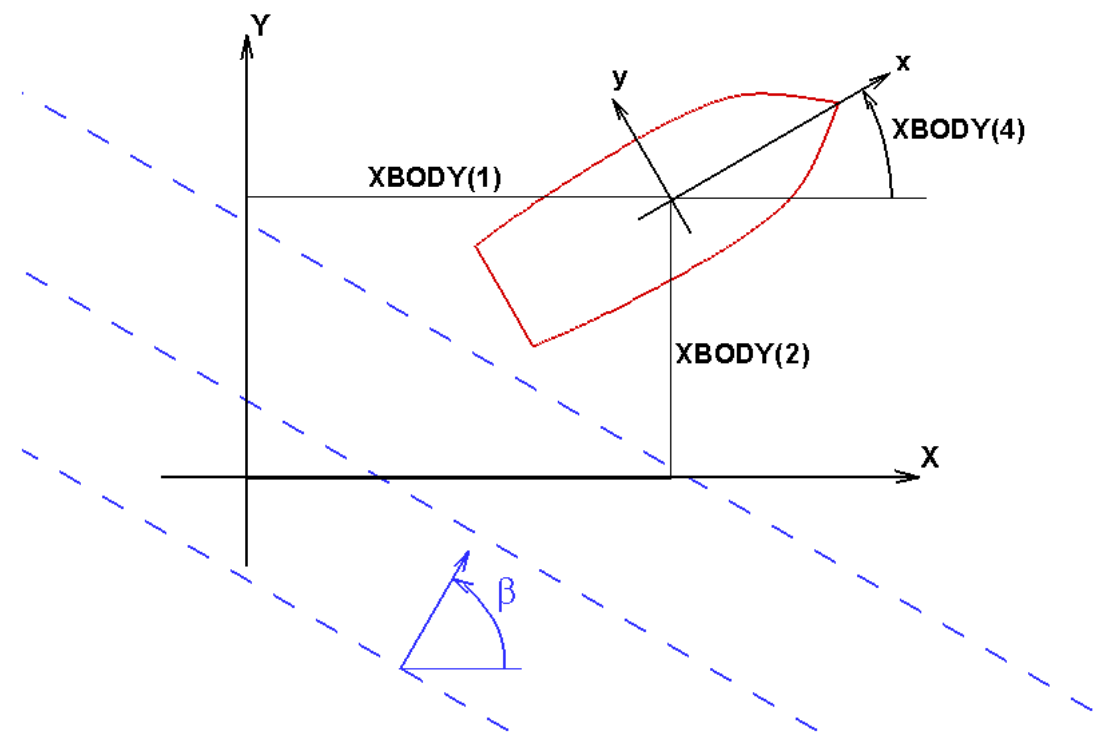

Source: (WAMIT, 2015).

Another important coordinate system is the one used in the directional spectrum polar plot, Figure 8 . The concentric lines indicate the wave angular frequency, and the colors indicate the energy density of that wave component.

Figure 8 - Directional spectrum polar plot reference.

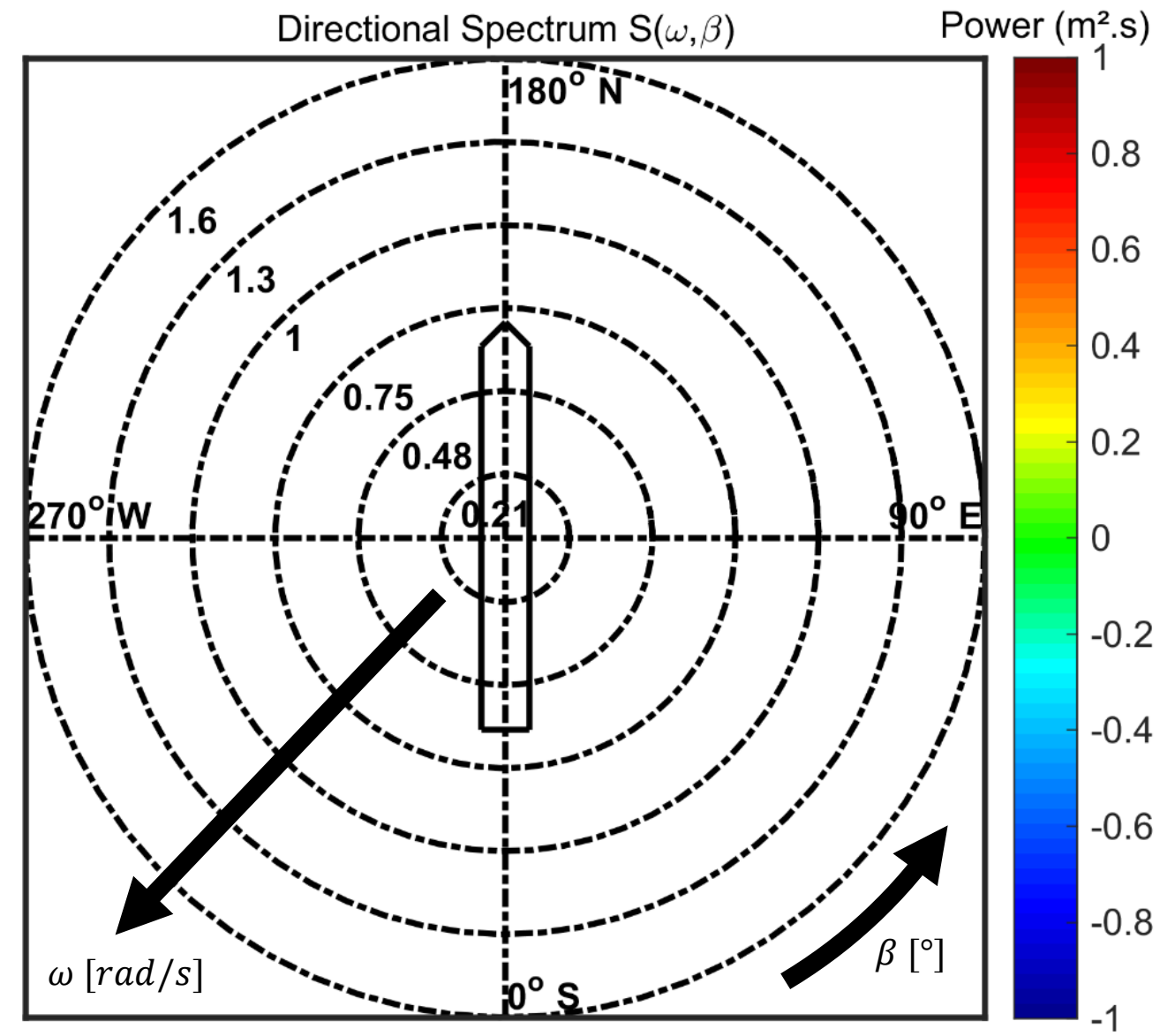

Source: Elaborated by the author. 


\subsection{Wave Theory}

The potential theory of gravity waves and the linear response of a free floating body are the bases of the dynamic system used in the estimation algorithm; a classical formulation, presented in fundamental hydrodynamic text books as (NEWMAN, 1999). This section describes only the consequences of the theory in the dynamics of floating bodies, but the unfamiliar reader may benefit from checking APPENDIX A - Potential Theory of Surface Waves for a theoretical introduction into the subject.

\subsubsection{Linear response model}

Under the ideal fluid hypothesis, the behavior of the water, including the behavior of waves propagating on its surface and the behavior of floating objects with small movements inside the domain, is described by a scalar potential function $\varphi(x, y, z, t)$, which determines the velocity vector $v(x, y, z, t)$ and the pressure $p(x, y, z, t)$ of the fluid, Equations (1) and (2), in which $\rho$ is the specific mass of the fluid, $\nabla$ the differential operator $\left[\frac{\partial}{\partial x}, \frac{\partial}{\partial y}, \frac{\partial}{\partial z}\right]$ and $\boldsymbol{g}$ the gravity vector acceleration, $\boldsymbol{g}=$ $[0,0,-g]=-\nabla g z$

$$
\begin{gathered}
\boldsymbol{v}(x, y, z, t)=\nabla \varphi(x, y, z, t) \\
\nabla\left(\frac{\partial \varphi(x, y, z, t)}{\partial t}+\frac{1}{2} \nabla \varphi(x, y, z, t) \cdot \nabla \varphi(x, y, z, t)+\frac{p(x, y, z, t)}{\rho}+g z\right)=\mathbf{0}
\end{gathered}
$$

The potential function is determined based on a second-order partial differential equation, the Laplace's equation, Equation (3), in which $\Delta$ is the Laplace's operator $\left[\frac{\partial^{2}}{\partial x^{2}}, \frac{\partial^{2}}{\partial y^{2}}, \frac{\partial^{2}}{\partial z^{2}}\right]$; and on boundary equations stating the constraints of the domain, the impermeability of the sea bottom, for example, Equation (4), in which the depth is $h$.

$$
\begin{gathered}
\Delta \varphi=0 \\
v_{z}(z=-h)=\left.0 \rightarrow \frac{\partial \varphi}{\partial z}\right|_{z=-h}=0
\end{gathered}
$$

When a moving floating body is inside the domain, subject to a regular wave plane progressive harmonic wave with a specified amplitude, frequency and direction 
- the resultant potential is described as a superposition of potentials, accounting effects from:

- Radiation: the potential caused by a unitary movement of the body in each degree of freedom, $\varphi_{j}$;

- Diffraction: the unperturbed potential of the incident waves plus the scattering disturbance caused by the fixed body, $\varphi_{A}=\varphi_{0}+\varphi_{7}$.

Assuming an oscillatory body movement, with complex amplitude $\xi_{j}$ in each degree of freedom (DoF), the final potential will be the one presented in Equation (5), with $A$ the amplitude of the wave and $\omega$ its angular frequency.

$$
\varphi(x, y, z, t)=\operatorname{Re}\left\{\left[\sum_{j=1}^{6} \xi_{j} \varphi_{j}(x, y, z)+A\left(\varphi_{0}(x, y, z)+\varphi_{7}(x, y, z)\right)\right] e^{i \omega t}\right\}
$$

This potential, along with the necessary boundary equation, can be solved by the panel method, which is used in the software Wamit ${ }^{\circledR}$. Essentially, the body surface is discretized in panels and a superposition of potentials is defined based on the discretization - usually, some simple potentials are positioned on the panels, with their strength being calibrated to satisfy all the boundary conditions. A complete theoretical reference can be found in (WAMIT, 2015).

After solving the potential problem, the pressure can be found and integrated over the floating body in order to find the overall forces. The calculated forces can be written in the matrix form, being divided in: added mass $I_{A}(\omega)$ - a term proportional to the acceleration of the body, $\dot{U}_{j}$; radiation damping $\boldsymbol{B}(\omega)$ - a dissipative term proportional to the velocity of the body, $U_{j}$; and wave-exciting forces $\operatorname{Re}\left\{A e^{i \omega t} \boldsymbol{X}(\omega)\right\}-$ a term proportional to the amplitude of the incident wave; Equations (6):

$$
\left[\begin{array}{l}
\boldsymbol{F} \\
\boldsymbol{M}
\end{array}\right]_{\text {hydrodynamic }}=\boldsymbol{I}_{\boldsymbol{A}}(\omega) \dot{U}_{j}+\boldsymbol{B}(\omega) U_{j}+\operatorname{Re}\left\{A e^{i \omega t} \boldsymbol{X}(\omega)\right\}
$$

If the body oscillates around its hydrostatic equilibrium with small movements, the hydrostatic forces can be linearized in order to be described by a simple restoration matrix $\boldsymbol{C}$, Equation (7). 


$$
\left[\begin{array}{l}
\boldsymbol{F} \\
\boldsymbol{M}
\end{array}\right]_{\text {hydrostatic }}=\operatorname{Re}\{\boldsymbol{C} \xi\}, \quad \xi=\left\{\xi_{j} e^{i \omega t}\right\}_{j=1,2, \ldots, 6}
$$

The final dynamic system, applying all the forces on the inertia $I$ of the body, is then defined by Equation (8):

$$
\left\{-\omega^{2}\left(\boldsymbol{I}+\boldsymbol{I}_{\boldsymbol{A}}(\omega)\right)+i \omega \boldsymbol{B}(\omega)+\boldsymbol{C}\right\} \boldsymbol{\xi}=\boldsymbol{A} \boldsymbol{X}(\omega)
$$

Since the system is linear, it can be described by a transfer function of each possible wave - function of the angular frequency $\omega$ and the incidence angle $\beta$. This function can be discretized in a vector called Response Amplitude Operators (RAOs), which completely determine the floating body dynamics, Equation (9).

$$
\boldsymbol{R A O}(\omega, \beta)=\frac{\xi(\omega, \beta)}{A}=\left\{-\omega^{2}\left(\boldsymbol{I}+\boldsymbol{I}_{\boldsymbol{A}}(\omega)\right)+i \omega \boldsymbol{B}(\omega)+\boldsymbol{C}\right\}^{-1} \boldsymbol{X}(\omega, \beta)
$$

\subsubsection{Irregular wave statistics and models}

Although the previous equations were based on regular waves, the sea surface elevation is random in nature. Despite that, the linear response model assures the validity of the superposition method, Figure 9, and the response can still be determined by the RAOs, Equation (10).

$$
\boldsymbol{\xi}(t)=\operatorname{Re}\left\{\iint \boldsymbol{R} \boldsymbol{A} \boldsymbol{O}(\omega, \beta) e^{i \omega t} d A(\omega, \beta)\right\}
$$

Figure 9 - Irregular sea, formed by the superposition of regular waves.

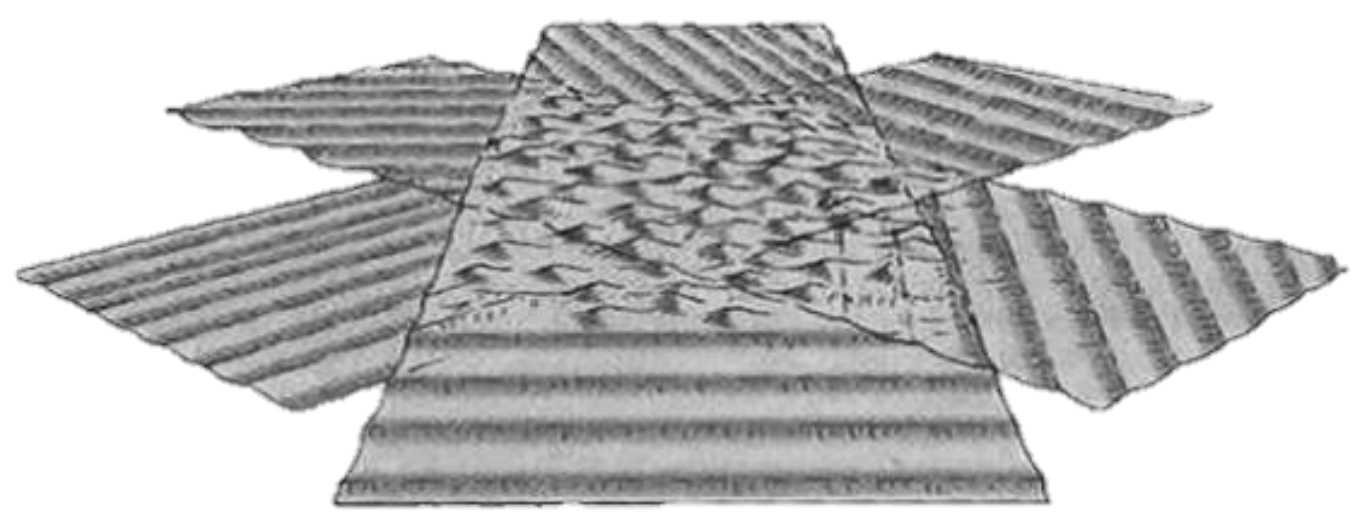

Source: (MARTINS, 2003) apud (CARNEIRO, 2012). 
In this case, it is not convenient anymore to talk about individual waves, each one with its own amplitude, and the most representative description of the sea will be the energy spectral density, $S(\omega)$, which distributes the overall energy in each component of the spectrum.

In a deterministic signal, the individual components are calculated via discretetime Fourier transform (DTFT), Equation (11), which transforms the time series $y(t)$ in its frequency decomposition $Y(\omega)$, Figure 10. Hence, the energy spectral density can be determined by Equation (12), and it is related to the 'amplitude' $A_{n}$ of each 'regular wave' that composes the irregular wave, as shown in Equation (13).

$$
\begin{gathered}
Y(\omega)=\sum_{t=-\infty}^{\infty} y(t) e^{-i \omega t} \\
S(\omega)=|Y(\omega)|^{2} \\
S\left(\omega_{n}\right) d \omega=\frac{1}{2} A_{n}^{2}
\end{gathered}
$$

Figure 10 - Energy spectral density determined by harmonic decomposition.

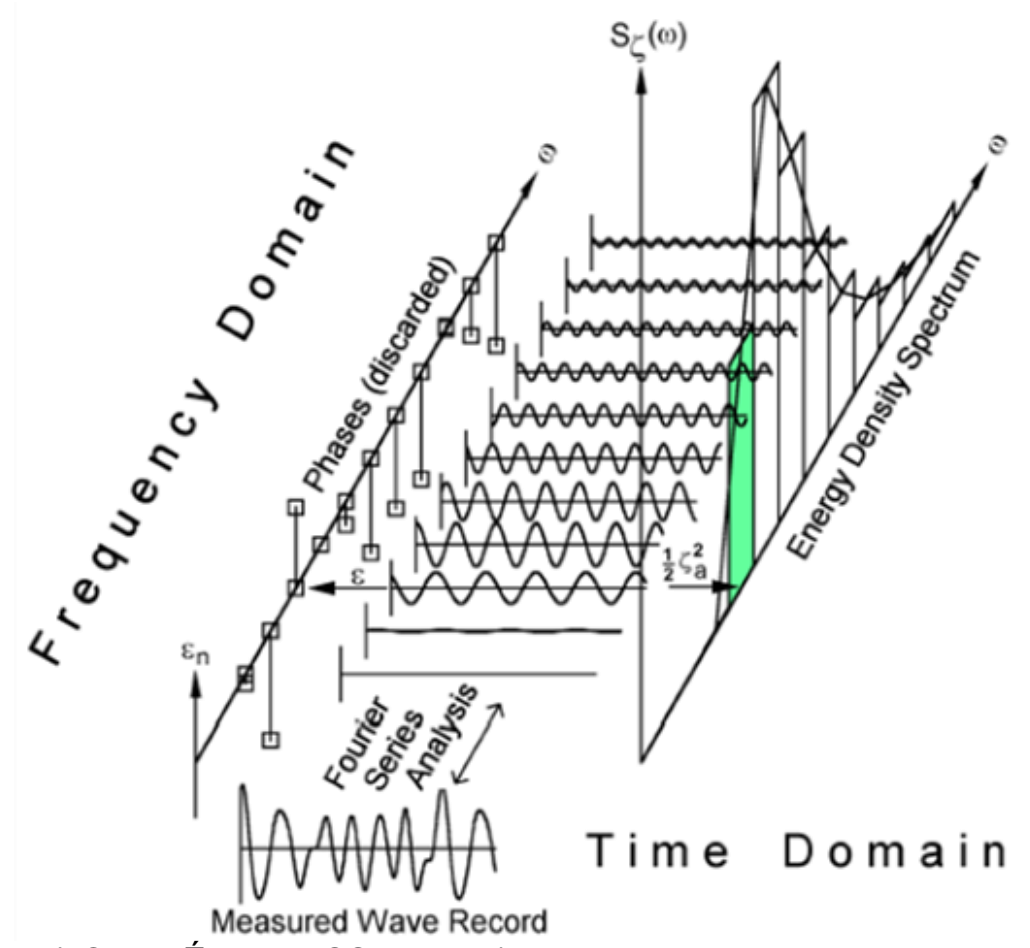

Source: (JOURNÉE \& MASSIE, 2011). 
However, it is possible to show, (Stoica \& Moses, 2005), that the same result can be obtained by the DTFT of the autocorrelation $\rho(k)$ of the signal, a more suitable approach for random signals, Equation (14).

$$
\rho(k)=\sum_{t=-\infty}^{\infty} y(t) y^{*}(t-k) \rightarrow \sum_{k=-\infty}^{\infty} \rho(k) e^{-i \omega k}=S(\omega)
$$

In addition, the energy spectral density can be further developed to account waves coming from different directions, as shown in Equation $(15)$, in which $S(\omega, \beta)$ is called directional energy spectrum.

$$
S(\omega)=\int_{0}^{2 \pi} S(\omega, \beta) d \beta
$$

Finally, the directional energy spectrum can be related to the vessel movements through the cross spectra between each degree of freedom, $\phi_{i j}(\omega)$, Equation (16).

$$
\phi_{i j}(\omega)=\int_{0}^{2 \pi} R A O_{i}(\omega, \beta) \cdot R A O_{j}^{*}(\omega, \beta) \cdot S(\omega, \beta) d \beta
$$

If the wave-elevation is supposed Gaussian, other interesting statistics can be derived by the energy spectral density. Defining the spectral moments $m_{k}$, it is possible to calculate: the significant wave height of the sea $H_{s}$ - the average of the highest one third of all the waves; and the mean centroid wave period $T_{1}$. The procedure is indicated in the Equations (17), (18) and (19).

$$
\begin{gathered}
m_{k}=\int_{0}^{\infty} \omega^{k} S(\omega) d \omega \\
H_{S}=4 \sqrt{m_{0}} \\
T_{1}=2 \pi \frac{m_{0}}{m_{1}}
\end{gathered}
$$

Based on semi-empirical relations, some standard directional energy spectra are used. The JONSWAP energy spectral density is, nowadays, the most common 
parametric model in the literature, and, consequently, the logical choice for benchmark comparisons, given by Equations (20) to (24). The parameter $T_{P}$ is called peak period.

$$
\begin{gathered}
S(\omega)=\frac{320 H_{S}^{2}}{T_{P}^{4}} \omega^{-5} e^{\left\{\frac{-1950}{T_{P}^{4}} \omega^{-4}\right\}} \gamma^{A} \\
\gamma=3.3 \\
\left.\left.A(\omega)=e^{\left\{-\left(\frac{\omega}{\omega_{P}}-1\right.\right.}\right)^{2}\right\} \\
\sigma=0.07 \text { if } \omega<\omega_{P}, \quad \sigma=0.09 \text { otherwise } \\
T_{P}=1.199 T_{1}
\end{gathered}
$$

Even though the spectrum is sometimes expanded, with a more complex function for $\gamma$, for example, $T_{P}$ and $H_{S}$ are sufficient to determine the $17^{\text {th }}$ ITTC (1984) recommended JONSWAP energy spectral density, (SIMOS, 2014).

The standard directional energy spectrum is obtained by multiplying the energy spectral density by a directional spread function in the form of a cosine-squared - also the most common parametric spread function adopted in the literature; as shown in Equations (25), (26) and (27).

$$
\begin{gathered}
S(\omega, \beta)=S(\omega) \cdot D(\omega, \beta) \\
D(\omega, \beta)=G(s) \cdot \cos ^{2 s}\left(\frac{\beta-\beta_{0}}{2}\right) \\
G(s) \rightarrow \int_{0}^{2 \pi} D(\omega, \beta) d \beta=1
\end{gathered}
$$

The spread function is completely defined by the parameters $s$, which determines the spread, and $\beta_{0}$, which determines the main direction. The function $G(s)$ is only a normalizing factor used to guarantee a unitary integral.

As a consequence of those choices, the seas used in this work, for validation and comparison purposes, will be defined, hereinafter, by a function $S(\omega, \beta)=$ $f\left(H_{S}, T_{P}, \beta_{0}, s\right)$. Examples of the different components are shown in Figure 11, Figure 12 and Figure 13. 
Figure 11 - Example of JONSWAP energy spectral density.

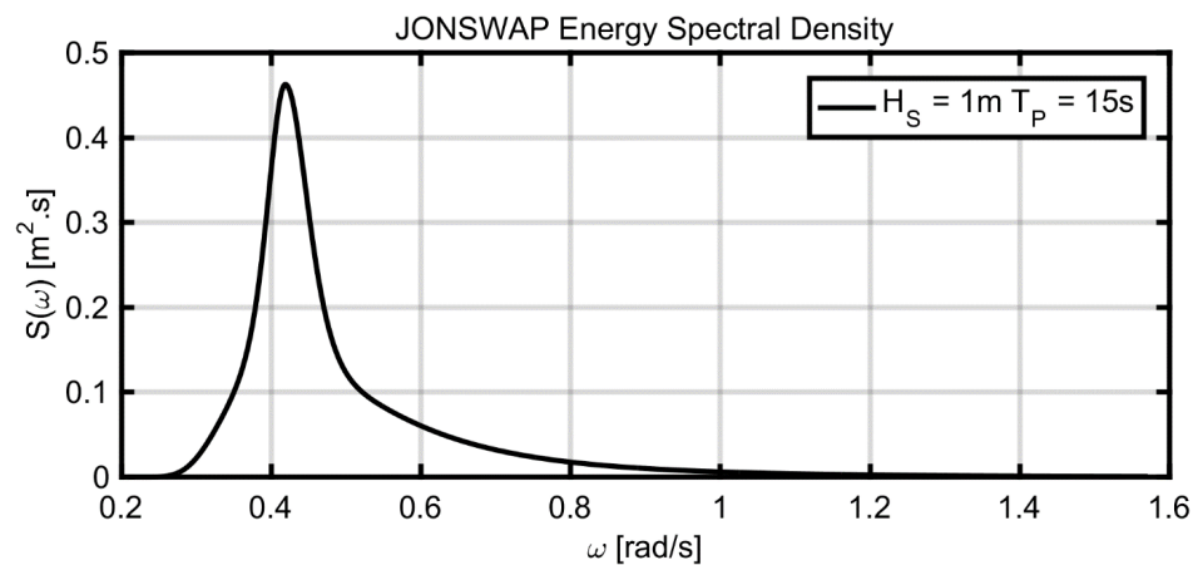

Source: Elaborated by the author.

Figure 12 - Example of cosine-squared directional spread function.

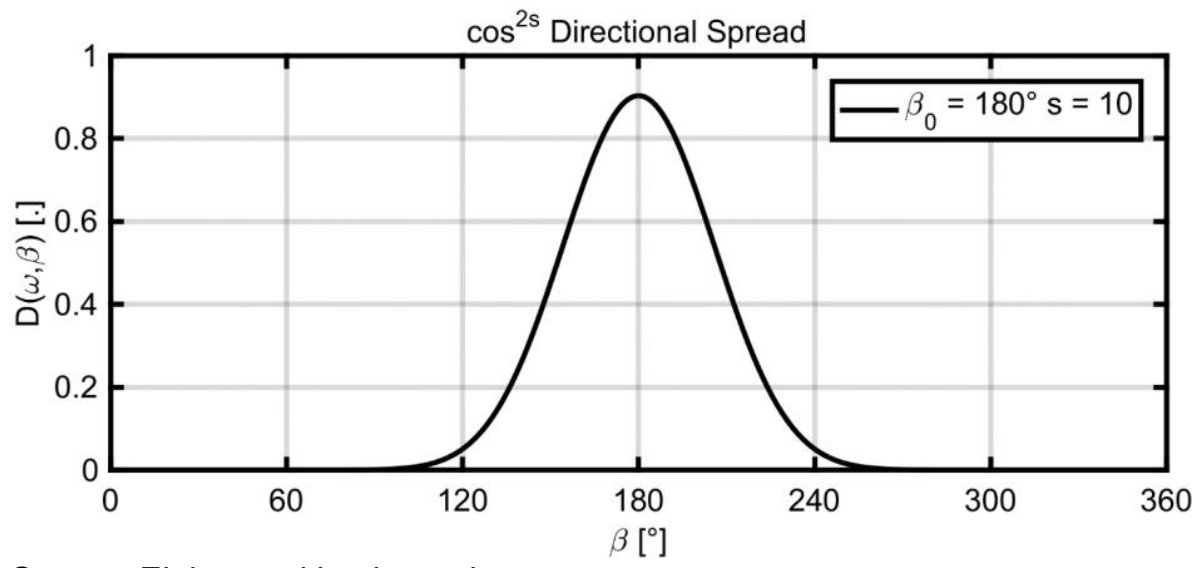

Source: Elaborated by the author.

Figure 13 - Example of directional energy spectrum.

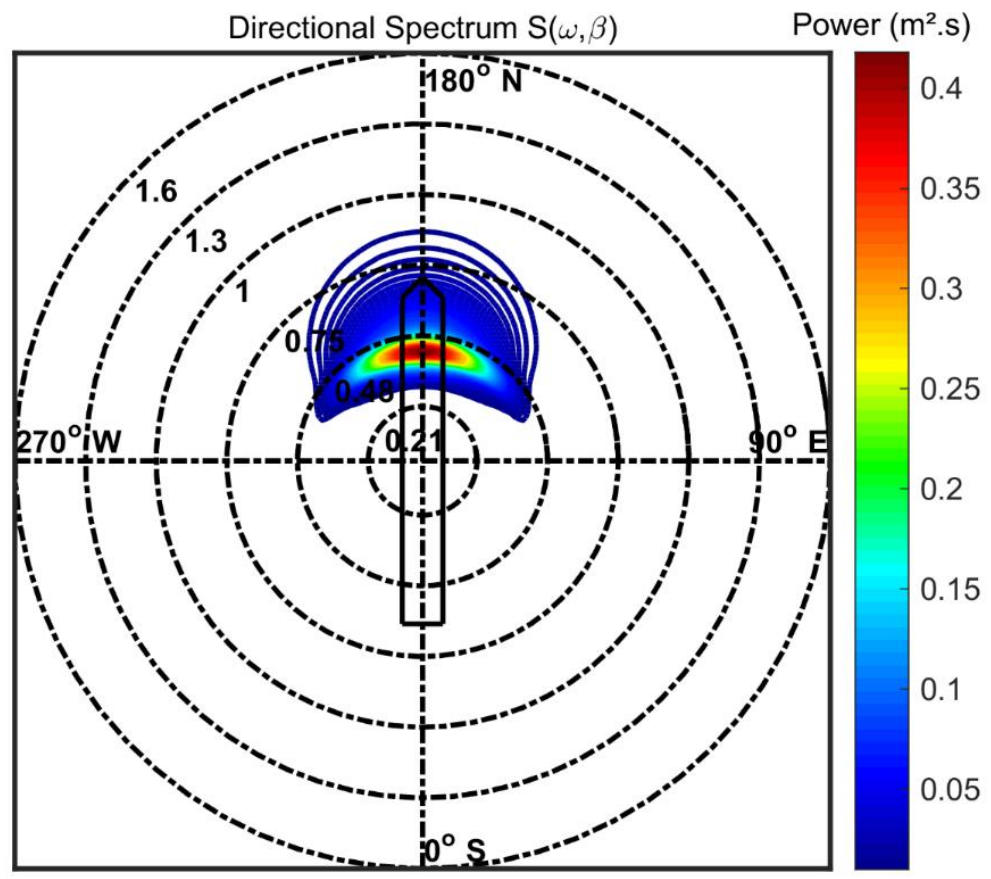

Source: Elaborated by the author. 


\subsection{Bayesian Estimation}

Although a number of different estimation procedures exist, this work focuses on the Bayesian based procedure. The main reasons are: it is easy to be extended to incorporate wave-probes measurements; it is easy to be solved by simple optimization algorithms, lowering the time demanded by simulations; and it has already been applied successfully to wave estimation problems with clear advantages against other methods. The method is described in the next sections, based on (BERGER, 1985), (ALPAYDIN, 2004) and (NIELSEN, 2005).

\subsubsection{General Bayesian estimation}

The Bayes' theorem is the probability law which determines a probability of some event $A$ after the known occurrence of other event $B$, or $P(A \mid B)$, as a function of the probability of the event $B$ after the known occurrence of the event $A$, or $P(B \mid A)$. Its formulation is easy to be shown as a restriction of the probability space $\Omega$, Figure 14, and it is described in Equations (28), (29) and (30).

$$
\begin{gathered}
P(A \mid B)=\frac{P(A \cap B)}{P(B)} \\
P(A \mid B) P(B)=P(B \mid A) P(A)=P(A \cap B) \\
P(A \mid B)=\frac{P(B \mid A) P(A)}{P(B)}
\end{gathered}
$$

Figure 14 - Probability space and Bayes rule.

$\Omega$

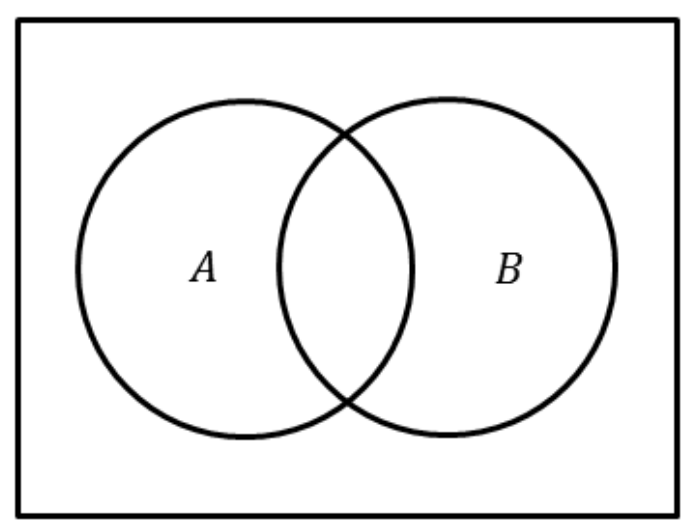

Source: Elaborated by the author.

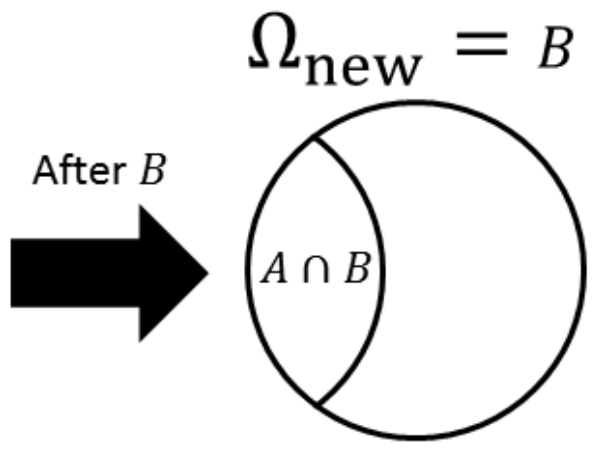


In the estimation area, those events are assumed to be the state $X$, that the method aims at estimate, and the measurement $Y$, that was made by a given sensor. Then, the procedure is to choose the state that has the maximum probability given the measurement, as stated in Equation (31).

$$
\max _{x} P(X=x \mid Y=y)=\frac{P(Y=y \mid X=x) P(X=x)}{P(Y=y)}
$$

As the measure was already made, the denominator is determined and constant for all possible $x$ values, so it is not necessary to know its value to perform the optimization procedure, Equation (32).

$$
\max _{x} P(X=x \mid Y=y) \equiv \max _{x} P(Y=y \mid X=x) P(X=x)
$$

The estimation function presented is called the posterior probability, $P(X=x \mid Y=y)$, and can be divided in two terms: the prior knowledge $P(X=x)$, related to the belief of the happening of state $x$; and the conditional probability, or likelihood, $P(Y=y \mid X=x)$, related to the possible sensor outcomes, mainly due to noise and non-modelled variables.

The latter, the likelihood, is easy to be obtained through sensor experiments, and usually is modelled as the actual state $x$, times a constant, plus a Gaussian noise with zero mean and known variance $\sigma^{2}, \mathcal{N}\left(\mu=0, \sigma^{2}\right)$, Equations (33), (34) and (35).

$$
\begin{gathered}
y=k x+\epsilon, \quad \epsilon=\mathcal{N}\left(\mu=0, \sigma^{2}\right) \\
P(\epsilon)=f\left(\epsilon \mid \mu=0, \sigma^{2}\right) d \epsilon=\frac{1}{\sqrt{2 \pi \sigma^{2}}} e^{-\frac{(\epsilon-\mu)^{2}}{2 \sigma^{2}}} d \epsilon \\
P(Y \mid X)=f\left(y \mid \mu=k x, \sigma^{2}\right) d y=\frac{1}{\sqrt{2 \pi \sigma^{2}}} e^{-\frac{(y-k x)^{2}}{2 \sigma^{2}}} d y
\end{gathered}
$$

The former, the prior function, however, is difficult to be obtained, since it would demand extensive measurement campaigns or, even, could not be obtained at all. To solve this problem, an approach is to use this function as a subjective belief about the state, giving preference for some outcomes despite others. 
One interesting example of subjective belief is to not give preference to any possible state value, and use a uniform distribution to represent $P(X)$. The resulting estimator, called the likelihood estimator, is presented in Equation (36).

$$
\max _{x} P(Y=y \mid X=x) P(X=x) \equiv \max _{x} \frac{1}{\sqrt{2 \pi \sigma^{2}}} e^{-\frac{(y-k x)^{2}}{2 \sigma^{2}}} \equiv \min _{x}(y-k x)^{2}
$$

The solution is equivalent to minimize the squared difference between the measurement and the estimation, and it is a known statistical procedure called the Least Square Method. The Bayesian procedure, in this case, stablishes a clear rationale behind the method: it is the best estimator when Gaussian measurement noise are experienced and nothing is known about the state of the measured variable. This is the origin of the Maximum Likelihood Method.

Other interesting example is to describe the state as the result of a second independent Gaussian random process - the result of a manufacturing process, for example. The estimator is presented in Equation (37).

$$
\begin{aligned}
& \max _{x} P(Y=y \mid X=x) P(X=x) \equiv \max _{x} \frac{1}{\sqrt{2 \pi \sigma_{y}^{2}}} e^{-\frac{(y-k x)^{2}}{2 \sigma_{y}^{2}}} \frac{1}{\sqrt{2 \pi \sigma_{x}^{2}}} e^{-\frac{\left(x-\mu_{x}\right)^{2}}{2 \sigma_{x}^{2}}} \\
& \equiv \max _{x} \frac{1}{\sqrt{2 \pi \sigma_{y}^{2}} \sqrt{2 \pi \sigma_{x}^{2}}} e^{-\left[\frac{(y-k x)^{2}}{2 \sigma_{y}^{2}}+\frac{\left(x-\mu_{x}\right)^{2}}{2 \sigma_{x}^{2}}\right]} \equiv \min _{x} \frac{(y-k x)^{2}}{2 \sigma_{y}^{2}}+\frac{\left(x-\mu_{x}\right)^{2}}{2 \sigma_{x}^{2}}
\end{aligned}
$$

This result is interesting because it is the base of stochastic filters, as the Kalman filter.

A third interesting example, and a more subjective one, is to penalize some possible states that differs from an expected one, formulating an idealized Gaussian function with a chosen penalty factor $\alpha$. Based on the same procedure shown previously, the result is presented in Equation (38).

$$
\begin{aligned}
\min _{x} \frac{(y-k x)^{2}}{2 \sigma_{y}^{2}} & +\frac{\left(x-\mu_{x}\right)^{2}}{2 \sigma_{x}^{2}} \equiv \min _{x}(y-k x)^{2}+\frac{\sigma_{y}^{2}\left(x-\mu_{x}\right)^{2}}{\sigma_{x}^{2}} \\
& \equiv \min _{x}(y-k x)^{2}+\alpha^{2}\left(x-\mu_{x}\right)^{2}
\end{aligned}
$$


The estimator is easily expanded for the multivariate case, more suitable for state space modelling of multi-degrees of freedom systems. In this case, the Gaussian multivariate distribution is used, been defined by a mean vector $\boldsymbol{\mu}=$ $\left[E\left[X_{1}\right], E\left[X_{2}\right], \ldots, E\left[X_{k}\right]\right]$ and the covariance matrix $\Sigma=\left[\operatorname{Cov}\left[X_{i}, X_{j}\right]\right]$, Equation (39).

$$
f(\boldsymbol{x})=\frac{1}{\sqrt{(2 \pi)^{k}|\Sigma|}} e^{\left(-\frac{1}{2}(\boldsymbol{x}-\boldsymbol{\mu})^{T} \boldsymbol{\Sigma}^{-1}(\boldsymbol{x}-\boldsymbol{\mu})\right)}
$$

Hence, assuming that each component of the measurement vector is independent and with same variance $\sigma_{y}^{2}$, adopting $\boldsymbol{\mu}_{\boldsymbol{x}}=\mathbf{0}$ and knowing that the inverse of the covariance matrix is always positive definite and can be decompose by the Cholesky factorization $-\boldsymbol{\Sigma}_{x}^{-1}=\boldsymbol{\Gamma}^{\mathrm{T}} \boldsymbol{\Gamma}$; the penalty approach is described by Equations (40), (41) and (42).

$$
\begin{gathered}
\min _{\boldsymbol{x}}(\boldsymbol{y}-\boldsymbol{K} \boldsymbol{x})^{T} \boldsymbol{\Sigma}_{\mathrm{y}}^{-1}(\boldsymbol{y}-\boldsymbol{K} \boldsymbol{x})+\boldsymbol{x}^{T} \boldsymbol{\Sigma}_{x}^{-1} \boldsymbol{x} \\
\min _{\boldsymbol{x}} \frac{1}{\sigma_{y}^{2}}(\boldsymbol{y}-\boldsymbol{K} \boldsymbol{x})^{T}(\boldsymbol{y}-\boldsymbol{K} \boldsymbol{x})+\boldsymbol{x}^{T} \boldsymbol{\Gamma}^{\mathrm{T}} \boldsymbol{\Gamma} \boldsymbol{x} \\
\min _{\boldsymbol{x}}\|\boldsymbol{K} \boldsymbol{x}-\boldsymbol{y}\|^{2}+\alpha^{2}\|\boldsymbol{\Gamma} \boldsymbol{x}\|^{2}
\end{gathered}
$$

The final result is also a known statistical procedure, called Tikhonov regularization. It is the most common regularization method, used when the least square problem is ill-posed, i.e., there are more variables than equations to solve the problem and the solution is non-unique.

The method essentially stablishes preferences for choosing the best solution of the problem. Some possible preferences are:

- Minimum energy:

$$
\boldsymbol{\Gamma}=\boldsymbol{I}_{k x k} \rightarrow \min _{\boldsymbol{x}}\|\boldsymbol{K} \boldsymbol{x}-\boldsymbol{y}\|^{2}+\alpha^{2}\|\boldsymbol{x}\|^{2}
$$

- Minimum energy in selected states:

$$
\Gamma_{i j}=1 \text { for some is and } j s \rightarrow \min _{\boldsymbol{x}}\|\boldsymbol{K} \boldsymbol{x}-\boldsymbol{y}\|^{2}+\alpha^{2}\left\|\left\{x_{i j}\right\}\right\|^{2} \text {; }
$$

- Minimum first derivative module:

$$
\boldsymbol{\Gamma} \boldsymbol{x}=\left\{\Delta x\left(x_{i+1}-x_{i}\right)\right\} \rightarrow \min _{\boldsymbol{x}}\|\boldsymbol{K} \boldsymbol{x}-\boldsymbol{y}\|^{2}+\alpha^{2}\left\|\left\{\Delta x\left(x_{i+1}-x_{i}\right)\right\}\right\|^{2} .
$$


Lastly, the Bayesian approach presented so far also has a great property. Without establishing any restrictions about the problem, but maintaining the general structure using Gaussian probability functions, Equations (43) and (44), the problem has a closed form solution, Equation (45).

$$
\begin{gathered}
\boldsymbol{b}=\boldsymbol{A} \boldsymbol{x}+\boldsymbol{\epsilon}, \quad \boldsymbol{\epsilon}=\mathcal{N}\left(\boldsymbol{\mu}=\mathbf{0}, \boldsymbol{\Sigma}_{\boldsymbol{y}}\right) \\
\max _{\boldsymbol{x}} P(\boldsymbol{Y}=\boldsymbol{b} \mid \boldsymbol{X}=\boldsymbol{x}) P(\boldsymbol{X}=\boldsymbol{x}) \equiv \\
\equiv \max _{\boldsymbol{x}} \frac{1}{\sqrt{(2 \pi)^{m}\left|\boldsymbol{\Sigma}_{\boldsymbol{y}}\right|}} e^{\left(-\frac{1}{2}(\boldsymbol{b}-\boldsymbol{A} \boldsymbol{x})^{T} \boldsymbol{\Sigma}_{\boldsymbol{y}}^{-1}(\boldsymbol{b}-\boldsymbol{A} \boldsymbol{x})\right)} \frac{1}{\sqrt{(2 \pi)^{n}\left|\boldsymbol{\Sigma}_{\boldsymbol{x}}\right|}} e^{\left(-\frac{1}{2}\left(\boldsymbol{x}-\boldsymbol{x}_{\mathbf{0}}\right)^{T} \boldsymbol{\Sigma}_{\boldsymbol{x}}^{-1}\left(\boldsymbol{x}-\boldsymbol{x}_{\mathbf{0}}\right)\right)} \equiv \\
\equiv \min _{\boldsymbol{x}}(\boldsymbol{A} \boldsymbol{x}-\boldsymbol{b})^{T} \boldsymbol{\Sigma}_{\boldsymbol{y}}^{-1}(\boldsymbol{A} \boldsymbol{x}-\boldsymbol{b})+\left(\boldsymbol{x}-\boldsymbol{x}_{\mathbf{0}}\right)^{T} \boldsymbol{\Sigma}_{\boldsymbol{x}}^{-1}\left(\boldsymbol{x}-\boldsymbol{x}_{\mathbf{0}}\right) \\
\hat{\boldsymbol{x}}=\left(\boldsymbol{A}^{T} \boldsymbol{P A}+\boldsymbol{Q}\right)^{-1}\left(\boldsymbol{A}^{T} \boldsymbol{P} \boldsymbol{b}+\boldsymbol{Q} \boldsymbol{x}_{\mathbf{0}}\right) \\
\text { or } \hat{\boldsymbol{x}}=\boldsymbol{x}_{\mathbf{0}}+\left(\boldsymbol{A}^{T} \boldsymbol{P} \boldsymbol{A}+\boldsymbol{Q}\right)^{-1}\left(\boldsymbol{A}^{T} \boldsymbol{P}\left(\boldsymbol{b}-\boldsymbol{A} \boldsymbol{x}_{\mathbf{0}}\right)\right) \\
\boldsymbol{P}=\boldsymbol{\Sigma}_{\boldsymbol{y}}^{-1} \text { and } \boldsymbol{Q}=\boldsymbol{\Sigma}_{\boldsymbol{x}}^{-1}
\end{gathered}
$$

The closed form solution means that, for each estimation, the algorithm needs only to perform simple matrix calculations, and reaches the global optimal solution of the problem. A great advantage compared to other estimation methods.

The main drawback, however, is that the linear system for Bayesian estimation is often ill-posed, possessing more variables than equations, classifying it as a highorder method. Consequently, a prior function must be used, with all the subjectivity and ad hoc hypothesis needed.

\subsubsection{Bayesian estimation applied to wave spectrum estimation}

In the particular case of directional wave spectrum estimation, the linear system is formulated by the discretization of the cross-spectra calculation previously presented. The discretization in $K$ directions is shown in Equation (46).

$$
\phi_{i j}(\omega) \approx \sum_{k=1}^{K} R A O_{i}(\omega, \beta) \cdot R A O_{j}^{*}(\omega, \beta) \cdot S(\omega, \beta) \Delta \beta, \quad \Delta \beta=\frac{2 \pi}{K}
$$


It is important to emphasize the complex nature of the quantities involved, which almost double the number of equations - one for the real part and other for the imaginary part. In this case, for a particular angular frequency $\omega_{m}$, the system, using $N$ degrees of freedom, is given by Equations (47)-(50).

$$
\begin{aligned}
& \boldsymbol{\phi}_{m}=\left[\begin{array}{c}
\phi_{11} \\
\phi_{22} \\
\vdots \\
\phi_{i i} \\
\vdots \\
\phi_{N N} \\
\operatorname{Re}\left\{\phi_{12}\right\} \\
\operatorname{Re}\left\{\phi_{13}\right\} \\
\vdots \\
\operatorname{Re}\left\{\phi_{23}\right\} \\
\vdots \\
\operatorname{Re}\left\{\phi_{i j}\right\} \\
\vdots \\
\operatorname{Re}\left\{\phi_{N-1 N}\right\} \\
\operatorname{Im}\left\{\phi_{12}\right\} \\
\vdots \\
\operatorname{Im}\left\{\phi_{i j}\right\} \\
\vdots \\
\operatorname{Im}\left\{\phi_{N-1 N}\right\}
\end{array}\right] \\
& \boldsymbol{A}_{\boldsymbol{m}} \cdot \frac{1}{\Delta \beta}= \\
& {\left[\begin{array}{ccccc}
R A O_{1}\left(\omega_{m}, \beta_{1}\right)^{2} & \ldots & R A O_{1}\left(\omega_{m}, \beta_{k}\right)^{2} & \ldots & R A O_{1}\left(\omega_{m}, \beta_{K}\right)^{2} \\
\vdots & & \vdots & & \vdots \\
R A O_{i}\left(\omega_{m}, \beta_{1}\right)^{2} & \ldots & R A O_{i}\left(\omega_{m}, \beta_{k}\right)^{2} & \ldots & R A O_{i}\left(\omega_{m}, \beta_{K}\right)^{2} \\
\vdots & \vdots & \vdots & \\
\operatorname{Re}\left\{R A O_{1}\left(\omega_{m}, \beta_{1}\right) R A O_{2}\left(\omega_{m}, \beta_{1}\right)^{*}\right\} & \ldots & \operatorname{Re}\left\{R A O_{1}\left(\omega_{m}, \beta_{k}\right) R A O_{2}\left(\omega_{m}, \beta_{k}\right)^{*}\right\} & \ldots & \operatorname{Re}\left\{R A O_{1}\left(\omega_{m}, \beta_{K}\right) R A O_{2}\left(\omega_{m}, \beta_{K}\right)^{*}\right\} \\
\vdots & & \vdots & \vdots \\
\operatorname{Re}\left\{R A O_{i}\left(\omega_{m}, \beta_{1}\right) R A O_{j}\left(\omega_{m}, \beta_{1}\right)^{*}\right\} & \ldots & \operatorname{Re}\left\{R A O_{i}\left(\omega_{m}, \beta_{k}\right) R A O_{j}\left(\omega_{m}, \beta_{k}\right)^{*}\right\} & \ldots & \operatorname{Re}\left\{R A O_{i}\left(\omega_{m}, \beta_{K}\right) R A O_{j}\left(\omega_{m}, \beta_{K}\right)^{*}\right\} \\
\vdots & & \vdots & \vdots \\
\operatorname{Re}\left\{R A O_{N-1}\left(\omega_{m}, \beta_{1}\right) R A O_{N}\left(\omega_{m}, \beta_{1}\right)^{*}\right\} & \ldots & \operatorname{Re}\left\{R A O_{N-1}\left(\omega_{m}, \beta_{k}\right) R A O_{N}\left(\omega_{m}, \beta_{k}\right)^{*}\right\} & \ldots & \operatorname{Re}\left\{R A O_{N-1}\left(\omega_{m}, \beta_{K}\right) R A O_{N}\left(\omega_{m}, \beta_{K}\right)^{*}\right\} \\
\vdots & \vdots & \vdots \\
\operatorname{Im}\left\{R A O_{i}\left(\omega_{m}, \beta_{1}\right) R A O_{j}\left(\omega_{m}, \beta_{1}\right)^{*}\right\} & \ldots & \operatorname{Im}\left\{R A O_{i}\left(\omega_{m}, \beta_{k}\right) R A O_{j}\left(\omega_{m}, \beta_{k}\right)^{*}\right\} & \ldots & \operatorname{Im}\left\{R A O_{i}\left(\omega_{m}, \beta_{K}\right) R A O_{j}\left(\omega_{m}, \beta_{K}\right)^{*}\right\} \\
\vdots & & \vdots & \vdots \\
\operatorname{Im}\left\{R A O_{N-1}\left(\omega_{m}, \beta_{1}\right) R A O_{N}\left(\omega_{m}, \beta_{1}\right)^{*}\right\} & \ldots & \operatorname{Im}\left\{R A O_{N-1}\left(\omega_{m}, \beta_{k}\right) R A O_{N}\left(\omega_{m}, \beta_{k}\right)^{*}\right\} & \ldots & \operatorname{Im}\left\{R A O_{N-1}\left(\omega_{m}, \beta_{K}\right) R A O_{N}\left(\omega_{m}, \beta_{K}\right)^{*}\right\}
\end{array}\right]} \\
& \boldsymbol{x}_{m}=\left[\begin{array}{c}
S\left(\omega_{m}, \beta_{1}\right) \\
\vdots \\
S\left(\omega_{m}, \beta_{k}\right) \\
\vdots \\
S\left(\omega_{m}, \beta_{K}\right)
\end{array}\right] \\
& \boldsymbol{\phi}_{m}=\boldsymbol{A}_{m} \boldsymbol{x}_{m}
\end{aligned}
$$

The equations can be further composed to describe a range of $M$ angular frequencies, resulting in a system with $N^{2} \cdot M$ equations and $K \cdot M$ variables, Equations (51) and (52). 


$$
\begin{gathered}
b=\boldsymbol{A} \boldsymbol{x} \\
\left\{\boldsymbol{\phi}_{m}\right\}=\left[\begin{array}{cccc}
\boldsymbol{A}_{\mathbf{1}} & & & \\
& \ddots & \boldsymbol{A}_{\boldsymbol{m}} & \\
\mathbf{0} & & \ddots & \boldsymbol{A}_{\boldsymbol{M}}
\end{array}\right]\left\{\boldsymbol{x}_{m}\right\}
\end{gathered}
$$

If three degrees of freedom are used, the most common formulation, $K$ greater than nine is enough to underdetermine the system. In effect, the number of variables greatly surpasses the number of equations, since $K$ is usually chosen to be greater than 36 - ten degree discretization. As a consequence, prior information is needed to guarantee the uniqueness of the solution.

The priors usually found in the literature follows (SIMOS, et al., 2009), who suggest a prior with three components to incentivize smoothness: a term penalizing the second order difference in frequencies, at a given direction; a term penalizing the second order difference in directions, at a given frequency; and a term penalizing energies at frequencies that do not cause relevant vessel movements.

The purpose of the first and the second ones is to decrease the noise of the spectrum, increasing its 'smoothness'. The third one, on the other hand, guarantees that the noise in extreme high or low frequencies - in which the rotational vessel response amplitude operator are low - will not be misinterpreted as extreme vessel movements, i.e., "if the system cannot sense, it is preferable to assume it is zero".

A specific penalty factor is assigned to each one of those priors, which are called hyperparameters $u_{1}, u_{2}$ and $u_{3}$ in the Bayesian context. The complete formulation is shown in Equations (53)-(57).

$$
\begin{gathered}
\varepsilon_{1 m k}=S\left(\omega_{m}, \beta_{k-1}\right)-2 S\left(\omega_{m}, \beta_{k}\right)+S\left(\omega_{m}, \beta_{k+1}\right) \\
\varepsilon_{2 m k}=S\left(\omega_{m-1}, \beta_{k}\right)-2 S\left(\omega_{m}, \beta_{k}\right)+S\left(\omega_{m+1}, \beta_{k}\right) \\
\sum \varepsilon_{1 m k}^{2}=\boldsymbol{x}^{T} \boldsymbol{H}_{1} \boldsymbol{x} \\
\sum \varepsilon_{2 m k}^{2}=\boldsymbol{x}^{T} \boldsymbol{H}_{2} \boldsymbol{x} \\
\sum_{k=1}^{K} \sum_{m=1}^{\text {low limit }} S\left(\omega_{m}, \beta_{k}\right)^{2}+\sum_{k=1}^{K} \sum_{m=\text { high limit }}^{M} S\left(\omega_{m}, \beta_{k}\right)^{2}=\boldsymbol{x}^{T} \boldsymbol{H}_{3} \boldsymbol{x}
\end{gathered}
$$


The resulting estimator is given in Equations (58) and (59).

$$
\begin{gathered}
\min _{\boldsymbol{x}} J(\boldsymbol{x})=\|\boldsymbol{A} \boldsymbol{x}-\boldsymbol{b}\|^{2}+\boldsymbol{x}^{T}\left[u_{1}^{2} \boldsymbol{H}_{1}+u_{2}^{2} \boldsymbol{H}_{2}+u_{3}^{2} \boldsymbol{H}_{3}\right] \boldsymbol{x} \\
\widehat{\boldsymbol{x}}=\left(\boldsymbol{A}^{T} \boldsymbol{A}+\left[u_{1}^{2} \boldsymbol{H}_{1}+u_{2}^{2} \boldsymbol{H}_{2}+u_{3}^{2} \boldsymbol{H}_{3}\right]\right)^{-1} \boldsymbol{A}^{T} \boldsymbol{b}
\end{gathered}
$$

\subsection{Clustering}

Other useful statistical method is the clustering; technique used to group together similar entities, accordingly to a specified metric. The procedure is applied in different parts of this work, and its utility will become evident in further sections.

The deductions presented are based on (ALPAYDIN, 2004), (BISHOP, 2006) and (HASTIE, et al., 2008).

\subsubsection{K-means clusters}

In the Euclidian metric space, the similarity measurement is the distance between data points, as illustrated in Figure 15.

Figure 15 - Euclidian space clusters.

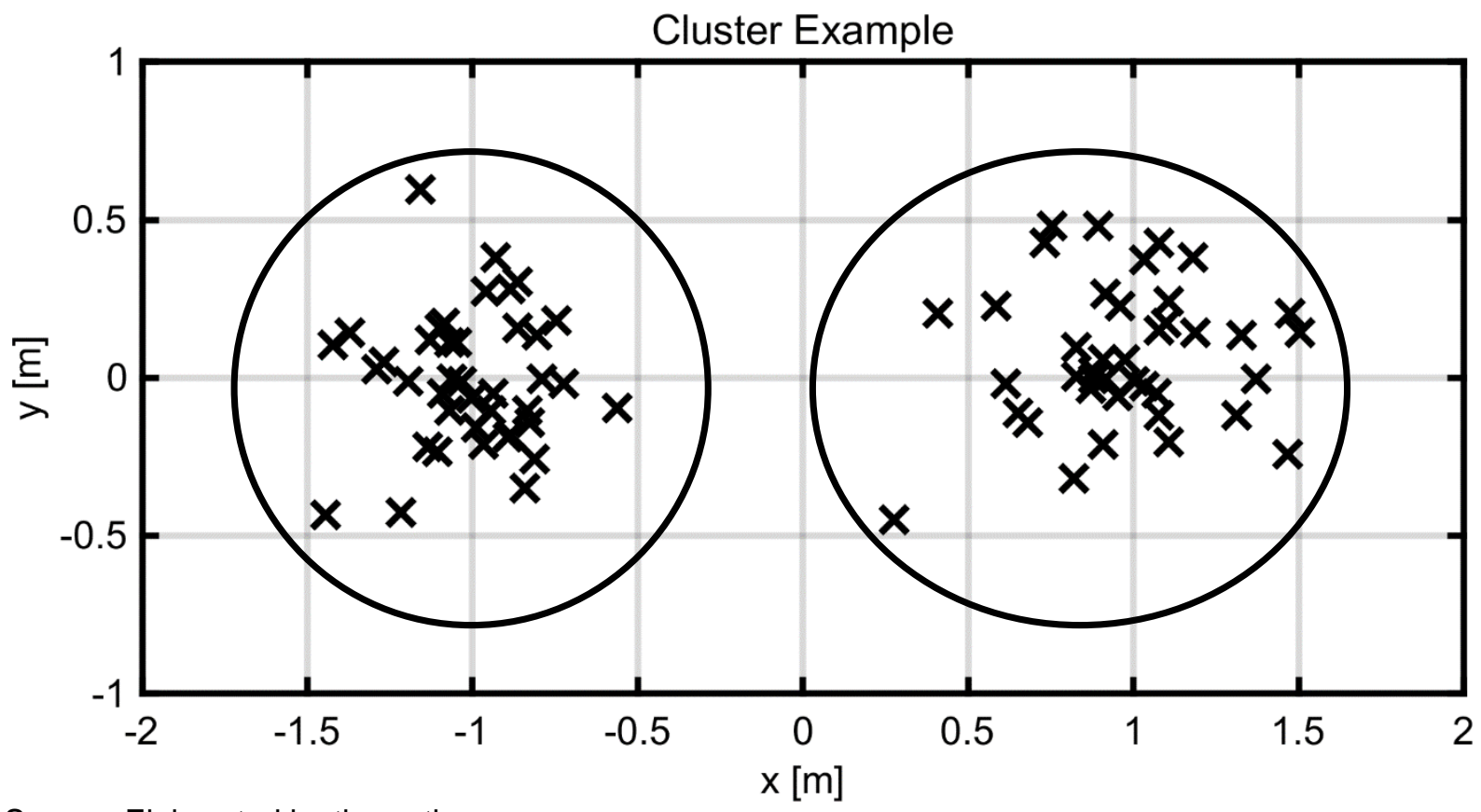

Source: Elaborated by the author.

In this context, the K-means procedure is the simplest method. It states that, given a number of clusters $K$, the best cluster assignment for $N$ data points is the one which minimizes the functional in Equation (62), which depends on the binary indicator variable $r_{n k}$, Equation (60), and on the centroids $\boldsymbol{\mu}_{k}$ of each cluster, Equation (61). 


$$
\begin{gathered}
\left\{\begin{array}{l}
r_{n k}=1, \quad \text { if point } n \text { belongs to cluster } k \\
r_{n k}=0, \quad \text { otherwise }
\end{array}\right. \\
\boldsymbol{\mu}_{k}=\frac{1}{\sum_{n=1}^{N} r_{n k}} \sum_{n=1}^{N} r_{n k} \boldsymbol{x}_{n} \\
J=\sum_{k=1}^{K} \sum_{n=1}^{N} r_{n k}\left\|\boldsymbol{x}_{n}-\boldsymbol{\mu}_{k}\right\|^{2}
\end{gathered}
$$

The formulation becomes clear when a new point is added to an existing configuration. In this case, the new point will be assigned in a way that it will represent the least increment in the sum of the 'inertias' of the clusters, Figure 16.

Figure 16 - Least increment in inertia.

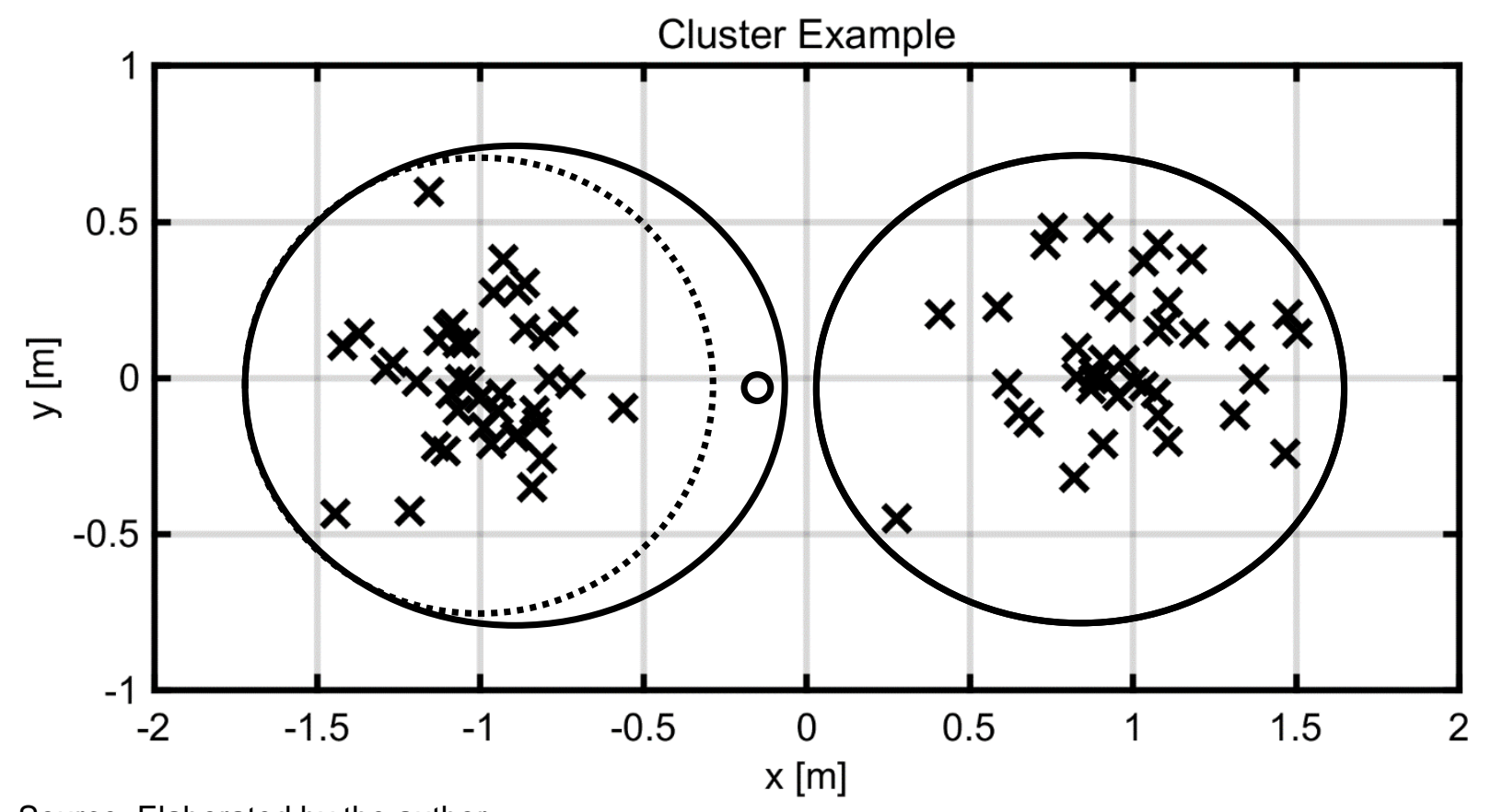

Source: Elaborated by the author.

The formulation will also give preference to clusters with the same size and number of data points. Comparing Figure 16 against Figure 17, it is possible to notice that the increase in the size of both clusters are quite similar; however, the first solution will be chosen by the algorithm due to the squared sum penalization, resulting in clusters almost equal. 
Figure 17 - Wrong solution with greater increment in inertia.

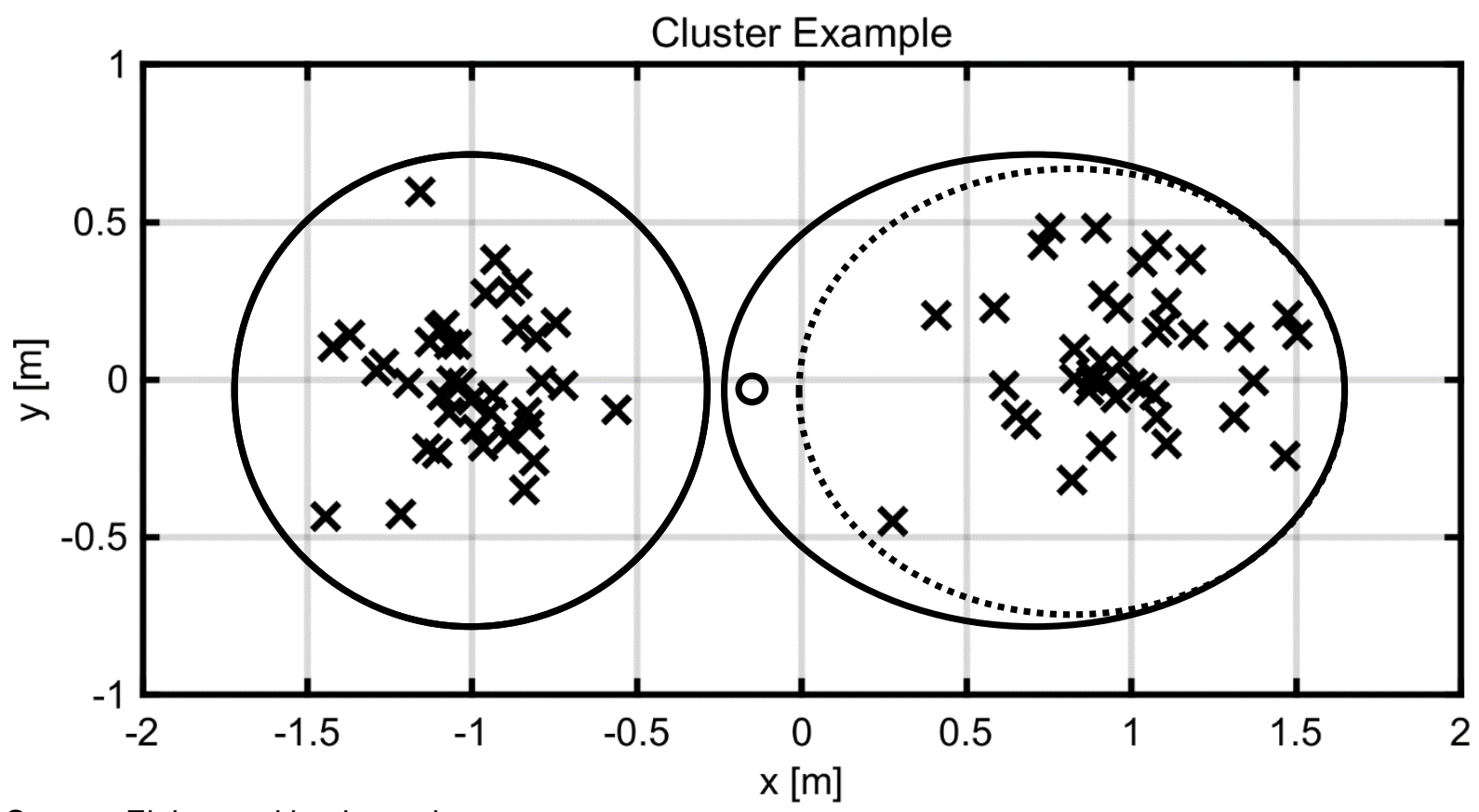

Source: Elaborated by the author.

The idea presented can be extended to any $\mathrm{N}$-dimensional space, with a proper reasoning about each dimension scale.

Although a number of algorithms and heuristics exist to solve the k-means optimization, which is NP-Hard, they will not be detailed in this text, and the default Matlab ${ }^{\circledR}$ solver will be used, with a trial and error configuration set for each case.

\subsection{2. 'Elbow' criterion}

The formulation of the k-mean clustering technique assumes that the number $K$ of clusters was provided. Even though this number is easy to infer up to the threedimensional case, it is not possible to visualize high-dimensional problems, and, as a consequence, the choice of the number of clusters is always a subject of controversy.

In this work, however, the exact number of clusters is not important, but only how different they are between them, and how similar the clustered data is. This allows the use of a known graphical subjective analysis called the 'elbow' criterion.

This analysis has a strong statistical base, interpreting the cluster squared sum as the variance of a Gaussian mixture process. In this sense, the sum of the variances will decrease until a linear plateau is found, in which the decrease is only due to the 
segmentation of the real groups. This moment can be identified by an 'elbow' in the squared sum graph. An example is given in Figure 18 to Figure 21.

Figure 18 - Example with five groups and four clusters, with high inertia.

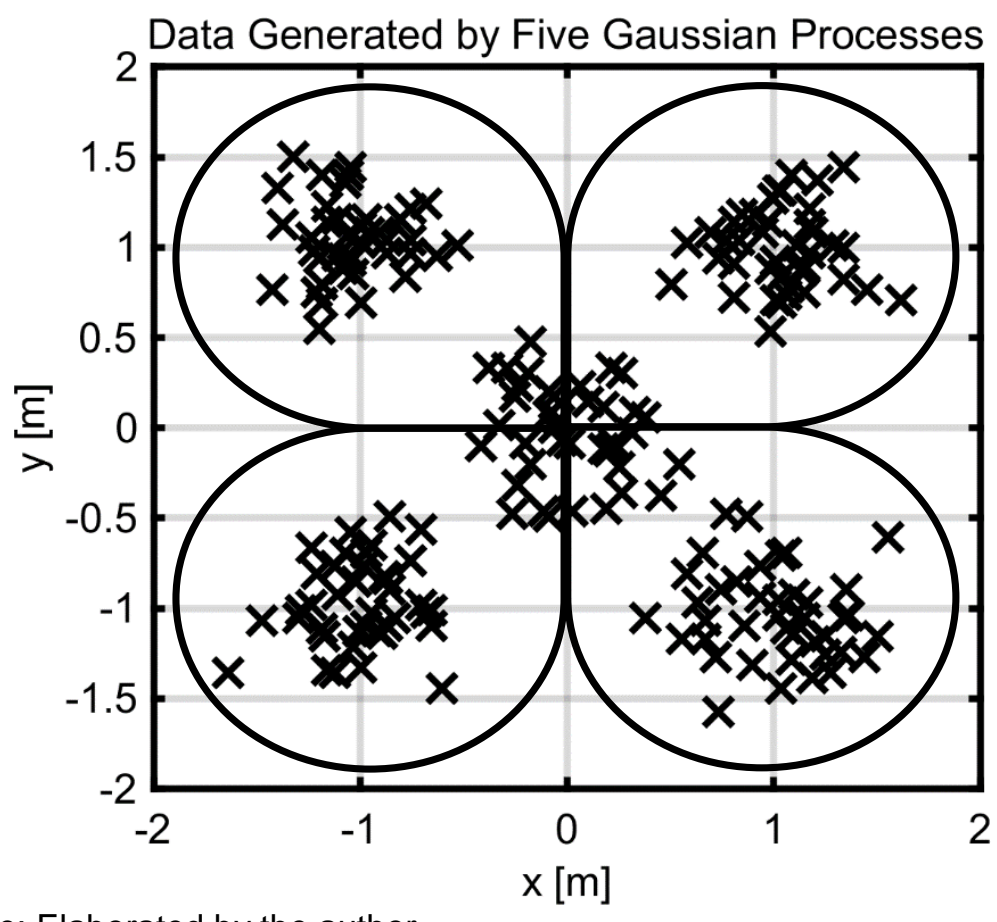

Source: Elaborated by the author.

Figure 19 - Same example with five clusters, and considerably less inertia.

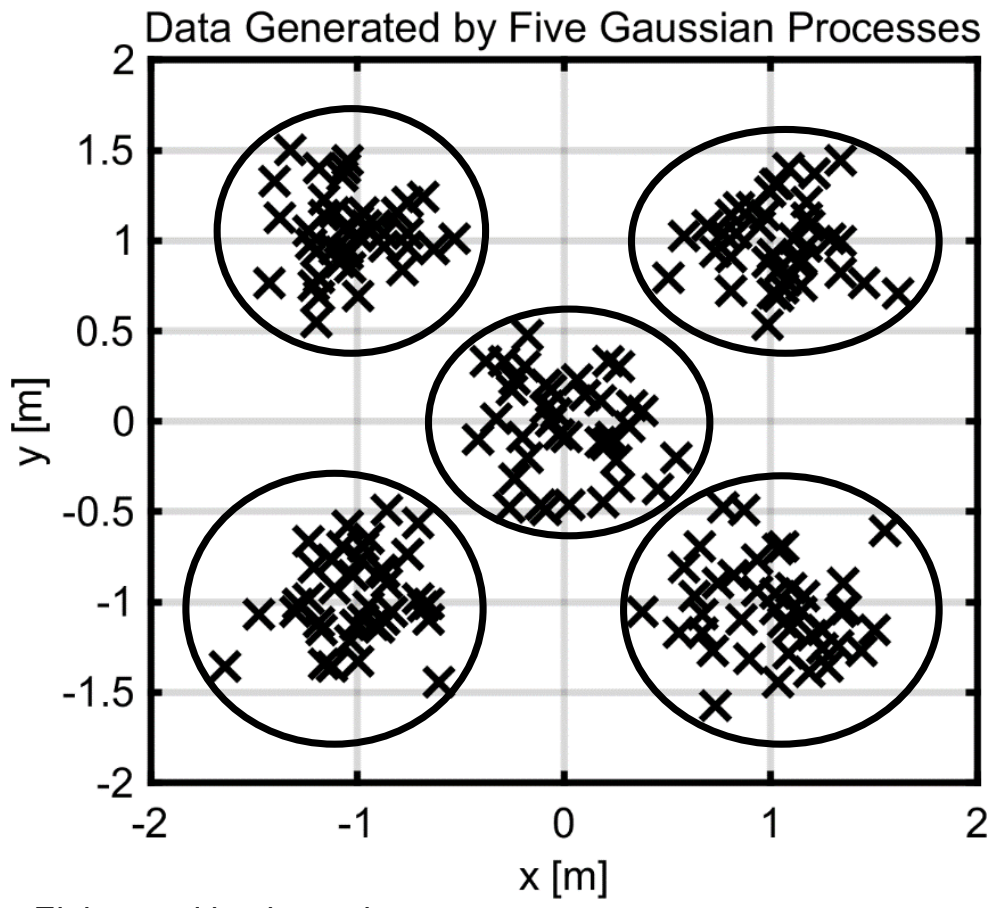

Source: Elaborated by the author. 
Figure 20 - Same example with six clusters, and slightly less inertia.

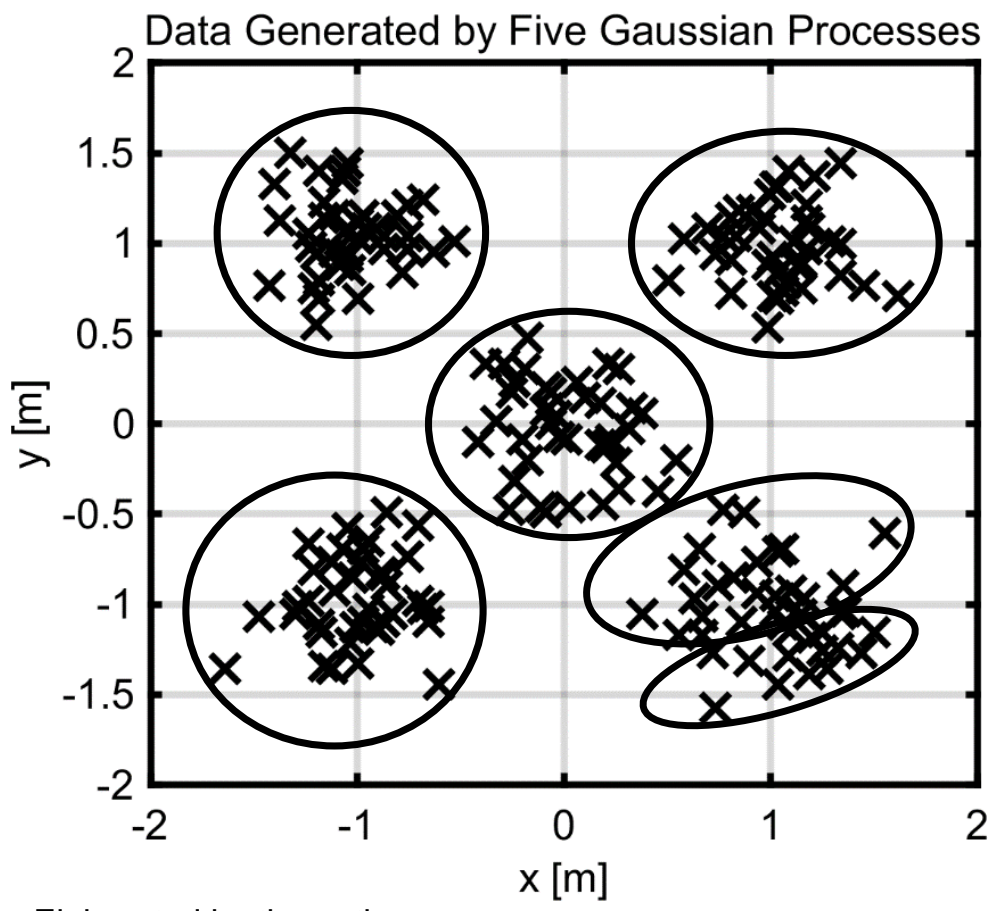

Source: Elaborated by the author.

Figure 21 - Five clusters selected, based on the 'elbow' criterion of the example.

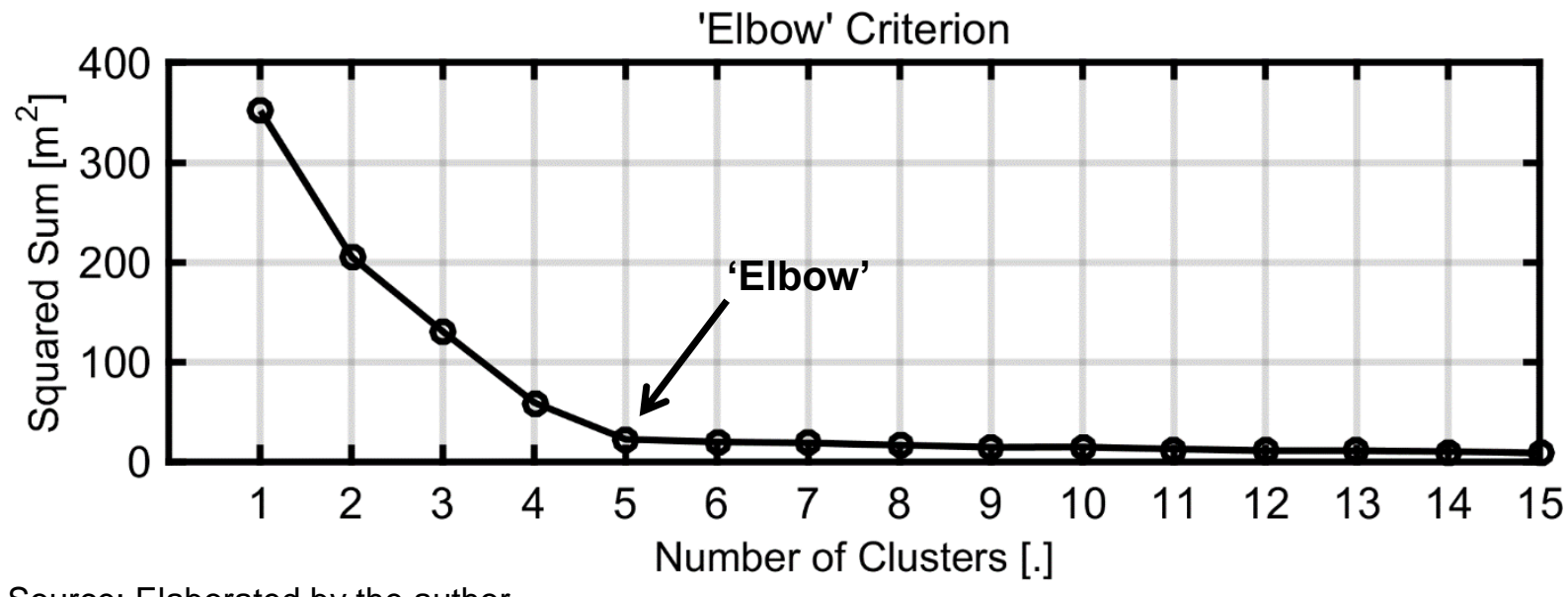

Source: Elaborated by the author.

It is also possible to interpret the method as a theoretical cost-benefit analysis, in which the number of cluster is the cost and the squared sum is the benefit. In this sense, a small number of clusters - consequently, a small cost; is able to achieve most of the benefit, and, at some point, adding another cluster will not improve almost anything, i.e., the marginal cost-benefit is almost zero. 


\subsection{Optimal Design of Experiments}

The last statistical procedure covered in this section is the optimal design of experiments. In the system identification context, the design of experiment guides the choice of experiments to be done in order to excite the most the dynamic system to be identified, mainly when limited resources cause extensive campaigns to be impossible.

In this text, the method will be used in a different, but analog, manner, in order to select the best position of the wave-probes, which will be explained in further sections. The deductions presented, valid for both applications, are based on (HABER, 2008) e (CHALONER \& VERDINELLI, 1995), and a general reference in the area is given by (PUKELSHEIM, 1948).

\subsubsection{Optimal design of experiments general theory}

It is possible to modify the linear dynamic model with Gaussian noise, Equation (63), to estimate, through experiments, the parameters of a dynamic system, using the same procedures shown in section 3.3 Bayesian Estimation. It is done by isolating the parameters of matrix $\boldsymbol{A}$ in a vector form $\boldsymbol{a}$, and mounting a number of different inputs and outputs of the system in the experiment matrix $\boldsymbol{X}$ - formed by $n$ experiments - and in the response vector $\boldsymbol{y}$, as shown in Equations (64) and (65).

$$
\begin{gathered}
\boldsymbol{A} \boldsymbol{x}=\boldsymbol{b}+\boldsymbol{\epsilon}, \quad \boldsymbol{\epsilon}=\mathcal{N}\left(\boldsymbol{\mu}=\mathbf{0}, \sigma^{2} \boldsymbol{I}\right) \\
\boldsymbol{y}_{i}=\boldsymbol{b}_{p \times 1}=\boldsymbol{A}_{p \times k} \boldsymbol{x}_{k \times 1}=\boldsymbol{X}_{i} \boldsymbol{a}=\left[\begin{array}{ccc}
\boldsymbol{x}^{T} & & \mathbf{0} \\
& \ddots & \\
\mathbf{0} & & \boldsymbol{x}^{T}
\end{array}\right]\left[\begin{array}{c}
a_{11} \\
\vdots \\
a_{1 k} \\
a_{21} \\
\vdots \\
a_{p 1} \\
\vdots \\
a_{p k}
\end{array}\right] \\
\boldsymbol{y}=\boldsymbol{X a}, \quad\left[\begin{array}{c}
\boldsymbol{y}_{1} \\
\vdots \\
\boldsymbol{y}_{i} \\
\vdots \\
\boldsymbol{y}_{n}
\end{array}\right]=\left[\begin{array}{c}
\boldsymbol{X}_{1} \\
\vdots \\
\boldsymbol{X}_{i} \\
\vdots \\
\boldsymbol{X}_{n}
\end{array}\right] \boldsymbol{a}
\end{gathered}
$$

The system parameters are, then, identified by an estimation procedure; the Tikhonov regularized least-square estimation, for example, Equation (66). 


$$
\widehat{\boldsymbol{a}}=\left(\boldsymbol{X}^{T} \boldsymbol{X}+\alpha^{2} \boldsymbol{\Gamma}^{T} \boldsymbol{\Gamma}\right)^{-1} \boldsymbol{X}^{T} \boldsymbol{b}
$$

In this context, the design of experiment is concerned about choosing the best experiment matrix $\boldsymbol{X}$ to guarantee the best estimation, mainly in an environment of limited resources and high cost experiments. One possible solution, for example, is to try to minimize the expected squared error between the real parameter and the estimation, Equation (67), which can be further developed, Equation (68), and decomposed in a bias and a variance, Equation (69).

$$
\begin{gathered}
E\left[\|\widehat{\boldsymbol{a}}-\boldsymbol{a}\|^{2}\right] \\
E\left[\left\|\left(\boldsymbol{X}^{T} \boldsymbol{X}+\alpha^{2} \boldsymbol{\Gamma}^{T} \boldsymbol{\Gamma}\right)^{-1} \boldsymbol{X}^{T} \boldsymbol{b}-\boldsymbol{a}\right\|^{2}\right]= \\
=E\left[\left\|\left(\boldsymbol{X}^{T} \boldsymbol{X}+\alpha^{2} \boldsymbol{\Gamma}^{T} \boldsymbol{\Gamma}\right)^{-1} \boldsymbol{X}^{T}(\boldsymbol{A} \boldsymbol{x}-\boldsymbol{\epsilon})-\boldsymbol{a}\right\|^{2}\right]= \\
=E\left[\left\|\left(\boldsymbol{X}^{T} \boldsymbol{X}+\alpha^{2} \boldsymbol{\Gamma}^{T} \boldsymbol{\Gamma}\right)^{-1} \boldsymbol{X}^{T}(\boldsymbol{X} \boldsymbol{a}-\boldsymbol{\epsilon})-\boldsymbol{a}\right\|^{2}\right]= \\
=E\left[\left\|\left(\boldsymbol{C}^{-1} \boldsymbol{X}^{T} \boldsymbol{X}-\boldsymbol{I}\right) \boldsymbol{a}-\boldsymbol{C}^{-1} \boldsymbol{X}^{T} \boldsymbol{\epsilon}\right\|^{2}\right], \quad \boldsymbol{C}=\left(\boldsymbol{X}^{T} \boldsymbol{X}+\alpha^{2} \boldsymbol{\Gamma}^{T} \boldsymbol{\Gamma}\right) \\
E\left[\|\widehat{\boldsymbol{a}}-\boldsymbol{a}\|^{2}\right]=\underbrace{\alpha^{4}\left\|\boldsymbol{C}^{-1} \boldsymbol{\Gamma}^{T} \boldsymbol{\Gamma} \boldsymbol{a}\right\|^{2}}_{\text {bias }}+\underbrace{\sigma^{2} \text { trace }\left(\boldsymbol{X} \boldsymbol{C}^{-2} \boldsymbol{X}^{T}\right)}_{\text {variance }}
\end{gathered}
$$

When the estimator is unbiased, which means $\alpha=0$, and the estimator is reduced to the least-square problem, the minimization implies Equation (70), a classical solution called the A-optimal design of experiment.

$$
\min _{\boldsymbol{X}} E\left[\|\widehat{\boldsymbol{a}}-\boldsymbol{a}\|^{2}\right] \equiv \min _{\boldsymbol{X}} \operatorname{trace}\left(\boldsymbol{X} \boldsymbol{C}^{-2} \boldsymbol{X}^{T}\right) \equiv \min _{\boldsymbol{X}} \operatorname{trace}\left(\left(\boldsymbol{X}^{T} \boldsymbol{X}\right)^{-1}\right)
$$

The unbiased estimator, however, is not appropriate for the ill-posed problem in this work, which demands a regularized estimator. Therefore, the procedure must be expanded.

\subsubsection{Bayesian optimal design of experiments}

The Bayesian optimal design starts before zeroing the parameter $\alpha$, which means the bias must be taken in account. If the parameter vector $\boldsymbol{a}$ is assumed Gaussian with covariance matrix $\boldsymbol{\Sigma}_{\boldsymbol{a}}$, the design criterion can be reduced to Equation (71). The equation can be further simplified if the inverse of the covariance matrix is taken being equal to the Tikhonov matrix, $\boldsymbol{\Sigma}_{\boldsymbol{a}}=\left(\boldsymbol{\Gamma}^{T} \boldsymbol{\Gamma}\right)^{-1}$ - reminding that this is the origin of the regularization matrix in the Bayesian perspective, as shown in section 3.3.1 General Bayesian estimation, Equation (41). The final result can be obtained by 
the generalized singular value decomposition of $\boldsymbol{X}$ and $\boldsymbol{\Gamma}$, achieving the A-optimal Bayesian design of experiments, Equation (72).

$$
\begin{gathered}
\min _{\boldsymbol{X}} E\left[\|\widehat{\boldsymbol{a}}-\boldsymbol{a}\|^{2}\right] \equiv \min _{\boldsymbol{X}} \alpha^{4} \operatorname{trace}\left(\boldsymbol{B} \boldsymbol{\Sigma}_{\boldsymbol{a}} \boldsymbol{B}^{T}\right)+\sigma^{2} \operatorname{trace}\left(\boldsymbol{X} \boldsymbol{C}^{-2} \boldsymbol{X}^{T}\right), \boldsymbol{B}=\boldsymbol{C}^{-1} \boldsymbol{\Gamma}^{T} \boldsymbol{\Gamma} \\
\min _{\boldsymbol{X}} E\left[\|\widehat{\boldsymbol{a}}-\boldsymbol{a}\|^{2}\right] \equiv \min _{\boldsymbol{X}} \operatorname{trace}\left(\boldsymbol{C}^{-1}\right)=\min _{\boldsymbol{X}} \operatorname{trace}\left(\left(\boldsymbol{X}^{T} \boldsymbol{X}+\alpha^{2} \boldsymbol{\Gamma}^{T} \boldsymbol{\Gamma}\right)^{-1}\right)
\end{gathered}
$$

It is important to emphasize that this result is based on the strong hypothesis that the prior is exactly the probability of each state, which was already discussed and does not hold true, since the prior can be used as regularization and only represents a subjective belief about the state, e.g., smoothness or low energy.

Despite this, the simple optimal design formulation is seductive, and it can be assumed that the subjective prior is similar enough to the real prior so as to produce good results and the same qualitative reasoning about the experiments, i.e., experiment $\eta_{1}$ will be better than $\eta_{2}$ either using the subjective prior or the real prior. Of course, the final verdict must be given by numerical and small scale experiments.

\subsubsection{Utility optimal design of experiments}

The squared error criterion is not necessarily the best criterion to select an experiment, and a general concept can be formulated as to maximize a utility function select appropriately for the experiment objectives.

In this sense, a design $\eta$ is chosen, a parameter $\boldsymbol{a}$ happens, and a measurement $\boldsymbol{y}$ is taken. After that, the experiment utility is verified as a function of each term, $U(\eta, \boldsymbol{a}, \boldsymbol{y})$. The optimal design is then the one that maximize the expected utility of the experiment, Equation (73).

$$
E[U(\eta)]=\iint U(\eta, \boldsymbol{a}, \boldsymbol{y}) p(\boldsymbol{y} \mid \boldsymbol{a}) p(\boldsymbol{a}) d \boldsymbol{a} d \boldsymbol{y}
$$

Using the utility framework, the A-optimal Bayesian design of experiment is obtained easily by defining $U(\eta, \boldsymbol{a}, \boldsymbol{y})=\|\widehat{\boldsymbol{a}}-\boldsymbol{a}\|^{2}$.

In this work, it is used a general utility in the form of a weighted error squared sum. Using this function, it is possible to give importance to the estimation of some 
parameters over others. The final expectation and the optimal design derived from it are shown in Equations (74), (75) and (76).

$$
\begin{gathered}
U(\eta, \boldsymbol{a}, \boldsymbol{y})=\|\boldsymbol{W}(\widehat{\boldsymbol{a}}-\boldsymbol{a})\|^{2}=(\widehat{\boldsymbol{a}}-\boldsymbol{a})^{T} \boldsymbol{W}_{2}(\widehat{\boldsymbol{a}}-\boldsymbol{a}), \quad \boldsymbol{W}_{2}=\boldsymbol{W}^{T} \boldsymbol{W} \\
E[U(\eta)]=-\iint(\widehat{\boldsymbol{a}}-\boldsymbol{a})^{T} \boldsymbol{W}_{2}(\widehat{\boldsymbol{a}}-\boldsymbol{a}) p(\boldsymbol{y} \mid \boldsymbol{a}) p(\boldsymbol{a}) d \boldsymbol{a} d \boldsymbol{y} \\
\max _{\eta} E[U(\eta)] \equiv \min _{\boldsymbol{X}} \operatorname{trace}\left(\boldsymbol{W}_{2} \boldsymbol{C}^{-1}\right)=\min _{\boldsymbol{X}} \operatorname{trace}\left(\boldsymbol{W}_{2}\left(\boldsymbol{X}^{T} \boldsymbol{X}+\alpha^{2} \boldsymbol{\Gamma}^{T} \boldsymbol{\Gamma}\right)^{-1}\right)
\end{gathered}
$$

This function is useful in a number of cases. A clear example happens when the parameters come from $l$ different and independent dynamic systems. In this case, defining $\alpha=0$ to simplify, the experiment matrix $\boldsymbol{X}$ will be a block diagonal matrix, Equations (77) and (78).

$$
\begin{aligned}
& \boldsymbol{X}=\left[\begin{array}{ccc}
\boldsymbol{X}_{1} & & \mathbf{0} \\
& \ddots & \\
\mathbf{0} & & \boldsymbol{X}_{l}
\end{array}\right] \rightarrow \boldsymbol{X}^{T}=\left[\begin{array}{ccc}
\boldsymbol{X}_{1}^{T} & & \mathbf{0} \\
& \ddots & \\
\mathbf{0} & & \boldsymbol{X}_{l}^{T}
\end{array}\right] \text { and } \boldsymbol{X}^{T} \boldsymbol{X}=\left[\begin{array}{ccc}
\boldsymbol{X}_{1}^{T} \boldsymbol{X}_{1} & & \mathbf{0} \\
& \ddots & \\
\mathbf{0} & & \boldsymbol{X}_{l}^{T} \boldsymbol{X}_{l}
\end{array}\right] \\
& \boldsymbol{X}^{T} \boldsymbol{X}=\left[\begin{array}{ccc}
\boldsymbol{X}_{1}^{T} \boldsymbol{X}_{1} & & \mathbf{0} \\
\mathbf{0} & & \boldsymbol{X}_{l}^{T} \boldsymbol{X}_{l}
\end{array}\right] \rightarrow\left(\boldsymbol{X}^{T} \boldsymbol{X}\right)^{-1}=\left[\begin{array}{ccc}
\left(\boldsymbol{X}_{1}^{T} \boldsymbol{X}_{1}\right)^{-1} & & \mathbf{0} \\
\mathbf{0} & \ddots & \left(\boldsymbol{X}_{l}^{T} \boldsymbol{X}_{l}\right)^{-1}
\end{array}\right]
\end{aligned}
$$

The weight matrix $\boldsymbol{W}$ can be chosen to be an identity matrix at a particular system position $i$, and zero elsewhere, Equation (79).

$$
\boldsymbol{W}=\left[\begin{array}{lll}
\mathbf{0} & & \mathbf{0} \\
& \boldsymbol{I}_{i} & \\
\mathbf{0} & & \mathbf{0}
\end{array}\right] \rightarrow \boldsymbol{W}_{2}=\boldsymbol{W}^{T} \boldsymbol{W}=\left[\begin{array}{lll}
\mathbf{0} & & \mathbf{0} \\
& \boldsymbol{I}_{i} & \\
\mathbf{0} & & \mathbf{0}
\end{array}\right]
$$

Consequently, the optimal design of experiments is given by Equation (80).

$$
\operatorname{trace}\left(\boldsymbol{W}_{2}\left(\boldsymbol{X}^{T} \boldsymbol{X}\right)^{-1}\right)=\operatorname{trace}\left(\left[\begin{array}{lll}
\mathbf{0} & & \mathbf{0} \\
& \left(\boldsymbol{X}_{i}^{T} \boldsymbol{X}_{i}\right)^{-1} & \\
\mathbf{0} & & \mathbf{0}
\end{array}\right]\right)=\operatorname{trace}\left(\left(\boldsymbol{X}_{i}^{T} \boldsymbol{X}_{i}\right)^{-1}\right)=
$$

This example illustrates how a proper choice of the weight matrix, when the dynamic system is composed by smaller independent systems, is capable of selecting a particular one and achieving the expected result, which would be obtainable by applying the A-optimal design only on the small system. 
In this work, the presented procedure allows the evaluation of the estimation capabilities for individual angular frequencies and individual directions, which is explained in further sections.

\subsubsection{Optimal design of experiments applied to input estimation}

Up to this point, the optimal design was applied to select the best experiment to estimate the parameters of the system. In this context, the procedure can be interpreted as choosing the inputs which excites the most the dynamic system, in order to have a glance of the influence of each parameter. In this work, however, the estimation is concerned about what was the input that caused a given measurement, and which degree of freedom to select to have the best estimation.

Since the mathematical formulations of both problems are equivalent, the optimal design can be reinterpreted as choosing the degrees of freedom most affected by the environment, in order to have a glance of the influence of each possible frequency and direction on the final response. Using this interpretation, it is possible to use the optimal design framework to select the degrees of freedom and positions of the wave-probes that result in the best estimation, reducing a problem naively solved by extensive simulations campaigns to a simple matrix calculation, Equations (81), (82) and (83).

$$
\begin{aligned}
\boldsymbol{A} \boldsymbol{x}=\boldsymbol{b}+\boldsymbol{\epsilon} & \equiv \boldsymbol{X} \boldsymbol{a}=\boldsymbol{y}+\boldsymbol{\epsilon} \\
\iint U(\eta, \boldsymbol{x}, \boldsymbol{b}) p(\boldsymbol{b} \mid \boldsymbol{x}) p(\boldsymbol{x}) d \boldsymbol{x} d \boldsymbol{b} & \equiv \iint U(\eta, \boldsymbol{a}, \boldsymbol{y}) p(\boldsymbol{y} \mid \boldsymbol{a}) p(\boldsymbol{a}) d \boldsymbol{a} d \boldsymbol{y} \\
\min _{\boldsymbol{A}} \operatorname{trace}\left(\boldsymbol{W}_{2}\left(\boldsymbol{A}^{T} \boldsymbol{A}+\alpha^{2} \boldsymbol{\Gamma}^{T} \boldsymbol{\Gamma}\right)^{-1}\right) & \equiv \min _{\boldsymbol{X}} \operatorname{trace}\left(\boldsymbol{W}_{2}\left(\boldsymbol{X}^{T} \boldsymbol{X}+\alpha^{2} \boldsymbol{\Gamma}^{T} \boldsymbol{\Gamma}\right)^{-1}\right)
\end{aligned}
$$

Finally, in the formulation used in this work, the optimal degrees-of-freedom selection is given by Equation (84).

$$
O D o F=\min _{\boldsymbol{A}} \operatorname{trace}\left(\boldsymbol{W}_{2}\left(\boldsymbol{A}^{T} \boldsymbol{A}+\left[u_{1}^{2} \boldsymbol{H}_{1}+u_{2}^{2} \boldsymbol{H}_{2}+u_{3}^{2} \boldsymbol{H}_{3}\right]\right)^{-1}\right)
$$

A general survey about optimal sensor placement, including the A-optimal Bayesian Design, can be found in (KRAUSE, et al., 2008). 


\section{PROPOSED METHODS}

In this section, the methods proposed in the present work are described, applying the theoretical background previously explained to solve the directional wave estimation problem.

\subsection{The Extended Linear Model}

The first problem which is solved by this work is how to incorporate the waveprobe measurements in the traditional Bayesian estimation algorithm.

The problem seems difficult when the estimation is interpreted as the inverse of the dynamical behavior of the vessel, but it is trivial if the vessel is simply thought as a sensor, with the RAOs as the state observer of the wave amplitudes. Using this perspective, the wave-probe measurements are new sensors, which need just to be added to the observation matrix.

At the same time, if the dynamic behavior is put into perspective, the waveprobes can be seen as new degrees of freedom of the system, with its own transfer function for each regular wave, in a way that the linear model must be simply expanded.

Reassuring the similarity between the wave-elevation pattern and the vessel dynamic response, it is convenient to recover the vessel dynamics, Equation (85), the general form of the domain potential, Equation (86), and the free surface elevation in a chosen point, Equation (87), using as input a single progressive plane wave without loss of generality, as the superposition principle is always valid for linear systems.

$$
\begin{gathered}
\xi=A \cdot \boldsymbol{R A O}(\omega, \beta) \\
\varphi(x, y, z, t)=\operatorname{Re}\left\{\left[\sum_{j=1}^{6} \xi_{j} \varphi_{j}(x, y, z)+A\left(\varphi_{0}(x, y, z)+\varphi_{7}(x, y, z)\right)\right] e^{i \omega t}\right\}= \\
=\operatorname{Re}\left\{\left[\left\{\varphi_{j}(x, y, z)\right\}^{T} \cdot \xi+A\left(\varphi_{0}(x, y, z)+\varphi_{7}(x, y, z)\right)\right] e^{i \omega t}\right\}= \\
=A \cdot \operatorname{Re}\left\{\left[\left\{\varphi_{j}(x, y, z)\right\}^{T} \cdot \boldsymbol{R A O}(\omega, \beta)+\left(\varphi_{0}(x, y, z)+\varphi_{7}(x, y, z)\right)\right] e^{i \omega t}\right\}= \\
=A \cdot \bar{\varphi}(x, y, z, t)
\end{gathered}
$$




$$
\zeta(x, y, t)=-\left.\frac{1}{g} \frac{\partial \varphi}{\partial t}\right|_{z=0}=A \cdot\left(-\left.\frac{1}{g} \frac{\partial \bar{\varphi}}{\partial t}\right|_{z=0}\right)=A \cdot f(\omega, \beta)
$$

The function $f(\omega, \beta)$, which linearly relates the surface elevation with the wave amplitude, is, by definition, a response amplitude operator. As a consequence of this fact, if arbitrary points are chosen to represent the surface elevation, the equations can be written as a single linear system, with a more general RAO matrix, Equation (88).

$$
\left[\begin{array}{l}
\xi \\
\zeta
\end{array}\right]=A \cdot\left[\begin{array}{c}
\boldsymbol{R A O}_{\text {vessel }} \\
\boldsymbol{R A O _ { \text { wave-elevation } }}
\end{array}\right]
$$

In the same way, it is possible to expand the cross-spectra formulation, but now with the general RAO matrix and the general cross-correlation, $\phi_{i j}(\omega)$, not only among the vessel DoFs but also among the wave-elevation measurements, Equation (89).

$$
\phi_{i j}(\omega) \approx \sum_{k=1}^{K} R A O_{i}(\omega, \beta) \cdot R A O_{j}^{*}(\omega, \beta) \cdot S(\omega, \beta) \Delta \beta, \quad i, j=\text { DoFs }+ \text { Probes }
$$

Even though the wave-elevation is entirely calculated by Wamit ${ }^{\circledR}$, the waveprobe measurements differ significantly from it due to the vessel movements. This happens because some of the vessel movements, mainly when transmitted to the wave-probes, have the same magnitude as the surface elevation, interfering directly with the height measured by the sensor. A simple example is when the vessel is excited by large period waves with wave length greater than the vessel length, and, as a consequence, the wave-hull elevation presents almost no variation, since the vessel follows the wave, Figure 22. On the other hand, for high frequencies, the vessel will present a small motion-response but the wave-hull elevation is very similar to the actual wave elevation; remembering that the wave elevation is the summation of the incoming wave amplitude, the diffraction and the irradiation, due to the presence of the vessel.

Figure 22 - Low-frequency waves versus high-frequency waves.
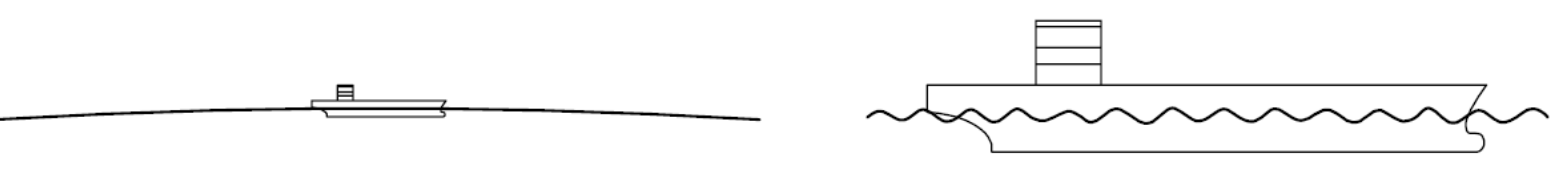

Source: (NIELSEN, 2005). 
The general influences of each degree of freedom in the wave-probe measurement are shown in Figure 23 to Figure 28, in which $W P_{\text {meas. }}$ is the wave-probe measurement, i.e., the distance between the sensor and the water surface, and it is a function of the wave elevation $\zeta$ and the vessel movements $\xi$.

Figure 23 - Wave-probe measurement affected by the surge movement.

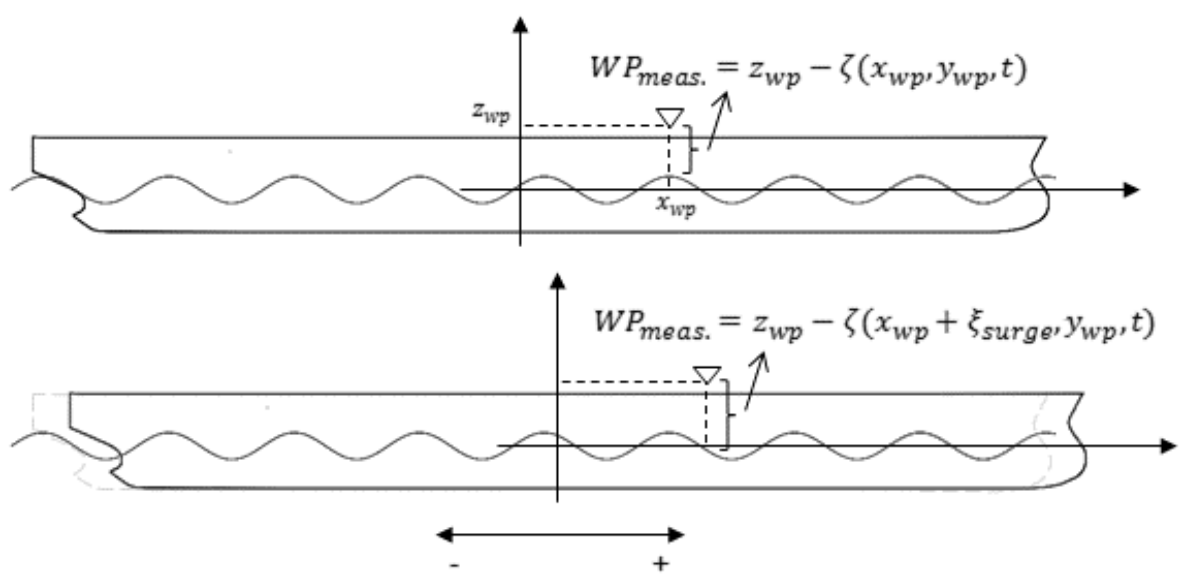

Source: Elaborated by the author.

Figure 24 - Wave-probe measurement affected by the sway movement.

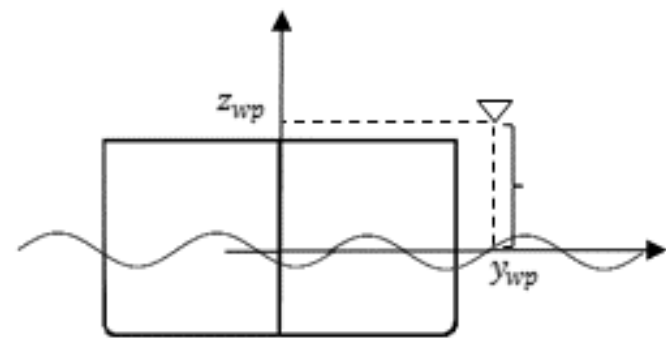

$W P_{\text {meas. }}=z_{w p}-\zeta\left(x_{w p}, y_{w p}, t\right)$

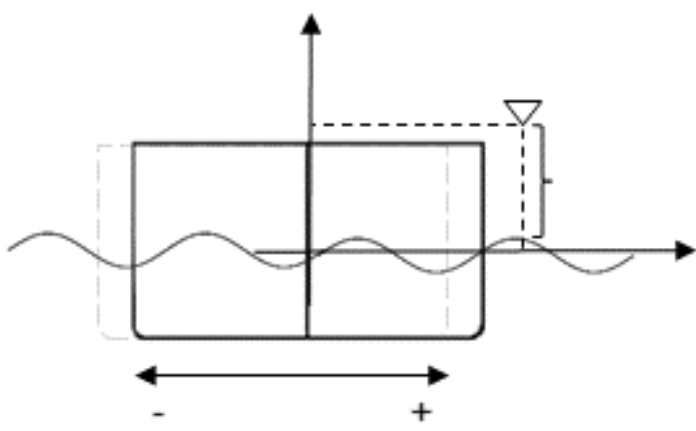

$W P_{\text {meas. }}=z_{w p}-\zeta\left(x_{w p}, y_{w p}+\xi_{\text {sway }}, t\right)$

Source: Elaborated by the author.

Figure 25 - Wave-probe measurement affected by the heave movement.

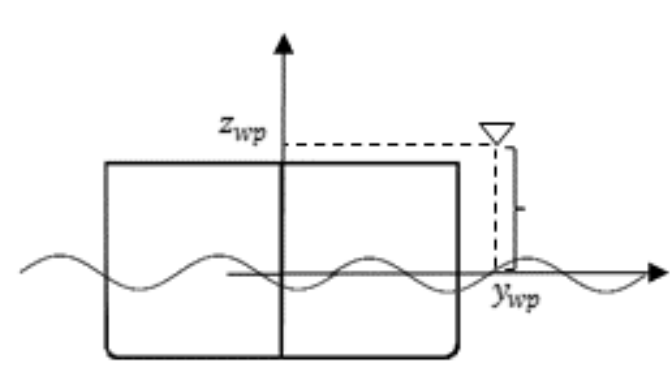

$W P_{\text {meas. }}=z_{w p}-\zeta\left(x_{w p}, y_{w p}, t\right)$

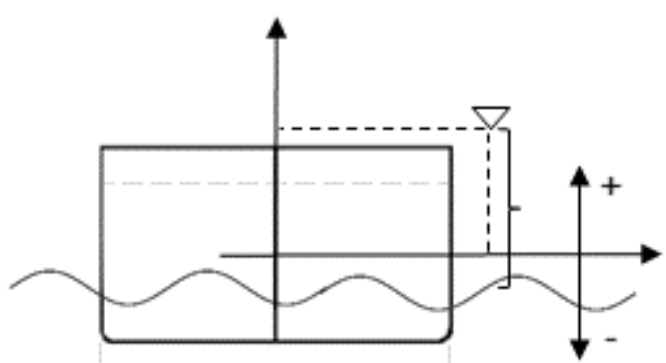

$W P_{\text {meas. }}=z_{w p}-\zeta\left(x_{w p}, y_{w p}, t\right)+\xi_{\text {heave }}$

Source: Elaborated by the author. 
Figure 26 - Wave-probe measurement affected by the roll movement.
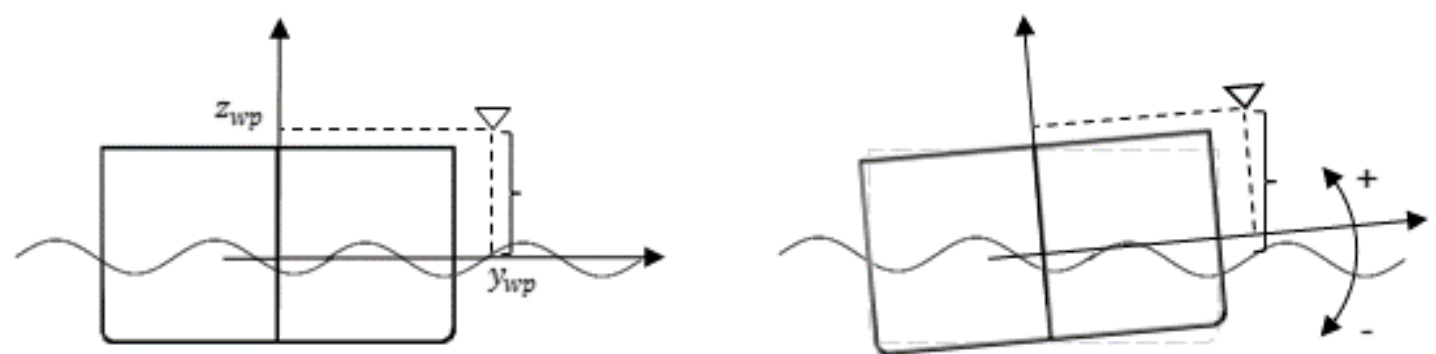

$$
W P_{\text {meas. }}=z_{w p}-\zeta\left(x_{w p}, y_{w p}, t\right) \quad W P_{\text {meas. }}=z_{w p}-\zeta\left(x_{w p}, y_{w p}, t\right)+\xi_{\text {roll }} \cdot y_{w p}
$$

Source: Elaborated by the author.

Figure 27 - Wave-probe measurement affected by the pitch movement.

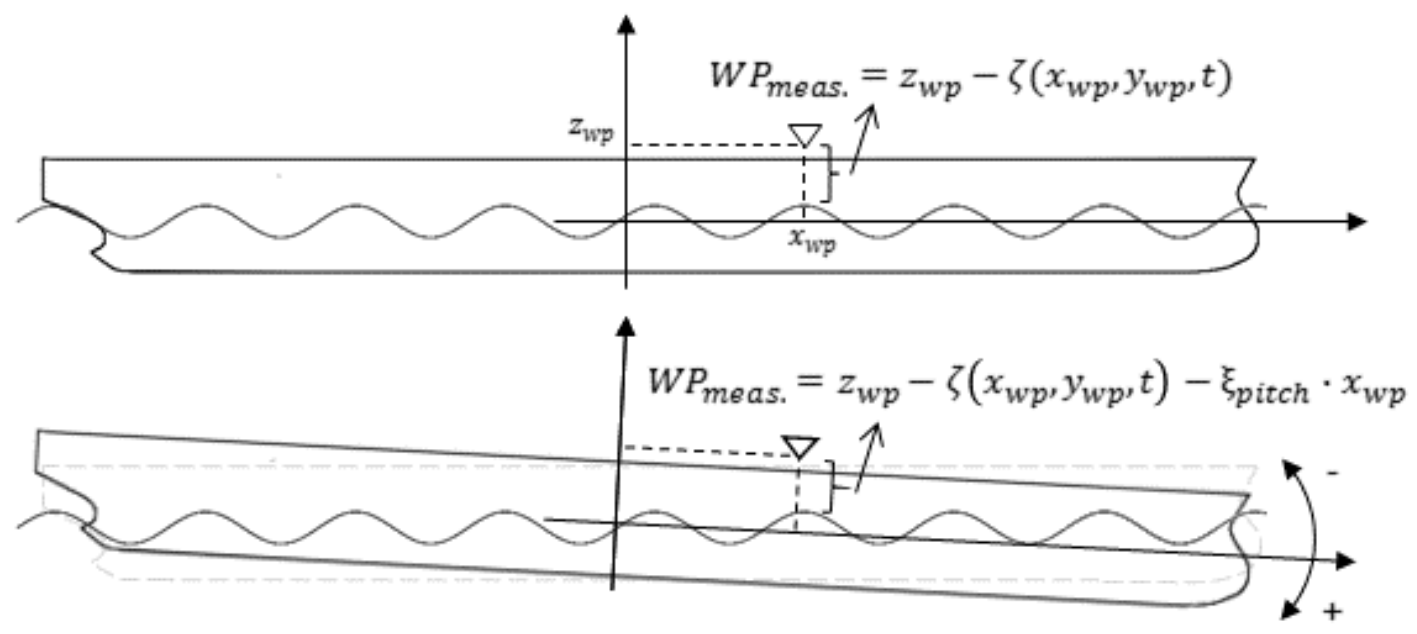

Source: Elaborated by the author.

Figure 28 - Wave-probe measurement affected by the yaw movement.

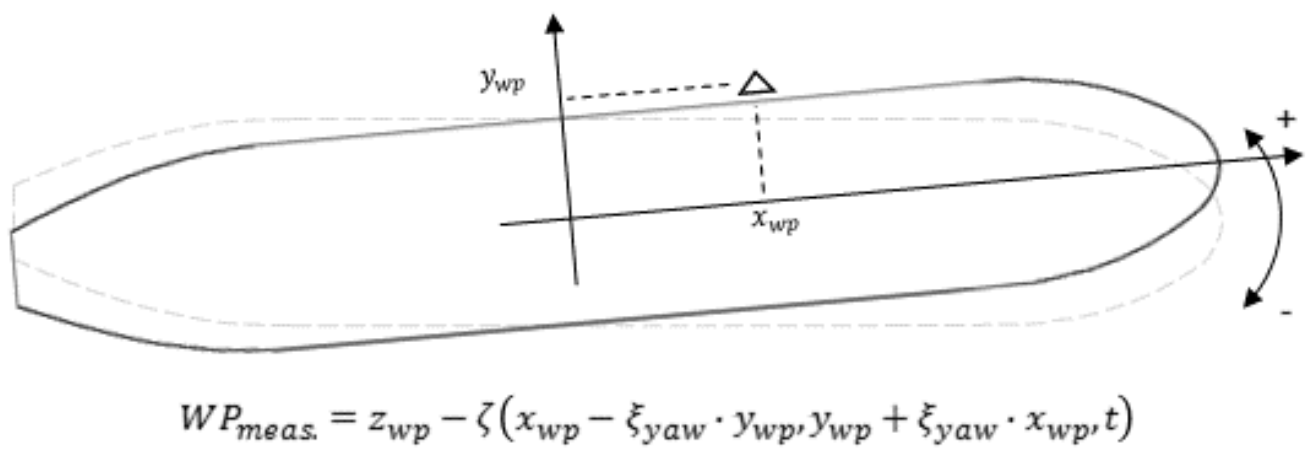

Source: Elaborated by the author. 
Despite the non-linearity of the influence of the vessel motions due to the change in the position of the wave elevation measurement, $\zeta$, it can be shown that only the vertical motions have contributions in the same order of magnitude of the wave elevation and the influence of them can be linearized. This approach, and the entire underlying hypothesis, are validated numerically in APPENDIX B - Superposition of Vessel Motions.

In conclusion, only the heave, the roll and the pitch movements are used, and linear corrections are enough to describe their influence. It means that a choice can be made: the wave-probe measurement time series can be processed and converted to the wave-elevation time series by subtracting the measurements from the other movements, in a way that the estimation is based on the wave-elevation RAOs; or RAOs for the wave-probe measurements can be previously calculated and directly incorporated into the estimation, dismissing the need of pre-processing the time series.

In this text, the wave-probe RAOs are used, avoiding errors due to phase shifts in the sensors series, and the conversion between the wave-elevation RAOs and the wave-probe RAOs are shown in Equations (90), (91) and (92).

$$
\begin{aligned}
& {\left[\begin{array}{l}
\xi_{\text {vessel }} \\
\xi_{\text {probe }}
\end{array}\right]=\left[\begin{array}{ll}
I & 0 \\
X & I
\end{array}\right]\left[\begin{array}{c}
\xi_{\text {vessel }} \\
\zeta_{\text {elevation }}
\end{array}\right]=A \cdot\left[\begin{array}{cc}
I & 0 \\
X & I
\end{array}\right]\left[\begin{array}{c}
R A O_{\text {vessel }} \\
R A O_{\text {elevation }}
\end{array}\right]=A \cdot\left[\begin{array}{l}
R A O_{\text {vessel }} \\
R A O_{\text {probe }}
\end{array}\right]}
\end{aligned}
$$

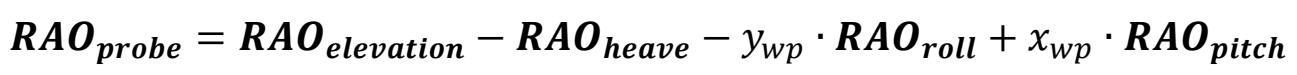

$$
\begin{aligned}
& \boldsymbol{X}_{i}=\left[\begin{array}{llllll}
0 & 0 & -1 & -y_{w p_{i}} & +x_{w p_{i}} & 0
\end{array}\right]
\end{aligned}
$$

\subsection{Positioning and Selecting the Number of Probes}

The second issue this work discusses is how to choose the best wave-probe positions, and how to decide, in a reasonable manner, how many probes to employ.

Restating the estimation algorithm, Equations (93) and (94), the problem can be reformulated as to choose the $\boldsymbol{A}$ matrix, formed by the RAOs of each generalized degree of freedom, which results in the best estimation. However, what is a "good estimation' will be always subjective, as it depends on the adopted criterion.

$$
\begin{gathered}
\min _{\boldsymbol{x}} J(\boldsymbol{x})=\|\boldsymbol{A} \boldsymbol{x}-\boldsymbol{b}\|^{2}+\boldsymbol{x}^{T}\left[u_{1}^{2} \boldsymbol{H}_{1}+u_{2}^{2} \boldsymbol{H}_{2}+u_{3}^{2} \boldsymbol{H}_{3}\right] \boldsymbol{x} \\
\widehat{\boldsymbol{x}}=\left(\boldsymbol{A}^{T} \boldsymbol{A}+\left[u_{1}^{2} \boldsymbol{H}_{1}+u_{2}^{2} \boldsymbol{H}_{2}+u_{3}^{2} \boldsymbol{H}_{3}\right]\right)^{-1} \boldsymbol{A}^{T} \boldsymbol{b}
\end{gathered}
$$


A simple criterion is the expected squared error, Equation (95), discussed in section 3.5.2 Bayesian optimal design of experiments, which is enough to choose which vessel degrees of freedom to employ in the usual estimation, as it is shown in a FPSO example in Table 1.

$$
\min _{\boldsymbol{A}} E\left[\|\widehat{\boldsymbol{x}}-\boldsymbol{x}\|^{2}\right]=\min _{\boldsymbol{A}} \operatorname{trace}\left(\left(\boldsymbol{A}^{T} \boldsymbol{A}+\left[u_{1}^{2} \boldsymbol{H}_{1}+u_{2}^{2} \boldsymbol{H}_{2}+u_{3}^{2} \boldsymbol{H}_{3}\right]\right)^{-1}\right)
$$

Table 1 - Trace criterion for all possible 3DoF combination of a FPSO employed in a conventional Bayesian estimation, with periods ranging from $8 \mathrm{sec}$. to $30 \mathrm{sec}$., from the best to the worst.

\section{Degrees of Freedom Combination}

Sway-Heave-Pitch

Sway-Heave-Surge

Roll-Heave-Pitch

Roll-Heave-Surge

Roll-Surge-Pitch

Sway-Surge-Pitch

Sway-Roll-Heave

Sway-Roll-Surge

Sway-Roll-Pitch

Heave-Surge-Pitch

\section{Trace Criterion}
$0.016010^{9}$

$0.021810^{9}$

$0.022010^{9}$

$0.023310^{9}$

$0.026210^{9}$

$0.027610^{9}$

$0.042910^{9}$

$0.046610^{9}$

$0.047110^{9}$

$0.703010^{9}$

Source: Elaborated by the author.

The example illustrates how the method is capable of suggesting bases similar to the ones obtained by (TANNURI, et al., 2003), (NIELSEN, 2005) and (LAJIC, 2010), using arguments based on the odd and even properties of each DoF. Furthermore, it classifies the different bases in a clear way, in which changing similar DoFs, e.g., rollsway or pitch-surge, does not imply much worsening, but keeping only DoFs that have similar properties, removing roll and sway, for example, causes a fast deterioration of the estimation capabilities, since the rest of the DoFs act as equations that are a linear combination of the others, and do not aggregate anything to the linear system. 
When wave-probes are added, however, the average criterion may not be enough to decide the best configuration, as it 'masks' some undesirable behaviors. One of them is to add probes that improve the most the estimation of periods that are inside the vessel estimation capabilities, which is against the main objective of enlarging the range of estimated frequencies. Other problem is probe configurations that have blind spots, i.e., some incoming directions that have poor estimations capabilities, and can only be perceived by intensive campaigns simulations, if an average criterion is used.

Those problems are solved by using the utility design of experiments to set a multi-objective criterion, which is capable of evaluating each angular frequency and incoming direction independently. The values calculated for each frequency are, then, grouped and three objective functions are defined: the maximum trace among all the frequencies, the standard deviation of the traces and the average trace; and the same is done for the incoming directions, totalizing six objective functions. After a proper choice of the optimum, this guarantees that the estimation will be well behaved in all the possible seas. The objective functions are presented in Equations (96) to (102).

$$
\begin{aligned}
& \min _{\boldsymbol{A}} \operatorname{MAX}\left(\operatorname{trace}\left(\boldsymbol{W}_{\boldsymbol{l}_{2}}\left(\boldsymbol{A}^{T} \boldsymbol{A}+\left[u_{1}^{2} \boldsymbol{H}_{1}+u_{2}^{2} \boldsymbol{H}_{2}+u_{3}^{2} \boldsymbol{H}_{3}\right]\right)^{-1}\right)\right) \\
& \min _{\boldsymbol{A}} \operatorname{AVERAGE}\left(\operatorname{trace}\left(\boldsymbol{W}_{\boldsymbol{l}_{2}}\left(\boldsymbol{A}^{T} \boldsymbol{A}+\left[u_{1}^{2} \boldsymbol{H}_{1}+u_{2}^{2} \boldsymbol{H}_{2}+u_{3}^{2} \boldsymbol{H}_{3}\right]\right)^{-1}\right)\right) \\
& \min _{\boldsymbol{A}} \operatorname{STD}\left(\operatorname{trace}\left(\boldsymbol{W}_{\boldsymbol{l}_{2}}\left(\boldsymbol{A}^{T} \boldsymbol{A}+\left[u_{1}^{2} \boldsymbol{H}_{1}+u_{2}^{2} \boldsymbol{H}_{2}+u_{3}^{2} \boldsymbol{H}_{3}\right]\right)^{-1}\right)\right) \\
& \boldsymbol{W}_{\boldsymbol{l}_{2}}=\boldsymbol{W}_{\boldsymbol{l}}^{T} \boldsymbol{W}_{\boldsymbol{l}}, \quad \boldsymbol{W}_{\boldsymbol{l}}=\left\{w_{i j}\right\} \\
& \boldsymbol{x}_{m}=\left[\begin{array}{c}
S\left(\omega_{m}, \beta_{1}\right) \\
\vdots \\
S\left(\omega_{m}, \beta_{k}\right) \\
\vdots \\
S\left(\omega_{m}, \beta_{K}\right)
\end{array}\right], \quad \boldsymbol{x}=\left\{\begin{array}{c}
\boldsymbol{x}_{1} \\
\vdots \\
\boldsymbol{x}_{m} \\
\vdots \\
\boldsymbol{x}_{M}
\end{array}\right\} \\
& w_{i j}=1 \text { if } \boldsymbol{l} \cdot K \leq i=j \leq \boldsymbol{l} \cdot(K+1), \quad \text { for the } \boldsymbol{l}^{\text {th }} \text { frequency } \\
& w_{i j}=1 \text { if } i=j=n K+\boldsymbol{l}, n=0, \ldots, M, \quad \text { for the } \boldsymbol{l}^{\text {th }} \text { direction }
\end{aligned}
$$

Lastly, the choice of the number of the probes is solved by using the 'elbow' criterion in a similar way to its usage for clusters, section 3.4.2 'Elbow' criterion. In this case, the value of the trace will decrease until the estimation system becomes fully 
determined. After that, a plateau happens, in which the trace decrease is only due to the addition of more redundancies, reducing the variance of the estimation, which is expected under the hypothesis of independent noise in the measurements.

Even if a completely determined system is not obtainable, because of the great number of sensors it would demand, for example, the 'elbow' plot can also be used to evaluate how much improvement one is not having by not employing a new sensor.

\subsection{Optimization Heuristic}

The proposed objective functions, and the optimal design of experiments in general, are discrete, highly non-linear and non-convex. It means that numerical gradient-based optimizations, as Sequential Quadratic Programming (SQP), cannot be applied in a satisfactory manner, since it can be stuck in local minima. Furthermore, those algorithms are not easily employed in multi-objective problems.

Since the exhaustive search is prohibitive, a number of different algorithms has been used, the so called global optimization algorithms: simulated annealing, genetic algorithms, tabu search and branch-and-bound approaches; (KRAUSE, et al., 2008). However, a heuristic approach is proposed in this text, which was found to perform better due to the idiosyncrasies of the problem.

In general, the presented algorithms work by evaluating a possible solution, or a set of possible solutions, and then figuring out, using a rationale, the next solution to be evaluated, probably best than the previous. Differently than the gradient-based algorithms, the global search algorithms usually accept worse solutions for a moment, accordingly to some criteria, avoiding being captured by a local minimum. Despite the name "global optimization algorithms", it is important to emphasize that there is no guarantee that the algorithms will, indeed, find the global minimum in a finite time.

The simulated annealing algorithm is based on thermodynamic ideas. Using the concept of 'temperature', the algorithm 'jumps' from one possible solution to other. The higher the temperature, the larger can be the jumps, and the worse can be the accepted new solutions. During each step the temperature is lowered, in a way that the algorithm will, hopefully, converge to the best solution, since the jumps will get lower and lower, and only the best solutions will start to be accepted. 
The genetic algorithm is based on the Darwinist evolution theory. Starting from a population of possible solutions, which is called the parents, its properties are combined to form a new population. A parent with a better objective function will have more probability of have its characteristics passed to a new generation, which provides the optimization property of the algorithm. Essentially, after some generations, the algorithm will have internally learned successfully strategies of the particular problem, and only the best individuals will be in the population.

On the one hand, the previously presented algorithms try to walk freely on the solution domain, choosing a path which will possibly reach the best solution; on the other hand, tabu search and branch-and-bound algorithms usually try to exhaust the domain in an intelligent way, avoiding the verification of regions that are known to be always worse. The tabu search stores in the memory previously visited solutions, avoiding unnecessary spending of resources; and branch-and-bound tries to enumerate all the solutions and to group similar ones together, dismissing bad groups by evaluating their maximum and minimum value, or its bounds. As a consequence of their methods, these algorithms are usually computationally expensive.

However, there is a property of the optimal design of experiments that can be used to accelerate the branch-and-bound algorithm: adding a new experiment, or DoF, that is a linear combination of the others will not modify the objective function significantly. It means that, if there is already some possible DoFs and the algorithm must choose the last DoF to be added, instead of verifying each of possible new DoFs, it is possible to verify if a group, consisted about the average of some of the remaining DoF values, will increase the objective function. If that is true, it is known that the best DoF will be inside the group; if it is false, it is known that the group can be dismissed. Essentially, a divide and conquer strategy.

A naively method would consist about using all the DoFs in a first evaluation, and then removing one by one selecting, in each step, the DoF that gives the least improvement in the objective function, until the desired number of DoFs is found. This would mean that, if $n$ DoFs are possible and $k$ DoFs are desired, $n+(n-1)+\cdots+$ $(k+1)=\frac{(n+k+1) \cdot(n-k)}{2}$ evaluations would be necessary, considerably less than all the possible combinations $\frac{n !}{(n-k) ! k !}$. If one hundred possible wave-probe positions were 
evaluated and five positions were desired, for example, the exhaustive search would mean 75.3 million cases, and the improved method only five thousand.

The approach, however, has two problems. The first one is that it will not always reach the best solution, as shown in the example in Table 2.

Table 2 - Example of the naive method.

\begin{tabular}{cc}
\hline Matrix & Trace Criterion \\
\hline $\boldsymbol{A}=\left[\begin{array}{c}\boldsymbol{A}_{1} \\
\vdots \\
\boldsymbol{A}_{i} \\
\vdots \\
\boldsymbol{A}_{n}\end{array}\right]=\left[\begin{array}{ccc}1 & 0 & 0 \\
0 & 1 & 0 \\
0 & 0 & 1 \\
0 & 0.8 & 0.8\end{array}\right]$ & $\operatorname{trace}\left(\left(\boldsymbol{A}^{T} \boldsymbol{A}+\alpha^{2} \boldsymbol{\Gamma}^{T} \boldsymbol{\Gamma}\right)^{-1}\right)=2.6424$ \\
$\alpha^{2} \cdot \boldsymbol{\Gamma}^{T} \boldsymbol{\Gamma}=0.01 \cdot \boldsymbol{I}_{3 \times 3}$ & \\
\hline
\end{tabular}

First Step: Remove the least significant DoF

$$
\begin{array}{ccc}
\boldsymbol{A} & =\left[\begin{array}{l}
\boldsymbol{A}_{1} \\
\boldsymbol{A}_{2} \\
\boldsymbol{A}_{3}
\end{array}\right]=\left[\begin{array}{lll}
1 & 0 & 0 \\
0 & 1 & 0 \\
0 & 0 & 1
\end{array}\right] & \mathrm{tr}=2.9703 \text { best } \\
\boldsymbol{A}=\left[\begin{array}{l}
\boldsymbol{A}_{1} \\
\boldsymbol{A}_{2} \\
\boldsymbol{A}_{4}
\end{array}\right] \text { or }\left[\begin{array}{l}
\boldsymbol{A}_{1} \\
\boldsymbol{A}_{3} \\
\boldsymbol{A}_{4}
\end{array}\right]=\left[\begin{array}{ccc}
1 & 0 & 0 \\
0 & 1 & 0 \\
0 & 0.8 & 0.8
\end{array}\right] & t r=4.4597 \\
\boldsymbol{A}=\left[\begin{array}{l}
\boldsymbol{A}_{2} \\
\boldsymbol{A}_{3} \\
\boldsymbol{A}_{4}
\end{array}\right]=\left[\begin{array}{ccc}
0 & 1 & 0 \\
0 & 0 & 1 \\
0 & 0.8 & 0.8
\end{array}\right] & t r=101.4268
\end{array}
$$

DoF 4 is the least significant DoF

Second Step: Remove the least significant DoF

$$
\boldsymbol{A}=\left[\begin{array}{l}
\boldsymbol{A}_{1} \\
\boldsymbol{A}_{2}
\end{array}\right] \text { or }\left[\begin{array}{l}
\boldsymbol{A}_{1} \\
\boldsymbol{A}_{3}
\end{array}\right] \text { or }\left[\begin{array}{l}
\boldsymbol{A}_{2} \\
\boldsymbol{A}_{3}
\end{array}\right]=\left[\begin{array}{lll}
1 & 0 & 0 \\
0 & 1 & 0
\end{array}\right] \quad \quad t r=101.9802
$$

All remaining solutions are equal

However, the best solution HAS the DoF 4

$$
\begin{array}{cc}
\boldsymbol{A}=\left[\begin{array}{l}
\boldsymbol{A}_{1} \\
\boldsymbol{A}_{2}
\end{array}\right] \text { or }\left[\begin{array}{l}
\boldsymbol{A}_{1} \\
\boldsymbol{A}_{3}
\end{array}\right] \text { or }\left[\begin{array}{l}
\boldsymbol{A}_{2} \\
\boldsymbol{A}_{3}
\end{array}\right]=\left[\begin{array}{lll}
1 & 0 & 0 \\
0 & 1 & 0
\end{array}\right] & \operatorname{tr}=101.9802 \\
\boldsymbol{A}=\left[\begin{array}{l}
\boldsymbol{A}_{1} \\
\boldsymbol{A}_{4}
\end{array}\right]=\left[\begin{array}{ccc}
1 & 0 & 0 \\
0 & 0.8 & 0.8
\end{array}\right] & \operatorname{tr}=101.7653 \text { best }
\end{array}
$$

Source: Elaborated by the author. 
This problem arises when, in an intermediate step, one DoF is the linear combination of other couple DoFs that are still in the group but will be removed later. Since only one DoF remains, the DoF that is the linear combination would give a better result, as the other DoFs need each other to complement their response.

The second problem arises when the computational cost of the algorithm is evaluated. It is much more expensive to evaluate a matrix with one hundred DoFs than a matrix with five DoFs, as it is shown in Figure 29 - evaluation of a single period, with 36 incoming directions. The computations were made in a notebook with Intel Core i75500U, 2.4GHz Dual-Core processor; 16Gb of DDR3 RAM, 1600MHz; Windows 10, 64 bits; and Matlab ${ }^{\circledR} 2016$ a.

Figure 29 - Experimental computation cost and polynomial fitting.

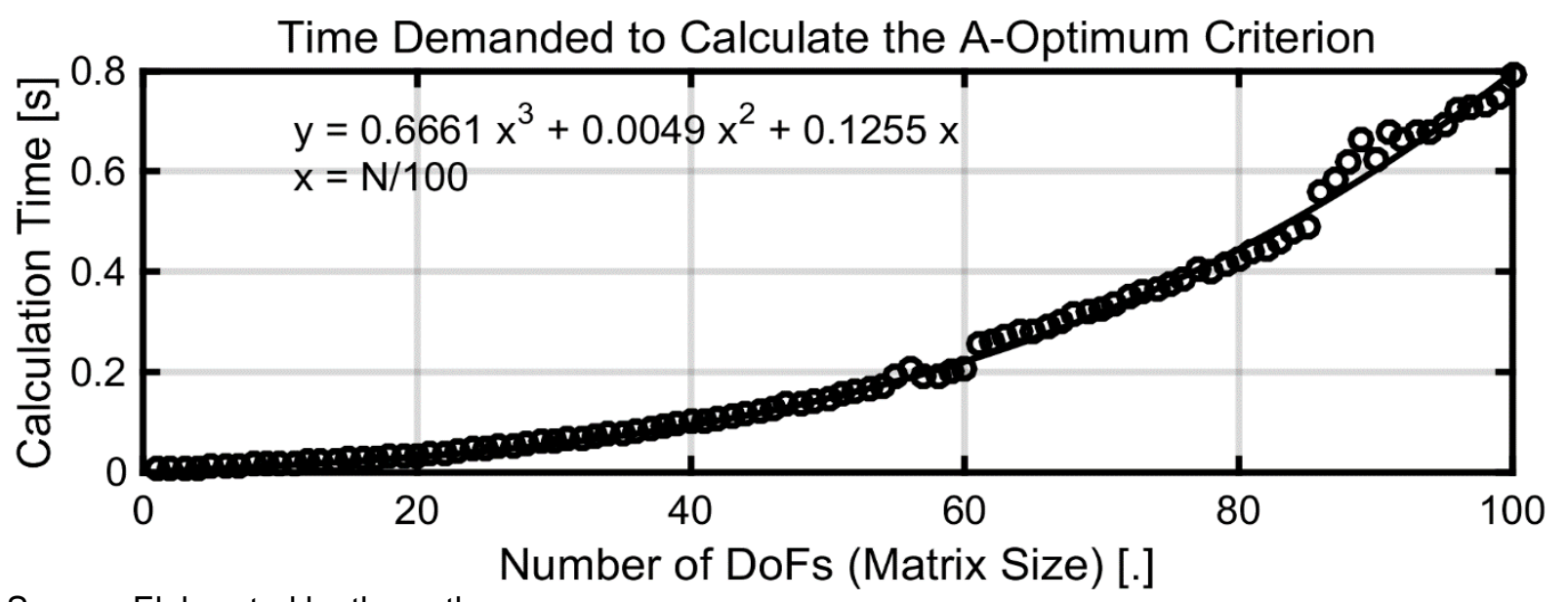

Source: Elaborated by the author.

Using the same previously illustrative example, if one hundred possible waveprobe positions were evaluated and five positions were desired, the total computation time to evaluate the five thousand cases would be around 1770.3 seconds times the number of wave periods, which is enough to evaluate 158486 matrices with 5-DoF combinations, more than 30 times more evaluations.

The heuristic proposed in this thesis is capable of lowering the optimization time using the same idiosyncrasy presented, but grouping together similar DoFs and evaluating all the combinations among the groups as if they, characterized by the average of its DoFs, were the real DoF. In that manner, only matrices with the needed number of DoFs are used and, even if the number of evaluated combinations is greater, the total computation time is lower. 
Essentially, two hypotheses are made:

1. The best solution will contain DoFs that have the most different responses among them: it means that, if there is a group of similar wave-probes, the best solution WILL NOT contain more than one of them, i.e., one is enough because adding another results in redundancy.

2. The average response of a group of similar DoFs is enough to represent the influence of the best of its DoFs: since the average is the linear combination of all the DoFs, if a particular response of one of the grouped DoFs is needed to determine the system, the average is enough to indicate that the needed response is among the DoFs of the group.

One possible example of the first hypothesis is to choose among the following DoFs: $\left[\begin{array}{lll}0 & 0 & 0.9\end{array}\right]$, $\left[\begin{array}{lll}0 & 0 & 1\end{array}\right]$ and $\left[\begin{array}{lll}0 & 0 & 1.1\end{array}\right]$. In a scenario of limited resource, i.e., more variables than equation, to choose ONLY the third one is the best approach, as the others will only add redundancy because their similarity, but will not add more estimation capability to the system, as they are very similar among them. The choice of the highest value is always justified because it results in the best information-noise ratio, since all the noise is independent and of equal amplitude in a well-conditioned estimation problem; if that is not true, a weigh matrix must be used in the likelihood to guarantee the probabilistic interpretation.

One possible example of the second hypothesis is a system that already has two DoFs defined and need to choose the last one. Using the system $\left[\begin{array}{lll}1 & 0 & 0 \\ 0 & 1 & 0\end{array}\right]$, and choosing among DoFs organized in two groups: $G_{1}=\left\{\left[\begin{array}{lll}0.9 & 0.1 & 0\end{array}\right],\left[\begin{array}{lll}1.1 & 0.04 & 0\end{array}\right]\right\}$ and $G_{2}=\left\{\left[\begin{array}{lll}0.1 & 0.9 & 0\end{array}\right],\left[\begin{array}{lll}0 & 0.85 & 0.03\end{array}\right],\left[\begin{array}{lll}0.2 & 0.95 & 0\end{array}\right]\right\}$, it is obvious that the best solution is achieved by choosing the second element of group 2, since it determines the system because it is the only one that has a value in the third column. In this case, if the average of each group is calculated, $\overline{G_{1}}=\left[\begin{array}{lll}1 & 0.07 & 0\end{array}\right]$ and $\overline{G_{2}}=$ $\left[\begin{array}{lll}0.1 & 0.9 & 0.01\end{array}\right]$, it becomes clear how using the average of each group can be used to evaluate the potential of their members, as all the possible information they can provide is taken in account. This example also illustrates the reason because the average is preferable against the simple summation, as the summation would benefit large groups, giving the wrong impression of a greater information-noise ratio. 
In the particular case of choosing the best wave-probe positions, the clustering technique is more intuitive, as it will group together wave-probes that are close to each other and the average response is, usually, very similar to the response of the waveprobe that is in the middle of the group. An example with one hundred possible positions grouped together in twenty four clusters is given in Figure 30, in which each cluster is represented by one color, and the average position of the cluster is represented by a black X.

Figure 30 - Example of clustered wave-probes.

Wave-Probe Clusters

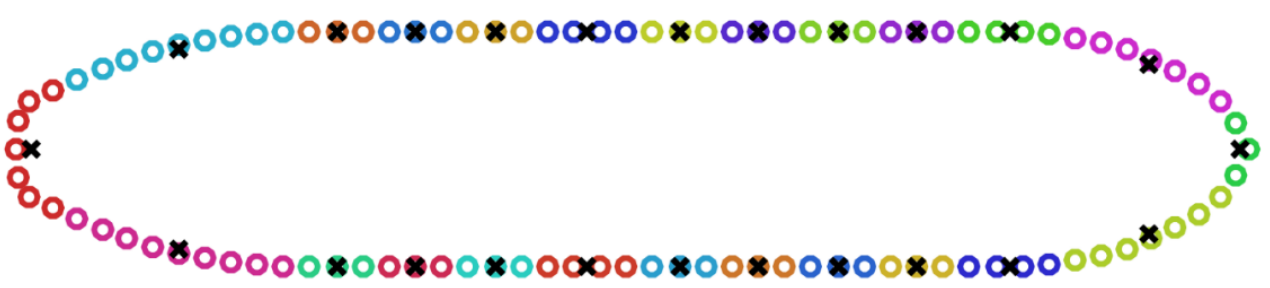

Source: Elaborated by the author.

The final algorithm is presented in the following block diagrams. The first one presents the pre-processing step, Figure 31.

Figure 31 - Pre-processing before the optimization algorithm.

PRE-PROCESSING

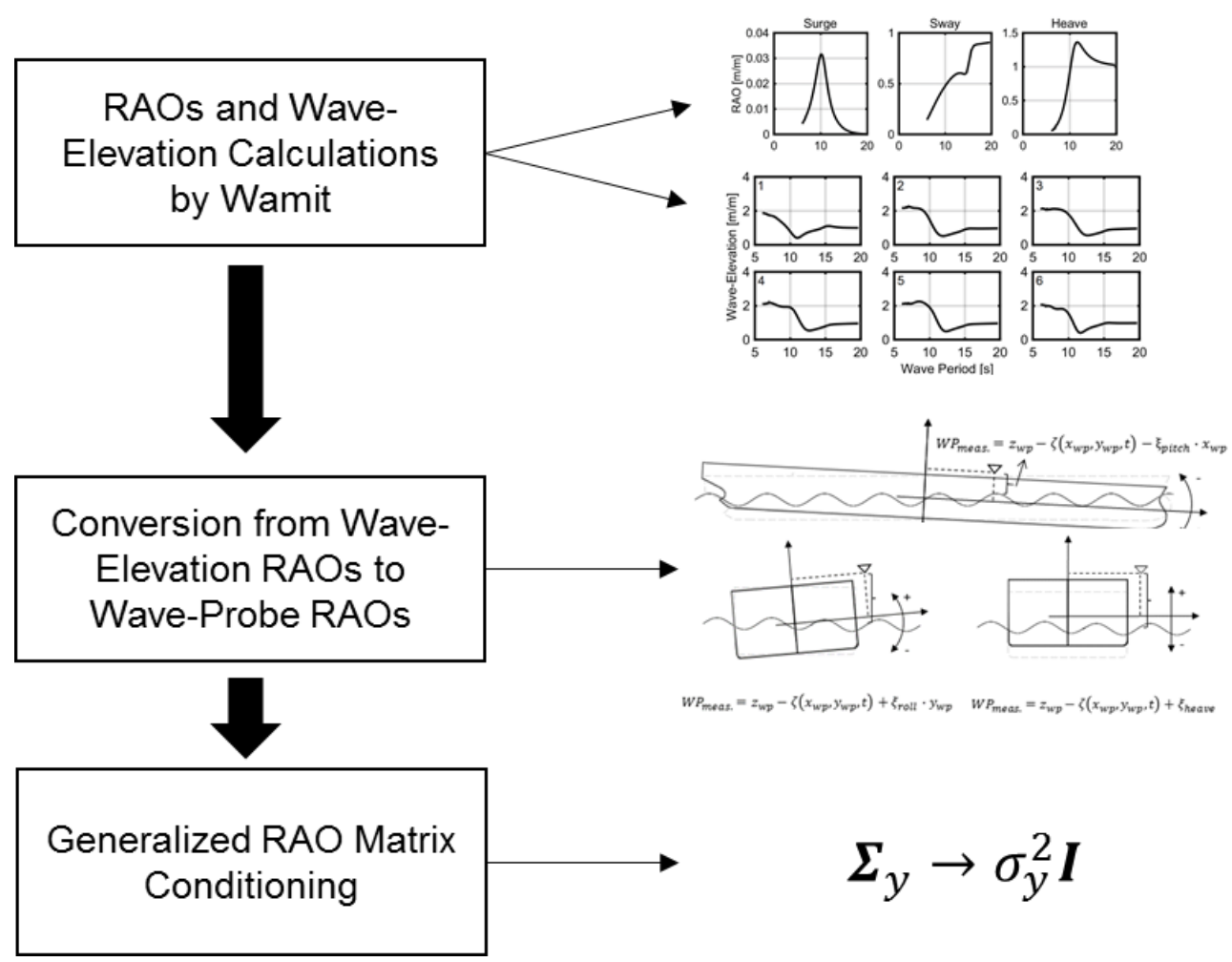

Source: Elaborated by the author. 
After the pre-processing, the possible positions are grouped together by similarity, in order to reduce the number of combinations to evaluate, Figure 32.

Figure 32 - Clustering by similarity.

\section{CLUSTERING}

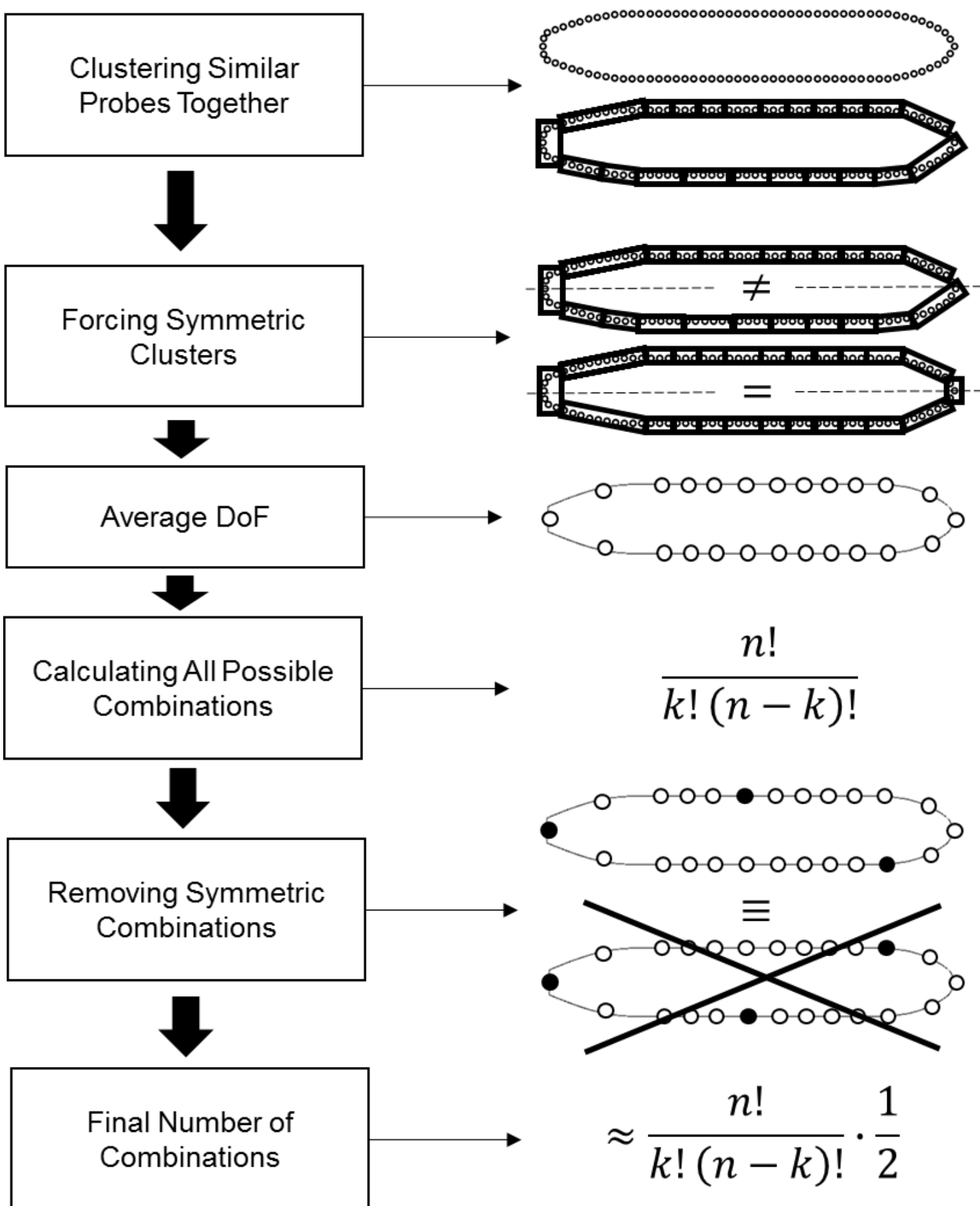

Source: Elaborated by the author. 
And, finally, the optimization algorithm selects the possible best candidates. The final optimal candidate must be chosen using the six objective functions, Figure 33.

Figure 33 - Optimization algorithm.

\section{OPTIMIZATION ALGORITHM}

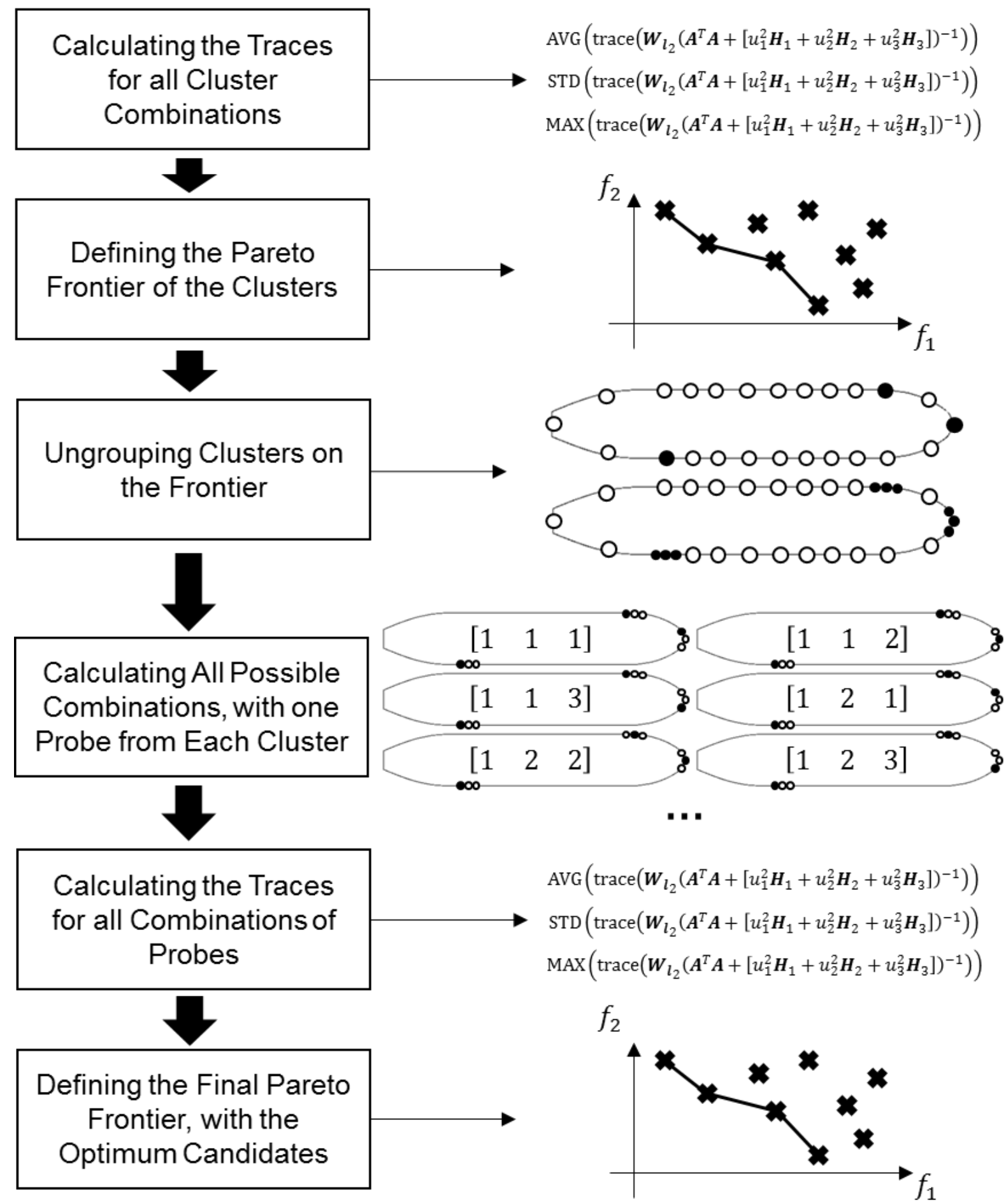

Source: Elaborated by the author. 
Before detailing each step of the algorithm, a simple prediction of its running time can be made. If there are one hundred possible positions, and it is desired five positions, twenty clusters can be made, resulting in 7752 combinations, already removing the symmetric ones. Selecting twelve candidates on the Pareto Frontier, the maximum possible combination of the probes inside them is $\left(\frac{n_{\text {probes }}}{n_{\text {clusters }}}\right)^{n_{\text {DoFs }}}=\left(\frac{100}{20}\right)^{5}=$ 3125 , totalizing $12 \cdot 3125+7752=45252$. It means that the algorithm is, at least, 3.5 times faster than the naïve algorithm. Considering that usually the clusters do not have the same quantity of probes, it is possible to verify that the previous calculation defines a really high upper limit. For example, one cluster can have 7 probes and other 3: 7 . $7 \cdot 5 \cdot 3 \cdot 3=2205$, totalizing $12 \cdot 2205+7752=34212,4.6$ times faster; or even combinations of clusters that have only three probes: $7 \cdot 7 \cdot 3 \cdot 3 \cdot 3=1323,23628$ evaluations, 6.7 times faster. The upper limit of the number of calculations of each objective function is given by Equation (103), in which $k_{s y m}$. is a factor which defines the number of combinations after removing the symmetric clusters, and its upper limit is $k_{\text {sym. }} \leq 0.5+\frac{1}{n_{\text {clusters }}}$, presented in further deductions.

$$
\frac{n_{\text {clusters }} !}{\left(n_{\text {clusters }}-n_{\text {DoFs }}\right) ! n_{\text {DoFs }} !} \cdot k_{\text {sym. }}+n_{\text {Pareto }} \cdot\left(\frac{n_{\text {probes }}}{n_{\text {clusters }}}\right)^{n_{\text {DoFs }}}
$$

The number of clusters which has the minimum upper-bound execution time will usually be between 22 and 26 , for a large range of parameters: $80 \leq n_{\text {probes }} \leq 200$, $1 \leq n_{\text {DoFs }} \leq 10$ and $6 \leq n_{\text {Pareto }} \leq 18$.

Some of the above steps deserve more attention, and are detailed in the further subsections.

\section{RAO MATRIX CONDITIONING}

The pre-conditioning of the RAO matrix is simply made by multiplying it by a weight matrix, and the ideal conditioning must be done by an experiment based evaluation of each sensor noise, to guarantee noise independence and noise scale.

Despite of this, a general procedure is possible if the sensor data is not directly obtained, which is based on normalizing the dimensions of the measurements. Essentially, as most of the DoFs, the probes and the translational motions of the 
vessel, are in meters - or a distance measurement - they will be taken as the standards, and their noise will be considered independent and equal in magnitude. The angular movements, however, need to be corrected, as they are expressed in radians, and the adopted procedure will be multiplying them by half of the vessel length, in the case of the pitch and the yaw movements; and half of the vessel beam, i.e. the width, in the case of the roll movement. It can be justified by the fact that those measurements are the translational value in the stern, or bow; and in the port, or starboard, of the vessel, so they can be measured by the same distance sensors employed to measure the translational movements.

It is interesting to notice how the wave-probe RAOs are automatically weighted by the wave-probe positions when converting from wave-elevation, which also gives support to the method.

\section{Clustering and Average RAOS}

The clustering technique used is the K-mean clustering, and the similarity among the wave-probes is measured taken the RAO of each period and frequency as a particular dimension, and measuring the summation of the squared distance of each RAO. The complex nature of the RAO values results in a distance in the format of Equation (104), and in a general clustering problem formulation in Equations (105), (106) and (107).

$$
\begin{gathered}
\left\|R A O_{i}\left(\omega_{m}, \beta_{k}\right)-R A O_{j}\left(\omega_{m}, \beta_{k}\right)\right\|^{2} \\
=\operatorname{Re}\left\{R A O_{i}\left(\omega_{m}, \beta_{k}\right)-R A O_{j}\left(\omega_{m}, \beta_{k}\right)\right\}^{2}+\operatorname{Im}\left\{R A O_{i}\left(\omega_{m}, \beta_{k}\right)-R A O_{j}\left(\omega_{m}, \beta_{k}\right)\right\}^{2} \\
\left\{\begin{array}{l}
r_{p c}=1, \quad \text { if probe p belongs to cluster } c \\
r_{p c}=0, \quad \text { otherwise }
\end{array}\right. \\
\boldsymbol{\mu}_{c}=\frac{1}{\sum_{p=1}^{n_{\text {clusters }}} r_{p c}} \sum_{p=1}^{n_{\text {clusters }}} r_{p c}\left[\begin{array}{c}
\operatorname{Re}\left\{R A O_{p}\left(\omega_{1}, \beta_{1}\right)\right\} \\
\operatorname{Im}\left\{R A O_{p}\left(\omega_{1}, \beta_{1}\right)\right\} \\
\vdots \\
\operatorname{Re}\left\{R A O_{p}\left(\omega_{m}, \beta_{k}\right)\right\} \\
\operatorname{Im}\left\{R A O_{p}\left(\omega_{m}, \beta_{k}\right)\right\} \\
\vdots
\end{array}\right]_{1 \times 2 \cdot M \cdot K} \\
\min _{r_{p c} J}=\sum_{c=1}^{n_{\text {clusters }}} \sum_{p=1}^{n_{p r o b e s}} r_{n k} \| \boldsymbol{R A O \boldsymbol { O } _ { p } - \boldsymbol { \mu } _ { c } \| ^ { 2 }}
\end{gathered}
$$


It is interesting to notice that no position value is included in the problem, but, as expected, the final groups always contain probes that are neighbors from each other.

The value $\boldsymbol{\mu}_{c}$ is taken as the RAOs of the average DoF, which is used in the first step of the optimization.

\section{ForCING SYMMETRIC ClusteRs AND REMOVING SYMMETRIC COMBINATIONS}

Even though the clusters contain neighbor probes, there is no guarantee that the calculated clusters will be the same at port and starboard, because the clustering problem has multiple solutions. In order to force symmetry, a simple algorithm identifies the side with more clusters and mirror it, resulting in a symmetric arrange. The symmetry is important because it can reduce the number of combinations to evaluate almost to the half, since it is easy to realize that two configurations, in which one is the mirror of the other, will have the same overall performance in the estimation.

If only one probe is used during the estimation, it is easy to see that there are $n_{\text {probes }}-2$ symmetric probes, as the stern and the bow probes are discounted,

resulting in a reduction of $0.5+\frac{1}{n_{\text {probes }}}$ in the number of combinations, or $\frac{n_{\text {probes }}-2}{2}+$ $2=n_{\text {probes }} \cdot\left(\frac{1}{2}+\frac{1}{n_{\text {probes }}}\right)$ final value. When more probes are used, there are combinations containing the stern and the bow probes that ARE symmetric, so only a percentage of the $\frac{1}{n_{\text {probes }}}$ will be non-symmetric and the reduction will be always greater, which establishes the upper bound of $k_{\text {sym. }}$.

\section{Calculating the Trace Criterion}

The complete trace criterion is very expensive to be calculated, but using some interesting block diagonal matrix properties, Equations (108)-(112), and knowing that all the matrix involved are block diagonal, the calculations can be simplified and the execution time is significantly lowered, mainly the calculation of the inverse of the functions. The algorithm can be further accelerated by techniques for sparse matrices. 


$$
\begin{aligned}
& A=\left[\begin{array}{ccc}
A_{1} & & 0 \\
& A_{m} & \\
0 & & A_{M}
\end{array}\right] \\
& \boldsymbol{A}^{T}=\left[\begin{array}{ccc}
\boldsymbol{A}_{\mathbf{1}}^{T} & & \mathbf{0} \\
& \boldsymbol{A}_{\boldsymbol{m}}^{T} & \\
\mathbf{0} & & \boldsymbol{A}_{\boldsymbol{M}}^{T}
\end{array}\right], \boldsymbol{A}^{T} \boldsymbol{A}=\left[\begin{array}{ccc}
\boldsymbol{A}_{\mathbf{1}}^{T} \boldsymbol{A}_{\mathbf{1}} & & \mathbf{0} \\
& \boldsymbol{A}_{\boldsymbol{m}}^{T} \boldsymbol{A}_{\boldsymbol{m}} & \\
\mathbf{0} & & \boldsymbol{A}_{\boldsymbol{M}}^{T} \boldsymbol{A}_{\boldsymbol{M}}
\end{array}\right] \\
& A^{-1}=\left[\begin{array}{ccc}
A_{1}^{-1} & & 0 \\
& A_{m}^{-1} & \\
0 & & A_{M}^{-1}
\end{array}\right] \\
& A+B=\left[\begin{array}{ccc}
A_{1}+B_{1} & & 0 \\
0 & A_{m}+B_{m} & A_{M}+A_{M}
\end{array}\right] \\
& \operatorname{trace}(\boldsymbol{A})=\sum_{\boldsymbol{m}=1}^{M} \operatorname{trace}\left(\boldsymbol{A}_{\boldsymbol{m}}\right)
\end{aligned}
$$

In the case of the objective functions for the independent frequencies, a further simplification can be made because the dynamic system is independent among the frequencies, and the utility design of experiments is reduced to the optimal design of experiment applied to each system, as explained in the example in section 3.5.3, Utility optimal design of experiments. The equivalence is summarized in Equation (113).

$$
\begin{gathered}
\operatorname{trace}\left(\boldsymbol{W}_{\boldsymbol{l}_{2}}\left(\boldsymbol{A}^{T} \boldsymbol{A}+\left[u_{1}^{2} \boldsymbol{H}_{1}+u_{2}^{2} \boldsymbol{H}_{2}+u_{3}^{2} \boldsymbol{H}_{3}\right]\right)^{-1}\right) \\
\equiv \\
\operatorname{trace}\left(\left(\boldsymbol{A}_{\boldsymbol{m}}^{T} \boldsymbol{A}_{\boldsymbol{m}}+\left[u_{1}^{2} \boldsymbol{H}_{1_{\boldsymbol{m}}}+u_{2}^{2} \boldsymbol{H}_{2 \boldsymbol{m}}+u_{3}^{2} \boldsymbol{H}_{3 \boldsymbol{m}}\right]\right)^{-1}\right)
\end{gathered}
$$

Finally, a great improvement can also be made if the independency of the individual blocks calculations is taken in account. Using this property, the problem can be solved using parallel techniques, since multi-core computers are now available even for domestic users.

\section{PARETO FRONTIER}

The Pareto Frontier is a useful tool for multi-objective optimizations that is used when there is not a rule that dictates the preference or relation among the objective functions. Since there is no preference, the idea is that a given solution $A$ is better than a solution $B$ only if ALL the objective function values are better; if just one is not better, 
the solutions are equally good. The frontier is, then, formed by the solutions that are not completely dominated by any other.

In the algorithm, since the solution which indicates the optimum clusters is an approximation, more layers of the Pareto-Frontier are used, lowering the risk of discarding a solution that can become the best one when the real evaluation is made.

A graphical explanation of the Pareto Frontier, and the idea of layers, is given in Figure 34.

Figure 34 - Example of a Pareto Frontier.

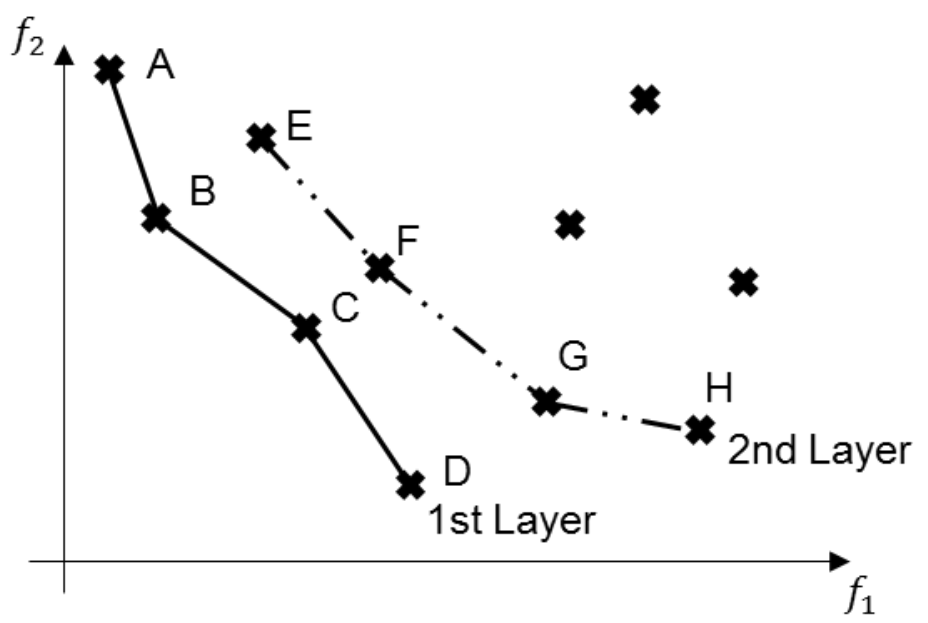

A, B, C and D are not completely dominated by any solution

B completely dominates $\mathrm{E}$ because $f_{1_{B}}<f_{1_{E}} A N D f_{2_{B}}<f_{2_{E}}$ $\mathrm{C}$ completely dominates $\mathrm{F}$ because $f_{1_{C}}<f_{1_{F}} A N D f_{2_{C}}<f_{2_{F}}$ But $\mathrm{B}$ does not dominate $\mathrm{F}$ because $f_{1_{B}}<f_{1_{F}} B U T f_{2_{B}}>f_{2_{F}}$ And $\mathrm{C}$ does not dominate $\mathrm{E}$ because $f_{2_{C}}<f_{2_{E}} B U T f_{1_{C}}>f_{1_{E}}$

Source: Elaborated by the author.

\subsection{Optimization of Degrees of Freedom Usage by Frequency}

One of the wave estimation utilities is the real-time prediction of waves around the vessel. This demands that the algorithm is as fast as possible, but without losing its reliability.

It is known that some DoFs respond better for some frequencies than others. The vessel response, for example, is preferable for wave periods ranging from 10 seconds to 30 seconds; and wave-elevations are preferable for wave periods below 8 seconds. It means that the matrix of RAOs, the $\boldsymbol{A}$ matrix, does not need to include all the chosen DoFs in each frequency matrix $\boldsymbol{A}_{m}$. If three wave-probes and three vessel 
DoFs are used, for example, instead of having an $\boldsymbol{A}$ matrix with $6^{2} \cdot M$ rows and $K \cdot M$ columns, it is possible to have an $\boldsymbol{A}$ matrix with $3^{2} \cdot M$ rows and $K \cdot M$ columns with almost the same performance, but using only the probes in the low period matrices, a mixing of probes and vessel DoFs in intermediate period matrices, and only vessel DoFs in the high period matrices. This can be done because the removed DoFs do not cause any degradation of the estimation capabilities, since their responses are negligible for the selected range of periods.

In this case, the optimization is done for each period, and the exhaustive search is possible. Comparing the resulting matrix with a matrix that demands the same computational effort during the estimation, it is possible to notice how the mixed-DoF matrix can use more different DoFs and has a lower trace-criterion. The proposal is summarized in Figure 35.

Figure 35 - Proposed RAO matrix.

$$
\begin{aligned}
& \boldsymbol{A}_{\boldsymbol{n}^{2} \cdot \boldsymbol{m} \times \boldsymbol{k} \cdot \boldsymbol{m}}=\left[\begin{array}{ccc}
\boldsymbol{A}_{1 n^{2} \times k} & & \mathbf{0} \\
\mathbf{0} & \cdots & \boldsymbol{A}_{m_{n^{2} \times k}}
\end{array}\right] \\
& m \text { periods } \\
& k \text { directions } \\
& n \text { DoFs in each matrix } \boldsymbol{A}_{m} \\
& \boldsymbol{A}_{\text {conventional }} \rightarrow \text { same } n \text { DoFs in every matrix } \boldsymbol{A}_{m} \\
& A_{\text {proposed }} \rightarrow \text { each matrix } A_{m} \text { with specific } n \text { DoFs }
\end{aligned}
$$

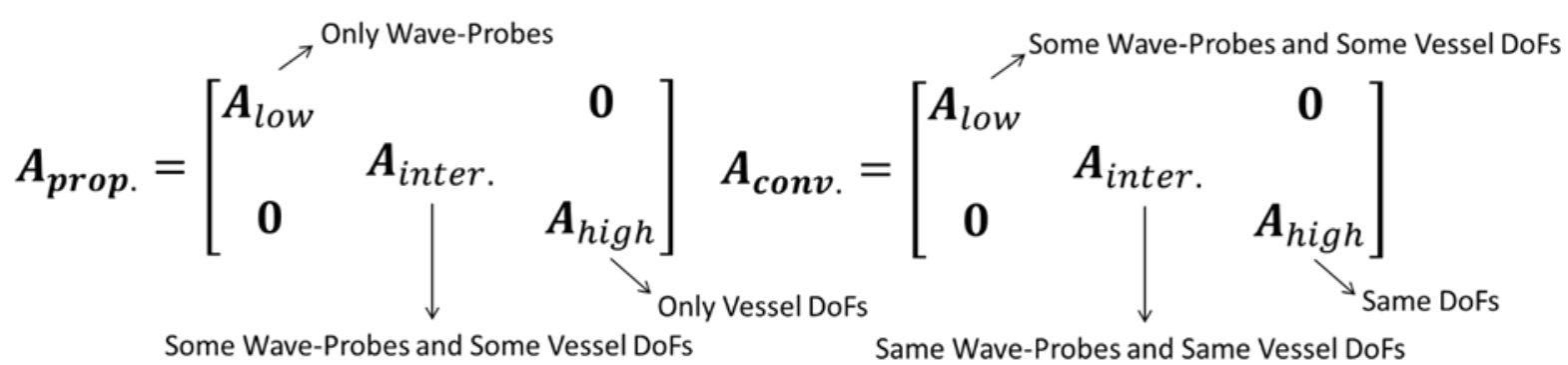

Source: Elaborated by the author.

The previous explanation would be unnecessary if the closed form of the estimation problem could be used. The matrices involved in the estimation, and the inverse of them, could be pre-calculated and an estimation matrix $\boldsymbol{E}$ could be defined, reducing the problem to a simple matrix multiplication, Equation (114). This procedure is so fast that further reductions would not be necessary. 


$$
\widehat{\boldsymbol{x}}=\left(\boldsymbol{A}^{T} \boldsymbol{A}+\left[u_{1}^{2} \boldsymbol{H}_{1}+u_{2}^{2} \boldsymbol{H}_{2}+u_{3}^{2} \boldsymbol{H}_{3}\right]\right)^{-1} \boldsymbol{A}^{T} \boldsymbol{b}=\boldsymbol{E} \boldsymbol{b}
$$

However, as the vector $\boldsymbol{x}$ represents an energy measurement, it cannot assume negative values, and an inequality constraint must be added to the optimization problem. It means that the closed form does not exist anymore and a quadratic programming algorithm must be used, Equations (115) to (118). The size of matrix $\boldsymbol{A}$ is very important in this case, producing a huge impact in the optimization computational cost.

$$
\begin{gathered}
\min _{\boldsymbol{x}, x_{i}>0} J(\boldsymbol{x})=\|\boldsymbol{A} \boldsymbol{x}-\boldsymbol{b}\|^{2}+\boldsymbol{x}^{T}\left[u_{1}^{2} \boldsymbol{H}_{1}+u_{2}^{2} \boldsymbol{H}_{2}+u_{3}^{2} \boldsymbol{H}_{3}\right] \boldsymbol{x} \\
\|\boldsymbol{A} \boldsymbol{x}-\boldsymbol{b}\|^{2}=(\boldsymbol{A} \boldsymbol{x}-\boldsymbol{b})^{T}(\boldsymbol{A} \boldsymbol{x}-\boldsymbol{b})=\boldsymbol{x}^{\boldsymbol{T}} \boldsymbol{A}^{\boldsymbol{T}} \boldsymbol{A} \boldsymbol{x}-2 \boldsymbol{b}^{\boldsymbol{T}} \boldsymbol{A} \boldsymbol{x}+\boldsymbol{b}^{\boldsymbol{T}} \boldsymbol{b} \\
\min _{\boldsymbol{x}, x_{i}>0} J(\boldsymbol{x}) \equiv \min _{\boldsymbol{x}, x_{i}>0} \frac{1}{2} \boldsymbol{x}^{\boldsymbol{T}}\left[\boldsymbol{A}^{\boldsymbol{T}} \boldsymbol{A}+u_{1}^{2} \boldsymbol{H}_{1}+u_{2}^{2} \boldsymbol{H}_{2}+u_{3}^{2} \boldsymbol{H}_{3}\right] \boldsymbol{x}-\left(\boldsymbol{A}^{\boldsymbol{T}} \boldsymbol{b}\right)^{\boldsymbol{T}} \boldsymbol{x} \\
\min _{\boldsymbol{x}, x_{i}>0} \frac{1}{2} \boldsymbol{x}^{\boldsymbol{T}} \boldsymbol{H} \boldsymbol{x}+\boldsymbol{f}^{\boldsymbol{T}} \boldsymbol{x}, \boldsymbol{H}
\end{gathered}
$$

Details about the settings of the quadratic programming algorithm can be found in APPENDIX C - Numerical Issues.

It is important to remember that the trace criterion assumes the solution in the closed form, which means that eventually two approximations were done: the prior was assumed equal to the real state distribution; and the closed form solution is close to the quadratic programming solution.

\subsection{Optimal Prior}

If there is some information about the possible states that one aims to estimate, and given some conditions, it is possible to estimate an optimal prior for a given criterion.

If the complete probability distribution of the states was known, it would be possible to calculate the exact prior using particle methods or general approximation tools, like neural networks. However, it is not interesting to abandon the quadratic form of the current prior: it guarantees solutions that can be achieved by fast computations and a global minimum, since all the functions involved are quadratic with only boundary constraints. 
The optimal quadratic prior can be estimated by a sample of seas using the Bayesian method with the following formulation, Equations (119) and (120):

$$
\begin{gathered}
\max _{\boldsymbol{\Gamma}, x_{\mathbf{0}}} P\left(\boldsymbol{\Gamma}, \boldsymbol{x}_{\mathbf{0}} \mid \boldsymbol{x}_{\text {sample }}\right)=P\left(\boldsymbol{x}_{\text {sample }} \mid \boldsymbol{\Gamma}, \boldsymbol{x}_{\mathbf{0}}\right) P\left(\boldsymbol{\Gamma}, \boldsymbol{x}_{\mathbf{0}}\right) / P\left(\boldsymbol{x}_{\text {sample }}\right) \\
\max _{\boldsymbol{\Gamma}, x_{\mathbf{0}}} P\left(\boldsymbol{\Gamma}, \boldsymbol{x}_{\mathbf{0}} \mid \boldsymbol{x}_{\text {sample }}\right) \propto P\left(\boldsymbol{x}_{\text {sample }} \mid \boldsymbol{\Gamma}, \boldsymbol{x}_{\mathbf{0}}\right) P\left(\boldsymbol{\Gamma}, \boldsymbol{x}_{\mathbf{0}}\right)
\end{gathered}
$$

The idea of the formulation is to choose the more probable $\boldsymbol{x}_{\mathbf{0}}$ vector and $\boldsymbol{\Gamma}$ matrix given the sample, i.e., which would be the more probable probability function, in a given format, that would have generated the sample.

If no preference is given to any $\boldsymbol{\Gamma}$ or $\boldsymbol{x}_{\mathbf{0}}, P\left(\boldsymbol{\Gamma}, \boldsymbol{x}_{\mathbf{0}}\right)=$ uniform distribution, the Bayesian problem is equivalent to maximize the likelihood function, i.e., what are the vector $x_{\mathbf{0}}$ and the matrix $\boldsymbol{\Gamma}$ that, if the sample was generated from them, the sample would have the maximum probability of happening? Essentially, Equation (121).

$$
\max _{\boldsymbol{\Gamma}, \boldsymbol{x}_{0}} P\left(\boldsymbol{\Gamma}, \boldsymbol{x}_{\mathbf{0}} \mid \boldsymbol{x}_{\text {sample }}\right) \equiv \max _{\boldsymbol{\Gamma}, \boldsymbol{x}_{\mathbf{0}}} P\left(\boldsymbol{x}_{\text {sample }} \mid \boldsymbol{\Gamma}, \boldsymbol{x}_{\mathbf{0}}\right)
$$

Recovering the general quadratic Tykhonov regularization problem, Equation (122), and guaranteeing a proper prior, i.e., $\int_{-\infty}^{+\infty} P\left(\boldsymbol{x} \mid \boldsymbol{\Gamma}, \boldsymbol{x}_{\mathbf{0}}\right) d \boldsymbol{x}=1$, the Tykhonov equivalent prior can be written as Equation (123). This form can be easily inferred when the Tykhonov regularization is interpreted as a Gaussian prior with $\boldsymbol{\Sigma}^{-1}=\boldsymbol{\Gamma}^{\mathrm{T}} \boldsymbol{\Gamma}$.

$$
\begin{gathered}
\min _{\boldsymbol{x}}\|\boldsymbol{A} \boldsymbol{x}-\boldsymbol{b}\|^{2}+\alpha^{2}\left\|\boldsymbol{\Gamma}\left(\boldsymbol{x}-\boldsymbol{x}_{\mathbf{0}}\right)\right\|^{2} \\
P\left(\boldsymbol{x} \mid \boldsymbol{\Gamma}, \boldsymbol{x}_{\mathbf{0}}\right)=\frac{1}{\sqrt{(2 \pi)^{n}\left|\left(\boldsymbol{\Gamma}^{\mathrm{T}} \boldsymbol{\Gamma}\right)^{-1}\right|}} e^{\left(-\frac{1}{2}\left(\boldsymbol{x}-\boldsymbol{x}_{\mathbf{0}}\right)^{T} \boldsymbol{\Gamma}^{\mathrm{T}} \boldsymbol{\Gamma}\left(\boldsymbol{x}-\boldsymbol{x}_{\mathbf{0}}\right)\right)}
\end{gathered}
$$

Renaming $\boldsymbol{\Gamma}^{\mathrm{T}} \boldsymbol{\Gamma}=\mathbf{Q}^{-1}$, for reasons that will be clear soon, the maximization problem, with a number $n_{\text {sample }}$ of seas samples, becomes Equation (124) and, finally, Equation (125). 


$$
\begin{gathered}
\max _{\mathbf{Q}, \boldsymbol{x}_{\mathbf{0}}} P\left(\boldsymbol{x}_{\text {sample }} \mid \mathbf{Q}, \boldsymbol{x}_{\mathbf{0}}\right)=\max _{\mathbf{Q}, \boldsymbol{x}_{\mathbf{0}}} P\left(\boldsymbol{x}_{\mathbf{1}} \cap \boldsymbol{x}_{\mathbf{2}} \cap \ldots \cap \boldsymbol{x}_{n_{\text {sample }}} \mid \mathbf{Q}, \boldsymbol{x}_{\mathbf{0}}\right)= \\
=\max _{\mathbf{Q}, \boldsymbol{x}_{\mathbf{0}}} \prod_{i=1}^{n_{\text {sample }}} \frac{1}{\sqrt{(2 \pi)^{n}|\boldsymbol{Q}|}} e^{\left(-\frac{1}{2}\left(\boldsymbol{x}_{i}-\boldsymbol{x}_{\mathbf{0}}\right)^{T} \mathbf{Q}^{-1}\left(\boldsymbol{x}_{\boldsymbol{i}}-\boldsymbol{x}_{\mathbf{0}}\right)\right)}
\end{gathered}
$$

This problem is a known maximum likelihood problem solved by general matrix calculus rules, with (DWYER, 1967) being an earlier reference.

Taking the natural logarithm of the objective function - the logarithm is a monotonically increasing function, which means that the maximum point of the original positive objective function will be the same of the logarithm of this function - the problem can be rewritten as Equation (126).

$$
\ln \left(f\left(\boldsymbol{Q}, \boldsymbol{x}_{\mathbf{0}}\right)\right)=\text { cte. }-\frac{n_{\text {sample }}}{2} \ln (|\boldsymbol{Q}|)-\frac{1}{2} \sum_{i=1}^{n_{\text {sample }}}\left(\boldsymbol{x}_{i}-\boldsymbol{x}_{\mathbf{0}}\right)^{T} \boldsymbol{Q}^{-1}\left(\boldsymbol{x}_{i}-\boldsymbol{x}_{\mathbf{0}}\right)
$$

Taking the derivatives with respect to $\boldsymbol{Q}$ and $\boldsymbol{x}_{\mathbf{0}}$, Equation (127) and (128), and equating both to zero, the parameters that define the maximum point can be found, Equation (129) and (130) - (DWYER, 1967) profs that this indeed a maximum point.

$$
\begin{gathered}
\frac{\partial \ln \left(f\left(\boldsymbol{Q}, \boldsymbol{x}_{\mathbf{0}}\right)\right)}{\partial \boldsymbol{x}_{\mathbf{0}}}=-\frac{1}{2} \sum_{i=1}^{n_{\text {sample }}}\left(-2 \boldsymbol{Q}^{-1}\left(\boldsymbol{x}_{i}-\boldsymbol{x}_{\mathbf{0}}\right)\right)=\boldsymbol{Q}^{-1} \sum_{i=1}^{n_{\text {sample }}}\left(\boldsymbol{x}_{i}-\boldsymbol{x}_{\mathbf{0}}\right) \\
\frac{\partial \ln \left(f\left(\boldsymbol{Q}, \boldsymbol{x}_{\mathbf{0}}\right)\right)}{\partial \boldsymbol{Q}}=-\frac{n_{\text {sample }}}{2} \boldsymbol{Q}^{-1}+\frac{1}{2} \boldsymbol{Q}^{-1} \sum_{i=1}^{n_{\text {sample }}}\left[\left(\boldsymbol{x}_{i}-\boldsymbol{x}_{\mathbf{0}}\right)\left(\boldsymbol{x}_{i}-\boldsymbol{x}_{\mathbf{0}}\right)^{T}\right] \boldsymbol{Q}^{-1} \\
\frac{\partial \ln \left(f\left(\boldsymbol{Q}, \boldsymbol{x}_{\mathbf{0}}\right)\right)}{\partial \boldsymbol{x}_{\mathbf{0}}}=0 \rightarrow \sum_{i=1}^{n_{\text {sample }}}\left(\boldsymbol{x}_{i}-\boldsymbol{x}_{\mathbf{0}}\right)=0 \rightarrow \boldsymbol{x}_{\mathbf{0}}=\sum_{i=1}^{n_{\text {sample }}} \boldsymbol{x}_{i}=\boldsymbol{\mu}_{\boldsymbol{x}} \\
\frac{\partial \ln \left(f\left(\boldsymbol{Q}, \boldsymbol{x}_{\mathbf{0}}\right)\right)}{\partial \boldsymbol{Q}}=0 \rightarrow \boldsymbol{Q}=\frac{\sum_{i=1}^{n_{\text {sample }}}\left[\left(\boldsymbol{x}_{\boldsymbol{i}}-\boldsymbol{x}_{\mathbf{0}}\right)\left(\boldsymbol{x}_{i}-\boldsymbol{x}_{\mathbf{0}}\right)^{T}\right]}{n_{\text {sample }}}=\boldsymbol{\Sigma}_{\boldsymbol{x}}
\end{gathered}
$$

It means that the maximum likelihood quadratic prior, calibrated by sample seas, is equivalent to a Gaussian probability function, using the sample mean and the sample covariance of the seas, Equation (131). The estimation problem can be re-written, Equations (132) and, if the covariance matrix of the measurement errors is properly normalized, Equation (133). 


$$
\begin{aligned}
& \boldsymbol{X}=\left[\begin{array}{llll}
\boldsymbol{x}_{1} & \boldsymbol{x}_{2} & \ldots & \boldsymbol{x}_{\boldsymbol{n}}
\end{array}\right], \boldsymbol{x}_{\mathbf{0}}=\operatorname{avg}(\boldsymbol{X}), \Sigma_{\boldsymbol{x}}=\operatorname{cov}(\boldsymbol{X}) \\
& \max _{\boldsymbol{x}} P(\boldsymbol{Y}=\boldsymbol{b} \mid \boldsymbol{X}=\boldsymbol{x}) P(\boldsymbol{X}=\boldsymbol{x}) \equiv \\
& \equiv \max _{x} \frac{1}{\sqrt{(2 \pi)^{m}\left|\Sigma_{y}\right|}} e^{\left(-\frac{1}{2}(\boldsymbol{b}-\boldsymbol{A x})^{T} \boldsymbol{\Sigma}_{y}^{-1}(\boldsymbol{b}-\boldsymbol{A x})\right)} \frac{1}{\sqrt{(2 \pi)^{n}\left|\boldsymbol{\Sigma}_{\boldsymbol{x}}\right|}} e^{\left(-\frac{1}{2}\left(\boldsymbol{x}-\boldsymbol{x}_{\mathbf{0}}\right)^{T} \boldsymbol{\Sigma}_{\boldsymbol{x}}^{-1}\left(\boldsymbol{x}-\boldsymbol{x}_{\mathbf{0}}\right)\right)} \equiv \\
& \equiv \min _{\boldsymbol{x}}(\boldsymbol{A} \boldsymbol{x}-\boldsymbol{b})^{T} \boldsymbol{\Sigma}_{\boldsymbol{y}}^{-1}(\boldsymbol{A} \boldsymbol{x}-\boldsymbol{b})+\left(\boldsymbol{x}-\boldsymbol{x}_{\mathbf{0}}\right)^{T} \boldsymbol{\Sigma}_{\boldsymbol{x}}^{-1}\left(\boldsymbol{x}-\boldsymbol{x}_{\mathbf{0}}\right) \\
& J(\boldsymbol{x})=(\boldsymbol{A} \boldsymbol{x}-\boldsymbol{b})^{T}(\boldsymbol{A} \boldsymbol{x}-\boldsymbol{b})+\left(\boldsymbol{x}-\boldsymbol{x}_{\mathbf{0}}\right)^{T} u^{2} \boldsymbol{H}\left(\boldsymbol{x}-\boldsymbol{x}_{\mathbf{0}}\right)
\end{aligned}
$$

It is important to emphasize that the resulting prior does not mean that the sample can indeed be represented by the Gaussian probability function. Actually, in seas estimation, the values in the vector $\boldsymbol{x}$ usually assume zeros with a higher probability than the Gaussian function suggests - it is simple to visualize it when it is known that when a wave comes from a particular direction, everywhere else is zero, it means that the values in a particular direction will happen approximately $\left(n_{\beta}-1\right) / n_{\beta}$ more often than the Gaussian function suggests.

Despite of this, the formulation is still valid, since it was desired a quadratic function that would best fit a given training data in the maximum likelihood sense. The maximum likelihood sense can also be though as the quadratic prior which is the closest to the equal probability of happening of each sample sea, i.e., maintains the shape of the spectrum without giving preference to any parameter.

Other criterion that can be used to find the optimal prior is to find a linear estimator $\boldsymbol{K}$ that minimizes the expected squared error between the estimation and the true state, Equation (134):

$$
\min _{\boldsymbol{K}} E\left[\left(\boldsymbol{x}-\boldsymbol{x}_{\text {est }}\right)^{2}\right], \boldsymbol{x}_{\boldsymbol{e s t}}=\boldsymbol{K} \cdot \boldsymbol{y}
$$

A simple unidimensional example can be used to illustrate the problem, Equations (135) to (139). Knowing that the measurement $y$ is connected with the state $x$ by a linear system, assuming that the measurement error $\epsilon$ and the state $x$ are uncorrelated, i.e., $E[x \epsilon]=0$, and adopting $E[x]=0$ : 


$$
\begin{gathered}
y=A x+\epsilon \\
\min _{k} E\left[(x-k y)^{2}\right] \equiv \min _{k} E\left[(x-k(A x+\epsilon))^{2}\right] \\
\min _{k} E\left[(x(1-k A)-k \epsilon)^{2}\right] \\
E\left[(x-k y)^{2}\right]=(1-k A)^{2} E\left[x^{2}\right]+k^{2} E\left[\epsilon^{2}\right]-2(1-k A) k \overbrace{E[x \epsilon]}^{0} \\
E\left[(x-k y)^{2}\right]=(1-k A)^{2} E\left[x^{2}\right]+k^{2} E\left[\epsilon^{2}\right]=(1-k A)^{2} \sigma_{x}^{2}+k^{2} \sigma_{y}^{2}
\end{gathered}
$$

Deriving the criterion by $k$ and equating to zero, Equation (140):

$$
\begin{gathered}
\frac{d E\left[(x-k y)^{2}\right]}{d k}=-2 A \sigma_{x}^{2}+2 k A^{2} \sigma_{x}^{2}+2 k \sigma_{y}^{2}=0 \\
k_{b e s t}=\frac{A \sigma_{x}^{2}}{A^{2} \sigma_{x}^{2}+\sigma_{y}^{2}} \\
x_{e s t}=k y=\left(A \sigma_{x}^{2} A+\sigma_{y}^{2}\right)^{-1}\left(A \sigma_{x}^{2} y\right)
\end{gathered}
$$

The resulting estimation, Equations (141) and (142), is exactly the closed form of the Bayesian estimation shown in Equation (45), i.e., without assuming anything about the probability of the state and anything about the probability of the measurement error, if a linear estimator is desired, the best estimation is equivalent to the Bayesian problem with the prior equals to the covariance matrix of the possible states. This is a known result from the linear stochastic filtering theory and the deduction of the complete matrix formula for it can be found in (DAVIS, 2013).

In conclusion, the covariance of a sample of known possible states is the optimal prior in those two reasonable criteria, i.e., if the estimation problem format is defined as linear in the resulting estimator or quadratic in the originating cost functional, with no prior hypothesis about the real probability distribution function. This strong result will be shown to hold true in experimental trials during this thesis. 


\section{NUMERICAL VALIDATION}

All the proposals so far are only based on the theory, so they need to be tested to be validated. As stated before, proposals as the optimal design of experiments - to select the best wave-probe position; and the utility design of experiments - to foresee the estimation capabilities of the final matrix $\boldsymbol{A}$; are based in some strong hypothesis concerning the prior distribution of the waves and the optimal estimation result. Despite the assumptions, the proposals are not related to the physical hypothesis concerning the model, which need to be tested experimentally, it means that a numerical validation is enough to give a preliminary proof of concept.

The numerical validation can also give an interesting comparison between the estimation with only vessel DoFs against the estimation with wave-probes, and provide important insights in the design of the physical experiments.

\subsection{Error Criteria}

The evaluation about how good the estimation algorithm is must follow a criterion, or a set of criteria, which is capable of quantifying how much the estimated sea differs from the inputted sea. Since it is interesting to compare the proposed methods against the standard practice in the area, all the error metrics that were found in the literature will be used.

The first set of error criteria quantifies the difference in each component of the directional sea spectrum independently, assuming that the directional spread function depends only on the direction. This is useful when the proposal is compared against methods that can only provide some of the components, e.g., radars, which are best to predict the directional spread function; or ship-borne wave recorders, which are best to predict the spectral energy density. They are shown in Equations (143) to (149).

$$
\begin{gathered}
\operatorname{Err}_{S_{\omega}}^{2}=\int_{0}^{\infty}(S(\omega)-\hat{S}(\omega))^{2} d \omega=\int_{0}^{\infty}\left(\int_{0}^{2 \pi} S(\omega, \beta) d \beta-\int_{0}^{2 \pi} \hat{S}(\omega, \beta) d \beta\right)^{2} d \omega \\
\left|\operatorname{Err}_{S_{\omega}}\right|=\int_{0}^{\infty}|S(\omega)-\hat{S}(\omega)| d \omega=\int_{0}^{\infty}\left|\int_{0}^{2 \pi} S(\omega, \beta) d \beta-\int_{0}^{2 \pi} \hat{S}(\omega, \beta) d \beta\right| d \omega
\end{gathered}
$$




$$
\begin{gathered}
\operatorname{Err}_{S_{\omega}} \%=\frac{\int_{0}^{\infty}|S(\omega)-\hat{S}(\omega)| d \omega}{\int_{0}^{\infty} S(\omega) d \omega}=\frac{\int_{0}^{\infty}\left|\int_{0}^{2 \pi} S(\omega, \beta) d \beta-\int_{0}^{2 \pi} \hat{S}(\omega, \beta) d \beta\right| d \omega}{\int_{0}^{\infty} \int_{0}^{2 \pi} S(\omega, \beta) d \beta d \omega} \\
\operatorname{Err}_{D_{\beta}}^{2}=\int_{0}^{2 \pi}(D(\beta)-\widehat{D}(\beta))^{2} d \beta \\
\left|\operatorname{Err}_{D_{\beta} \mid}=\int_{0}^{2 \pi}\right| D(\beta)-\widehat{D}(\beta) \mid d \beta \\
\operatorname{Err}_{D_{\beta}} \%=\frac{\int_{0}^{2 \pi}|D(\beta)-\widehat{D}(\beta)| d \beta}{\int_{0}^{2 \pi} D(\beta) d \beta} \\
\boldsymbol{S}_{\boldsymbol{\omega} \boldsymbol{\beta}_{M \cdot K \times 1}}=\boldsymbol{M}\left(\boldsymbol{S}_{\boldsymbol{\omega}}\right) \times \boldsymbol{D}_{\boldsymbol{\beta}_{K \times 1}} \rightarrow \widehat{\boldsymbol{D}}_{\boldsymbol{\beta}}=\operatorname{pinv}\left(\boldsymbol{M}\left(\widehat{\boldsymbol{S}}_{\boldsymbol{\omega}}\right)\right) \times \widehat{\boldsymbol{S}}_{\boldsymbol{\omega} \boldsymbol{\beta}} \text { (matrix form) }
\end{gathered}
$$

The second set of errors is that concerning the parameters of the standard seas, which can be useful when comparing the proposed approach against parametric estimation algorithms. They are listed in Equations (150) to (157).

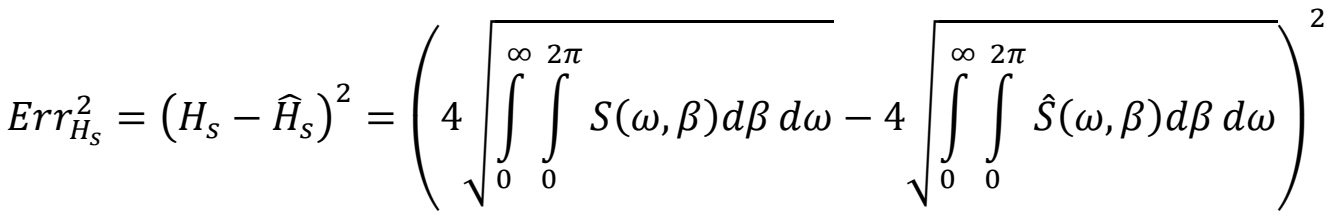

$$
\begin{aligned}
& \left|\operatorname{Err}_{H_{S}}\right|=\left|H_{S}-\widehat{H}_{S}\right|, \operatorname{Err}_{H_{S}} \%=\frac{\left|H_{S}-\widehat{H}_{S}\right|}{H_{S}} \\
& \operatorname{Err}_{T_{1}}^{2}=\left(T_{1}-\widehat{T}_{1}\right)^{2}=\left(2 \pi \frac{\int_{0}^{\infty} \int_{0}^{2 \pi} S(\omega, \beta) d \beta d \omega}{\int_{0}^{\infty} \omega \int_{0}^{2 \pi} S(\omega, \beta) d \beta d \omega}-2 \pi \frac{\int_{0}^{\infty} \int_{0}^{2 \pi} \hat{S}(\omega, \beta) d \beta d \omega}{\int_{0}^{\infty} \omega \int_{0}^{2 \pi} \hat{S}(\omega, \beta) d \beta d \omega}\right)^{2} \\
& \left|\operatorname{Err}_{T_{1}}\right|=\left|T_{1}-\widehat{T}_{1}\right|, \operatorname{Err}_{T_{1}} \%=\frac{\left|T_{1}-\widehat{T}_{1}\right|}{T_{1}} \\
& \operatorname{Err}_{T_{P}}=1.199 \operatorname{Err}_{T_{1}}, \quad \text { assuming ITTC JONSWAP } \\
& \left|\operatorname{Err}_{\beta_{0}}\right|=\left|\beta_{0}-\hat{\beta}_{0}\right|=\left|\operatorname{atan}\left[\frac{\int_{0}^{\infty} \int_{0}^{2 \pi} S(\omega, \beta) \sin (\beta) d \beta d \omega}{\int_{0}^{\infty} \int_{0}^{2 \pi} S(\omega, \beta) \cos (\beta) d \beta d \omega}\right]-\operatorname{atan}\left[\frac{\int_{0}^{\infty} \int_{0}^{2 \pi} \hat{S}(\omega, \beta) \sin (\beta) d \beta d \omega}{\int_{0}^{\infty} \int_{0}^{2 \pi} \hat{S}(\omega, \beta) \cos (\beta) d \beta d \omega}\right]\right| \\
& \hat{s}=\min _{s, s \in \mathbb{Z} \mid 1 \leq s \leq 100}\left[\widehat{\boldsymbol{S}}_{\boldsymbol{\omega} \boldsymbol{\beta}_{M \cdot K \times 1}}-\boldsymbol{M}\left(\widehat{\boldsymbol{S}}_{\boldsymbol{\omega}}\right) \times \boldsymbol{D}\left(G(s) \cdot \cos ^{2 s}\left(\frac{\beta-\hat{\beta}_{0}}{2}\right)\right)\right]^{\mathbf{2}} \\
& \left|E r r_{s}\right|=|s-\hat{s}|, E r r_{s} \%=\frac{|s-\hat{s}|}{s}
\end{aligned}
$$

Other set of error criteria are described and proposed by (BISPO, et al., 2012), and are metrics related to the high-resolution difference between the estimated and 
the inputted spectra. The first error quantifies a quadratic difference in each region of the polar plot, and the second one tries to measure how dislocated the spectra are from each other, using a normalizing factor to mask possible magnitude differences. They are shown in Equations (158) and (159).

$$
\begin{gathered}
\operatorname{Err}_{S_{\omega \beta}}^{2}=\int_{0}^{\infty} \int_{0}^{2 \pi}(S(\omega, \beta)-\hat{S}(\omega, \beta))^{2} d \beta d \omega \\
\operatorname{Err}_{\bar{S}(\omega, \beta), \text { norm. }}^{2}=\int_{0}^{\infty} \int_{0}^{2 \pi}(\bar{S}(\omega, \beta)-\hat{S}(\omega, \beta))^{2} d \beta d \omega=\int_{0}^{\infty} \int_{0}^{2 \pi}\left(\frac{S(\omega, \beta)}{\max (S(\omega, \beta))}-\frac{\widehat{S}(\omega, \beta)}{\max (\widehat{S}(\omega, \beta))}\right)^{2} d \beta d \omega
\end{gathered}
$$

It is also possible to expand the concepts to define a high-resolution absolute error and a high-resolution percent error, Equations (160) and (161).

$$
\begin{aligned}
\left|\operatorname{Err}_{S_{\omega \beta}}\right| & =\int_{0}^{\infty} \int_{0}^{2 \pi}|S(\omega, \beta)-\hat{S}(\omega, \beta)| d \beta d \omega \\
\operatorname{Err}_{S_{\omega \beta}} \% & =\frac{\int_{0}^{\infty} \int_{0}^{2 \pi}|S(\omega, \beta)-\hat{S}(\omega, \beta)| d \beta d \omega}{\int_{0}^{\infty} \int_{0}^{2 \pi} S(\omega, \beta) d \beta d \omega}
\end{aligned}
$$

Finally, it is interesting to define errors related to the numerical aspect of the method, without considering the model behind it, Equations (162), (163) and (164).

$$
\begin{gathered}
E r r_{x}^{2}=\|x-\widehat{x}\|=\sum_{i=1}^{M \cdot K}\left(x_{i}-\hat{x}_{i}\right)^{2} \\
\left|E r r_{x}\right|=\sum_{i=1}^{M \cdot K}\left|x_{i}-\hat{x}_{i}\right| \\
\operatorname{Err}_{x} \%=\frac{\sum_{i=1}^{M \cdot K}\left|x_{i}-\hat{x}_{i}\right|}{\sum_{i=1}^{M \cdot K} x_{i}}
\end{gathered}
$$

There are some important numerical aspects in those calculations. Firstly, the denominators of the percent errors are always positive, since they represent energy, so they do not need to be put in absolute values. Lastly, the numerical integration in $\beta$ must be done carefully, since numerical problems can happen, as discussed in APPENDIX C - Numerical Issues. 


\subsection{Wave-Probe Positioning}

In order to validate the trace criterion, only a small number of positions were defined as possible, since the reduced number would allow the evaluation of all the combinations among them. Twelve positions were defined, since this is the number of gauges available for the physical experiments, and they were chosen using cluster techniques. The idea was to define twelve clusters and select the mean position of each one, in order to guarantee probes with the more diverse response among them, enlarging the possible trace criterion outcomes, Figure 36 and Table 3.

Figure 36 - Position of the wave-probes for the experiments.

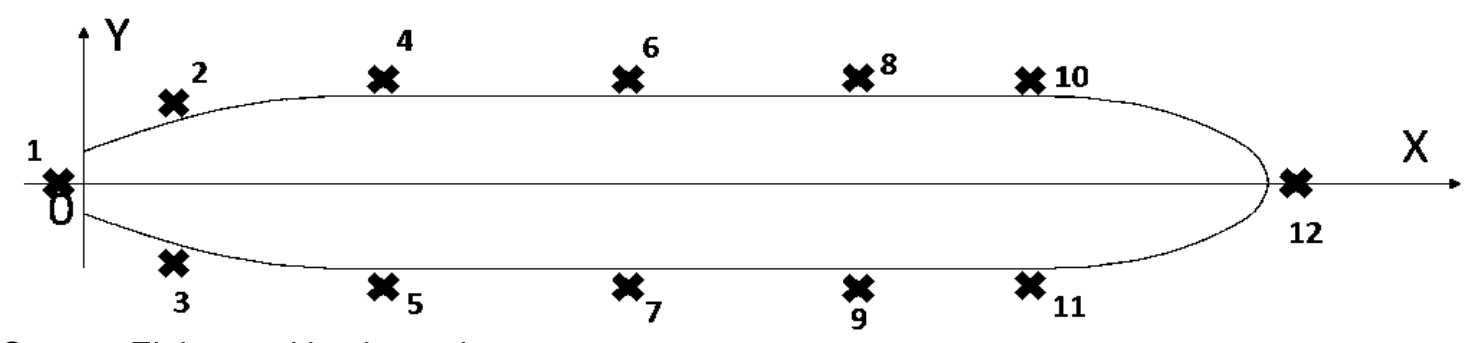

Source: Elaborated by the author.

Table 3 - Position of the wave-probes in the model scale, in millimeters.

\begin{tabular}{ccccccccccccc}
\hline & $\mathbf{1}$ & $\mathbf{2}$ & $\mathbf{3}$ & $\mathbf{4}$ & $\mathbf{5}$ & $\mathbf{6}$ & $\mathbf{7}$ & $\mathbf{8}$ & $\mathbf{9}$ & $\mathbf{1 0}$ & $\mathbf{1 1}$ & $\mathbf{1 2}$ \\
\hline $\mathrm{X}$ & -12 & 322 & 322 & 945 & 945 & 1697 & 1697 & 2410 & 2410 & 2925 & 2925 & 3679 \\
$\mathrm{Y}$ & 0 & 237 & -237 & 323 & -323 & 323 & -323 & 323 & -323 & 320 & -320 & 0 \\
\hline
\end{tabular}

Source: Elaborated by the author.

\subsection{Description of the Campaign}

The objective of the numerical validation campaign is to provide information for the following questions:

- Which error criteria can be correctly predicted by the trace criterion?

- What are the possible improvements of adding wave-probes to the estimation algorithm?

The answers, however, have a strong dependency with the sea statistics, and would only be valid for the specific tested conditions.

Usually, the sea statistics are raised experimentally in regions of interest, and are organized in metocean reports, which summarizes meteorological and sea state information. It is safe to assume that the reports will contain some kind of JONSWAP- 
based spectral density function, some kind of cosine-squared-based spread function, a possible frequency table with the probability of occurrence of a doublet $H_{S} \times T_{P}$, a possible frequency table with the probability concerning the incoming directions $\beta_{0}$, and a possible frequency table with some kind of spread factor $s$.

In this text, an idealized metocean is used, with a frequency table in the format shown in Table 4 . The parameter $\beta_{0}$ can assume any value between $0^{\circ}$ and $360^{\circ}$ with uniform probability; and the parameter $s$ can assume integers values between 1 and 100 , also with uniform probability.

Table 4 - Idealized joint occurrence of $H_{S}$ and $T_{P}$.

\begin{tabular}{|c|c|c|c|c|c|c|c|c|c|c|c|c|c|c|c|c|c|c|}
\hline $\begin{array}{c}\mathrm{Tp} \rightarrow \\
\mathrm{Hs} \downarrow\end{array}$ & 3 & 4 & 5 & 6 & 7 & 8 & 9 & 10 & 11 & 12 & 13 & 14 & 15 & 16 & 17 & 18 & 19 \\
\hline & 4 & 5 & 6 & 7 & 8 & 9 & 10 & 11 & 12 & 13 & 14 & 15 & 16 & 17 & 18 & 19 & 20 \\
\hline 0,5 & 1,0 & 0 & 0 & 0 & 0 & 0 & 0 & 0 & 0 & 0 & 0 & 0 & 0 & 0 & 0 & 0 & 0 & 0 \\
\hline 1,0 & 1,5 & 0 & 100 & 100 & 100 & 100 & 100 & 100 & 100 & 100 & 100 & 100 & 100 & 100 & 100 & 100 & 100 & 0 \\
\hline 1,5 & 2,0 & 0 & 100 & 100 & 100 & 100 & 100 & 100 & 100 & 100 & 100 & 100 & 100 & 100 & 100 & 100 & 100 & 0 \\
\hline 2,0 & 2,5 & 0 & 100 & 100 & 100 & 100 & 100 & 100 & 100 & 100 & 100 & 100 & 100 & 100 & 100 & 100 & 100 & 0 \\
\hline 2,5 & 3,0 & 0 & 100 & 100 & 100 & 100 & 100 & 100 & 100 & 100 & 100 & 100 & 100 & 100 & 100 & 100 & 100 & 0 \\
\hline 3,0 & 3,5 & 0 & 100 & 100 & 100 & 100 & 100 & 100 & 100 & 100 & 100 & 100 & 100 & 100 & 100 & 100 & 100 & 0 \\
\hline 3,5 & 4,0 & 0 & 100 & 100 & 100 & 100 & 100 & 100 & 100 & 100 & 100 & 100 & 100 & 100 & 100 & 100 & 100 & 0 \\
\hline 4,0 & 4,5 & 0 & 0 & 0 & 0 & 0 & 0 & 0 & 0 & 0 & 0 & 0 & 0 & 0 & 0 & 0 & 0 & 0 \\
\hline
\end{tabular}

Source: Adapted from (PETROBRAS, 2010).

The frequency table is used to seed a Monte-Carlo simulation, producing 1000 different seas. The seas are, then, estimated by different matrices $\boldsymbol{A}$, and the results are summarized in the next sections.

\subsection{Verification of the Error Metrics}

Before running estimation procedures, it is interesting to perform a verification of the error metrics. It allows the verification of bias and alias in the estimation of the parameters and in the decomposition of the seas. The importance of this section will become evident with the results presented.

Each one of the sea vectors are generated by the procedure in Equation (165) and their parameters are estimated by the procedure in Equation (166). The errors are, then, calculated comparing the parameters used to seed the sea against the parameters estimated using the sea generated, without adding any kind of noise. 


$$
\begin{gathered}
\boldsymbol{x}=f\left(H_{S}, T_{P}, \beta_{0}, s\right)=S(\omega) \cdot D(\beta), \quad S(\omega)=g\left(H_{S}, T_{P}\right), \quad D(\beta)=h\left(\beta_{0}, s\right) \\
{\left[\widehat{H}_{S}, \widehat{T}_{P}, \hat{\beta}_{0}, \hat{s}, \hat{S}(\omega), \widehat{D}(\beta)\right]=\text { parameterEstimation }(\boldsymbol{x})}
\end{gathered}
$$

For the first trial, a discretization of 40 values of $\omega$, varying from $2 \pi / 30$ to $2 \pi / 4$, and 40 values of $\beta$ was used.

The numerical errors $E r r_{x}^{2},\left|E r r_{x}\right|$ and $E r r_{x} \%$ are, of course, exactly zero, since the vector is the same. The high resolution errors $\operatorname{Err}_{S_{\omega \beta}}^{2},\left|\operatorname{Err}_{S_{\omega \beta}}\right|, \operatorname{Err}_{S_{\omega \beta}} \%$ and $\operatorname{Err}_{\bar{S}(\omega, \beta), n o r m .}^{2}$ are also exactly zero, since $S(\omega, \beta)$ is just the vector $x$ reshaped.

The decomposition of the vector in the components $S(\omega)$ and $D(\beta)$ however, are not exactly zero, since some calculations are necessary to estimate each part. Despite of this, the errors $\operatorname{Err}_{S_{\omega}}^{2},\left|\operatorname{Err}_{S_{\omega}}\right|, \operatorname{Err}_{S_{\omega}} \%, \operatorname{Err}_{D_{\beta}}^{2},\left|\operatorname{Err}_{D_{\beta}}\right|$ and $\operatorname{Err}_{D_{\beta}} \%$ are always smaller than $10^{-12}$, indicating that the difference is only due to numerical truncation.

The parametric errors, however, are non-negligible for some parameters, and selected error metrics can be seen in Figure 37.

Figure 37 - Parametric errors during verification with $40 \omega$ and $40 \beta$.

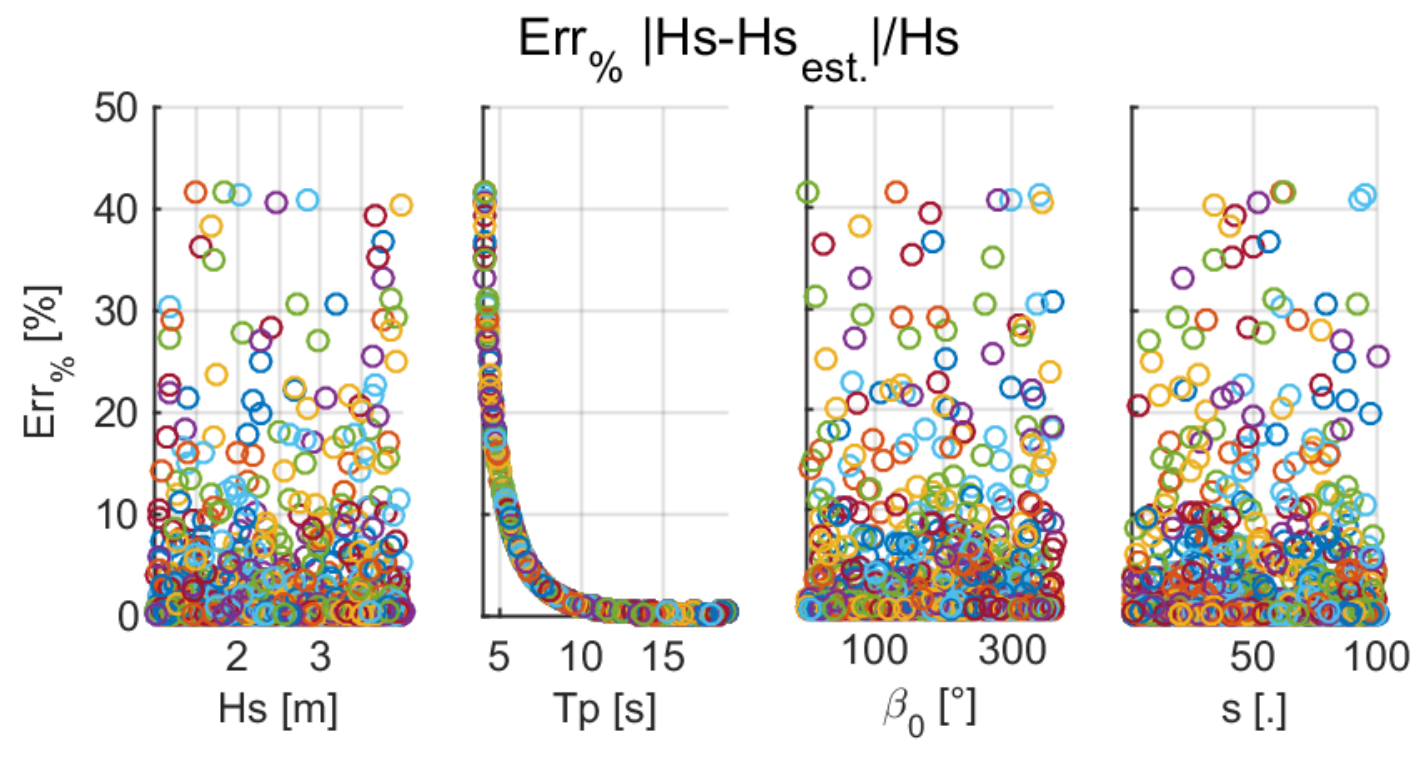




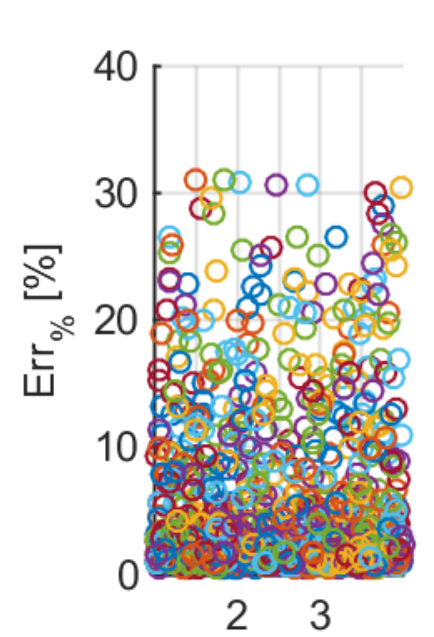

Hs [m]
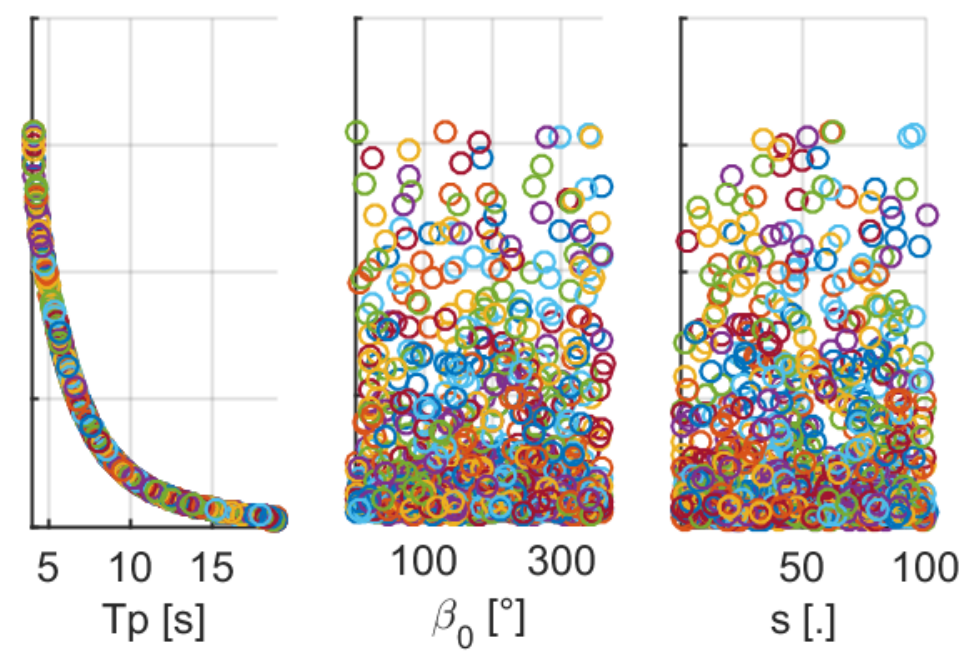

Err $_{\text {abs. }}\left|\beta_{0}-\beta_{0}\right|$
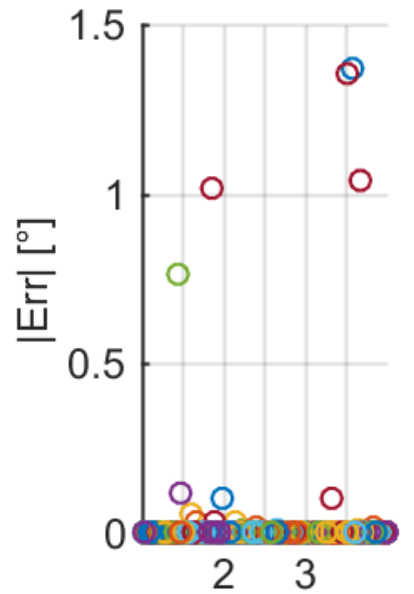

Hs [m]
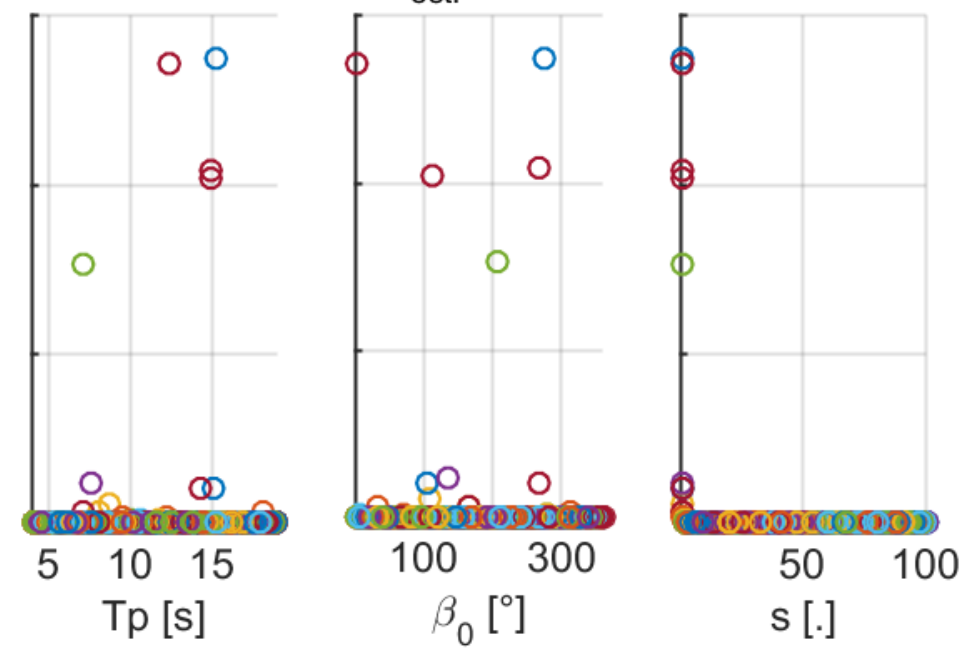

$\mathrm{Err}_{\text {abs. }}\left|\mathrm{s}^{\mathrm{s}} \mathrm{s}_{\text {est. }}\right|$
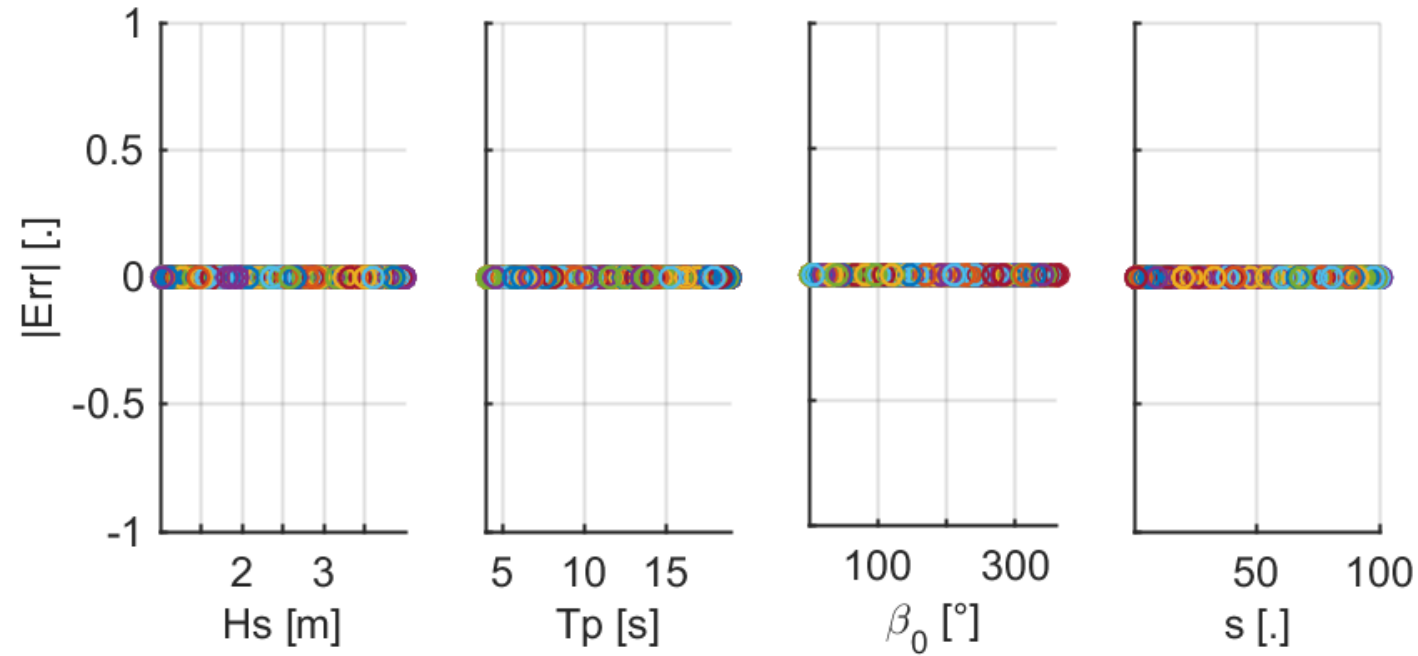

Source: Elaborated by the author. 
The errors in $H_{S}$ and $T_{P}$ have a clear relation with the $T_{P}$ of the generated sea. It happens because of the upper limit of the values $\omega$ in the discretization - lower limit of the values of the period. The theoretical JONSWAP spectrum are defined for an infinite range of frequencies; when the range is constrained, some of the energy of the spectrum will be lost, so the parameters calculated by integral formulations will be always smaller than the real ones. Lower $T_{P}$ spectra are close to the truncation point, so they will experience more error. Since the error is large and deterministic, it can be classified as a bias, and can be simply corrected even when noise is applied.

The errors in the $s$ estimation are exactly zero, without any bias. However, the errors in $\beta_{0}$ can reach around $1.5^{\circ}$ when the seas present spread factor equals to one. In this case, the error can be seen as an alias, i.e., there is a range of the parameter that causes almost the same output response due to discretization. It makes sense that this happens for small values of $s$, since in these cases the spread function will be almost a flat plateau around the incoming direction, and small variations of $\beta_{0}$ will cause almost no variation. In this case, the error may not be simply corrected when noise is applied, since it is related with the small difference between the correct value and, thus, can be classified as an alias.

The discretization used, however, are too high for estimation purposes. A second trial is, then, performed reducing the discretization of $\beta$ to 20 . The selected metrics for this case are shown in Figure 38.

Figure 38 - Parametric errors during verification with $40 \omega$ and $20 \beta$.

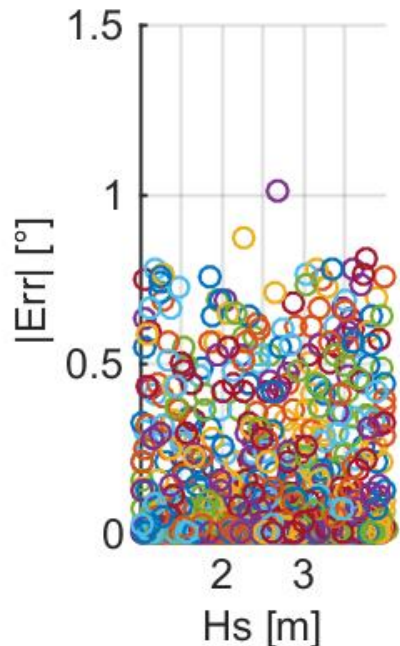

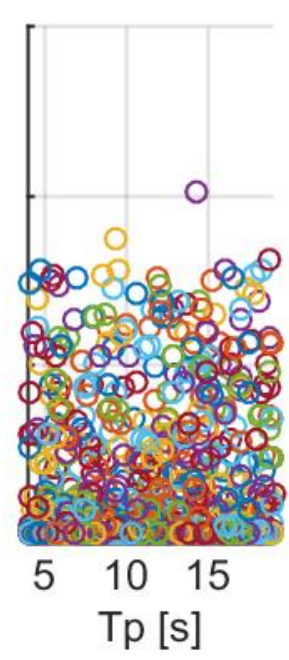

$\mathrm{Tp}$ [s]
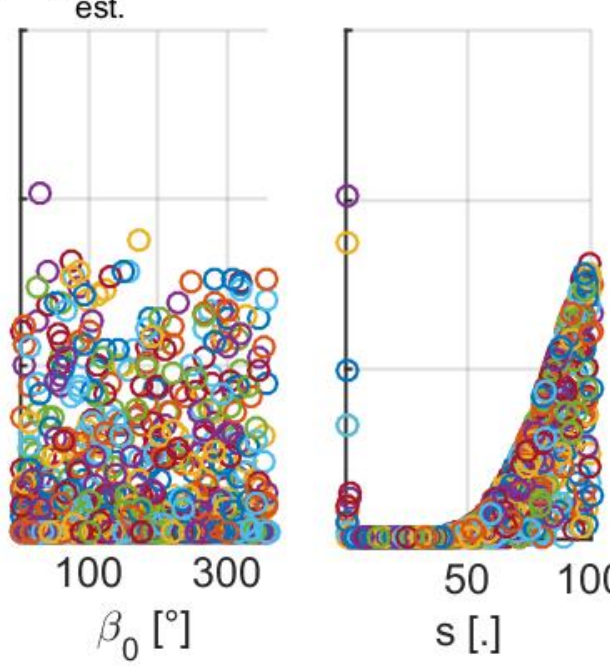

$50 \quad 100$ s [.] 

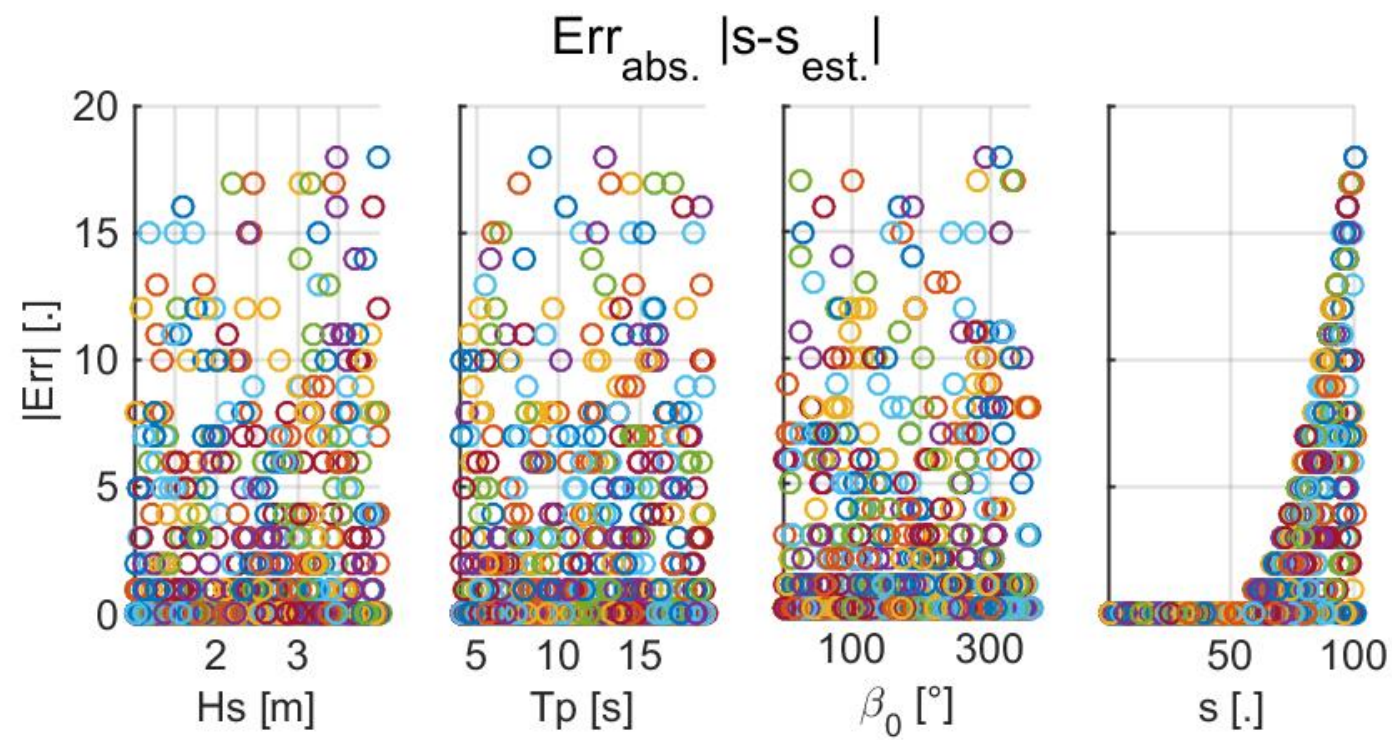

Source: Elaborated by the author.

In this case, a new kind of directional parameters error starts to appear for spread factors greater than 50 . With a detailed analysis, it is possible to see that this kind of error happens when the sea $\beta_{0}$ is between two points in the discretization. In these cases, when the spread factor is greater than $50, D(\beta)$ has a high pronounced peak, and a small difference in the incoming direction cause huge differences in the shape of the response, meaning that the estimated spread factor will have to be corrected to account for this difference. Finally, the two estimated parameters are able to reach a value that results in a $D(\beta)$ with almost no difference against the real function, but with significantly different parameters, a clear case of alias. Figure 39 shows an example. In spite of the difference in $s$, there is almost no variation in the function shape. Again, this error is not corrected easily, and is classified as an alias.

Figure 39 - Example of alias in $D(\beta)$.

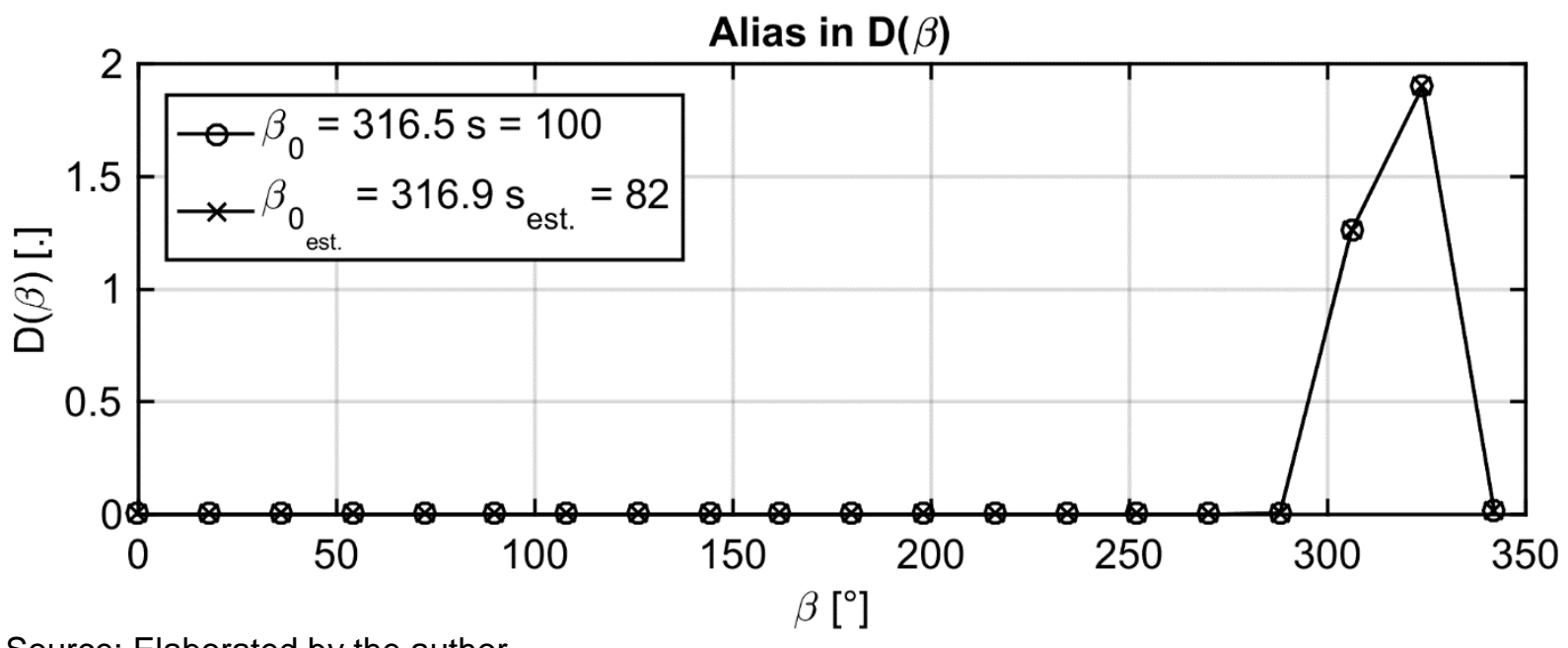

Source: Elaborated by the author. 
Finally, a third trial is done with both discretizations equal to 20 . The only difference is that the errors in $H_{S}$ and $T_{P}$ are not monotonically descending, but oscillates when the $T_{P}$ value is between the discretization points. The error, however, can still be classified as a bias, and easily corrected.

Figure 40 - Parametric errors during verification with $20 \omega$ and $20 \beta$.

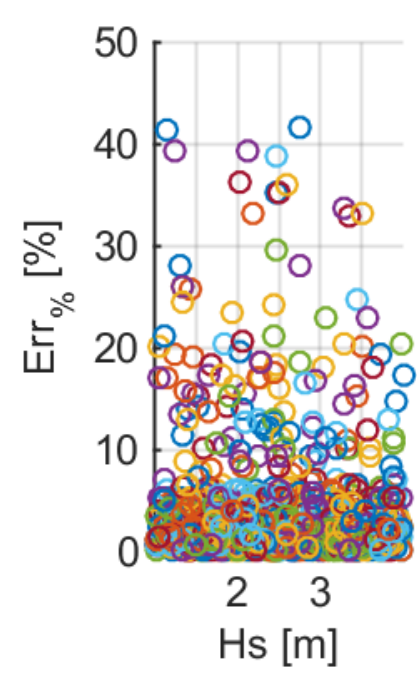

$$
\mathrm{Err}_{\%} \mid \mathrm{Hs}-\mathrm{Hs} \text { est. } \mid / \mathrm{Hs}
$$
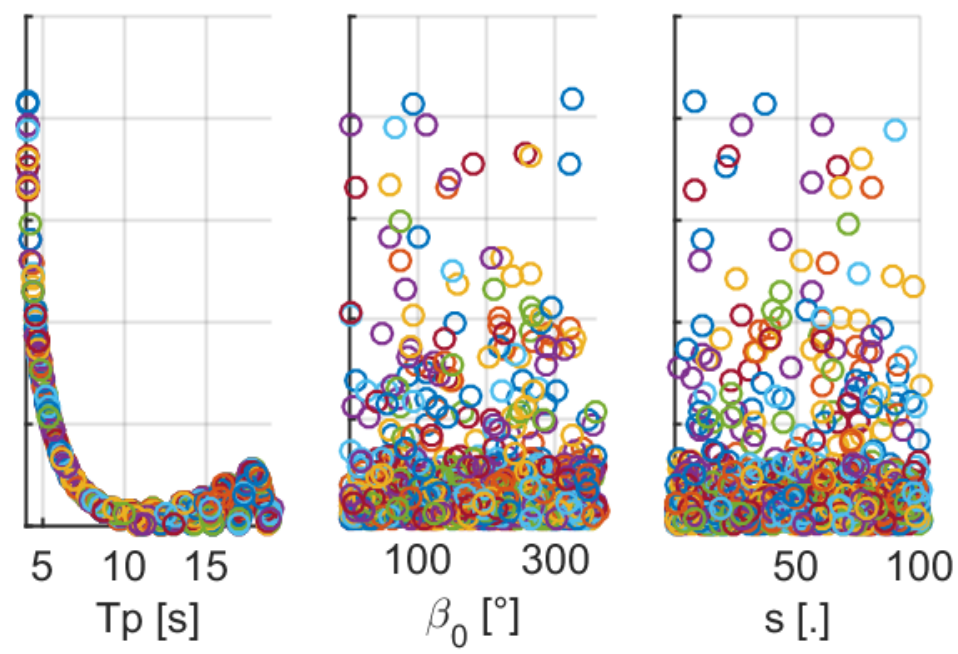

$$
\mathrm{Err}_{\%}\left|\mathrm{Tp}-\mathrm{Tp} \mathrm{est.}_{\mathrm{T}}\right| / \mathrm{Tp}
$$
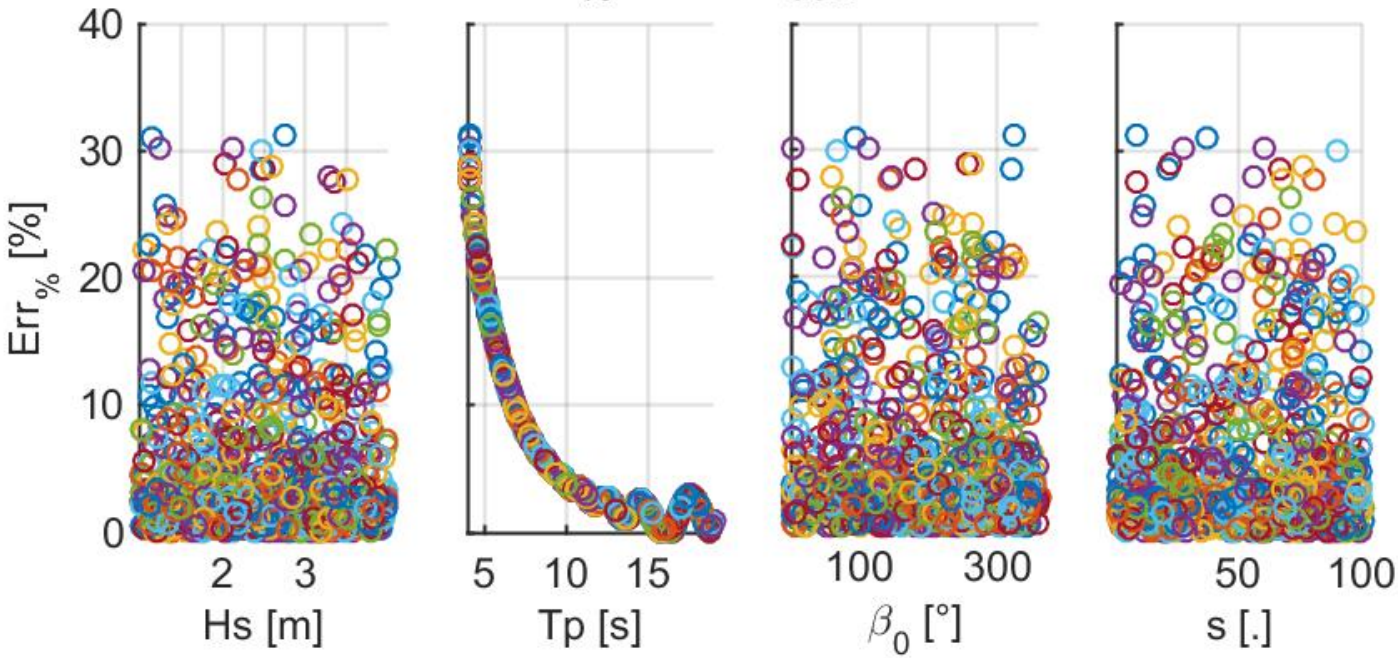

Source: Elaborated by the author.

Despite the alias in the estimation of the directional parameters, it can be argued that the alias does not cause a sensible change in the characterization of the spectrum, so it is not a real error, which is true. However, knowing that an alias can happen, and in which cases it happens, is important when analyzing the possible improvements by employing wave-probes in the estimation. 
It is interesting to perform a final verification, adding some noise to the estimated sea. The purpose of the study is to verify if it is really possible to correct the bias in the estimation. Firstly, a sea is generated, Equation (167), and the bias is calculated estimating the parameters of the sea without noise, Equation (168); secondly, noise is added, Equation (169), and the parameters are estimated for the noisy sea, Equation (170); lastly the error metrics are calculated comparing the noise sea estimation against the biased real parameters. The results are shown in Figure 41 and Figure 42.

$$
\begin{gathered}
\boldsymbol{x}=f\left(H_{S}, T_{P}, \beta_{0}, s\right) \\
{\left[H_{S}+b_{H_{S}}, T_{P}+b_{T_{P}}, \beta_{0}+b_{\beta_{0}}, s+b_{S}\right]=\operatorname{parameterEstimation}(\boldsymbol{x})} \\
\boldsymbol{x}^{*}=\boldsymbol{x}+\left|0.001 \cdot \boldsymbol{\epsilon}_{\mathbf{1}}\right|+0.1 \cdot \boldsymbol{I} \times \boldsymbol{\epsilon}_{\mathbf{2}}^{T} \times \boldsymbol{x}, \quad \boldsymbol{\epsilon}_{\mathbf{1}} \text { and } \boldsymbol{\epsilon}_{\mathbf{2}} \operatorname{are} \mathcal{N}(\boldsymbol{\mu}=\mathbf{0}, \boldsymbol{\Sigma}=\boldsymbol{I}) \\
{\left[H_{S}^{*}, T_{P}^{*}, \beta_{0}^{*}, s^{*}\right]=\text { parameterEstimation }\left(\boldsymbol{x}^{*}\right)}
\end{gathered}
$$

Figure 41 - Parameter errors without removing bias.

$\mathrm{Err}_{\%}\left|\mathrm{Hs}-\mathrm{Hs}{ }_{\text {est. }}\right| / \mathrm{Hs}$
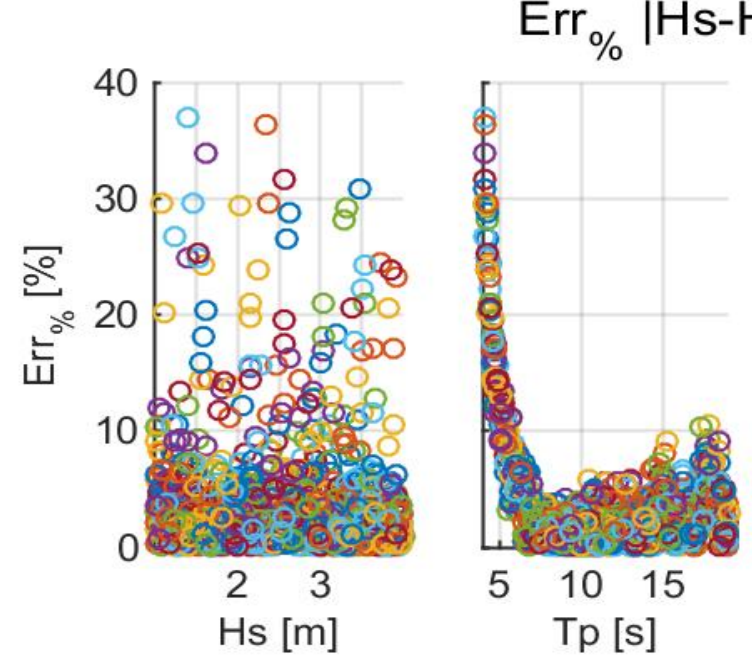

$5 \quad 1015$

$\mathrm{Tp}[\mathrm{s}]$

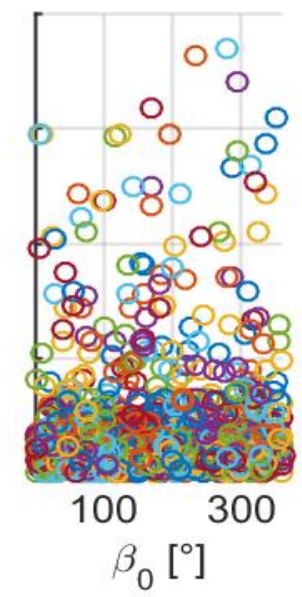

$\mathrm{Err}_{\%}\left|\mathrm{Tp}-\mathrm{Tp} \mathrm{est}_{\text {el }}\right| / \mathrm{Tp}$

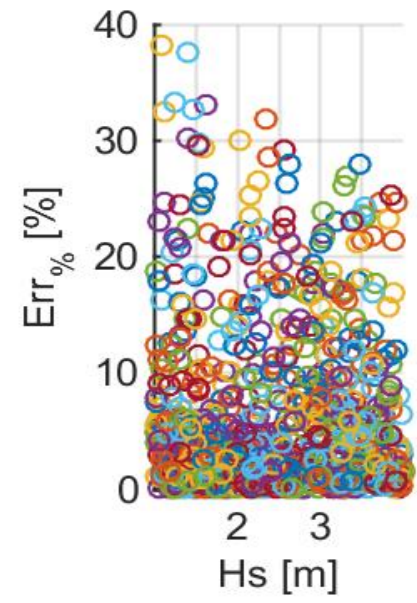

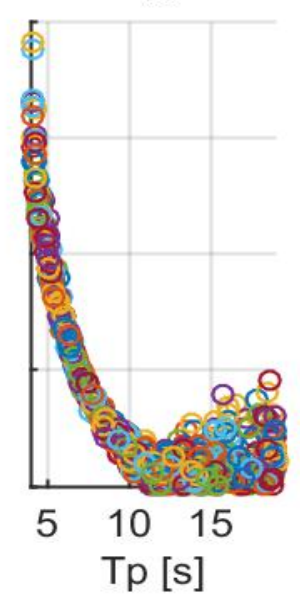

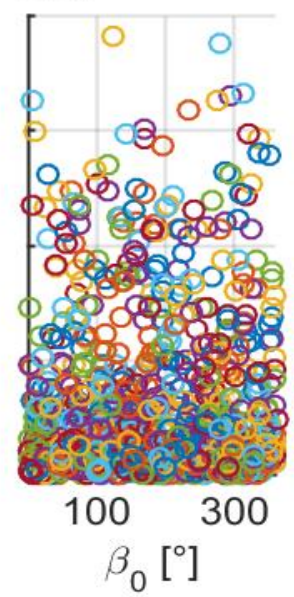

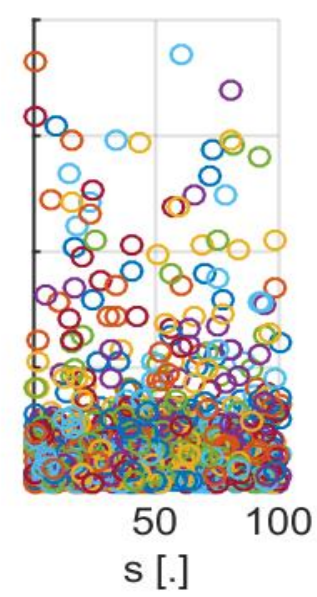

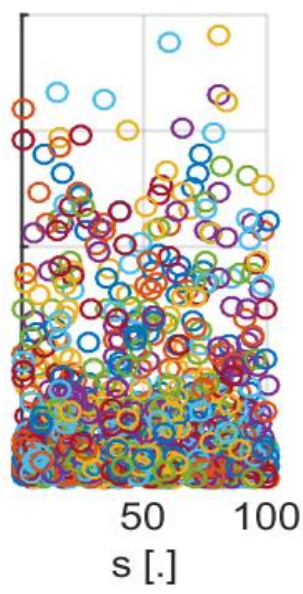



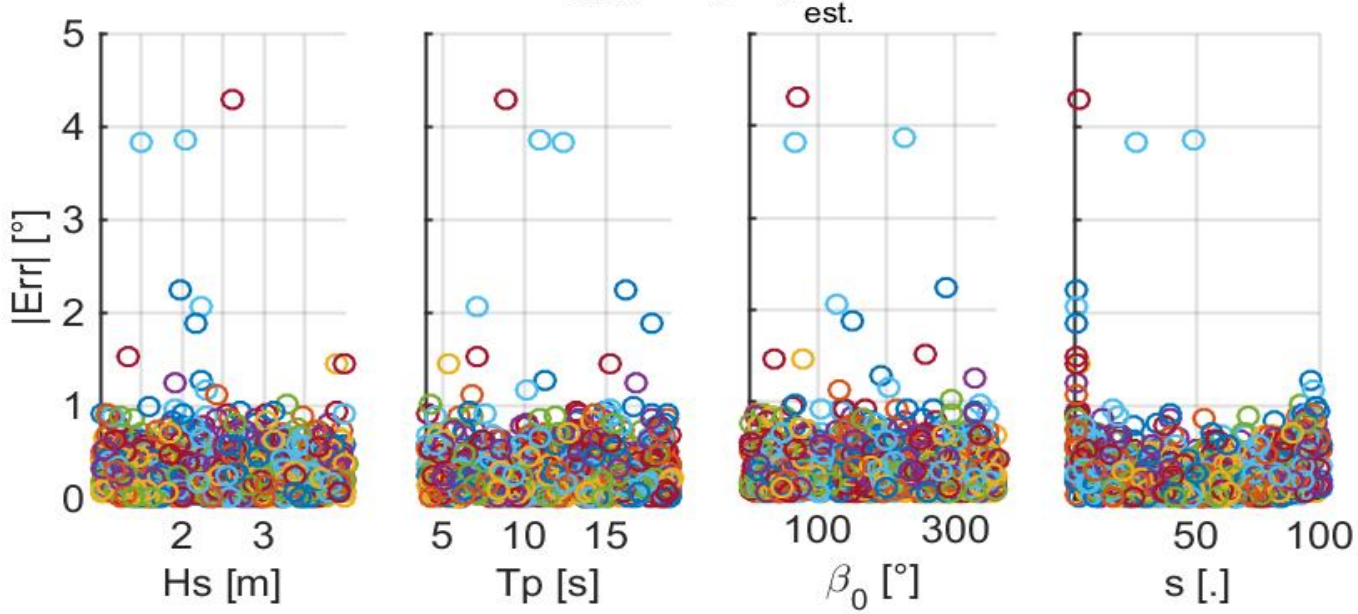

Err $_{\text {abs. }}\left|\mathrm{s} \mathrm{s}_{\text {est. }}\right|$
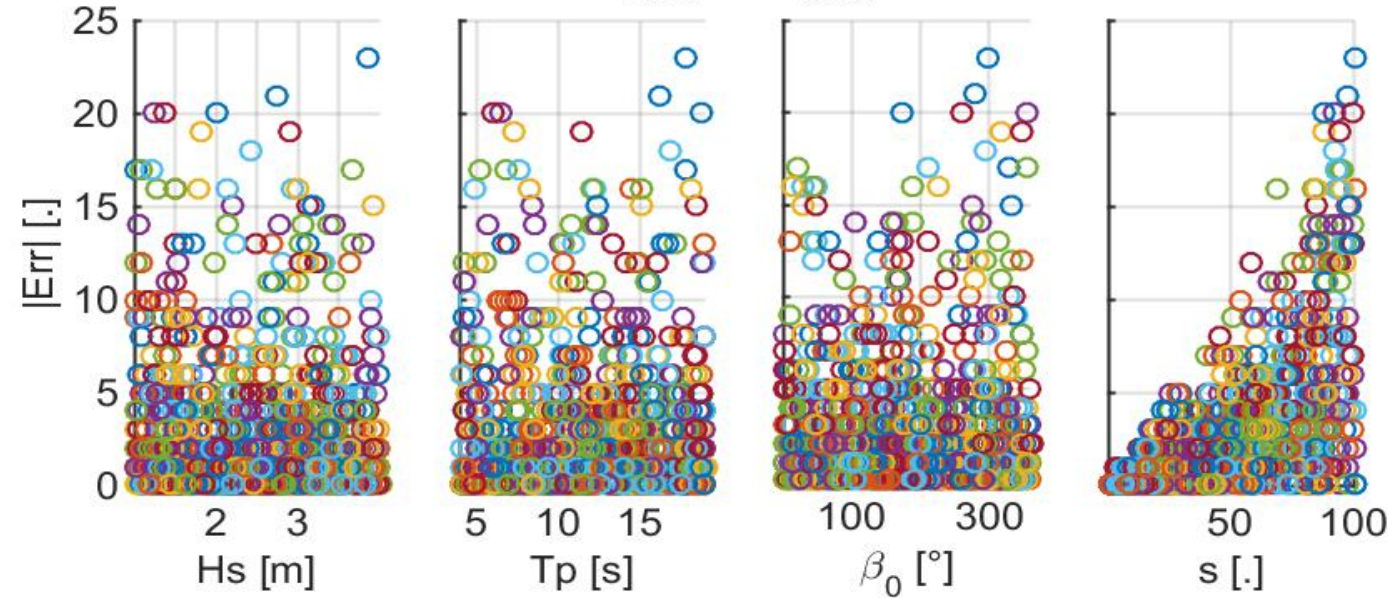

Source: Elaborated by the author.

Figure 42 - Parameter errors removing bias.

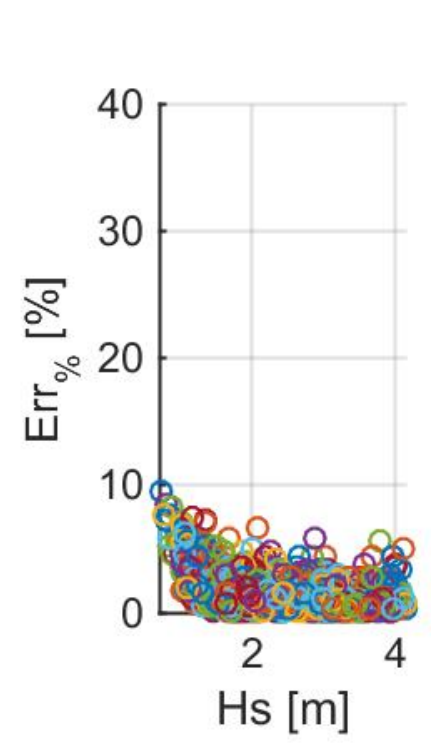

$\mathrm{Err}_{\%}|\mathrm{Hs}-\mathrm{Hs} \mathrm{est}| / \mathrm{Hs}$
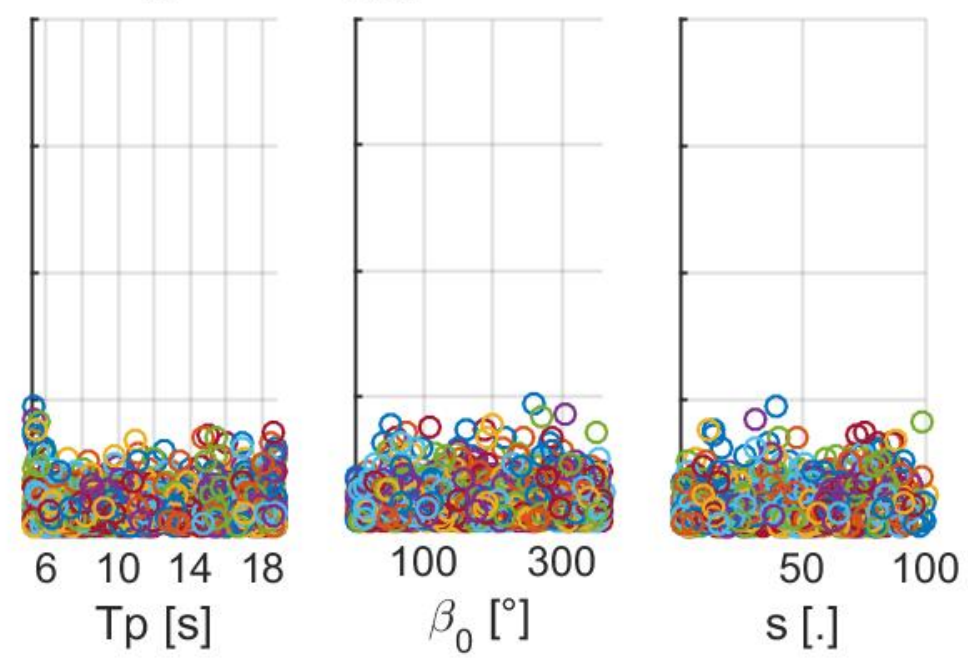


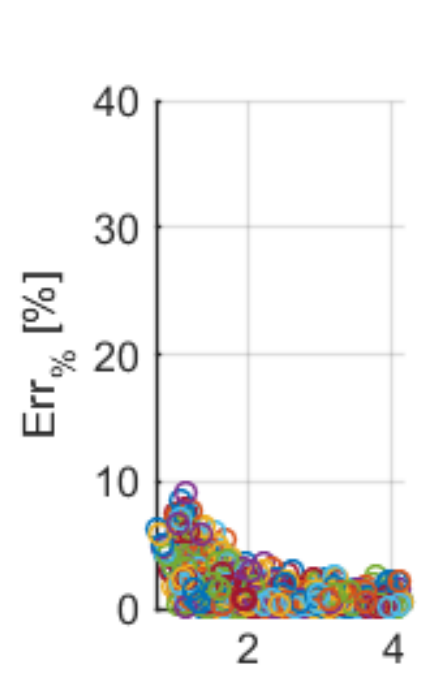

$\mathrm{Hs}[\mathrm{m}]$

Err $_{\%}\left|T p-T p_{\text {est. }}\right| / T p$
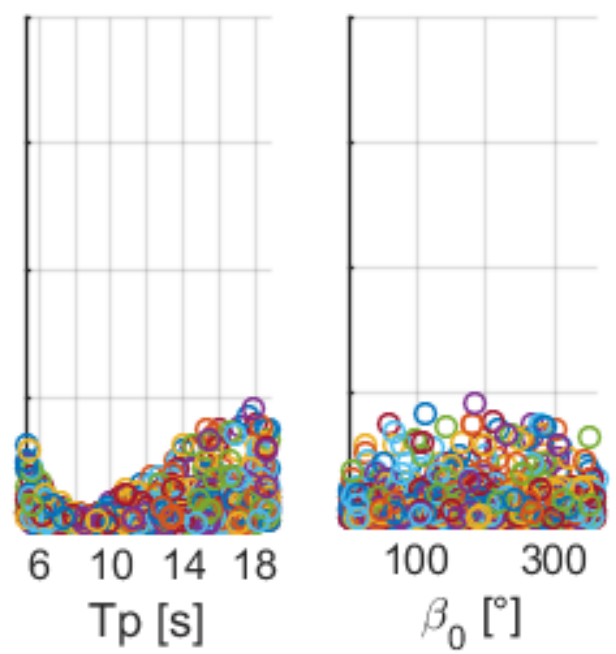

Err $_{\text {abs. }}\left|\beta_{0}-\beta_{0}\right|$
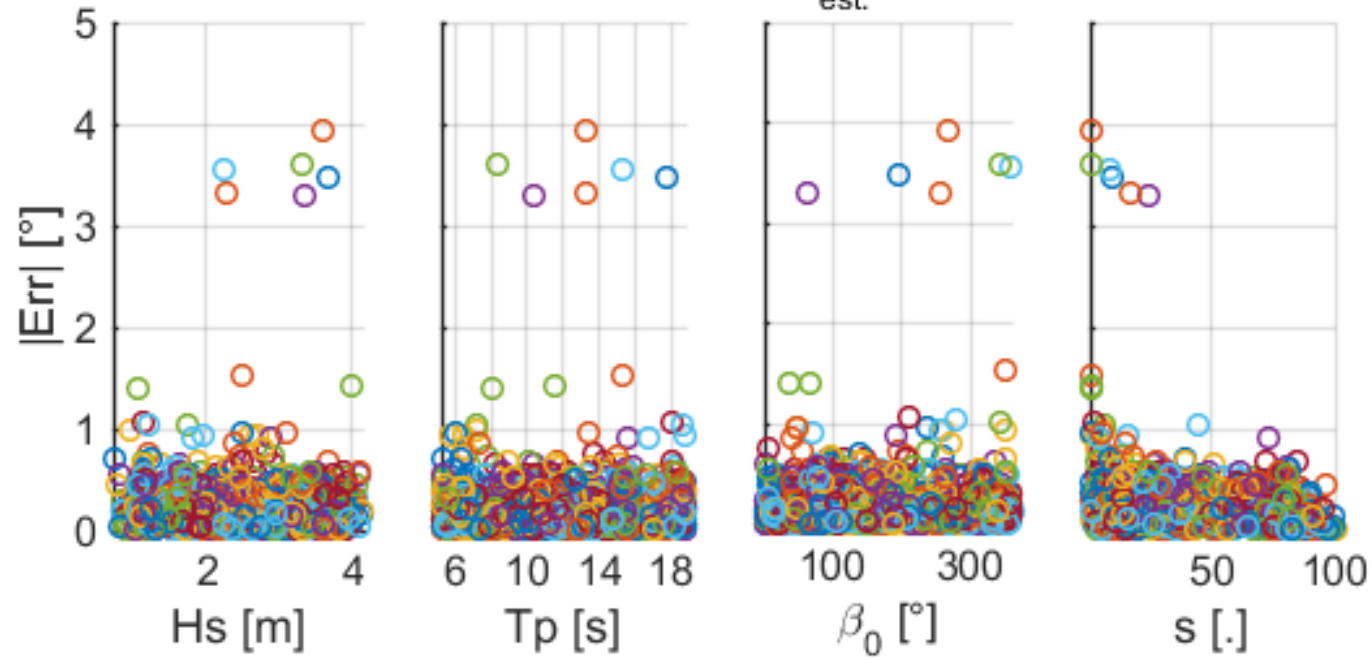

$\mathrm{Err}_{\text {abs. }}\left|\mathrm{s}^{\mathrm{s}} \mathrm{s}_{\text {est. }}\right|$

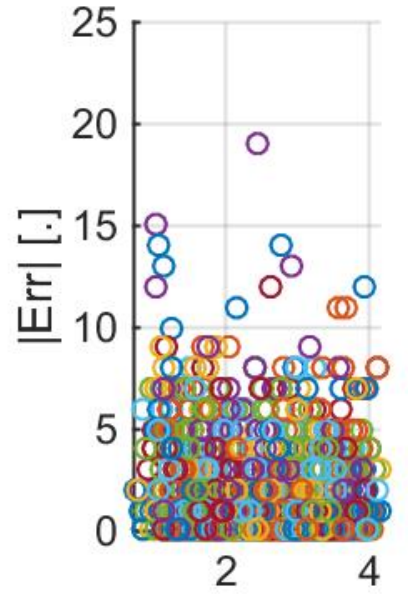

$\mathrm{Hs}[\mathrm{m}]$

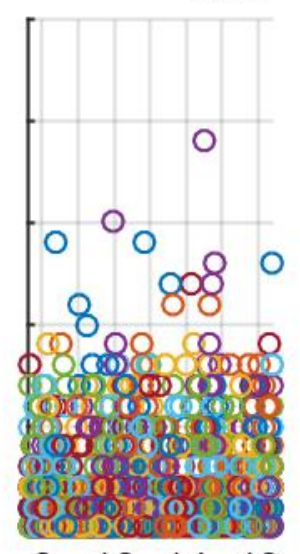

$\begin{array}{llll}6 & 10 & 14 & 18\end{array}$

$\mathrm{Tp}$ [s]
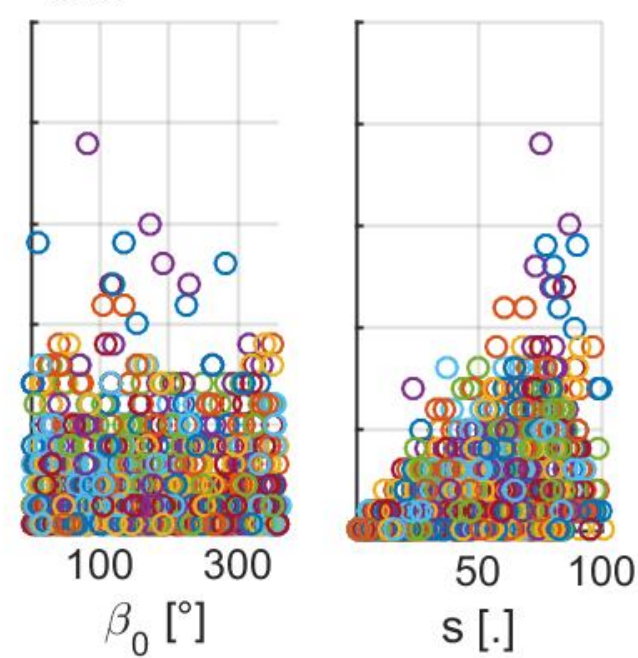

Source: Elaborated by the author. 
In conclusion, as expected, the numerical bias could be removed from the estimations of $H_{S}$ and $T_{P}$, but not from the estimations of $\beta_{0}$ and $s$, since the last two errors are caused by alias related with the problem discretization; notwithstanding, the estimation of the directional parameters also had a slight improvement.

\subsection{Validation of the Trace Criterion}

After correcting the numerical bias, and knowing the possible alias in the direction estimation, it is possible to perform the simulations in order to validated the proposals made. The first validating results are those concerning the trace criterion.

This trial of numerical tests was performed with all the combinations among the twelve possible wave-probe positions. The vessel DoFs used were the sway-heavepitch combination, as proposed by (SIMOS, et al., 2009), with the hyperparameters defined by (BISPO, et al., 2012). The number of wave-probes was also fixed in three, since the objective of this trial is to validate the hypothesis: "the least the trace criterion of a combination, the best is the estimation"; totalizing $\frac{12 !}{3 ! 9 !}=220$ combinations.

For each combination, 1000 seas were generated and estimated, and the trace criterion was calculated. The errors of the estimation of each sea were, then, calculated, and their means were compared against the trace criterion.

\subsubsection{Noiseless simulation}

Firstly, simulations without the addition of noise were performed, Figure 43.

Figure 43 - Trace criterion versus different error metrics in noiseless estimation.

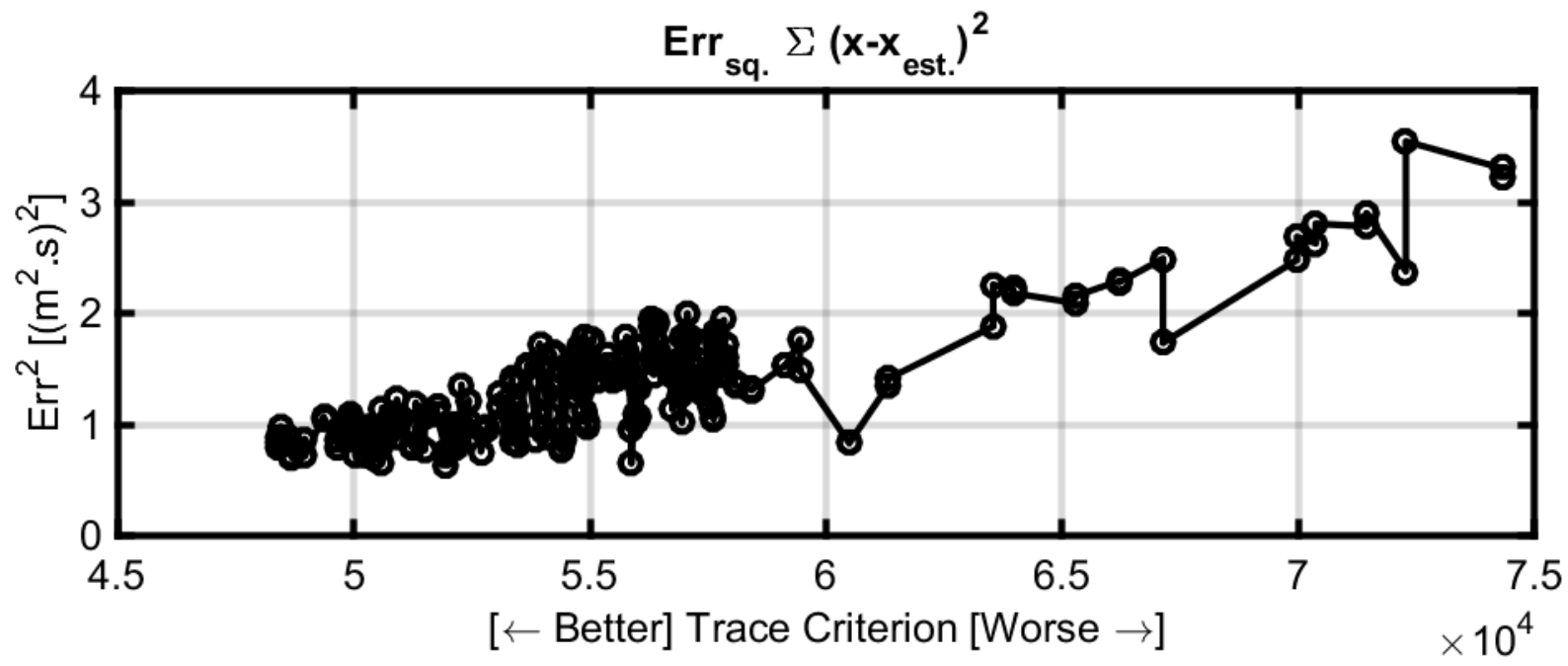



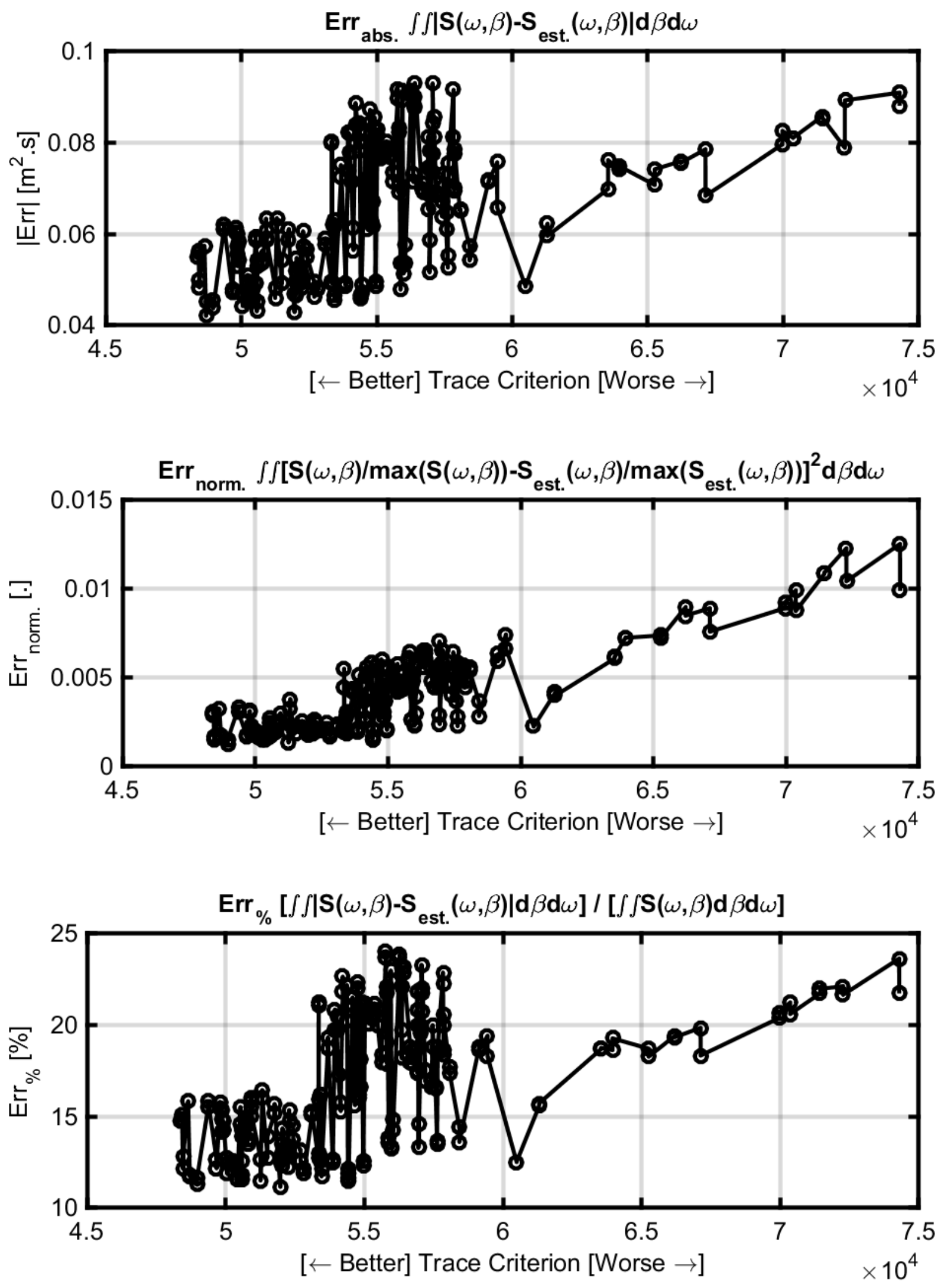

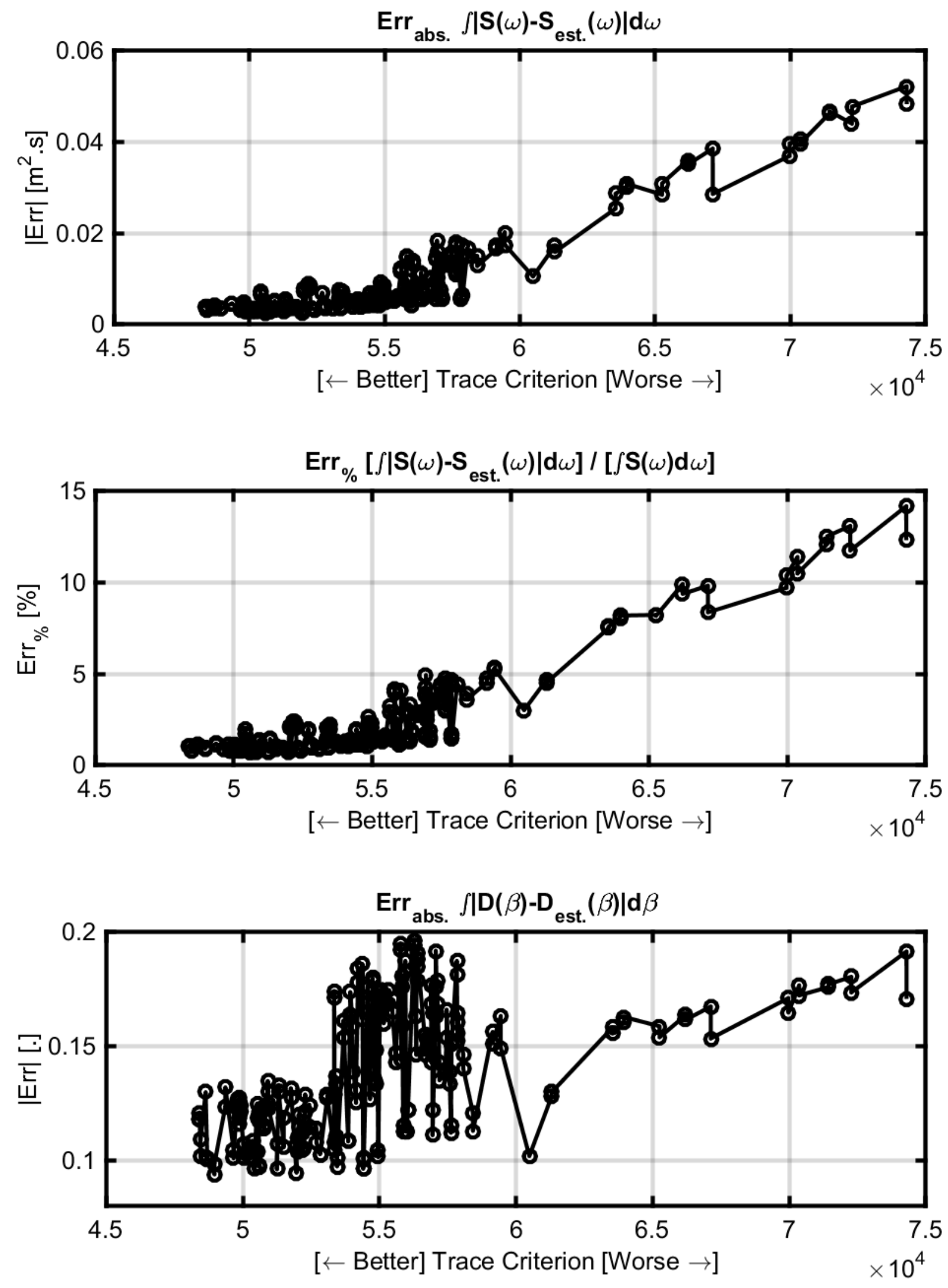

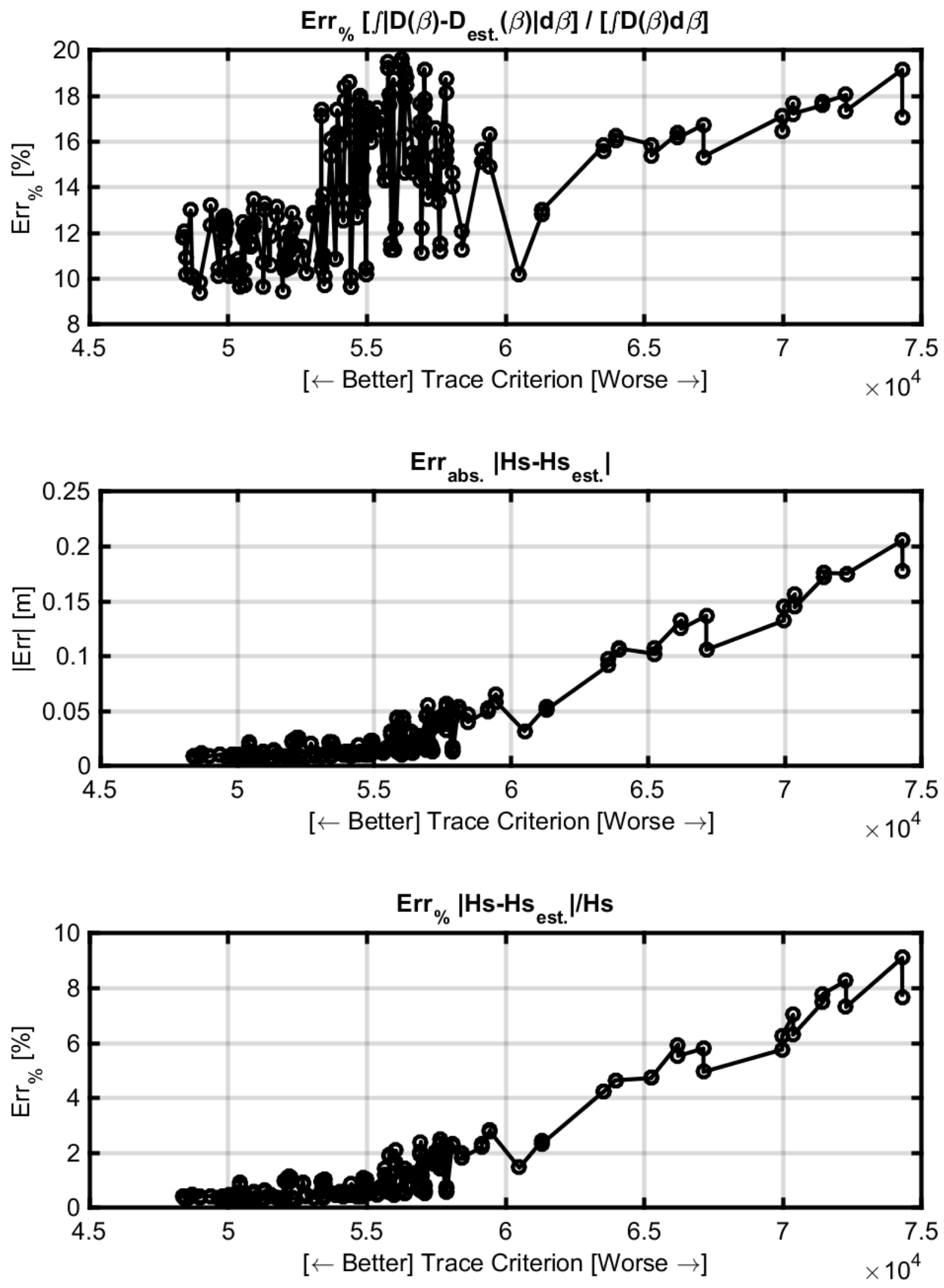

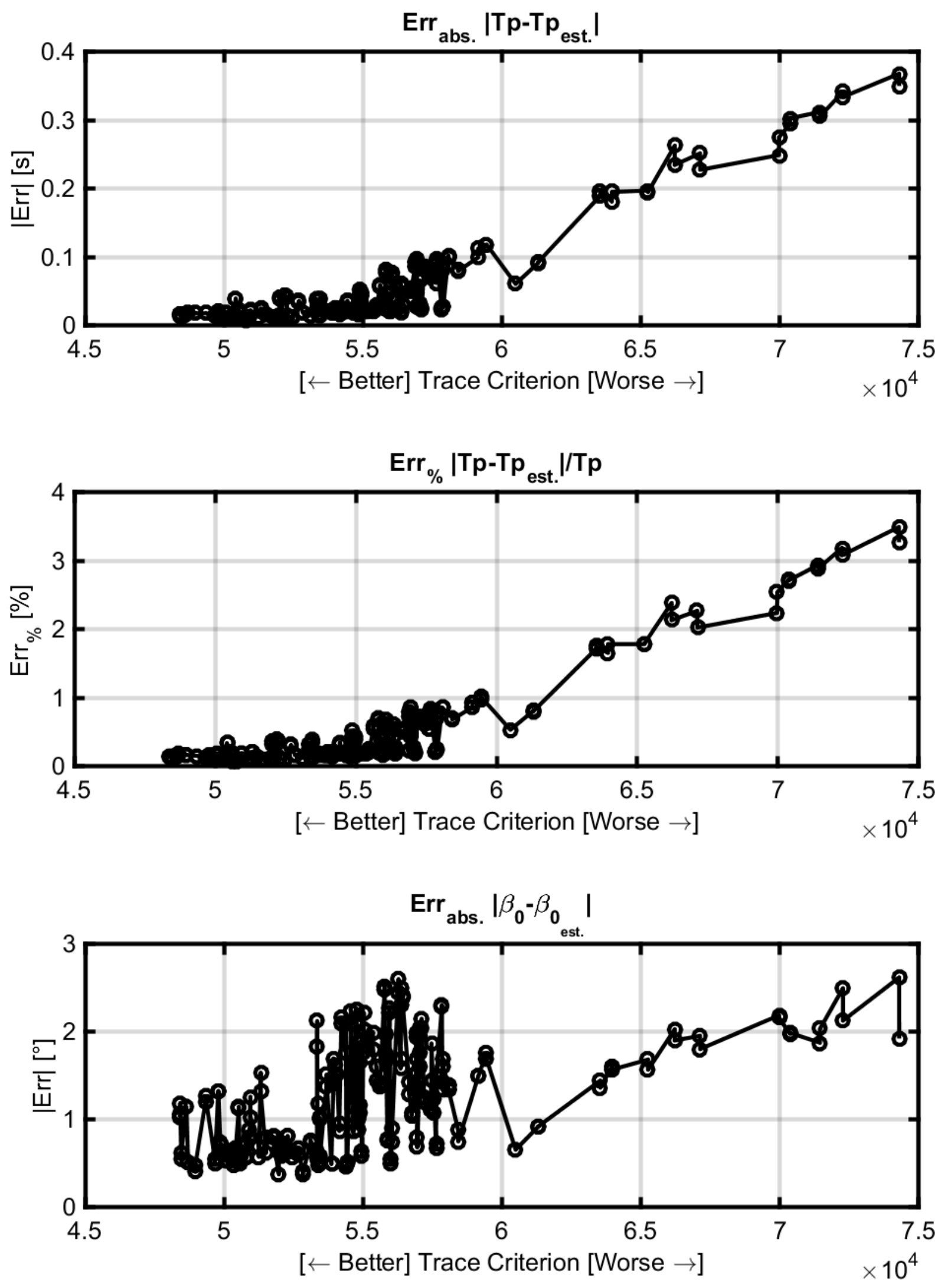

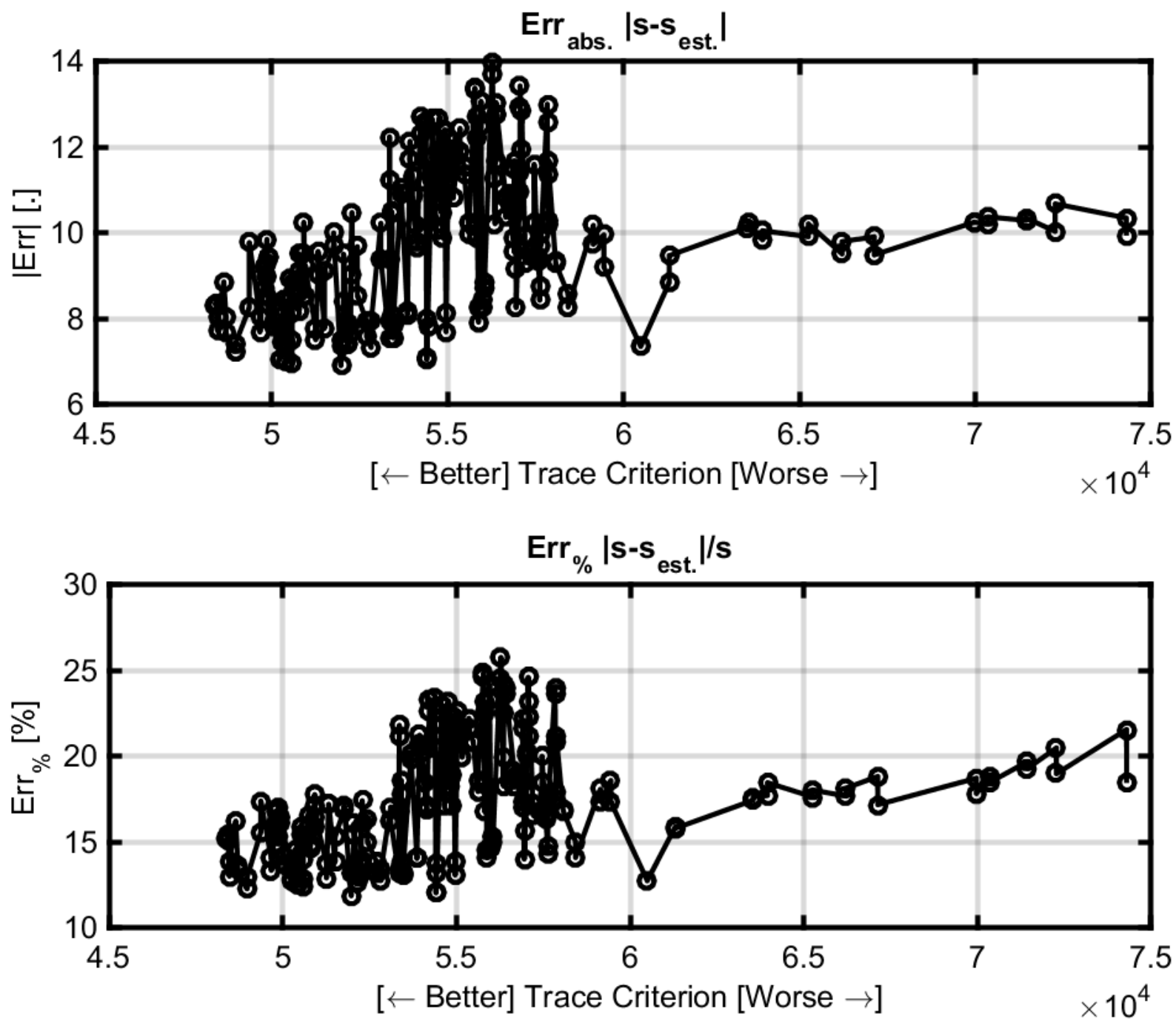

Source: Elaborated by the author.

The first conclusion is that there is a clear tendency indicating that low trace values generate low mean errors in all the error metrics proposed, even with the strong hypothesis made that the prior is exactly the probability distribution of the sea and that the optimal solution calculated by the closed form is equal to the one calculated by the quadratic programming with constraints.

The relation with $\operatorname{Err}_{x}^{2}$ is almost linear, which is expected by the theory; and metrics related with the spectrum energy shape have a strong relation with the criterion: $\left|E r r_{S \omega \beta}\right|, \operatorname{Err}_{S \omega \beta} \%,\left|\operatorname{Err}_{S \omega}\right|, \operatorname{Err}_{S \omega} \%,\left|\operatorname{Err}_{H S}\right|, \operatorname{Err}_{H S} \%,\left|\operatorname{Err}_{T p}\right|$ and $\operatorname{Err}_{T p} \%$.

Metrics related with the directional spread function do not have a tendency so strong with the criterion; notwithstanding, it is possible to affirm that if you take a value in a region with low trace, it probably will present a low error and the maximum possible 
error will be small if compared against regions with higher trace, see, for example, Figure 44. This happens in $\left|\operatorname{Err}_{D \beta}\right|, \operatorname{Err}_{D \beta} \%,\left|\operatorname{Err}_{S}\right|$ and $E r r_{S} \%$.

Figure 44 - Regions with low trace have lower worst-case.

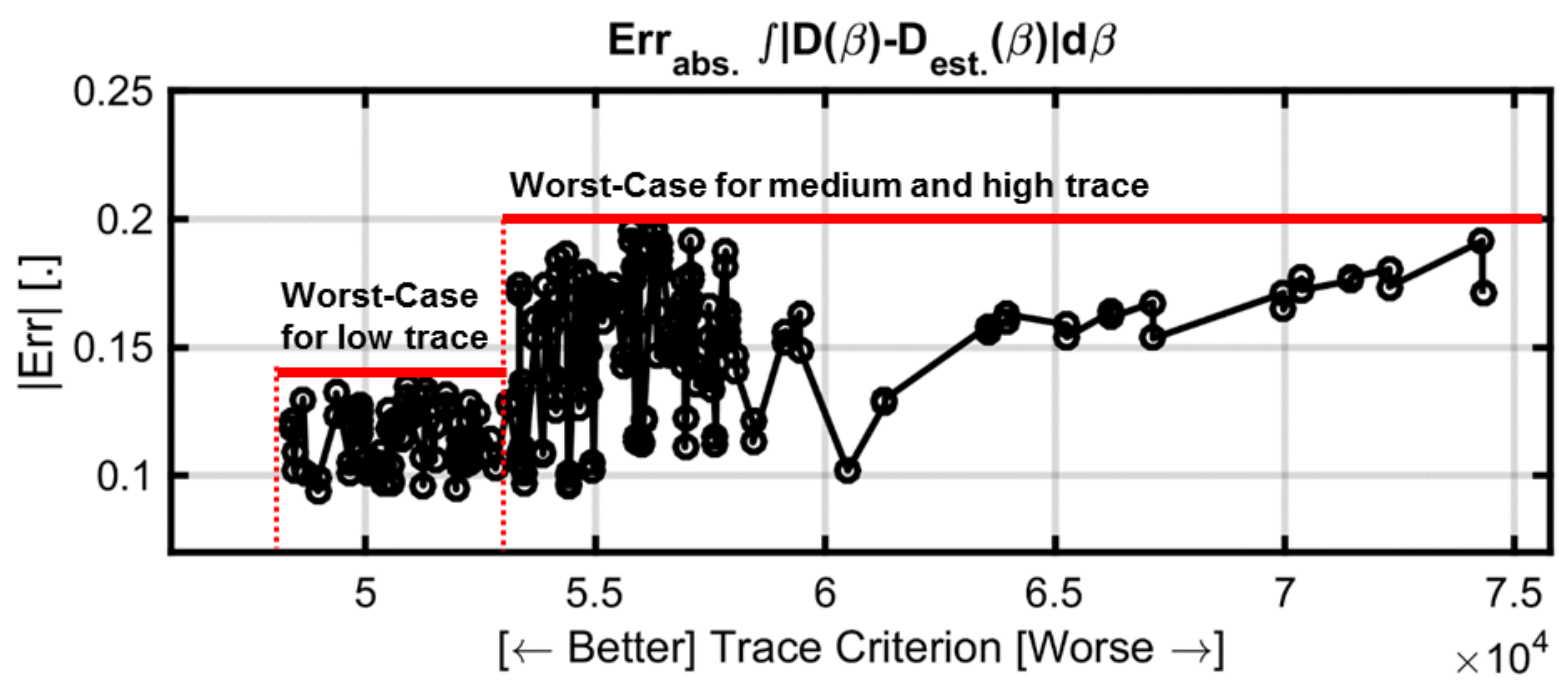

Source: Elaborated by the author.

If it is desired to minimize the errors in those criterions, the trace criterion can be used to calculate some optimal candidates, which can be then simulated until the best one is found. This is particular interesting if the computational time is put in perspective, since the trace calculation is between 8000 and 12000 faster than the simulation of 1000 seas, i.e., even if only 500 seas were used, which is in the limit of the convergence of the Monte-Carlo simulations, an optimization routine that takes 5 minutes with the trace criterion would take around 17 days to be completed. If the trace criterion is used to select ten best candidates, which are, then, simulated, the optimization would take no more than 10 minutes.

The error $\left|\operatorname{Err}_{\beta o}\right|$, however, has a region in which the errors seem to be smaller than in the low-trace region. Despite of that, there are some candidates in the low-trace region that have errors close to the optimal candidate, so the low-trace region remains a good starting point to optimize this particular metric.

As a consequence of the previous analysis, the trace criterion is also a good starting point of a multi-objective optimization that uses a combination of different mean error metrics.

Until now, the mean of each error metric was observed, but that does not reflect completely the estimation capabilities of each combination. Twenty combinations were 
chosen, equally spaced, to perform a more detailed analysis, Figure 45. The combination ID indicates how the probes were chosen, the ID 1, for example, represents the probes 1-2-3, the ID 2, 1-2-4, and so on.

Figure 45 - Chosen combination for detailed analysis.

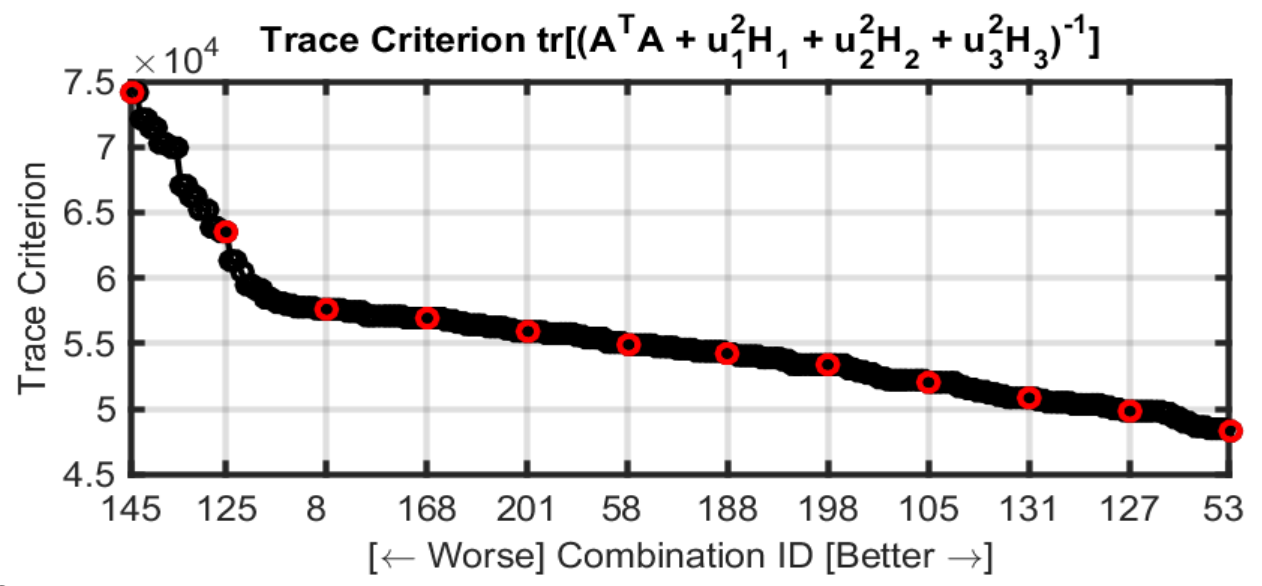

Source: Elaborated by the author.

The detailed analysis is done by a histogram type of graphic, allowing the visualization not only of the mean value but also of the cumulative distribution for each error. Essentially, it is possible to verify what is the maximum error - the error that $100 \%$ of the cases are below - what is the error that $90 \%$ of the cases are below, what is the error that $80 \%$ are below and so on. Only some error metrics are shown, since the others tend to follow them, Figure 46.

Figure 46 - Histogram of the combinations performing noiseless estimation.

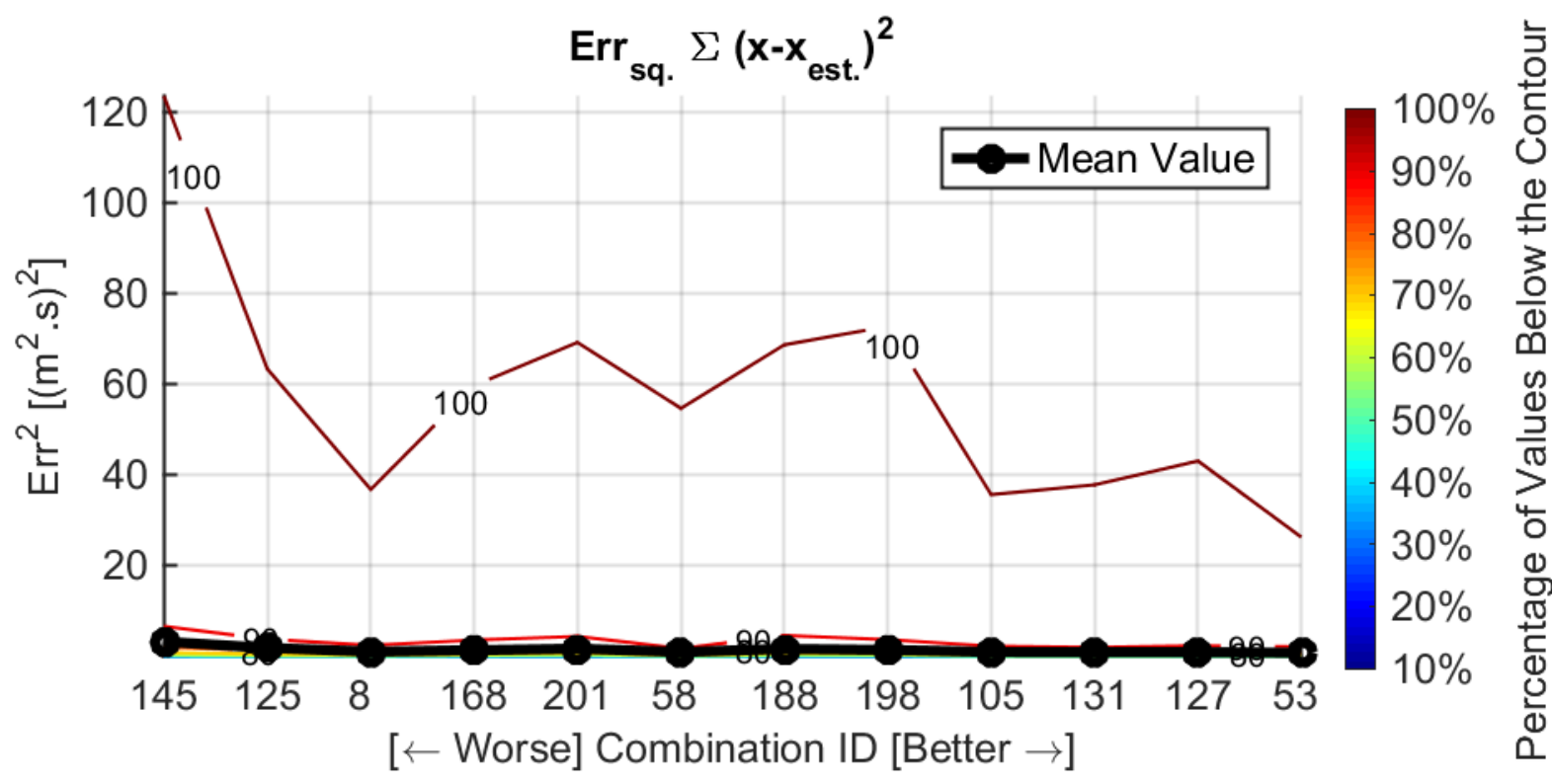



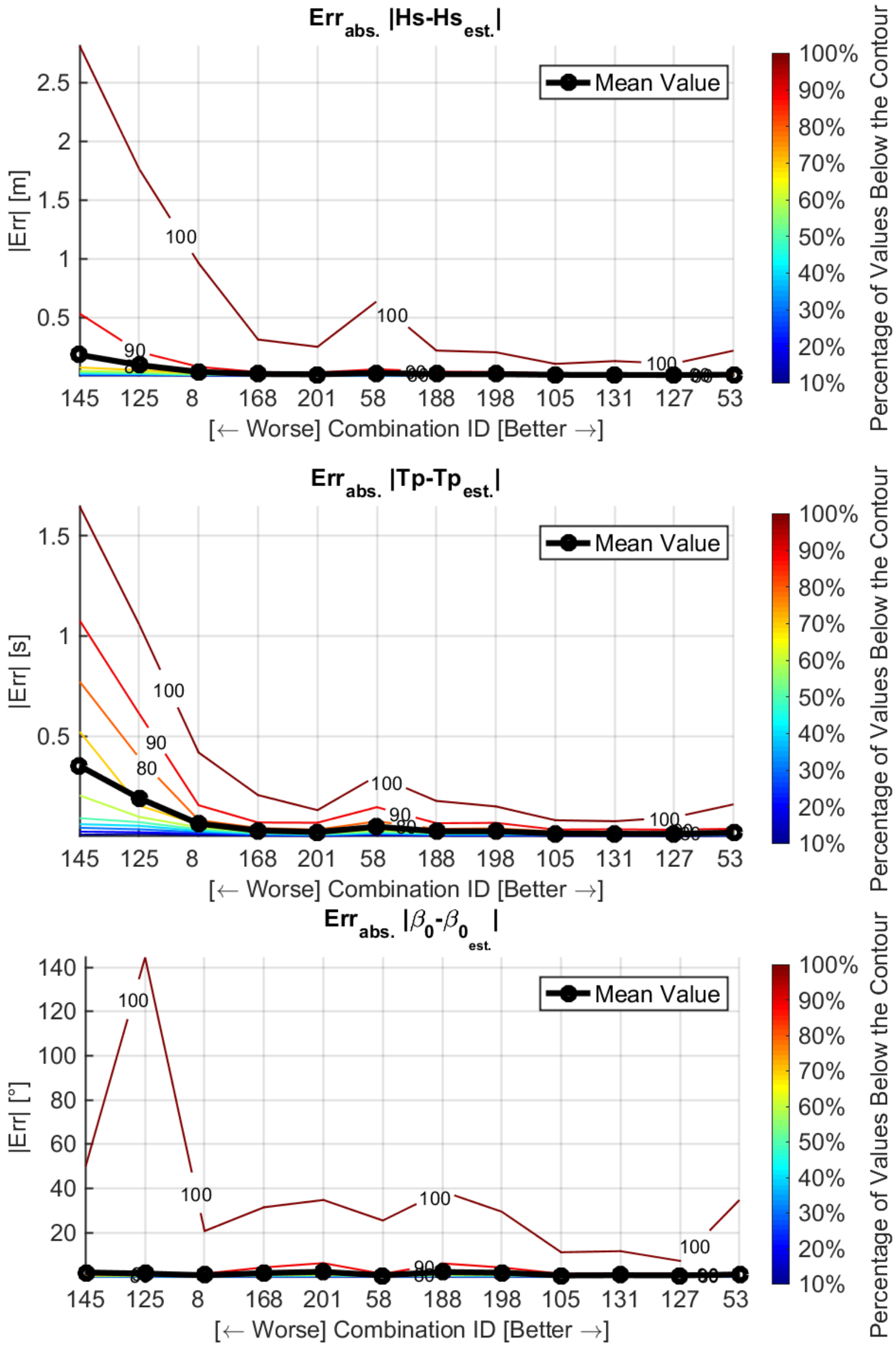


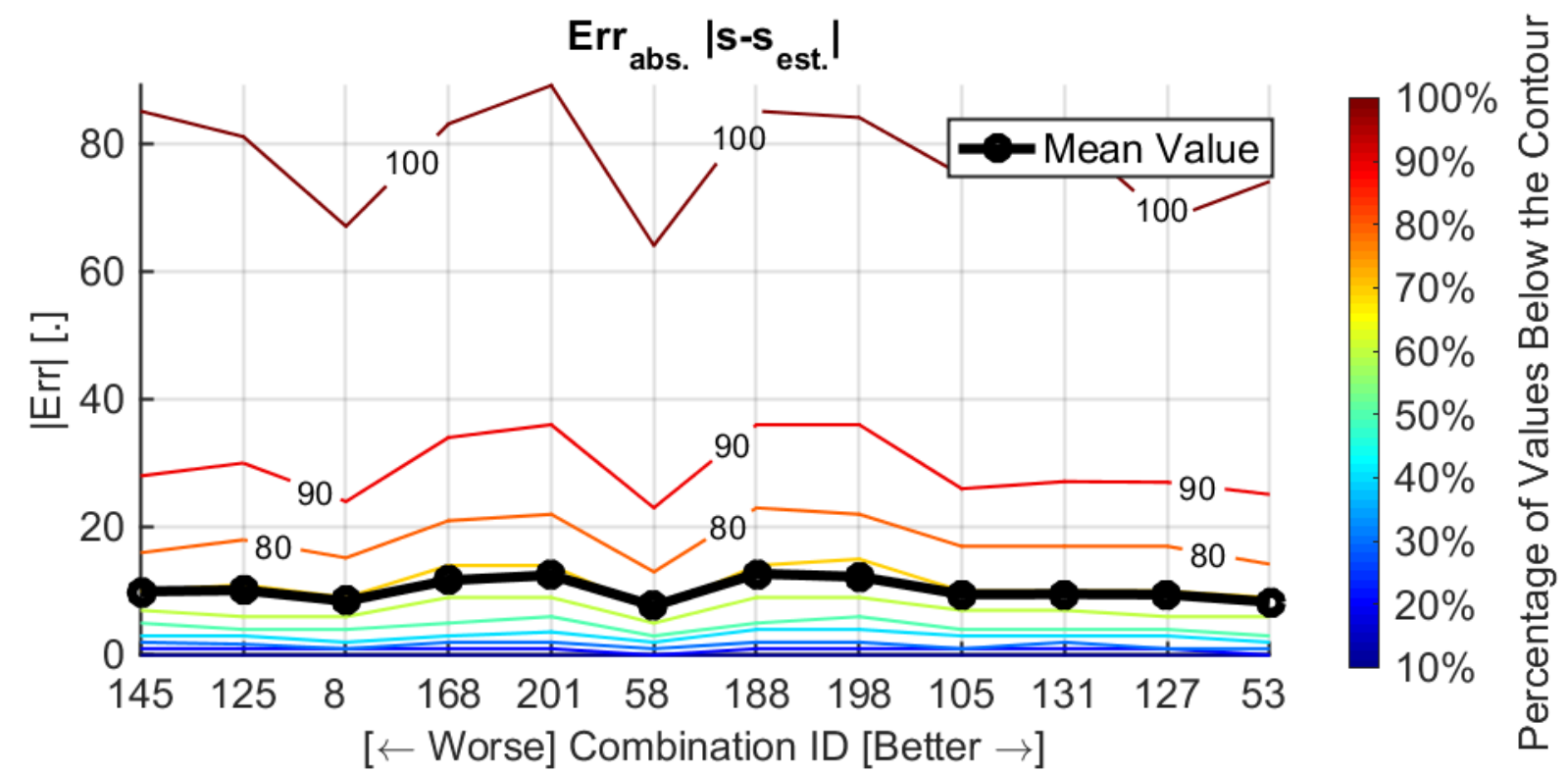

Source: Elaborated by the author.

For the metrics $\operatorname{Err}_{x}^{2}$ and $\left|\operatorname{Err}_{H}\right|$, the $10 \%$ largest errors greatly surpass the error of the other $90 \%$ of the cases, but the maximum value also tends to decrease with the decrease of the trace. The metric $\left|\operatorname{Err}_{T p}\right|$ also has a strong tendency with the trace criterion, not only the mean value but all the observed errors.

The mean direction metric, $\left|\operatorname{Err}_{\beta o}\right|$ also has the 10\% largest errors greatly surpassing the error of the other $90 \%$ of the cases, with a slightly tendency to follow the trace decrease. Despite of this, it seems that the most important thing for $\operatorname{Err}_{\beta o}$ is, actually, to avoid positions with high trace.

Finally, the metric $\left|E r r_{s}\right|$ does not seem to have a tendency, using the chosen combinations.

\subsubsection{Simulations with noise}

In order to validate the method, it is important to add noise to the measurements. The model assumes an error with normal distribution and same magnitude in each position of the response vector, so this formulation will be used in the simulations, Equation (171).

$$
\boldsymbol{b}=\boldsymbol{A} \boldsymbol{x}+\boldsymbol{\epsilon}, \quad \boldsymbol{\epsilon}=\mathcal{N}\left(\boldsymbol{\mu}=\mathbf{0}, \boldsymbol{\Sigma}=\sigma^{2} \boldsymbol{I}\right)
$$

Different orders of error magnitudes were tested, and the mean error of the parameters were acquired for the combination with the minor trace, Table 5 . It is 
possible to conclude that $\boldsymbol{\epsilon}=\mathcal{N}\left(\boldsymbol{\mu}=\mathbf{0}, \boldsymbol{\Sigma}=(0.1)^{2} \boldsymbol{I}\right)$ was the noise with the most reasonable effect, causing errors close to the errors observed in real applications; being so, that was the noise used in this numerical trials.

Table 5 - Comparison of the estimation errors for different noise magnitudes.

\begin{tabular}{ccccc}
\hline$\sigma$ & $\left|\boldsymbol{E r r}_{\boldsymbol{H} \boldsymbol{s}}\right|$ & $\left|\boldsymbol{E r r}_{\boldsymbol{T} \boldsymbol{p}}\right|$ & $\left|\operatorname{Err}_{\boldsymbol{\beta} \boldsymbol{o}}\right|$ & $\left|\operatorname{Err}_{\boldsymbol{s}}\right|$ \\
\hline 0.01 & 0.02 & 0.1 & 1 & 9 \\
0.10 & 0.2 & 0.9 & 5 & 18 \\
1.00 & 1.5 & 2.9 & 40 & 43 \\
\hline
\end{tabular}

Source: Elaborated by the author.

The results are shown in Figure 47.

Figure 47 - Trace criterion versus different error metrics in estimation with noise.
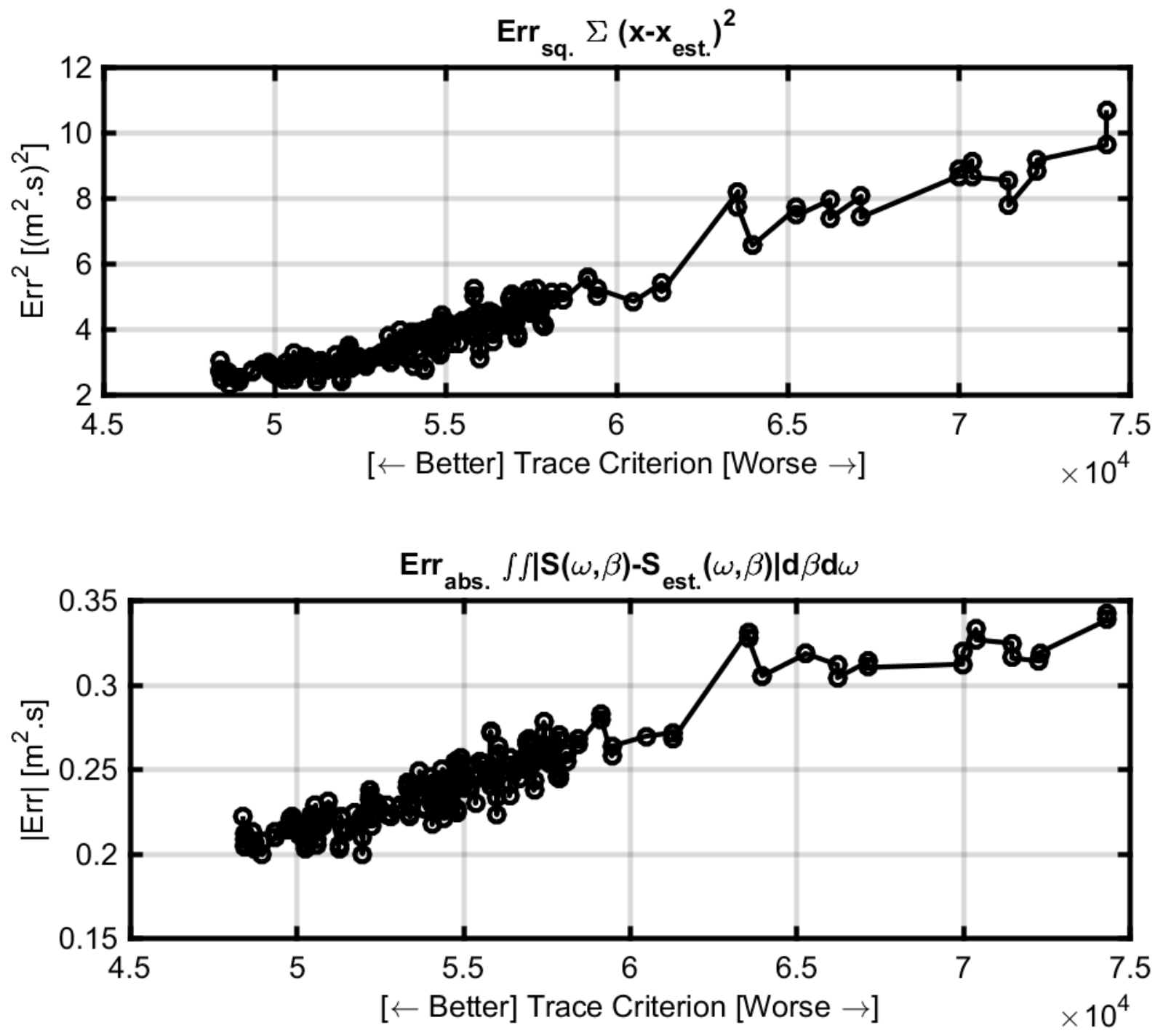

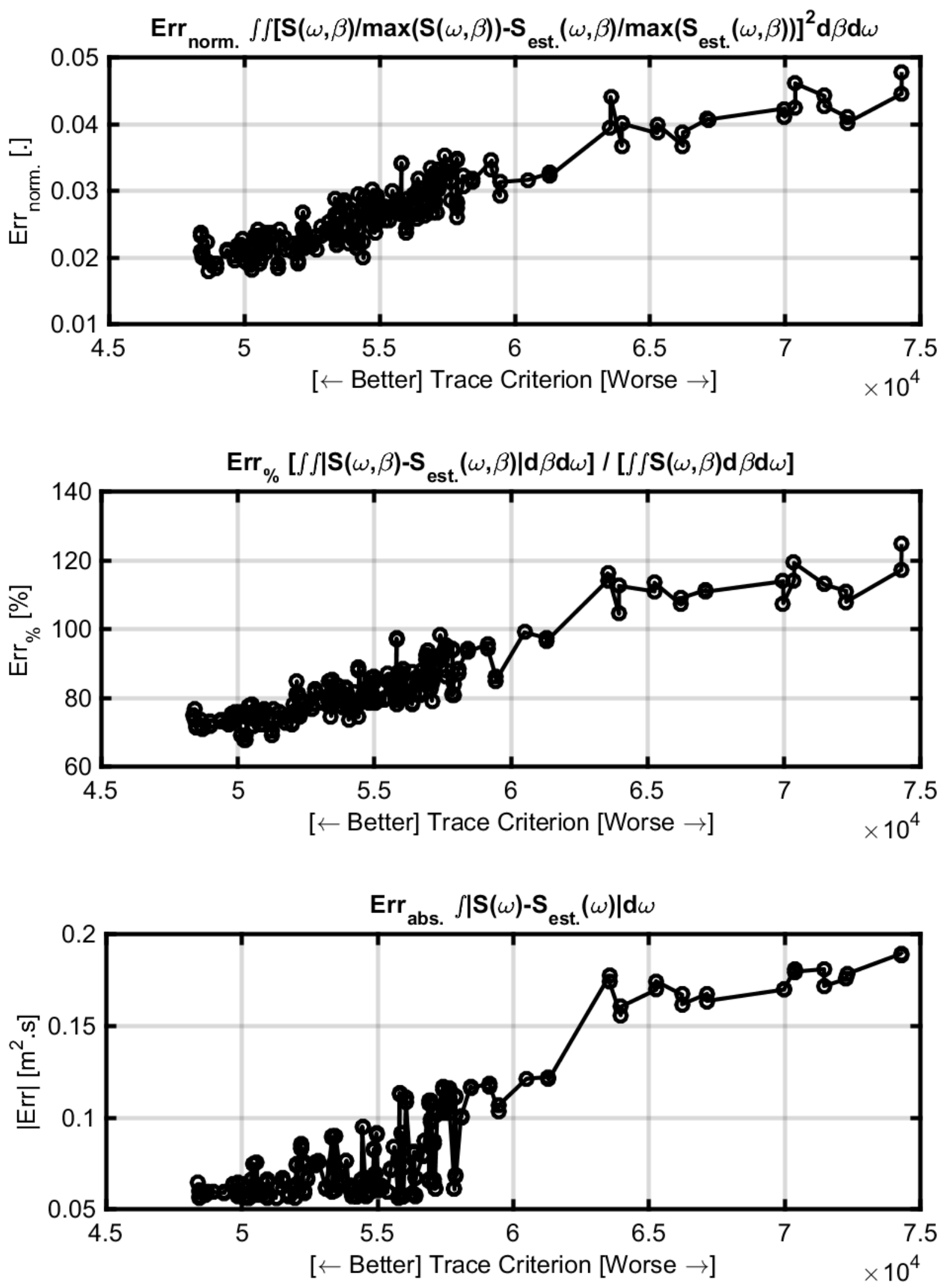

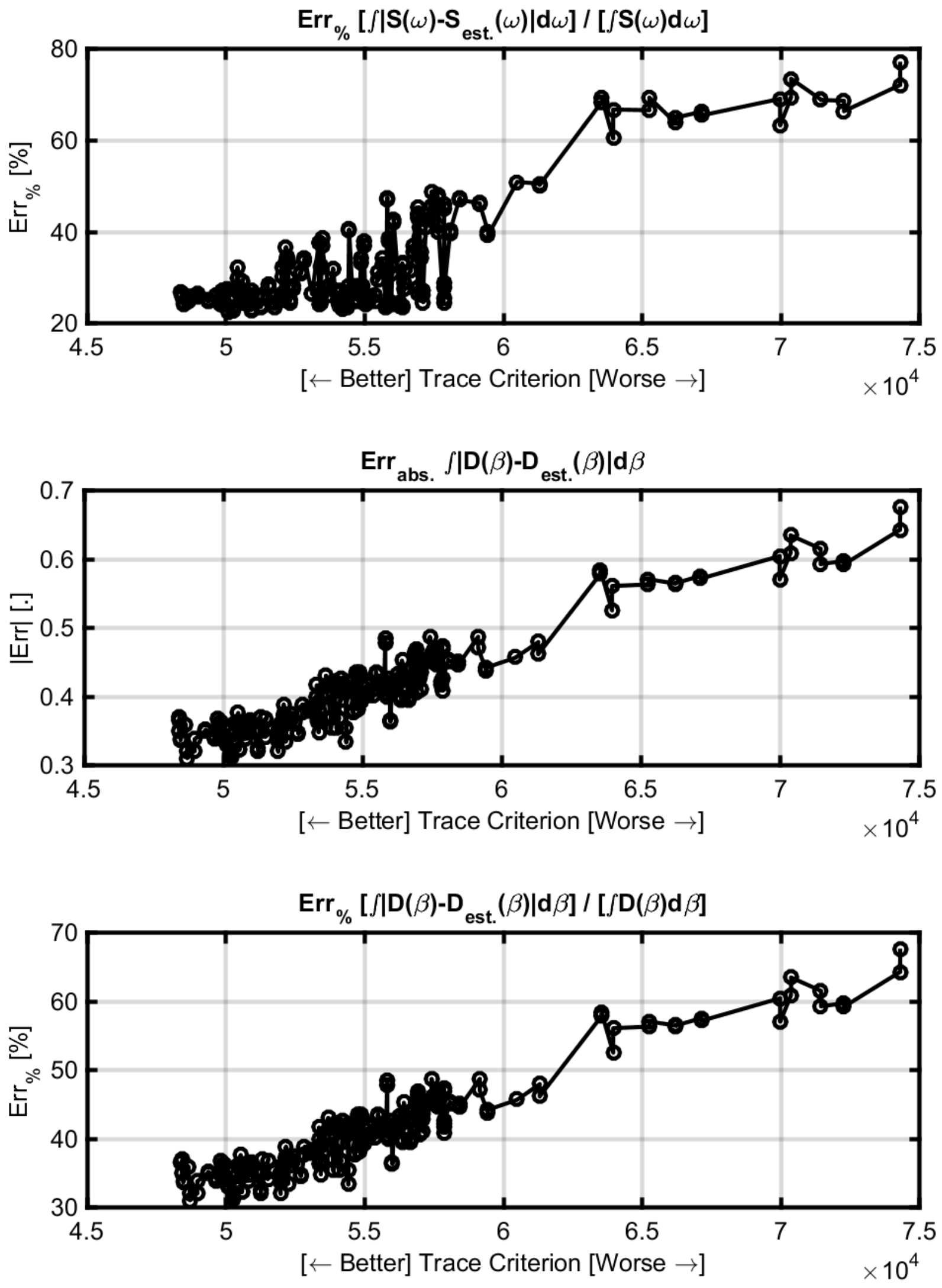

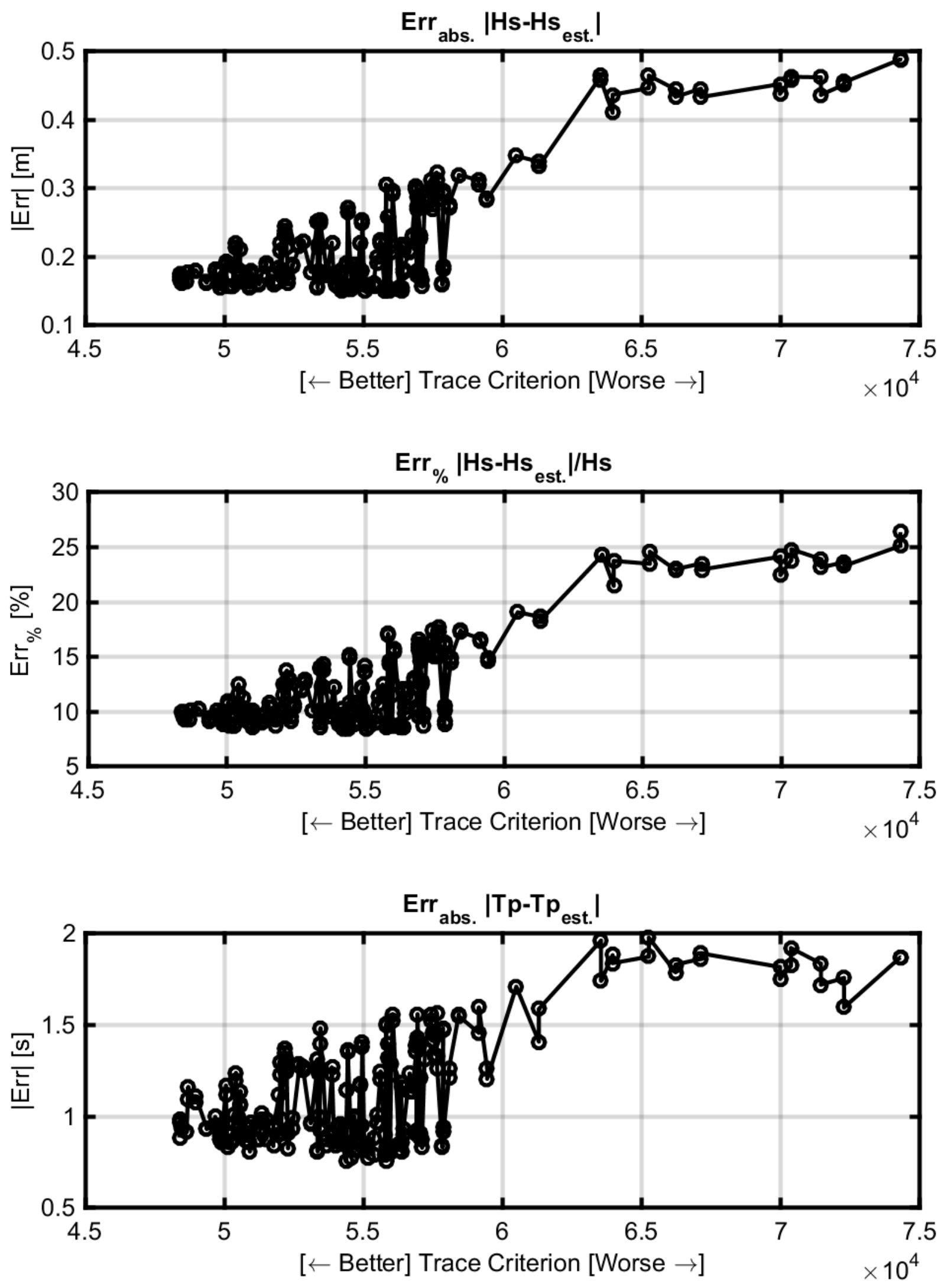

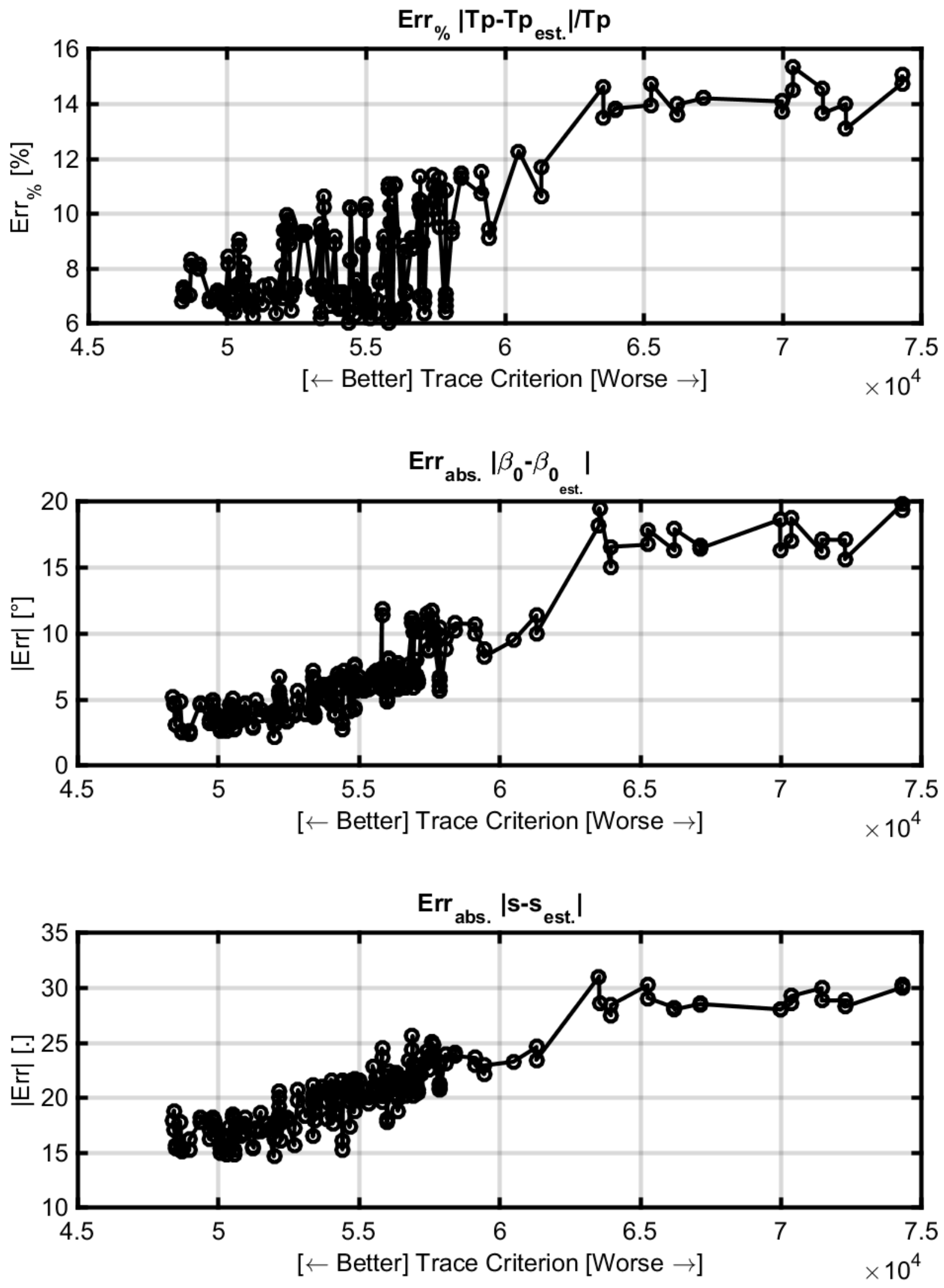


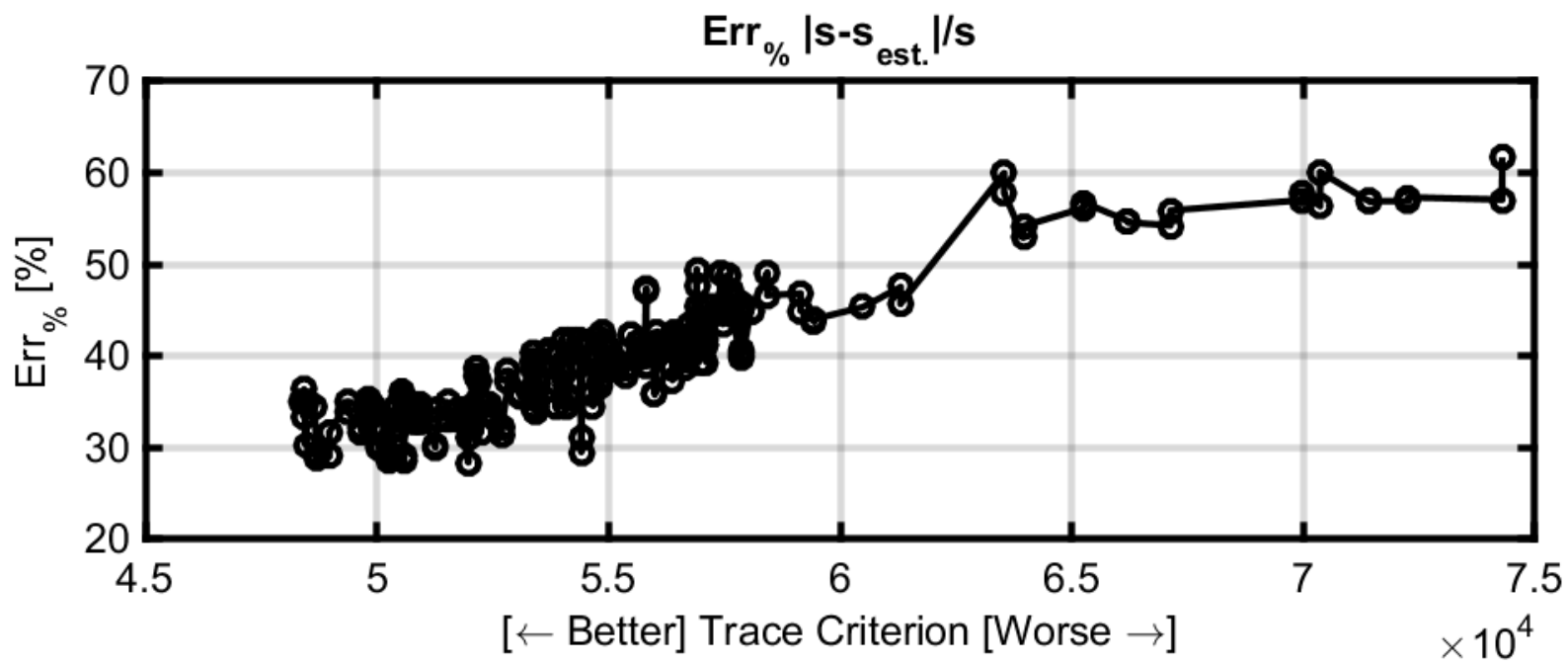

Source: Elaborated by the author.

Comparing the results of the estimation with noise against the results of the noiseless estimation, it is possible to notice that the relation with the trace criterion is stronger with noise, for all the error metrics proposed.

The relation with $E r r_{x}^{2}$ remains almost linear, following the theory; and metrics related to the directional spread function start to have a strong relation too: $\left|\operatorname{Err}_{D \beta}\right|$, $\operatorname{Err}_{D \beta} \%,\left|E r r_{s}\right|, E r r_{s} \%$ and, even, $\left|\operatorname{Err}_{\beta o}\right|$. The errors related with the spectrum density energy, $\left|\operatorname{Err}_{S \omega}\right|, \operatorname{Err}_{S \omega} \%,\left|\operatorname{Err}_{H S}\right|, \operatorname{Err}_{H S} \%,\left|\operatorname{Err}_{T p}\right|$ and $\operatorname{Err}_{T p} \%$, become more noisy than in the noiseless simulation, but the tendency is strong too; furthermore, the maximum possible error in a specified region decreases with the trace, giving support to the hybrid "trace criterion-simulation" approach, Figure 48.

Figure 48 - Regions with low trace also have lower worst-case.

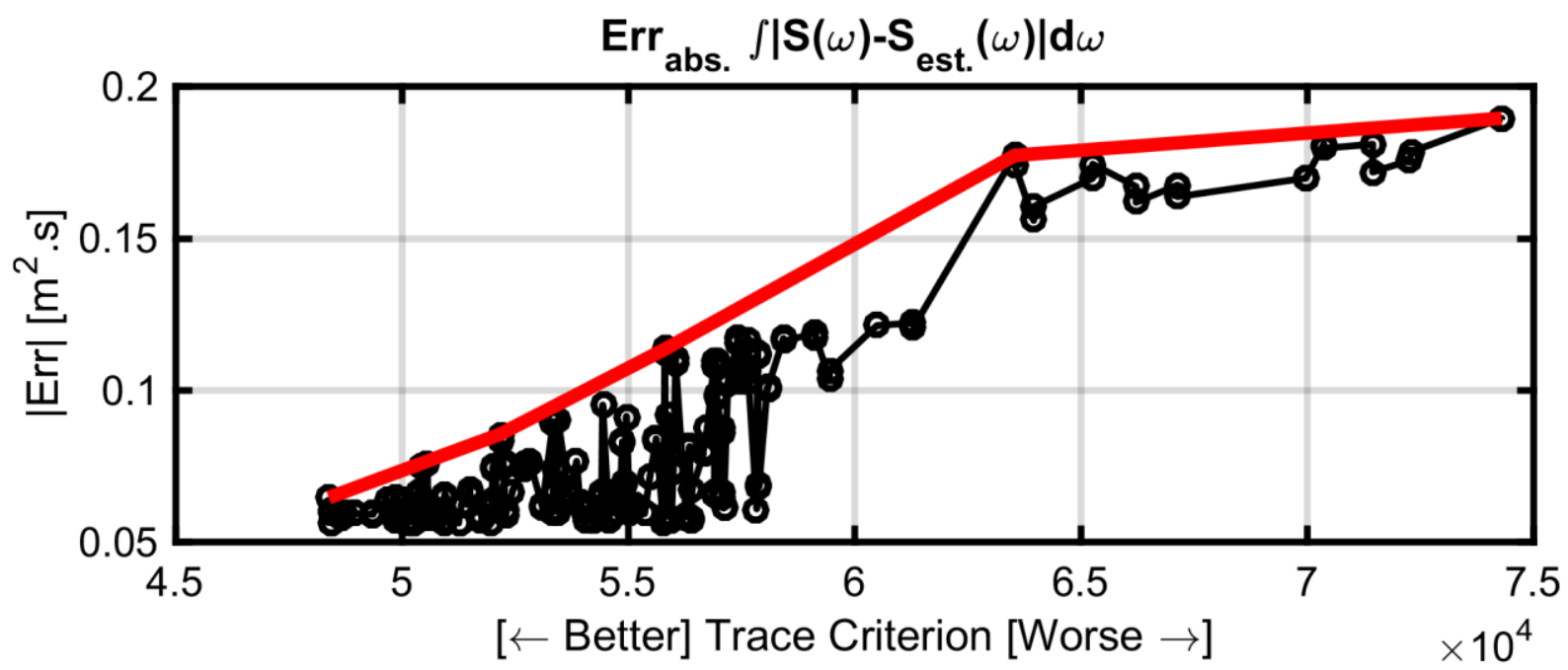

Source: Elaborated by the author. 
There is a strong mathematical explanation for this behavior, Eq. (172) to (177).

$$
\begin{aligned}
& \operatorname{Err}_{S_{\omega}}^{2}=\int_{0}^{\infty}(S(\omega)-\hat{S}(\omega))^{2} d \omega=\int_{0}^{\infty}\left(\int_{0}^{2 \pi} S(\omega, \beta) d \beta-\int_{0}^{2 \pi} \hat{S}(\omega, \beta) d \beta\right)^{2} d \omega \\
& = \\
& =\int_{0}^{\infty}\left(\int_{0}^{2 \pi}(S(\omega, \beta)-\hat{S}(\omega, \beta)) d \beta\right)^{2} d \omega=\int_{0}^{\infty}\left(\int_{0}^{2 \pi} \operatorname{Err}(\omega, \beta) d \beta\right)^{2} d \omega \\
& \text { trace criterion } \equiv \operatorname{Err}_{S_{\omega \beta}}^{2}=\int_{0}^{\infty} \int_{0}^{2 \pi}(S(\omega, \beta)-\hat{S}(\omega, \beta))^{2} d \beta d \omega= \\
& =\int_{0}^{\infty}\left(\int_{0}^{2 \pi}(\operatorname{Err}(\omega, \beta))^{2} d \beta\right) d \omega \\
& \text { Schwarz's inequality } \rightarrow\left(\int_{a}^{b} \psi_{1}(x) \psi_{2}(x) d x\right)^{2} \leq \int_{a}^{b} \psi_{1}(x)^{2} d x \cdot \int_{a}^{b} \psi_{2}(x)^{2} d x \\
& \left(\int_{0}^{2 \pi} \operatorname{Err}(\omega, \beta) d \beta\right)^{2} \leq \int_{0}^{2 \pi}(\operatorname{Err}(\omega, \beta))^{2} d \beta \cdot \int_{0}^{2 \pi} 1^{2} d \beta \\
& =2 \pi \int_{0}^{2 \pi}(\operatorname{Err}(\omega, \beta))^{2} d \beta \\
& \int_{0}^{\infty}\left(\int_{0}^{2 \pi} \operatorname{Err}(\omega, \beta) d \beta\right)^{2} d \omega \leq 2 \pi \int_{0}^{\infty}\left(\int_{0}^{2 \pi}(\operatorname{Err}(\omega, \beta))^{2} d \beta\right) d \omega \\
& \operatorname{Err}_{S_{\omega}}^{2} \leq 2 \pi \cdot \operatorname{Err}_{S_{\omega \beta}}^{2}
\end{aligned}
$$


The previous equations prove that an optimal trace criterion, which is expected to minimize $\operatorname{Err}_{S_{\omega \beta}}^{2}$, limits the upper bound of $\operatorname{Err}_{S_{\omega}}^{2}$. Similar results can be obtained for the other error metrics.

Again, more detailed analysis can be made using histogram graphs, Figure 49, using the same combinations that were shown in the previous case.

Figure $49-$ Histogram of the combinations performing estimation with noise.
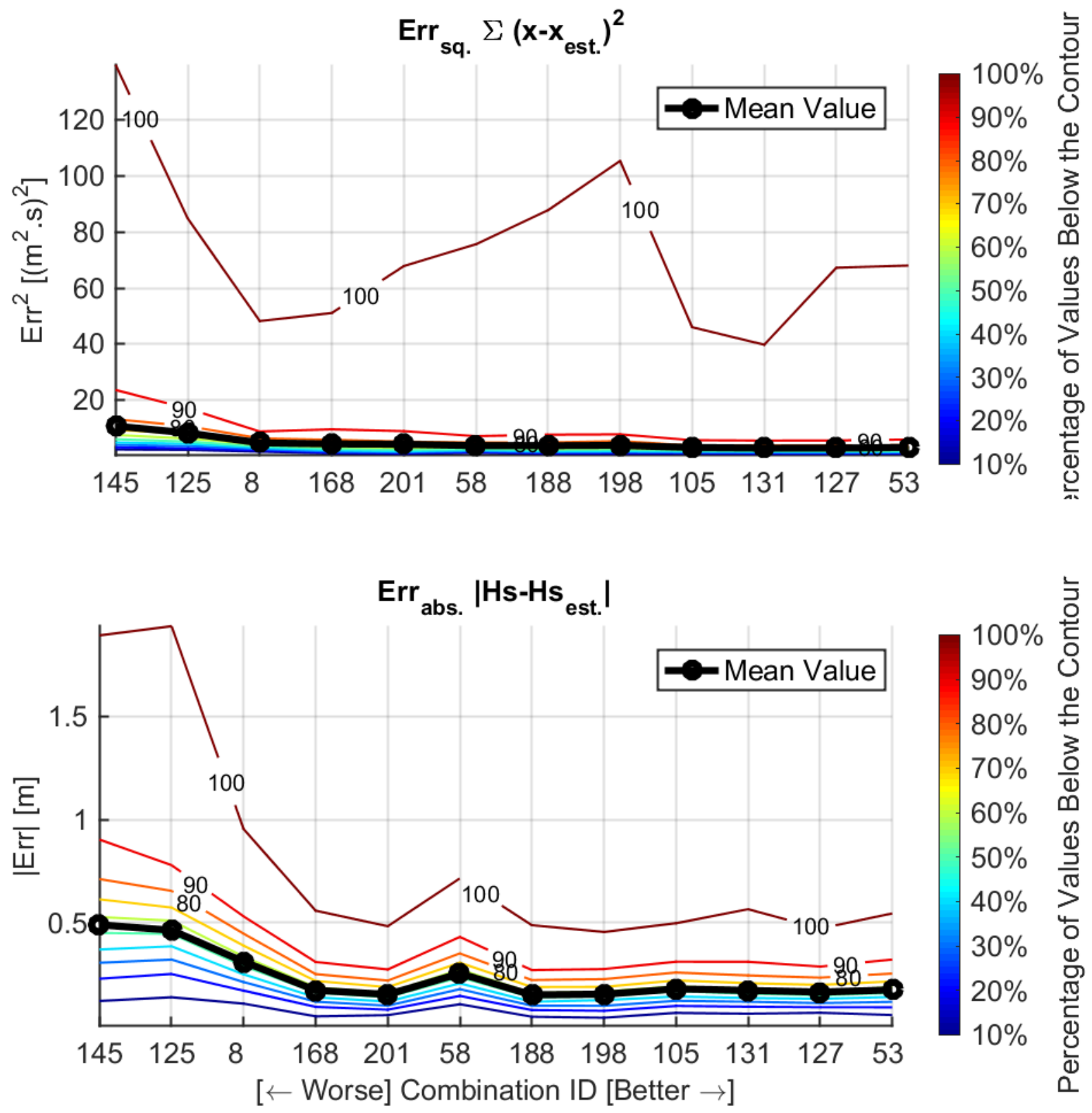

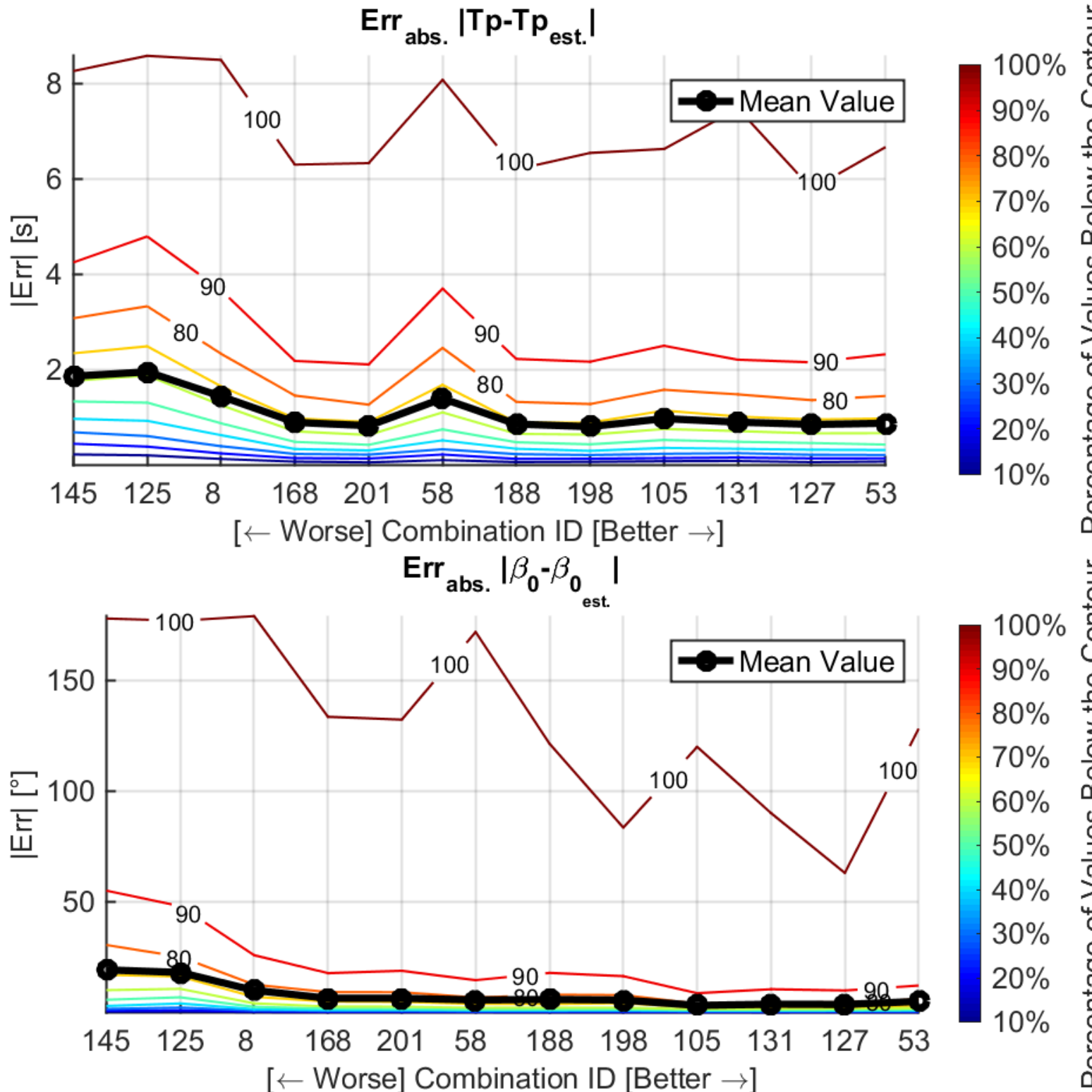

Err $_{\text {abs. }} \mid \mathbf{s}-\mathrm{s}_{\text {est. }}$ |

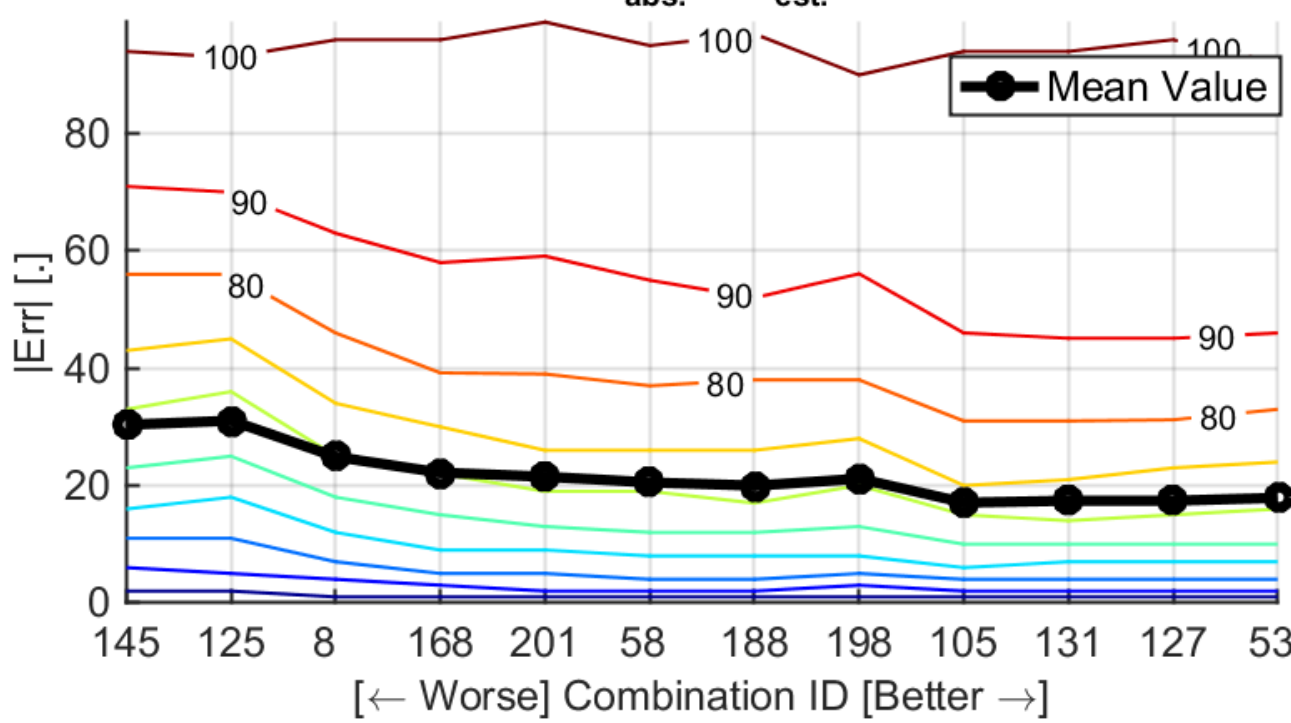

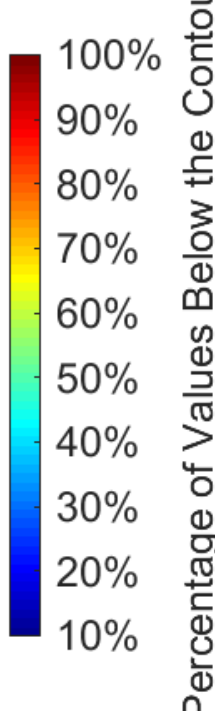

Source: Elaborated by the author. 
It is interesting to notice, in the graphs, that the mean error trend represents the trend of most of the waves, as put in evidence by the tendency of all the contour lines. The maximum error, however, only possesses a strong relation with the trace in the $H_{S}$ estimation, indicating that, perhaps, if the maximum error is the objective, more probes will have to be employed.

In conclusion, observing all the available data, the trace criterion proposal is strongly validated, for any error metric among the presented ones. It means that the optimization of the probes position can be made by simple algorithms in a fast manner, and extensive simulation campaigns are not necessary.

Although the data also suggests that the trace criterion is enough to predict the behavior in all possible waves, even if it is a mean criterion, it may not be true when fewer wave-probes are used, so more analysis are further presented.

\subsection{Study of the Usage of Different Number of Wave-Probes}

Another interesting study that can be done is the impact of the usage of different number of wave-probes in the estimation result.

In this set, the number of possible wave probes was varied from 0 to 12 , and the combination of positions with the best trace was used in each case. The trace result is shown in Figure 50 and the best positions are shown in Figure 51. The noise amplitude is equal to the used in the previous section, 0.1 .

Figure 50 - Best trace criterion for different number of wave-probes.

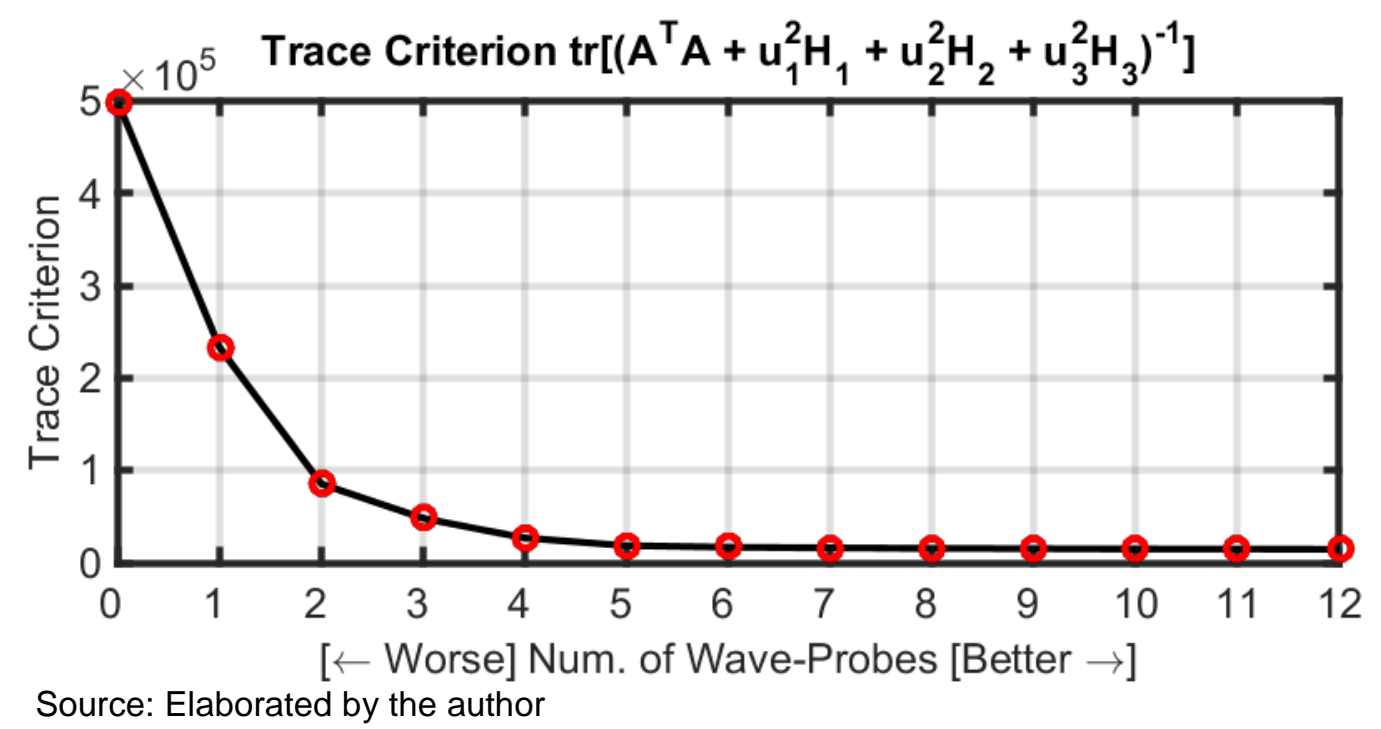

Source: Elaborated by the author 
Figure 51 - Best position of the wave-probes for different number of gauges.

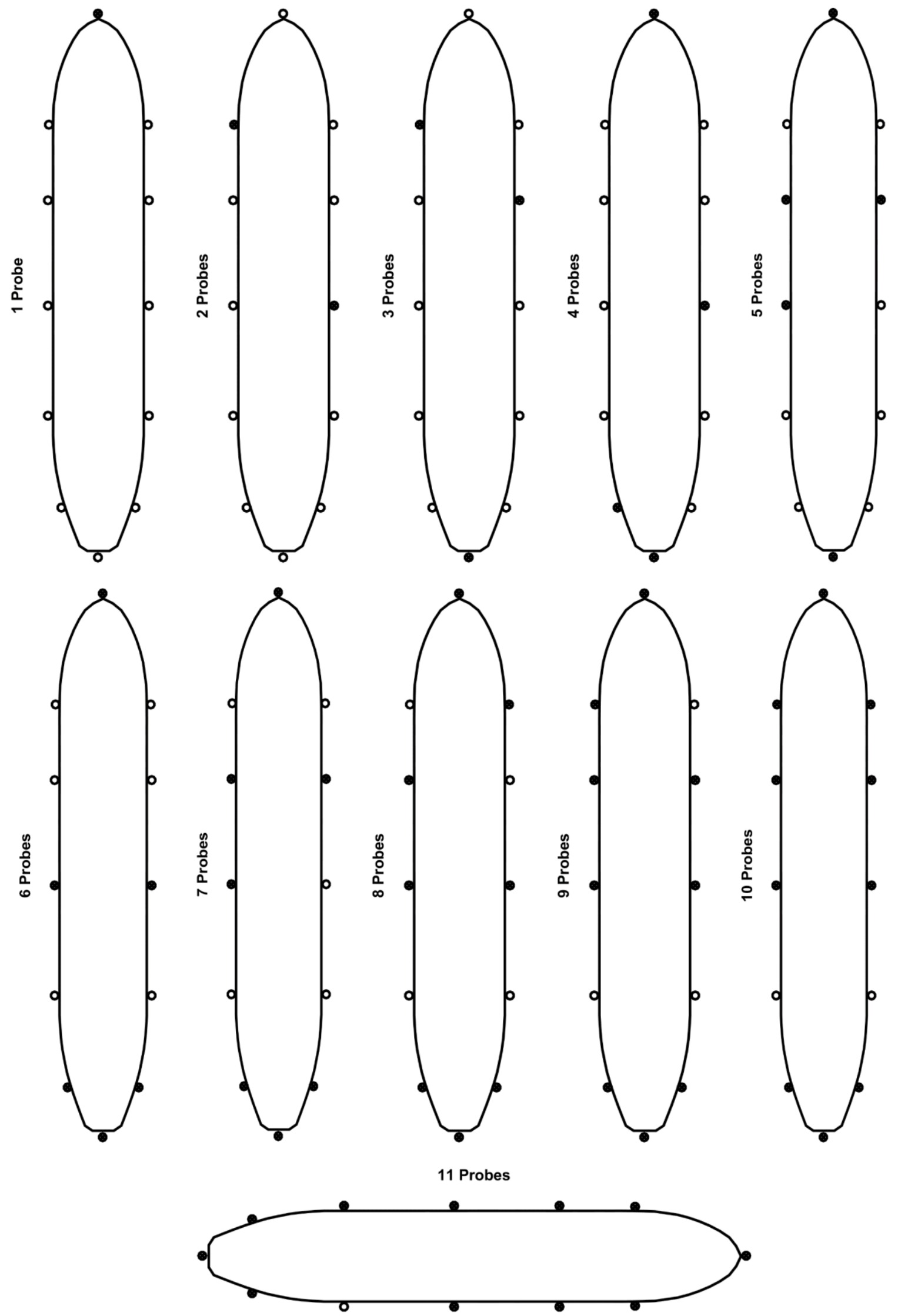

Source: Elaborated by the author. 
The results of this simulation are shown in Figure 52 and Figure 53.

Figure 52 - Error metrics for different number of wave-probes.
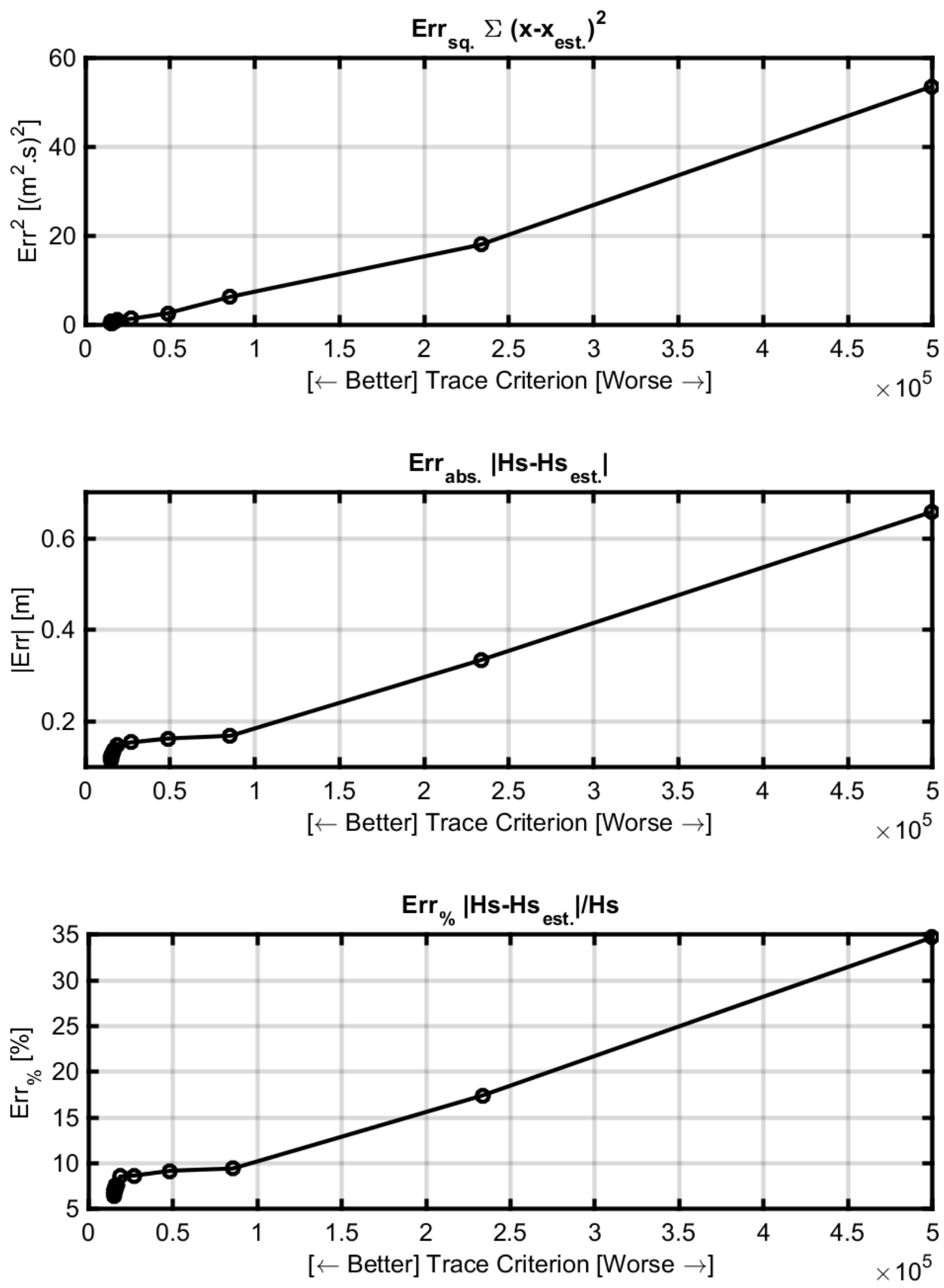

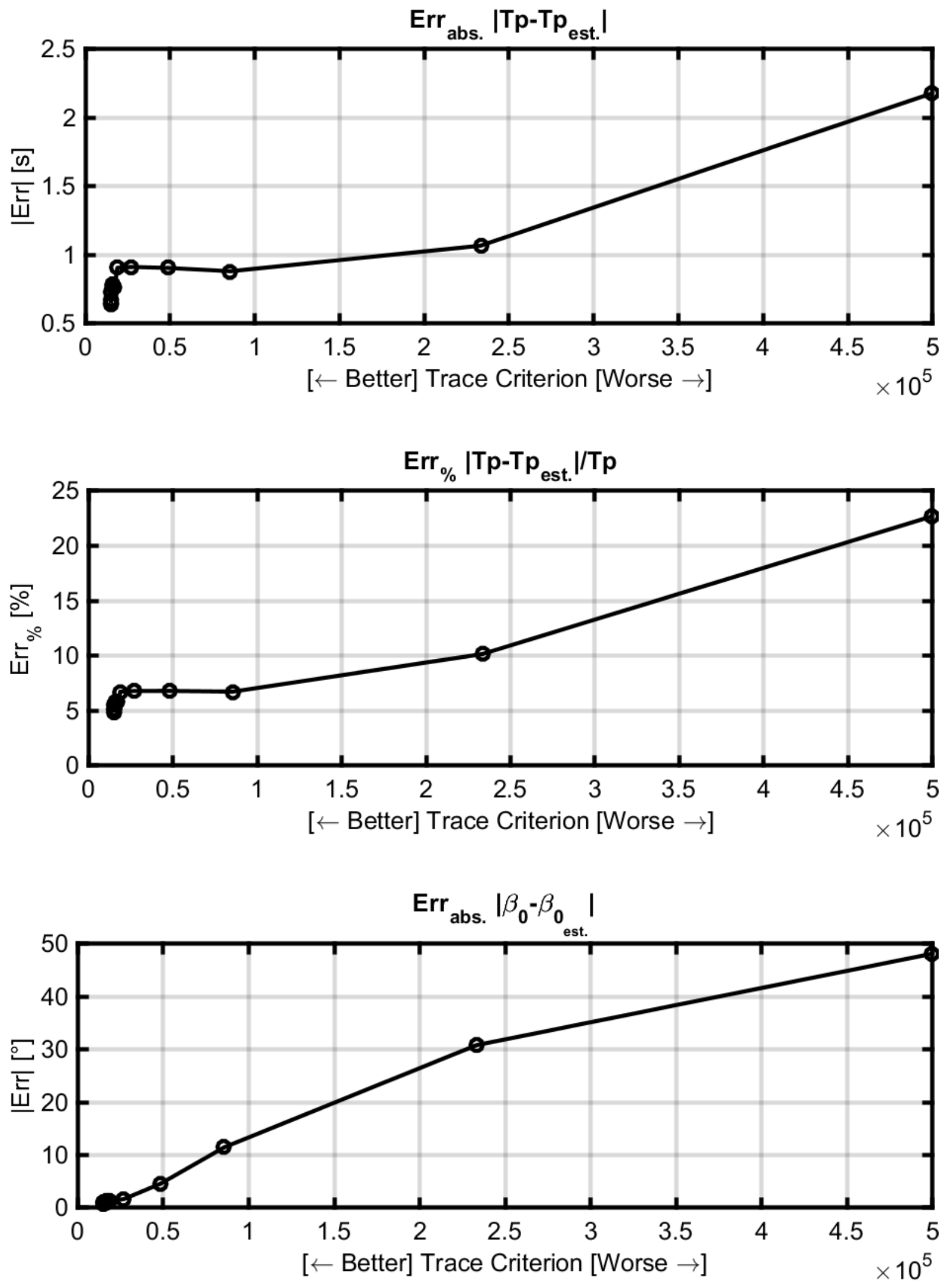

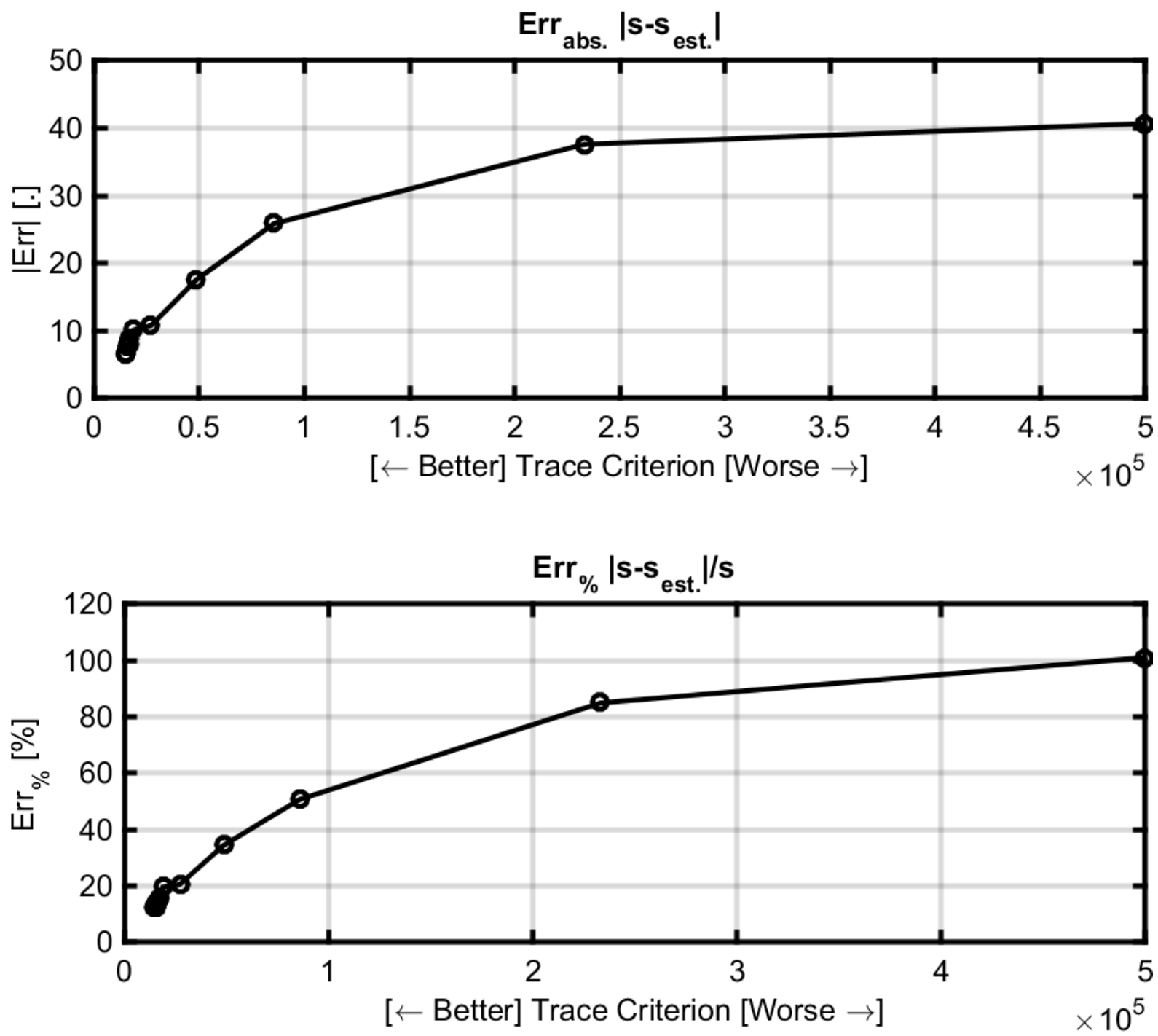

Source: Elaborated by the author.

Figure 53 - Histogram of the errors for different number of wave-probes.

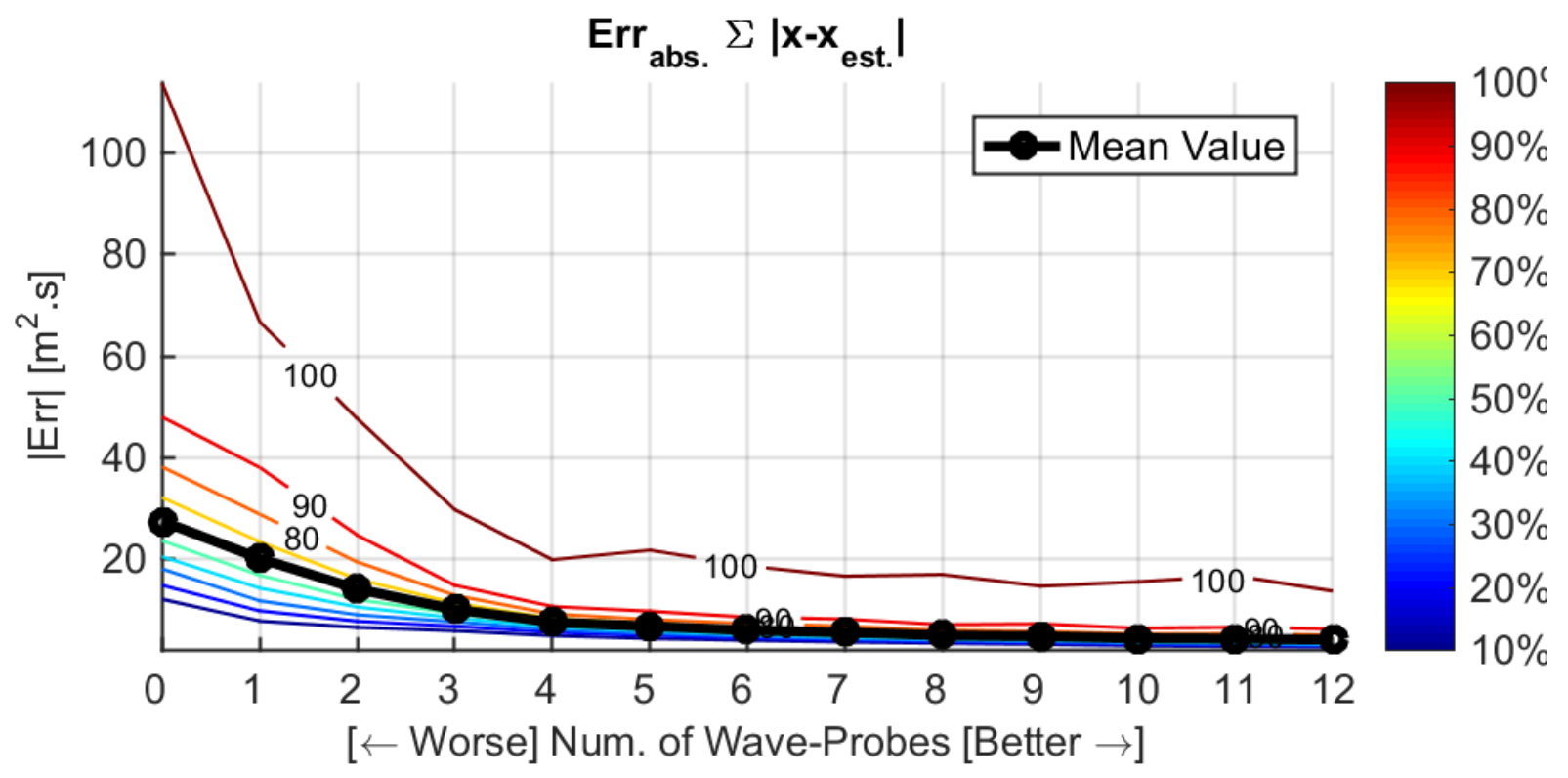



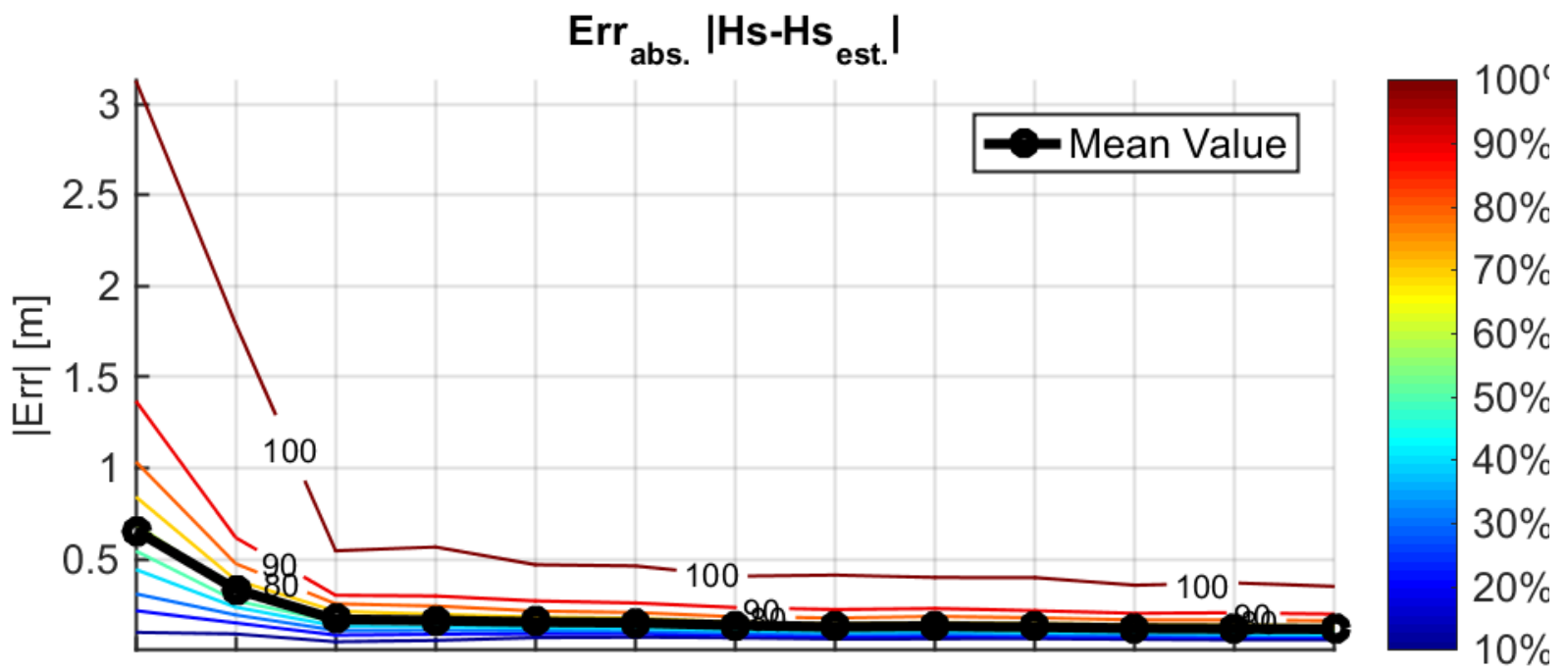

$\begin{array}{lllllllllllll}0 & 1 & 2 & 3 & 4 & 5 & 6 & 7 & 8 & 9 & 10 & 11 & 12\end{array}$

$[\leftarrow$ Worse] Num. of Wave-Probes [Better $\rightarrow$ ]

$\mathrm{Err}_{\%} \mid \mathrm{Hs}-\mathrm{Hs}$ est. $\mid / \mathrm{Hs}$

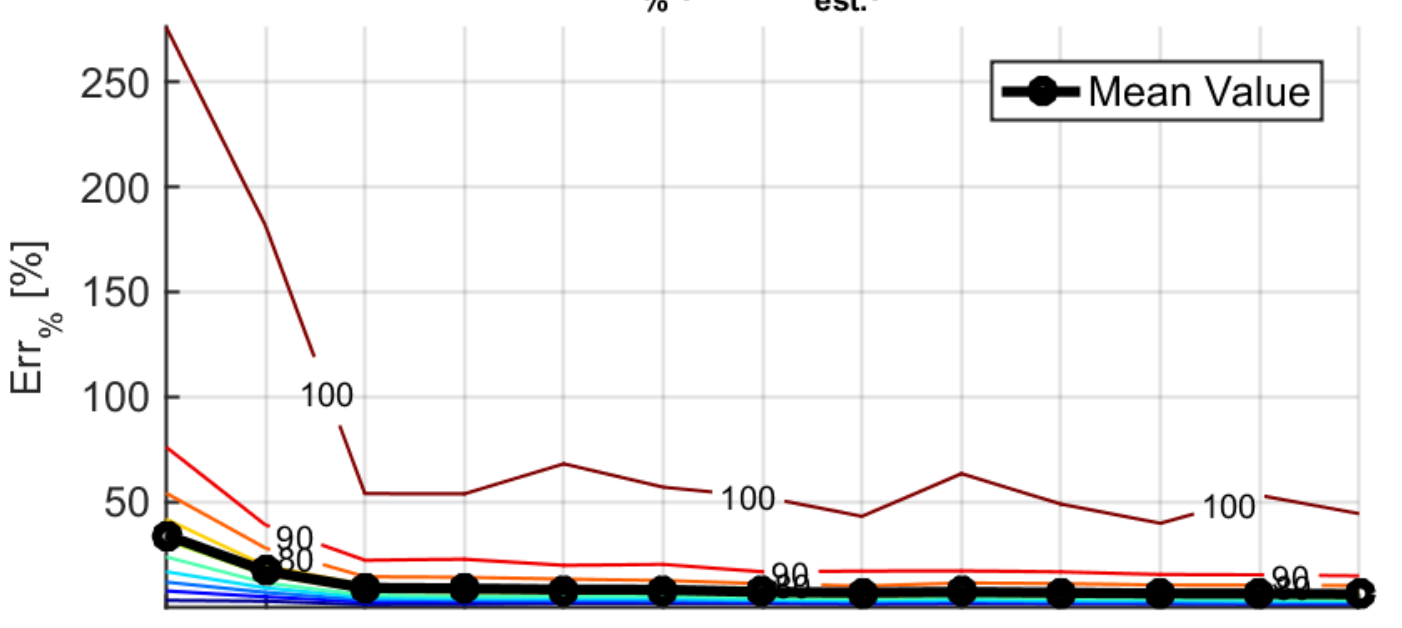

$100^{\circ}$

$90 \%$

$80 \%$

$70 \%$

$60 \%$

$50 \%$

$40 \%$

$30 \%$

$20 \%$

$10 \%$

$\begin{array}{lllllllllllll}0 & 1 & 2 & 3 & 4 & 5 & 6 & 7 & 8 & 9 & 10 & 11 & 12\end{array}$

$[\leftarrow$ Worse] Num. of Wave-Probes [Better $\rightarrow$ ]

Err $_{\text {abs. }}$ |Tp-Tp est. |

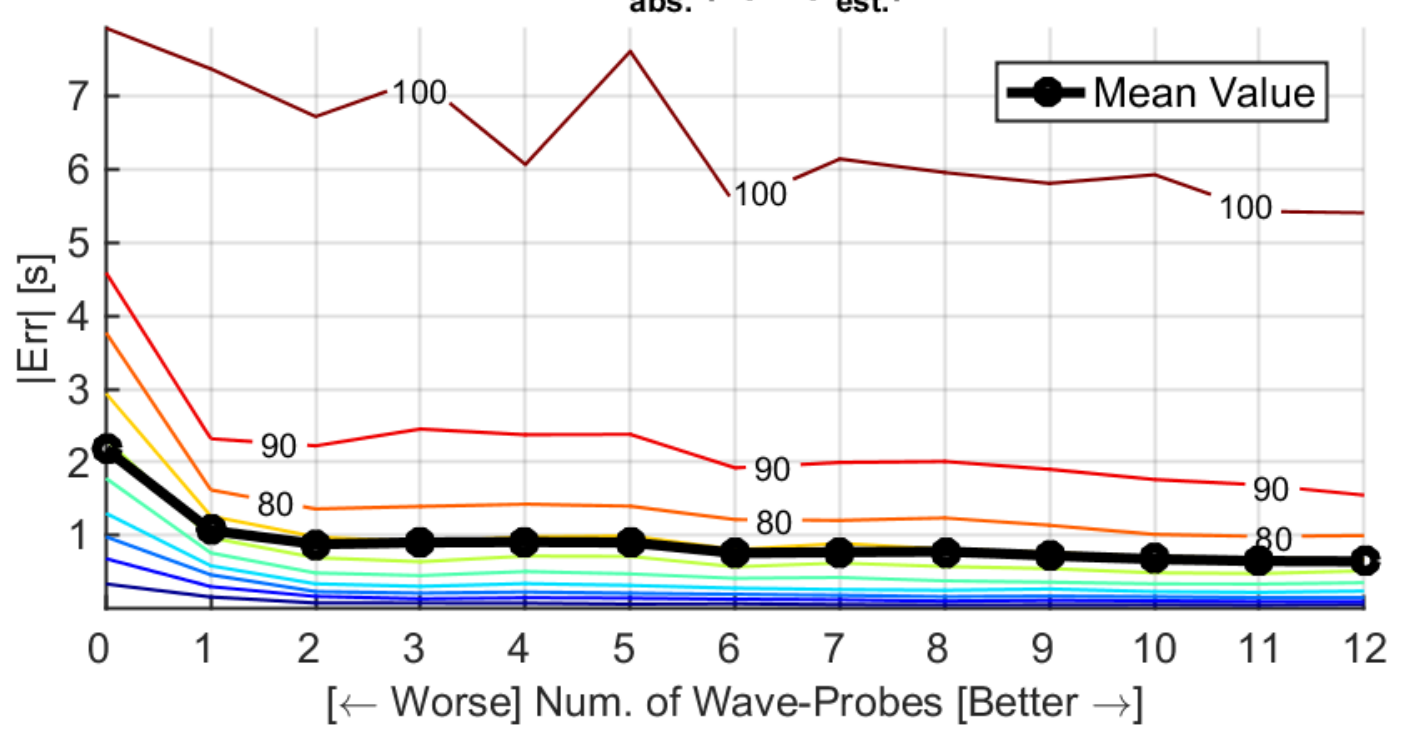

100

$90 \%$

$80 \%$

$70 \%$

$60 \%$

$50 \%$

$40 \%$

$30 \%$

$20 \%$

$10 \%$ 

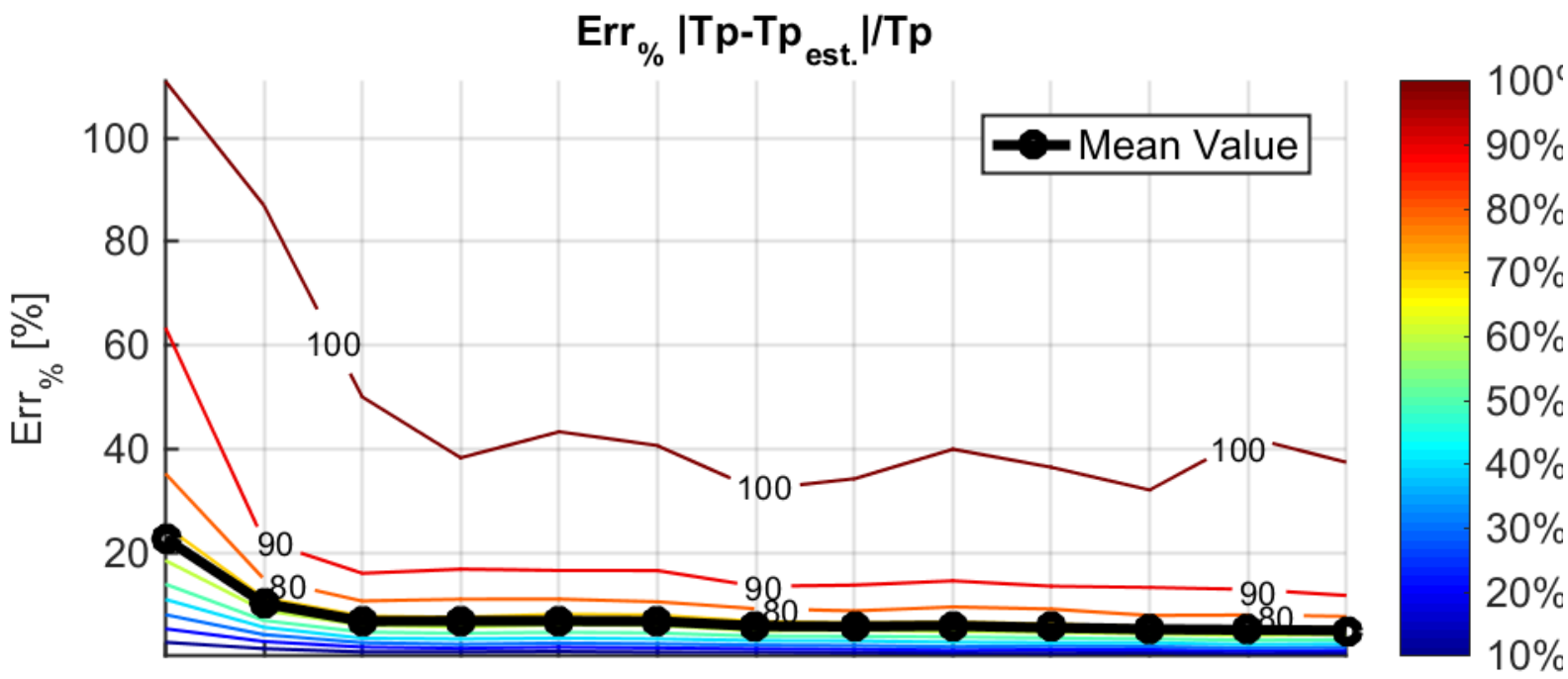

$\begin{array}{lllllllllllll}0 & 1 & 2 & 3 & 4 & 5 & 6 & 7 & 8 & 9 & 10 & 11 & 12\end{array}$

$[\leftarrow$ Worse] Num. of Wave-Probes [Better $\rightarrow$ ]

$\mathrm{Err}_{\text {abs. }}\left|\beta_{0}-\beta_{0}\right|$

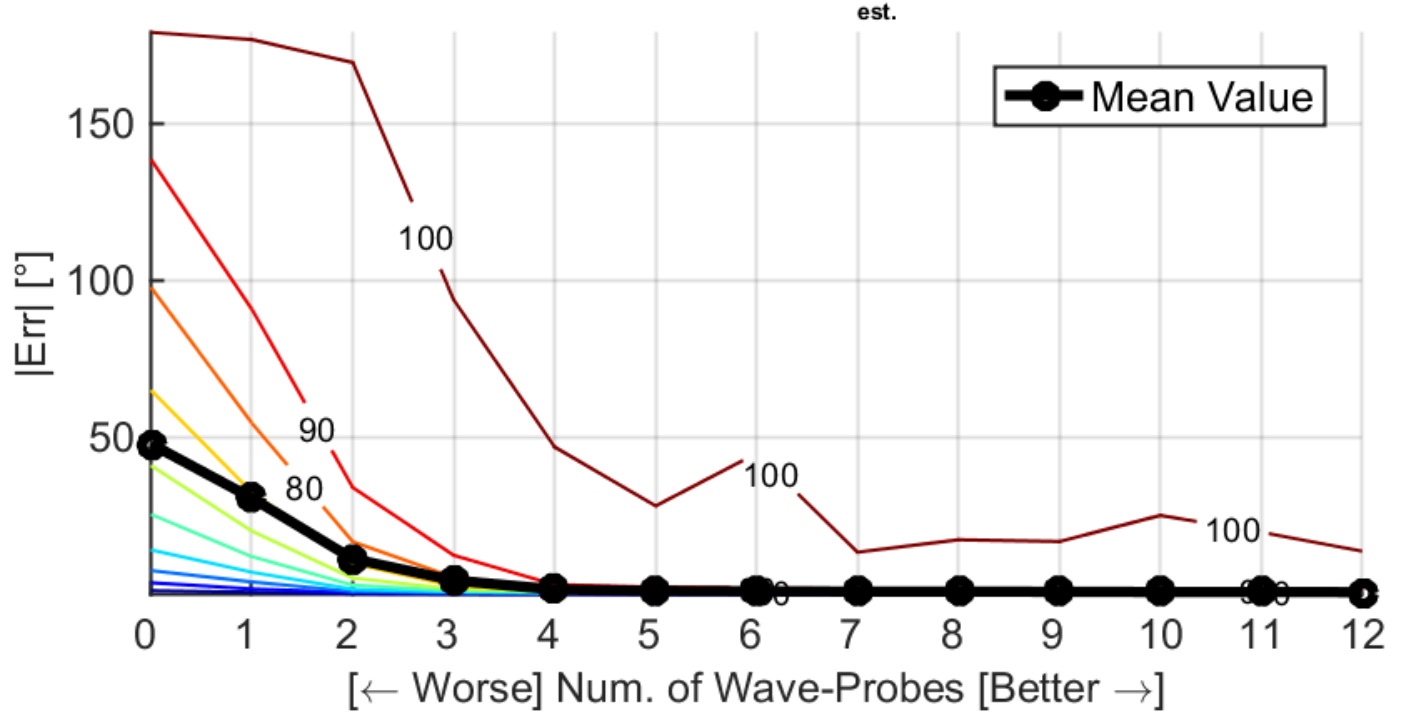

$100^{\prime}$

$90 \%$

$80 \%$

$70 \%$

$60 \%$

$50 \%$

$40 \%$

$30 \%$

$20 \%$

$10 \%$

Err $_{\text {abs. }} \mid \mathbf{s}^{-s_{\text {est. }} \mid}$

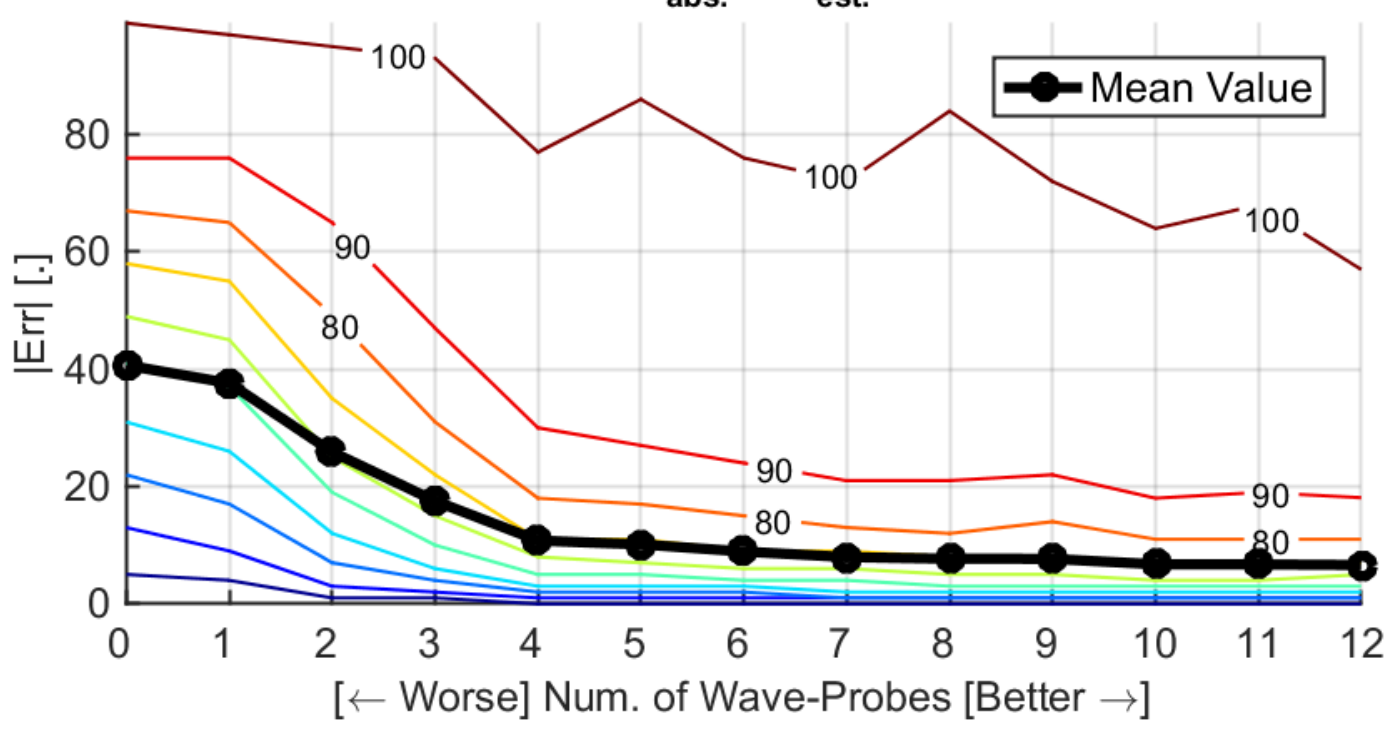

$100^{\circ}$

$90 \%$

$80 \%$

$70 \%$

$60 \%$

$50 \%$

$40 \%$

$30 \%$

$20 \%$

$10 \%$

$[\leftarrow$ Worse] Num. of Wave-Probes [Better $\rightarrow$ ] 


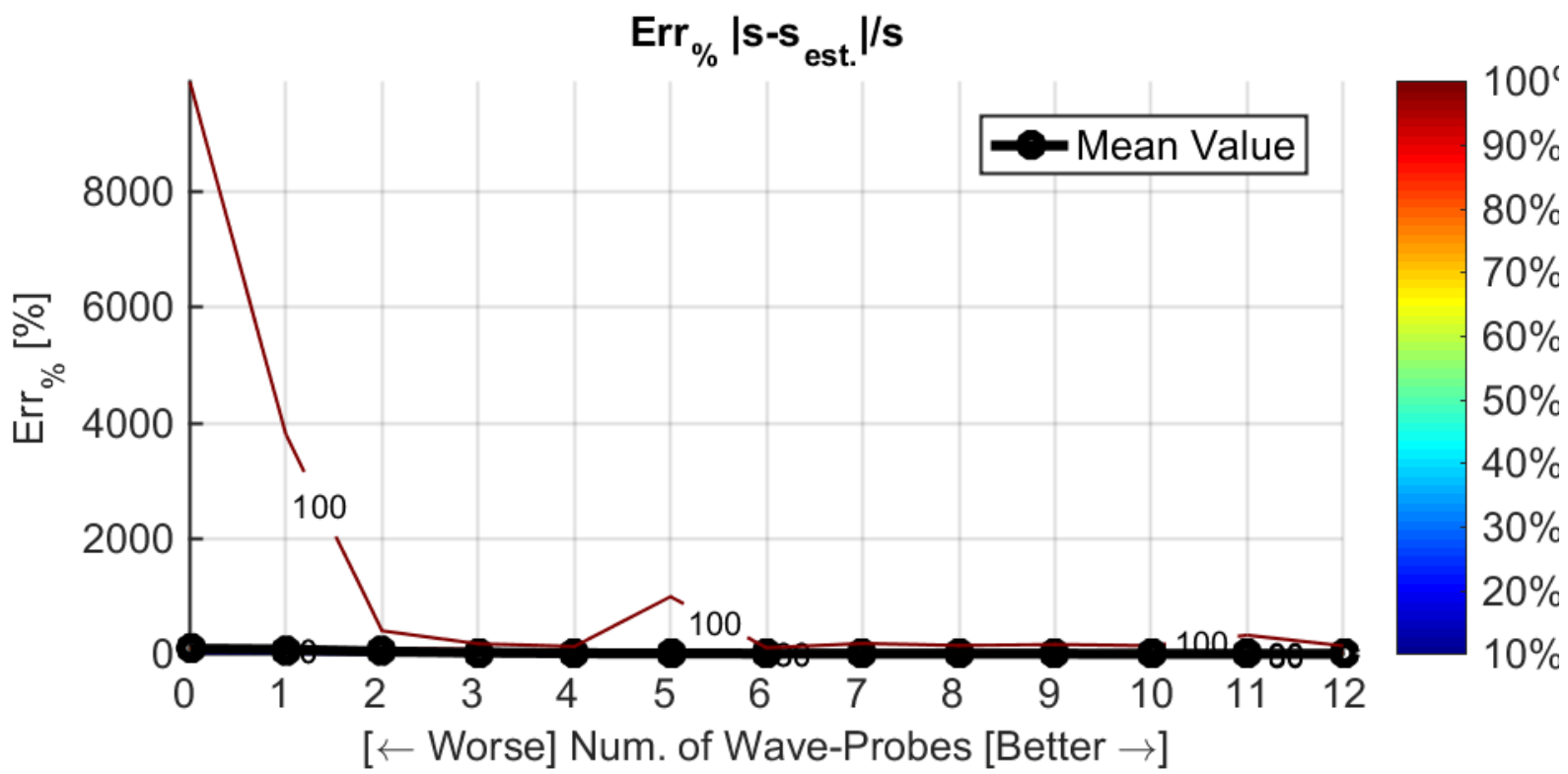

Source: Elaborated by the author.

The first important information provided by the data is the validation of the 'elbow' criterion. By applying the criterion in the trace vs number of probes figures, it is possible to estimate the best number of probes as something between 2 and 4 .

The usage of two wave-probes seems to be the best choice for energy related parameters, $H_{S}$ and $T_{P}$, which can be visualized by the mean error vs trace criterion curves; while the usage of four probes would be recommended to improve the estimation of directional parameters, $\beta_{0}$ and $s$. This is also perceived in the histogram graphs, in which 'elbow' shape curves can be seen in each contour, at least up to the $90 \%$ contour, suggesting the same number of probes discussed.

An interesting conclusion of the data is that the energy estimation of the directional spectrum converges earlier than the spread function, which is expected. The second interesting conclusion is that the 'elbow', indeed, happens when the system is fully determined: if 20 directions are used, it would be necessary 20 equations in each frequency; using two probes plus three vessel movements $N^{2}=25$ equations are determined, i.e., it is expected that the system will be fully determined if, at least, two probes are employed. Of course the number of equations do not guarantee that they will be linearly independent, so two wave-probes would be the minimum necessary.

The last conclusion can possibly suggest a rationale guiding the choice of the discretization. While the frequency discretization can be guided by the sample 
frequency and its impact in the spectral estimation with the Welch's method, which are described in the experimental section, the direction discretization could be determined by the number of degrees of freedom used in the estimation algorithm.

\subsection{Validation of the Heuristic}

In this section, an analysis of the heuristic results is provided.

Following the proposed algorithm, the wave-probe RAOs for the one hundred positions were calculated by the software $\mathrm{Wamit}^{\circledR}$, and were grouped together in 24 clusters, Figure 54.

Figure 54 - One hundred positions grouped together in 24 clusters.

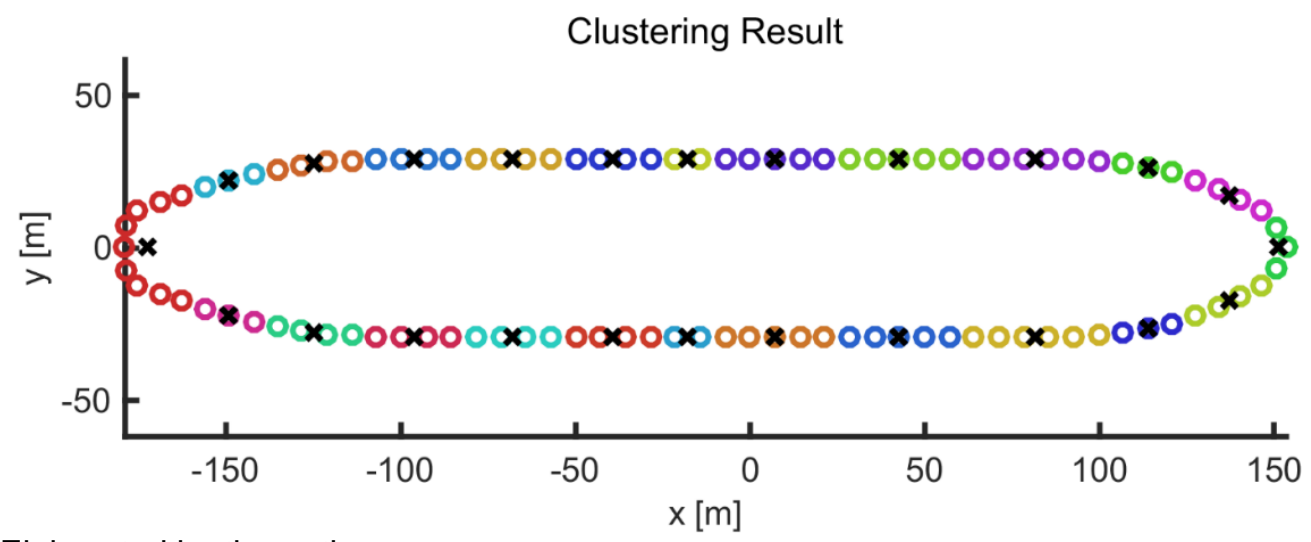

Source: Elaborated by the author.

Using those clusters, two cases were calculated: the addition of one wave-probe and the addition of three wave-probes. The first case, the addition of one wave-probe, results in the first layer of the Pareto Frontier with only three candidates. More layers were added to provide 11 possible wave-probe combinations, or 11 matrices $\boldsymbol{A}$. The trace results are shown in Figure 55.

Figure 55 - Values obtained for the addition of one wave-probe.

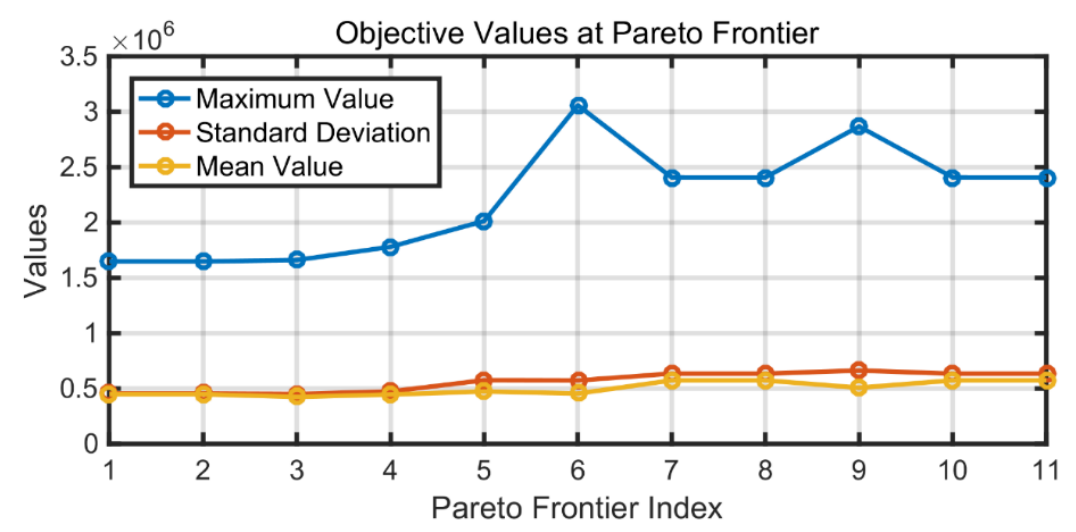

Source: Elaborated by the author. 
The second case, the addition of three wave-probes, results in the first layer of the Pareto Frontier with 26 candidates. The trace results are shown in Figure 56.

Figure 56 - Values obtained for the addition of three wave-probes.

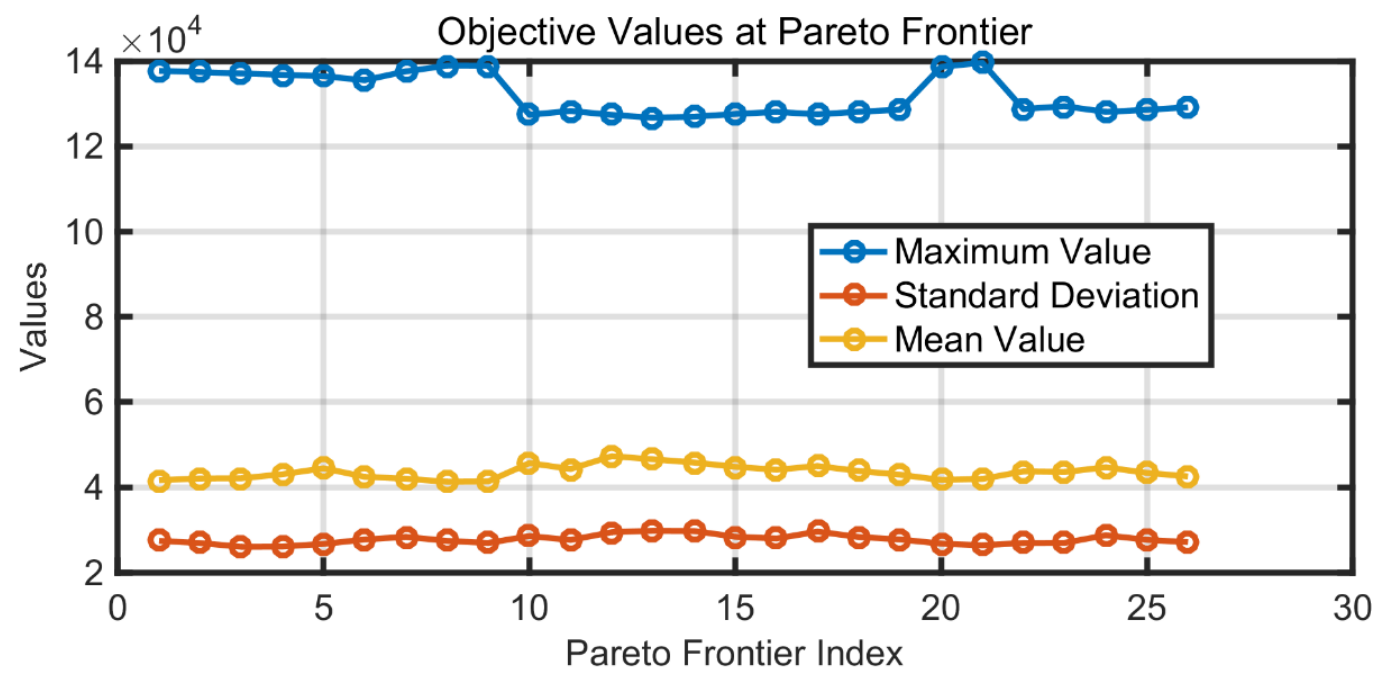

Source: Elaborated by the author.

Comparing the results, it is possible to notice that the addition of three waveprobes resulted in a great decrease in the trace values, as expected.

The positions suggested by the algorithm for the one-wave-probe problem are shown in Figure 57. It interesting to notice that, as expected, symmetric positions perform equally well when the algorithm evaluates both. In this case, some symmetric positions are evaluated because the clusters at the stern and at the bow have mirrored internal components, which are not removed by the symmetrical filtering.

Figure 57 - Positions suggested by the algorithm for a single wave-probe.

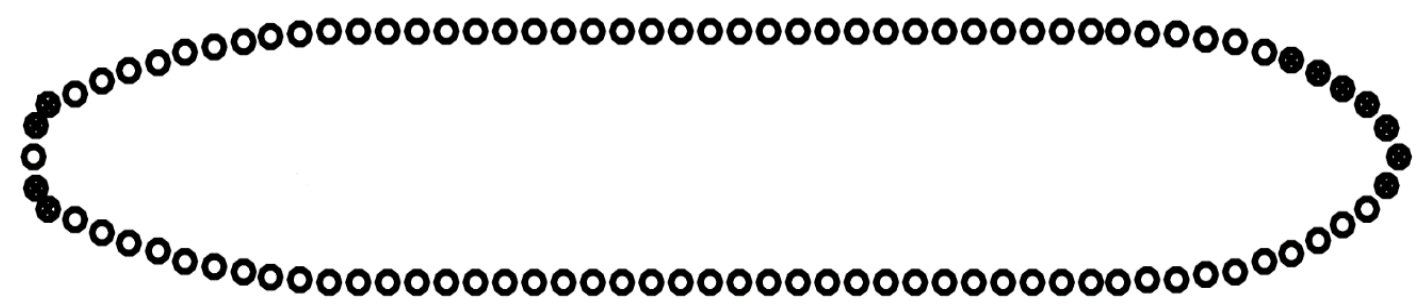

Source: Elaborated by the author.

For the three-wave-probe problem, the cases with indices equal to 1 and equal to 10 are shown in Figure 58. 
Figure 58 - Suggested solutions for the three-wave-probe problem.

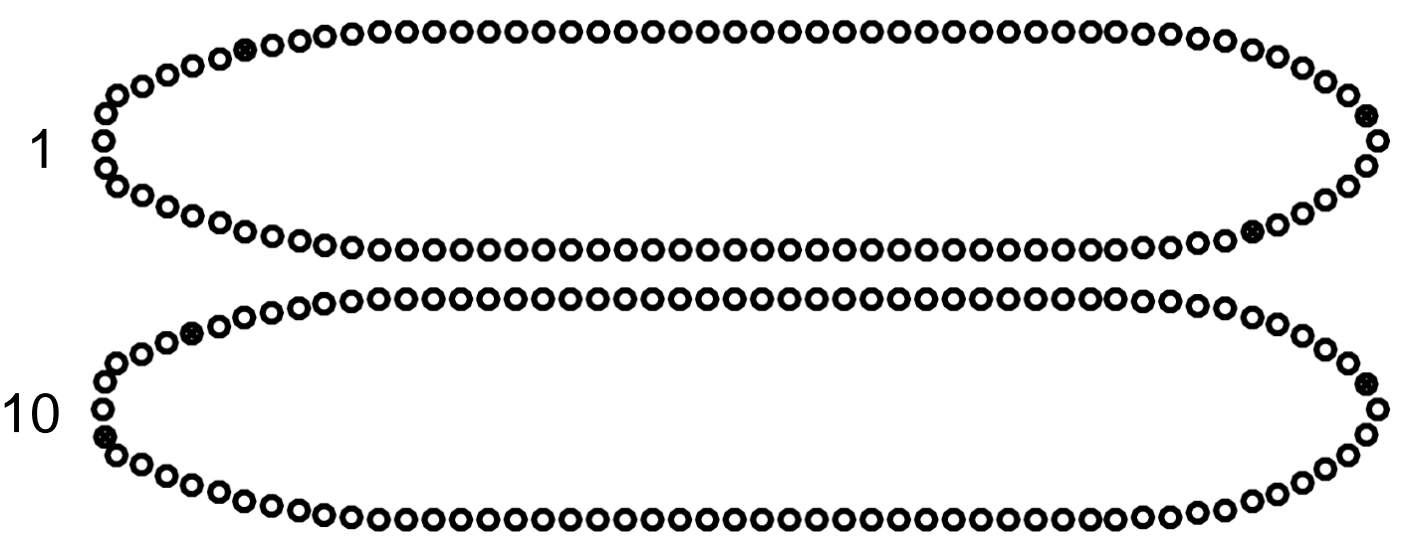

Source: Elaborated by the author.

A simple comparison was made against a genetic algorithm, in which only the total trace criterion was used, and each gene was configured to be the selected waveprobe position. The average results are shown in Table 6.

Table 6 - Comparison between the algorithms.

\begin{tabular}{ccc}
\hline Algorithm & Final Optimal Total Trace Criterion & Execution Time \\
\hline Heuristic & 0.23 & 5 minutes \\
Genetic Algorithm & 0.25 & 16 hours \\
\hline
\end{tabular}

Source: Elaborated by the author.

It is important to emphasize that a specific optimized genetic algorithm probably would have a performance more close to the heuristic than the presented one. The overall hypothesis of the heuristic, however, have a strong validation with the results. 


\subsection{Validation of the Optimal Prior}

During the trace criterion validation, it was emphasized that the main hypothesis is that the prior functions defined are similar to the real prior of the sea. The prior functions suggested in the literature have general smoothing properties, and do not account for the problem idiosyncrasies, so it was expected that the trace criterion would not be a perfect metric to measure the errors.

In this section, the optimal prior based on sample seas will be validated, based on the shape information of the expected spectra. If it is assumed that the spectrum is almost always a JONSWAP type spectrum, and no preference is given to the parameters $H_{S}, T_{P}, \beta_{0}$ and $s$, it is possible to calibrate a generic prior using MonteCarlo simulations. To avoid the inherent random nature of the Monte-Carlo, it is possible to generate a set of seas with parameters varying accordingly with the discretization of the problem, Table 7. If it is used a $20 \times 20$ discretization, 360000 possible seas are generated.

Table 7 - Proposed discretization to calculate the new prior.

\begin{tabular}{ccccc}
\hline & $\boldsymbol{H}_{\boldsymbol{S}}$ & $\boldsymbol{\omega}_{\boldsymbol{P}}=\mathbf{2 \pi} / \boldsymbol{T}_{\boldsymbol{P}}$ & $\boldsymbol{\beta}_{\mathbf{0}}$ & $\boldsymbol{s}$ \\
\hline Minimum & 1 & $\min \left(\omega_{i}\right)$ & $\min \left(\beta_{i}\right)$ & 1 \\
Interval & 0.5 & $\Delta \omega$ & $\Delta \beta$ & 1 \\
Maximum & 5 & $\max \left(\omega_{i}\right)$ & $\max \left(\beta_{i}\right)$ & 100 \\
\hline
\end{tabular}

Source: Elaborated by the author.

Formally, the generated sample of the seas does not pass in any of the multivariate normality tests in the package developed by (KORKMAZ, et al., 2014). Despite of this, as discussed in section 4.5 Optimal Prior, the formulation is still valid, since it was desired a quadratic function that would best fit a given training data in the maximum likelihood sense. The maximum likelihood sense can also be though as the quadratic prior which is the closest to the equal probability of happening of each sample sea, i.e., maintains the shape of the spectrum without giving preference to any 
parameter; and a third interpretation is that the proposed optimal prior results in the best linear estimator of the problem.

If the prior is generated directly by the sample, however, a problem can happen. The sample mean is not equal zero, since all the sea energy is positive, but equal to a constant value, since no preference was given to any direction and any $T_{P}$. This constant value, however, can deliver wrong results with vectors close to the null space of the RAO matrix. If $\boldsymbol{A} \boldsymbol{x}_{\text {null }}=\mathbf{0}$, the best value will be determined by the prior and $x^{*}=x_{0}=$ cte., despite any measurement being made.

This can be easily solved by forcing $\boldsymbol{x}_{\mathbf{0}}=\mathbf{0}$, which is equivalent to duplicate the sample of the seas with the negative values of them, Equation (178). It makes sense, since the purpose of the prior is to describe the general shape of the sea vector values, which will be maintained to $-\boldsymbol{x}_{\text {sample }}$. Moreover, the quadratic programming nonnegative constraint limits the prior probability, and it is clear, by the general histogram of the sea vector values, that the quadratic prior, with non-negative constraints, must be centralized in zero to represent the best the probability, Figure 59 .

$$
\left.\frac{\sum_{i=1}^{n_{\text {sample }}}\left[\left(\boldsymbol{x}_{i}-\boldsymbol{x}_{\mathbf{0}}\right)\left(\boldsymbol{x}_{i}-\boldsymbol{x}_{\mathbf{0}}\right)^{T}\right]}{n_{\text {sample }}}\right|_{\boldsymbol{x}_{\mathbf{0}}=\mathbf{0}}=\frac{\sum_{i=1}^{n_{\text {sample }}}\left[\left(\boldsymbol{x}_{i}\right)\left(\boldsymbol{x}_{i}\right)^{T}\right]}{2 n_{\text {sample }}}+\frac{\sum_{i=1}^{n_{\text {sample }}}\left[\left(-\boldsymbol{x}_{i}\right)\left(-\boldsymbol{x}_{i}\right)^{T}\right]}{2 n_{\text {sample }}}
$$

Figure 59 - Histogram in a particular sea vector row. The occurrence of zero values is greater than shown in the histogram, it was cropped for visualization purposes.

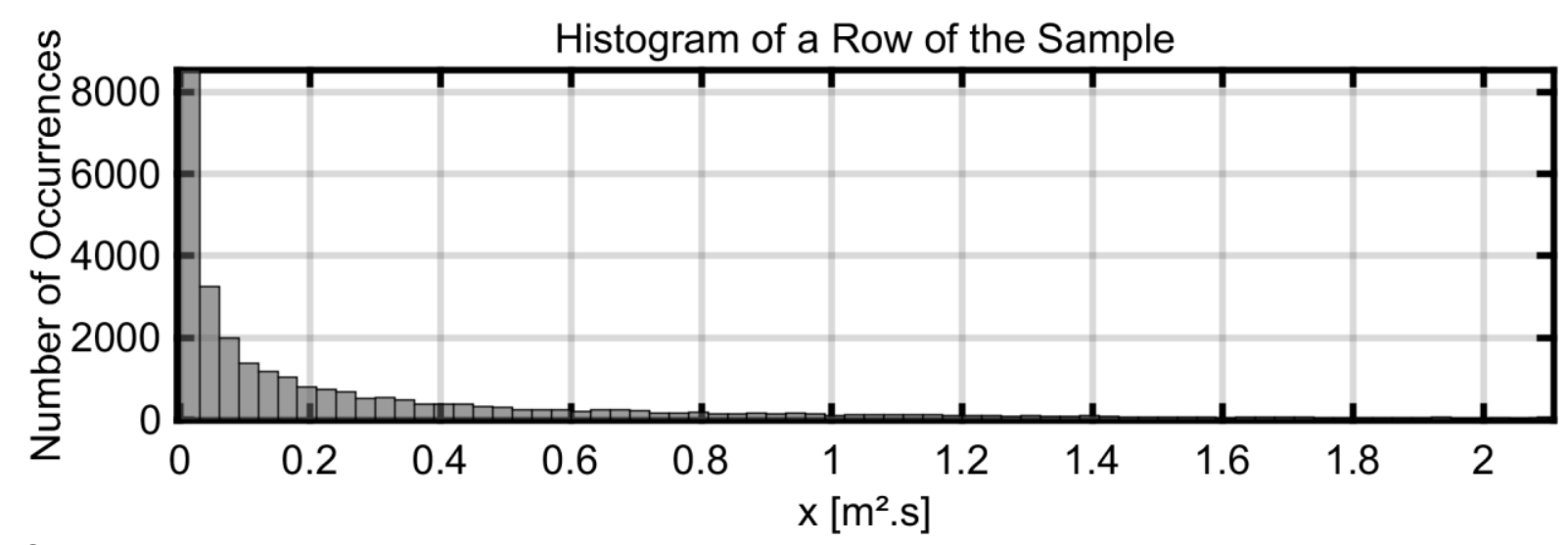

Source: Elaborated by the author. 
The maximum likelihood quadratic prior is given by Equations (179) and (180).

$$
\begin{gathered}
\boldsymbol{H}_{M L Q P}=\operatorname{inv}\left(\operatorname{cov}\left(\left[\boldsymbol{X}_{\text {sample }} ;-\boldsymbol{X}_{\text {sample }}\right]\right)\right) \\
\boldsymbol{\Gamma}_{M L Q P}=\text { cholesky decomposition }\left(\boldsymbol{H}_{M L Q P}\right)
\end{gathered}
$$

Despite the high number of seas, the computer is able to handle the data with no problem, and the generation of the set only takes a few seconds. If memory is a problem, the mean and covariance can be estimated by online algorithms, so the generated seas do not need to be stored.

To evaluate the new proposal, it is interesting to plot it against the prior proposed in the literature, taking in account the hyperparameters. The values for the literature hyperparameters are the same used in the numerical trials, and the hyperparameter of the new prior was calibrated in order to give approximately the same magnitude of values in the analyzed region, Figure 60.

Figure 60 - Comparison between the column of the priors.

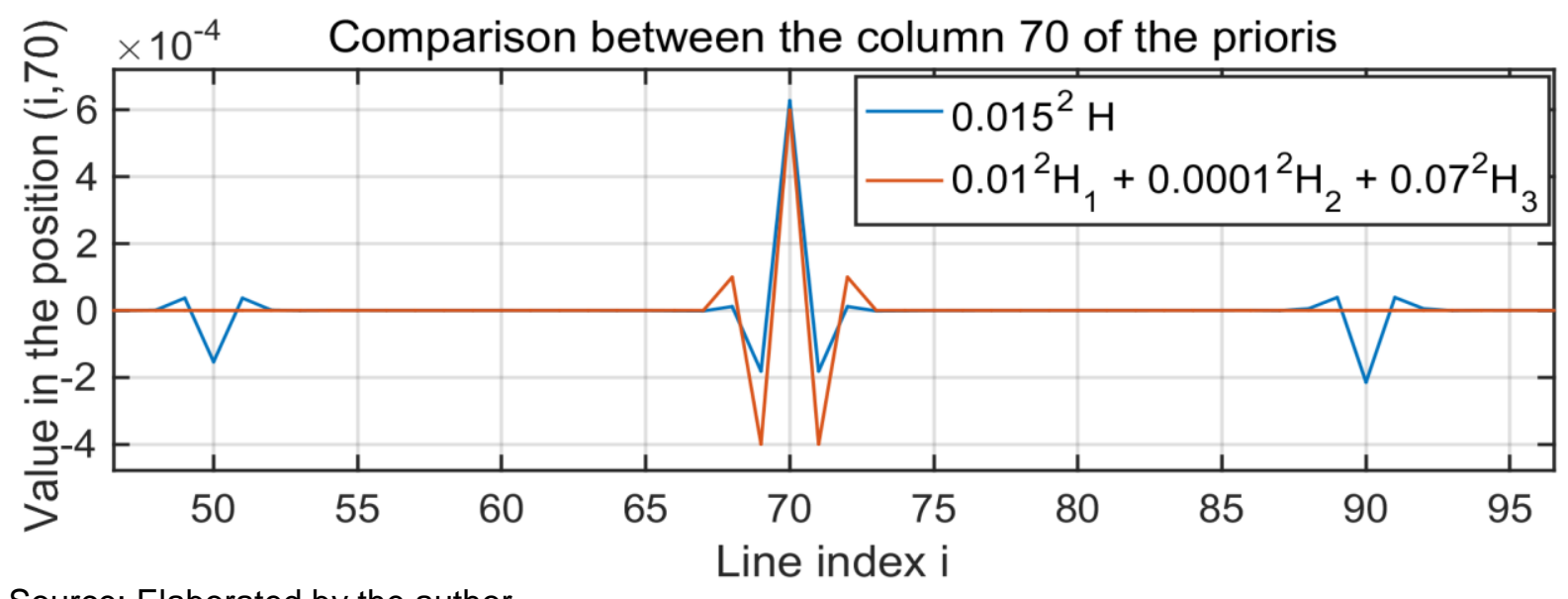

Source: Elaborated by the author.

The above figure shows a strong conclusion: the two priors are similar. It is interesting to notice, however, how the new prior have non-zero covariance in some other positions of the matrix, since not only the local relation is taken into account - in the sense of neighbor $\omega_{i}$ values - but the overall spectrum. This actually can be a drawback, since spectra with other shapes can happen, like bimodal spectra, but this 
is easily solved adding more spectra types in the simulation or zeroing the covariance in positions far from the diagonal, i.e., maintaining only the local relation.

The main advantage of this prior is that only one hyperparameter needs to be calibrated, as if this only hyperparameter is a base value and the hyperparameters of the literature prior are automatically weighed inside the new matrix. Moreover, with further developments, this parameter may be automatically adjusted by the measurement errors, since that, in the exact Bayesian formulation, $u^{2}=\sigma_{y}^{2}$.

There is other advantage, concerning how the prior penalizes noisy seas possibilities. In order to evaluate that, it is interesting to evaluate the proportion between the penalization of the ideal sea and a sea contaminated by some kind of noise. Some test seas were generated using the values in Table 8 , and the sea with added noise is $\boldsymbol{x}_{\text {noise }}=\boldsymbol{x}_{\text {ideal }}+0.1 \cdot\left[\begin{array}{lll}\varepsilon_{1} & & 0 \\ & \ddots & \\ 0 & & \varepsilon_{n}\end{array}\right] \cdot \boldsymbol{x}_{\text {ideal }}, \boldsymbol{\varepsilon}=\mathcal{N}(\boldsymbol{\mu}=\mathbf{0}, \boldsymbol{\Sigma}=\boldsymbol{I})$, i.e., for each position of the $x$ vector it is possible to happen up to $10 \%$ of error of the value in this position.

Table 8 - Proposed test set.

\begin{tabular}{ccccc}
\hline & $\boldsymbol{H}_{\boldsymbol{S}}$ & $\boldsymbol{\omega}_{\boldsymbol{P}}=\mathbf{2 \pi} / \boldsymbol{T}_{\boldsymbol{P}}$ & $\boldsymbol{\beta}_{\mathbf{0}}$ & $\boldsymbol{s}$ \\
\hline Minimum & 1 & $\min \left(\omega_{i}\right)$ & $0^{\circ}$ & 1 \\
Interval & - & $\Delta \omega$ & - & 10 \\
Maximum & 1 & $\max \left(\omega_{i}\right)$ & $0^{\circ}$ & 100 \\
\hline
\end{tabular}

Source: Elaborated by the author.

Essentially, the $H_{S}$ value is kept at one, since its variation will only cause an amplification of all priors; the $\beta_{0}$ value is kept at $0^{\circ}$, since the priors are indifferent to the incoming direction; and the $s$ value is varied with bigger interval, to improve visualization using less points. The results for each prior can be seen in Figure 61 and Figure 62, the higher the proportion between the penalization of the noisy sea and the penalization of the ideal sea, the better. 
Figure 61 - Penalization of the conventional prior.

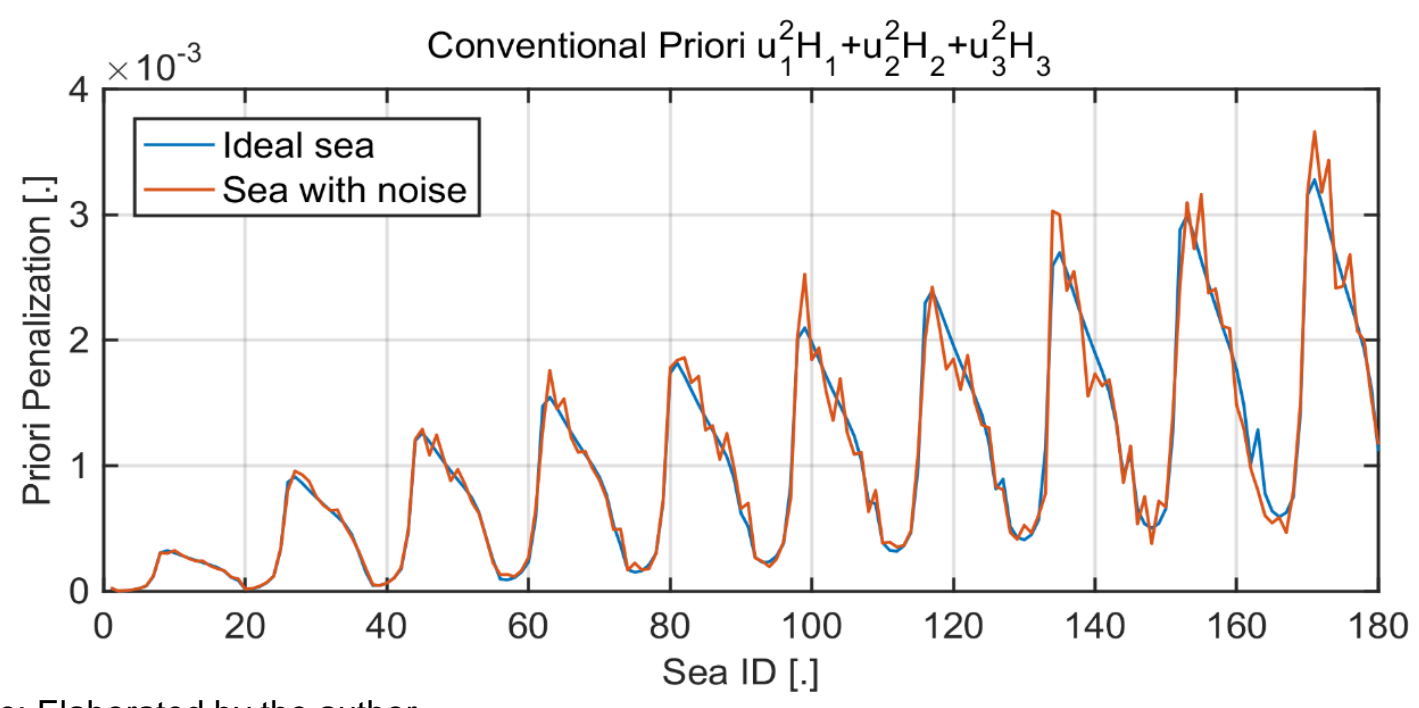

Source: Elaborated by the author.

Figure 62 - Penalization of the maximum likelihood quadratic prior (MLQP).

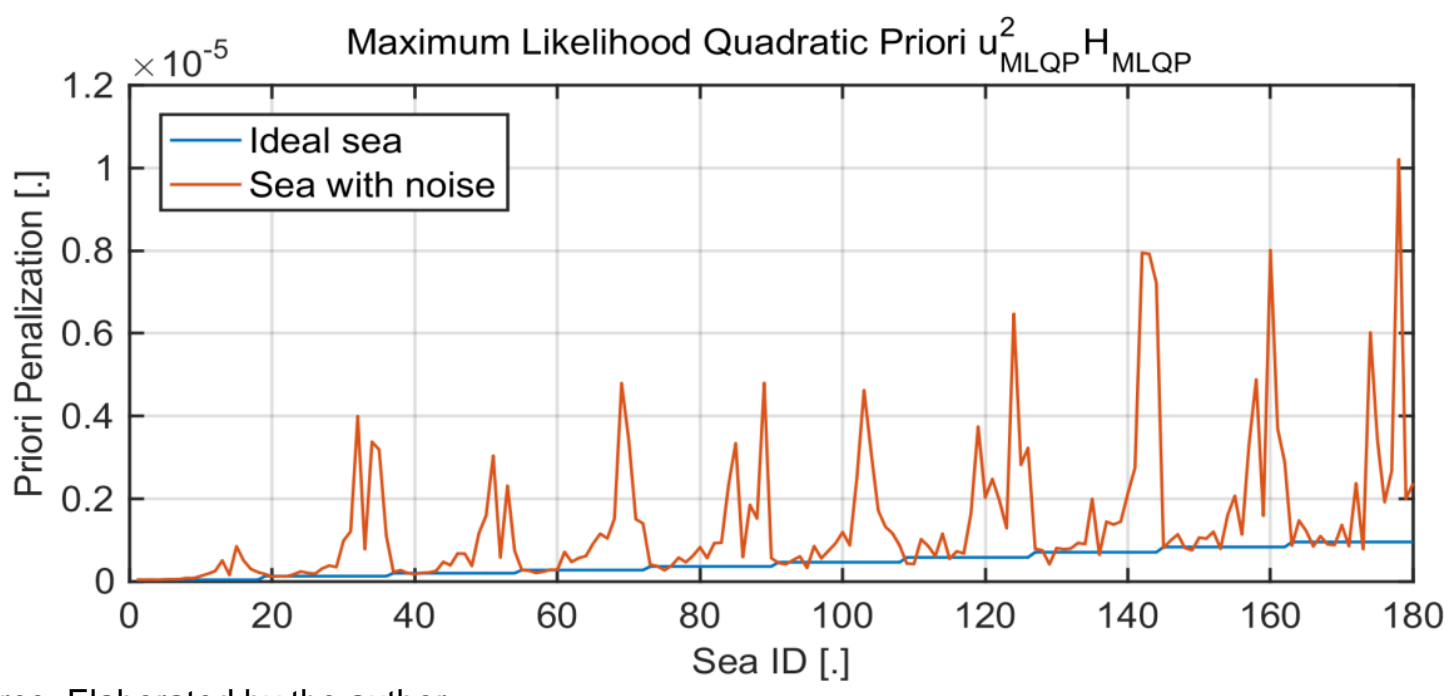

Source: Elaborated by the author.

It can be seen that the optimal prior is capable of identifying noisy seas better than the conventional prior, probably due to its global perspective, versus the local perspective of the second derivative.

The optimal prior also changes the optimal positioning result obtained by the trace criterion. In order to evaluate the relation among the new trace criterion and the error metrics, numerical simulations were performed for all possible combinations among three wave-probes with sway-heave-pitch vessel movements; and with measurement noise equals to 0.01 - the second most reasonable error magnitude, to avoid replicate the result of the numerical trials. Finally, the results were compared against the results obtained by the conventional prior, Figure 63. 
Figure 63 - Trace criterion versus errors for different priors.
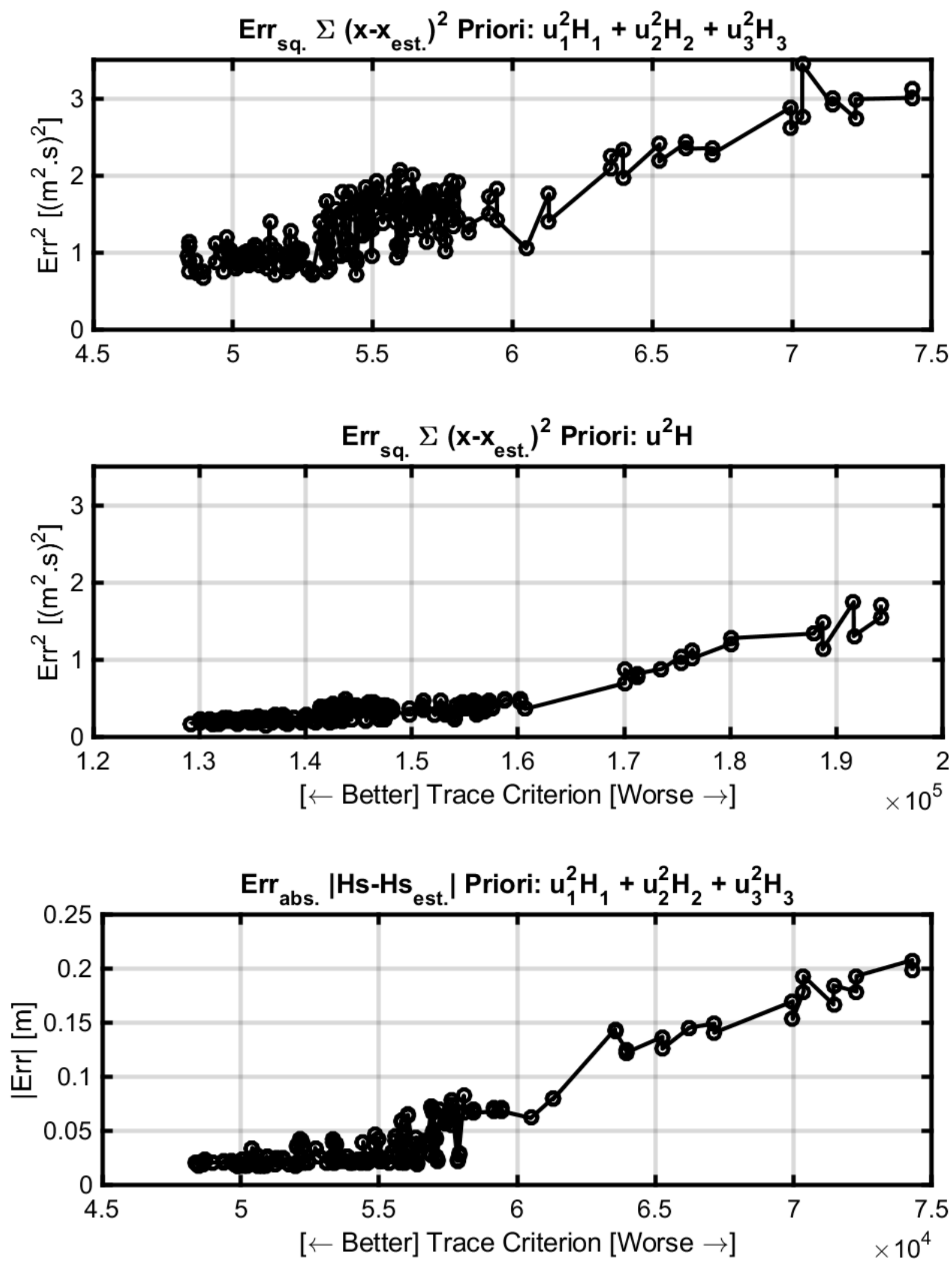

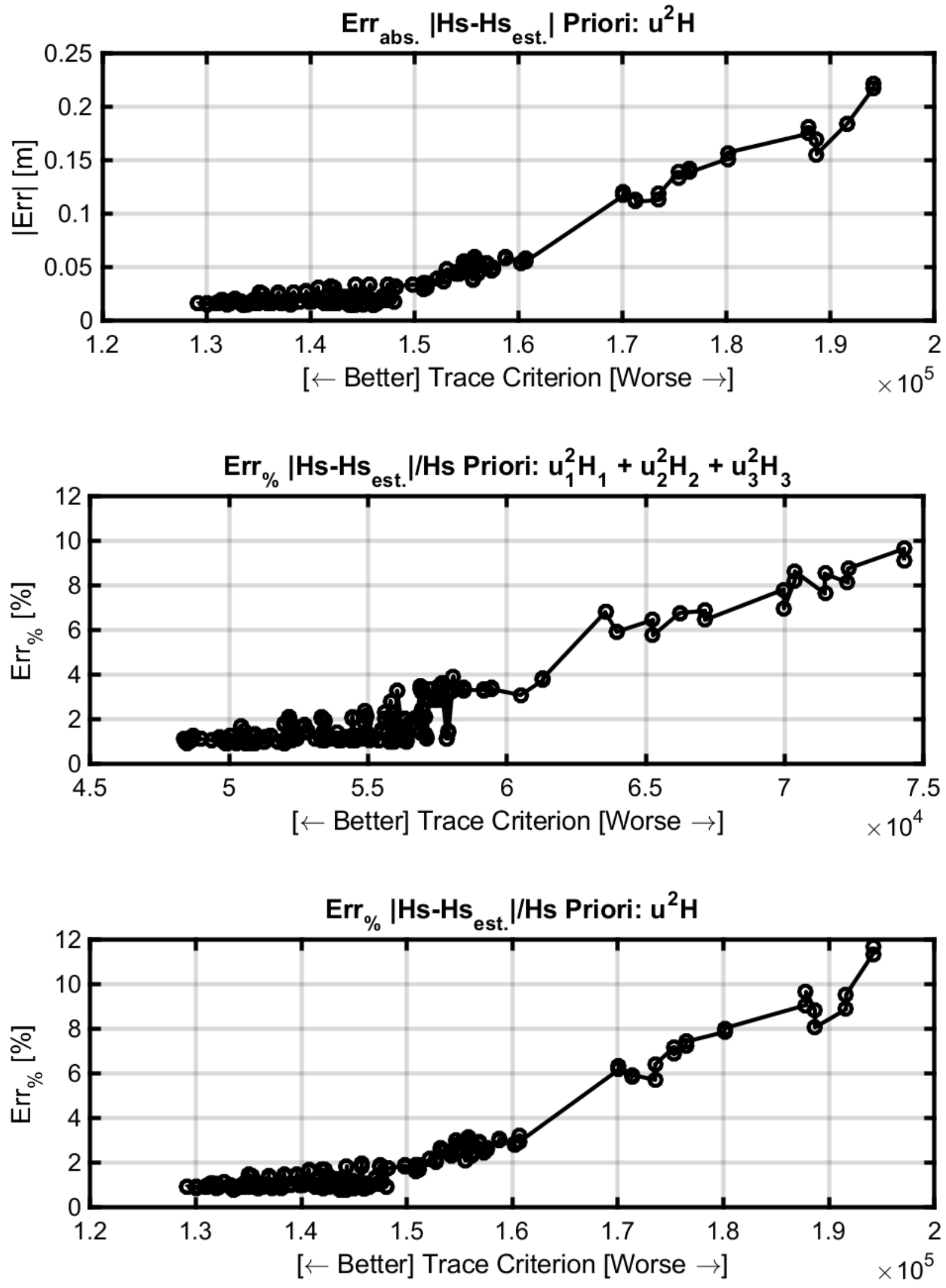

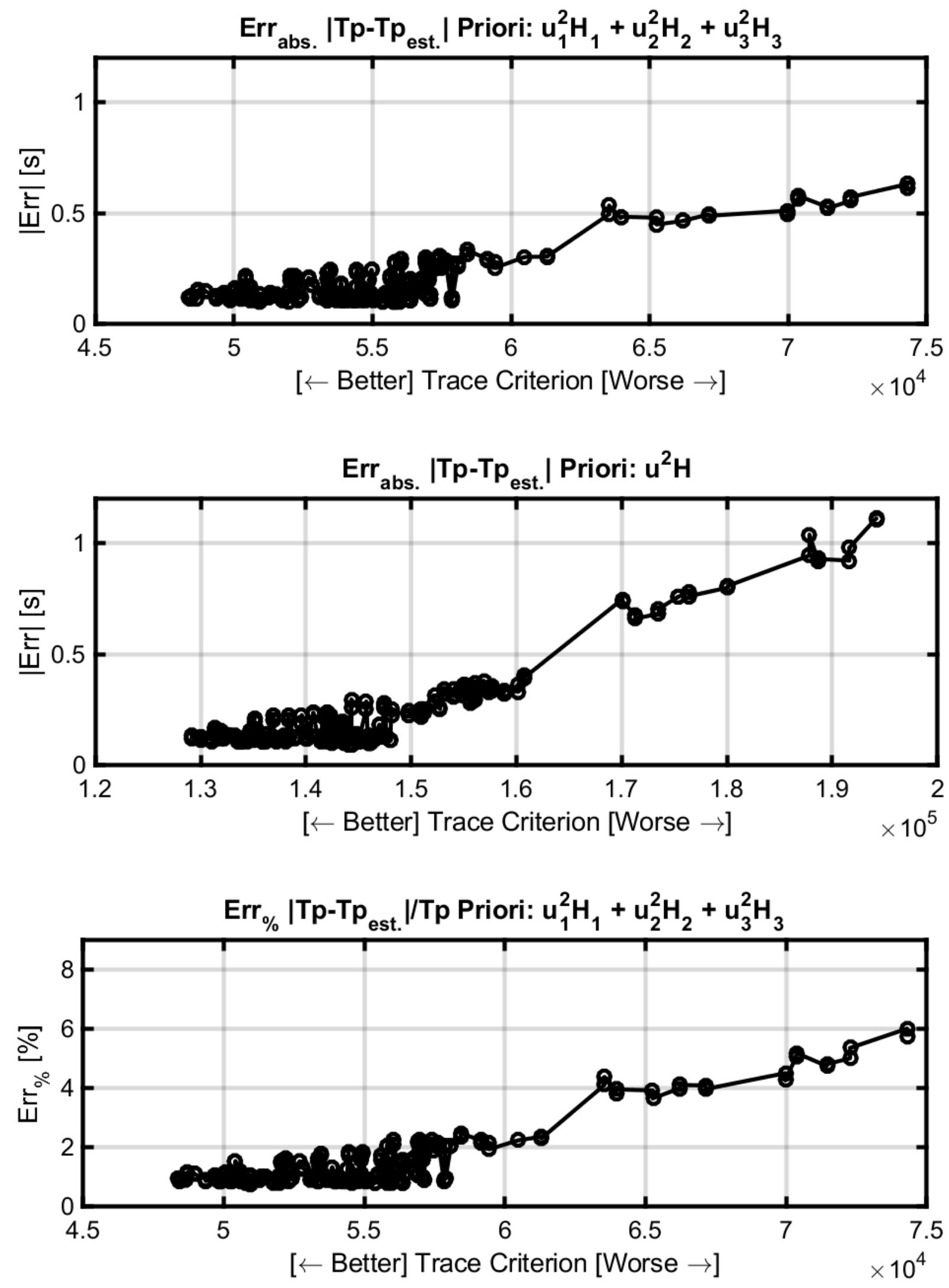

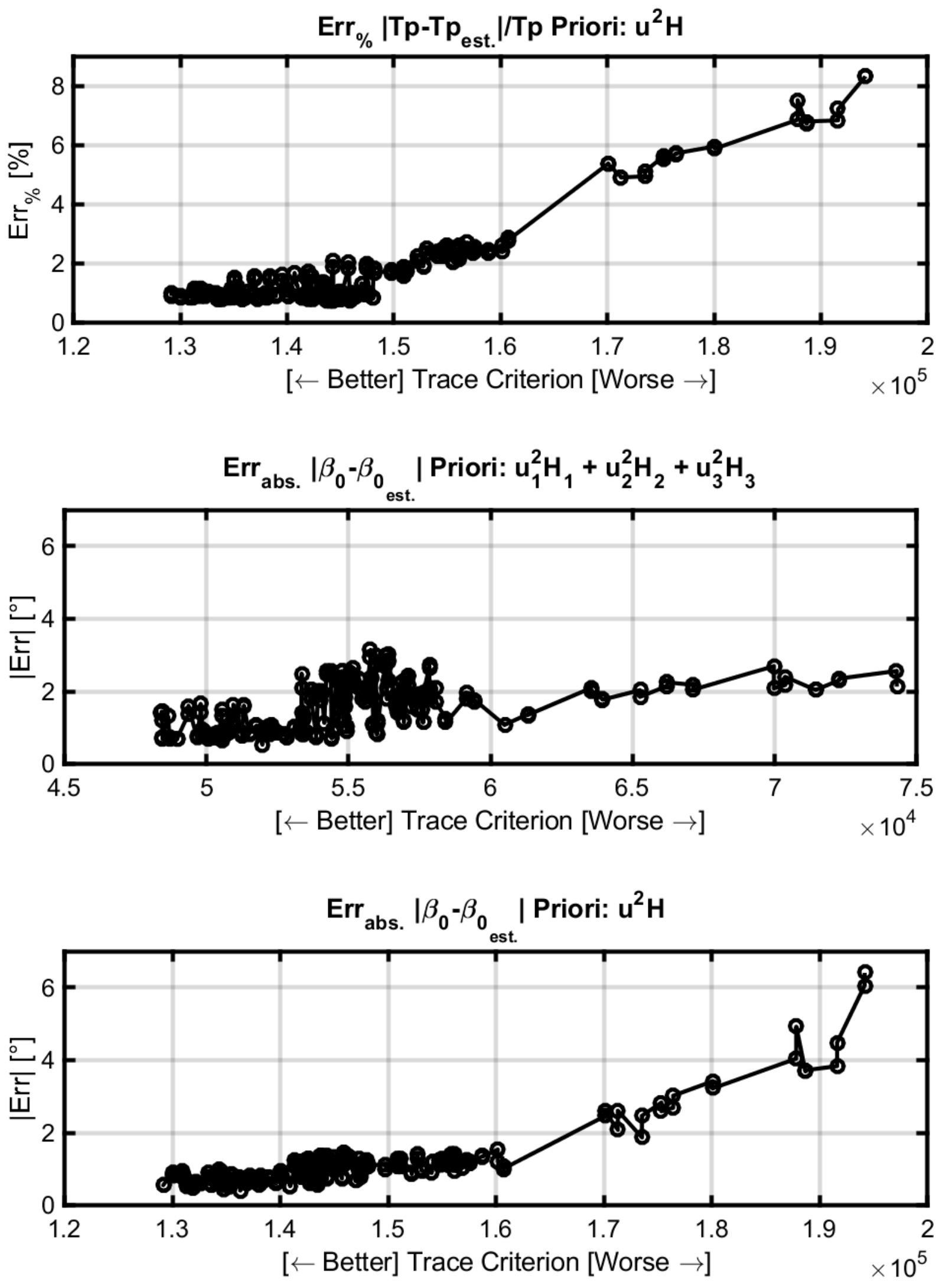

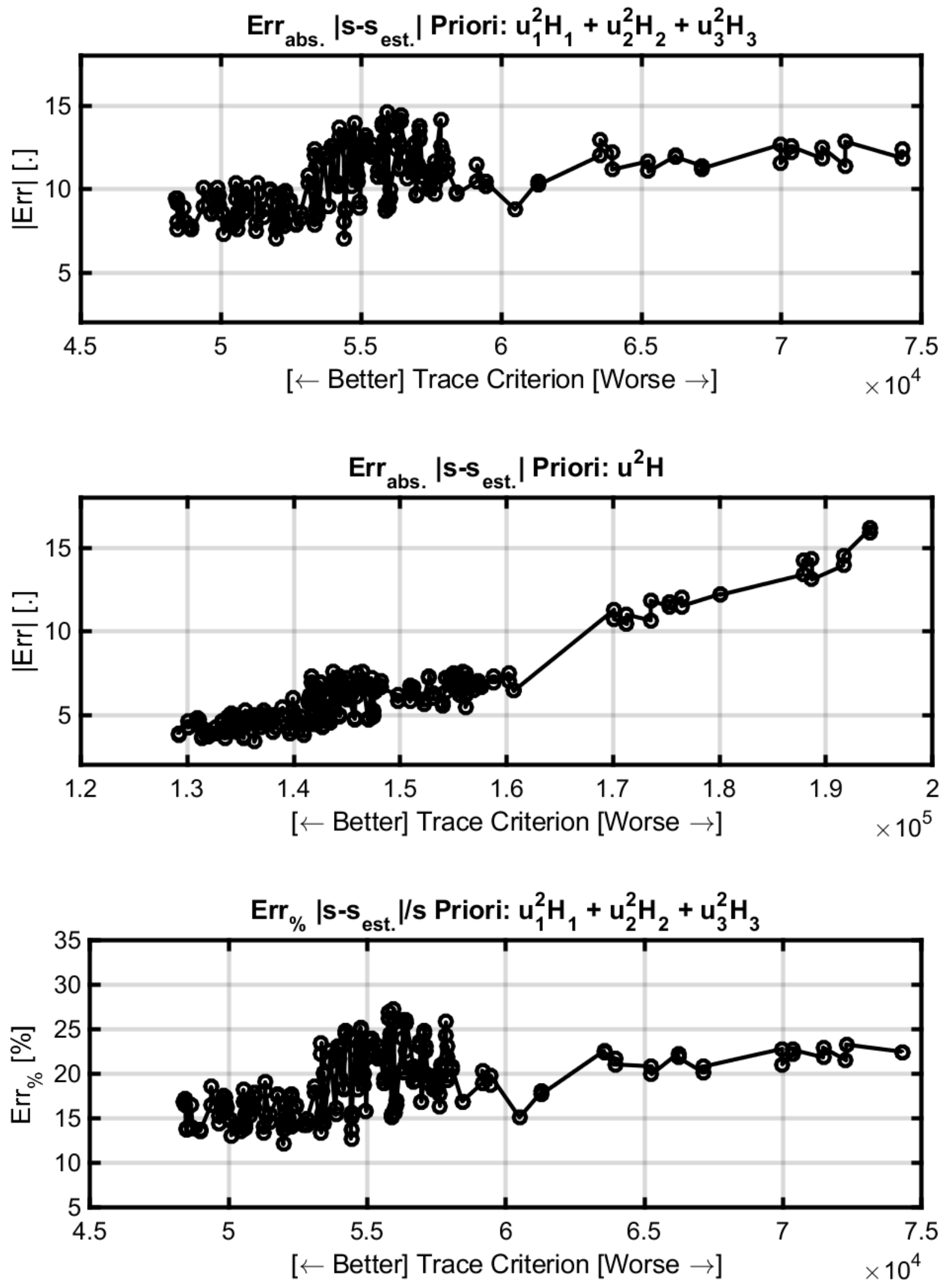


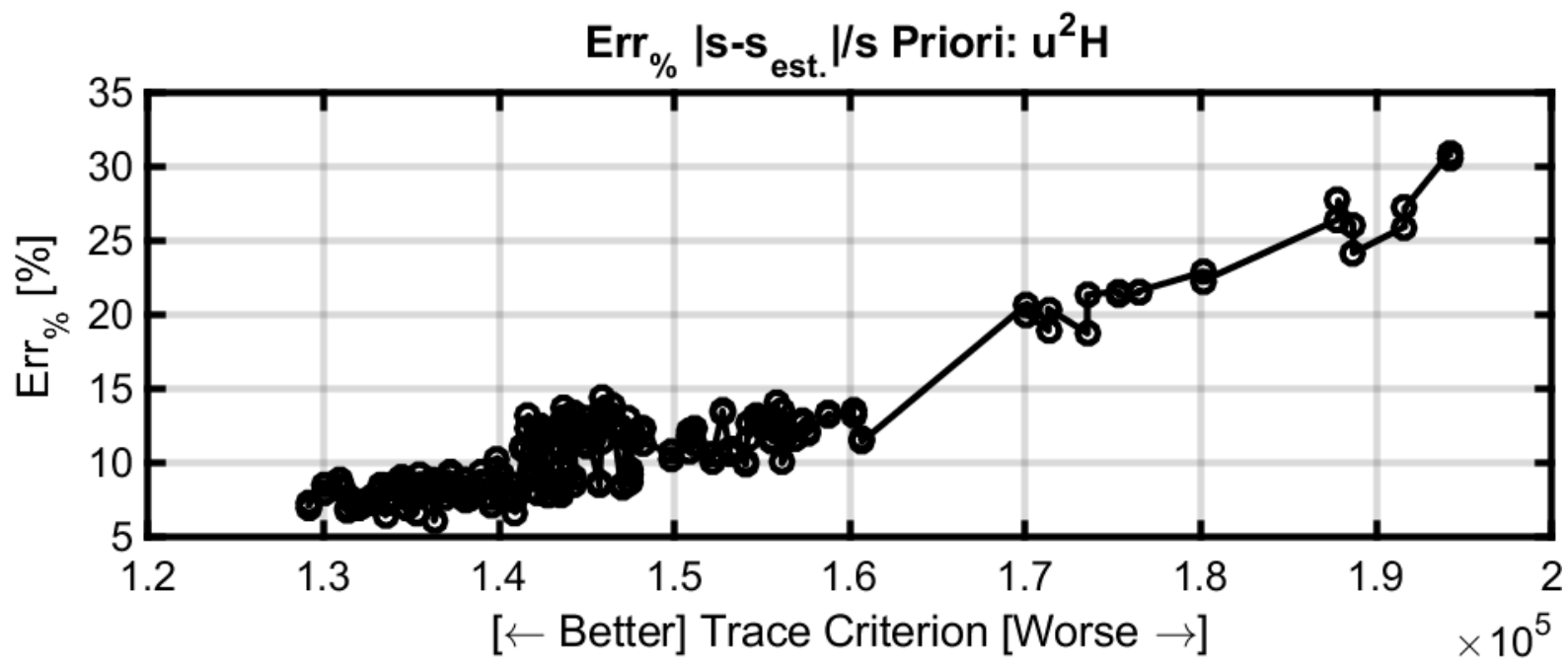

Source: Elaborated by the author.

The most evident conclusion of the graphs is that the tendency with the trace criterion is stronger with the optimal prior, even for directional parameters. Other important conclusion is that the best combination was able to perform equally well or better with this new prior, when compared against the literature prior.

In conclusion, the optimal prior seems to be really better than the usual prior in the literature; and even if the optimal prior provided no gain against the literature prior, it would be still the most suitable one to be used to calculate the trace criterion for optimal sensor placement purposes. 


\section{EXPERIMENTAL VALIDATION}

The numerical simulations are not able to provide information about the limitations of the assumed models of the vessel and the wave-elevation pattern; consequently, an experimental validation is necessary.

\subsection{Experimental Proposal}

The experiments are designed to answer the following questions:

- In the context of wave estimation, do the vessel movements and the waveprobe RAOs behave as predicted by the linear theory?

- The usage of wave-probes is indeed capable of improving the estimation capabilities of the method, even in other conditions as extreme events, bimodal seas and other drafts?

- Does the optimal prior really improves the results of the estimation?

\subsubsection{Experimental setup}

The experimental campaign was conducted under the coordination of Pedro Mello, with a small scale VLCC model provided by IPT, with a scale 1:90, Figure 64 .

Figure 64 - Vessel model.

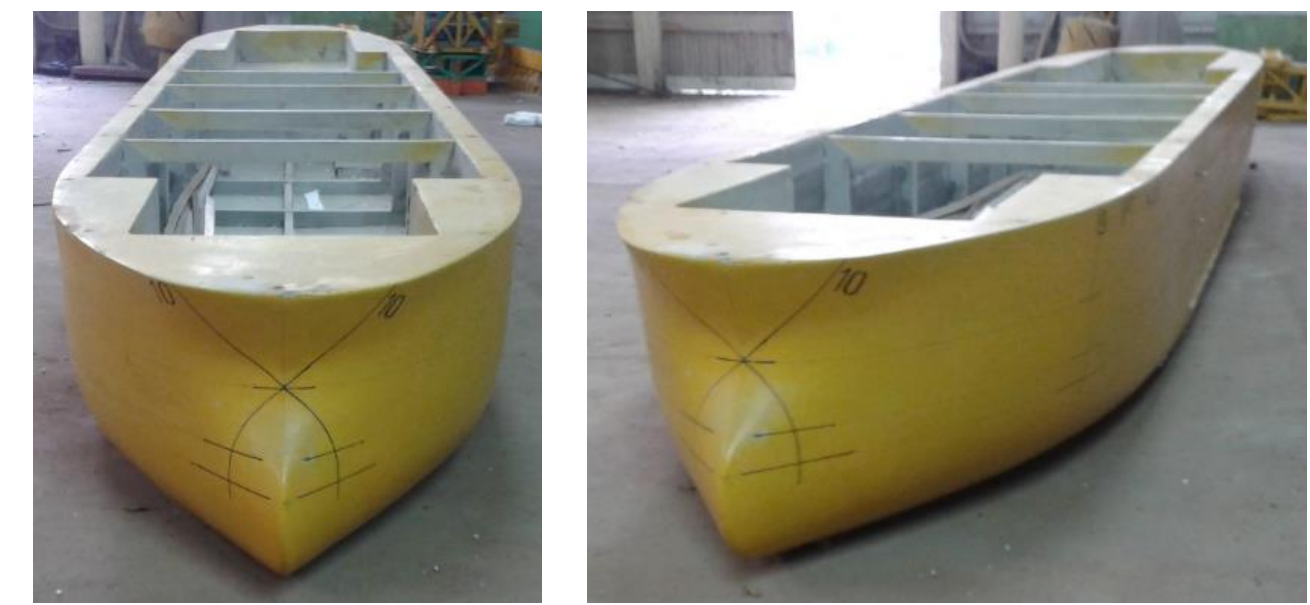

Source: Photographed by Pedro Mello.

The main dimensions and the center of gravity of the real vessel, on which the model is based, are shown in Figure 65. 
Figure 65 - Main dimensions of the real vessel.

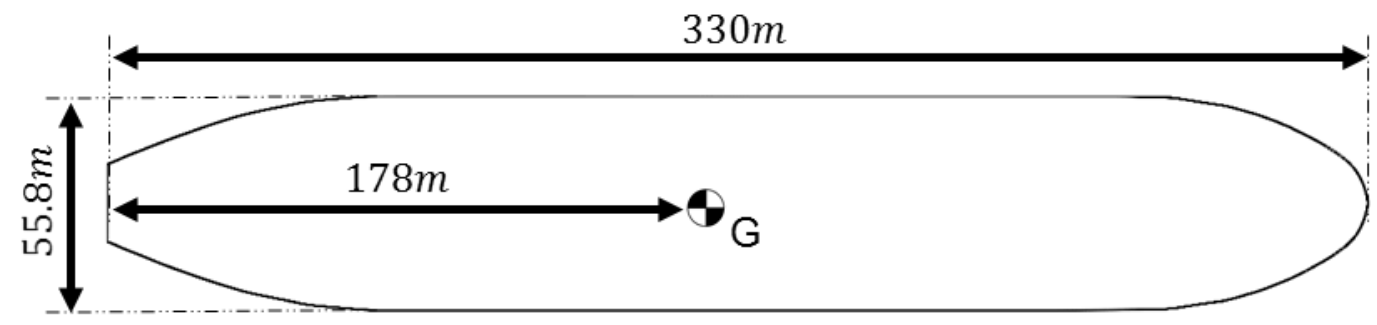

Source: Elaborated by the author.

Despite the fact that multiple drafts were used in the experiment, the longitudinal variation of the center of gravity is around one meter, which is negligible. The other properties, however, are not negligible and are listed in Table 9. The parameter $z_{C G}$ is the position of the center of mass along the $z$ direction, measured from the keel; the paremeters $R_{i i}$ are the radius of gyration, normalized by the length $L$ or the width $B$ of the vessel; and 'displacement' is the mass of the vessel, which is equal to the mass of the displaced water body in hydrostatic equilibrium, and it is given in tons.

Table 9 - Inertia and draft properties of the vessel.

\begin{tabular}{ccccccc}
\hline Draft & Draft [m] & $\mathbf{z}_{C G}[\mathrm{~m}]$ & $\frac{\boldsymbol{R}_{x x}}{\boldsymbol{B}}$ & $\frac{\boldsymbol{R}_{y y}}{\boldsymbol{L}}$ & $\frac{\boldsymbol{R}_{z z}}{\boldsymbol{L}}$ & Displacement [ton] \\
\hline $\begin{array}{c}\text { Ballasted } \\
\text { (empty) }\end{array}$ & 10 & 16.9 & 0.40 & 0.27 & 0.26 & 141921 \\
Intermediate & 15 & 13.9 & 0.39 & 0.25 & 0.24 & 220403 \\
Full & 18.5 & 13.9 & 0.37 & 0.25 & 0.23 & 278294 \\
\hline
\end{tabular}

Source: Elaborated by the author.

The properties of the model were estimated by CAD drawings with experimental correction: 3-point weight measurement, to determine the center of gravity; and bifilar torsional pendulum test, to determine the inertia of the hull, (HINRICHSEN, 2014); and the desired inertia properties were obtained by adding standard solid ballasts. The final properties were validated by measuring the natural period of oscillation in roll, pitch and heave with the vessel on the water, throughout decay tests, which determines the vessel dynamics; and static inclination, to guarantee the center of gravity position.

The wave basin that was used is the Hydrodynamic Calibrator at Numerical Towing Tank (CH-TPN). Its dimensions are $14 \mathrm{~m} \times 14 \mathrm{~m} \times 4.1 \mathrm{~m}$ depth, being a wave basin dedicated to small scale experiments. It is capable of generating waves along all 
its perimeter, using 148 moving wave generators, or flaps, and has active wave absorption, Figure 66. A complete description is found in (MELLO, et al., 2013).

Figure 66 - Wave basin drawing.

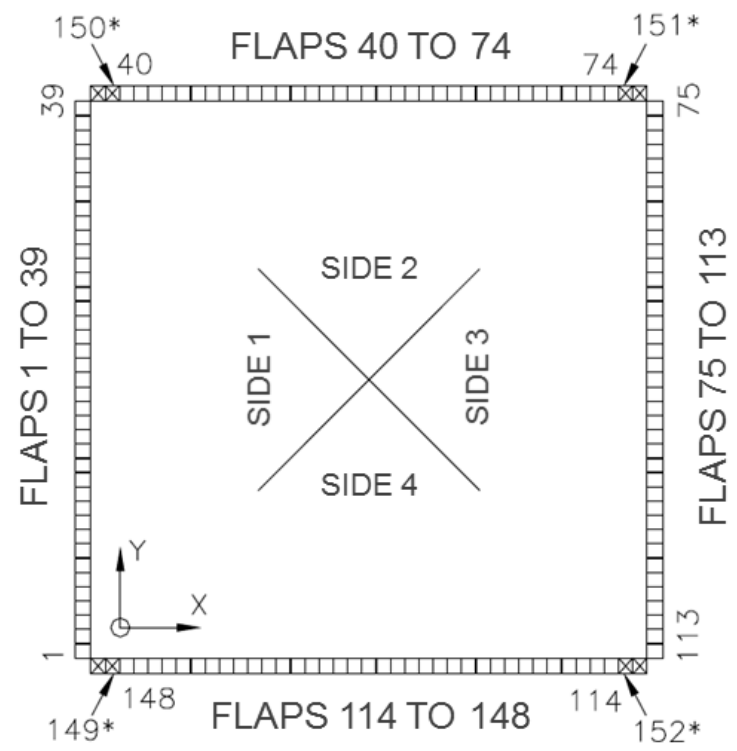

Source: Adapted from (CARNEIRO, 2012).

The system is capable of generating regular waves with frequencies from $0.4 \mathrm{~Hz}$ to $2.0 \mathrm{~Hz}$, and maximum wave height of $0.4 \mathrm{~m}$ at $0.75 \mathrm{~Hz}$. The maximum allowed steepness of the waves, $\frac{H_{S}}{\lambda_{P}}=\frac{H_{S}}{\frac{T_{P}^{2} g}{2 \pi}}$, is $5 \%$, and final constraints are imposed by physical limitations of the actuators: the stroke of the flaps and the limited screw velocity. The resulting valid region is shown in Figure 67.

Figure 67 - Valid region due to physical constraints.

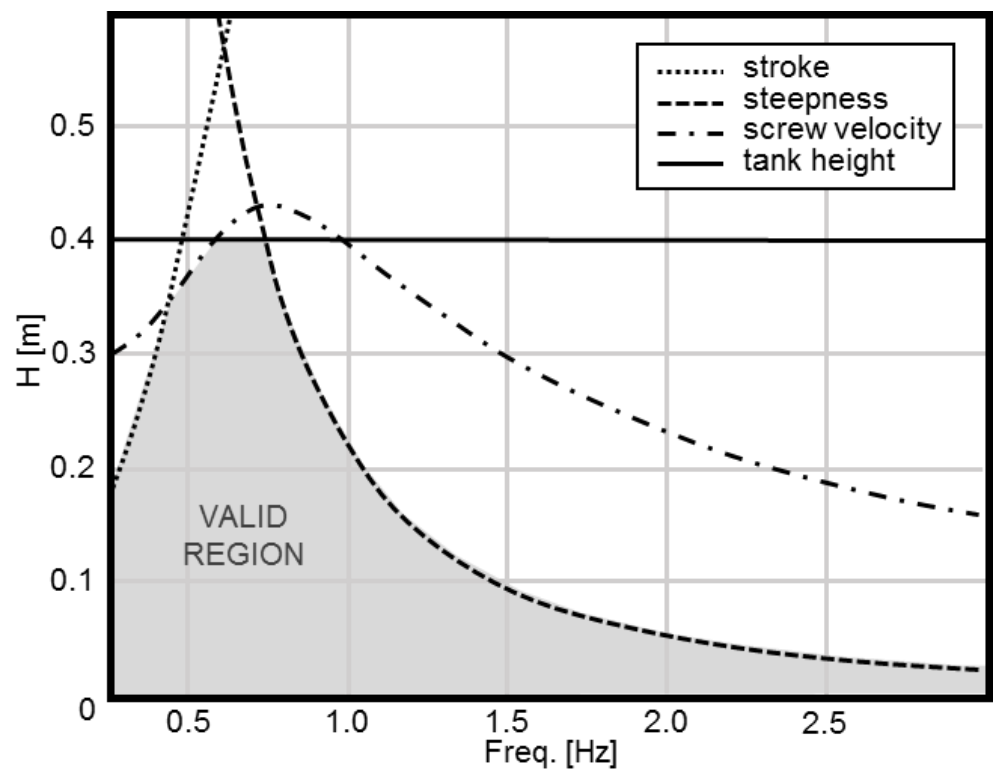

Source: Adapted from (CARNEIRO, 2012). 
The vessel movements were measured by a Qualisys ${ }^{\circledR}$ optical tracking system, which monitors reflective dummies attached to the hull, a non-intrusive method with linear displacement uncertainty around $0.1 \mathrm{~mm}$. Components of the system are shown in Figure 68.

Figure 68 - Reflective dummy and Qualisys ${ }^{\circledR}$ camera.
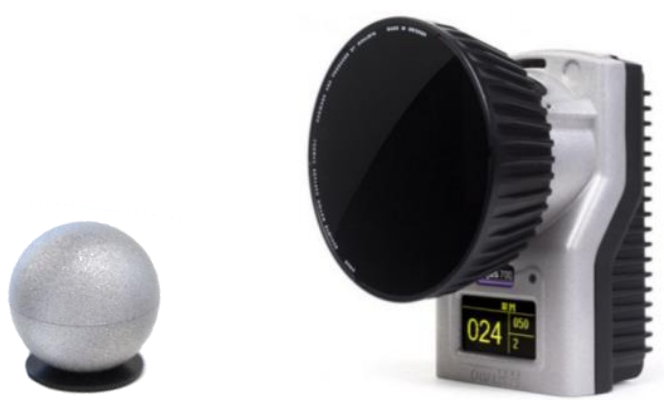

Source: (QUALISYS, 2016)

The wave-elevation was measured by twelve wave-probes positioned around the vessel, Figure 69. They are capacitive sensors with uncertainty around $1 \mathrm{~mm}$, Figure 70, with dynamic response shown in Figure 71.

Figure $69-C A D$ drawing of the model test with the wave-probes.

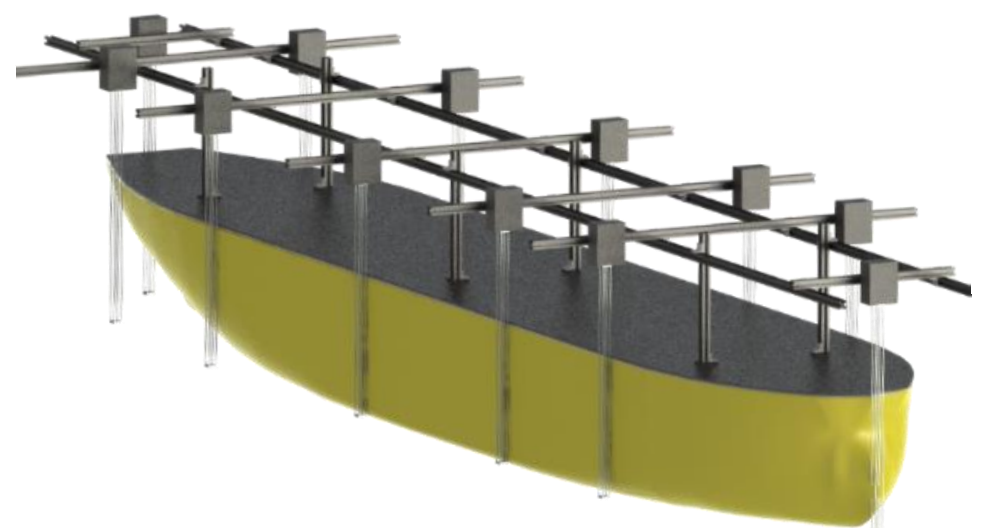

Source: Elaborated by the author.

Figure 70 - Wave-probes.

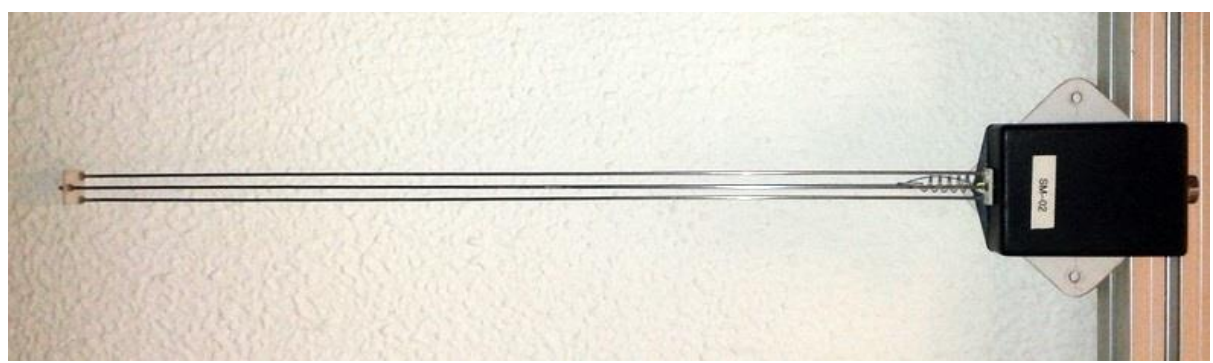

Source: Photographed by the author. 
Figure 71 - Wave-probes dynamic response, in real scale.
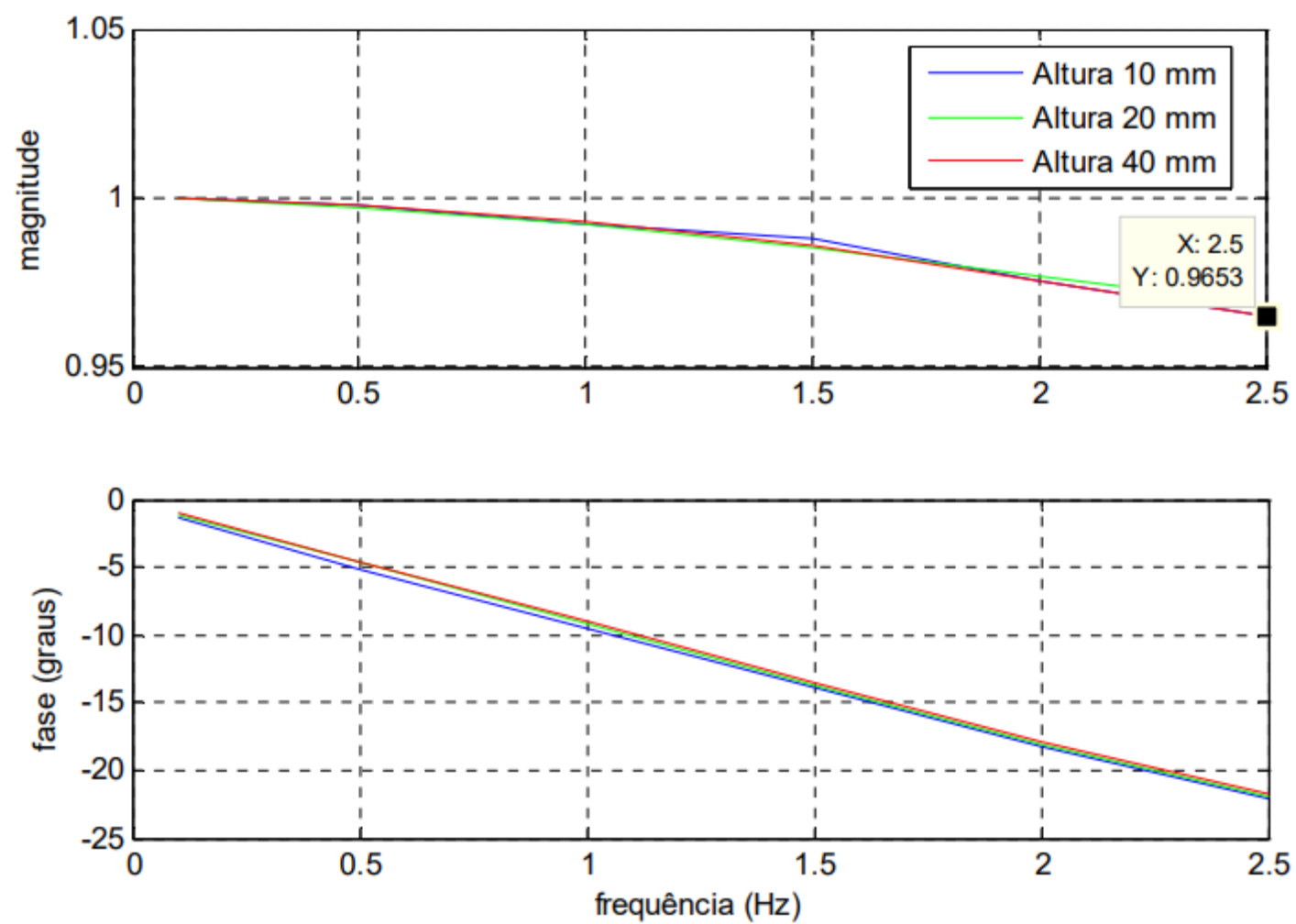

Source: (MELLO, 2012).

A similar test with the complete system can be seen in Figure 72. In the figure, differently than what is proposed, the probes are not installed on the vessel. The real test can be seen in Figure 73, with the twelve probes installed on the hull.

Figure 72 - Test with similar configuration.

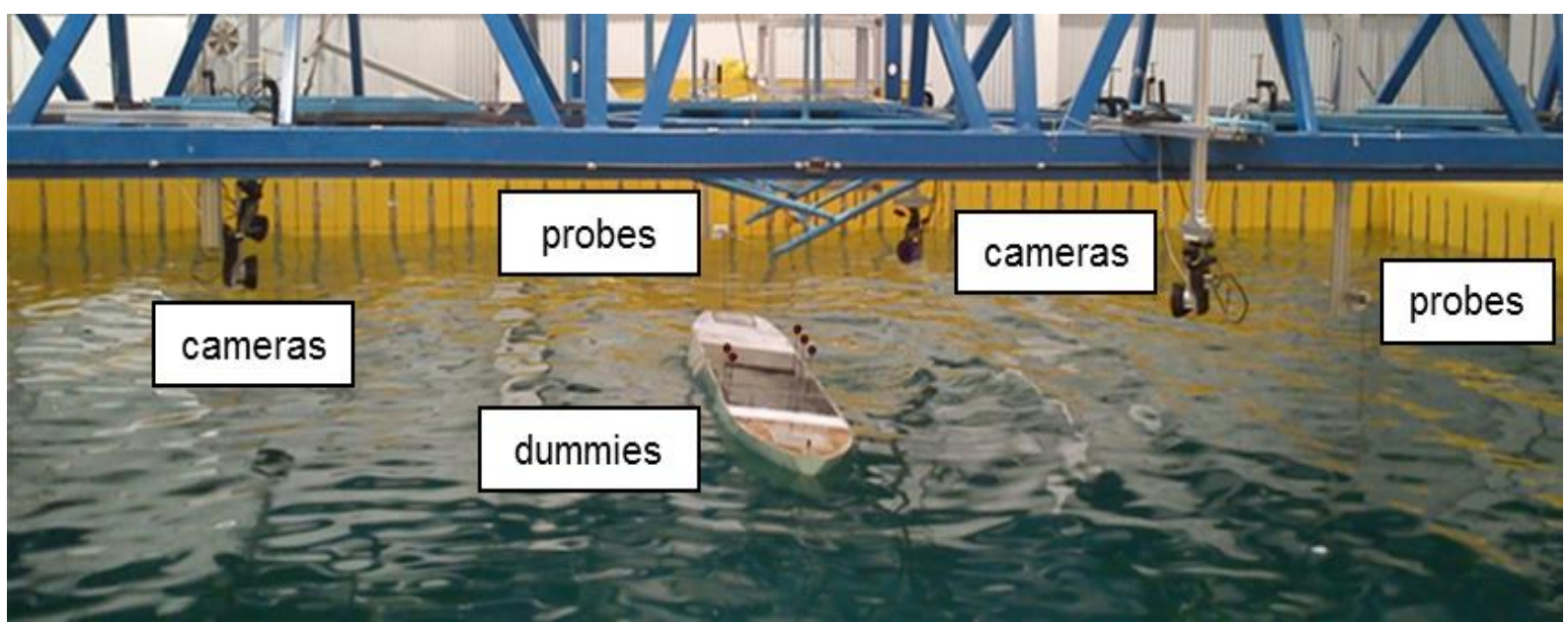

Source: Adapted from (MELLO, 2012). 
Figure 73 - Real test setup.

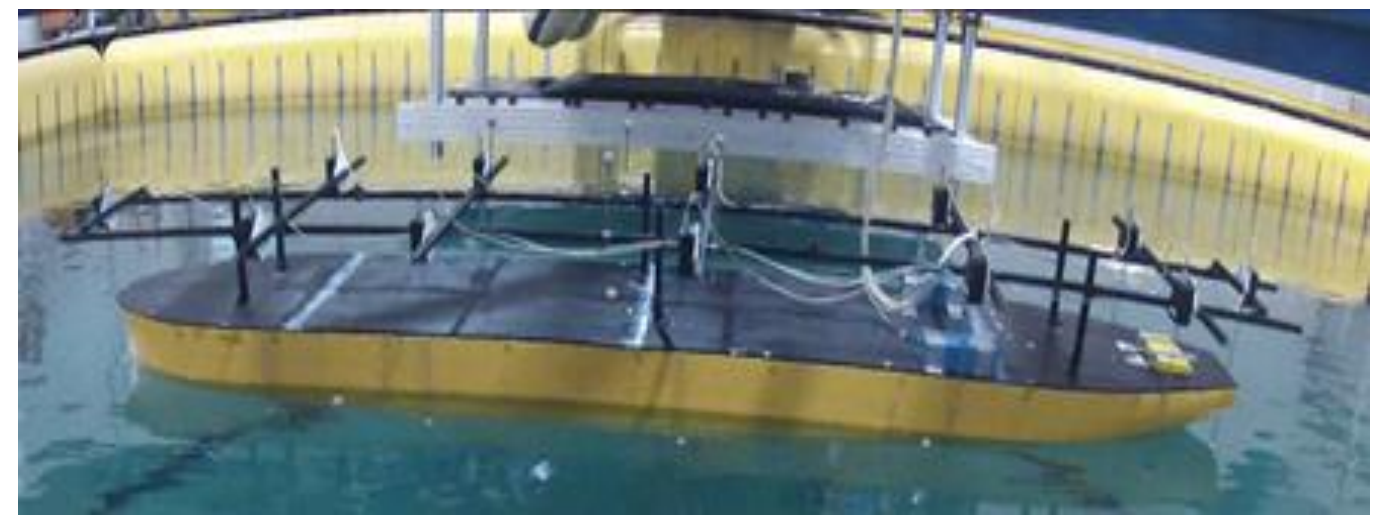

Source: Photographed by Pedro Mello.

Although it is not possible to see, the vessel is moored in four points with soft springs, which guarantees the translational positioning without major interference in the dynamic response.

The positions of the wave-probes were defined equal to the ones in the numerical trials, using cluster techniques, allowing a comparison between the experimental outcome and the numerical predictions.

\subsubsection{Wave selection}

A considerable number of tests is necessary to answer the proposed questions, but the resources, e.g., wave basin time allocation, are limited; consequently, a wave selection method must be applied.

The proposed method divides the experiments in some sets: statistical set, used to validate the prior hypothesis using real metocean data; extreme sea set, used to validate the linear model using real extreme wave data; bimodal set, designed to evaluate the principle of superposition and the capabilities of the algorithm to estimate bimodal seas, i.e., waves coming from different directions; and multiple drafts set, aiming at evaluating the robustness of the method against extreme drafts, ballasted and full. In all the sets, the experimental directional spectrum was obtained using the maximum entropy method with an array of 12 wave-probes, distributed in the towing tank without the vessel model.

\section{Metocean SeT}

As stated before, the metocean reports are the references for the real probability of the seas, so they need to be used in order to validate prior hypothesis assumed for 
the optimal design of experiments techniques. In this thesis, the metocean of Bacia de Campos - Campos Basin - is used, originated from a region located on the southeast Brazilian coast in the state of Rio de Janeiro.

As assumed, the report proposes a JONSWAP type spectrum with some modifications, which was not included in the experiment. No spread function is proposed, so a spread factor equals to $\infty$ was used, i.e., unidirectional experiment.

Even though a probability distribution of the sea directions is provided, it was assumed that the vessel can encounter the waves with any heading, so every test was

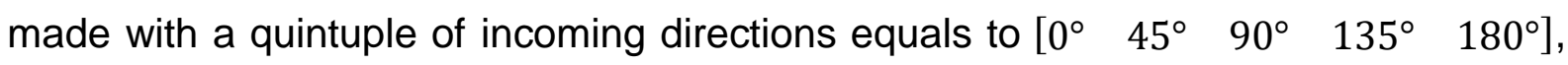
or the mean directions of the following sea, the quartering sea, the beam sea, the bow sea and the head sea.

Finally, for this first set of experiments, only the average draft was used, in order to maximize the possible duplets $\left[\begin{array}{ll}H_{S} & T_{P}\end{array}\right]$ that could be tested. The complete joint occurrence of the duplets is found in Table 10. It is important to emphasize that the frequency of each duplet did not need to be reproduced by the experiments, since each sea trial has multiple possible estimations intervals and a weighted statistic can be calculated.

Table 10 - Joint occurrence of $H_{S}$ and $T_{P}$.

\begin{tabular}{|c|c|c|c|c|c|c|c|c|c|c|c|c|c|c|c|c|c|c|}
\hline \multirow{2}{*}{\multicolumn{2}{|c|}{$\begin{array}{l}\mathrm{Tp} \rightarrow \\
\mathrm{Hs} \downarrow\end{array}$}} & 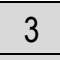 & 4 & 5 & 6 & 7 & 8 & 9 & 10 & 11 & 12 & 13 & 14 & 15 & 16 & 17 & 18 & 19 \\
\hline & & 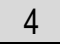 & 5 & 6 & 7 & 8 & 9 & 10 & 11 & 12 & 13 & 14 & 15 & 16 & 17 & 18 & 19 & 20 \\
\hline 0,0 & 0,5 & 34 & 185 & 40 & 8 & 10 & 10 & 35 & 72 & 88 & 88 & 130 & 75 & 73 & 11 & 13 & 7 & 5 \\
\hline 0,5 & 1,0 & 160 & 1358 & 775 & 265 & 245 & 128 & 139 & 272 & 298 & 224 & 148 & 64 & 55 & 11 & 1 & 0 & 1 \\
\hline 1,0 & 1,5 & 3 & 458 & 998 & 724 & 558 & 491 & 291 & 259 & 267 & 152 & 61 & 26 & 14 & 1 & 1 & 1 & 0 \\
\hline 1,5 & 2,0 & 0 & 13 & 307 & 563 & 413 & 374 & 268 & 191 & 196 & 118 & 80 & 11 & 5 & 3 & 1 & 0 & 0 \\
\hline 2,0 & 2,5 & 0 & 0 & 25 & 150 & 250 & 178 & 138 & 131 & 156 & 111 & 60 & 9 & 7 & 1 & 0 & 0 & 0 \\
\hline 2,5 & 3,0 & 0 & 0 & 1 & 15 & 74 & 70 & 69 & 68 & 87 & 52 & 39 & 13 & 8 & 4 & 0 & 0 & 0 \\
\hline 3,0 & 3,5 & 0 & 0 & 0 & 3 & 14 & 29 & 22 & 22 & 42 & 35 & 16 & 2 & 5 & 2 & 0 & 0 & 0 \\
\hline 3,5 & 4,0 & 0 & 0 & 0 & 0 & 1 & 2 & 14 & 23 & 20 & 15 & 19 & 3 & 3 & 2 & 1 & 0 & 0 \\
\hline 4,0 & 4,5 & 0 & 0 & 0 & 0 & 0 & 1 & 7 & 9 & 7 & 12 & 10 & 10 & 3 & 0 & 0 & 0 & 0 \\
\hline 4,5 & 5,0 & 0 & 0 & 0 & 0 & 0 & 0 & 1 & 2 & 2 & 2 & 6 & 3 & 2 & 0 & 0 & 0 & 0 \\
\hline 5,0 & 5,5 & 0 & 0 & 0 & 0 & 0 & 0 & 0 & 1 & 2 & 1 & 3 & 1 & 4 & 0 & 0 & 0 & 0 \\
\hline 5,5 & 6,0 & 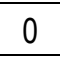 & 0 & 0 & 0 & 0 & 0 & 0 & 0 & 1 & 0 & 0 & 0 & 0 & 0 & 0 & 0 & 0 \\
\hline 6,0 & 6,5 & 0 & 0 & 0 & 0 & 0 & 0 & 0 & 0 & 0 & 0 & 1 & 0 & 0 & 0 & 0 & 0 & 0 \\
\hline 6,5 & 7,0 & 0 & 0 & 0 & 0 & 0 & 0 & 0 & 0 & 0 & 0 & 1 & 0 & 0 & 0 & 0 & 0 & 0 \\
\hline
\end{tabular}

Source: (PETROBRAS, 2010). 
In order to select the waves that represent the most the metocean, it is interesting to separate the duplets in wave families, and use an average criterion to select only one member to represent the entire family. This is done by the K-mean clustering algorithm.

Firstly, only $99 \%$ of the waves are maintaining, since some extreme events are easily identified and could potentially degrade the algorithm response. This is enough to remove sea states with annual occurrence equal or lower than nine.

For the K-mean calculations, the spectral density in each period is an independent dimension, and the responses of the vessel are calculated for pitch, roll and heave, for each of the incoming directions. Each period, in each response, for each direction, is also taken as an independent dimension. In the end, each possible sea is characterizes by a $M+3 \cdot 5 \cdot M$ vector, with $M$ equal the number of discretized periods, the first one being due to the spectral density; the number 3 due to the vessel movements; and the number 5 due to the directions used. It is important to use both the vessel movements and the sea spectrum because some similar vessel responses can occur for quite different seas, mainly low period seas; and similar seas can cause quite different vessel responses, mainly close to the resonance of some DoFs.

Using the 'elbow' criterion, 25 clusters would be enough to represent the metocean, Figure 74. Despite of that, 30 clusters were chosen, in order to guarantee some redundancy.

Figure 74 - 'Elbow' criterion applied to the clustering of the metocean data.

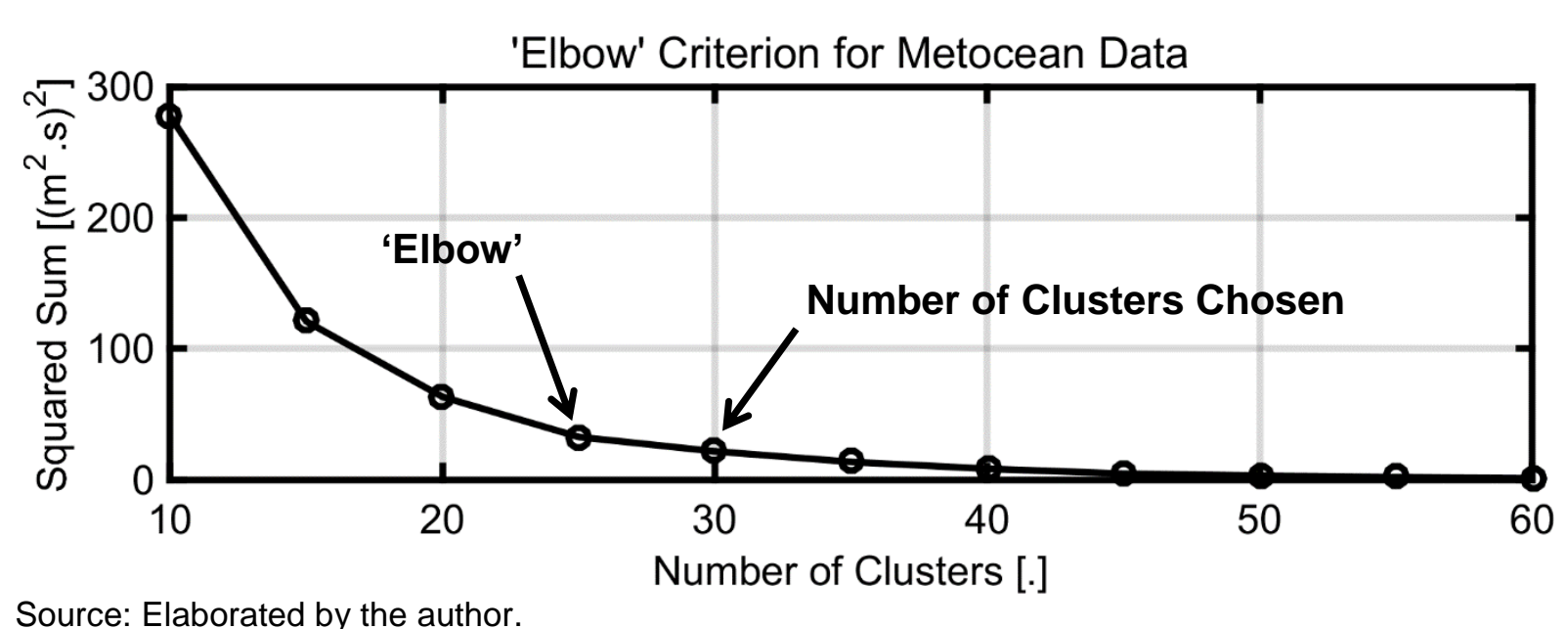

Source: Elaborated by the author. 
It is interesting to notice that no information was provided concerning the values of $H_{S}$ and $T_{P}$, but the clusters obtained by the algorithm are formed by neighbor cells, as expected, Table 11.

Table 11 - Clustered joint occurrence of $H_{S}$ and $T_{P}$.

\begin{tabular}{|c|c|c|c|c|c|c|c|c|c|c|c|c|c|c|c|c|c|c|}
\hline \multirow{2}{*}{\multicolumn{2}{|c|}{$\begin{array}{l}\mathrm{Tp} \rightarrow \\
\mathrm{Hs} \downarrow\end{array}$}} & 3 & 4 & 5 & 6 & 7 & 8 & 9 & 10 & 11 & 12 & 13 & 14 & 15 & 16 & 17 & 18 & 19 \\
\hline & & 4 & 5 & 6 & 7 & 8 & 9 & 10 & 11 & 12 & 13 & 14 & 15 & 16 & 17 & 18 & 19 & 20 \\
\hline 0,0 & 0,5 & 34 & 185 & 40 & 8 & 10 & 10 & 35 & 72 & 88 & 88 & 130 & 75 & 73 & 11 & 13 & 7 & 5 \\
\hline 0,5 & 1,0 & 160 & 1358 & 775 & 265 & 245 & 128 & 139 & 272 & 298 & 224 & 148 & 64 & 55 & 11 & 1 & 0 & 1 \\
\hline 1,0 & 1,5 & 3 & 458 & 998 & 724 & 558 & 491 & 291 & 259 & 267 & 152 & 61 & 26 & 14 & 1 & 1 & 1 & 0 \\
\hline 1,5 & 2,0 & 0 & 13 & 307 & 563 & 413 & 374 & 268 & 191 & 196 & 118 & 80 & 11 & 5 & 3 & 1 & 0 & 0 \\
\hline 2,0 & 2,5 & 0 & 0 & 25 & 150 & 250 & 178 & 138 & 131 & 156 & 111 & 60 & 9 & 7 & 1 & 0 & 0 & 0 \\
\hline 2,5 & 3,0 & 0 & 0 & 1 & 15 & 74 & 70 & 69 & 68 & 87 & 52 & 39 & 13 & 8 & 4 & 0 & 0 & 0 \\
\hline 3,0 & 3,5 & 0 & 0 & 0 & 3 & 14 & 29 & 22 & 22 & 42 & 35 & 16 & 2 & 5 & 2 & 0 & 0 & 0 \\
\hline 3,5 & 4,0 & 0 & 0 & 0 & 0 & 1 & 2 & 14 & 23 & 20 & 15 & 19 & 3 & 3 & 2 & 1 & 0 & 0 \\
\hline 4,0 & 4,5 & 0 & 0 & 0 & 0 & 0 & 1 & 7 & 9 & 7 & 12 & 10 & 10 & 3 & 0 & 0 & 0 & 0 \\
\hline 4,5 & 5,0 & 0 & 0 & 0 & 0 & 0 & 0 & 1 & 2 & 2 & 2 & 6 & 3 & 2 & 0 & 0 & 0 & 0 \\
\hline 5,0 & 5,5 & 0 & 0 & 0 & 0 & 0 & 0 & 0 & 1 & 2 & 1 & 3 & 1 & 4 & 0 & 0 & 0 & 0 \\
\hline 5,5 & 6,0 & 0 & 0 & 0 & 0 & 0 & 0 & 0 & 0 & 1 & 0 & 0 & 0 & 0 & 0 & 0 & 0 & 0 \\
\hline 6,0 & 6,5 & 0 & 0 & 0 & 0 & 0 & 0 & 0 & 0 & 0 & 0 & 1 & 0 & 0 & 0 & 0 & 0 & 0 \\
\hline 6,5 & 7,0 & 0 & 0 & 0 & 0 & 0 & 0 & 0 & 0 & 0 & 0 & 1 & 0 & 0 & 0 & 0 & 0 & 0 \\
\hline
\end{tabular}

Source: Elaborated by the author.

The similarity among the waves in the same cluster can be seen in Figure 75. The colors in the figure do not match the colors in the table, for the sake of clarity.

Figure 75 - Spectra density in each cluster.
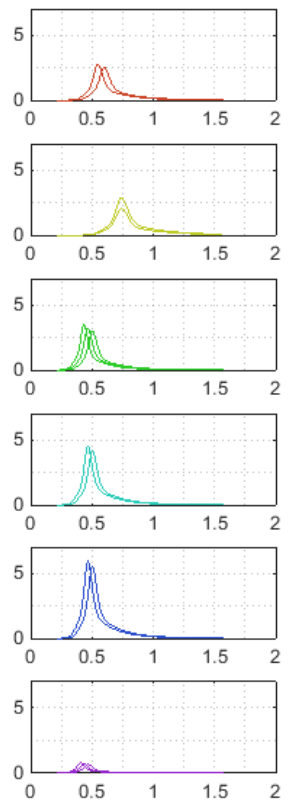

Source: Elaborated by the author.
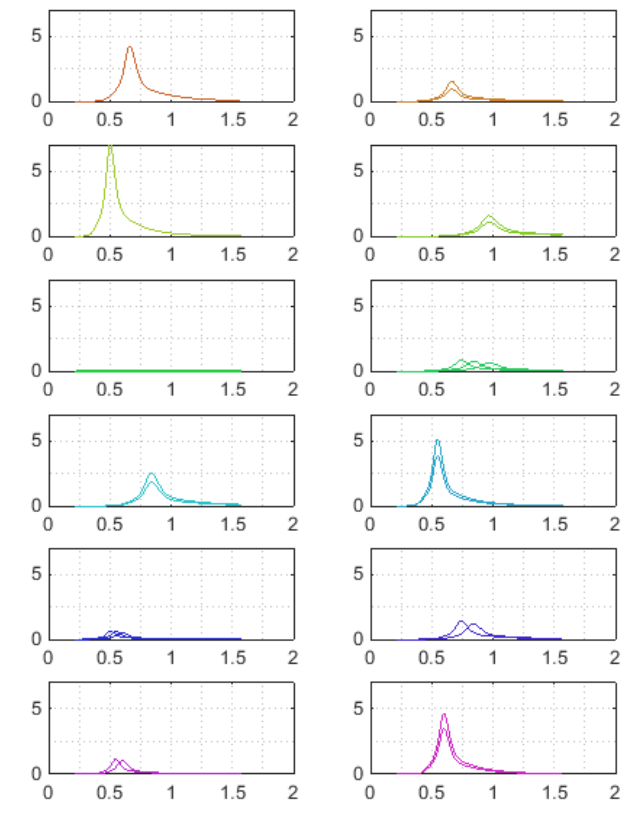
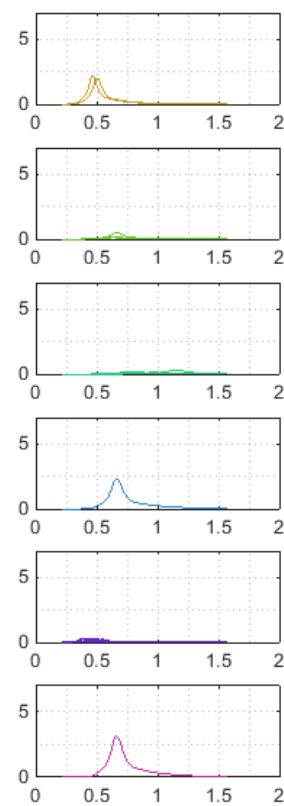
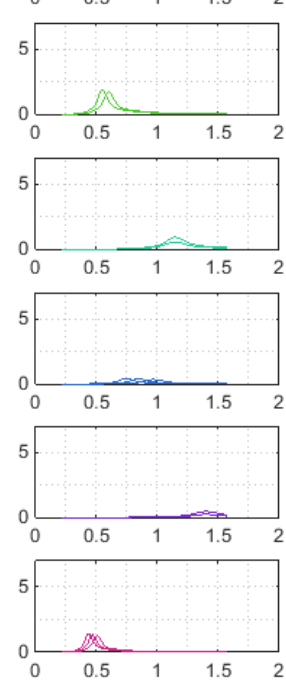
Due to the physical limitations of the tank, the cluster with the smallest $H_{S}$, the red one in the table, and the clusters with $T_{P}$ lower than $7 \mathrm{~s}$ could not be generated, resulting in 27 final duplets, or $27 \times 5=135$ experiments, accounting the five incoming directions. The final duplets are shown in Table 12.

Table 12 - Selected duplets: the averages of each cluster.

\begin{tabular}{cccccccccccccc}
\hline Tp & 7.1 & 7.2 & 8.1 & 8.1 & 8.1 & 8.5 & 9.0 & 9.9 & 9.9 & 9.9 & 9.9 & 10.1 & 10.9 \\
Hs & 0.9 & 2.4 & 1.3 & 1.7 & 3.0 & 2.2 & 3.0 & 2.0 & 2.8 & 3.3 & 3.8 & 1.0 & 3.5 \\
\hline 11.3 & 11.3 & 11.3 & 11.8 & 11.8 & 12.8 & 13.2 & 13.2 & 13.2 & 13.7 & 13.7 & 14 & 14.2 & 14.7 \\
1.8 & 2.3 & 2.8 & 1.3 & 3.5 & 4.3 & 2.3 & 3.3 & 3.8 & 1.8 & 2.8 & 0.8 & 4.3 & 1.3 \\
\hline
\end{tabular}

Source: Elaborated by the author.

The obtained values are not the average $\left[\begin{array}{ll}H_{S} & T_{P}\end{array}\right]$ of the cluster, but the duplet obtained when the parameters of the average spectrum density are calculated. This guarantees a final spectrum more centralized inside its family.

\section{EXTREME WAVES SET}

The second set of experiments is concerned about testing the limits of the linear system of equations, i.e., the vessel and the wave-probe RAOs.

The extreme events were taken from the same metocean used previously, as the waves with returning period equals to ten and one hundred years, Table 13.

Table 13 - Decenary and centenary waves.

\begin{tabular}{|c|c|c|c|c|}
\hline \multirow{2}{*}{ DIRECTION } & \multirow{2}{*}{\multicolumn{2}{|c|}{ PARAMETER }} & \multicolumn{2}{|c|}{ RETURN PERIOD (YEARS) } \\
\hline & & & 10 & 100 \\
\hline \multirow{2}{*}{$\mathbf{N}$} & Hs: & SIGNIFICANT WAVE HEIGHT (m) & 5.20 & 6.30 \\
\hline & TP: & PEAK PERIOD (s) & 9.28 & 10.00 \\
\hline \multirow{2}{*}{ NE } & Hs: & SIGNIFICANT WAVE HEIGHT (m) & 4.70 & 5.40 \\
\hline & TP: & PEAK PEBIOD (s) & 8.99 & 9.40 \\
\hline \multirow{2}{*}{$E$} & Hs: & SIGNIFICANT WAVE HEIGHT (m) & 4.20 & 4.70 \\
\hline & TP: & PEAK PERIOD (s) & 9.05 & 9.21 \\
\hline \multirow{2}{*}{ SE } & Hs: & SIGNIFICANT WAVE HEIGHT (m) & 5.50 & 6.70 \\
\hline & TP: & PEAK PERIOD (s) & 10.76 & 11.35 \\
\hline \multirow{2}{*}{ S } & Hs: & SIGNIFICANT WAVE HEIGHT (m) & 6.10 & 7.00 \\
\hline & TP: & PEAK PEBIOD (s) & 14.00 & 14.70 \\
\hline \multirow{2}{*}{ sw } & Hs: & SIGNIFICANT WAVE HEIGHT (m) & 6.90 & 7.80 \\
\hline & TP: & PEAK PERIOD (s) & 14.62 & 15.35 \\
\hline \multirow{2}{*}{ W/NW } & Hs: & SIGNIFICANT WAVE HEIGHT (m) & 4.00 & 4.60 \\
\hline & TP: & PEAK PERIOD (s) & 8.14 & 8.19 \\
\hline
\end{tabular}

Source: (PETROBRAS, 2010). 
In order to reduce the number of directions to be tested, two mooring systems were assumed as possible: the spread mooring system and the turret mooring system.

The turret mooring system, Figure 76, allows the vessel to rotate around a fixed position. It means that is reasonable to expect that the vessel will be aligned with the extreme conditions, i.e., the extreme conditions will be always head seas.

Figure 76 - Turret mooring system.

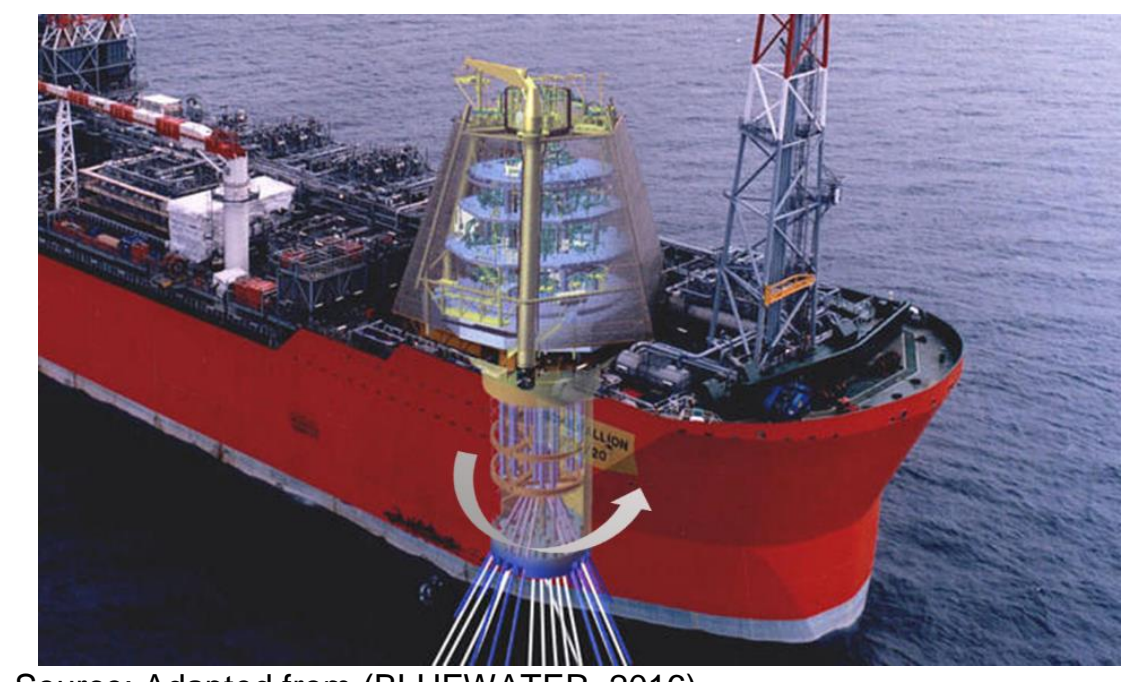

Source: Adapted from (BLUEWATER, 2016).

The spread mooring system, on its turn, is fixed in a constant heading, usually pointing to southwest. It means that each extreme sea does not need to be tested in all possible directions, but only in its incoming direction in relation with the southwest heading; reducing the number of experiments considerably. The mooring system is shown in Figure 77.

Figure 77 - Spread mooring system.

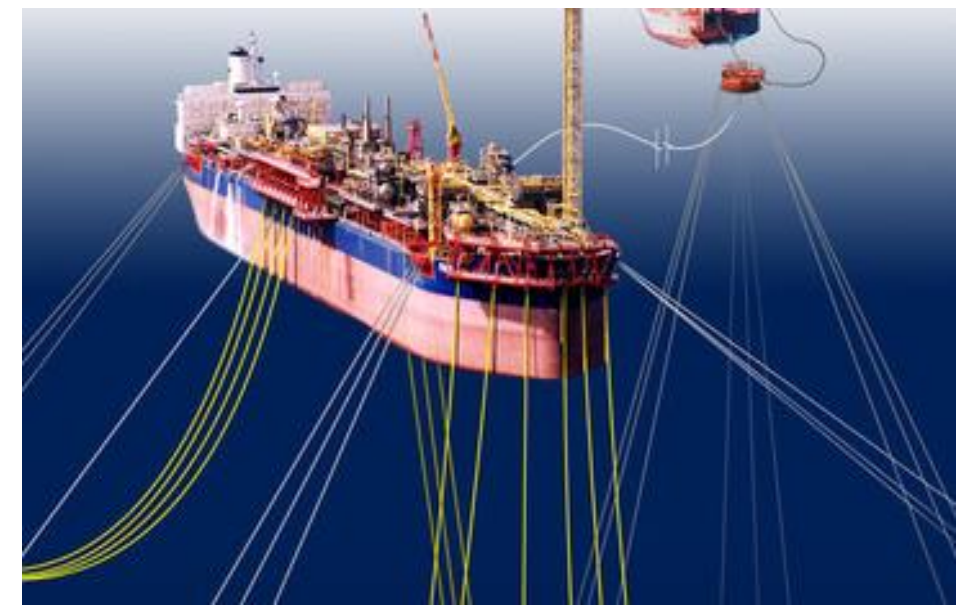

Source: (FUKY MarinTech, 2016). 
Merging the triplet $\left[\begin{array}{lll}\beta_{0} & H_{S} & T_{P}\end{array}\right]$ for each case, and combining with similar seas in the metocean set, a final set with 18 experiments is obtained, Table 14.

Table 14 - Extreme seas set of experiments.

\begin{tabular}{|c|c|c|}
\hline $\boldsymbol{\beta}_{0}\left[^{\circ}\right]$ & $H_{S}[m]$ & $T_{P}[s]$ \\
\hline $180^{\circ}$ & 4.3 & 8.5 \\
\hline $180^{\circ}$ & 5.3 & 9.5 \\
\hline $180^{\circ}$ & 6.5 & 10.0 \\
\hline $180^{\circ}$ & 5.5 & 11.0 \\
\hline $180^{\circ}$ & 6.5 & 11.5 \\
\hline $180^{\circ}$ & 6.0 & 14.0 \\
\hline $180^{\circ}$ & 7.0 & 14.5 \\
\hline $180^{\circ}$ & 8.0 & 15.5 \\
\hline $135^{\circ}$ & 4.3 & 8.0 \\
\hline $135^{\circ}$ & 6.0 & 14.0 \\
\hline $135^{\circ}$ & 7.0 & 15.0 \\
\hline $90^{\circ}$ & 4.3 & 8.0 \\
\hline $90^{\circ}$ & 5.5 & 11 \\
\hline $90^{\circ}$ & 6.5 & 11.5 \\
\hline $45^{\circ}$ & 5.0 & 9.0 \\
\hline $45^{\circ}$ & 6.5 & 10 \\
\hline $0^{\circ}$ & 4.5 & 9.0 \\
\hline $0^{\circ}$ & 5.5 & 9.5 \\
\hline
\end{tabular}

Source: Elaborated by the author. 


\section{BIMODAl SET}

This set of experiments is proposed to validate the superposition principle in the context of directional spectrum estimation.

The sea conditions are assumed to be $90^{\circ}$ apart from each order, simplifying the generation, since the main sea can be produced from side 1 to side 3 of the wave basin, and the second sea from side 2 to side 4 .

The vessel heading is varied among the values $\left[\begin{array}{lll}225^{\circ} & 180^{\circ} 135^{\circ}\end{array}\right]$, by the same argument used for the turret mooring systems, i.e., the vessel probably faces the main environmental force.

The spectrum energy density parameters were taken among the waves defined in the metocean set, and are $S_{1}=\left[H_{S}=2.8, T_{P}=9.9\right] ; S_{2}=\left[H_{S}=4.3, T_{P}=12.8\right] ; S_{3}=$ $\left[H_{S}=2.8, T_{P}=13.7\right]$. The idea was to take two sets with similar $H_{S}-S_{1}$ and $S_{3}-$ and two sets with similar $T_{P}-S_{2}$ and $S_{3}$; and to combine them to generate 6 possible seas: $\left(S_{1}, S_{1}\right),\left(S_{1}, S_{2}\right),\left(S_{1}, S_{3}\right),\left(S_{2}, S_{2}\right),\left(S_{2}, S_{3}\right)$ and $\left(S_{3}, S_{3}\right)$. Totalizing $6 \cdot 3=18$ tests.

\section{MULTIPLE DRAFTS}

The multiple drafts experiment was designed to evaluate if the method is robust against extreme draft variations. Two drafts were used: the ballasted draft, equals to $10 \mathrm{~m}$; and the full draft, equals to $18.5 \mathrm{~m}$. Those extreme situations can result in nonnegligible non-linearity, so two significant heights are used with three optimal peak period set. In this case, all the standard directions are necessary,

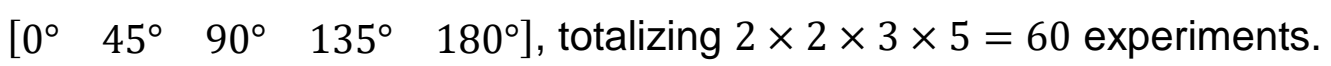

The duplets were also selected among the waves defined in the metocean set, but using the optimal design of experiments framework. The waves were classified in families with similar $H_{S}$, and for each one all the possible combinations of three $T_{P}$ were tested, until finding the best one accordingly with the trace criterion - in this case, the trace criterion is applied in the input matrix $\boldsymbol{X}$, in order to excite the most the RAOs. Lastly, the two most promising categories were selected, achieving not only excitations in different frequencies but also with different amplitudes. The selected duplets are shown in Table 15. 
Table 15 - Selected duplets for the multiple drafts set.

\begin{tabular}{ccc}
\hline Category & $\boldsymbol{H}_{S}[\boldsymbol{m}]$ & $\boldsymbol{T}_{\boldsymbol{P}}[\boldsymbol{s}]$ \\
\hline$I$ & 3.0 & 8.1 \\
$I$ & 2.8 & 9.9 \\
$I$ & 2.8 & 13.7 \\
$I I$ & 4.3 & 8.0 \\
$I I$ & 4.3 & 9.9 \\
$I I$ & 4.3 & 14.2 \\
\hline
\end{tabular}

Source: Elaborated by the author.

\subsection{Validation of the Extended Linear Model}

\subsubsection{Validation of the extended linear model for intermediate draft}

The most important objective of the experiment is to confirm if the linear models of the vessel and of the wave-probe responses are valid. If the models fail to describe the observed behaviors, the algorithm developed so far is useless.

Firstly, the RAOs were experimentally obtained through spectral system identification applied on the irregular wave responses, (ISERMANN \& MUNCHOF, 2011). Essentially, the transfer function is defined as the ratio of the cross-spectra between the input wave and the DoF output - and the spectral energy density; which is easily done by the Matlab ${ }^{\circledR}$ function "tfestimate".

For the spectral calculations, standard Matlab functions were used, employing the Welch's method with Hamming window, with the length defined as the greatest before the spectra starts to oscillate.

The RAOs were calculated for each experiment, but only the estimates in frequencies that present more than $20 \%$ of the energy of the peak of the spectrum were considered, since the estimates outside of this region tend to be just noise.

In order to put in evidence a possible non-linearity, the results obtained by seas with different $H_{S}$ were separated in categories identified by colors in the graphs. The 
significant heights were divided in three groups: I. $H_{S} \leq 2, I I .2<H_{S} \leq 3$ and $I I I .3<$ $H_{S}$, respectively blue, green and red.

The Wamit ${ }^{\circledR}$ 's model was recalibrated to account for roll differences in the natural period, probably due to imprecise inertia calibrations; and damping coefficient, which was calibrated by the wave with the smallest amplitude, following the linear model expected valid region. Selected results for the intermediate draft are shown in Figure 78 to Figure 80.

Figure 78 - Selected RAOs for $\beta=0^{\circ}$.
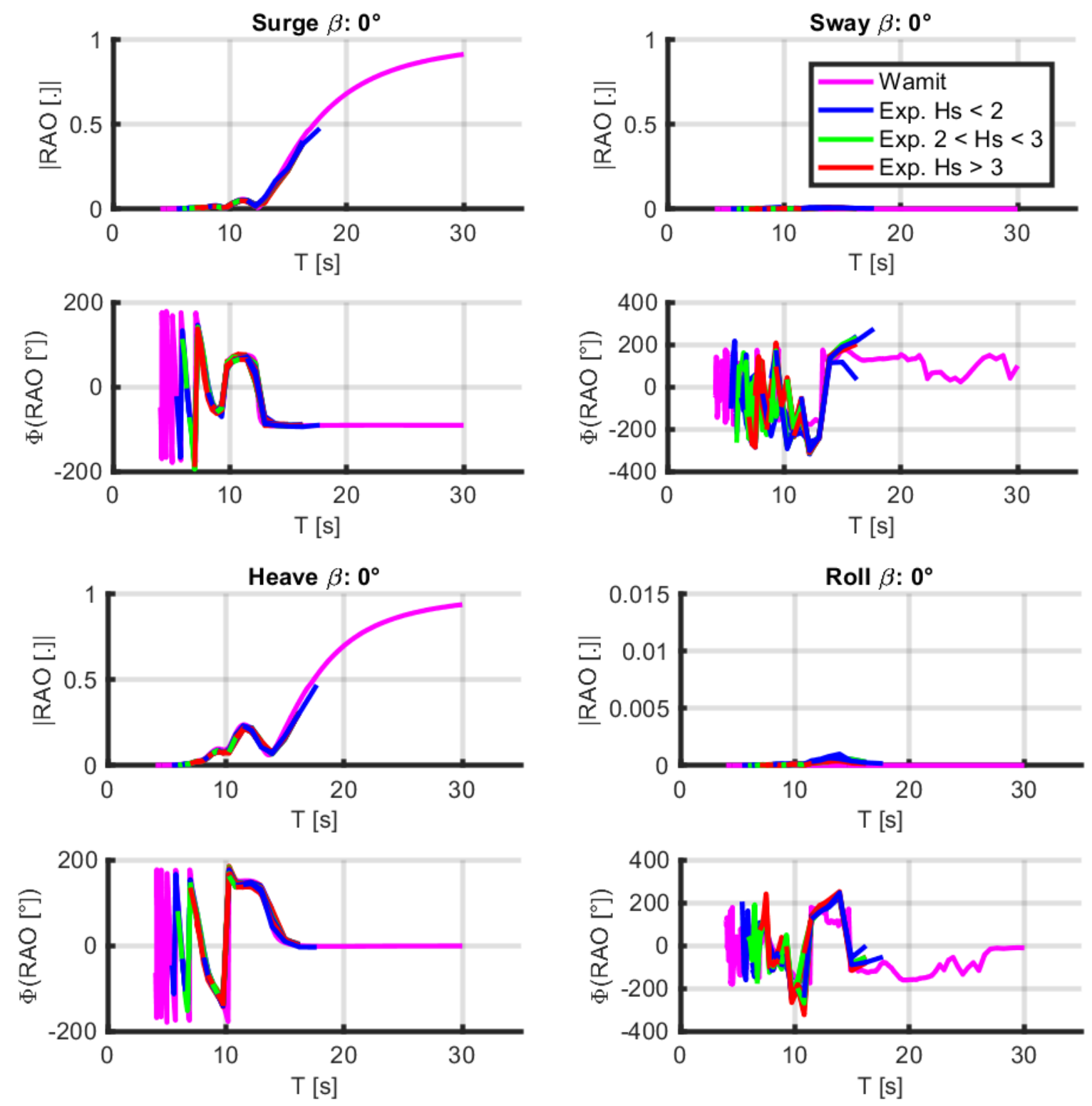

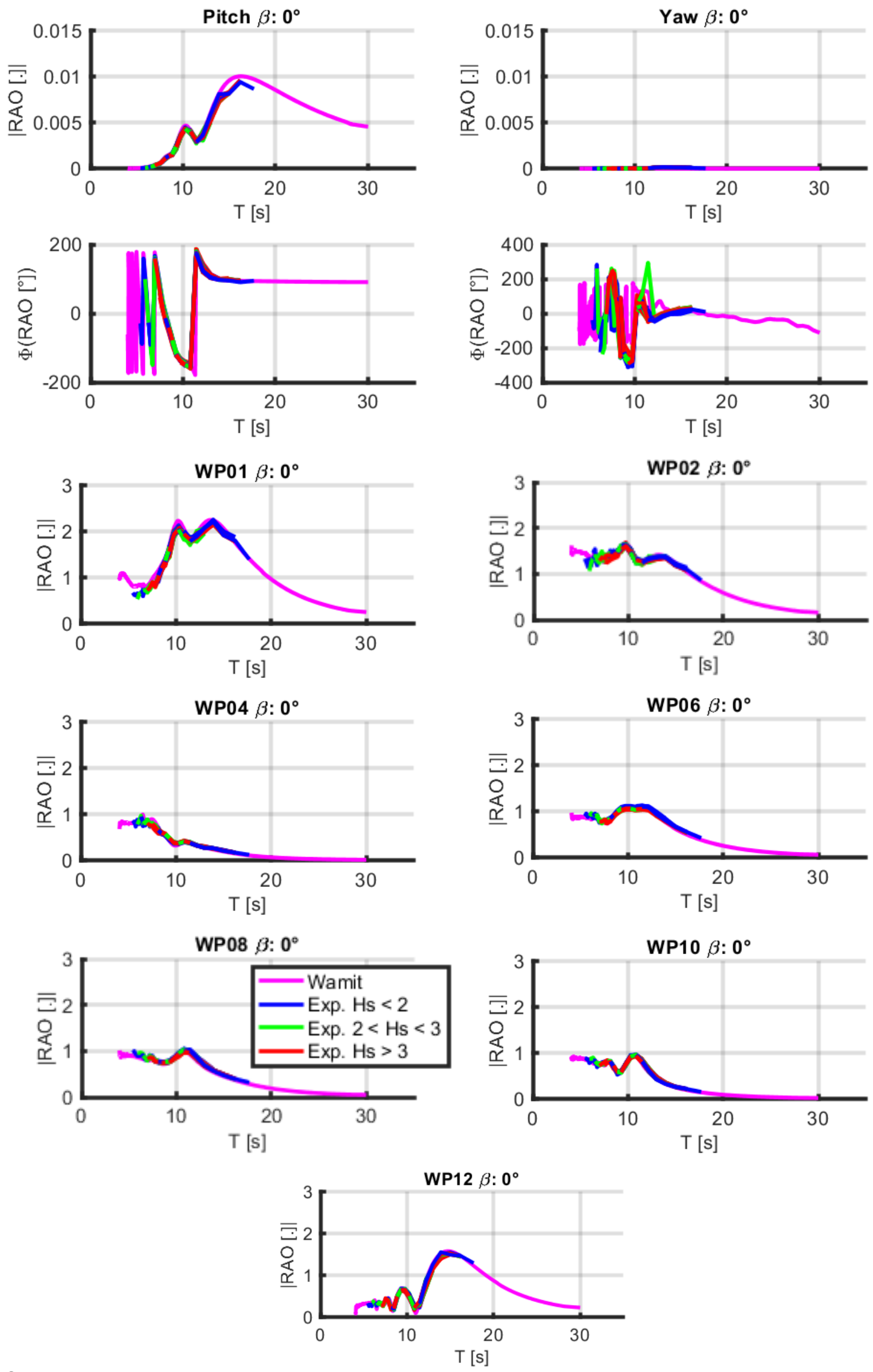

Source: Elaborated by the author. 
Figure 79 - Selected RAOs for $\beta=45^{\circ}$.
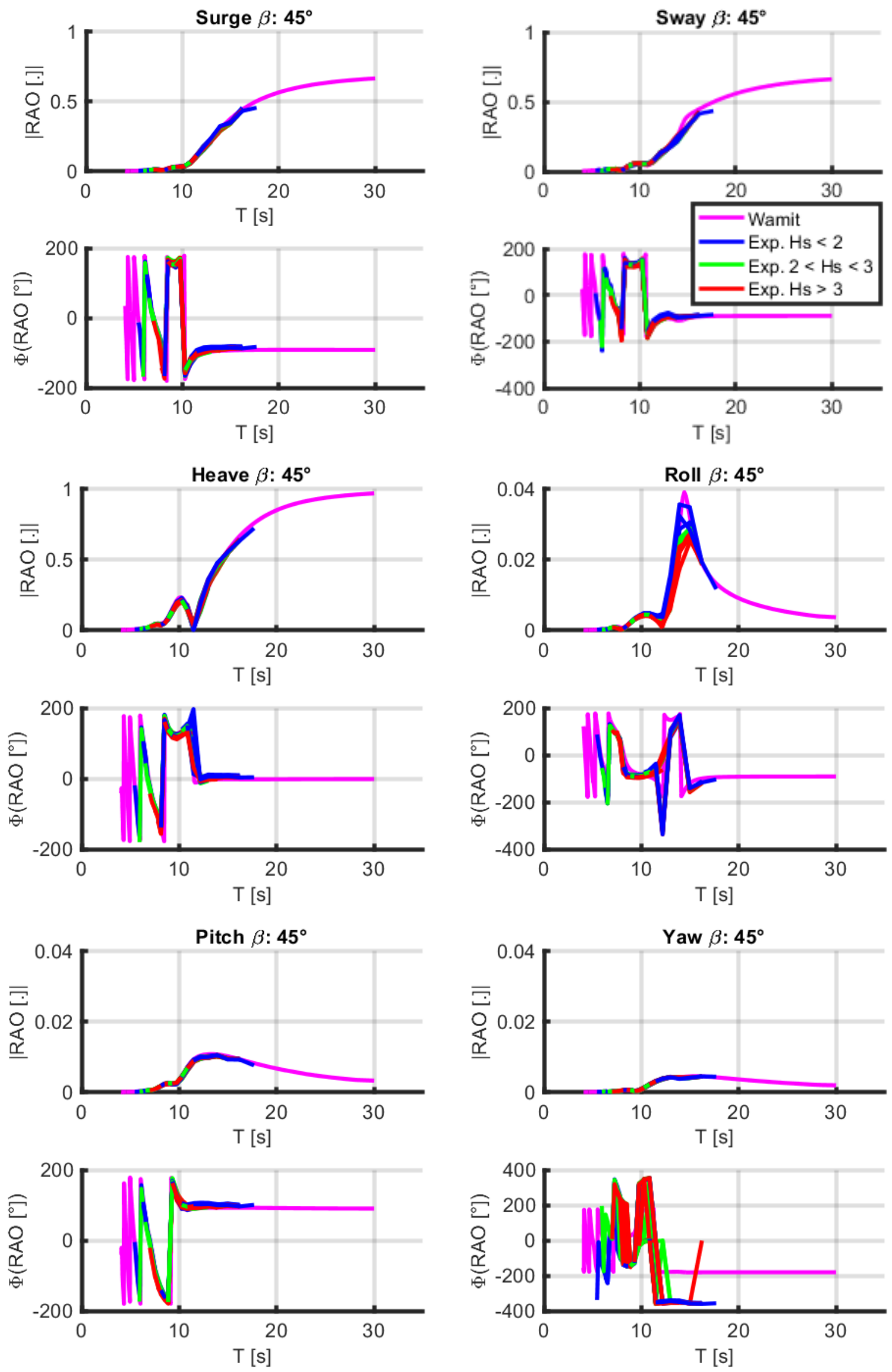

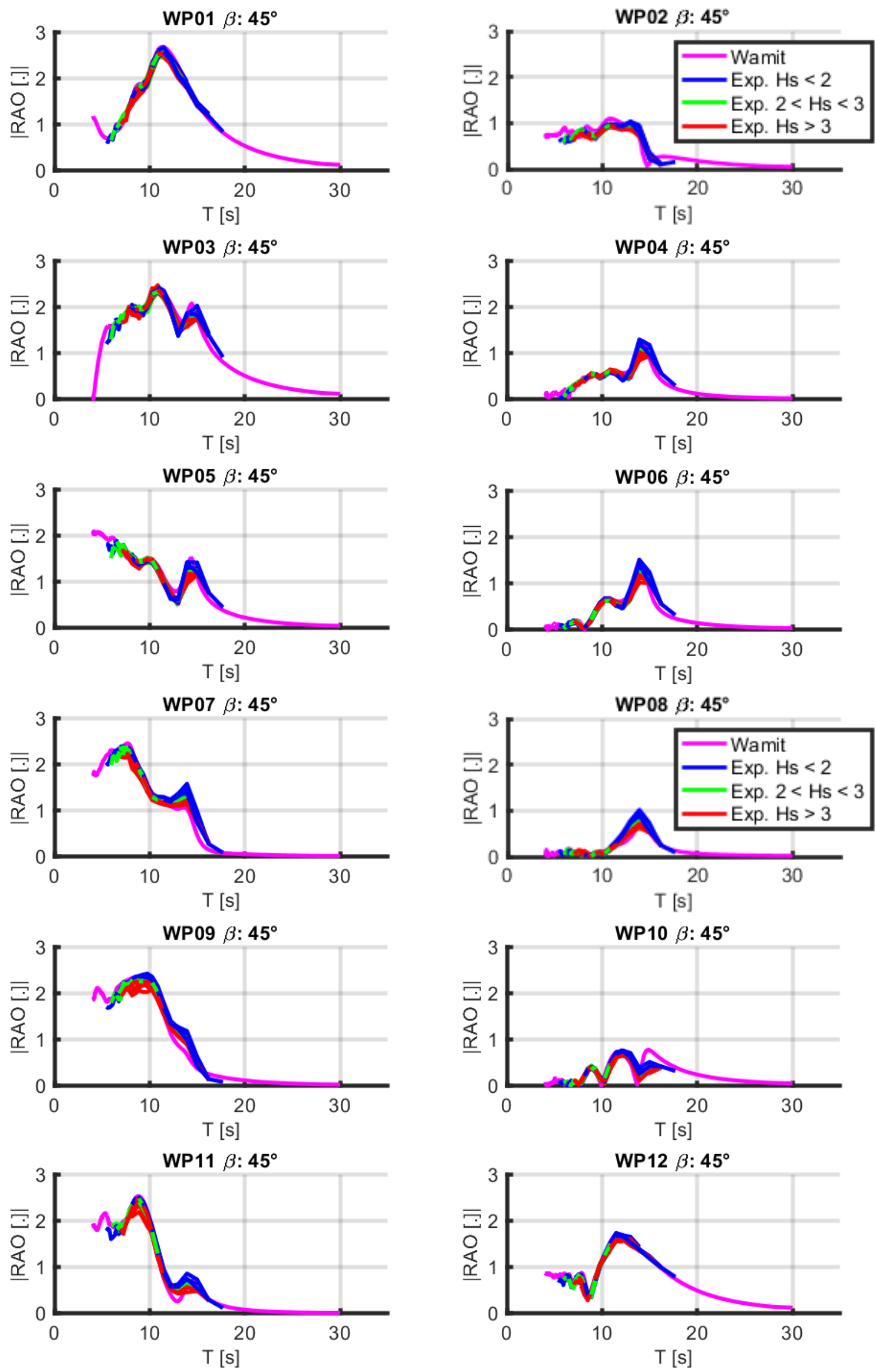

Source: Elaborated by the author.

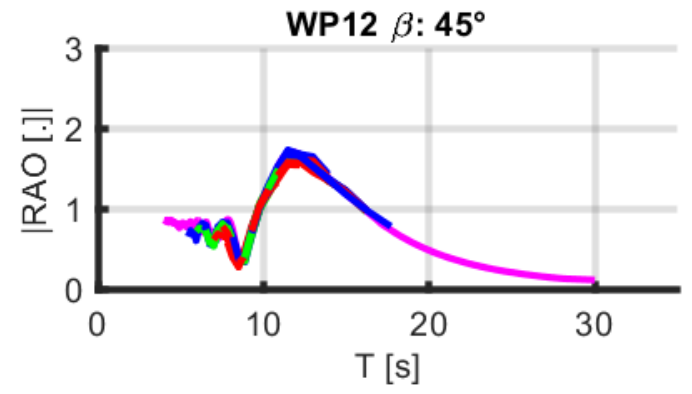


Figure 80 - Selected RAOs for $\beta=90^{\circ}$.
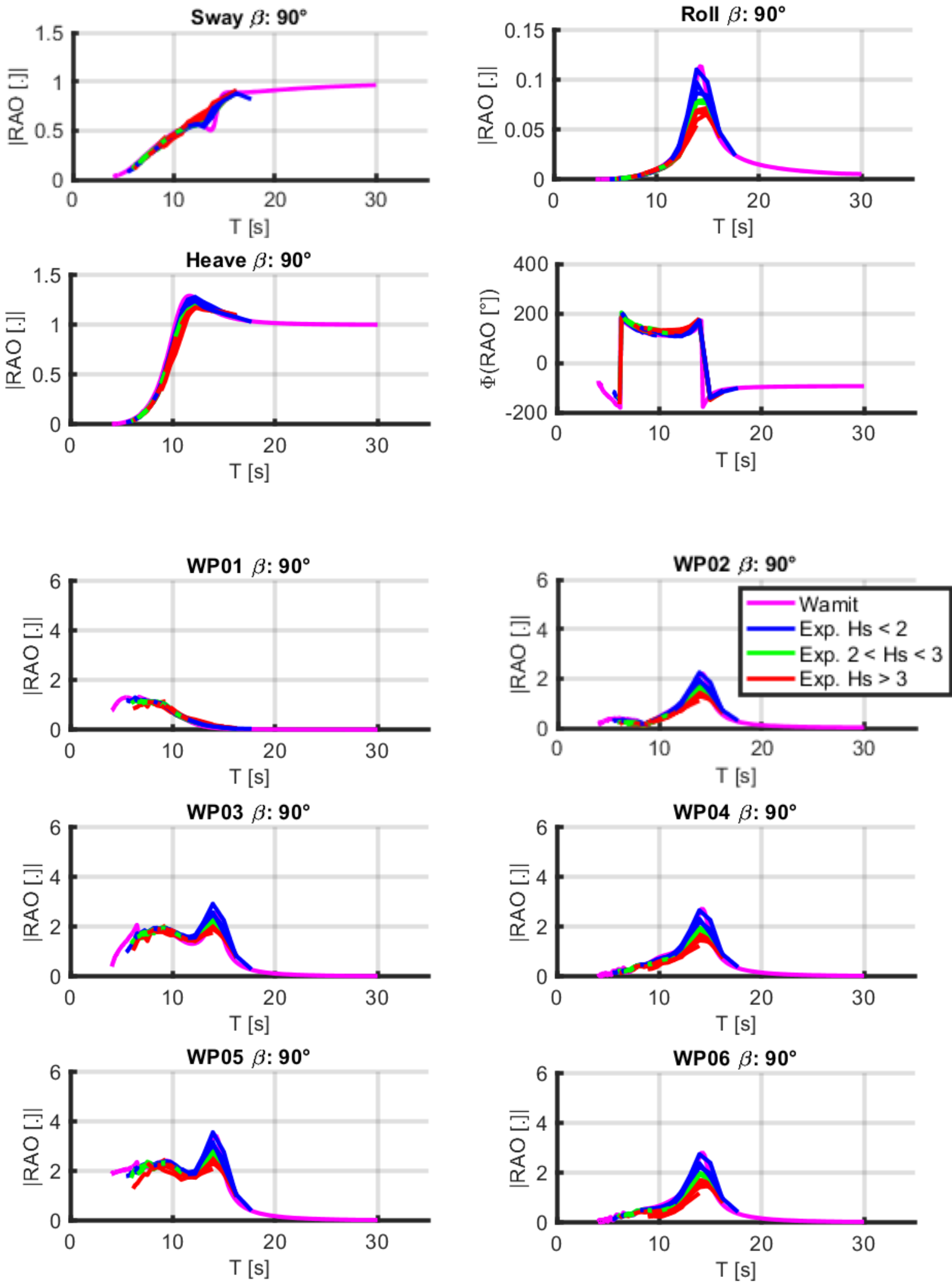

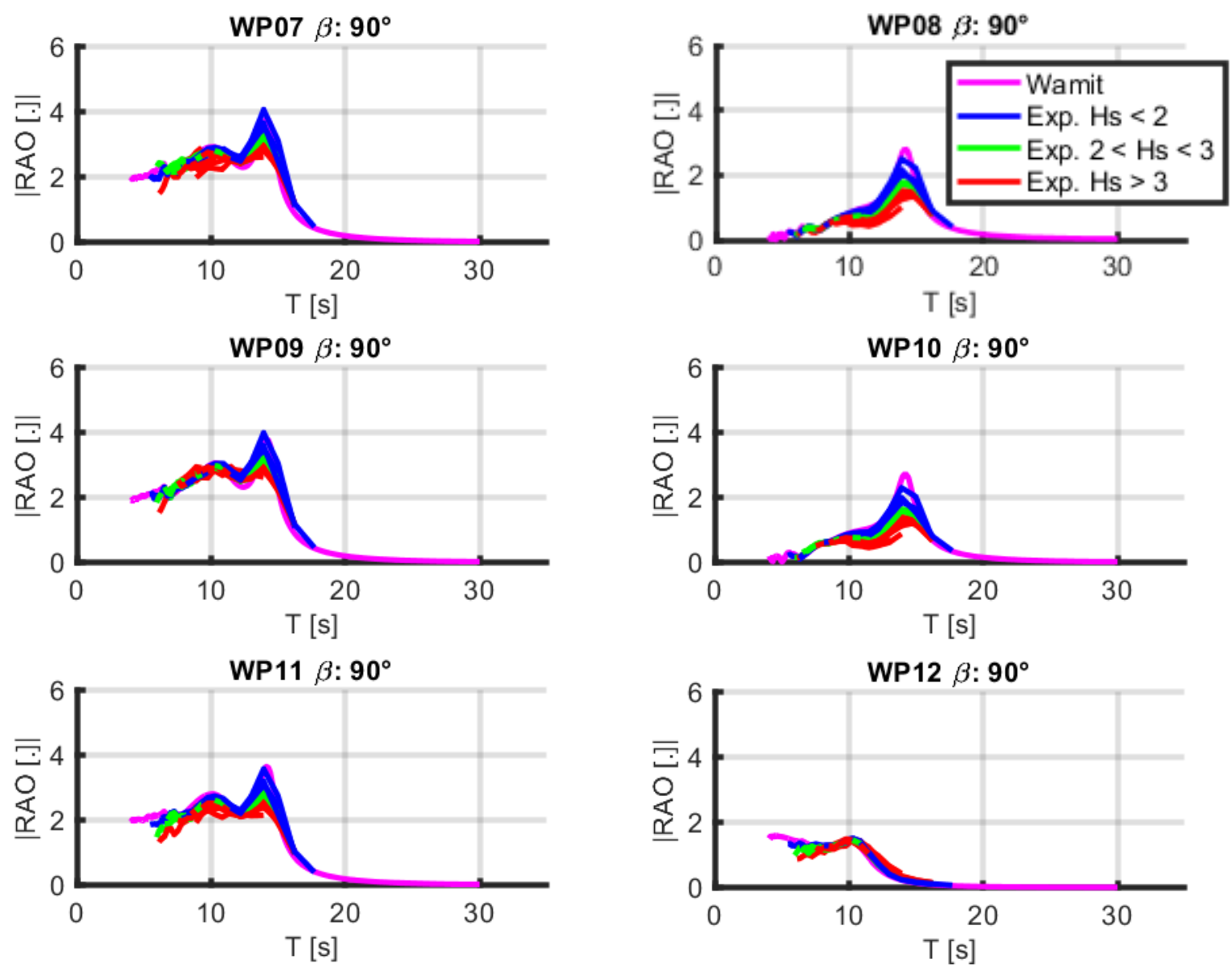

Source: Elaborated by the author.

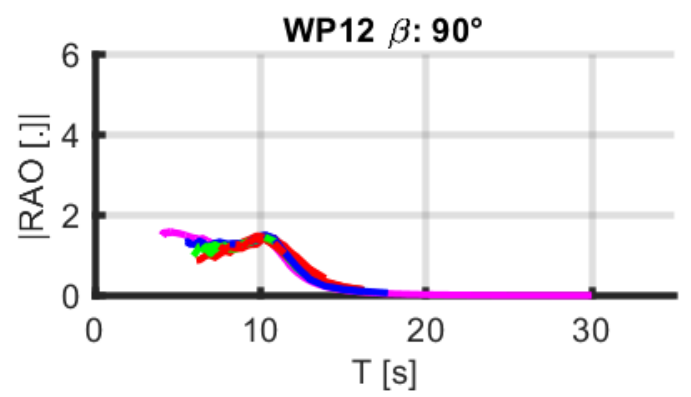

In general, for the intermediate draft, the linear model of the vessel response was validated experimentally, for most of the vessel movements. For the roll movement, however, there is a significant change in the RAOs due to the variation of the $H_{S}$ of the wave, probably due to viscous non-linear damping effects, since the response tends to get lower with higher movement amplitude, Figure 81.

This effect was already expected, and motivates the usage of the sway movement in the estimation with only vessel DoFs in works like (TANNURI, et al., 2003). The idea was to avoid the roll motion without loosing its ability to discriminate among different incoming directions. 
Figure 81 - Non-linearity in roll response.
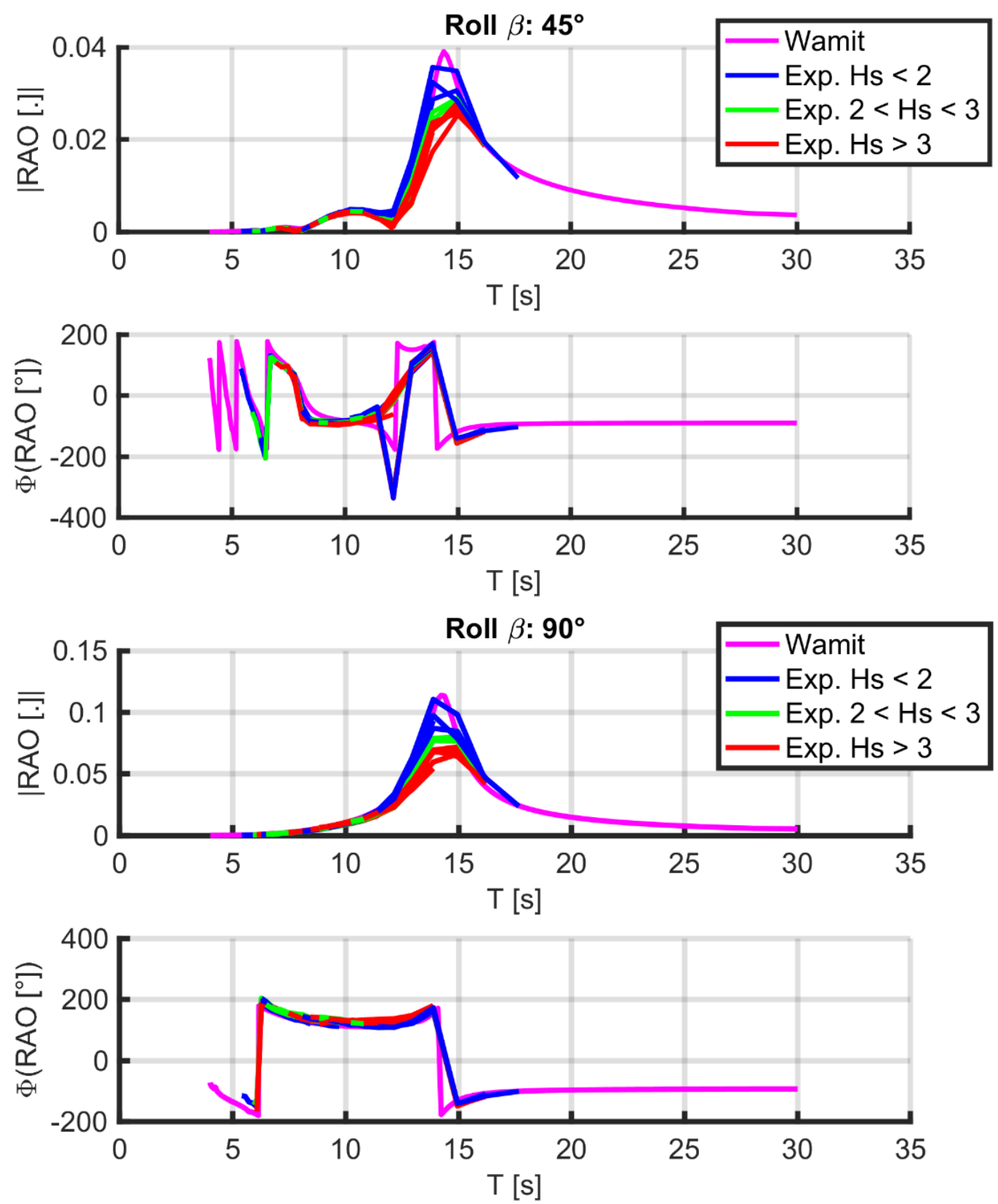

Source: Elaborated by the author.

The extended linear model, i.e., the wave-probe response linear model, is also validated for most of the situations. For beam seas, however, almost all wave-probes present non-linearity, since they are affected by the roll motion, Figure 82. 
Figure 82 - Example of wave-probe response affected by the roll non-linearity.
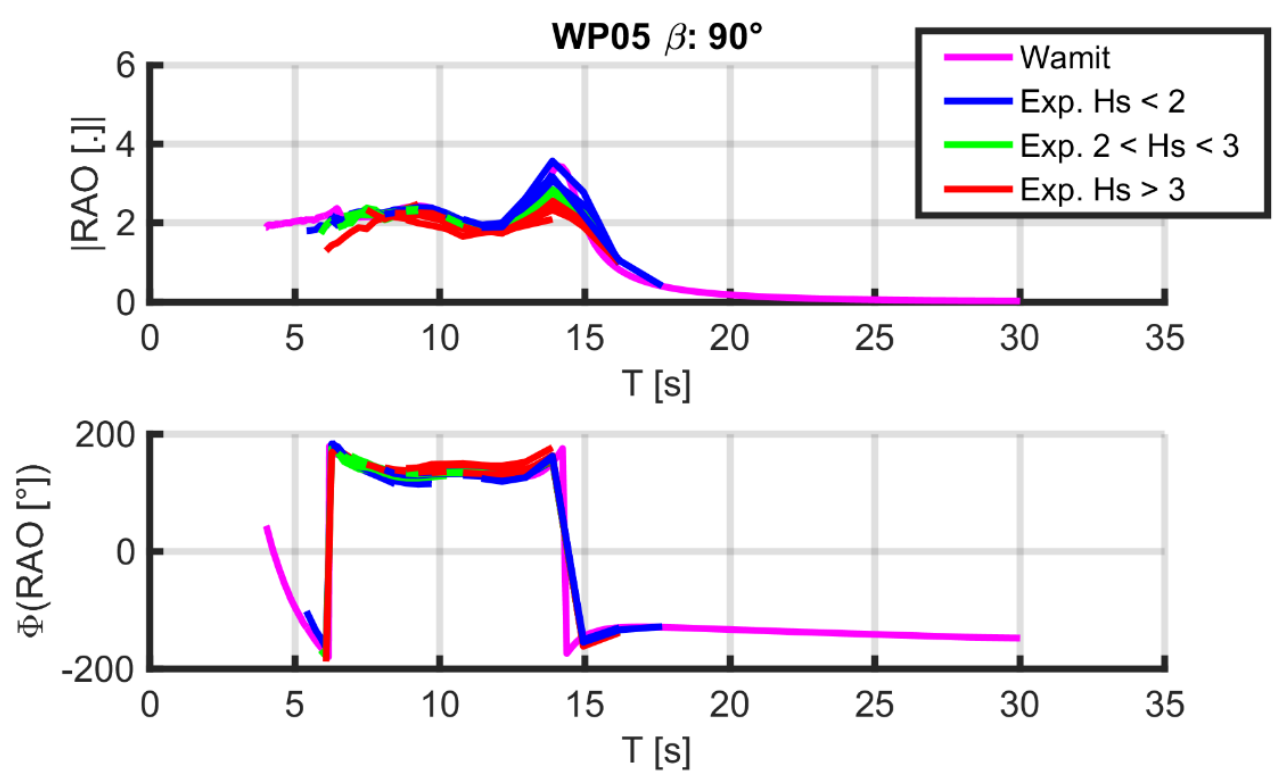

Source: Elaborated by the author.

Other anomalous situation happens with some RAOs at $135^{\circ}$, Figure 83.

Figure 83 - RAOs with discrepancy at $135^{\circ}$.
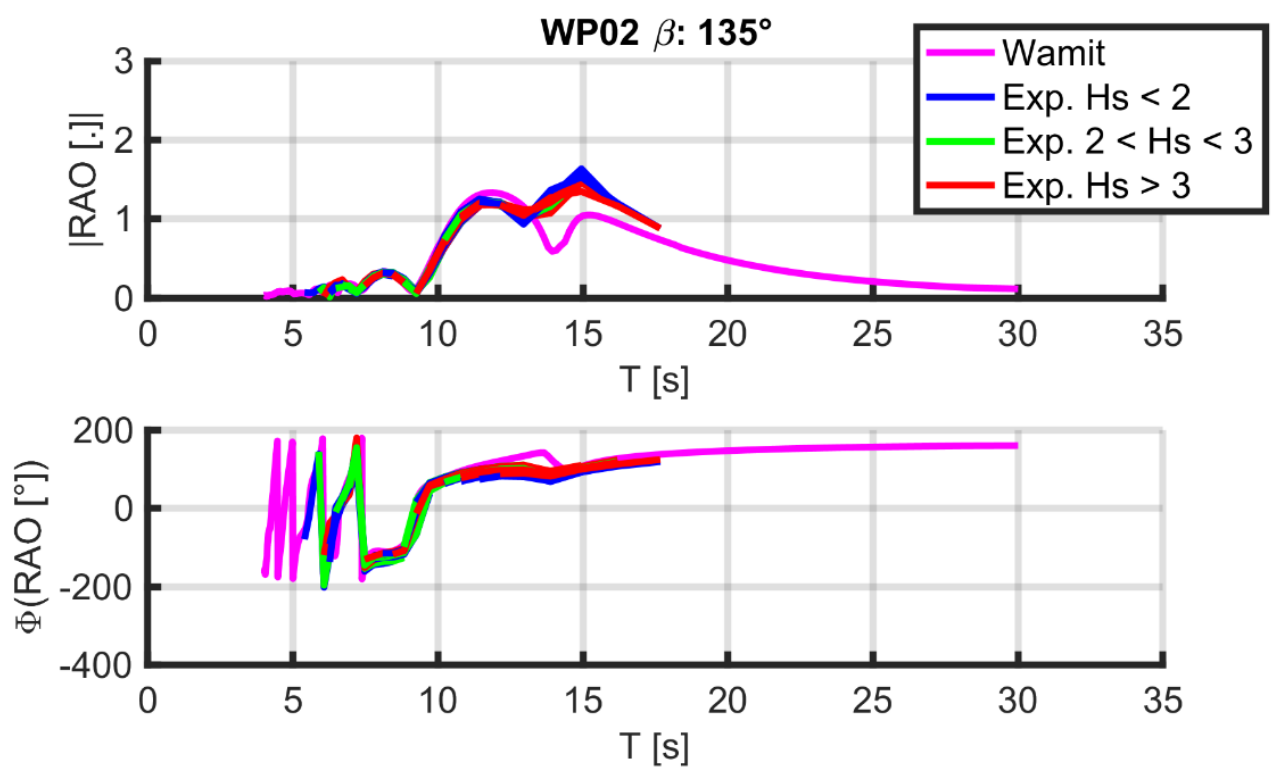

Source: Elaborated by the author.

At first sight, the $135^{\circ}$ error does not seem to be related with the roll nonlinearity, since it does not vary to much with $H_{S}$. A more detailed analysis reveals that the cancelation around 14s happens because the phase of the roll response, the predominant effect of the vessel interference, is almost in the opposite phase of the wave-elevation in the wave-probe position. However, as can be seen in Figure 84, there is a discrepancy in the theoretical and experimental roll phases around $70^{\circ}$. 
Figure 84 - Discrepancy in roll phases.
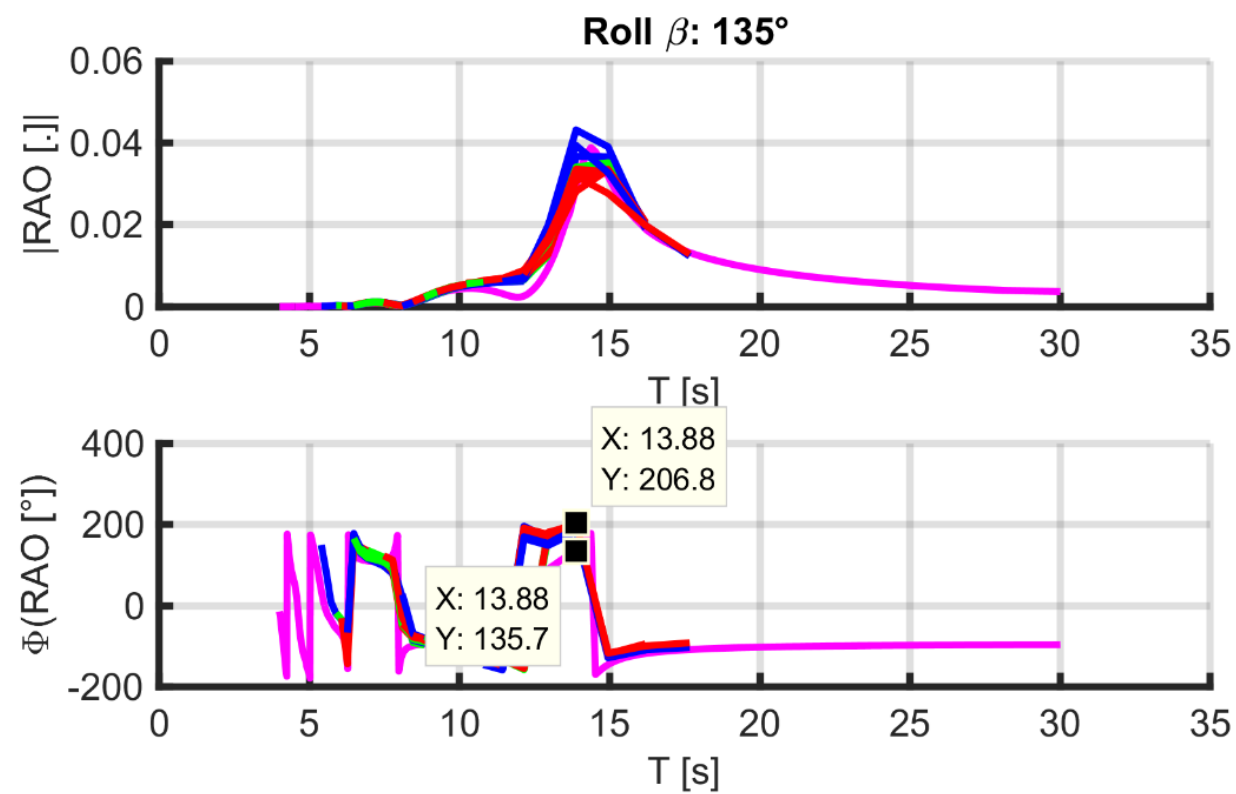

Source: Elaborated by the author.

If the expanded linear model is recalculated using the roll RAO with a phase shift of $70^{\circ}$, the new theoretical versus experimental result is given in Figure 85 .

Figure 85 - Expanded linear model with roll phase shift.
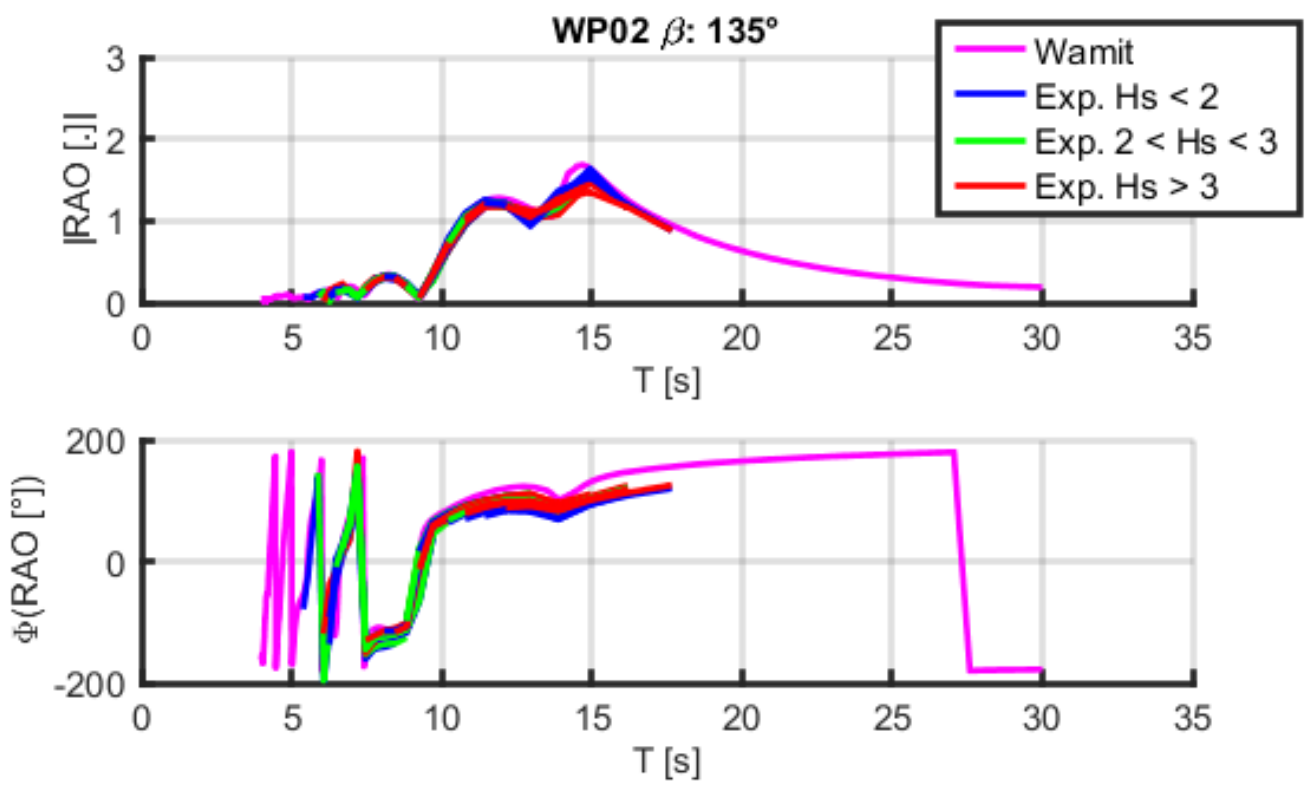

Source: Elaborated by the author. 
It is possible to see that the wave-probe response discrepancy was completely corrected, i.e., the anomalous behavior is also an effect of the roll model mismatch, but not due to non-linear viscous damping.

In conclusion, the extended linear model was almost completely validated during the experiments, and most of the discrepancies were caused by effects from the roll movement.

One possible approach to solve this problem is given in the section 4.4, Optimization of Degrees of Freedom Usage by Frequency, and consist in avoiding the usage of wave-probes around 12s, avoiding the roll influence. In that case, the estimation of waves with peak around 12s will be similar to the estimations using only vessel DoFs, which are already good in this range.

\subsubsection{Validation of the extended linear model for extreme draft}

So far, the linear model was only validated by the intermediate draft. Extreme draft, however, can present a non-negligible non-linear behavior even in other vessel DoFs than roll, so they need to be tested separately.

Vessels in the ballasted draft condition, i.e., with the minimum displacement, are highly susceptible to external forces, which can result in higher accelerations, higher velocities and, consequently, an increased non-linear viscous effect.

Vessels in the full draft condition, i.e., with the maximum displacement, have large underwater body volume, and their movement can cause non-negligible drag in translational movements. The roll movement is also affect negatively by the larger underwater body volume, and even the linearity of the hydrostatic restoration matrix can be affected.

The results for the ballasted draft are shown in Figure 86 to Figure 88, and the results for the full draft are shown in Figure 89 to Figure 91. 
Figure 86 - Selected RAOs for the ballasted condition, $\beta=0^{\circ}$.
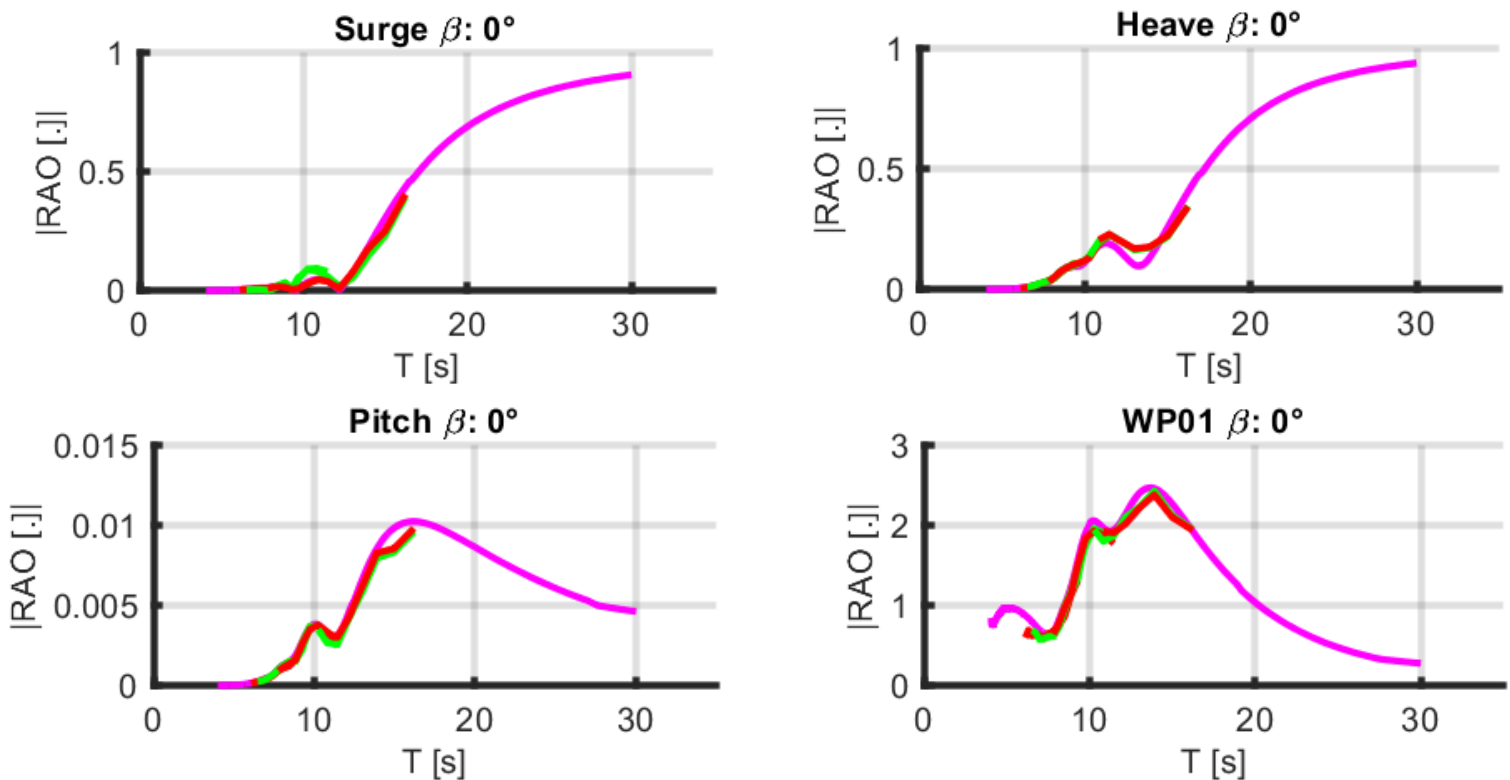

WP02 $\beta: 0^{\circ}$
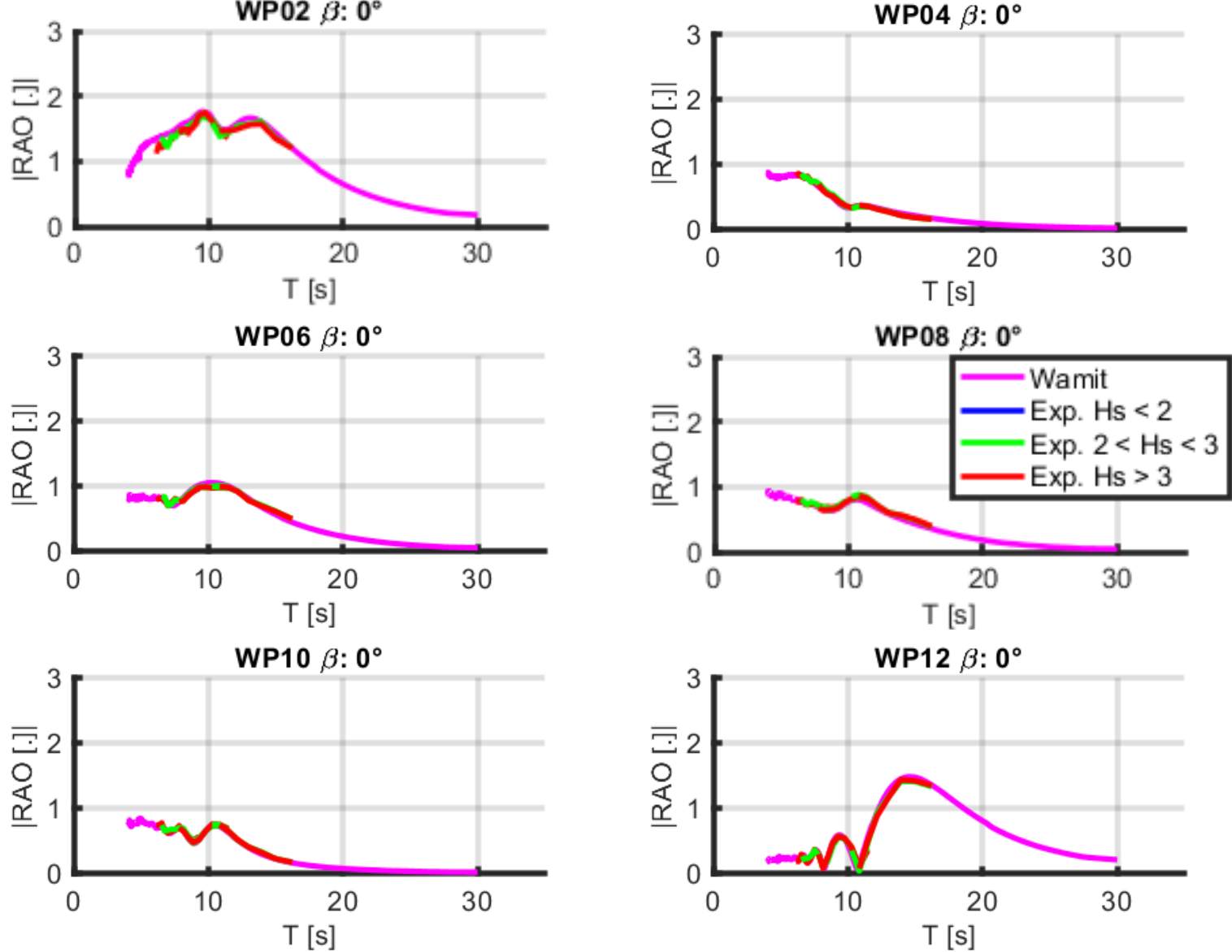

Source: Elaborated by the author.

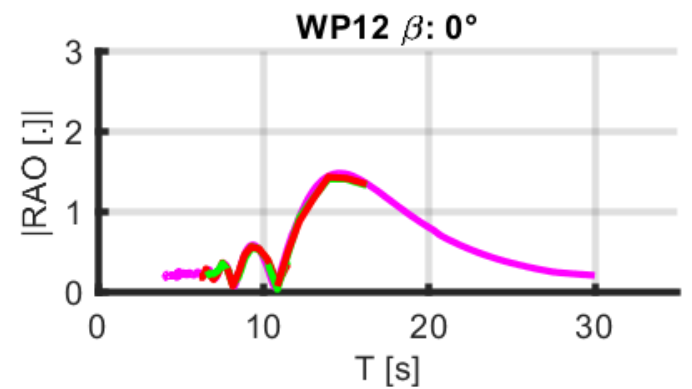


Figure 87 - Selected RAOs for the ballasted condition, $\beta=45^{\circ}$.
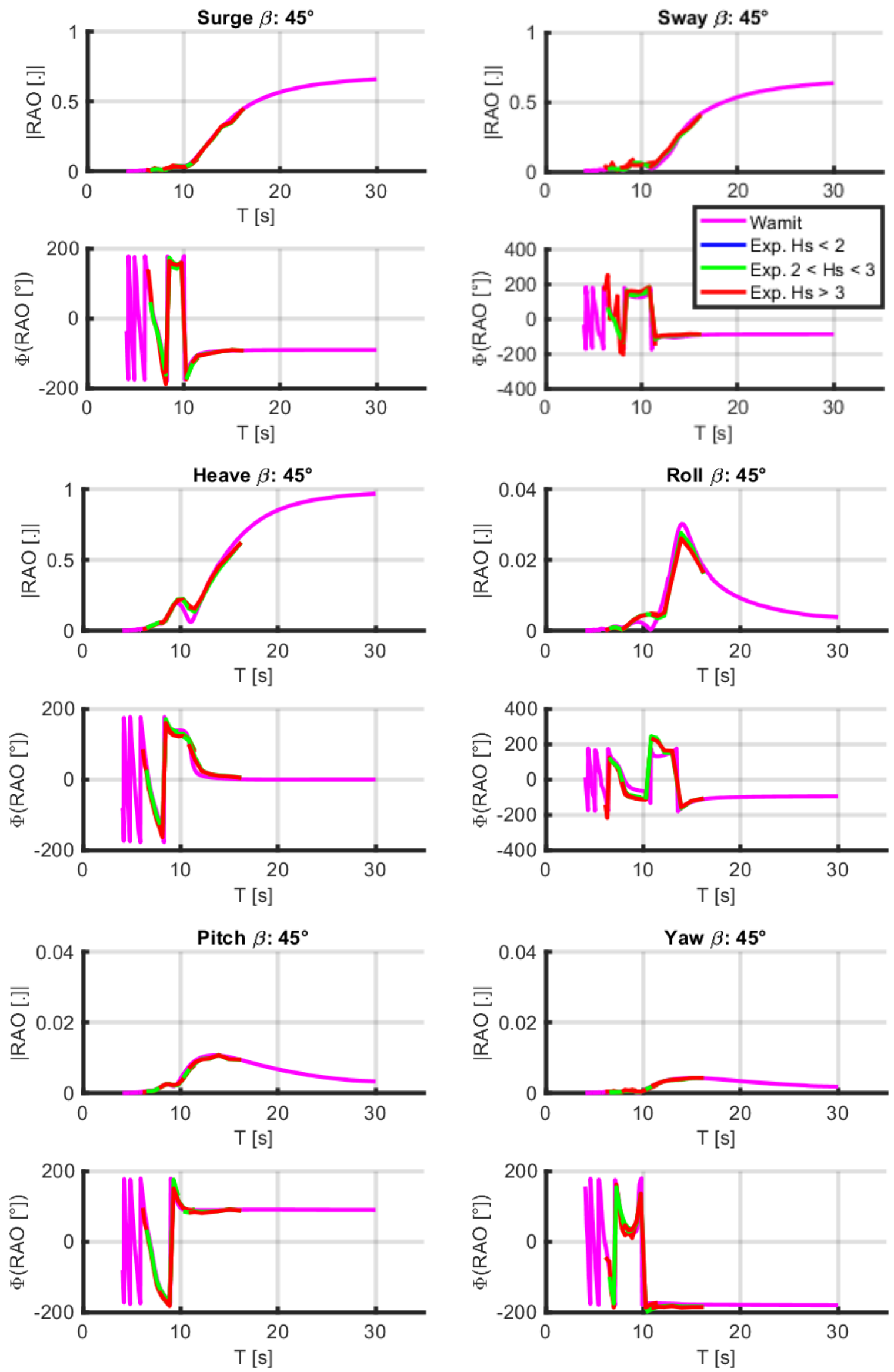

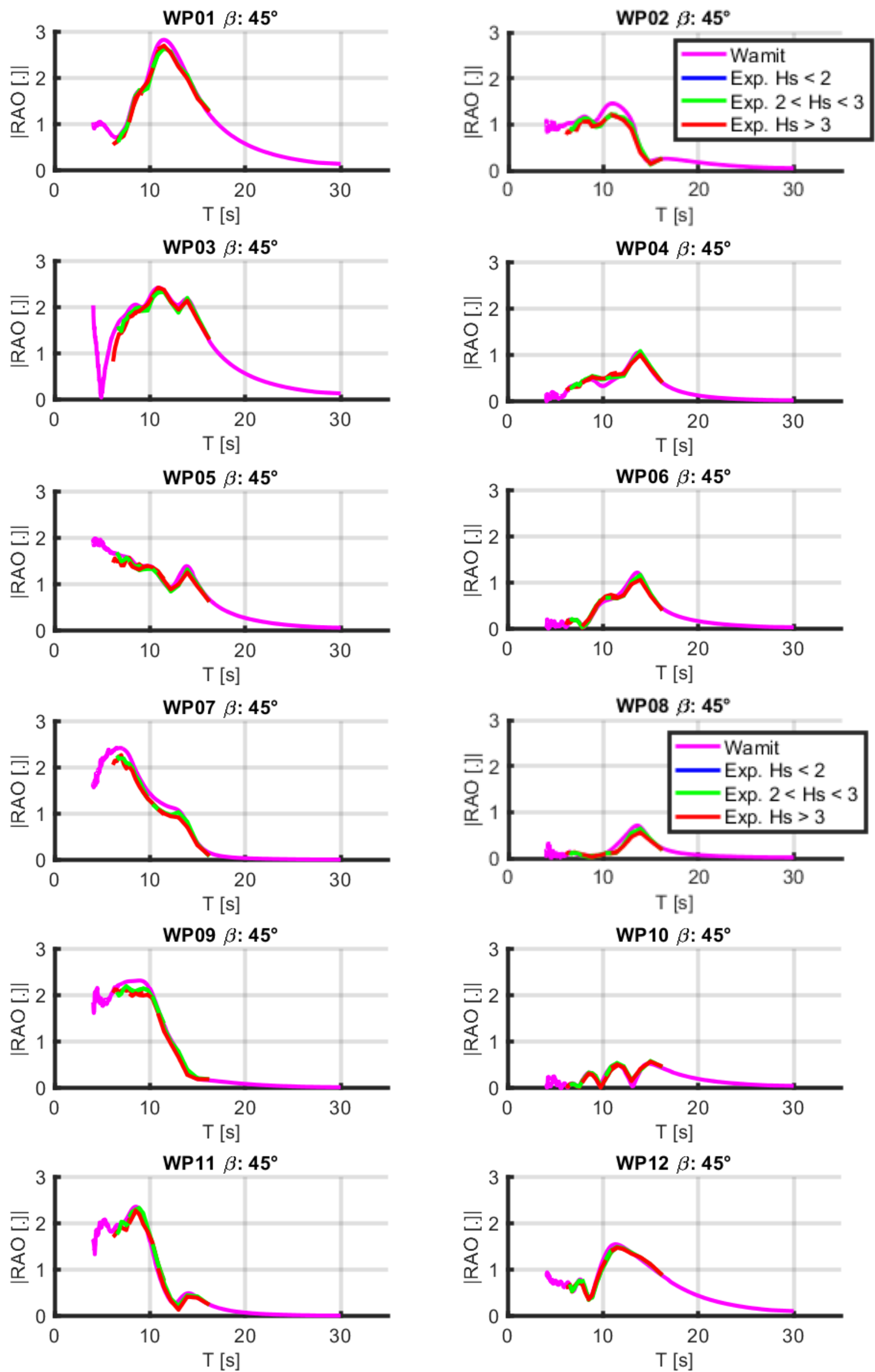

Source: Elaborated by the author.

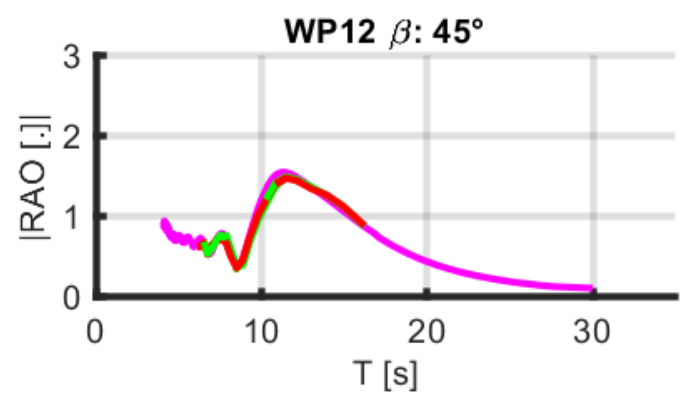


Figure 88 - Selected RAOs for the ballasted condition, $\beta=90^{\circ}$.
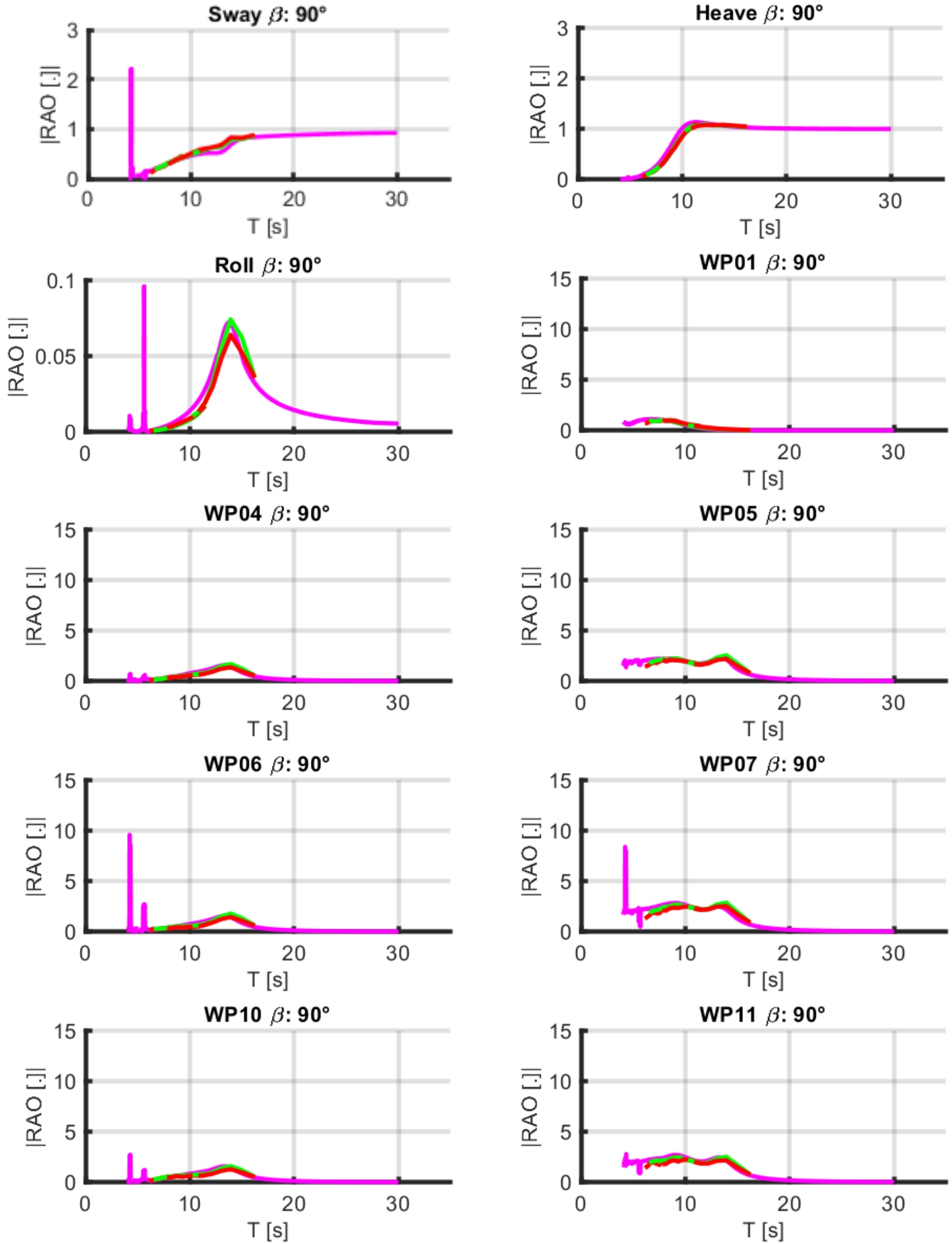

Source: Elaborated by the author.

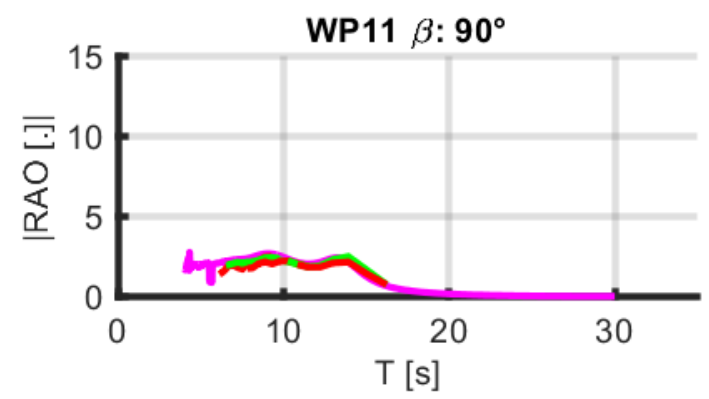


Figure 89 - Selected RAOs for the full condition, $\beta=0^{\circ}$.
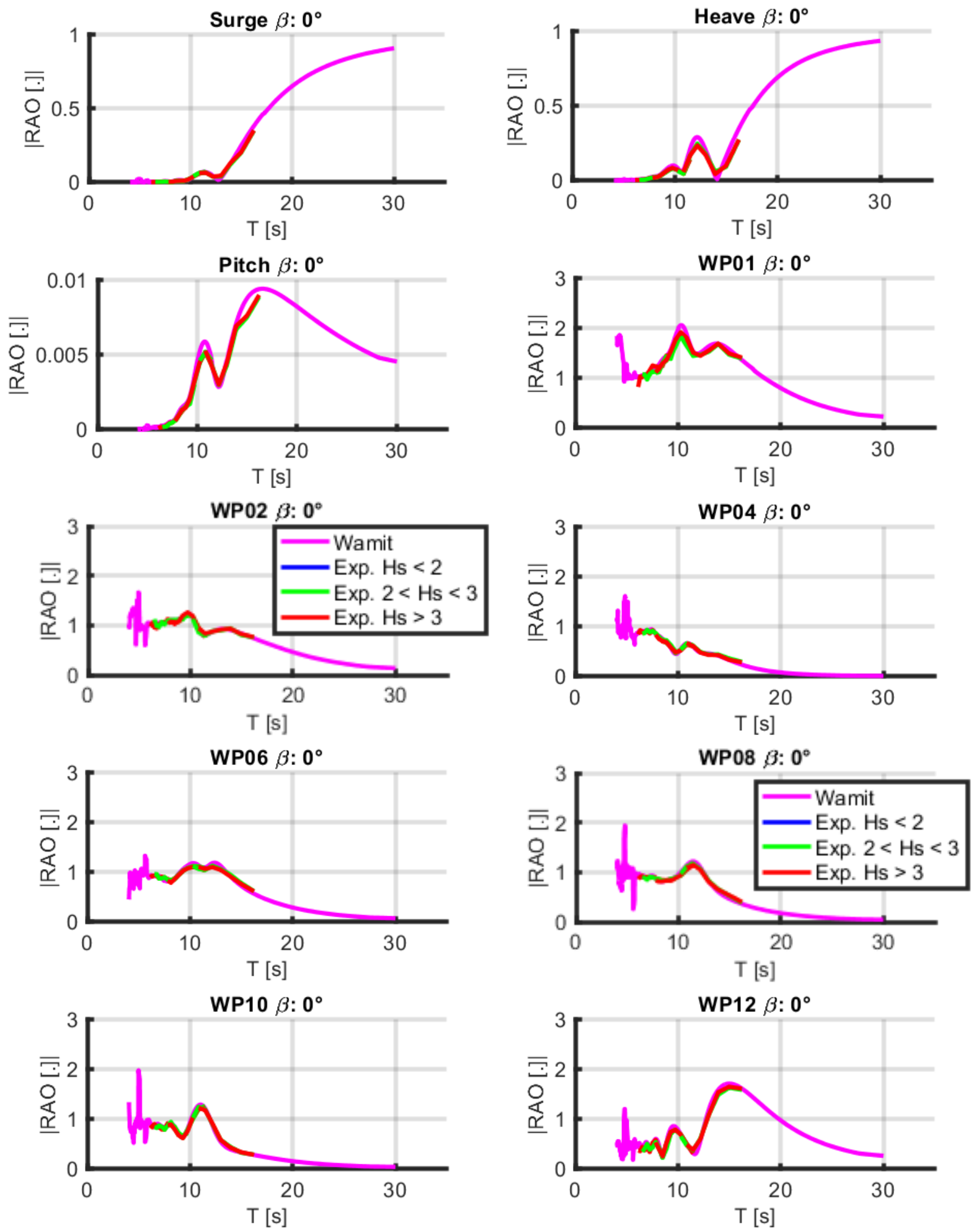

Source: Elaborated by the author. 
Figure $90-$ Selected RAOs for the full condition, $\beta=45^{\circ}$.
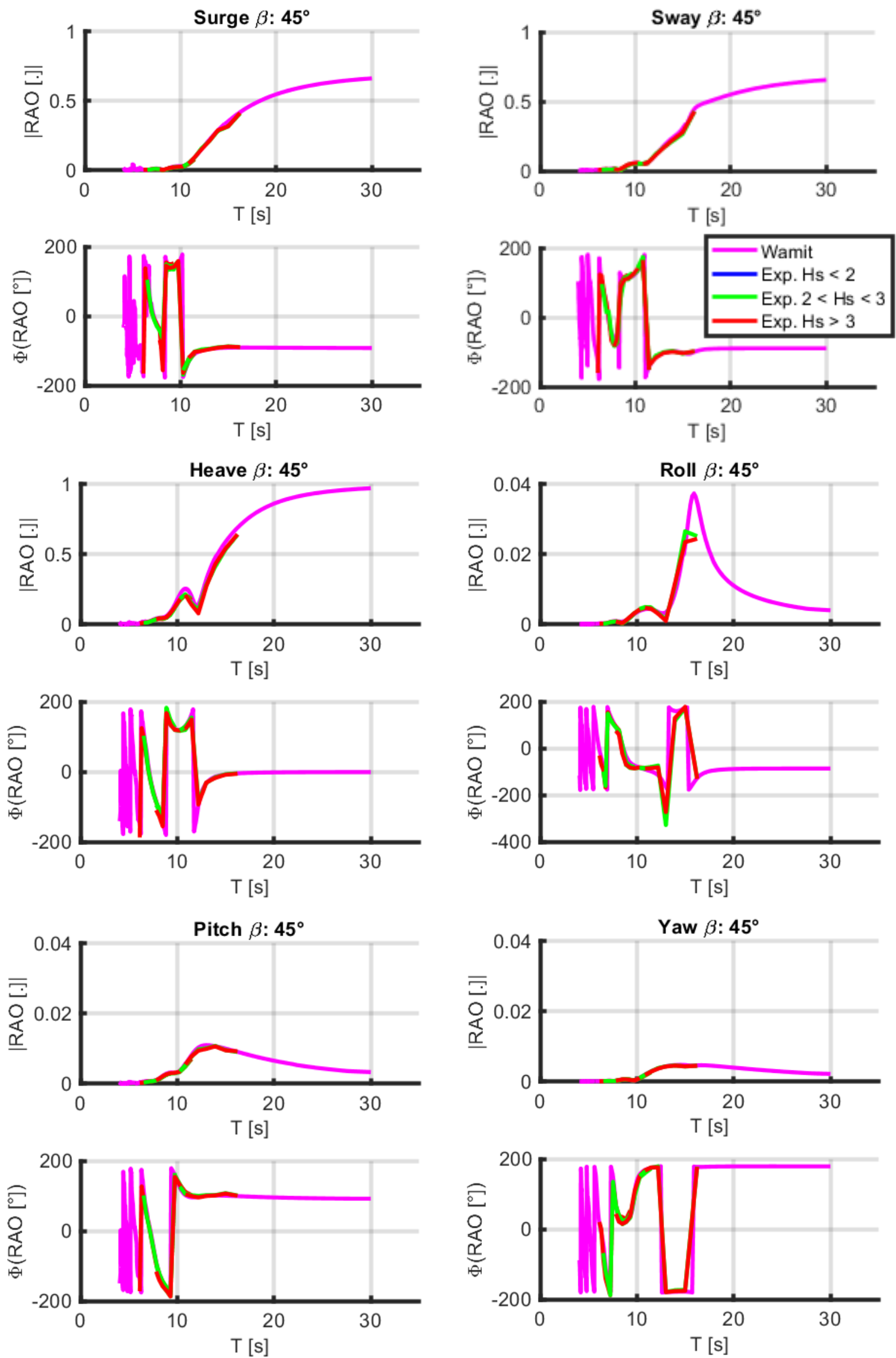

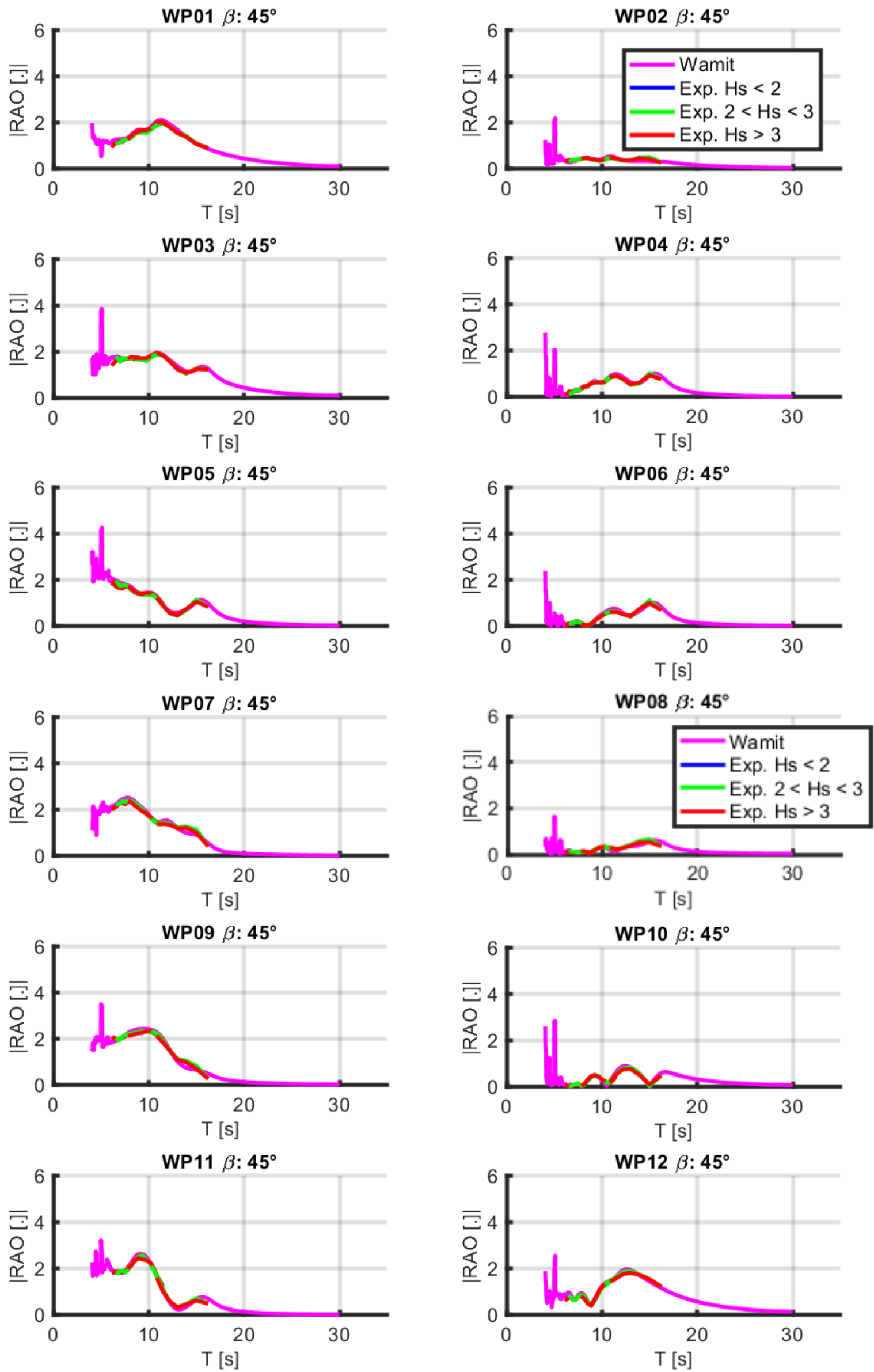

Source: Elaborated by the author.

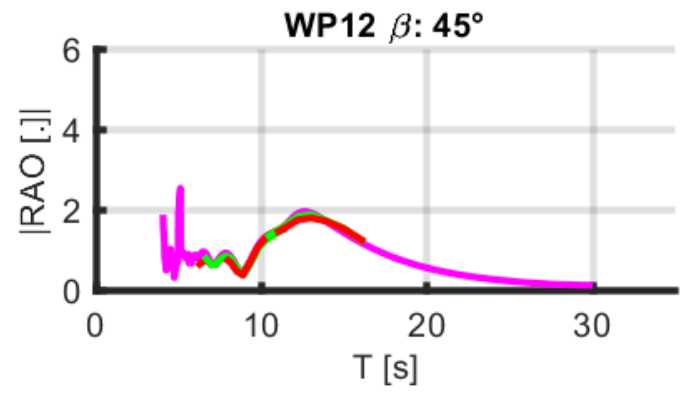


Figure 91 - Selected RAOs for the full condition, $\beta=90^{\circ}$.
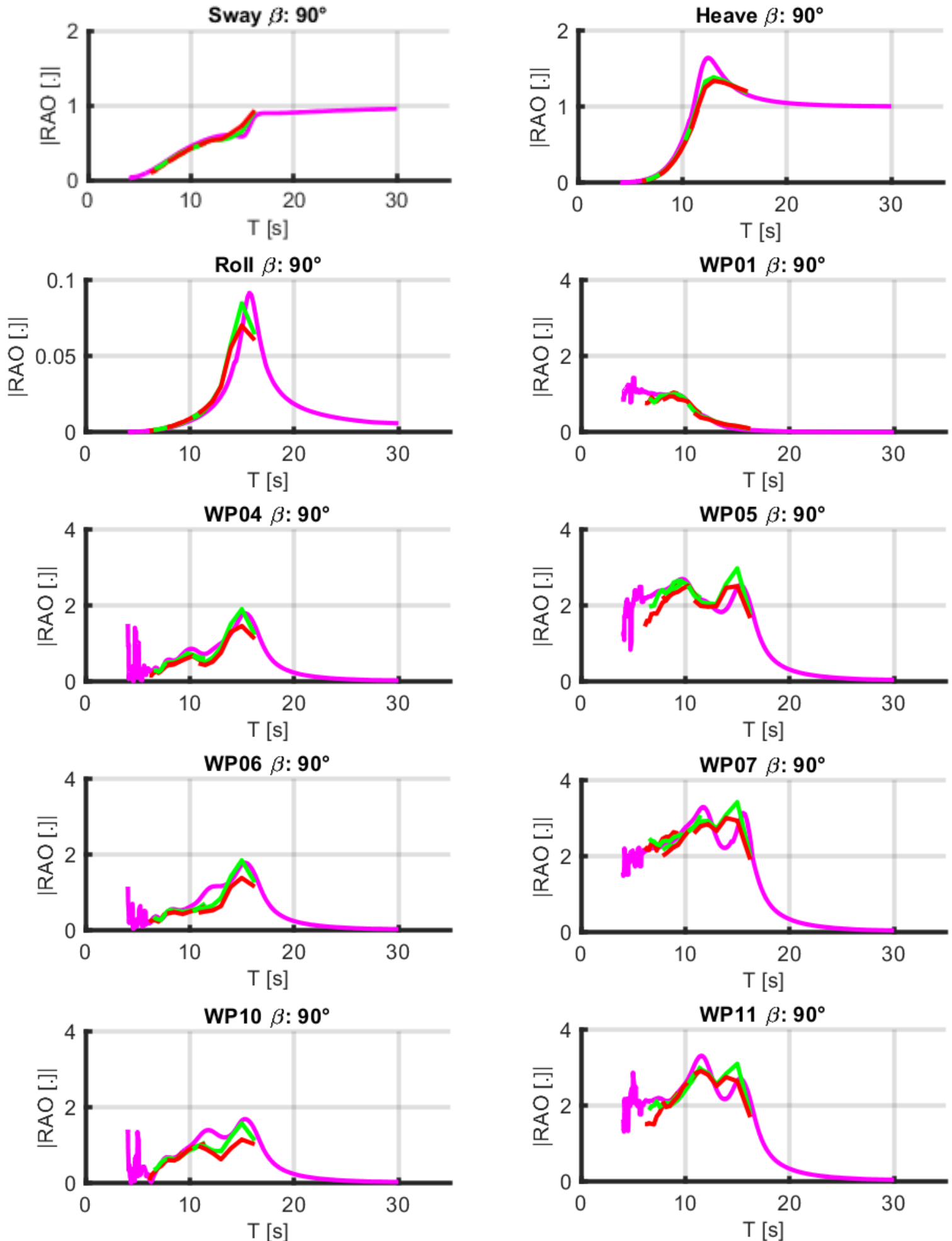

Source: Elaborated by the author. 
The results for the ballasted condition are better than the results for the intermediate draft. The non-linear viscous effects are less evident, but the roll phase shift effects are still present in the wave-probe responses. In general, the wave-probe response is accurate up to $12 \mathrm{~s}$.

For the full condition, however, the results seem to be worse than the results for the intermediate draft. At $90^{\circ}$, the heave damping is significantly underestimated by the linear model, and non-linear viscous effects in roll are evident. The wave-probe responses seem to have a smaller validity region, only up to $10 \mathrm{~s}-11 \mathrm{~s}$, and the phase shift effects become more apparent, being also affected by the heave discrepancy.

In conclusion, it can be stated that for all the drafts the vessel movements, excluding the roll movement, agree with the linear model, with some discrepancies for the heave movement at $90^{\circ}$ in full conditions. The wave-probe response also has a good agreement with the linear expanded model, however this agreement happens only up to $12 \mathrm{~s}$, since, after this period, roll effects affect the response.

\subsection{Validation of the Usage of the Wave-Probes}

\subsubsection{Validation of the wave-probes usage for intermediate draft}

In order to validate the estimation algorithm, and evaluate the possible improvements brought by the wave-probe employment, the number of wave-probes used in the estimations were varied from 0 to 6 , and, for each number, the best combination of them were determined by the single-objective trace criterion, Figure 51 , in the section 5.6 Study of the Usage of Different Number of Wave-Probes. The probes measurements were incorporated in all frequencies, despite the known discrepancies after $12 \mathrm{~s}$, in order to evaluate if these discrepancies affect the estimation outcome.

The estimation procedure follows the method described in the numerical trials. The vessel DoFs were fixed in Sway-Heave-Pitch, and the hyperparameters were kept the same used in the Only-DoFs case. It means that the following results can be potentially obtained only by adding wave-probe measurements in the estimation, without any modification or recalibration in the algorithm.

The results are expressed in histogram graphs, already presented in the numerical section, summarizing the results inside a given set of waves. The errors in 
the parameters $-H_{S}, T_{P}, \beta_{0}$ and $s$ - are used as an estimation quality measurement, since this allows comparisons not only against high-order methods but also against parametric methods.

The histograms, Figure 92, are plotted using the absolute and the percent error as metrics. In the significant height case, the percent error reflects the fact that the uncertainties in the estimation follows the increase in the height, the greater the height the grater the uncertainty, so the errors must be weighted. In the peak period case, the percent error reflects the desire to estimate better low period peak period, giving more weight to them. The estimation of $\beta_{0}$, by its turn, has no 'preference' or 'weighting factor', using the absolute value; the same for the $s$ parameter.

Figure 92 - Histograms of the parametric errors.
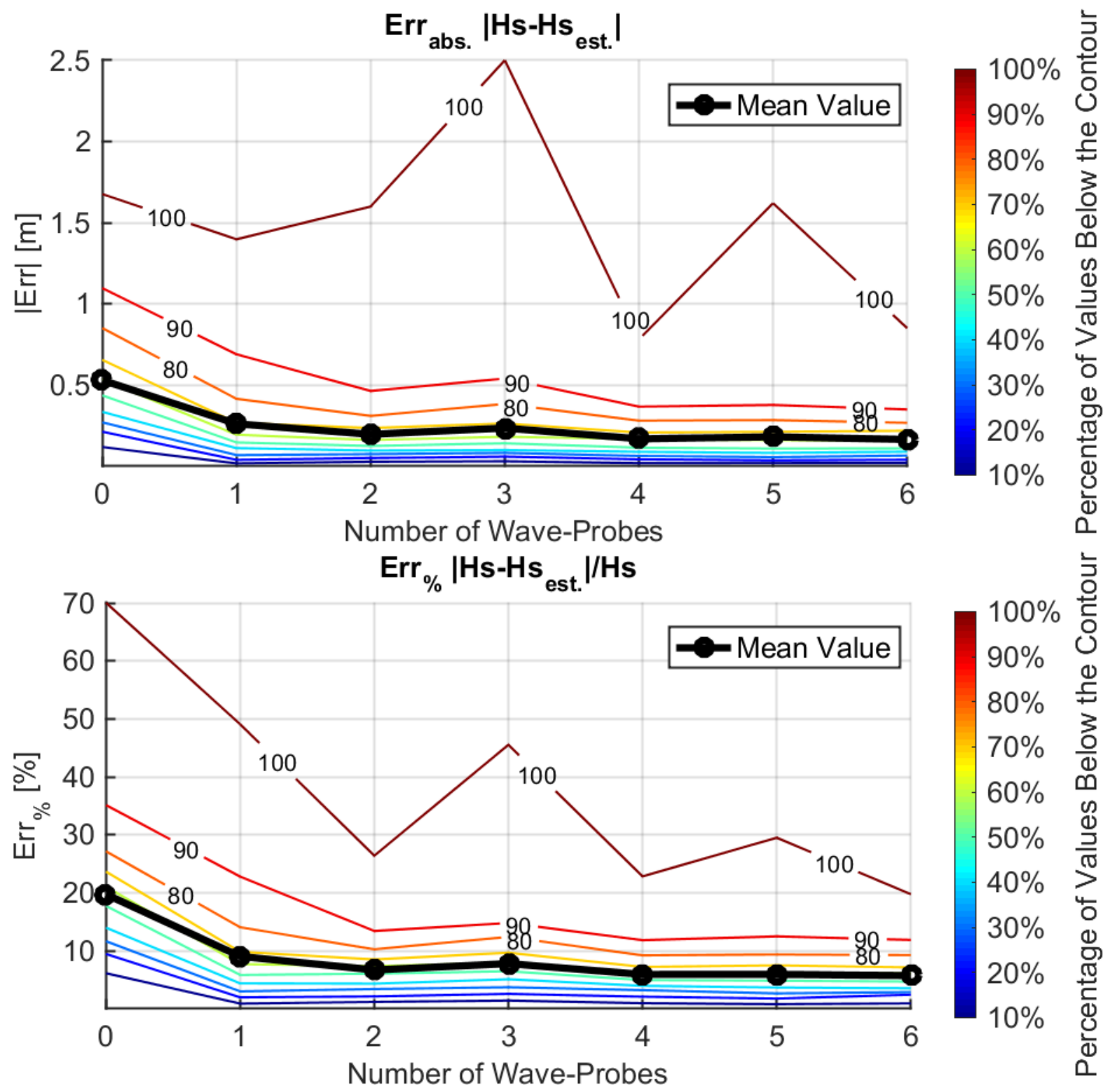

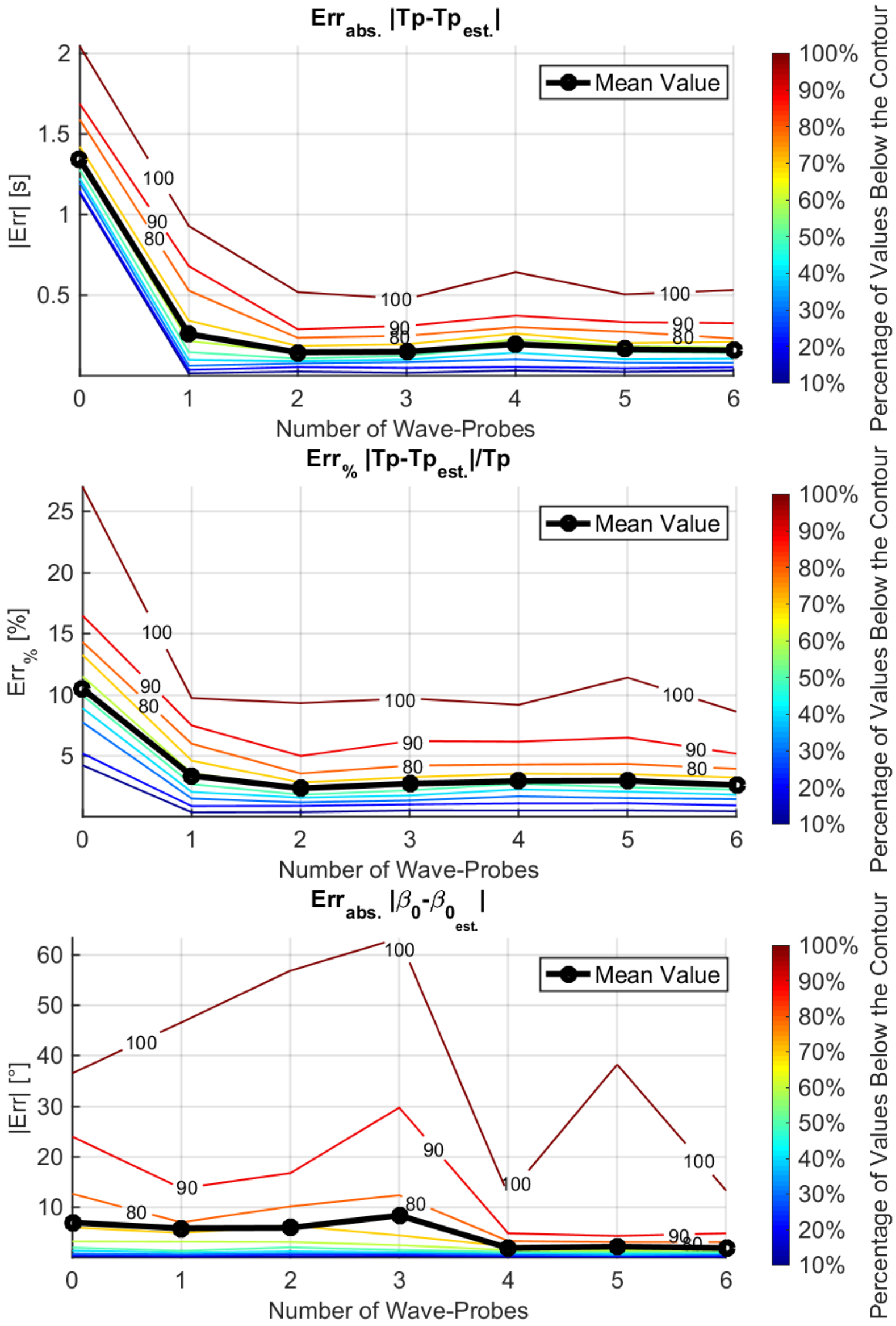


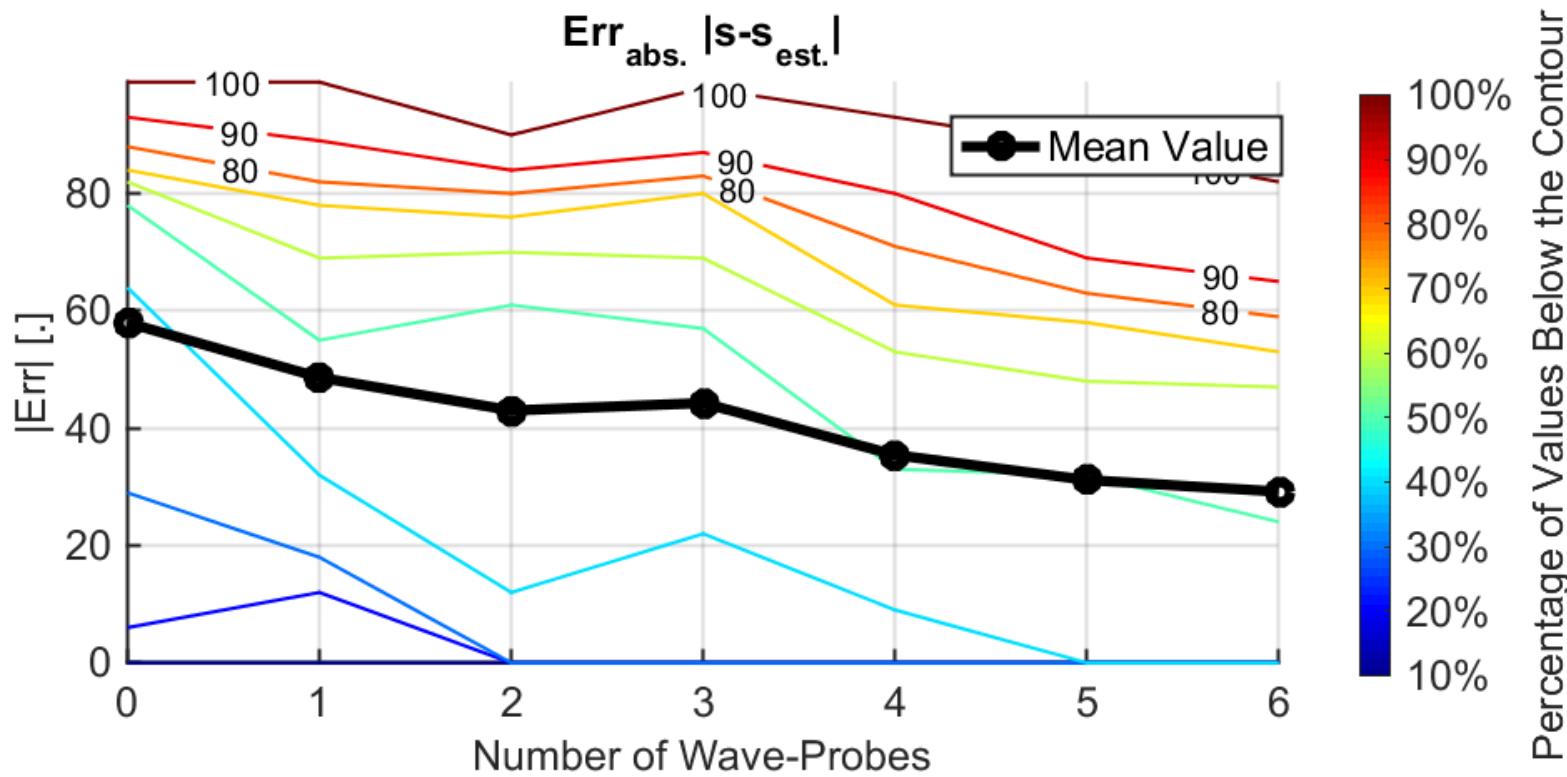

Source: Elaborated by the author.

In the $H_{S}$ histograms, it is possible to notice that most of the improvement is achieved when using two probes, with the trend presenting an 'elbow' shape, as hypothesized previously. The maximum absolute error does not present a clear trend, but in the percent error the trend is clear. It is also interesting how the addition of an odd number of wave-probes seems to be worse than the addition of an even number, which can be justified by the difference of estimation between the starboard and the port estimation, when an odd number is used.

The $T_{P}$ histograms follow the $H_{S}$ histograms closely, what was expected since both are energy related metrics. The 'elbow' shape is even more pronounced, with two wave-probes being a stabilization point.

Combining the conclusions for these first two parameters, the addition of just one wave-probe is already enough to decrease the percent error of $90 \%$ of the waves from $35 \%$ to $22 \%$ in the $H_{S}$ estimation, and from $16.5 \%$ to $7.5 \%$ in the $T_{P}$ estimation; approximately a $37 \%-55 \%$ improvement in the parameters estimations. The addition of two or more probes decreases the error from $35 \%$ to $13.5 \%$ in the $H_{S}$ estimation, and from $16.5 \%$ to $5 \%$ in the $T_{P}$ estimation; approximately a $62 \%-65 \%$ improvement in the parameters estimations.

The $\beta_{0}$ histogram, instead of the others, does not present a clear 'elbow' shape, or trend of improvement. Some possible reasons of that will be raised when discussing particular results, but, for now, although the maximum error becomes greater with the 
addition of 2 probes, an improvement happens in $90 \%$ of the sea states; furthermore, with 4 and 6 probes a great improvement can be noticed in the direction estimation, with the maximum error below $18^{\circ}$, the discretization used during the estimation.

The directional spread factor, $s$, has a slightly tendency to improve with the increase of the number of wave-probes, however it is known, from section, 5.4 Verification of the Error Metrics, that there is an alias phenomenon that deteriorates the estimation of this parameter. In order to overcome this, the histogram of the error in the directional spread function can be used, to verify the improvement in the directional aspect of the estimation, Figure 93.

Figure 93 - Histogram of the directional spread function error.

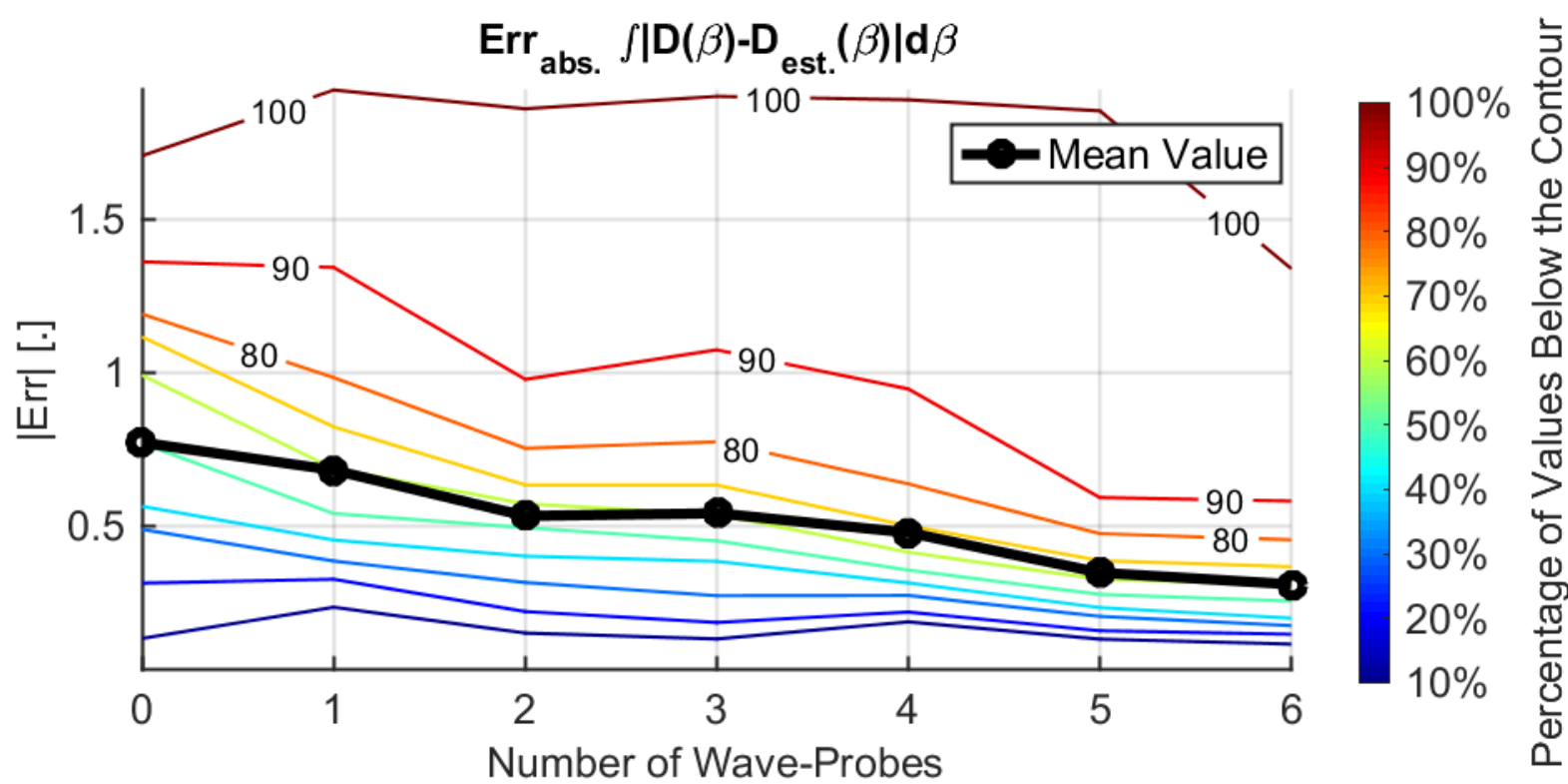

Source: Elaborated by the author.

Using the directional metric above the tendency becomes clear. Even though most of the improvement can be achieved with two and four probes, the metric seems to suggest that there is still a proportional improvement when six wave-probes are employed.

Summarizing the results so far: the usage of just one wave-probe is already able to achieve a considerably improvement in all the criteria; the usage of at least 2 waveprobes is recommended if it is desired to improve the most the spectral energy density estimation, which is related with the $H_{S}$ and $T_{P}$ parameters; the usage of at least 4 wave-probes is recommended if it is desired to know the mean direction of the incoming wave; and the usage of 6 probes is recommended for directional estimation. 
Some particular results are able to illustrate the behavior of the histogram plots. The estimation results are compared against the experimental spectrum provided by the tank, which was estimated using an array of probes by the method of the Maximum Entropy with a finer frequency-direction grid; the rediscretization of the experimental spectra provided is discussed in APPENDIX C - Numerical Issues.

Firstly, it is important to analyze cases that represent the main objective of this work, which is to improve the estimation of waves with low $T_{P}$. The estimation for waves coming from $0^{\circ}$ are shown in Figure 94 to Figure 98.

Figure $94-$ Low $T_{P}$ wave, coming from $0^{\circ}$, estimated without wave-probes.
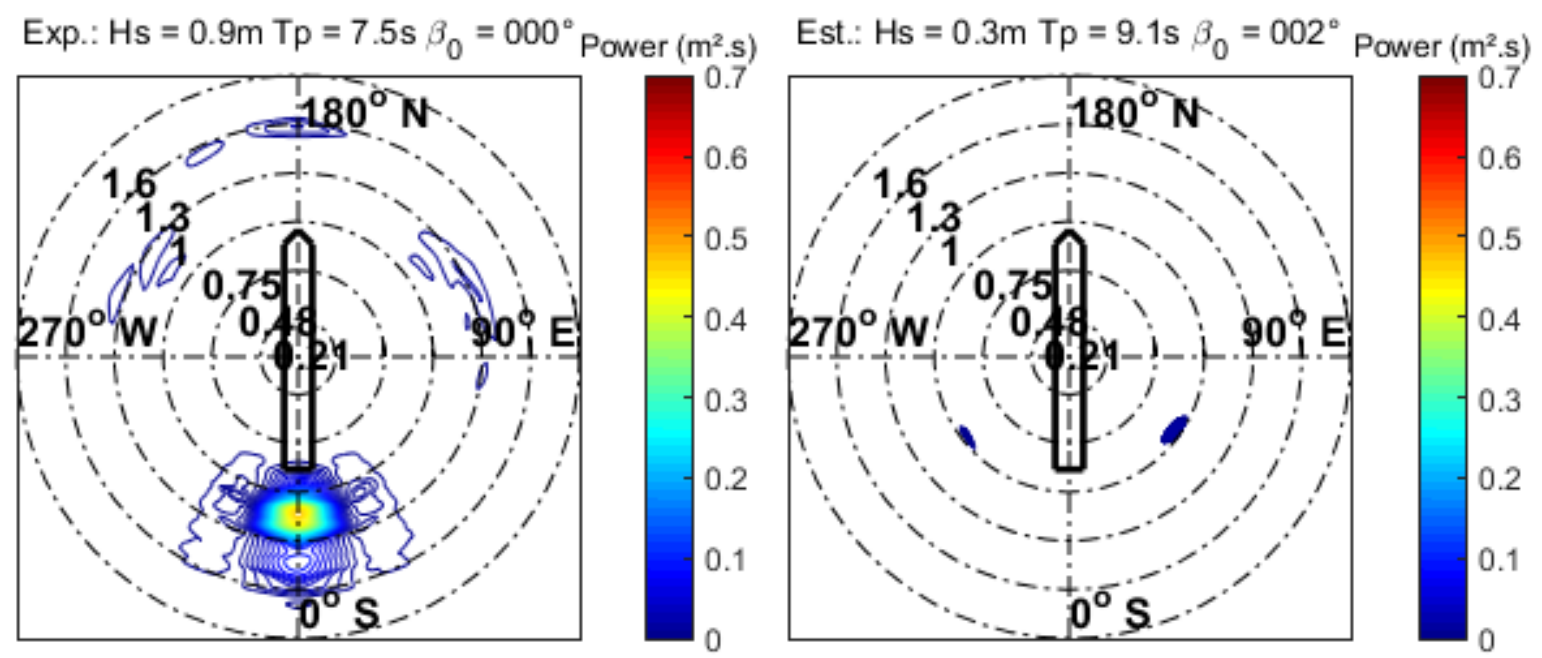

Source: Elaborated by the author.

Figure 95 - Low $T_{P}$ wave, coming from $0^{\circ}$, estimated with 1 wave-probe.

Exp.: $\mathrm{Hs}=0.9 \mathrm{~m} \mathrm{Tp}=7.5 \mathrm{~s} \beta_{0}=000^{\circ}$ Power $\left(\mathrm{m}^{2} . \mathrm{s}\right)$
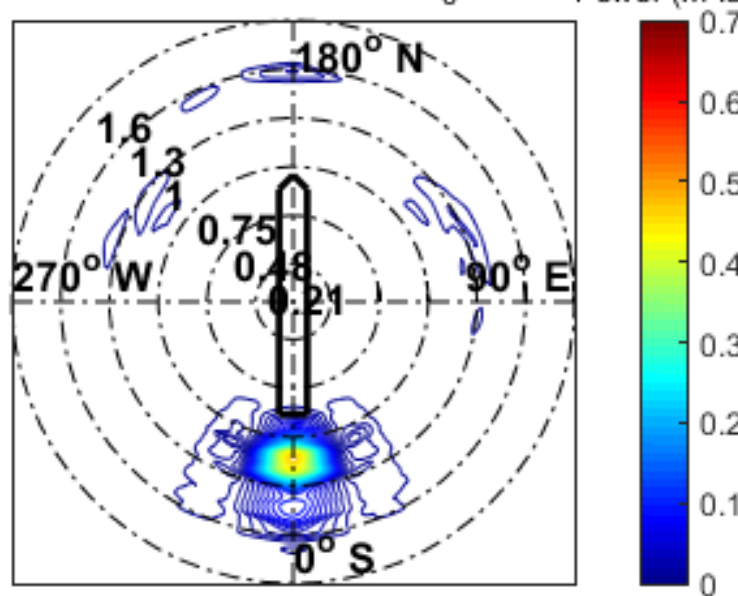

Est.: $\mathrm{Hs}=0.4 \mathrm{~m} \mathrm{Tp}=7.6 \mathrm{~s} \beta_{0}=001^{\circ}$ Power $\left(\mathrm{m}^{2} . \mathrm{s}\right)$

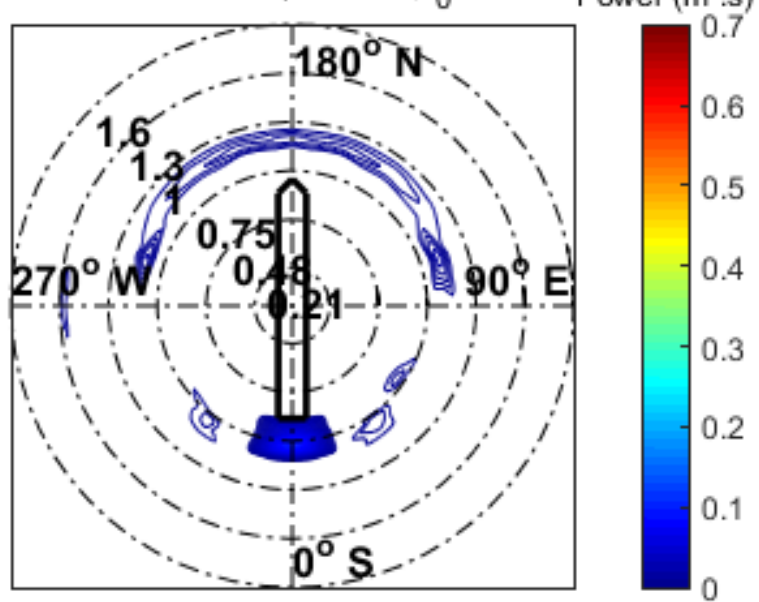

Source: Elaborated by the author. 
Figure 96 - Low $T_{P}$ wave, coming from $0^{\circ}$, estimated with 2 wave-probes.

Exp.: $\mathrm{Hs}=0.9 \mathrm{~m} \mathrm{Tp}=7.5 \mathrm{~s} \beta_{0}=000^{\circ}$ Power $\left(\mathrm{m}^{2} . \mathrm{s}\right)$
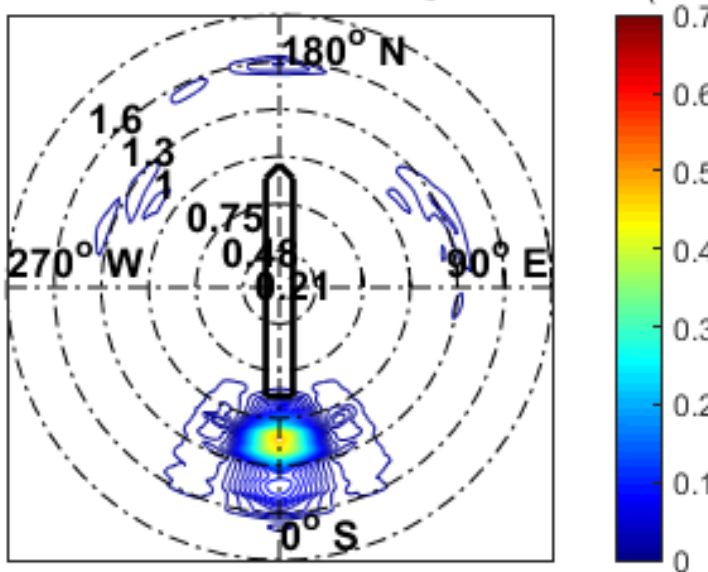

Source: Elaborated by the author.

Figure 97 - Low $T_{P}$ wave, coming from $0^{\circ}$, estimated with 4 wave-probes.

Exp.: $\mathrm{Hs}=0.9 \mathrm{~m} \mathrm{Tp}=7.5 \mathrm{~s} \beta_{0}=000^{\circ}$ Power $\left(\mathrm{m}^{2} . \mathrm{s}\right)$
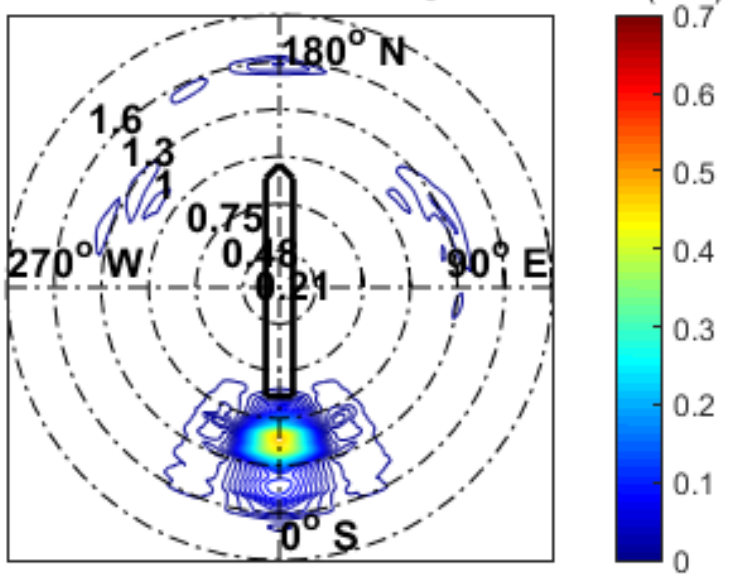

Est.: $\mathrm{Hs}=0.8 \mathrm{~m} \mathrm{Tp}=7.5 \mathrm{~s} \beta_{0}=001^{\circ}$ Power $\left(\mathrm{m}^{2} \mathrm{~s}\right)$

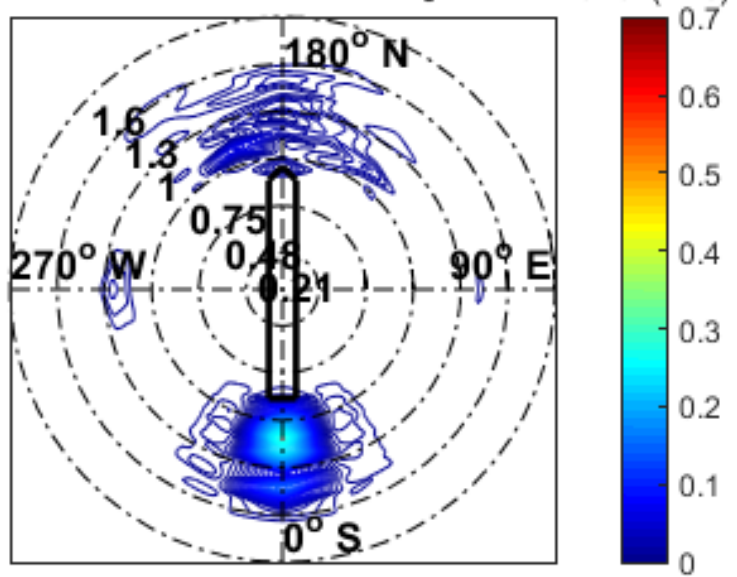

Est.: $\mathrm{Hs}=0.9 \mathrm{~m} \mathrm{Tp}=7.4 \mathrm{~s} \beta_{0}=357^{\circ}$ Power $\left(\mathrm{m}^{2} \mathrm{~s}\right)$
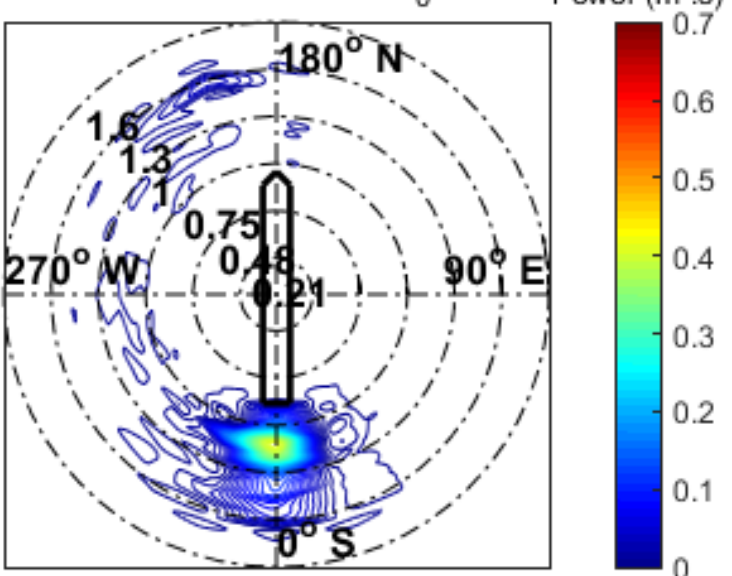

Source: Elaborated by the author.

Figure 98 - Low $T_{P}$ wave, coming from $0^{\circ}$, estimated with 6 wave-probes.

Exp.: $\mathrm{Hs}=0.9 \mathrm{~m} \mathrm{Tp}=7.5 \mathrm{~s} \beta_{0}=000^{\circ}$

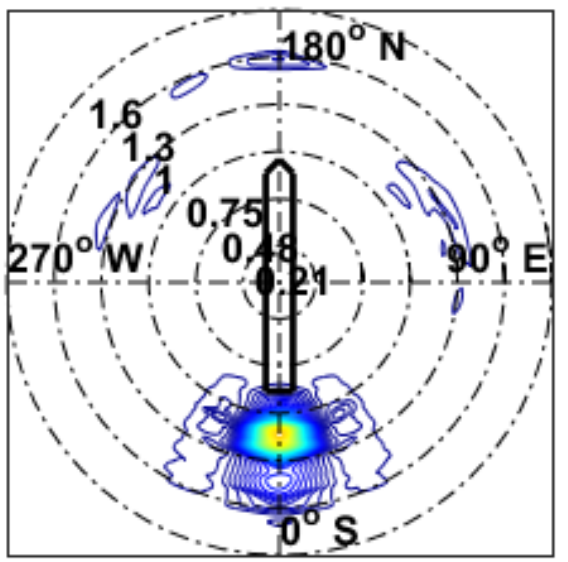

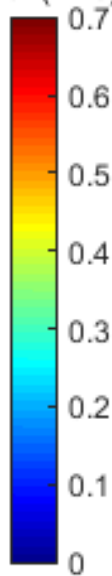

Est.: $\mathrm{Hs}=0.9 \mathrm{~m} \mathrm{Tp}=7.4 \mathrm{~s} \beta_{0}=358^{\circ}$

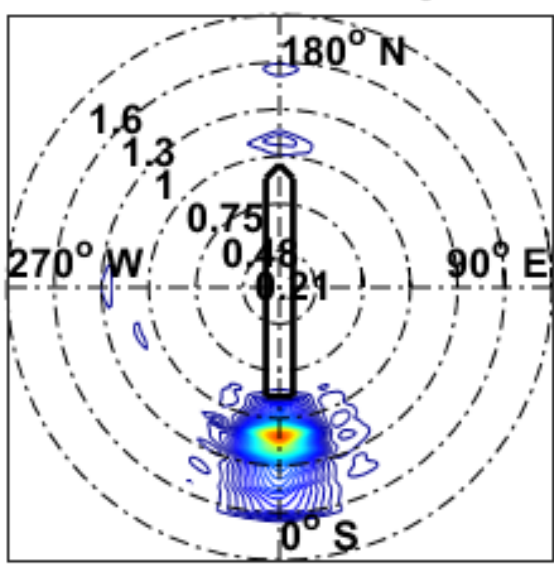
Power $\left(m^{2} . s\right)$

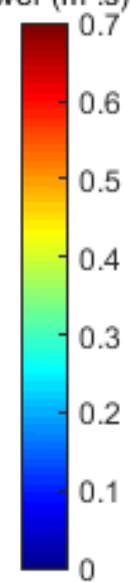

Source: Elaborated by the author. 
The results are a clear representation of the histogram conclusions. The addition of one probe already results in an improvement of the estimation, with $T_{P}$ improving faster than $H_{S}$; the addition of two probes already recovers most of the energy of the spectrum; and the addition of four to six probes improves the directional estimation.

In this particular case, the addition of two wave-probes already give a reasonable directional estimation, which is also the case for waves coming from $90^{\circ}$ and $180^{\circ}$, Figure 99 to Figure 102.

Figure $99-$ Low $T_{P}$ wave, coming from $90^{\circ}$, estimated without wave-probes.
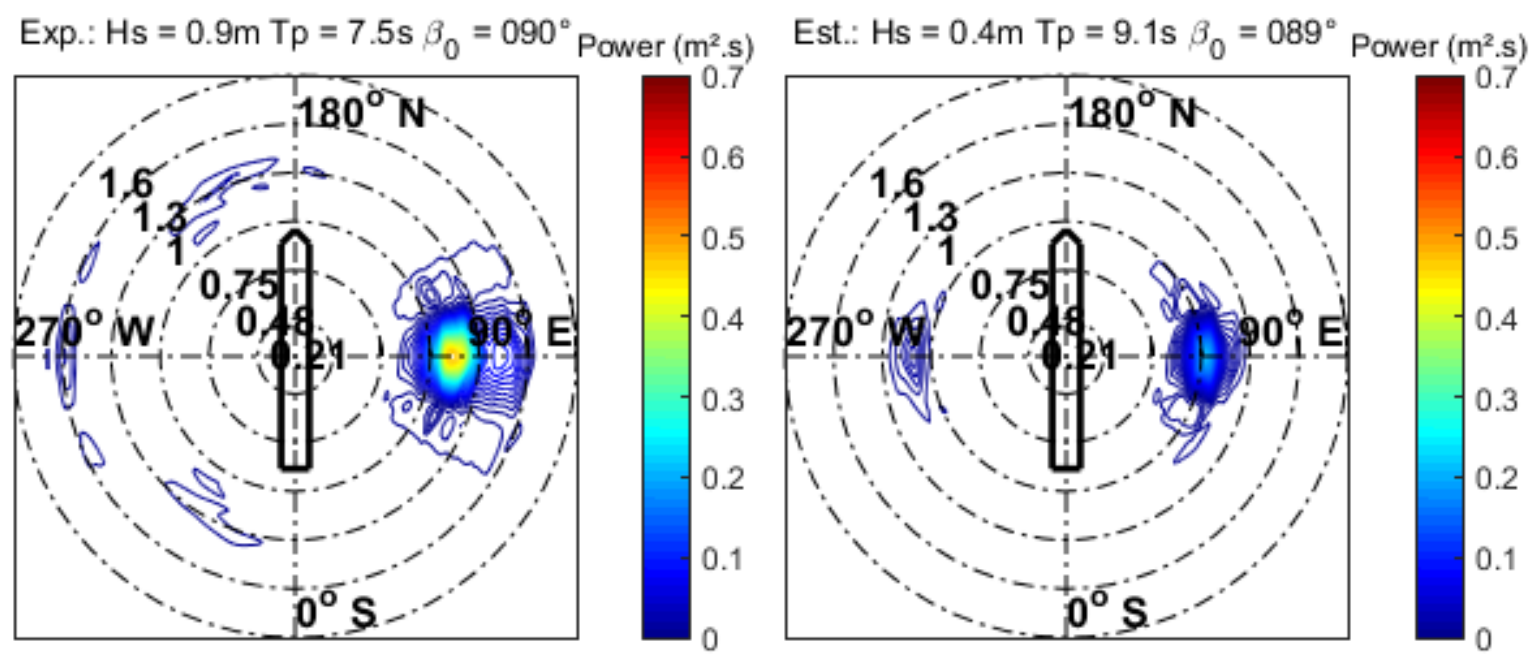

Source: Elaborated by the author.

Figure 100 - Low $T_{P}$ wave, coming from $90^{\circ}$, estimated with 2 wave-probes.

Exp.: $\mathrm{Hs}=0.9 \mathrm{~m} \mathrm{Tp}=7.5 \mathrm{~s} \beta_{0}=090^{\circ}$ Power $\left(\mathrm{m}^{2} . \mathrm{s}\right)$
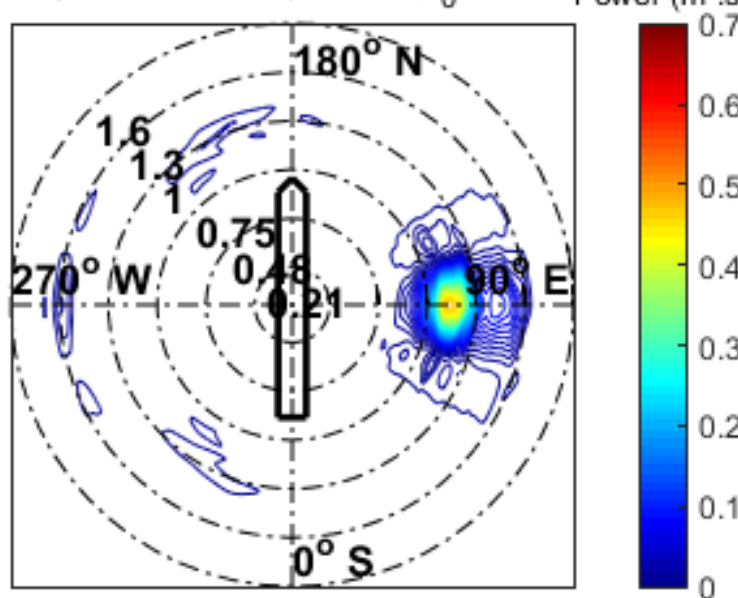

Est.: $\mathrm{Hs}=0.9 \mathrm{~m} \mathrm{Tp}=7.5 \mathrm{~s} \beta_{0}=090^{\circ}$ Power $\left(\mathrm{m}^{2} . \mathrm{s}\right)$

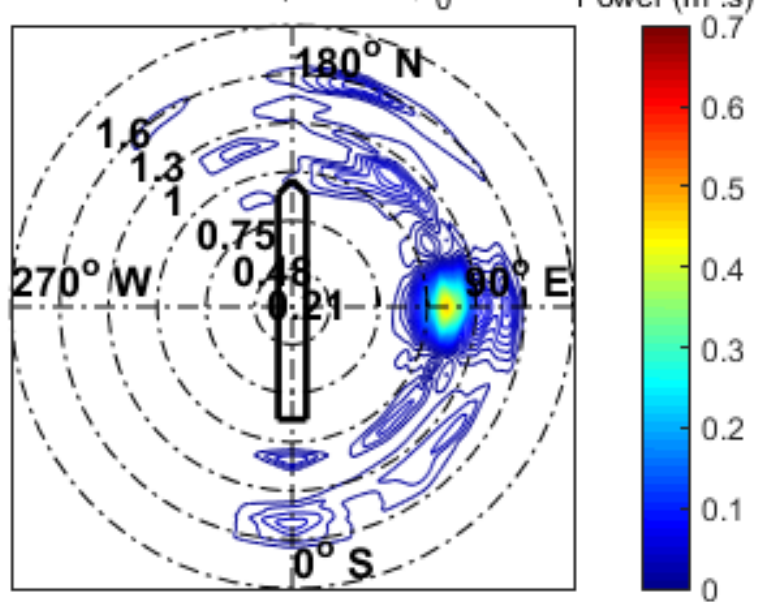

Source: Elaborated by the author. 
Figure 101 - Low $T_{P}$ wave, coming from $180^{\circ}$, estimated without wave-probes.
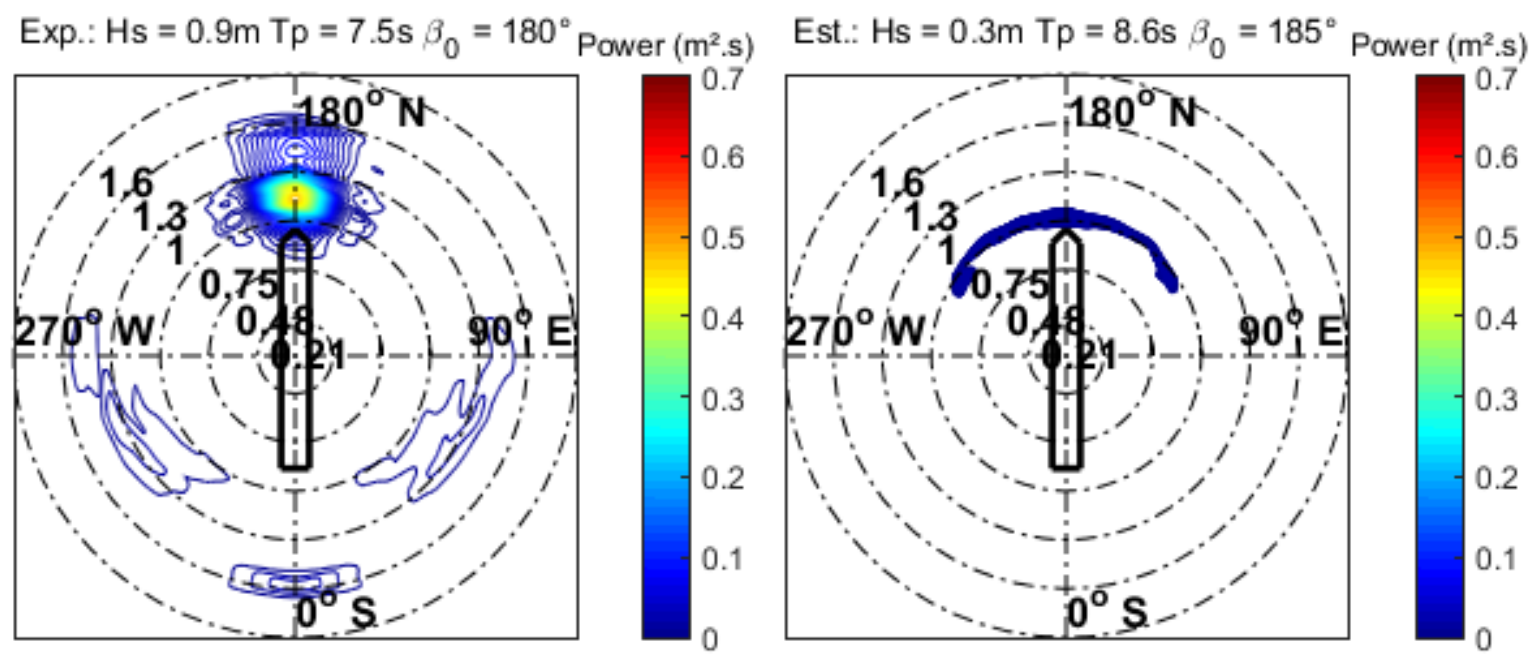

Source: Elaborated by the author.

Figure 102 - Low $T_{P}$ wave, coming from $180^{\circ}$, estimated with 2 wave-probes.
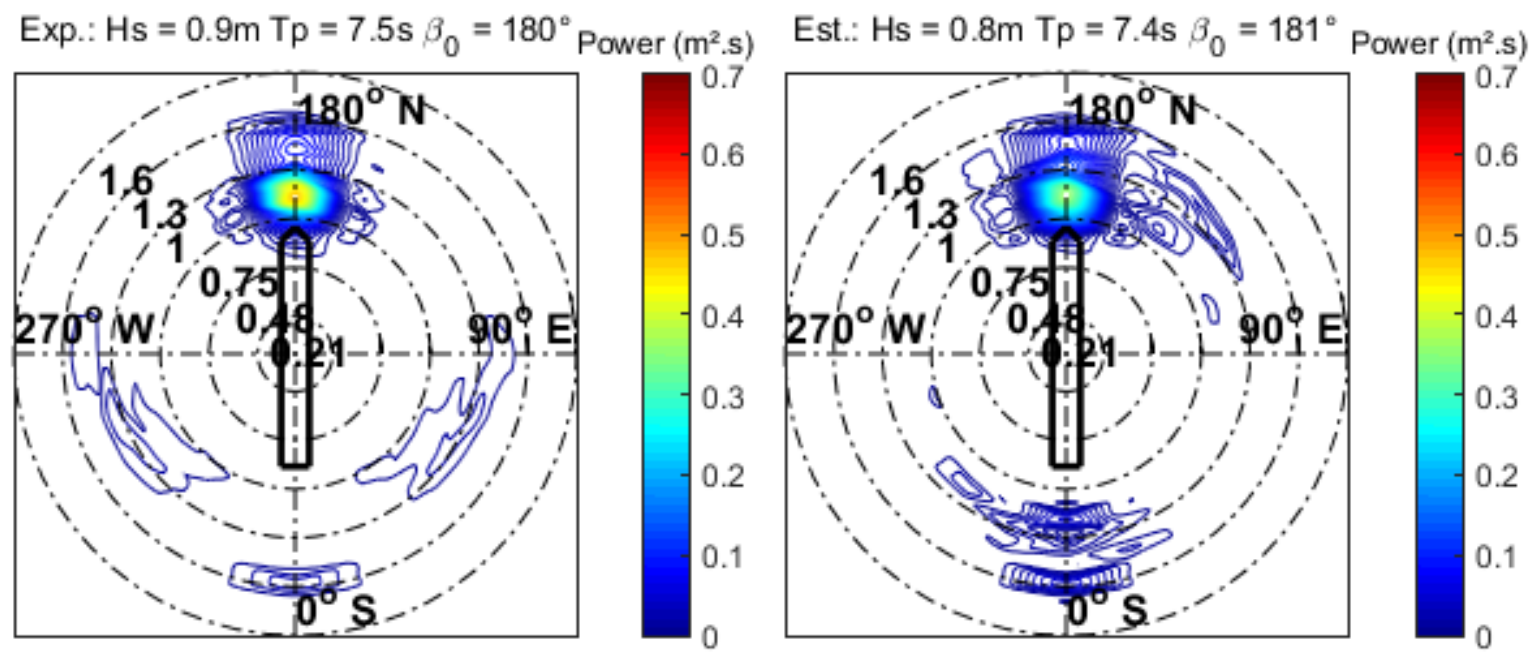

Source: Elaborated by the author.

The sufficiency of two probes, however, is not true for quartering and bow seas, i.e., waves coming respectively from $45^{\circ}$ and $135^{\circ}$, Figure 103 to Figure 110 .This is expected, since there is, at the same time, non-negligible movements from sway and pitch, preventing a correct direction estimation by the vessel movements; and when two or four wave probes are employed the behavior is similar to a wave encountering a wall with a single wave-probe, which is not capable of providing a proper direction discrimination, only the energy measurement Figure 111. 
Figure 103 - Low $T_{P}$ wave, coming from $45^{\circ}$, estimated without probes.

Exp.: $\mathrm{Hs}=0.9 \mathrm{~m} \mathrm{Tp}=7.4 \mathrm{~s} \beta_{0}=046^{\circ}$ Power $\left(\mathrm{m}^{2} \mathrm{~s}\right)$
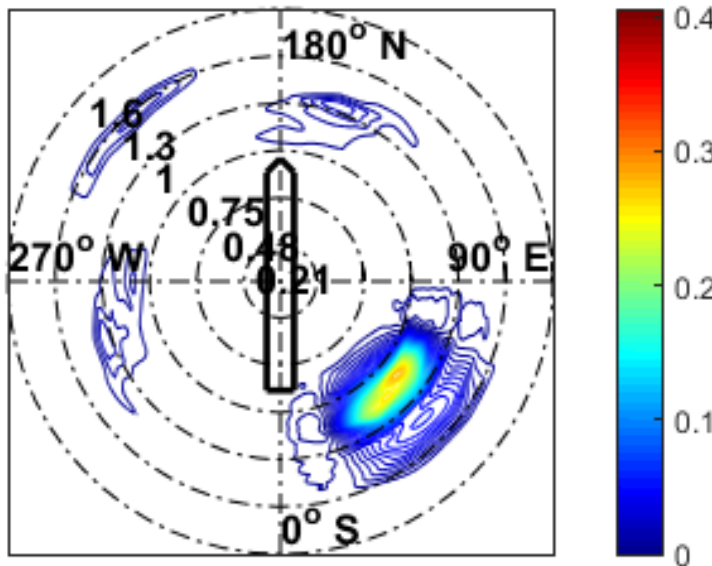

Est.: $\mathrm{Hs}=0.4 \mathrm{~m} \mathrm{Tp}=9.2 \mathrm{~s} \beta_{0}=013^{\circ}$ Power $\left(\mathrm{m}^{2} . \mathrm{s}\right)$
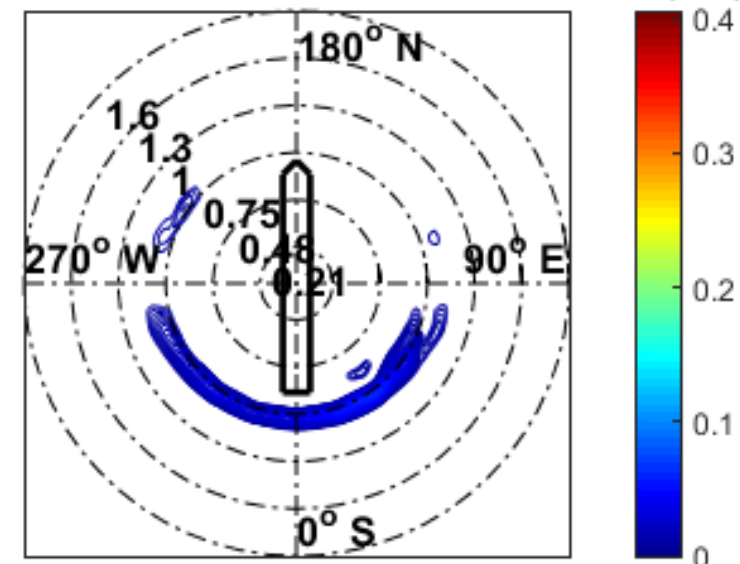

Source: Elaborated by the author.

Figure 104 - Low $T_{P}$ wave, coming from $45^{\circ}$, estimated with 2 wave-probes.

Exp.: $\mathrm{Hs}=0.9 \mathrm{~m} \mathrm{Tp}=7.4 \mathrm{~s} \beta_{0}=046^{\circ}$ Power $\left(\mathrm{m}^{2} . \mathrm{s}\right)$
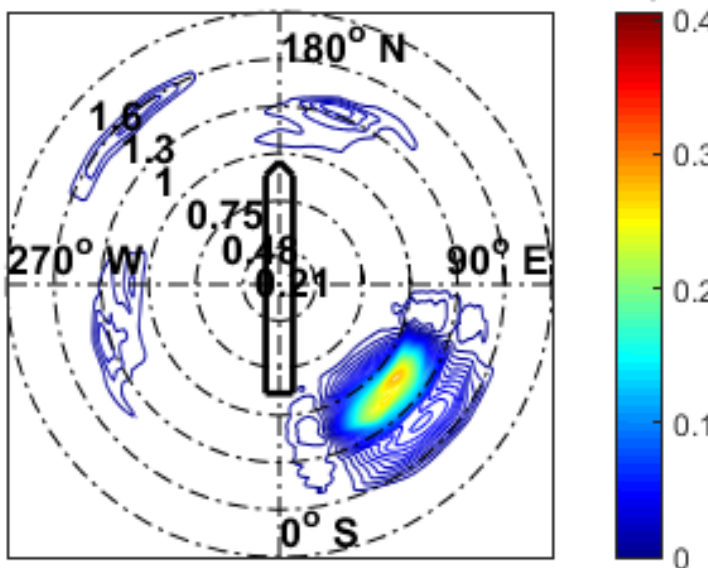

Est.: $\mathrm{Hs}=1.0 \mathrm{~m} \mathrm{Tp}=7.5 \mathrm{~s} \beta_{0}=067^{\circ}$

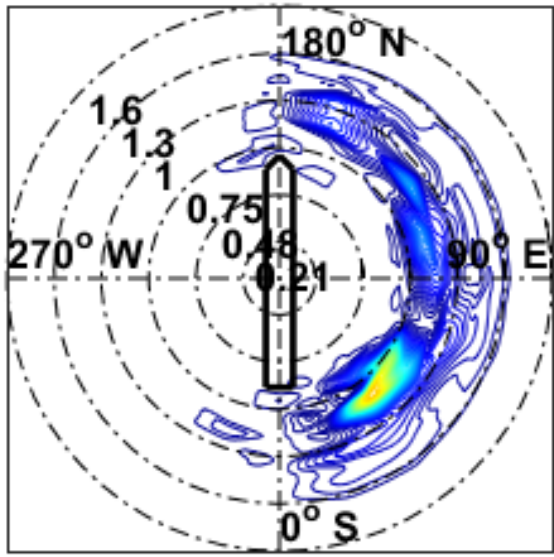

Power $\left(m^{2} . s\right)$

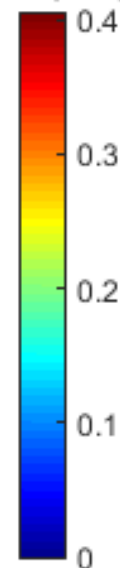

Source: Elaborated by the author.

Figure 105 - Low $T_{P}$ wave, coming from $45^{\circ}$, estimated with 4 wave-probes.

Exp.: Hs $=0.9 \mathrm{~m} \mathrm{Tp}=7.4 \mathrm{~s} \beta_{0}=046^{\circ}$ Power $\left(\mathrm{m}^{2} . \mathrm{s}\right)$
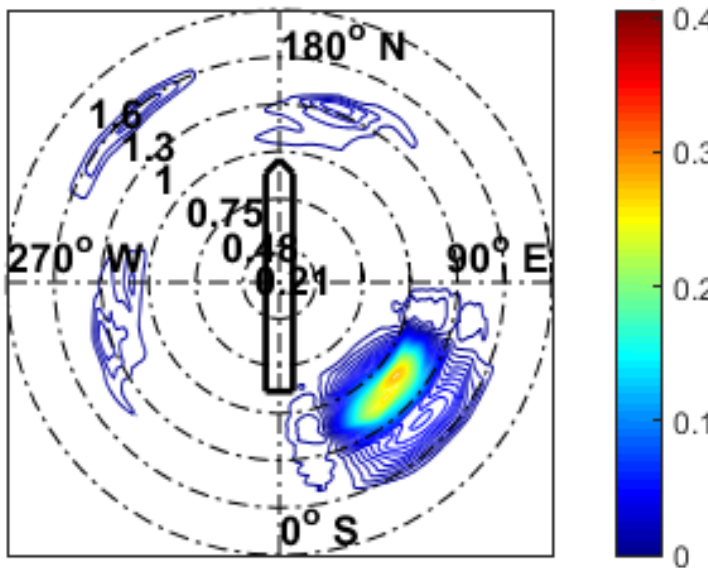

Est.: $\mathrm{Hs}=1.0 \mathrm{~m} \mathrm{Tp}=7.5 \mathrm{~s} \beta_{0}=059^{\circ}$ Power $\left(\mathrm{m}^{2} . \mathrm{s}\right)$

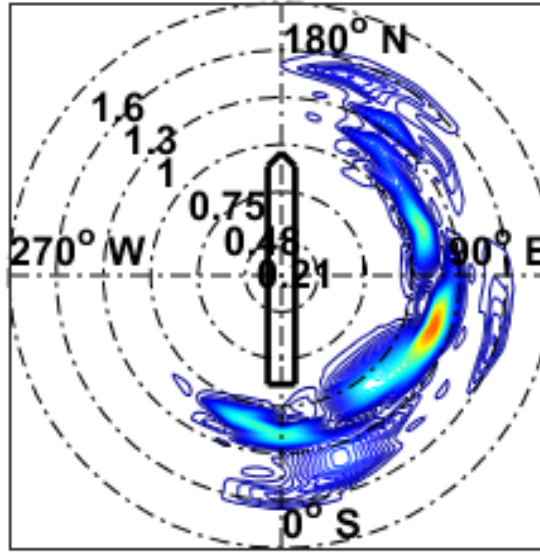

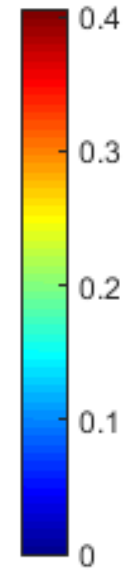

Source: Elaborated by the author. 
Figure 106 - Low $T_{P}$ wave, coming from $45^{\circ}$, estimated with 6 wave-probes.

Exp.: $\mathrm{Hs}=0.9 \mathrm{~m} \mathrm{Tp}=7.4 \mathrm{~s} \beta_{0}=046^{\circ}$ Power $\left(\mathrm{m}^{2} \mathrm{~s}\right)$
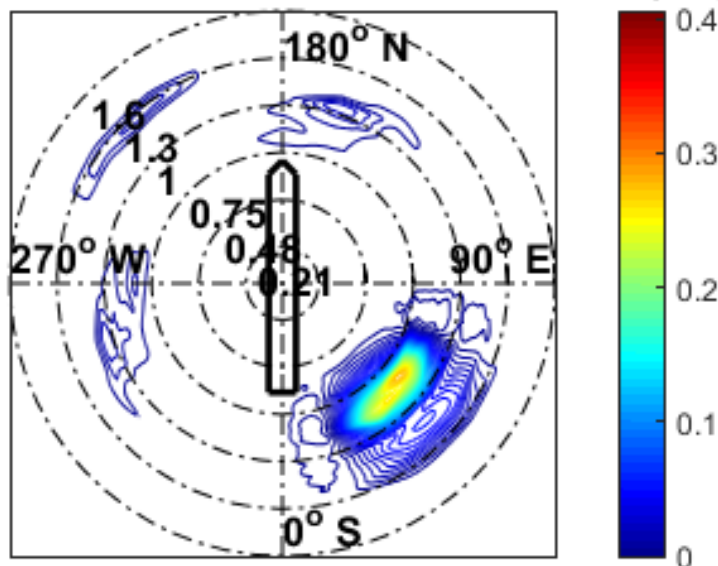

Est.: $\mathrm{Hs}=1.0 \mathrm{~m} \mathrm{Tp}=7.5 \mathrm{~s} \beta_{0}=056^{\circ}$ Power $\left(\mathrm{m}^{2} . \mathrm{s}\right)$
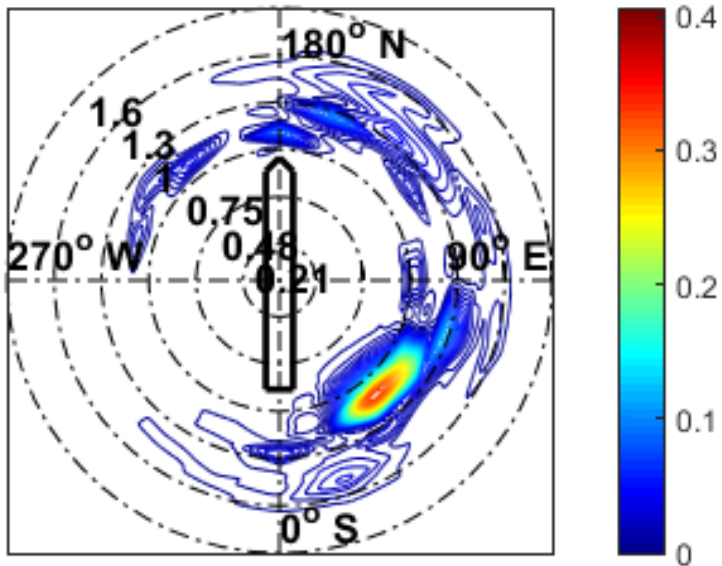

Source: Elaborated by the author.

Figure 107 - Low $T_{P}$ wave, coming from $135^{\circ}$, estimated without wave-probes.

Exp.: $\mathrm{Hs}=0.9 \mathrm{~m} \mathrm{Tp}=7.4 \mathrm{~s} \beta_{0}=136^{\circ}$ Power $\left(\mathrm{m}^{2} . \mathrm{s}\right)$
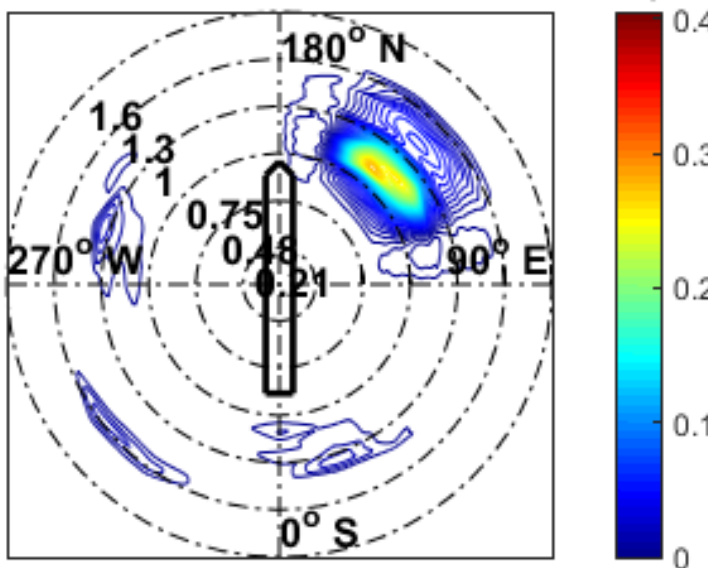

Est.: $\mathrm{Hs}=0.4 \mathrm{~m} \mathrm{Tp}=9.3 \mathrm{~s} \beta_{0}=153^{\circ}$ Power $\left(\mathrm{m}^{2} . \mathrm{s}\right)$
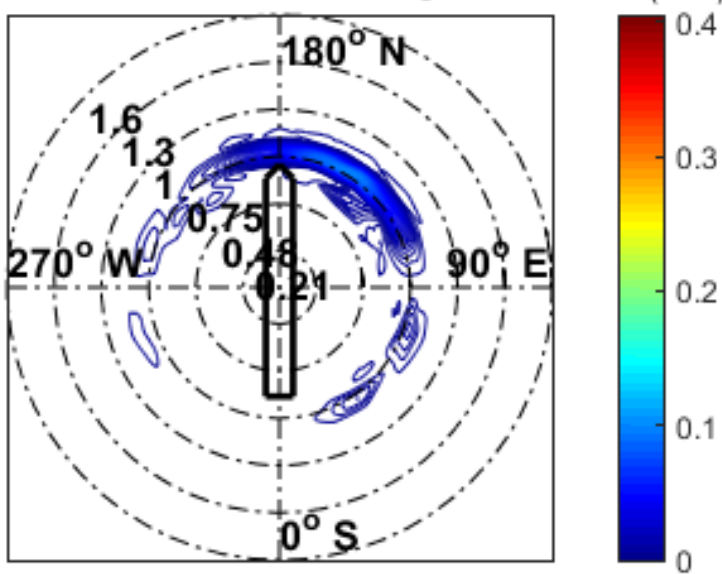

Source: Elaborated by the author.

Figure 108 - Low $T_{P}$ wave, coming from $135^{\circ}$, estimated with 2 wave-probes.

Exp.: $\mathrm{Hs}=0.9 \mathrm{~m} \mathrm{Tp}=7.4 \mathrm{~s} \beta_{0}=136^{\circ}$ Power $\left(\mathrm{m}^{2} . \mathrm{s}\right)$
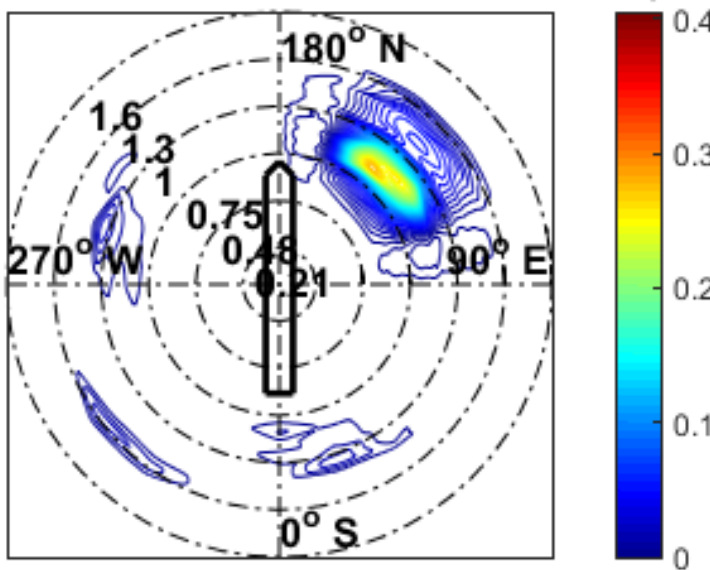

Est.: $\mathrm{Hs}=1.0 \mathrm{~m} \mathrm{Tp}=7.7 \mathrm{~s} \beta_{0}=079^{\circ}$ Power $\left(\mathrm{m}^{2} . \mathrm{s}\right)$

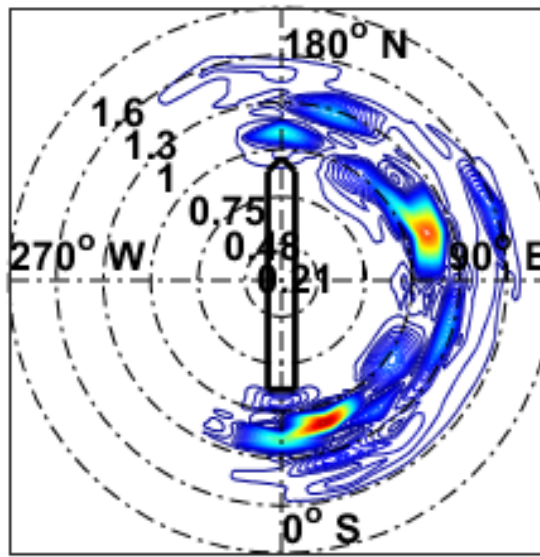

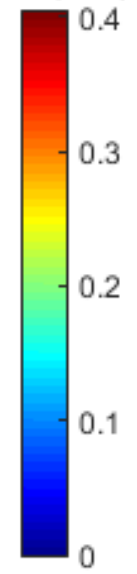

Source: Elaborated by the author. 
Figure 109 - Low $T_{P}$ wave, coming from $135^{\circ}$, estimated with 4 wave-probes.

Exp.: $\mathrm{Hs}=0.9 \mathrm{~m} \mathrm{Tp}=7.4 \mathrm{~s} \beta_{0}=136^{\circ}$ Power $\left(\mathrm{m}^{2} \mathrm{~s}\right)$
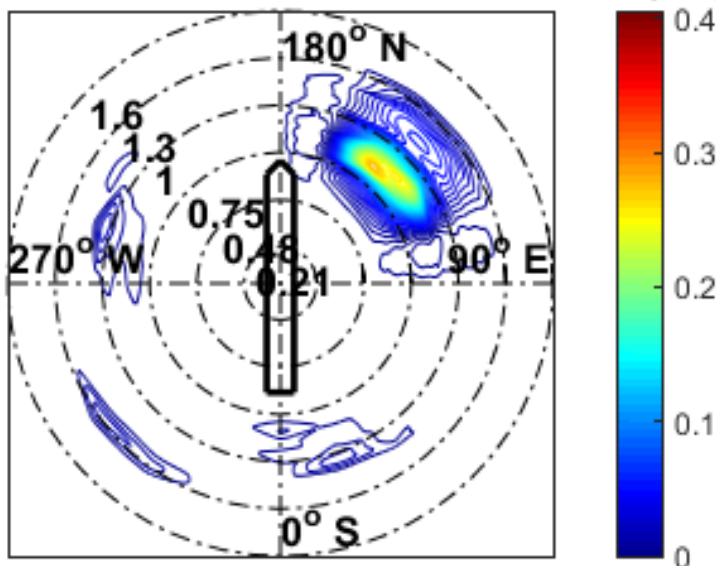

Est.: $\mathrm{Hs}=1.0 \mathrm{~m} \mathrm{Tp}=7.5 \mathrm{~s} \beta_{0}=133^{\circ}$ Power $\left(\mathrm{m}^{2} \mathrm{~s}\right)$

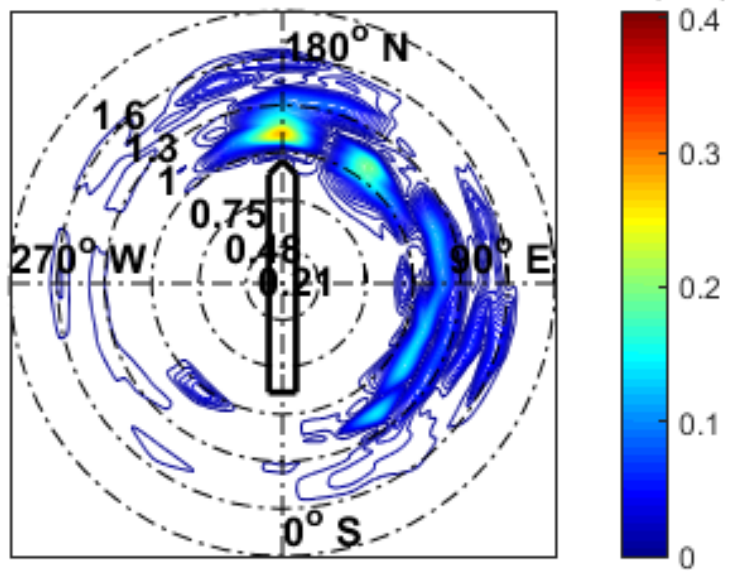

Source: Elaborated by the author.

Figure 110 - Low $T_{P}$ wave, coming from $135^{\circ}$, estimated with 6 wave-probes.

Exp.: $\mathrm{Hs}=0.9 \mathrm{~m} \mathrm{Tp}=7.4 \mathrm{~s} \beta_{0}=136^{\circ}$ Power $\left(\mathrm{m}^{2} . \mathrm{s}\right)$
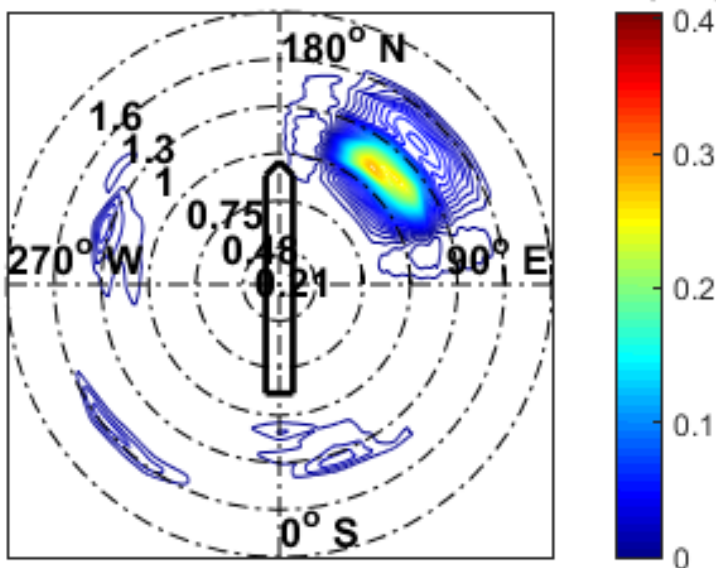

Est.: $\mathrm{Hs}=1.0 \mathrm{~m} \mathrm{Tp}=7.5 \mathrm{~s} \beta_{0}=123^{\circ}$ Power $\left(\mathrm{m}^{2} . \mathrm{s}\right)$
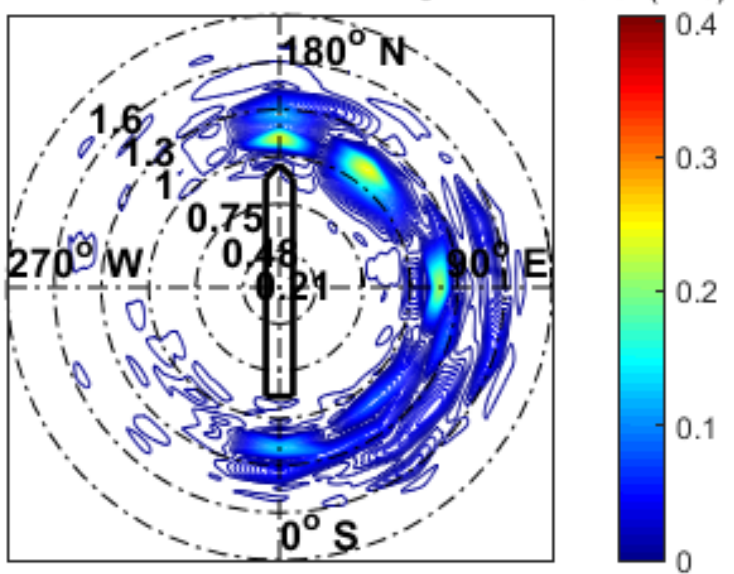

Source: Elaborated by the author.

Figure 111 - Energy spectral density with 0 and 2 probes.

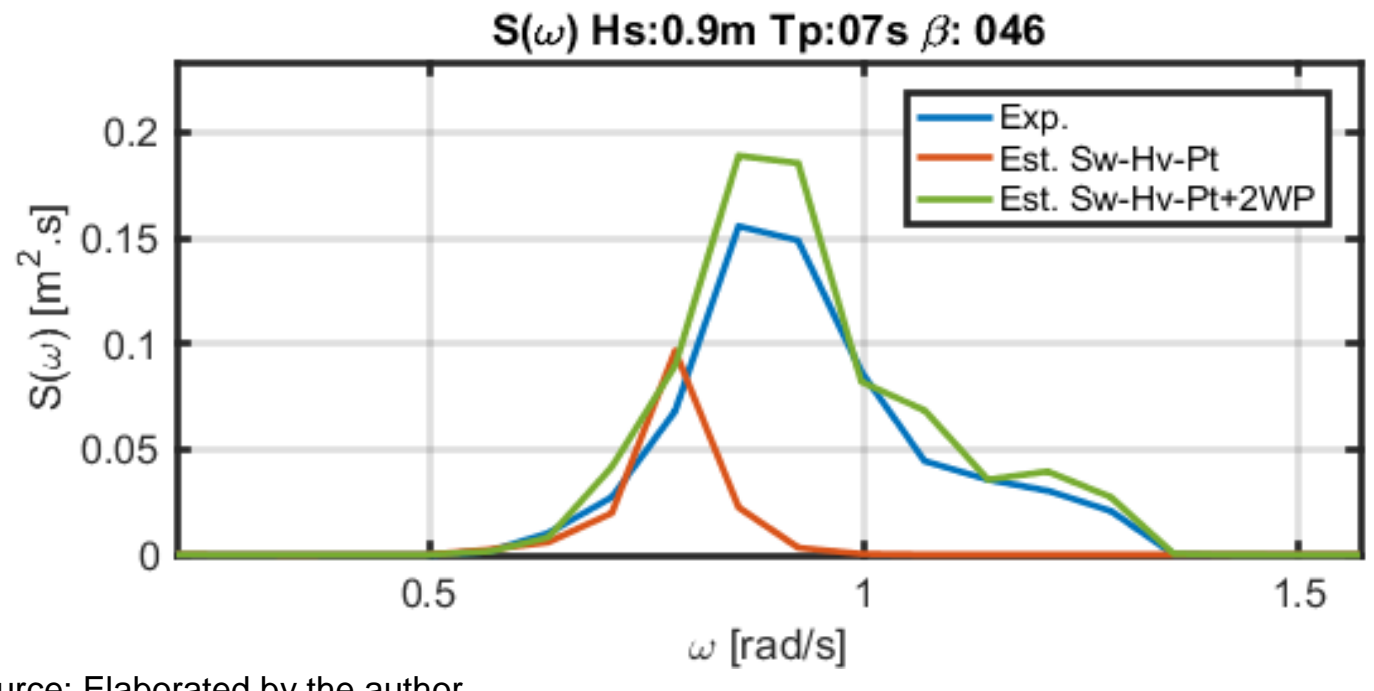

Source: Elaborated by the author. 
In conclusion, although two wave-probes are already capable of recovering the energy of the incoming wave, it is necessary six wave-probes to recover its direction characteristic, i.e., two wave-probes at the same side of the vessel.

Other interesting conclusion is how the wave-probes are capable of recovering the energy values beyond what is possible using only vessel DoFs. In Figure 111, for example, the energy beyond $\omega>1 \mathrm{rad} / \mathrm{s} \rightarrow T<6.3 \mathrm{~s}$ is completely recovered, even the tail due to the tank cut-off frequency after $2 \mathrm{~Hz}-\sim 1.26 \mathrm{rad} / \mathrm{s}$ in the real scale - an information that could not have come from the prior, but only from the measurements.

The results at $135^{\circ}$ are not completely satisfactory, for this wave with the lowest tested $T_{P}$, however the energy is positioned correctly in the first quadrant of the polar plot, which is already enough for a preliminary estimation of the drift forces, and the result improves for waves with higher $T_{P} \mathrm{~s}$, Figure 115 to Figure 117, for example.

The results shown so far, for the lowest $T_{P}$, are replicated for higher $T_{P}$ waves and different $H_{S}$; with two probes delivering most of the improvement, and six probes giving the best directional estimation. The improvements happen up to $12 \mathrm{~s}$, following the expected valid region of the expanded linear model, as discussed when analyzing the RAOs. The estimations of a medium $T_{P}$ wave coming from $0^{\circ}$ are shown in Figure 112 to Figure 114; and for the same medium $T_{P}$ wave coming from $135^{\circ}$ in Figure 115 to Figure 117.

Figure 112 - Medium $T_{P}$ wave, coming from $0^{\circ}$, estimated without wave-probes.
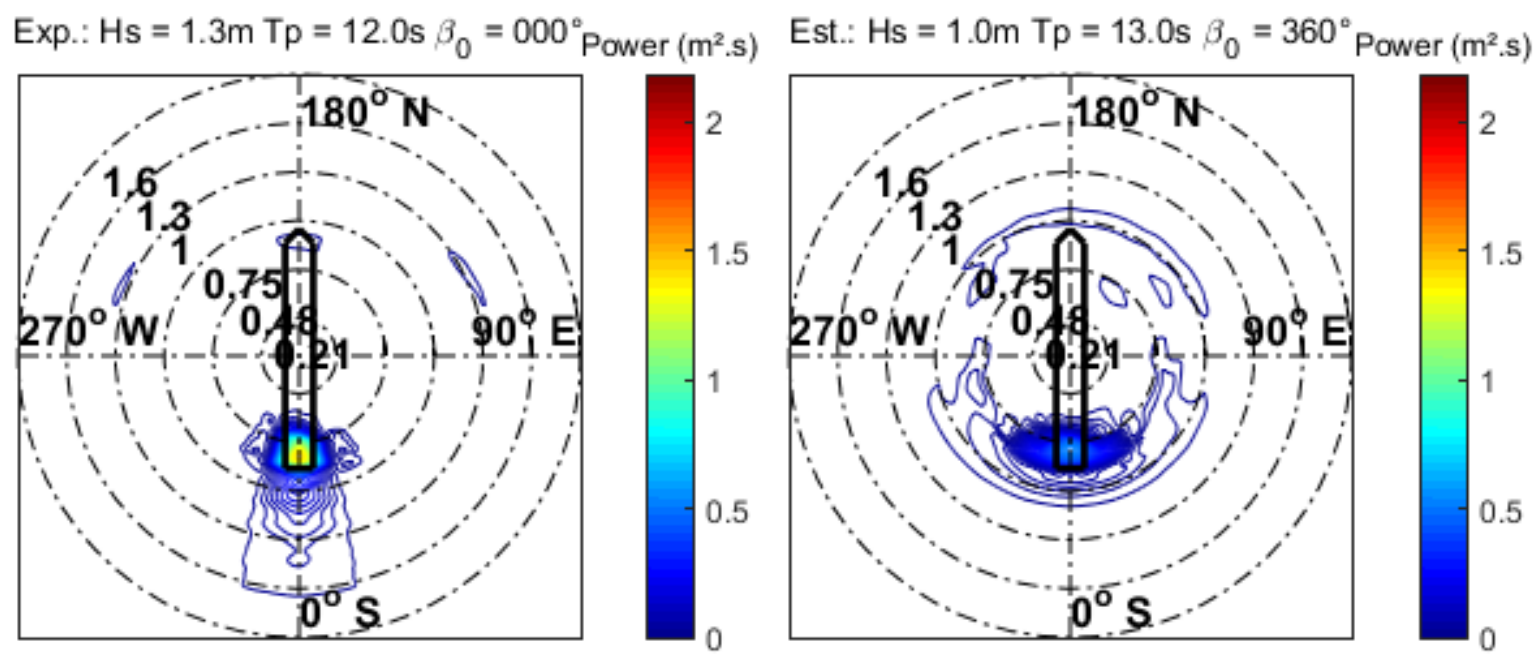

Source: Elaborated by the author. 
Figure 113 - Medium $T_{P}$ wave, coming from $0^{\circ}$, estimated with 2 wave-probes.

Exp.: $\mathrm{Hs}=1.3 \mathrm{~m} \mathrm{Tp}=12.0 \mathrm{~s} \beta_{0}=000^{\circ}$ Power $\left(\mathrm{m}^{2} . \mathrm{s}\right)$

Est.: $\mathrm{Hs}=1.2 \mathrm{~m} \mathrm{Tp}=11.9 \mathrm{~s} \beta_{0}=358^{\circ}$ Power $\left(\mathrm{m}^{2} . \mathrm{s}\right)$
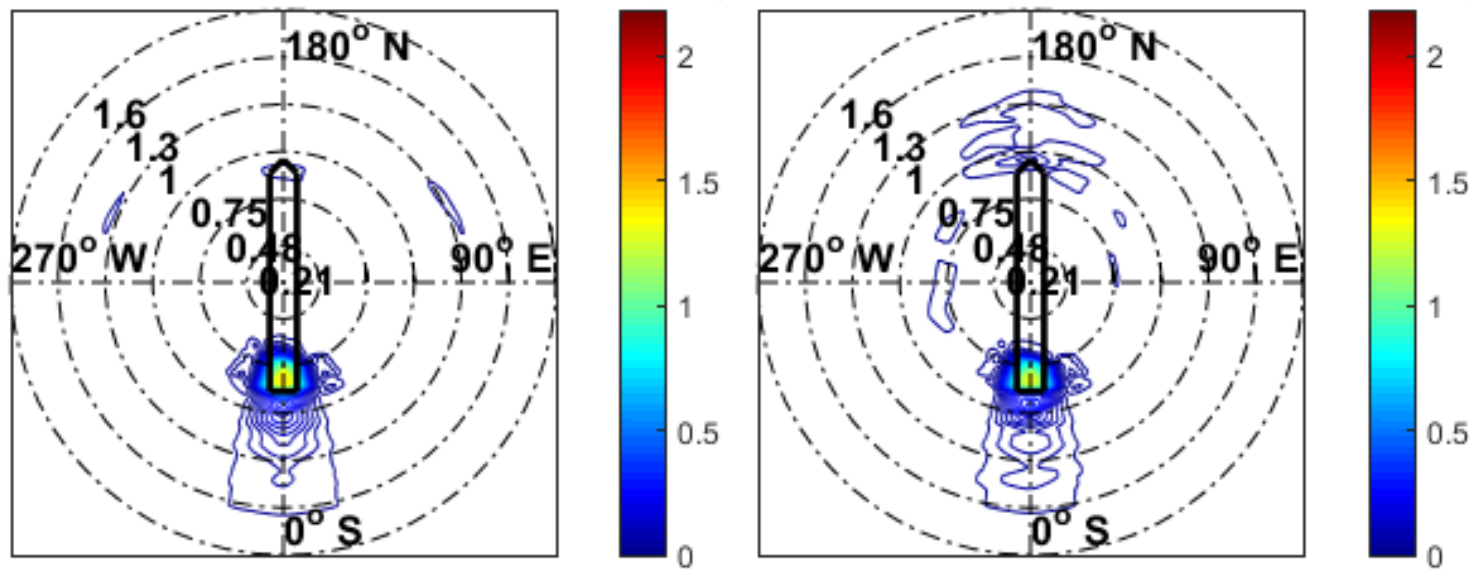

Source: Elaborated by the author.

Figure 114 - Medium $T_{P}$ wave, coming from $0^{\circ}$, estimated with 6 wave-probes.

Exp.: $\mathrm{Hs}=1.3 \mathrm{~m} \mathrm{Tp}=12.0 \mathrm{~s} \beta_{0}=000^{\circ}$ Power $\left(\mathrm{m}^{2} . \mathrm{s}\right)$

Est.: $\mathrm{Hs}=1.3 \mathrm{~m} \mathrm{Tp}=11.7 \mathrm{~s} \beta_{0}=359^{\circ}$ Power $\left(\mathrm{m}^{2} . \mathrm{s}\right)$
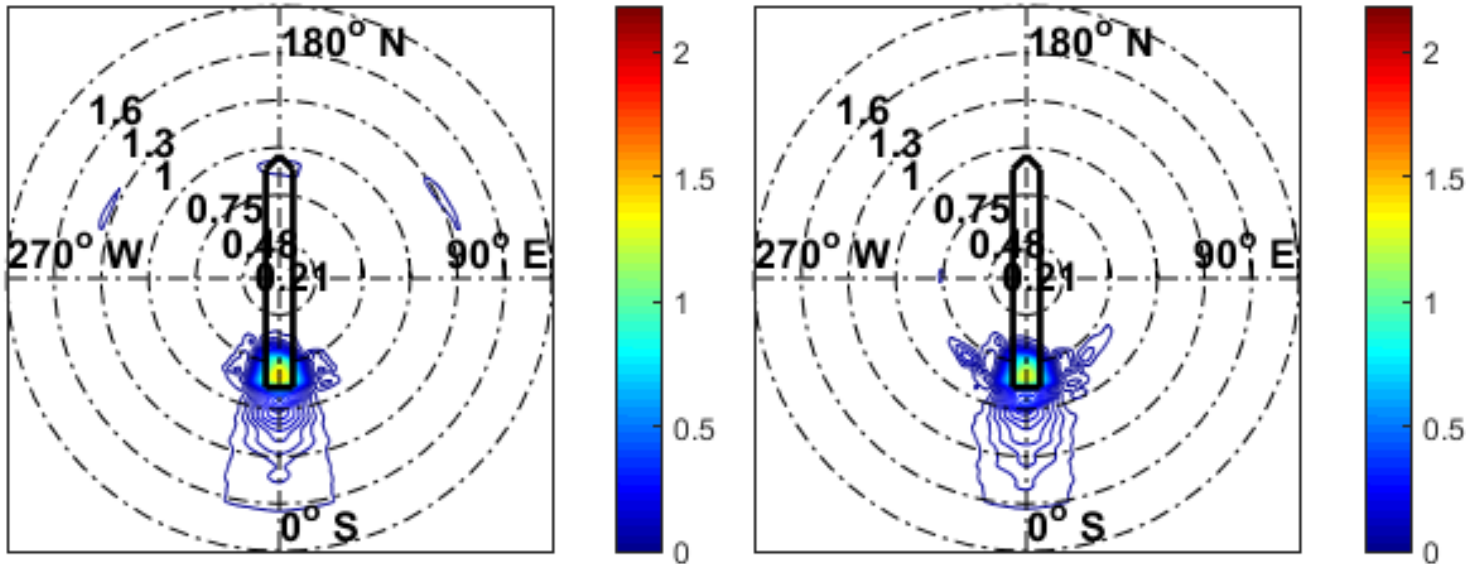

Source: Elaborated by the author.

Figure 115 - Medium $T_{P}$ wave, at $135^{\circ}$, estimated without wave-probes.

Exp.: $\mathrm{Hs}=1.3 \mathrm{~m} \mathrm{Tp}=12.0 \mathrm{~s} \beta_{0}=134^{\circ}$ Power $\left(\mathrm{m}^{2} . \mathrm{s}\right)$
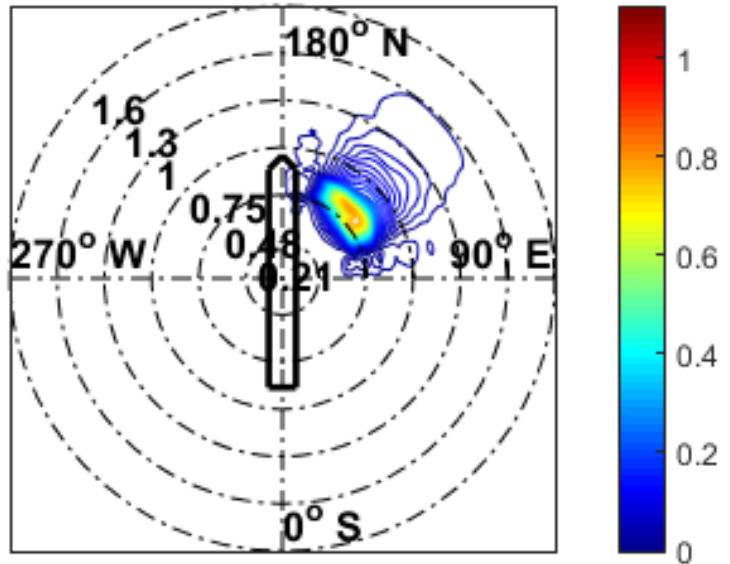

Est.: $\mathrm{Hs}=1.2 \mathrm{~m} \mathrm{Tp}=13.4 \mathrm{~s} \beta_{0}=153^{\circ}$ Power $\left(\mathrm{m}^{2} . \mathrm{s}\right)$
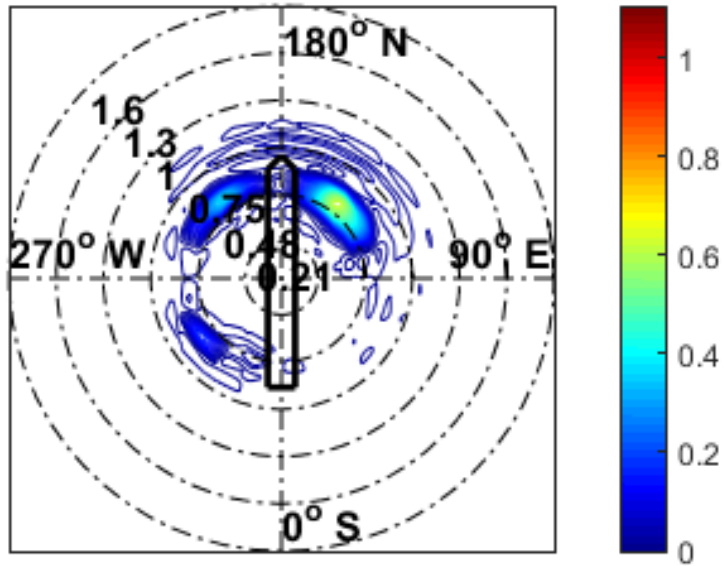

Source: Elaborated by the author. 
Figure 116 - Medium $T_{P}$ wave, at $135^{\circ}$, estimated with 2 wave-probes.

Exp.: $\mathrm{Hs}=1.3 \mathrm{~m} \mathrm{Tp}=12.0 \mathrm{~s} \beta_{0}=134^{\circ}$ Power $\left(\mathrm{m}^{2} . \mathrm{s}\right)$
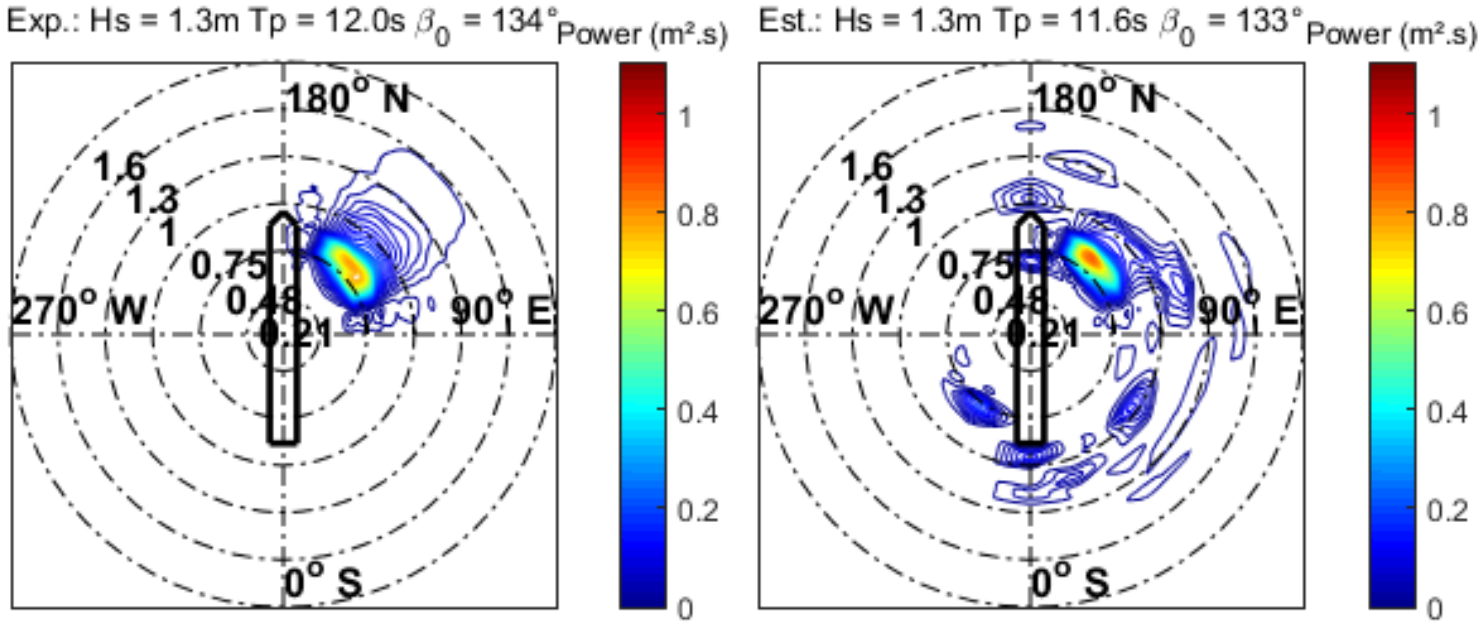

Source: Elaborated by the author.

Figure 117 - Medium $T_{P}$ wave, at $135^{\circ}$, estimated with 6 wave-probes.

Exp.: $\mathrm{Hs}=1.3 \mathrm{~m} \mathrm{Tp}=12.0 \mathrm{~s} \beta_{0}=134^{\circ}$ Power $\left(\mathrm{m}^{2} . \mathrm{s}\right)$
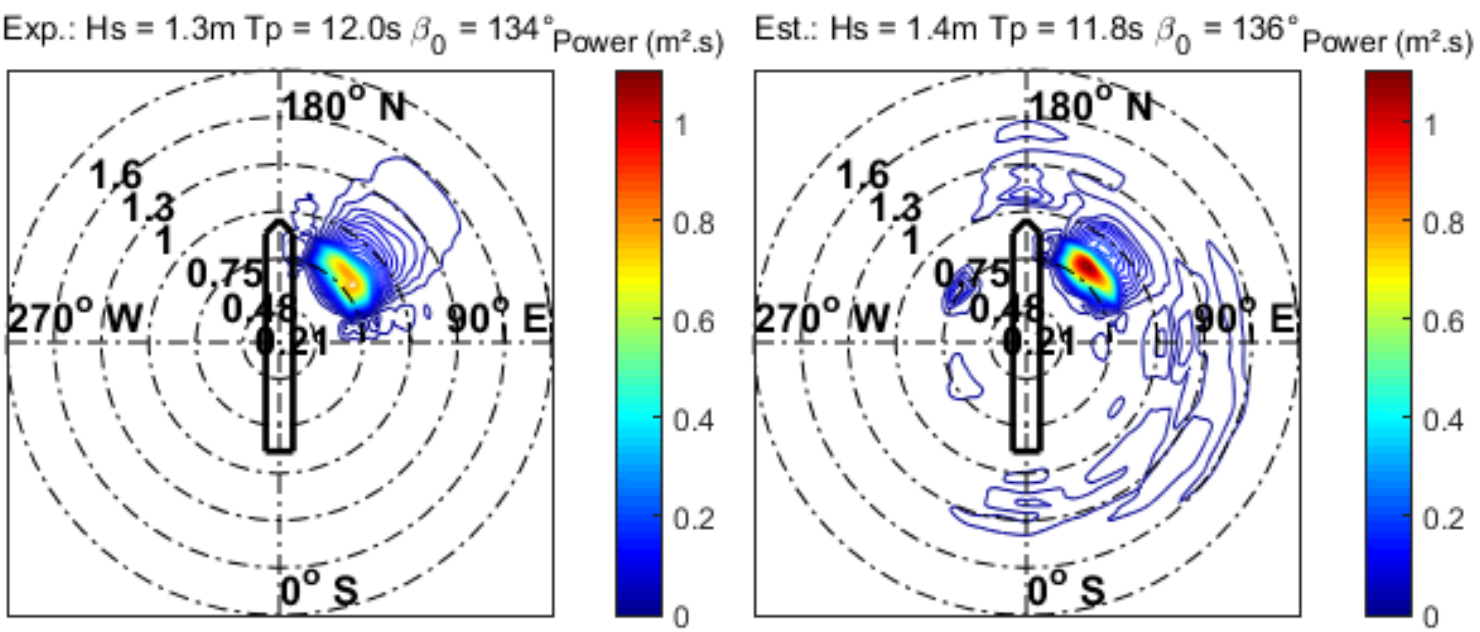

Source: Elaborated by the author.

For waves with peak period higher than $12 \mathrm{~s}$, coming from complicated directions, like $135^{\circ}$, the usage of a small number of wave-probes can lead to an estimation worse than estimation with only Sway-Heave-Pitch, due to the differences caused by the roll interference. This is easily solved by employing the wave-probes in frequencies until $0.52 \mathrm{rad} / \mathrm{s}, T=12 \mathrm{~s}$, using the procedure described in section 4.4, Optimization of Degrees of Freedom Usage by Frequency. Furthermore, if more waveprobes are used the problem is attenuated, and the algorithm provides good estimations. 
Figure 118 - High $T_{P}$ wave, coming from $135^{\circ}$, estimated without wave-probes.

Exp.: $\mathrm{Hs}=1.2 \mathrm{~m} \mathrm{Tp}=14.6 \mathrm{~s} \beta_{0}=133^{\circ}$ Power $\left(\mathrm{m}^{2} . \mathrm{s}\right)$

Est.: $\mathrm{Hs}=1.2 \mathrm{~m} \mathrm{Tp}=15.9 \mathrm{~s} \beta_{0}=138^{\circ}$ Power $\left(\mathrm{m}^{2} . \mathrm{s}\right)$
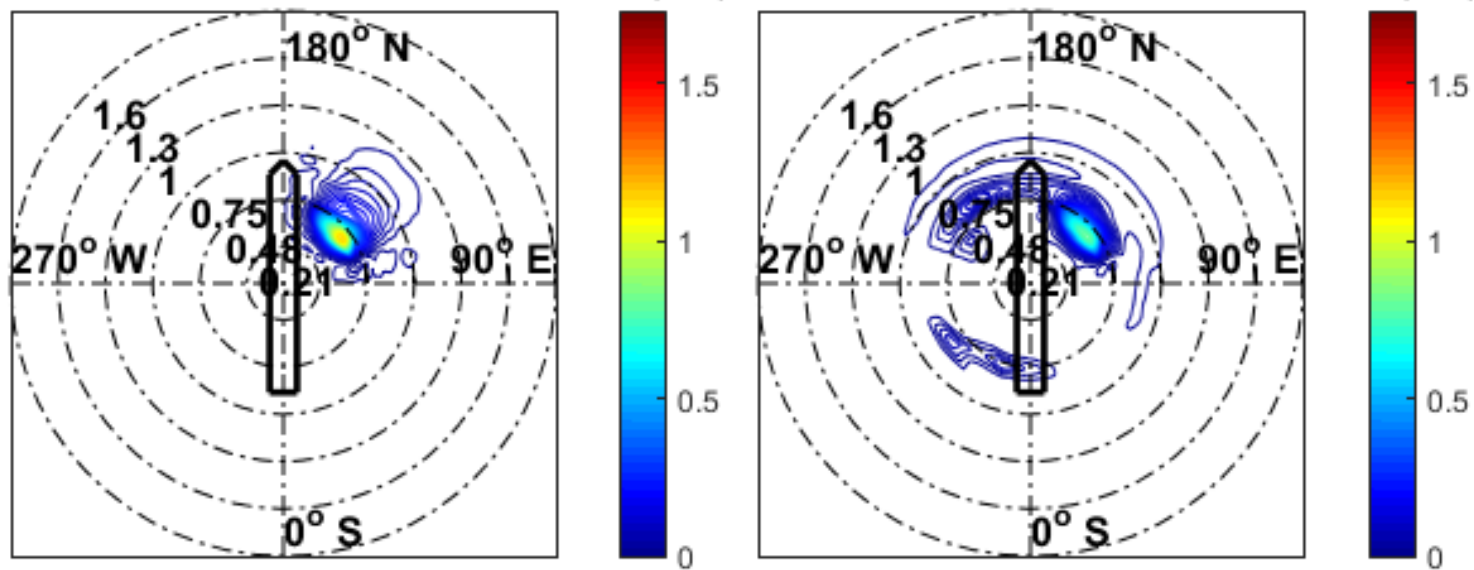

Source: Elaborated by the author.

Figure 119 - High $T_{P}$ wave, coming from $135^{\circ}$, estimated with 2 wave-probes.

Exp.: $\mathrm{Hs}=1.2 \mathrm{~m} \mathrm{Tp}=14.6 \mathrm{~s} \beta_{0}=133^{\circ}$ Power $\left(\mathrm{m}^{2} . \mathrm{s}\right)$
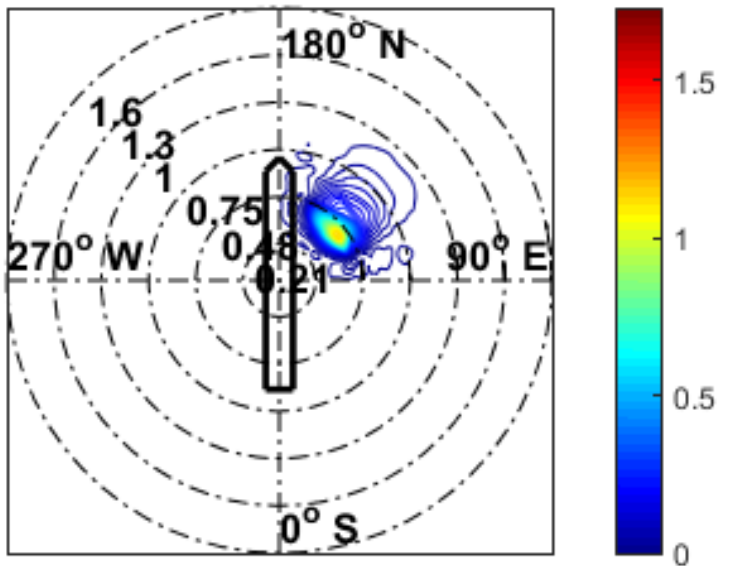

Est.: $\mathrm{Hs}=1.3 \mathrm{~m} \mathrm{Tp}=14.2 \mathrm{~s} \beta_{0}=152^{\circ}$ Power $\left(\mathrm{m}^{2} . \mathrm{s}\right)$
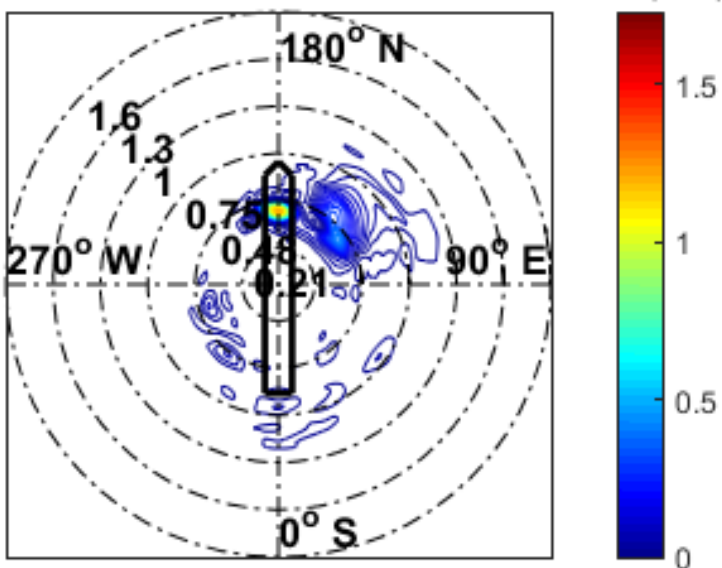

Source: Elaborated by the author.

Figure $120-$ High $T_{P}$ wave, coming from $135^{\circ}$, estimated with 6 wave-probes.

Exp.: $\mathrm{Hs}=1.2 \mathrm{~m} \mathrm{Tp}=14.6 \mathrm{~s} \beta_{0}=133^{\circ}$ Power $\left(\mathrm{m}^{2} . \mathrm{s}\right)$
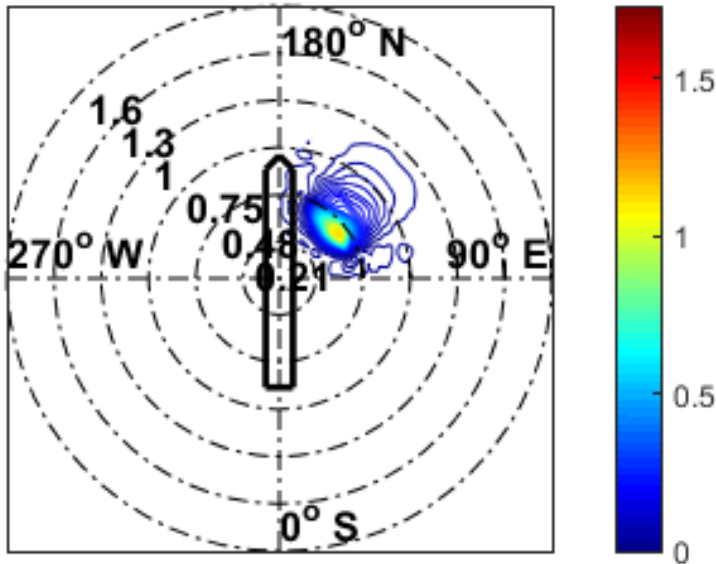

Est.: $\mathrm{Hs}=1.3 \mathrm{~m} \mathrm{Tp}=14.3 \mathrm{~s} \beta_{0}=141^{\circ}$ Power $\left(\mathrm{m}^{2} . \mathrm{s}\right)$
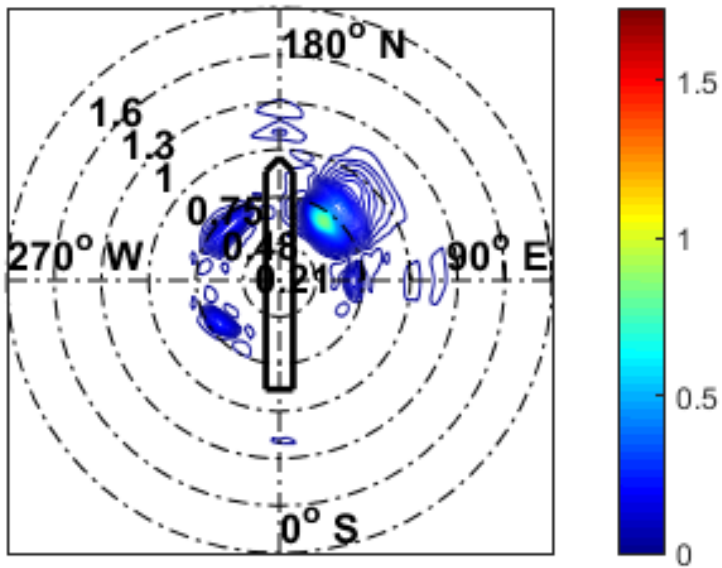

Source: Elaborated by the author. 


\subsubsection{Validation of the wave-probes usage for extreme draft}

The same analysis can be made for the extreme draft set of experiments.

Figure 121 - Histograms for the ballasted condition.
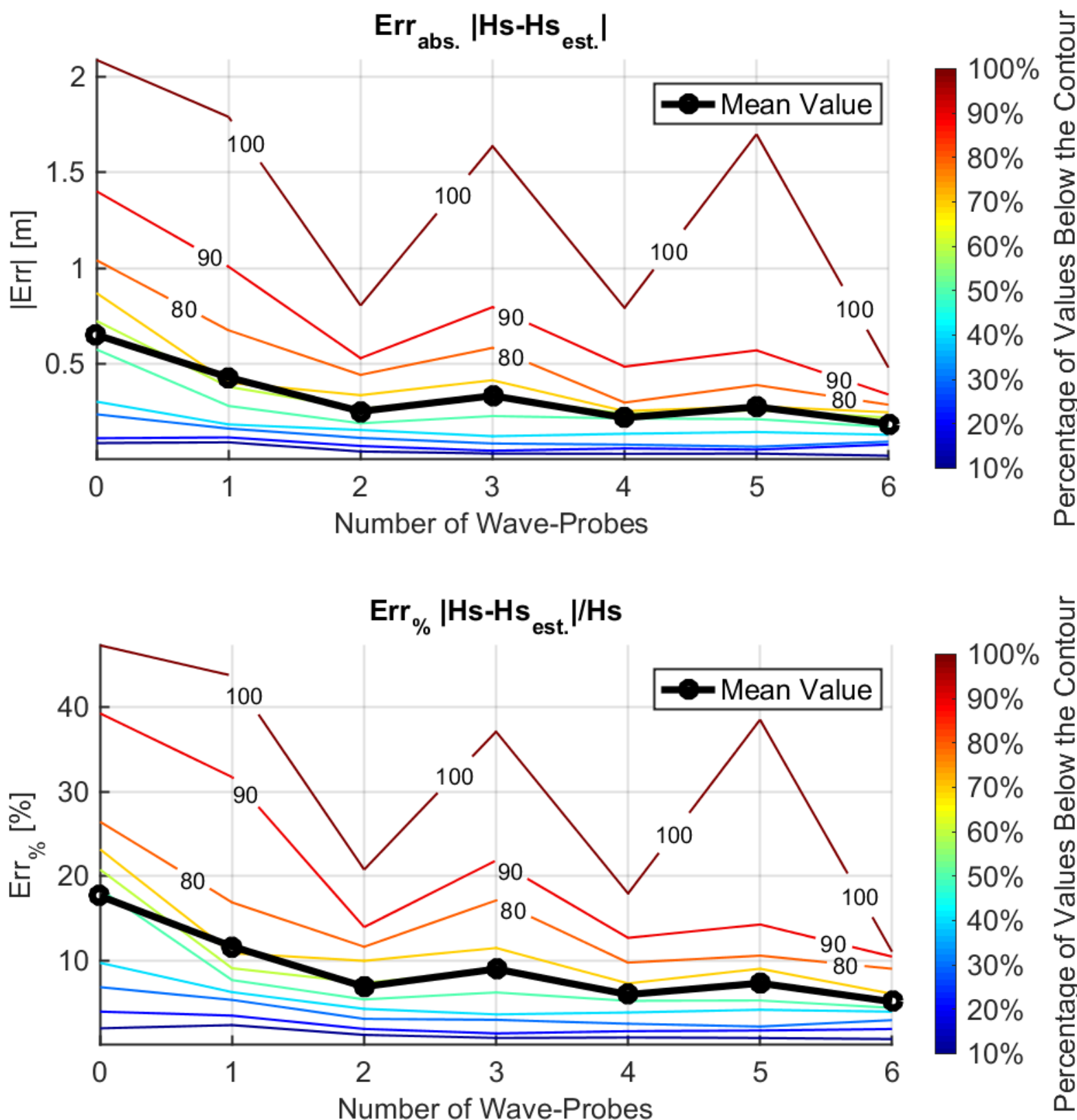

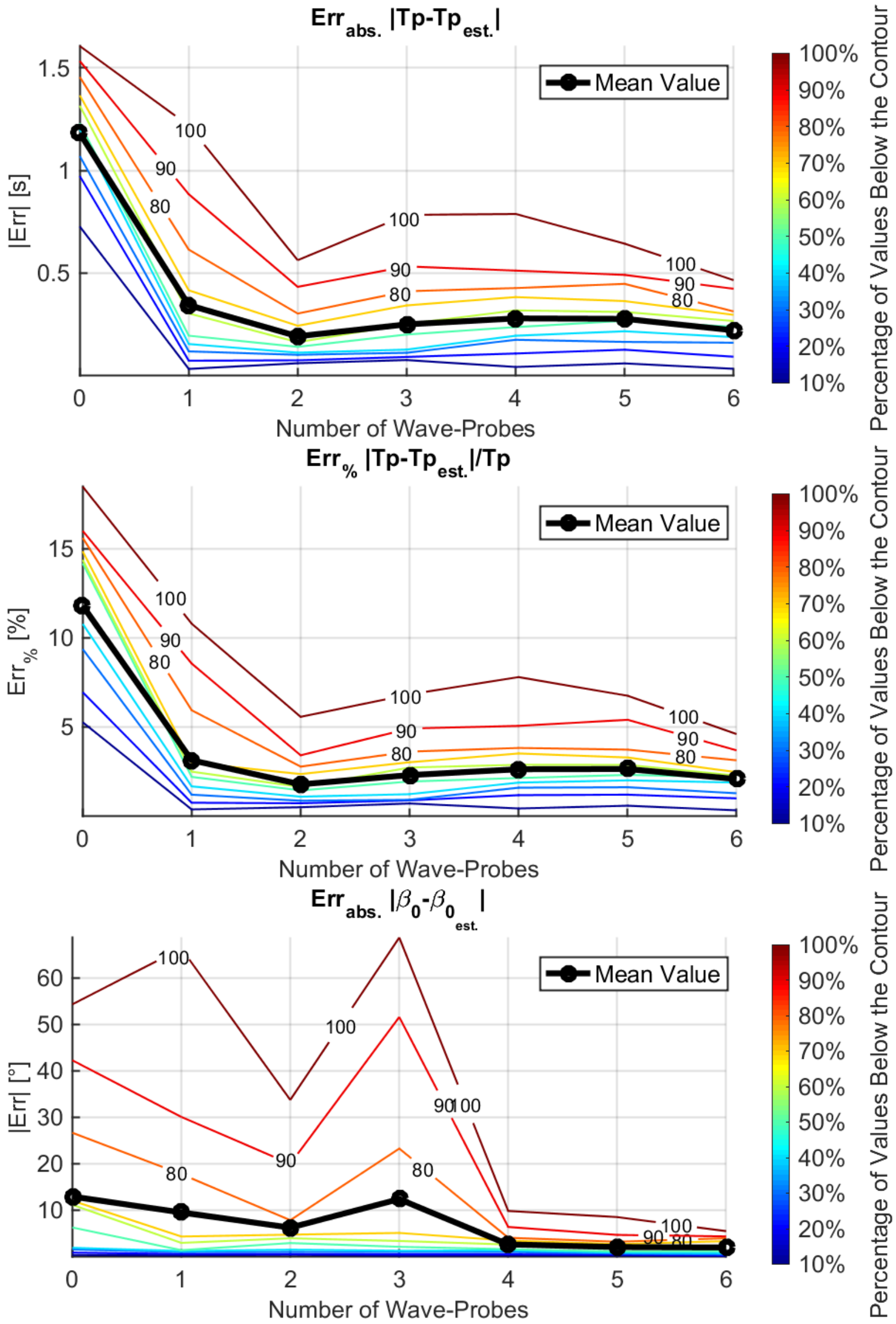

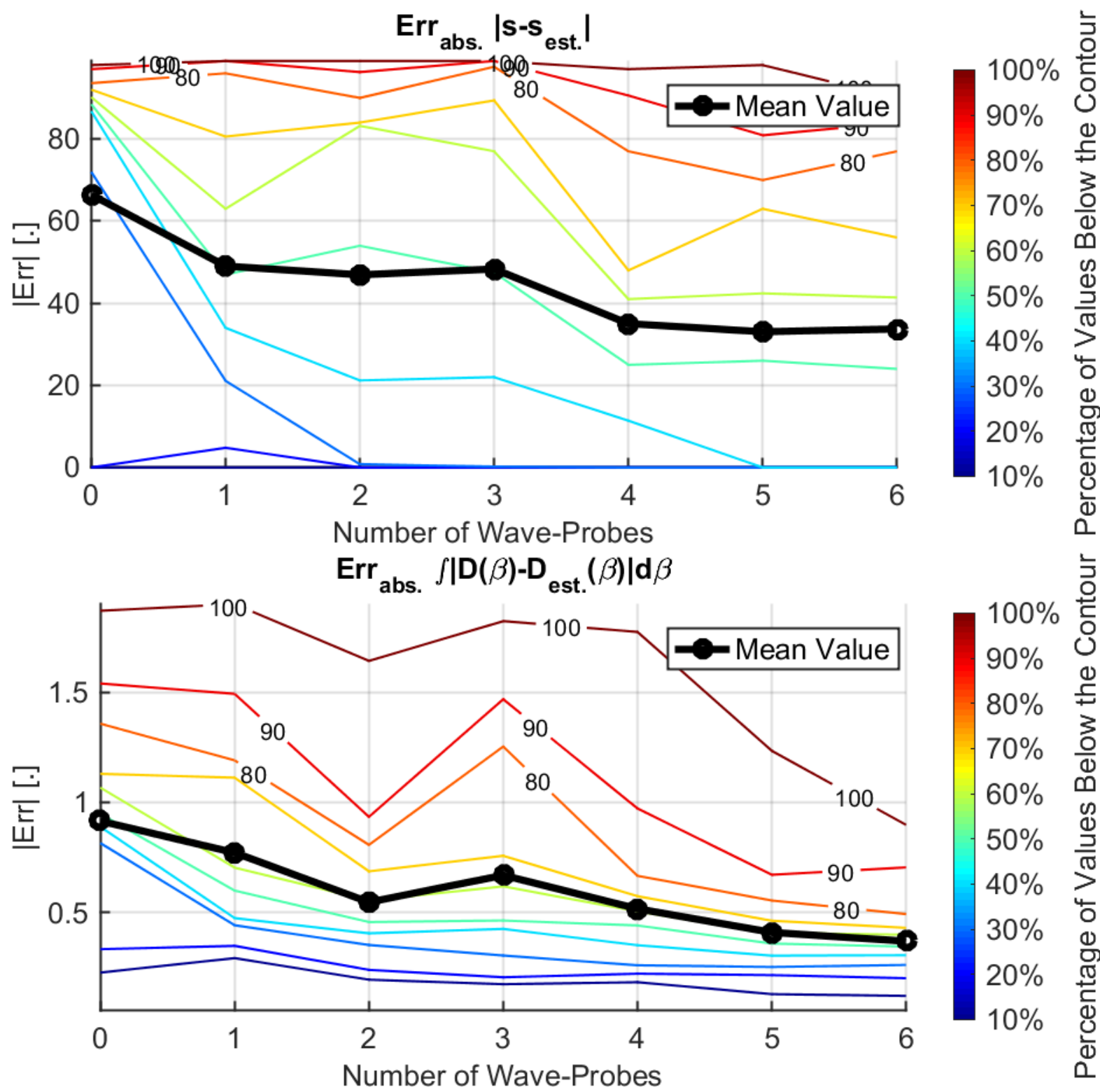

Source: Elaborated by the author.

For the ballasted condition, i.e., low draft, the conclusions are quite equivalent to the conclusions drawn for the intermediate draft, Figure 121. The usage of just one wave-probe is already able to achieve a considerably improvement, in all the criteria; the usage of at least 2 wave-probes is recommended if it is desired to improve the most the energy spectral density estimation; the usage of at least 4 wave-probes is recommended if it is desired to know the mean direction of the incoming wave; and the usage of 6 probes is recommended for directional estimation.

The general result is consistent with the intermediate case, Figure 122 to Figure 124. Furthermore, Following the RAO analysis, even for a small number of waveprobes, waves with $T_{P}>12 \mathrm{~s}$ are well estimated, Figure 125 to Figure 127. 
Figure 122 - Ballasted condition, medium $T_{P}$, without probes.

Exp.: $\mathrm{Hs}=2.8 \mathrm{~m} \mathrm{Tp}=10.1 \mathrm{~s} \beta_{0}=135^{\circ}$ Power $\left(\mathrm{m}^{2} . \mathrm{s}\right)$
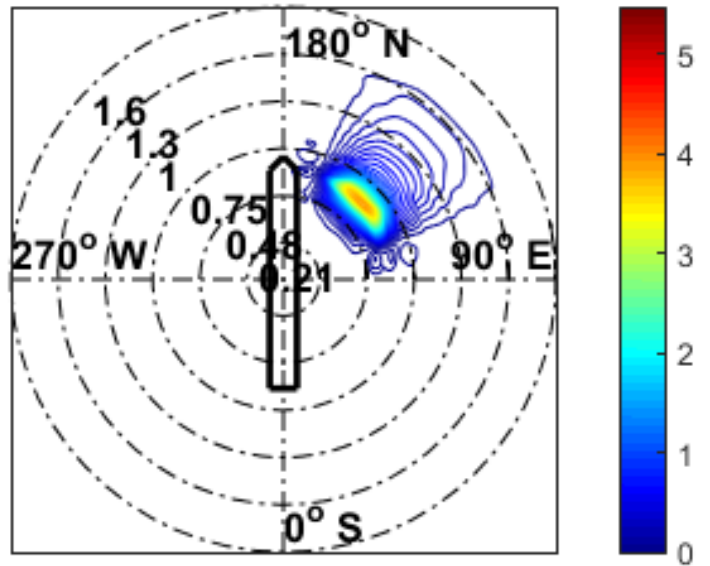

Est.: $\mathrm{Hs}=2.2 \mathrm{~m} \mathrm{Tp}=11.6 \mathrm{~s} \beta_{0}=180^{\circ}$ Power $\left(\mathrm{m}^{2} . \mathrm{s}\right)$

Source: Elaborated by the author.

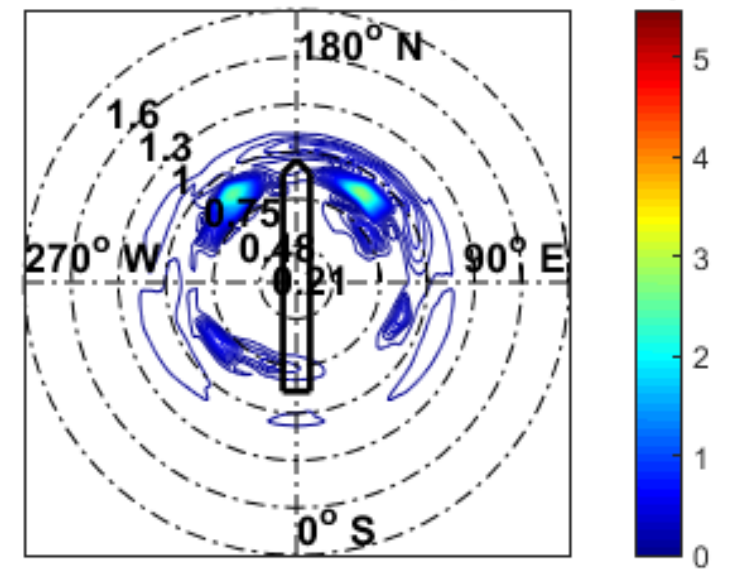

Figure 123 - Ballasted condition, medium $T_{P}$, with 2 probes.

Exp.: $\mathrm{Hs}=2.8 \mathrm{~m} \mathrm{Tp}=10.1 \mathrm{~s} \beta_{0}=135^{\circ}$ Power $\left(\mathrm{m}^{2} . \mathrm{s}\right)$

Est.: $\mathrm{Hs}=3.2 \mathrm{~m} \mathrm{Tp}=10.0 \mathrm{~s} \beta_{0}=125^{\circ}$ Power $\left(\mathrm{m}^{2} . \mathrm{s}\right)$
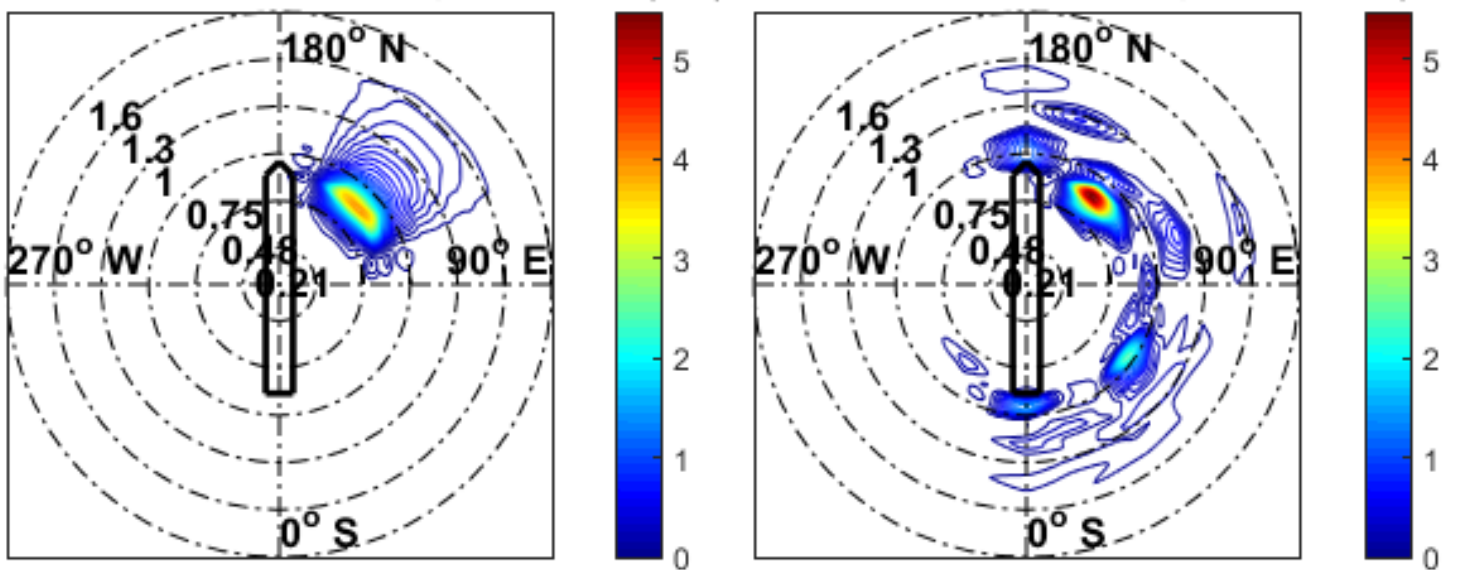

Source: Elaborated by the author.

Figure 124 - Ballasted condition, medium $T_{P}$, with 6 probes.

Exp.: $\mathrm{Hs}=2.8 \mathrm{~m} \mathrm{Tp}=10.1 \mathrm{~s} \beta_{0}=135^{\circ}$ Power $\left(\mathrm{m}^{2} . \mathrm{s}\right)$

Est.: $\mathrm{Hs}=3.1 \mathrm{~m} \mathrm{Tp}=10.0 \mathrm{~s} \beta_{0}=141^{\circ}$ Power $\left(\mathrm{m}^{2} . \mathrm{s}\right)$
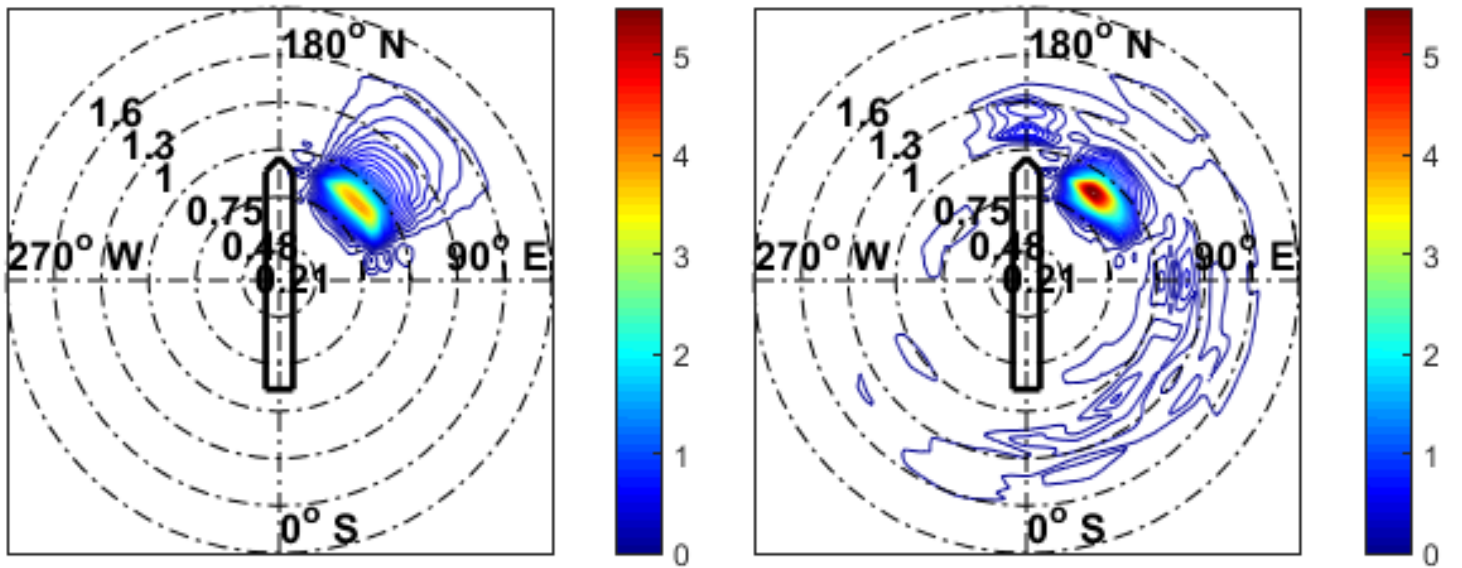

Source: Elaborated by the author. 
Figure 125 - Ballasted condition, high $T_{P}$, without probes.

Exp.: $\mathrm{Hs}=2.8 \mathrm{~m} \mathrm{Tp}=13.8 \mathrm{~s} \beta_{0}=135^{\circ}$ Power $\left(\mathrm{m}^{2} . \mathrm{s}\right)$
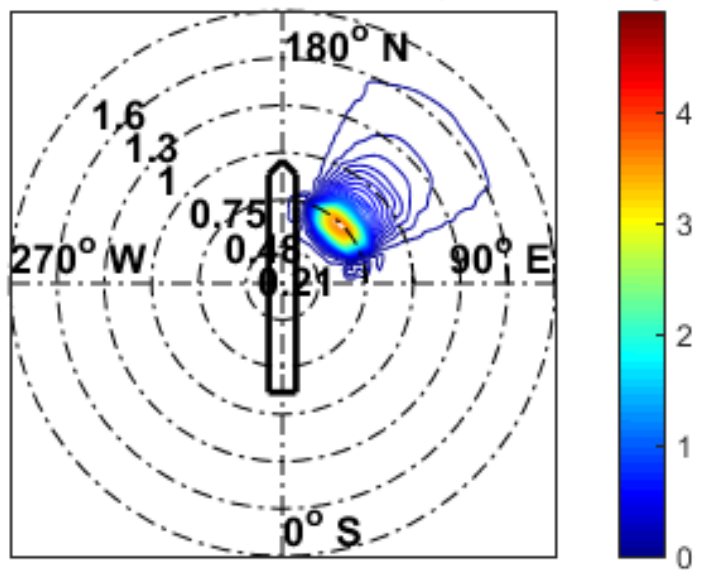

Est.: $\mathrm{Hs}=2.7 \mathrm{~m} \mathrm{Tp}=14.7 \mathrm{~s} \beta_{0}=124^{\circ}$ Power $\left(\mathrm{m}^{2} . \mathrm{s}\right)$

Source: Elaborated by the author.

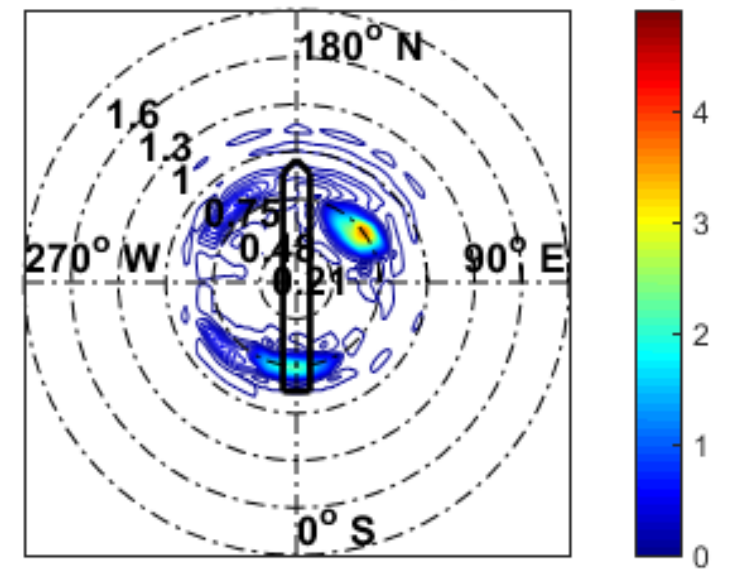

Figure 126 - Ballasted condition, high $T_{P}$, with 2 probes.

Exp.: $\mathrm{Hs}=2.8 \mathrm{~m} \mathrm{Tp}=13.8 \mathrm{~s} \beta_{0}=135^{\circ}$ Power $\left(\mathrm{m}^{2} . \mathrm{s}\right)$

Est.: $\mathrm{Hs}=3.0 \mathrm{~m} \mathrm{Tp}=13.3 \mathrm{~s} \beta_{0}=139^{\circ}$ Power $\left(\mathrm{m}^{2} . \mathrm{s}\right)$
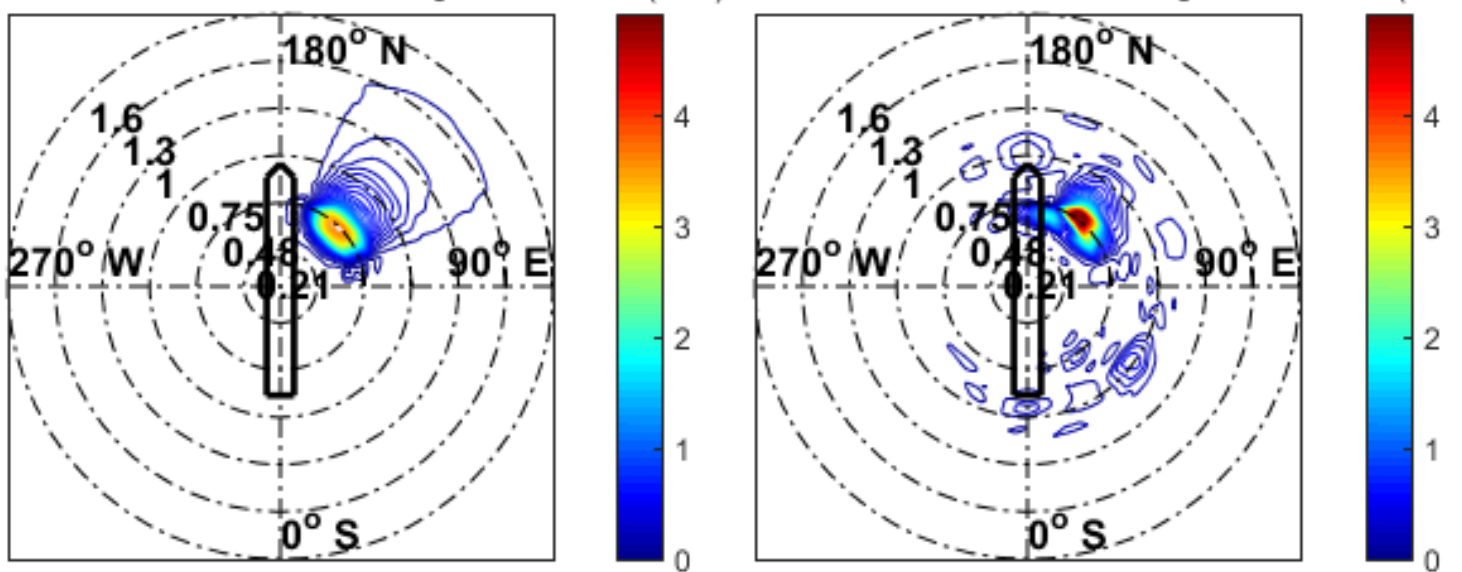

Source: Elaborates by the author.

Figure 127 - Ballasted condition, high $T_{P}$, with 6 probes.

Exp.: $\mathrm{Hs}=2.8 \mathrm{~m} \mathrm{Tp}=13.8 \mathrm{~s} \beta_{0}=135^{\circ}$ Power $\left(\mathrm{m}^{2} . \mathrm{s}\right)$

Est.: $\mathrm{Hs}=3.0 \mathrm{~m} \mathrm{Tp}=13.5 \mathrm{~s} \beta_{0}=139^{\circ}$ Power $\left(\mathrm{m}^{2} . \mathrm{s}\right)$
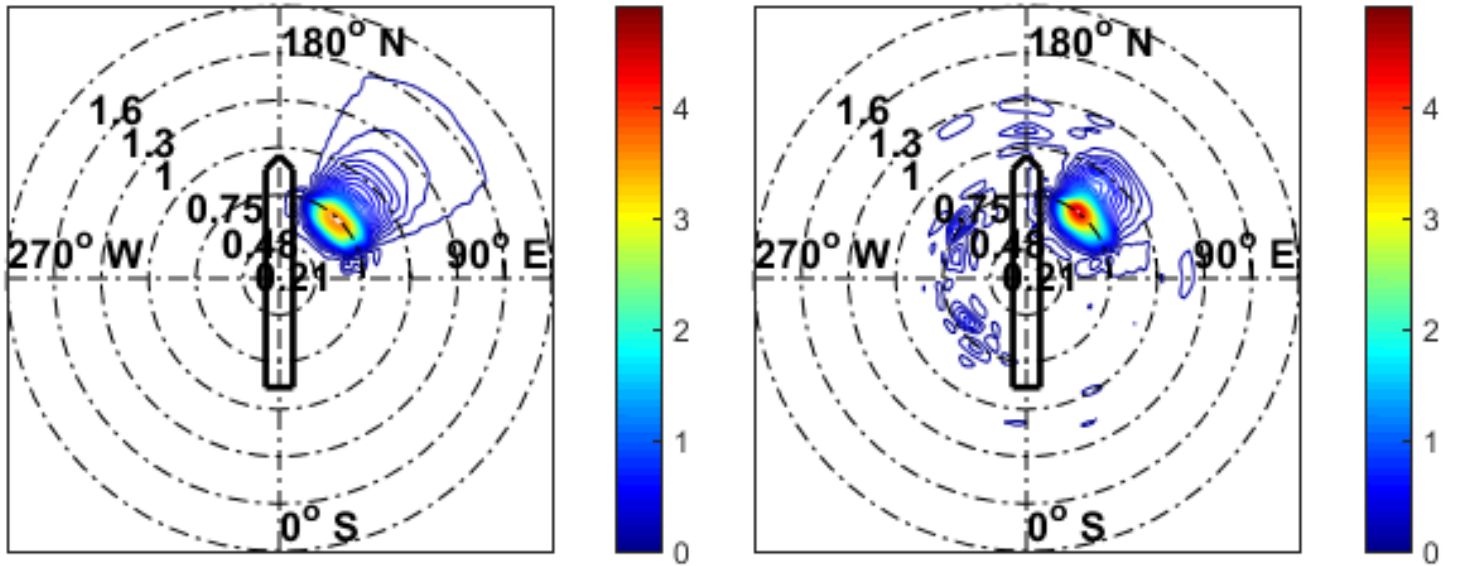

Source: Elaborated by the author. 
Figure 128 - Histograms for the full draft condition.

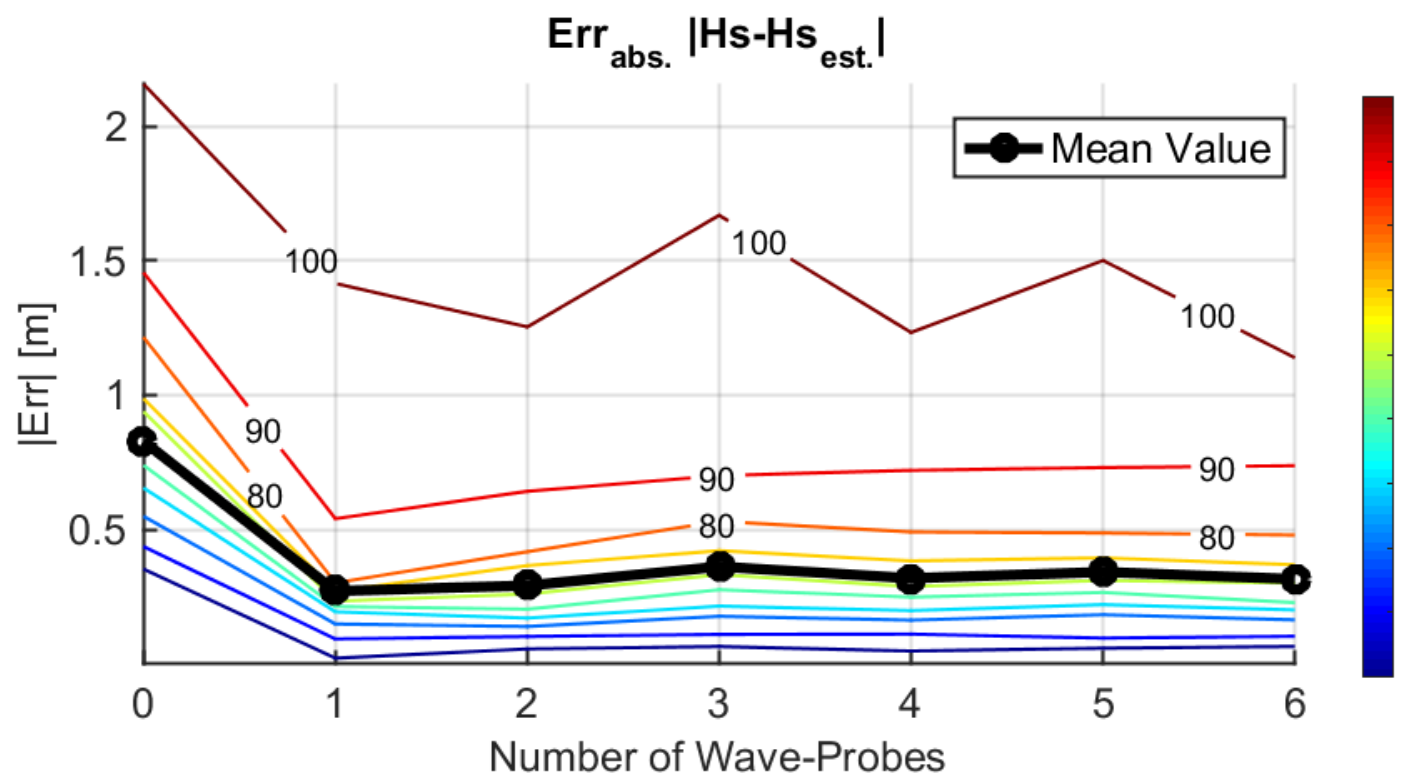

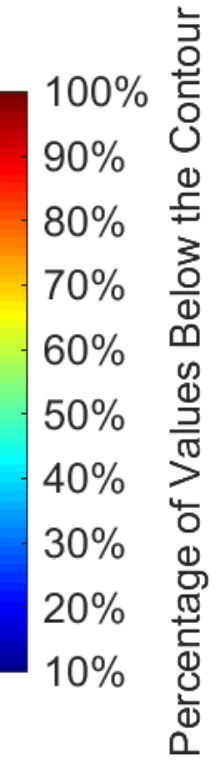

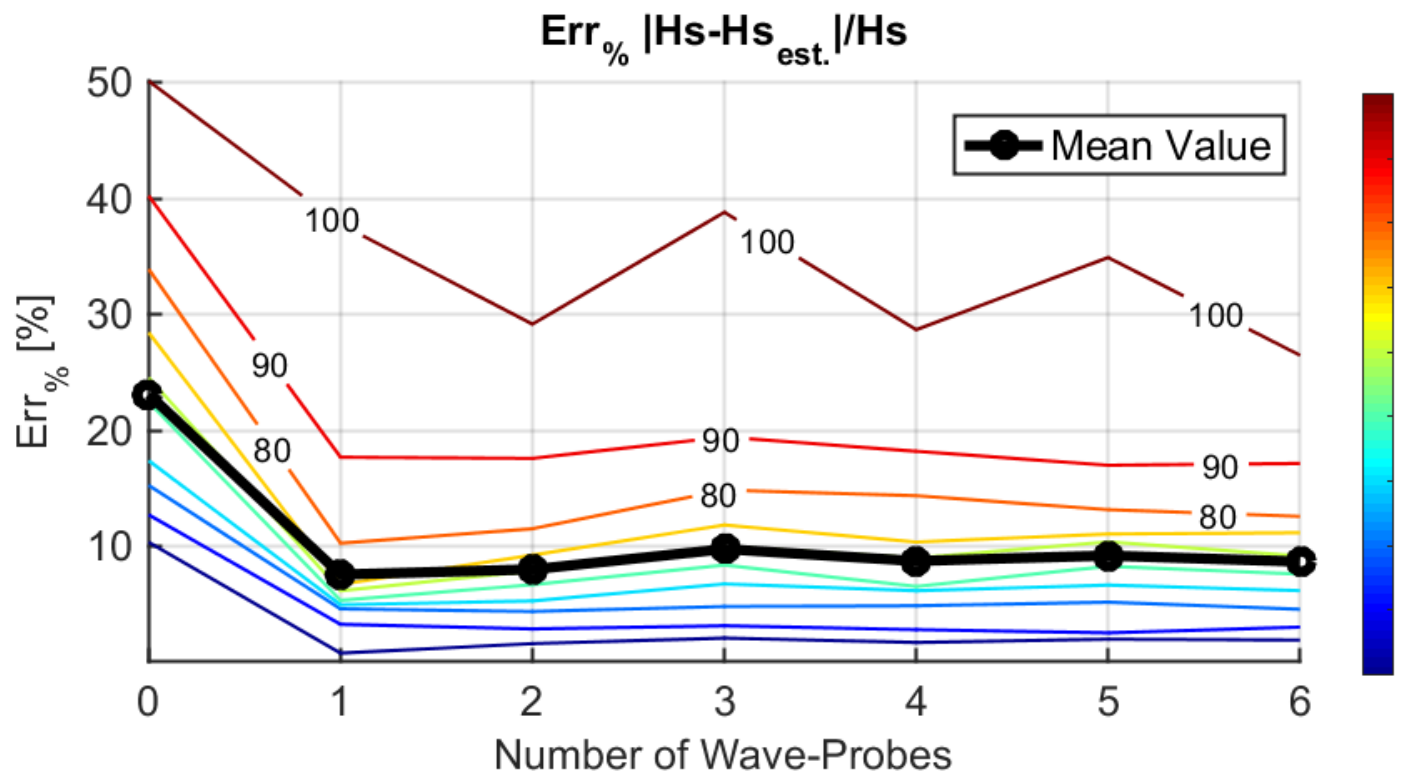

$100 \%$
$90 \%$
$80 \%$
$70 \%$
$60 \%$
$50 \%$
$40 \%$
$30 \%$
$20 \%$
$10 \%$

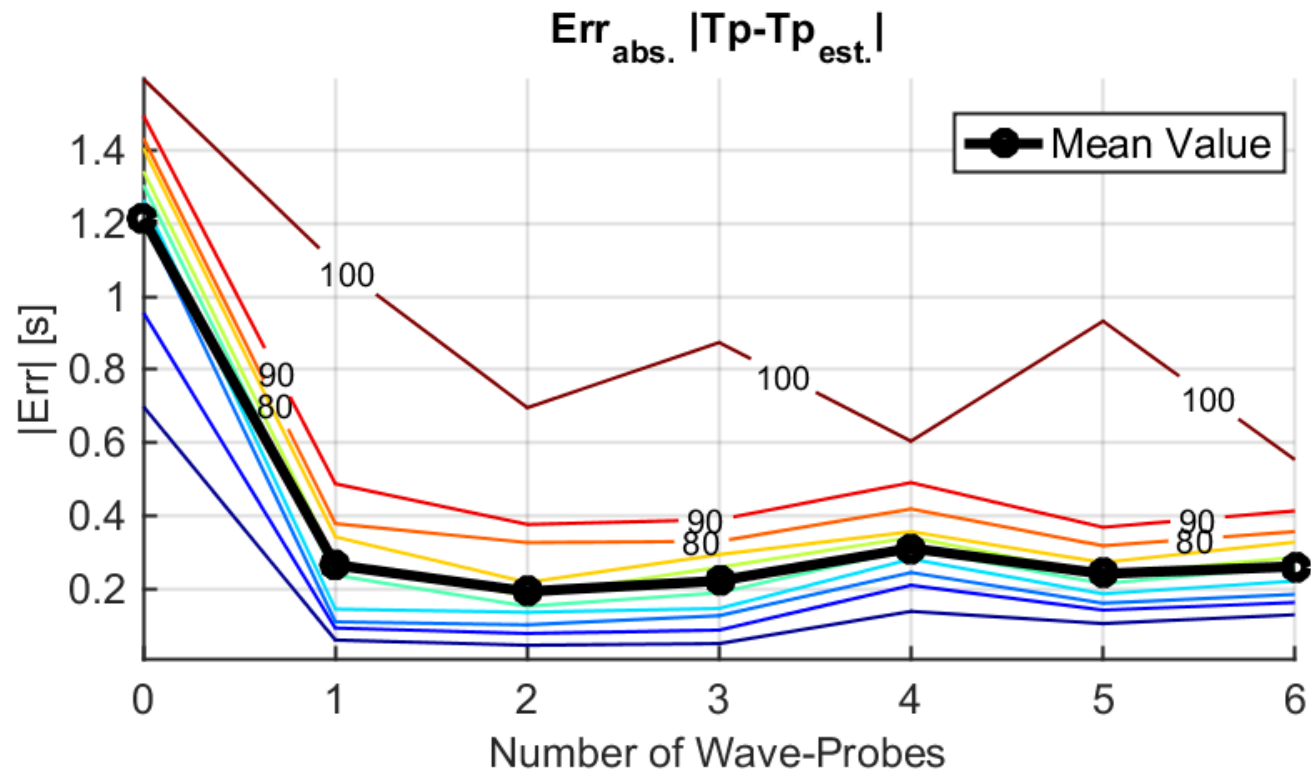



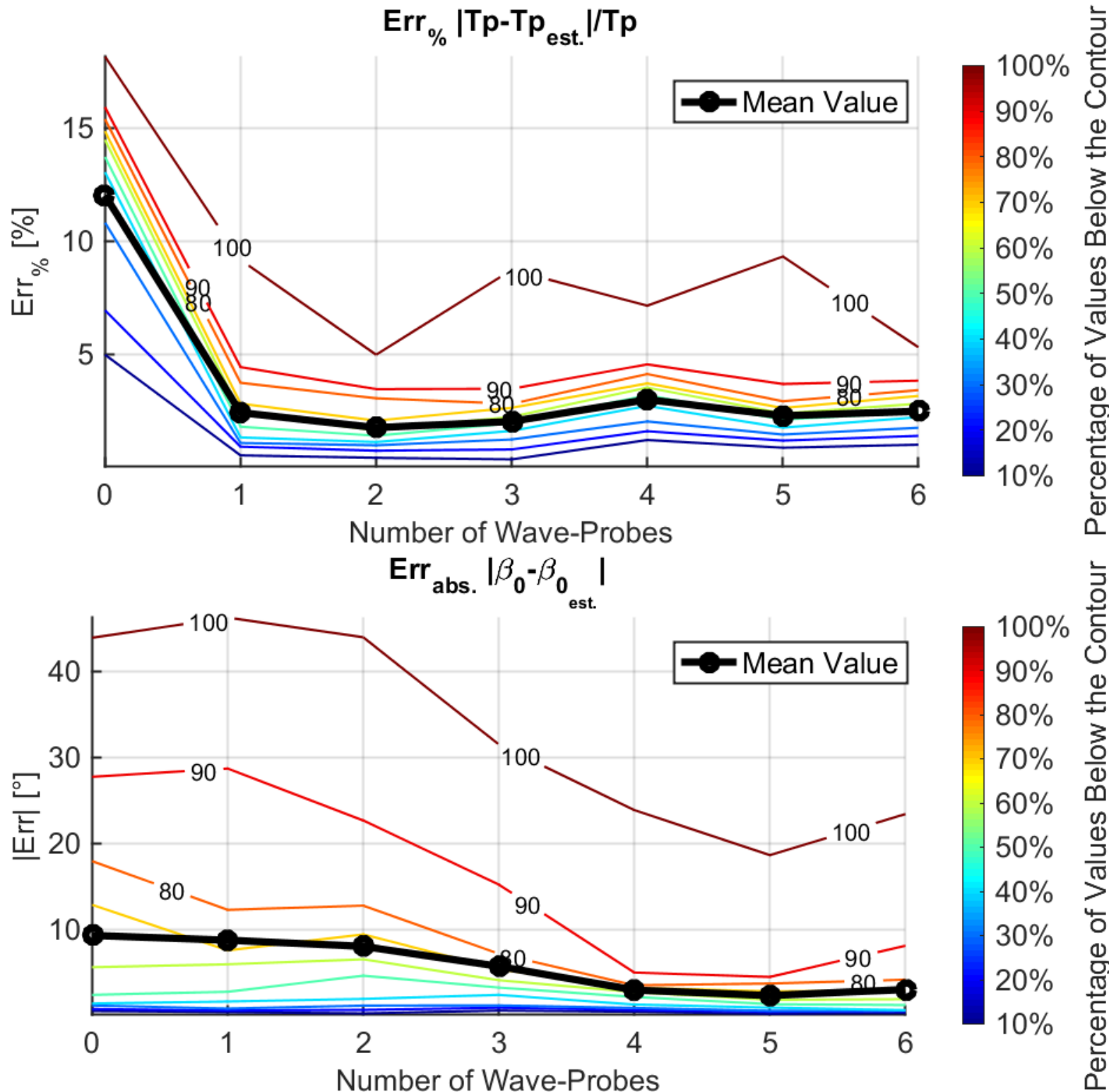

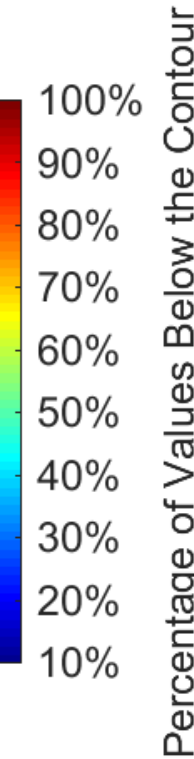

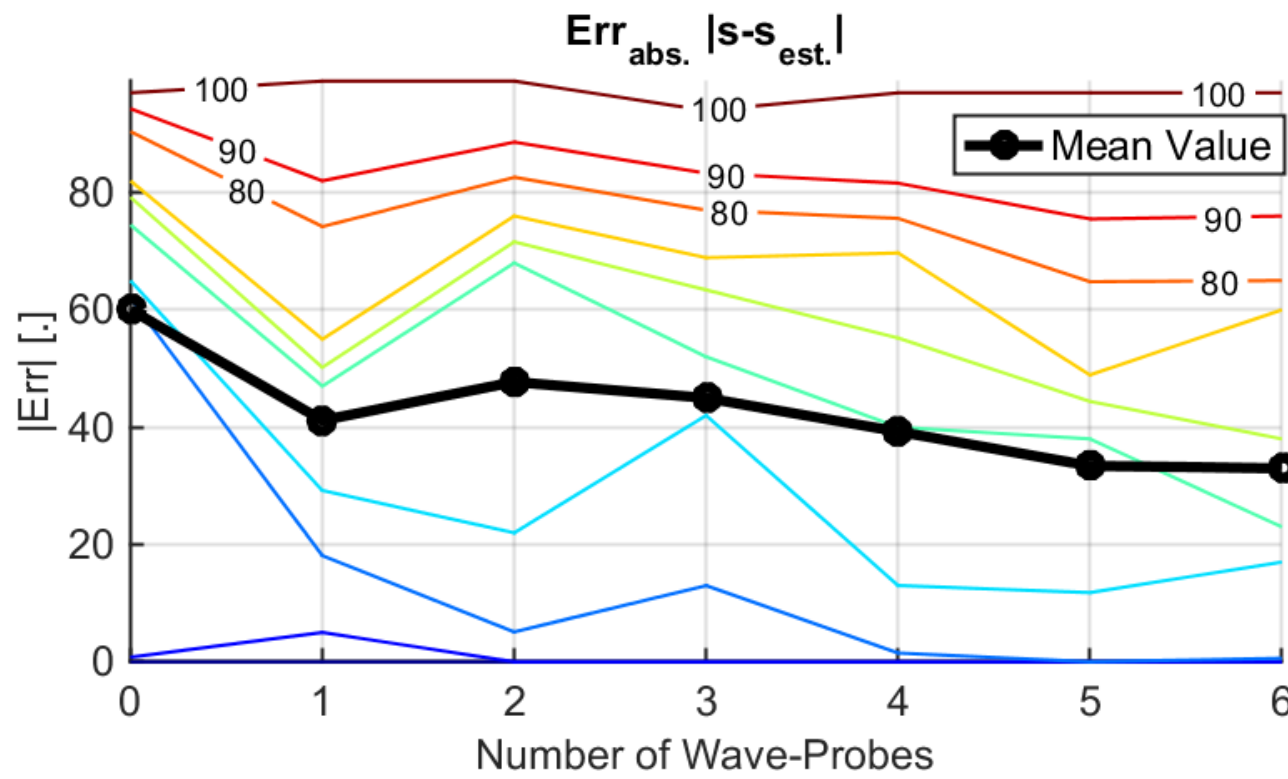




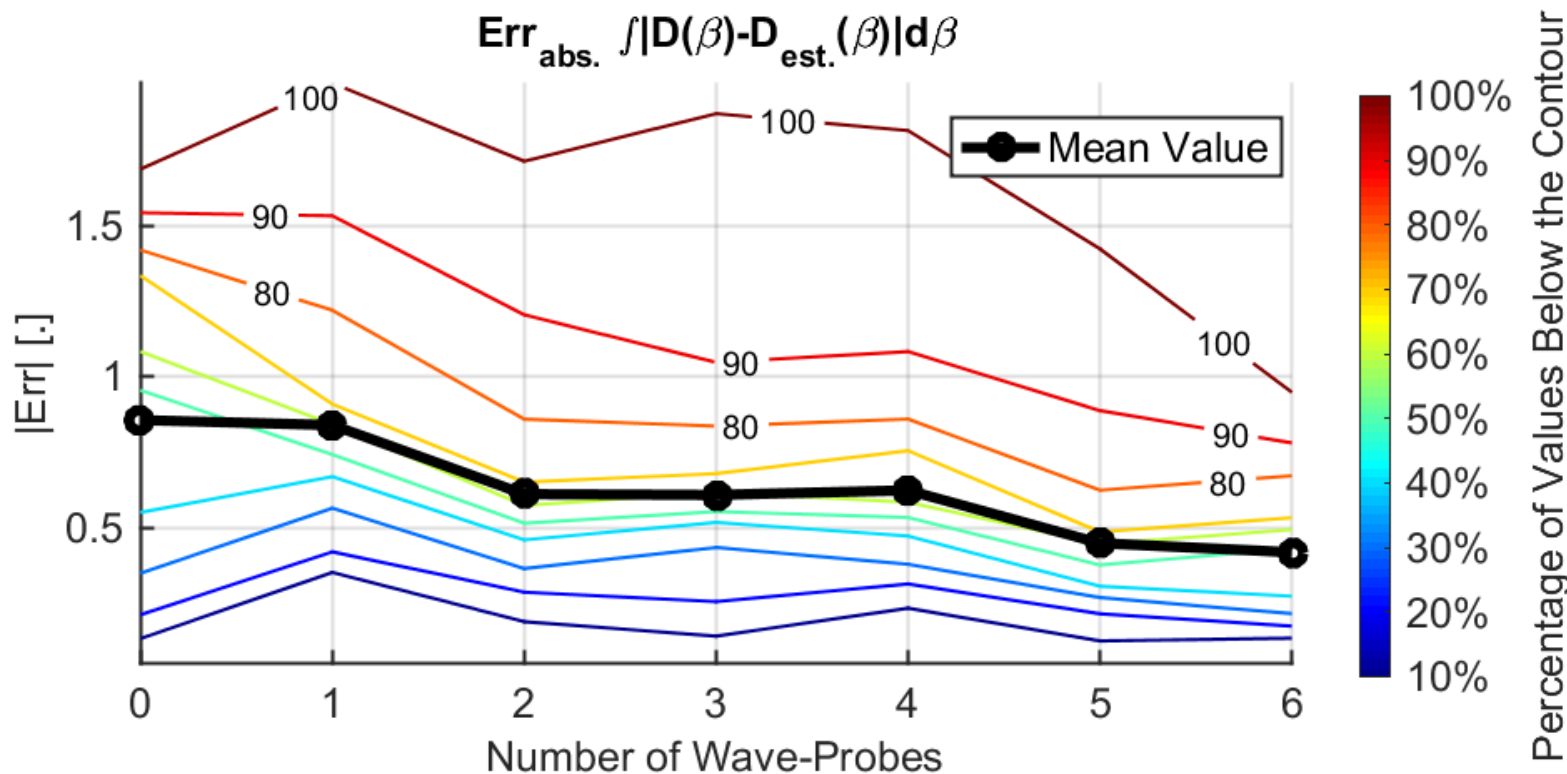

Source: Elaborated by the author.

For the full draft condition, the convergence for $H_{S}$ and $T_{P}$ seems to happen faster, with only one wave-probe; with the mean direction still converging to four probes; and the directional estimation converging to six probes. In general, a behavior also following the conclusions of the intermediate draft. In this draft situation, however, one probe is already enough to estimate waves with medium and high $T_{P}$; although for low $T_{P}$ one probe is still insufficient, Figure 129 to Figure 134 .

Figure 129 - Full draft condition, low $T_{P}$, without probes.
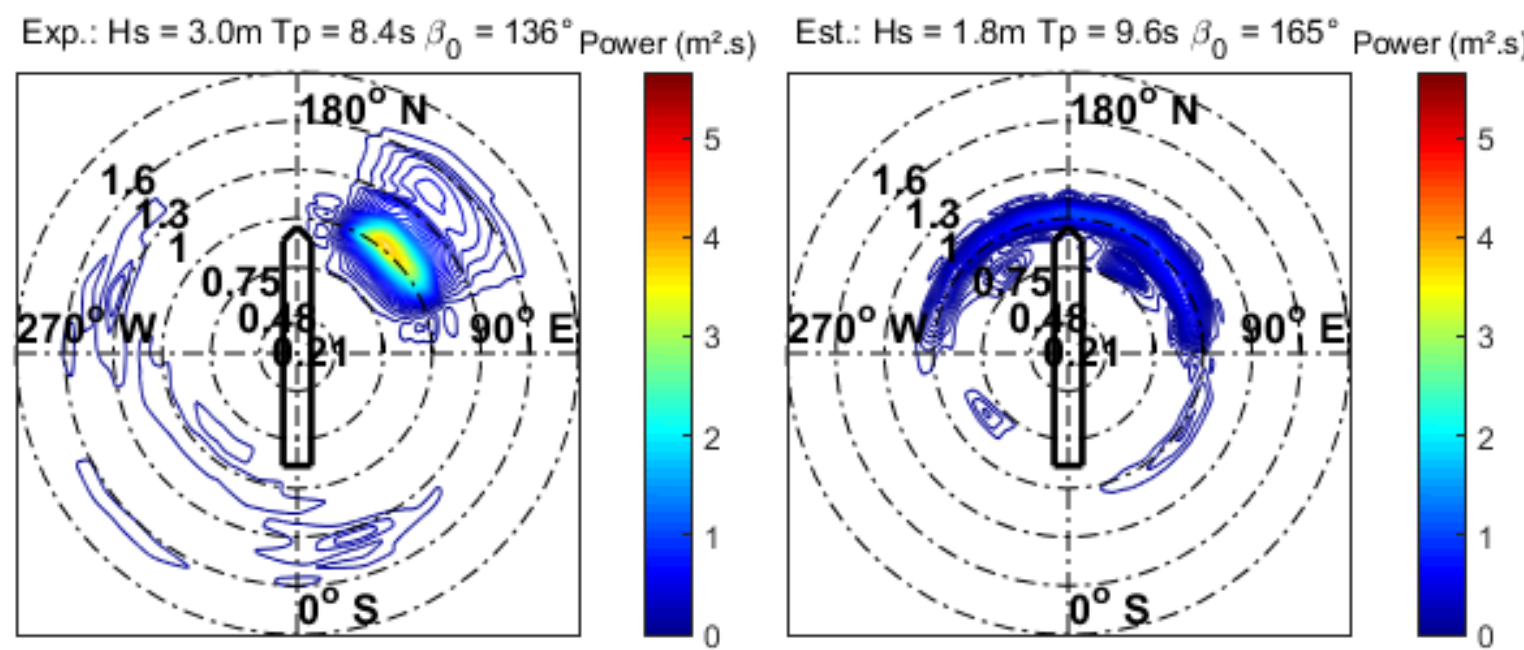

Source: Elaborated by the author. 
Figure 130 - Full draft condition, low $T_{P}$, with 1 probe.

Exp.: $\mathrm{Hs}=3.0 \mathrm{~m} \mathrm{Tp}=8.4 \mathrm{~s} \beta_{0}=136^{\circ}$ Power $\left(\mathrm{m}^{2} . \mathrm{s}\right)$
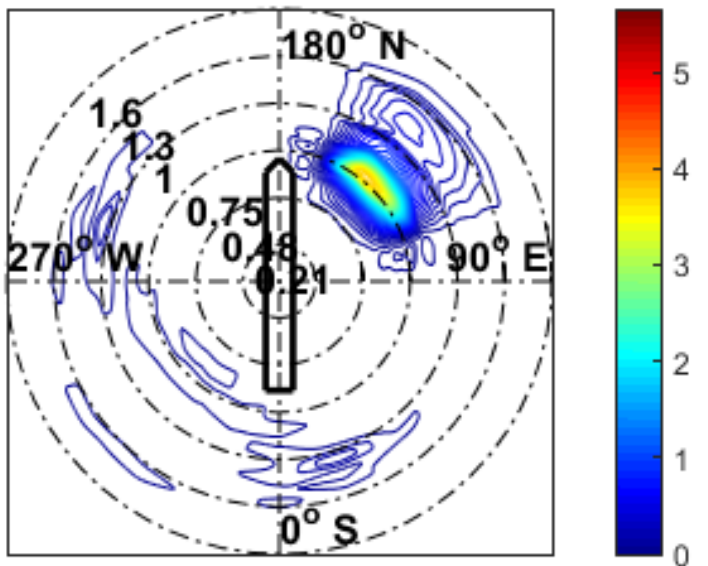

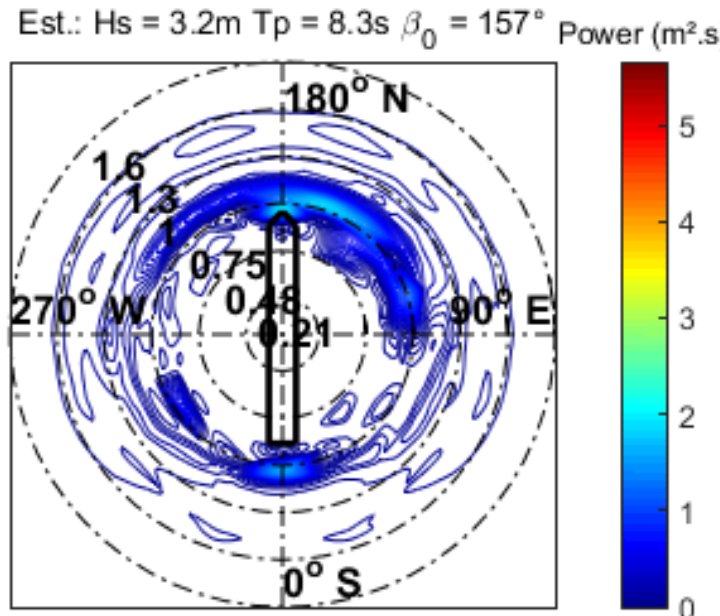

Source: Elaborated by the author.

Figure 131 - Full draft condition, medium $T_{P}$, without probes.

Exp.: $\mathrm{Hs}=2.8 \mathrm{~m} \mathrm{Tp}=10.1 \mathrm{~s} \beta_{0}=134^{\circ}$ Power $\left(\mathrm{m}^{2} . \mathrm{s}\right)$

Est.: $\mathrm{Hs}=1.8 \mathrm{~m} \mathrm{Tp}=11.7 \mathrm{~s} \beta_{0}=149^{\circ}$ Power $\left(\mathrm{m}^{2} . \mathrm{s}\right)$
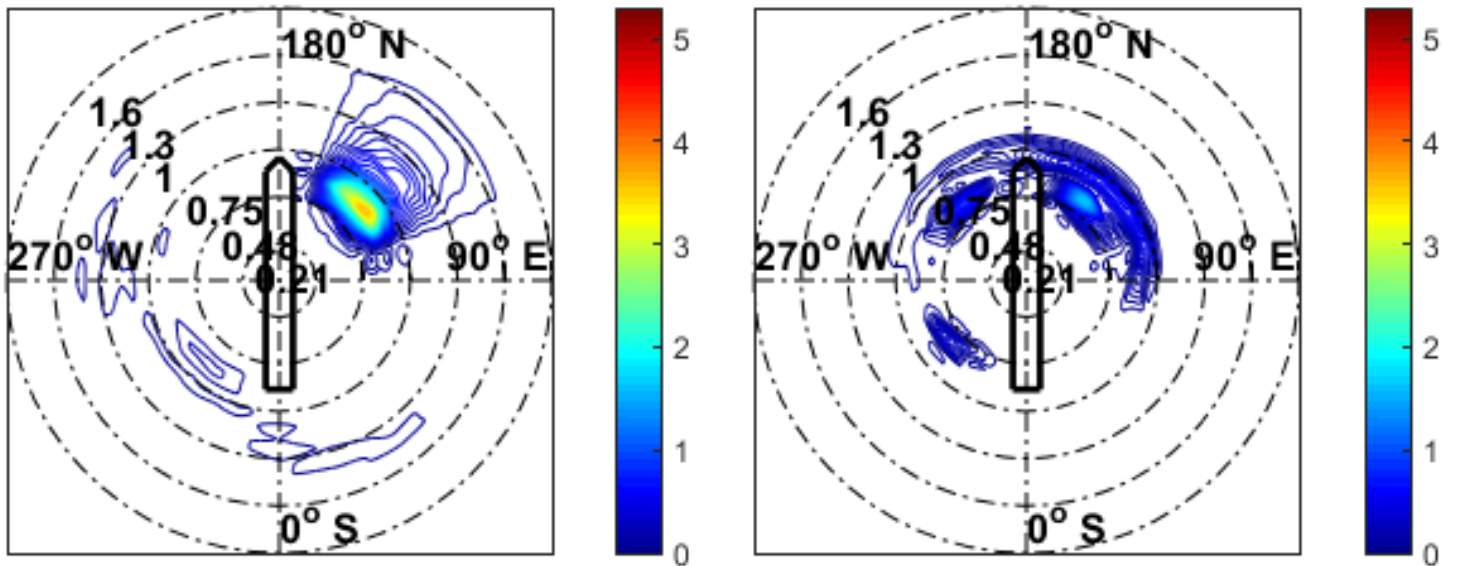

Source: Elaborated by the author.

Figure 132 - Full draft condition, medium $T_{P}$, with 1 probe.

Exp.: $\mathrm{Hs}=2.8 \mathrm{~m} \mathrm{Tp}=10.1 \mathrm{~s} \beta_{0}=134^{\circ}$ Power $\left(\mathrm{m}^{2} . \mathrm{s}\right)$

Est.: Hs $=3.1 \mathrm{~m} \mathrm{Tp}=10.1 \mathrm{~s} \beta_{0}=144^{\circ}$ Power $\left(\mathrm{m}^{2} . \mathrm{s}\right)$
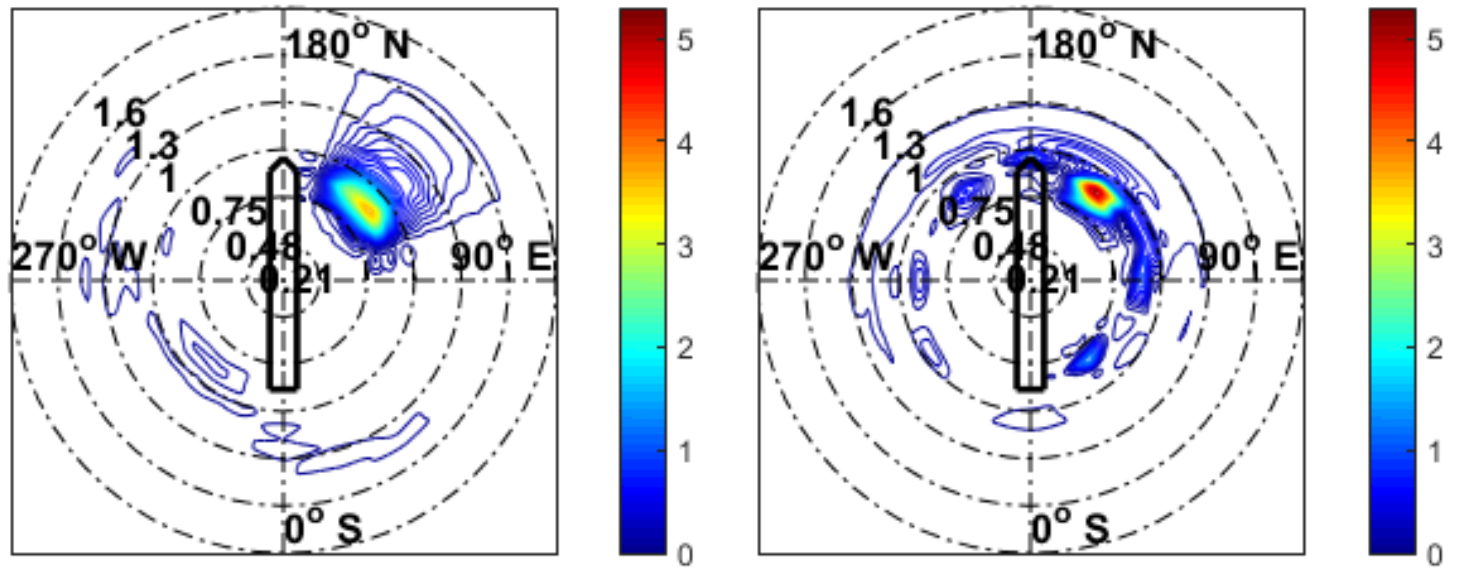

Source: Elaborated by the author. 
Figure 133 - Full draft condition, high $T_{P}$, without probes.

Exp.: $\mathrm{Hs}=2.9 \mathrm{~m} \mathrm{Tp}=14.0 \mathrm{~s} \beta_{0}=133^{\circ}$ Power $\left(\mathrm{m}^{2} . \mathrm{s}\right)$
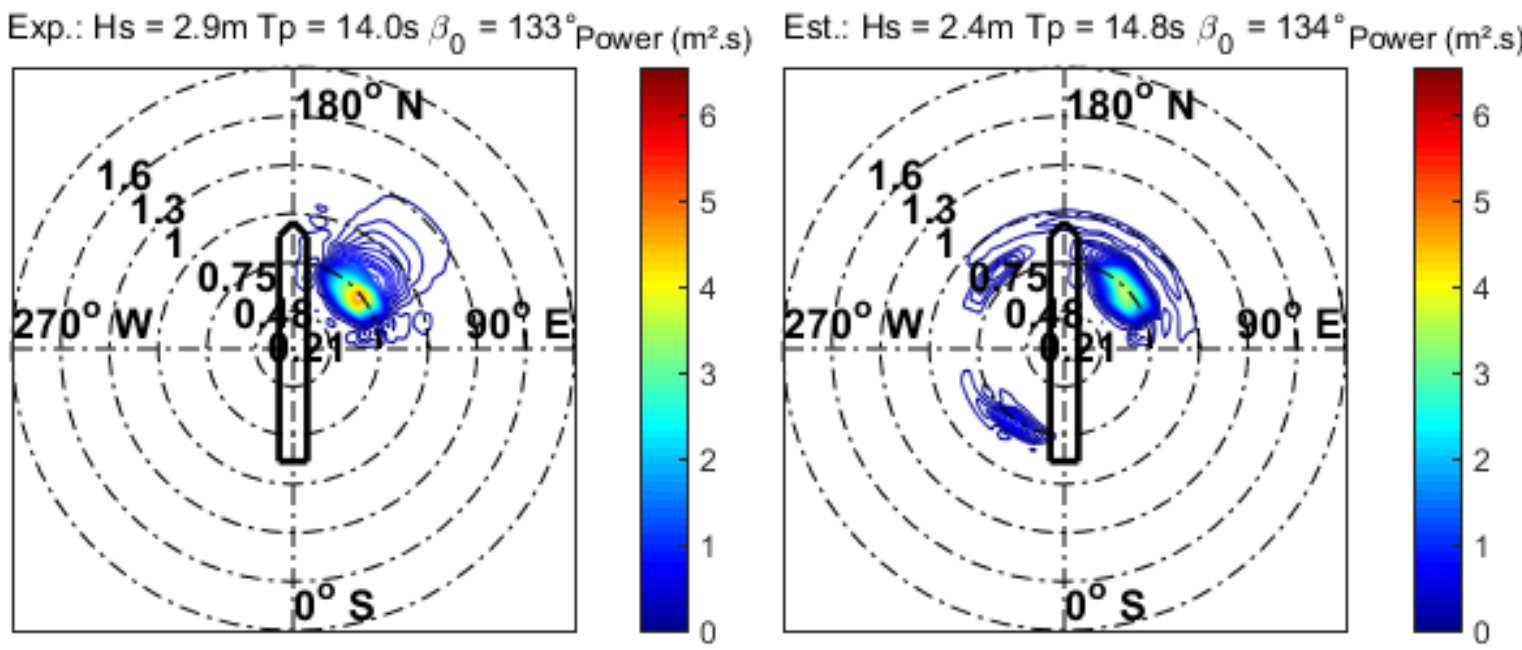

Source: Elaborated by the authors.

Figure 134 - Full draft condition, high $T_{P}$, with 1 probe.

Exp.: $\mathrm{Hs}=2.9 \mathrm{~m} \mathrm{Tp}=14.0 \mathrm{~s} \beta_{0}=133^{\circ}$ Power $\left(\mathrm{m}^{2} . \mathrm{s}\right)$

Est.: $\mathrm{Hs}=2.9 \mathrm{~m} \mathrm{Tp}=13.7 \mathrm{~s} \beta_{0}=135^{\circ}$ Power $\left(\mathrm{m}^{2} . \mathrm{s}\right)$
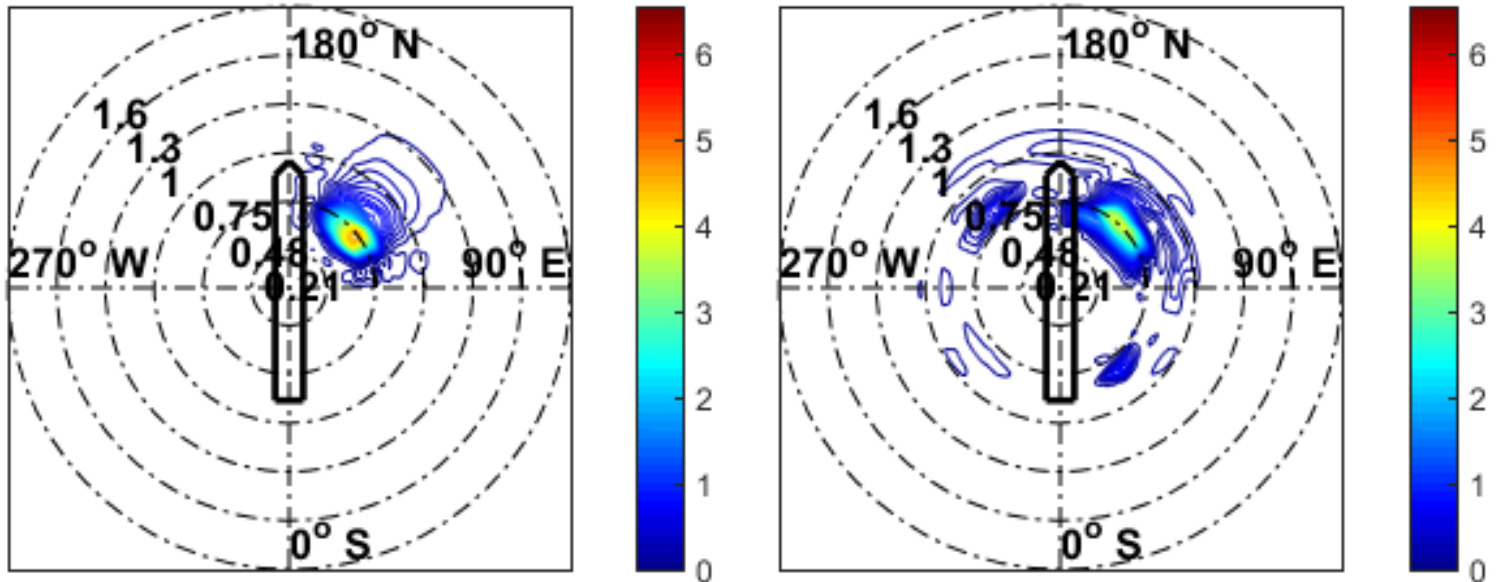

Source: Elaborated by the author.

\subsubsection{Validation of the wave-probes usage for bimodal seas}

The final analysis concerns the validation of the estimation of bimodal seas. In this case, the parametric estimation for each peak is not a simple task, so only the global parameters $H_{S}, T_{P}$ and $\beta_{0}$ will be taken in account. The histograms are shown in Figure 135. 
Figure 135 - Histograms of the estimation of bimodal seas.

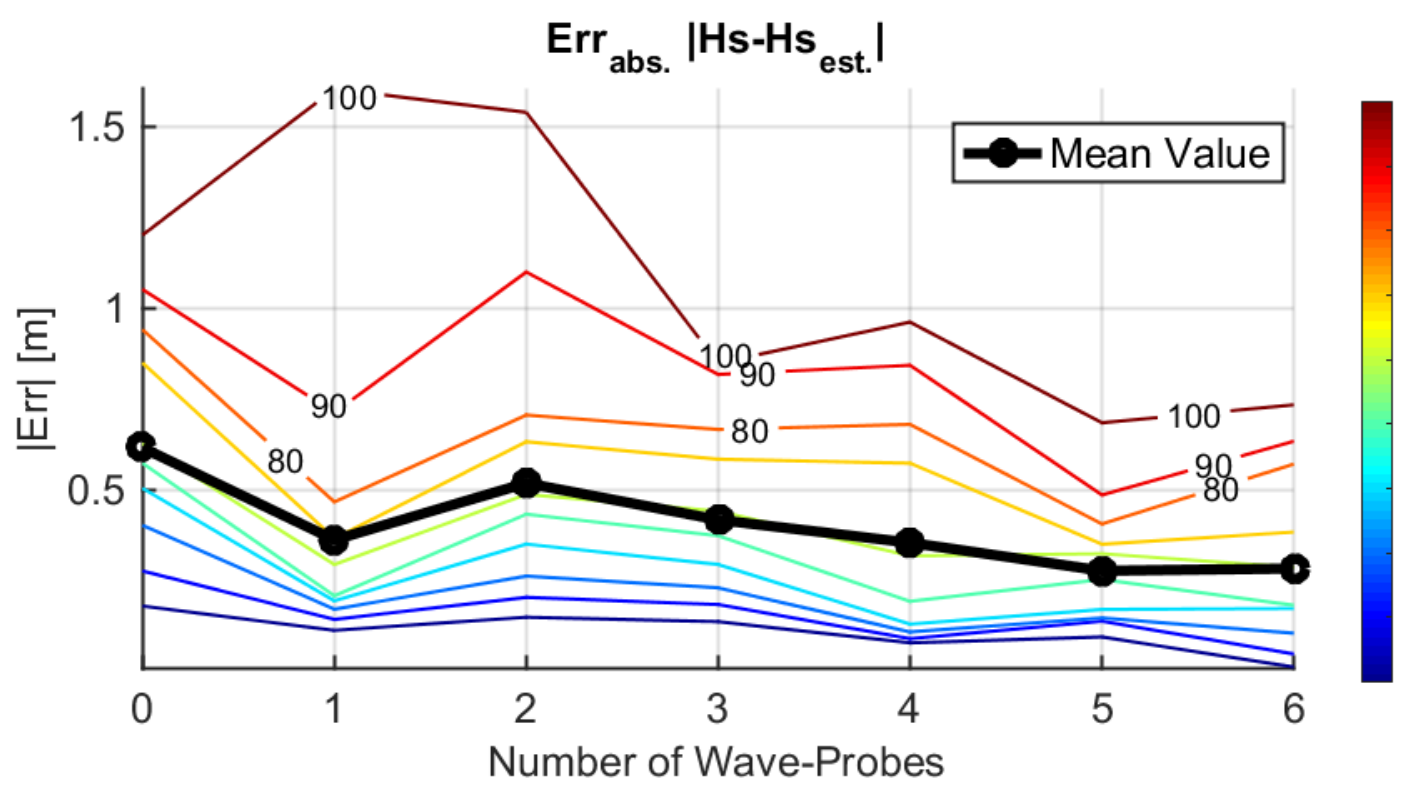

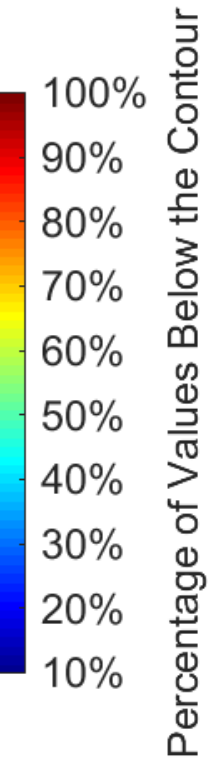

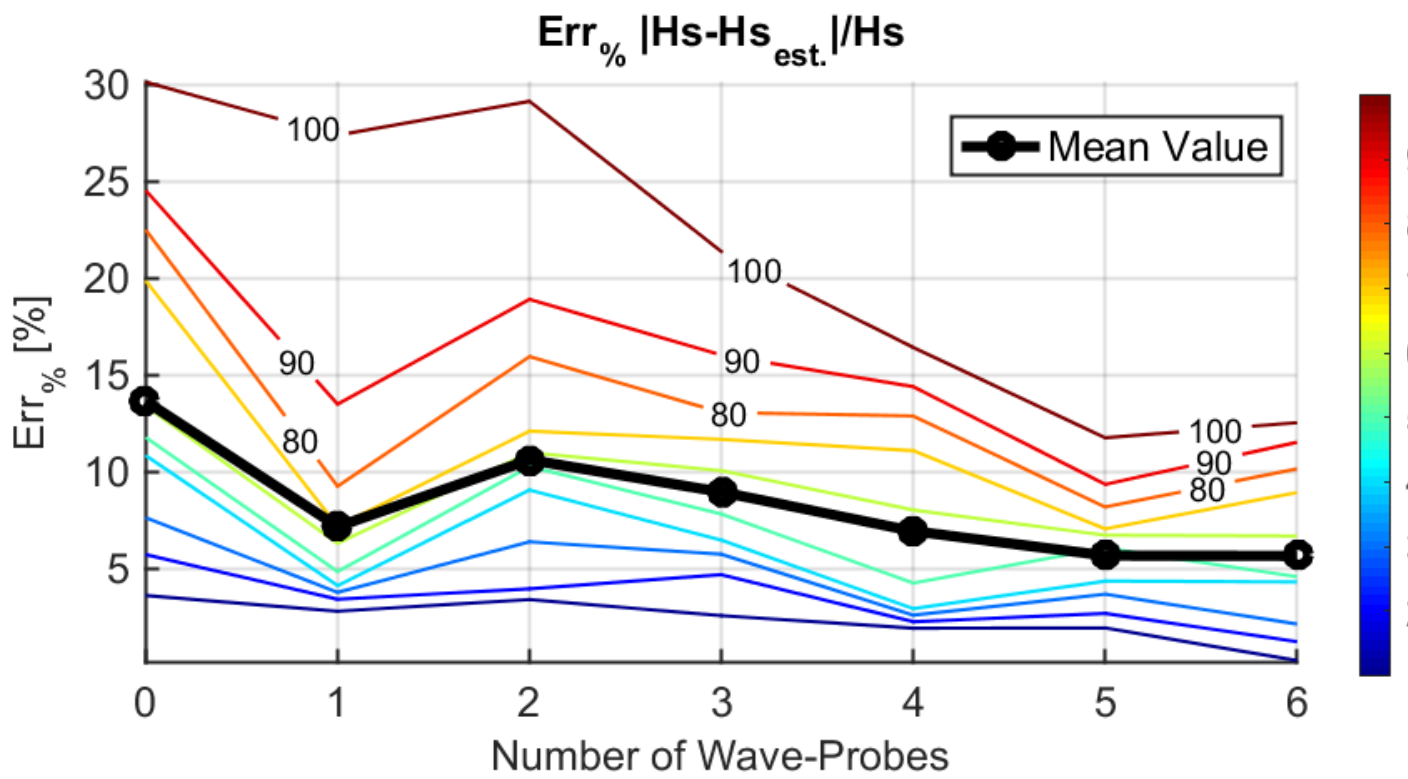

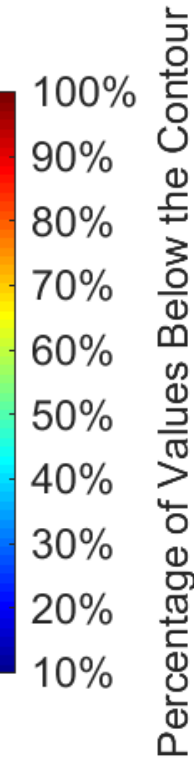

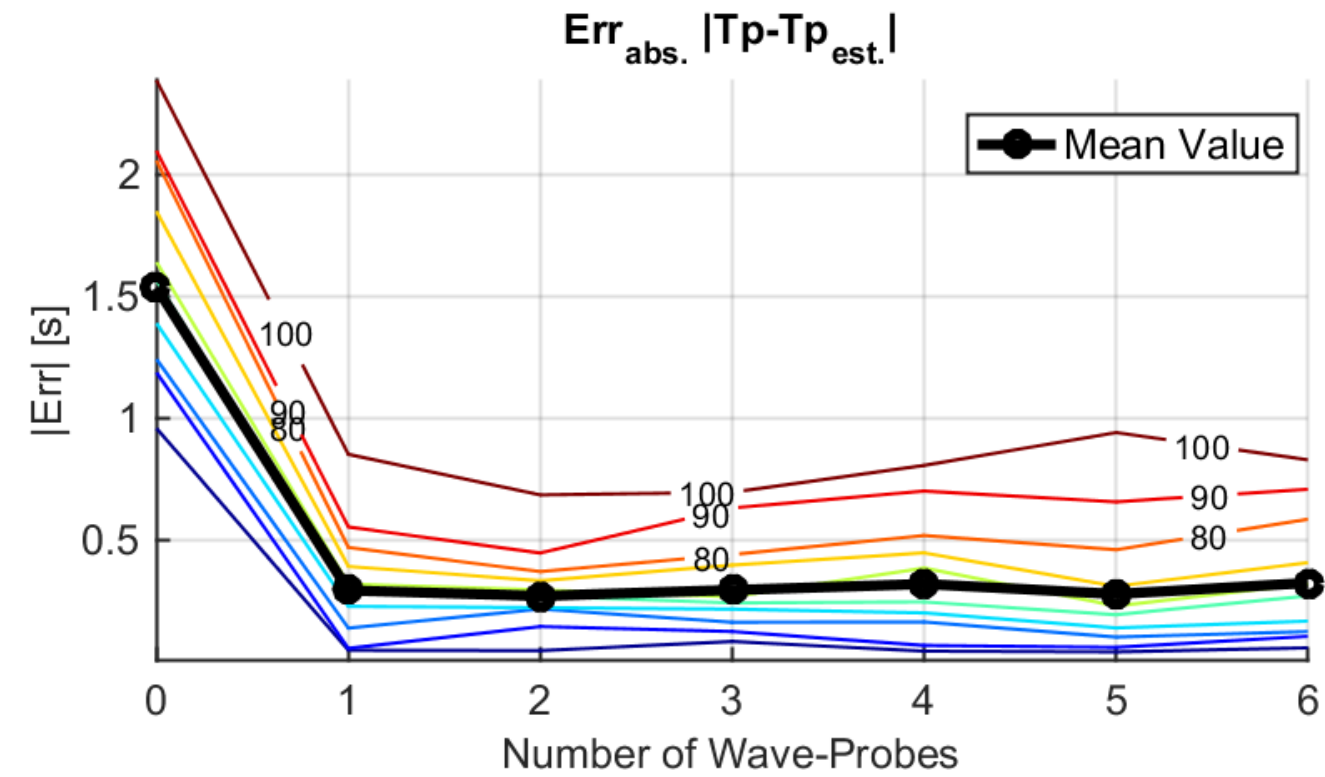

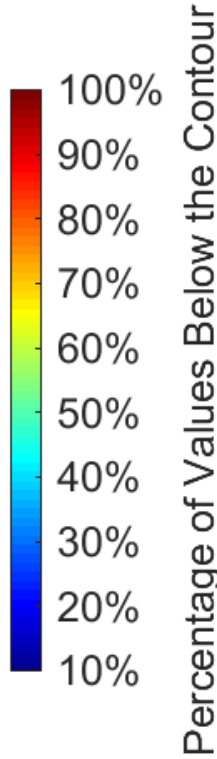




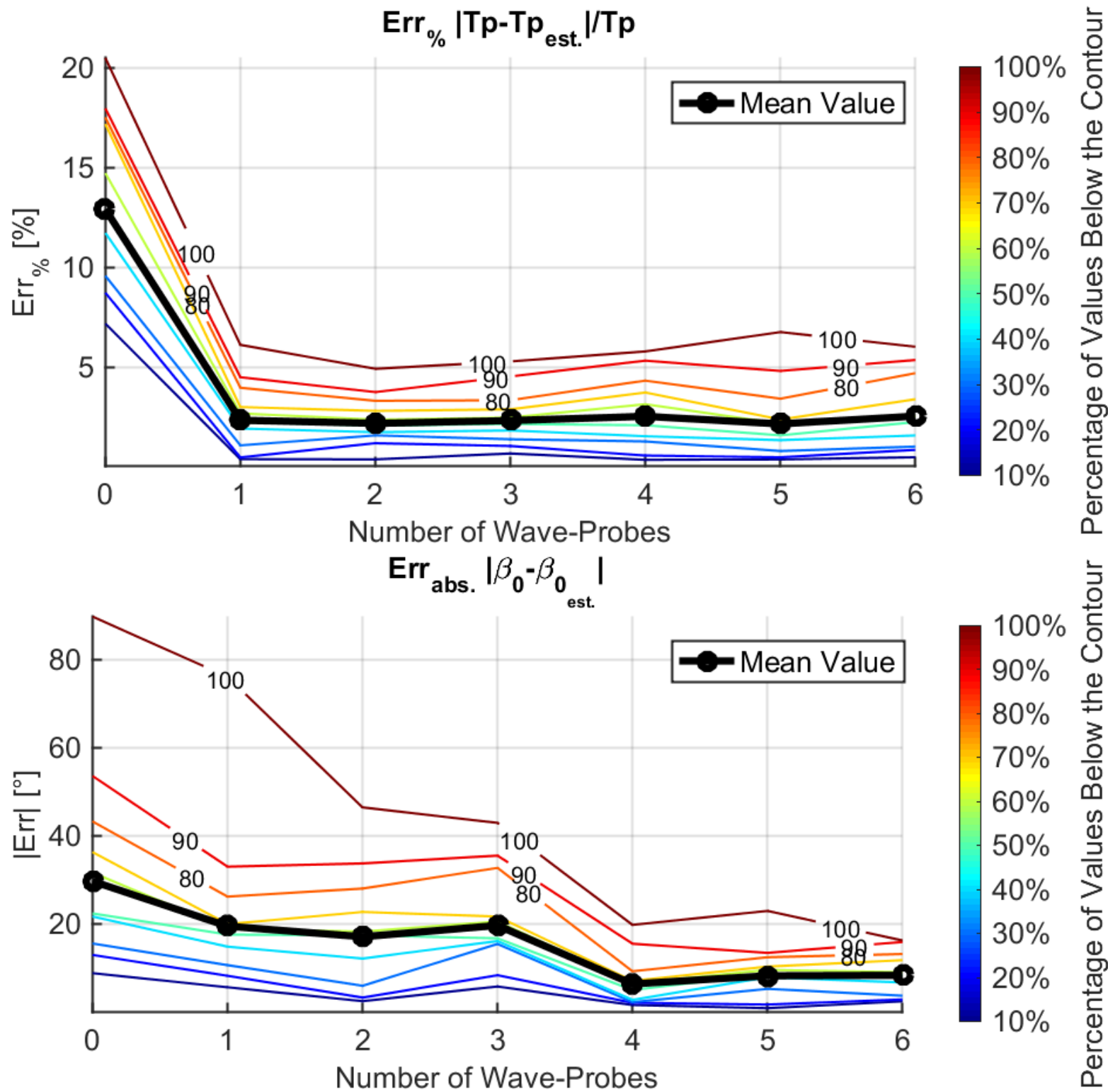

Source: Elaborated by the author.

It is important to remind that the bimodal set was generated by the following duplets: $\left(H_{S}=2.8, T_{P}=9.9\right),\left(H_{S}=4.3, T_{P}=12.8\right)$ and $\left(H_{S}=2.8, T_{P}=13.7\right)$. It means that $5 / 6$ of the seas have components with $T_{P}>12 s$, i.e., regions that are outside of the wave-probe RAO valid region. This explains the reason why for 3 to 4 probes there is a worsening in the $H_{S}$ metric, which is better only for 1 probe - which does not have roll influence - and for 5 and 6 probes - a higher number of probes that is known to not be affected so much by the discrepancies after 12 s.

Knowing that, the effective analysis of the bimodal set can only be done when wave-probes are employed just up to 12s. The following case, with all the energy peaks below 12s, illustrates the potential improvement Figure 136 to Figure 147. 
Figure 136 - Bow-Quartering, Low $T_{P}$, without wave-probes.

Exp.: $\mathrm{Hs}=3.9 \mathrm{~m} \mathrm{Tp}=10.0 \mathrm{~s} \beta_{0}=091^{\circ}$ Power $\left(\mathrm{m}^{2} . \mathrm{s}\right)$

Est.: $\mathrm{Hs}=2.9 \mathrm{~m} \mathrm{Tp}=11.9 \mathrm{~s} \beta_{0}=001^{\circ}$ Power $\left(\mathrm{m}^{2} . \mathrm{s}\right)$
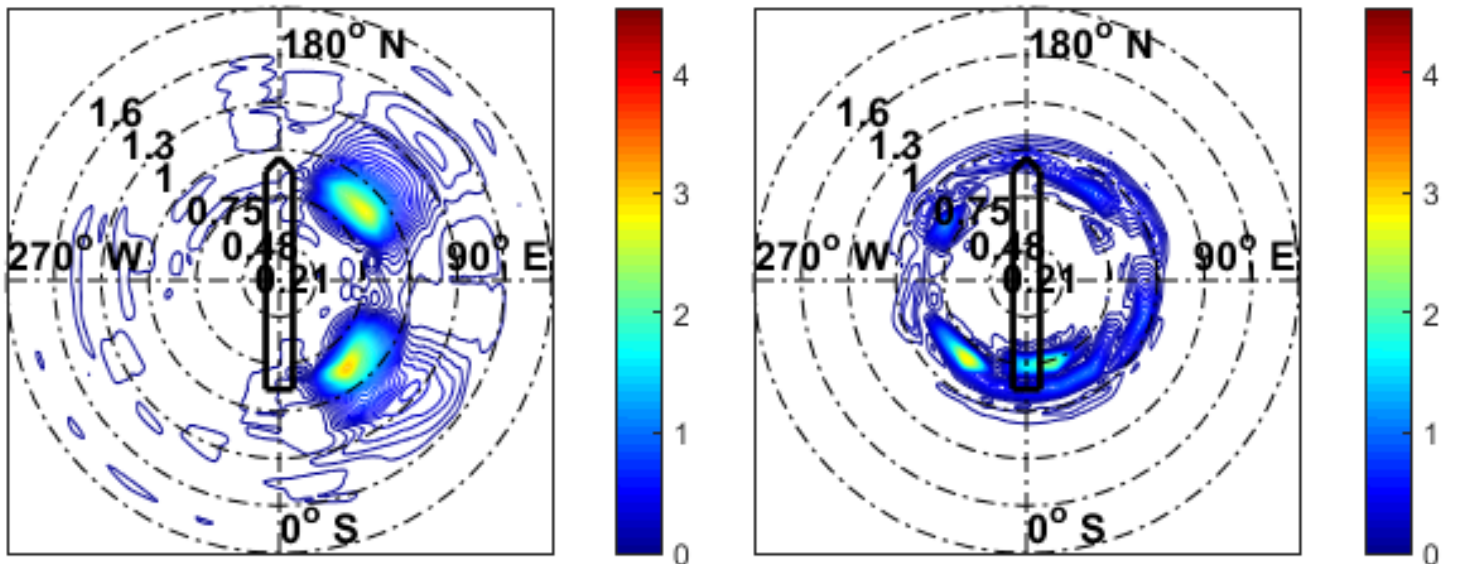

Source: Elaborated by the author.

Figure 137 - Bow-Quartering, Low $T_{P}$, with 2 wave-probes.

Exp.: $\mathrm{Hs}=3.9 \mathrm{~m} \mathrm{Tp}=10.0 \mathrm{~s} \beta_{0}=091^{\circ}$ Power $\left(\mathrm{m}^{2} . \mathrm{s}\right)$

Est.: $\mathrm{Hs}=4.3 \mathrm{~m} \mathrm{Tp}=9.7 \mathrm{~s} \beta_{0}=105^{\circ}$ Power $\left(m^{2} . s\right)$
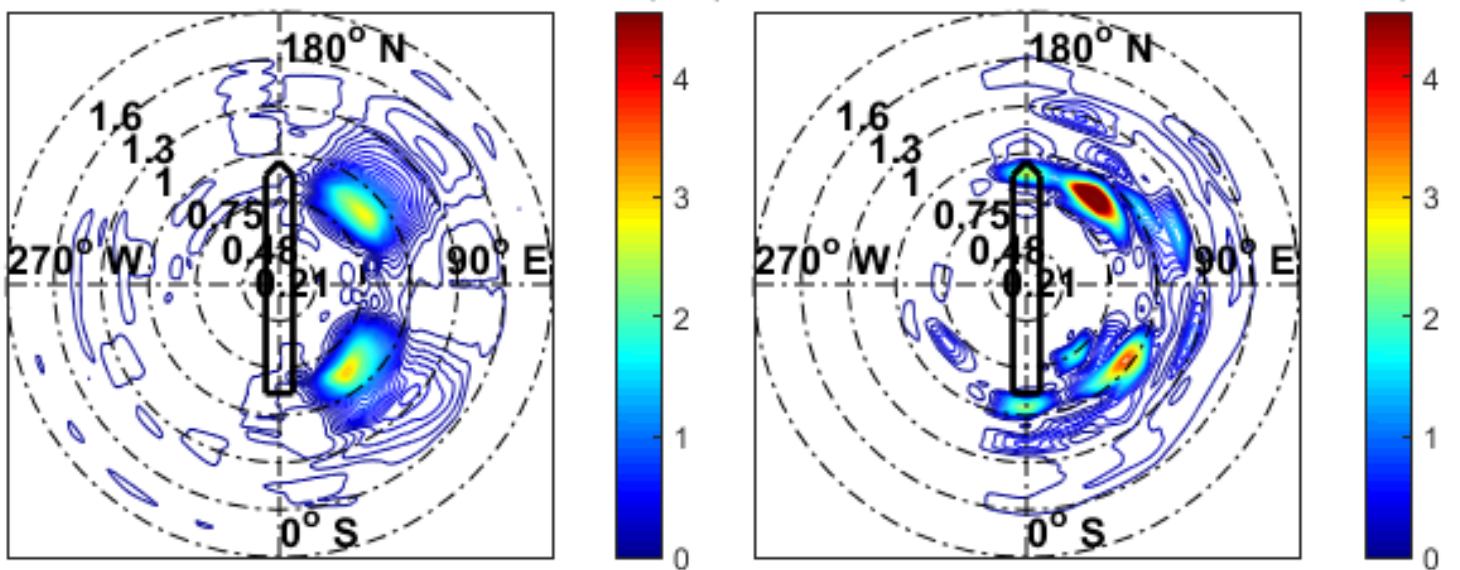

Source: Elaborated by the author.

Figure 138 - Bow-Quartering, Low $T_{P}$, with 4 wave-probes.

Exp.: $\mathrm{Hs}=3.9 \mathrm{~m} \mathrm{Tp}=10.0 \mathrm{~s} \beta_{0}=091^{\circ}$ Power $\left(\mathrm{m}^{2} . \mathrm{s}\right)$
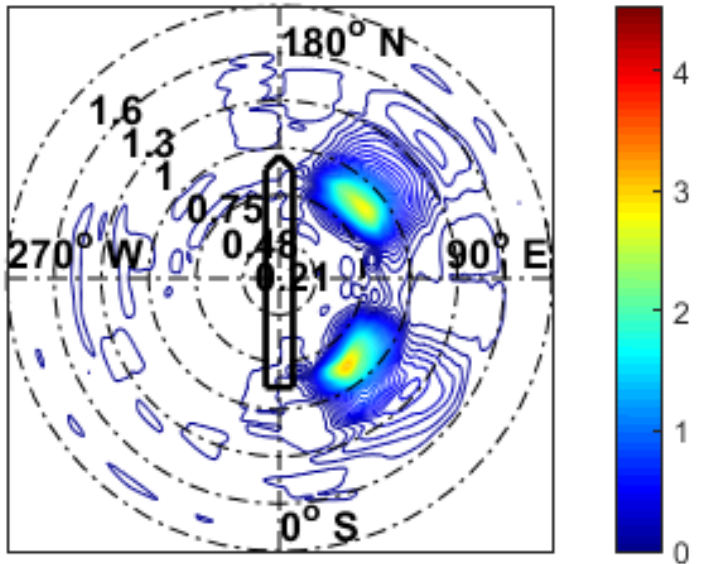

Est.: $\mathrm{Hs}=4.2 \mathrm{~m} \mathrm{Tp}=9.8 \mathrm{~s} \beta_{0}=091^{\circ}$ Power $\left(\mathrm{m}^{2} . \mathrm{s}\right)$
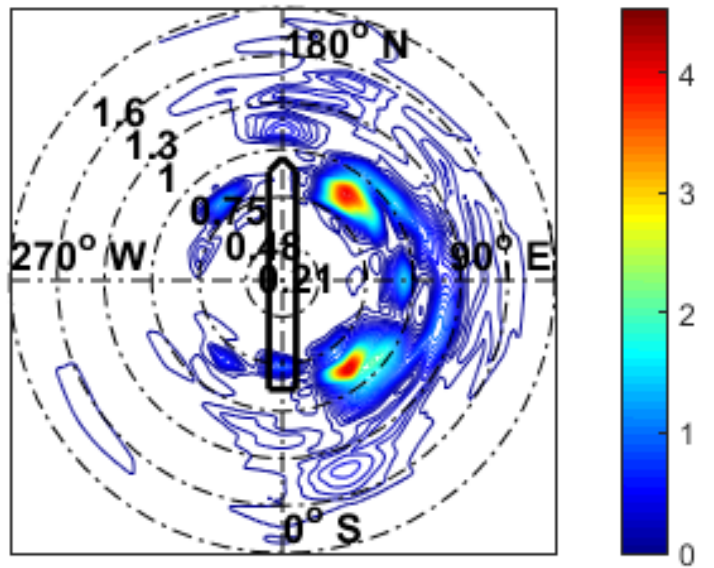

Source: Elaborated by the author. 
Figure 139 - Bow-Quartering, Low $T_{P}$, with 6 wave-probes.

Exp.: $\mathrm{Hs}=3.9 \mathrm{~m} \mathrm{Tp}=10.0 \mathrm{~s} \beta_{0}=091^{\circ}$ Power $\left(\mathrm{m}^{2} . \mathrm{s}\right)$
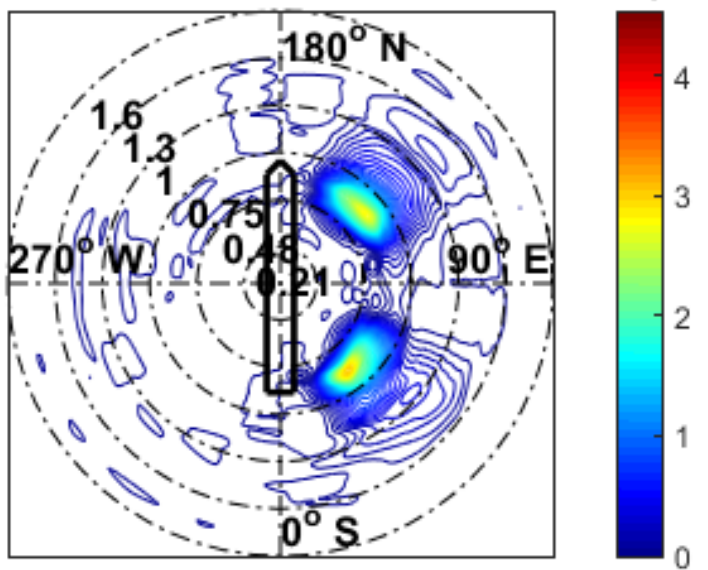

Est.: $\mathrm{Hs}=4.2 \mathrm{~m} \mathrm{Tp}=9.9 \mathrm{~s} \beta_{0}=093^{\circ}$ Power $\left(\mathrm{m}^{2} . \mathrm{s}\right)$

Source: Elaborated by the author.

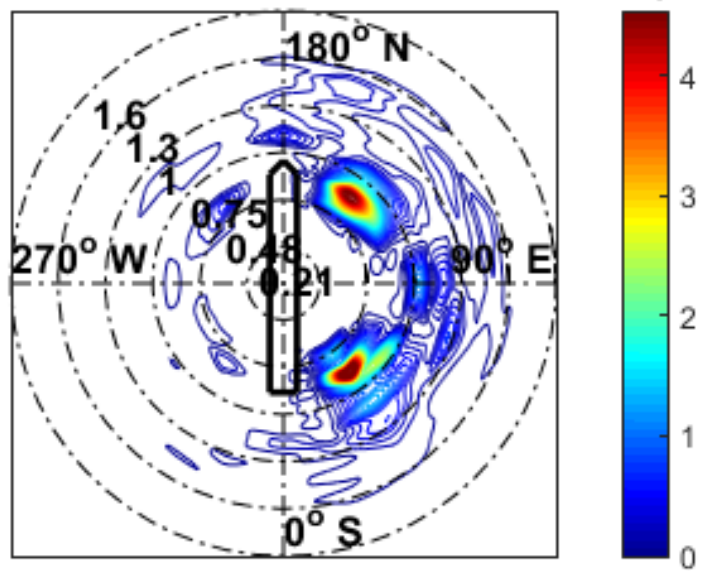

Figure 140 - Head-Beam, Low $T_{P}$, without wave-probes.

Exp.: $\mathrm{Hs}=3.9 \mathrm{~m} \mathrm{Tp}=10.0 \mathrm{~s} \beta_{0}=136^{\circ}$ Power $\left(\mathrm{m}^{2} . \mathrm{s}\right)$ Est.: $\mathrm{Hs}=2.7 \mathrm{~m} \mathrm{Tp}=11.3 \mathrm{~s} \beta_{0}=088^{\circ}$ Power $\left(\mathrm{m}^{2} . \mathrm{s}\right)$
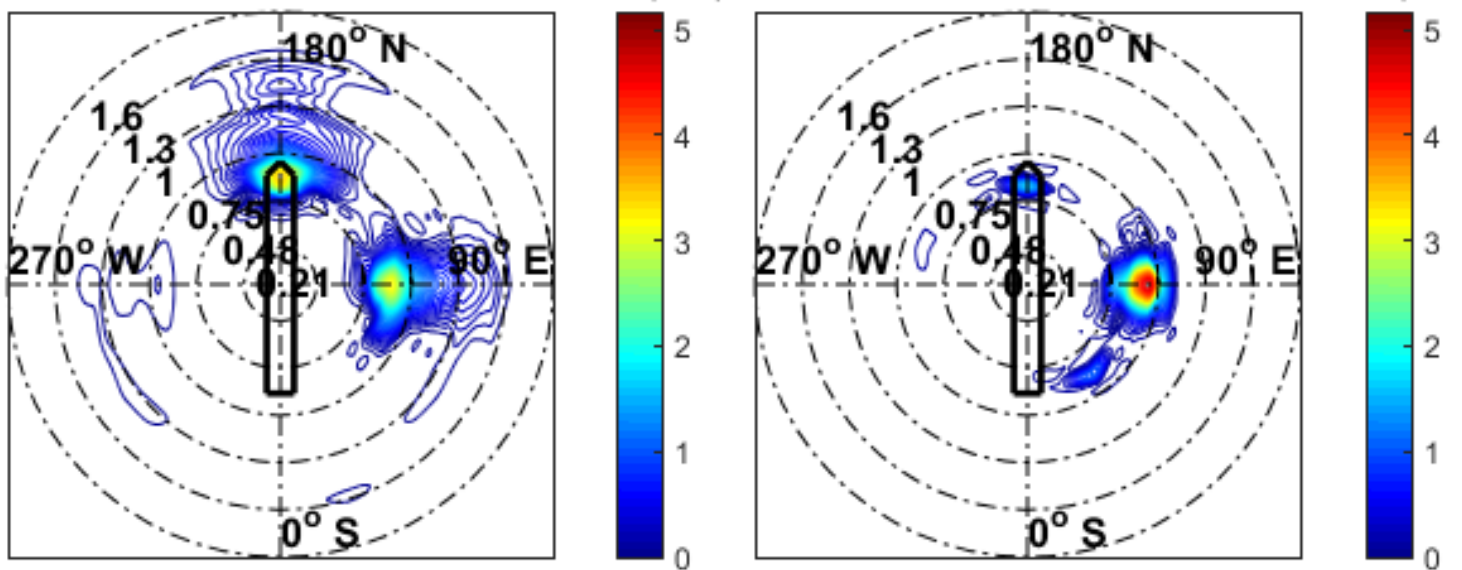

Source: Elaborated by the author.

Figure 141 - Head-Beam, Low $T_{P}$, with 2 wave-probes.

Exp.: $\mathrm{Hs}=3.9 \mathrm{~m} \mathrm{Tp}=10.0 \mathrm{~s} \beta_{0}=136^{\circ}$ Power $\left(\mathrm{m}^{2} . \mathrm{s}\right)$

Est.: $\mathrm{Hs}=4.1 \mathrm{~m} \mathrm{Tp}=10.4 \mathrm{~s} \beta_{0}=132^{\circ}$ Power $\left(\mathrm{m}^{2} . \mathrm{s}\right)$
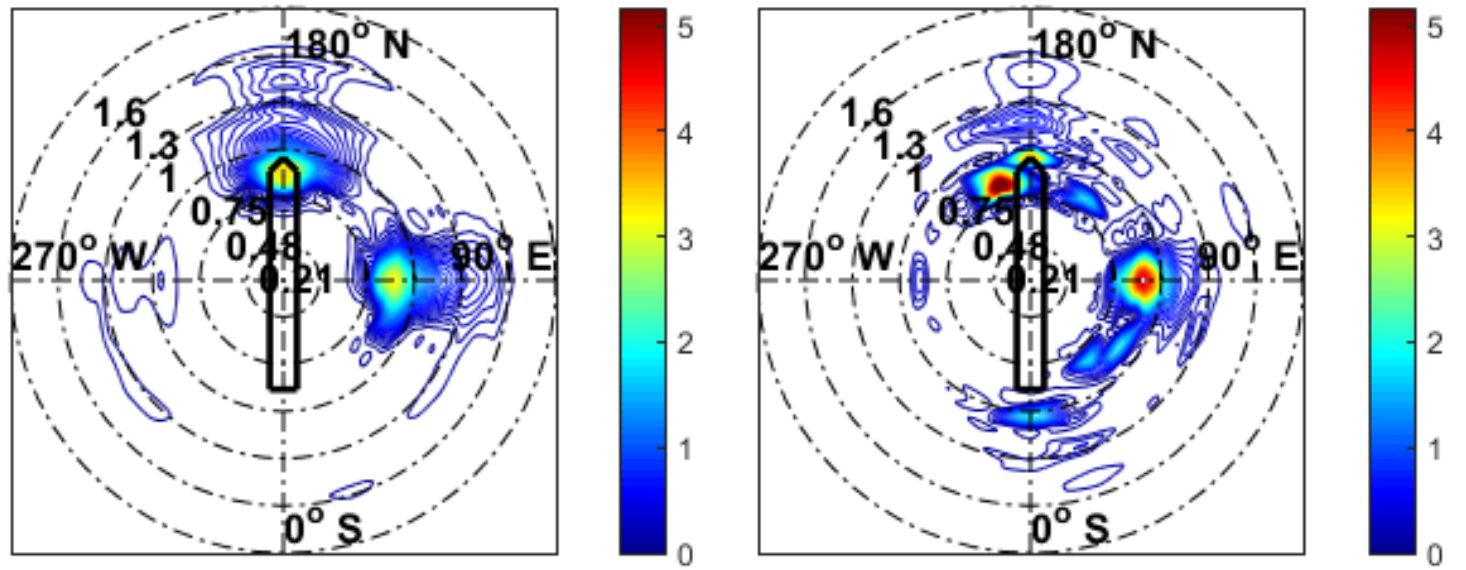

Source: Elaborated by the author. 
Figure 142 - Head-Beam, Low $T_{P}$, with 4 wave-probes.

Exp.: $\mathrm{Hs}=3.9 \mathrm{~m} \mathrm{Tp}=10.0 \mathrm{~s} \beta_{0}=136^{\circ}$ Power $\left(\mathrm{m}^{2} . \mathrm{s}\right)$
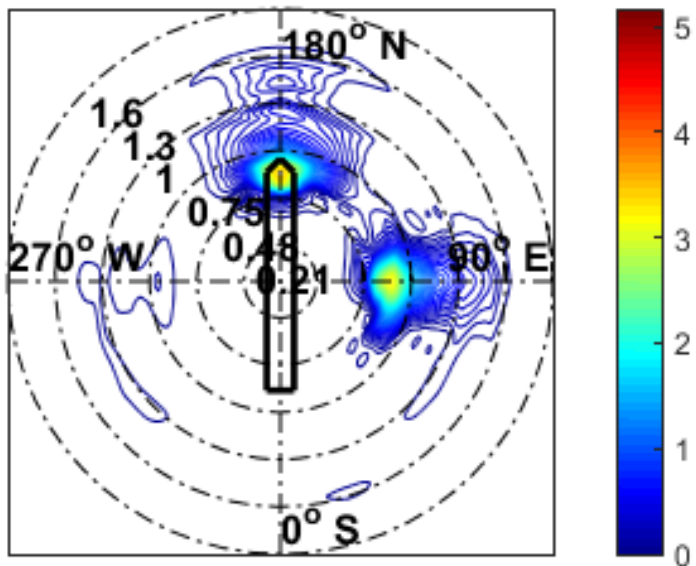

Est.: $\mathrm{Hs}=4.0 \mathrm{~m} \mathrm{Tp}=10.0 \mathrm{~s} \beta_{0}=129^{\circ}$ Power $\left(\mathrm{m}^{2} . \mathrm{s}\right)$

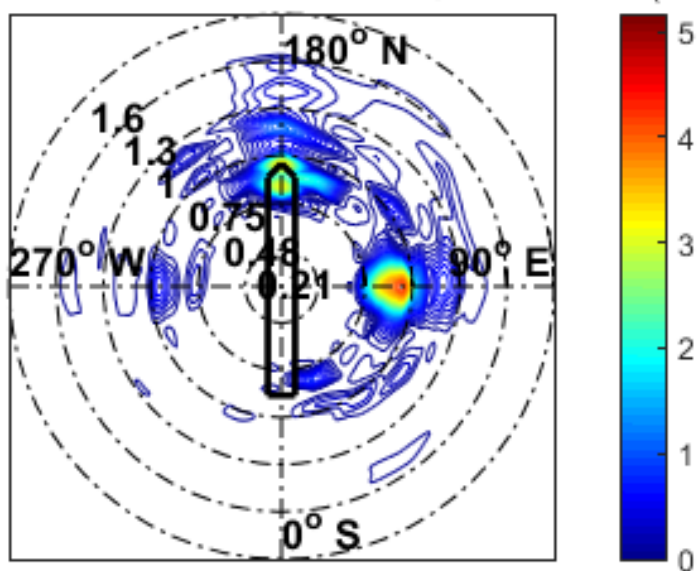

Source: Elaborated by the author.

Figure 143 - Head-Beam, Low $T_{P}$, with 6 wave-probes.

Exp.: $\mathrm{Hs}=3.9 \mathrm{~m} \mathrm{Tp}=10.0 \mathrm{~s} \beta_{0}=136^{\circ}$ Power $\left(\mathrm{m}^{2} . \mathrm{s}\right)$
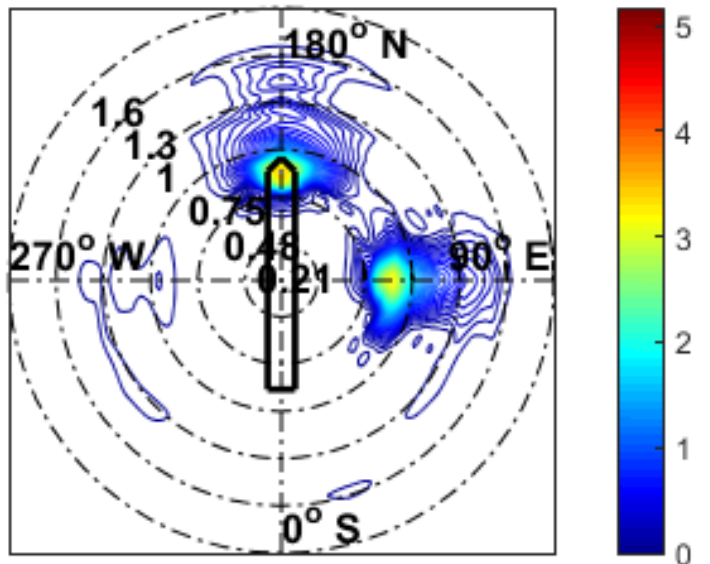

Est.: $\mathrm{Hs}=3.9 \mathrm{~m} \mathrm{Tp}=10.0 \mathrm{~s} \beta_{0}=127^{\circ}$ Power $\left(\mathrm{m}^{2} . \mathrm{s}\right)$

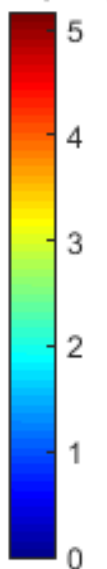

Source: Elaborated by the author.

Figure 144 - Bow-Bow, Low $T_{P}$, without wave-probes.

Exp.: $\mathrm{Hs}=3.9 \mathrm{~m} \mathrm{Tp}=10.0 \mathrm{~s} \beta_{0}=181^{\circ}$ Power $\left(\mathrm{m}^{2} . \mathrm{s}\right)$
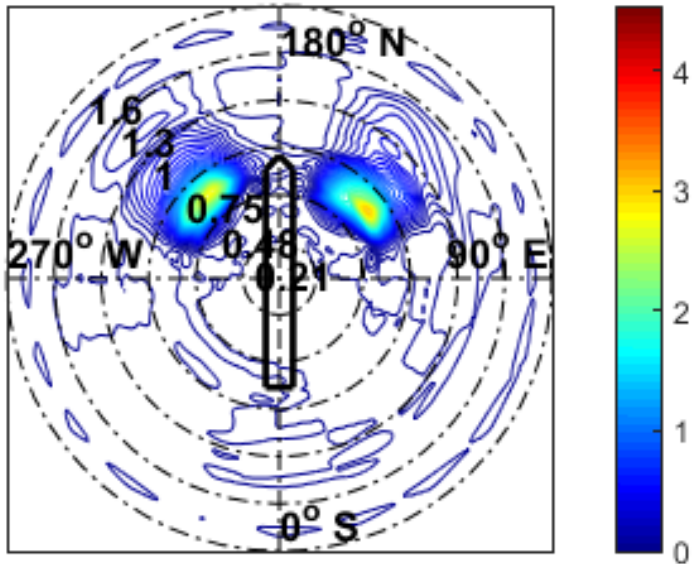

Est.: $\mathrm{Hs}=2.9 \mathrm{~m} \mathrm{Tp}=11.7 \mathrm{~s} \beta_{0}=181^{\circ}$ Power $\left(\mathrm{m}^{2} . \mathrm{s}\right)$

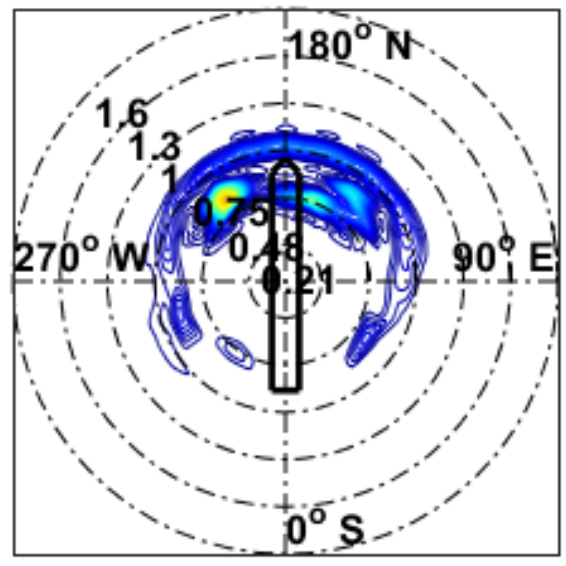

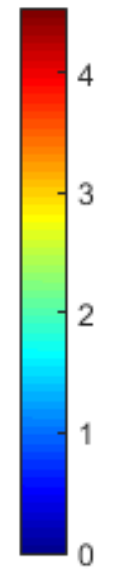

Source: Elaborated by the author. 
Figure 145 - Bow-Bow, Low $T_{P}$, with 2 wave-probes.

Exp.: $\mathrm{Hs}=3.9 \mathrm{~m} \mathrm{Tp}=10.0 \mathrm{~s} \beta_{0}=181^{\circ}$ Power $\left(\mathrm{m}^{2} . \mathrm{s}\right)$
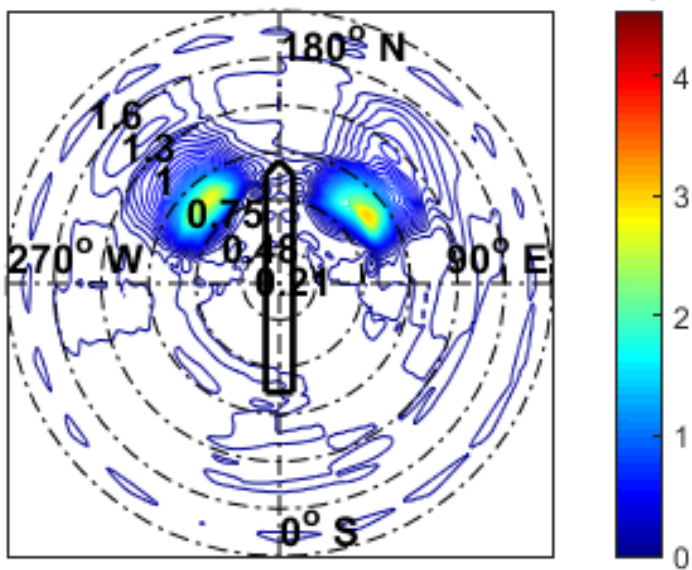

Est.: $\mathrm{Hs}=4.2 \mathrm{~m} \mathrm{Tp}=10.0 \mathrm{~s} \beta_{0}=182^{\circ}$ Power $\left(\mathrm{m}^{2} . \mathrm{s}\right)$

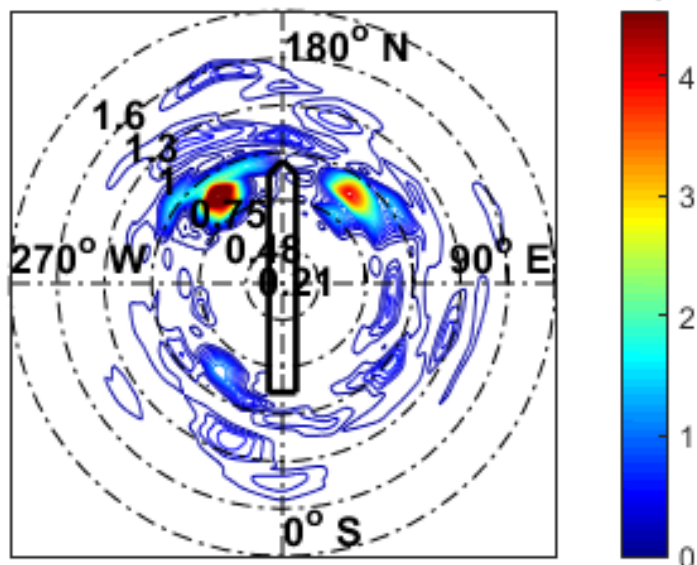

Source: Elaborated by the author.

Figure 146 - Bow-Bow, Low $T_{P}$, with 4 wave-probes.

Exp.: $\mathrm{Hs}=3.9 \mathrm{~m} \mathrm{Tp}=10.0 \mathrm{~s} \beta_{0}=181^{\circ}$ Power $\left(\mathrm{m}^{2} . \mathrm{s}\right)$
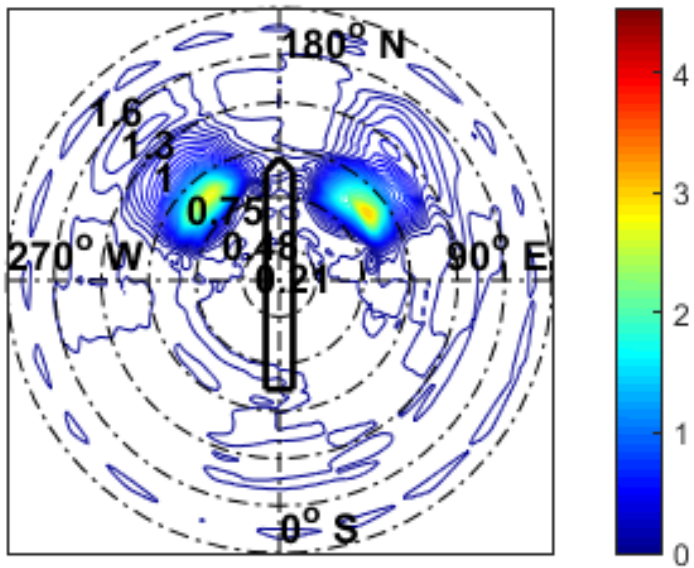

Est.: $\mathrm{Hs}=4.0 \mathrm{~m} \mathrm{Tp}=9.7 \mathrm{~s} \beta_{0}=172^{\circ}$ Power $\left(m^{2} . s\right)$
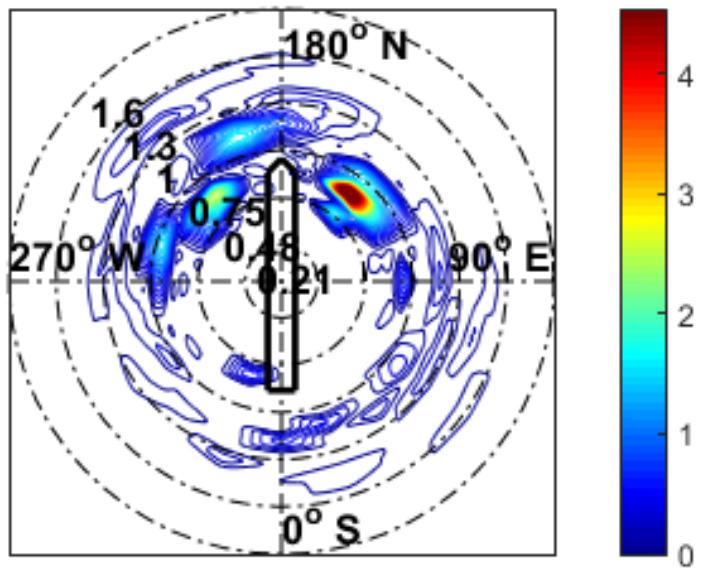

Source: Elaborated by the author.

Figure 147 - Bow-Bow, Low $T_{P}$, with 6 wave-probes.

Exp.: $\mathrm{Hs}=3.9 \mathrm{~m} \mathrm{Tp}=10.0 \mathrm{~s} \beta_{0}=181^{\circ}$ Power $\left(\mathrm{m}^{2} . \mathrm{s}\right)$

Est.: $\mathrm{Hs}=4.0 \mathrm{~m} \mathrm{Tp}=9.8 \mathrm{~s} \beta_{0}=183^{\circ}$ Power $\left(\mathrm{m}^{2} . \mathrm{s}\right)$
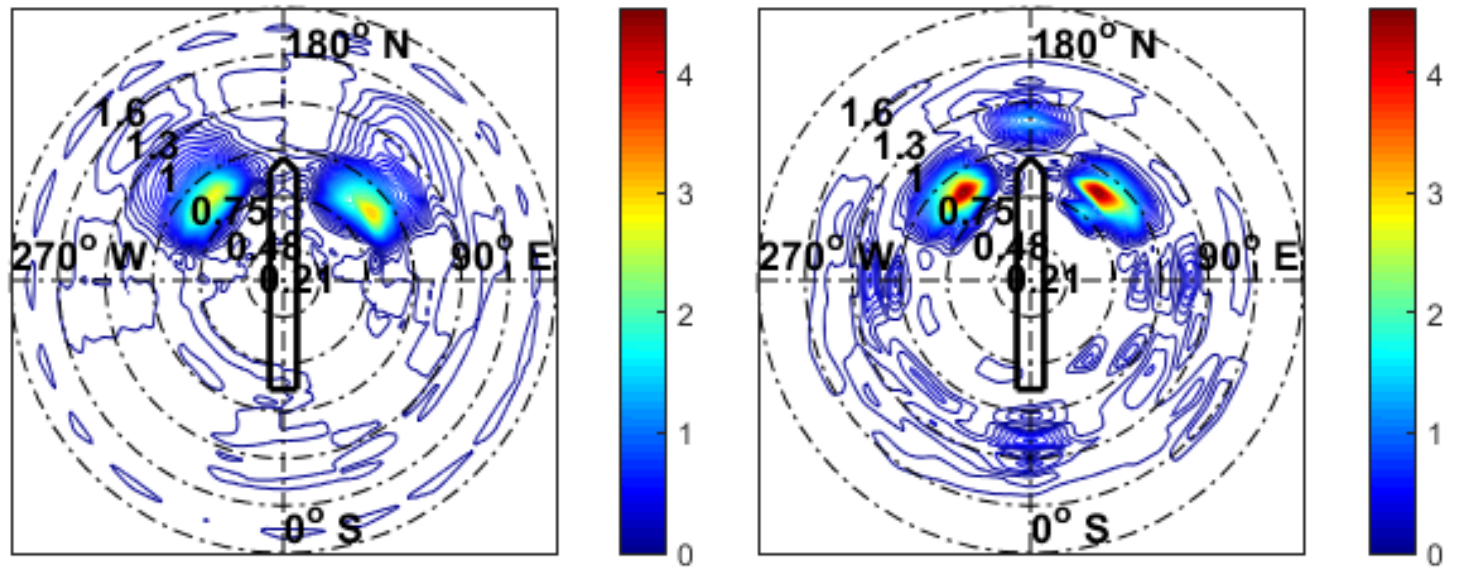

Source: Elaborated by the author. 
In general, the low $T_{P}$ spectrum is not correctly estimated using only vessel movements; two wave-probes are already able to recover most of the expected result; four wave probes are able to provide good mean direction estimation; and six waveprobes are able to achieve a good agreement with the experimental spectrum. It means that the conclusions remain the same as those for the intermediate draft.

\subsection{Validation of the Optimal Prior}

For the experimental evaluation, the optimal prior was implemented in the algorithm using a value of hyperparameter such as to minimize the metric $\left\|u^{2} \boldsymbol{H}_{M L Q P}-\left[u_{1}^{2} \boldsymbol{H}_{1}+u_{2}^{2} \boldsymbol{H}_{2}+u_{3}^{2} \boldsymbol{H}_{3}\right]\right\|^{2}$. The histograms for the intermediate draft, for the conventional and optimal priors, are given in Figure 148.

Figure 148 - Histograms comparing the conventional and the MLQP prior.

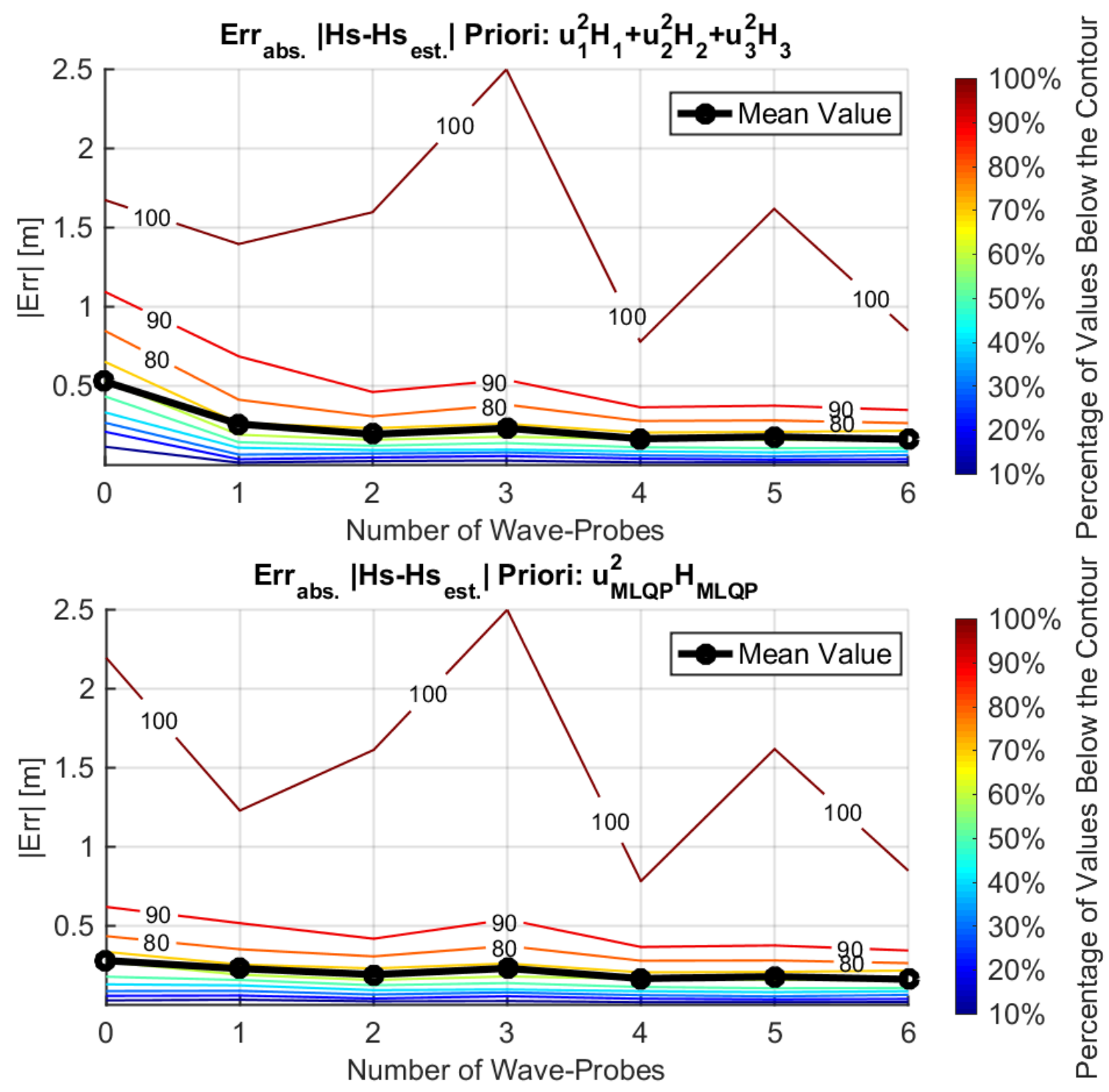




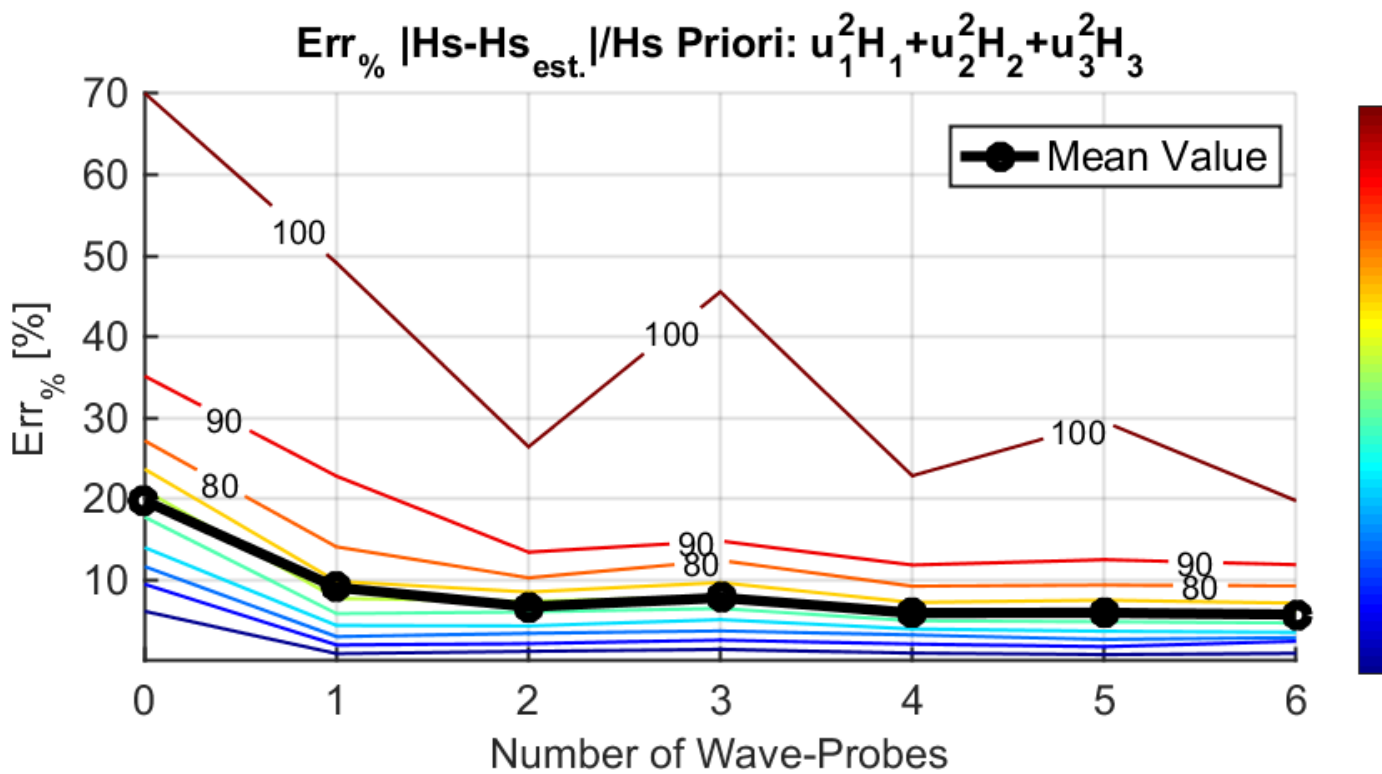

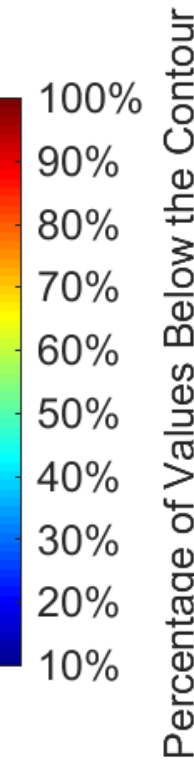

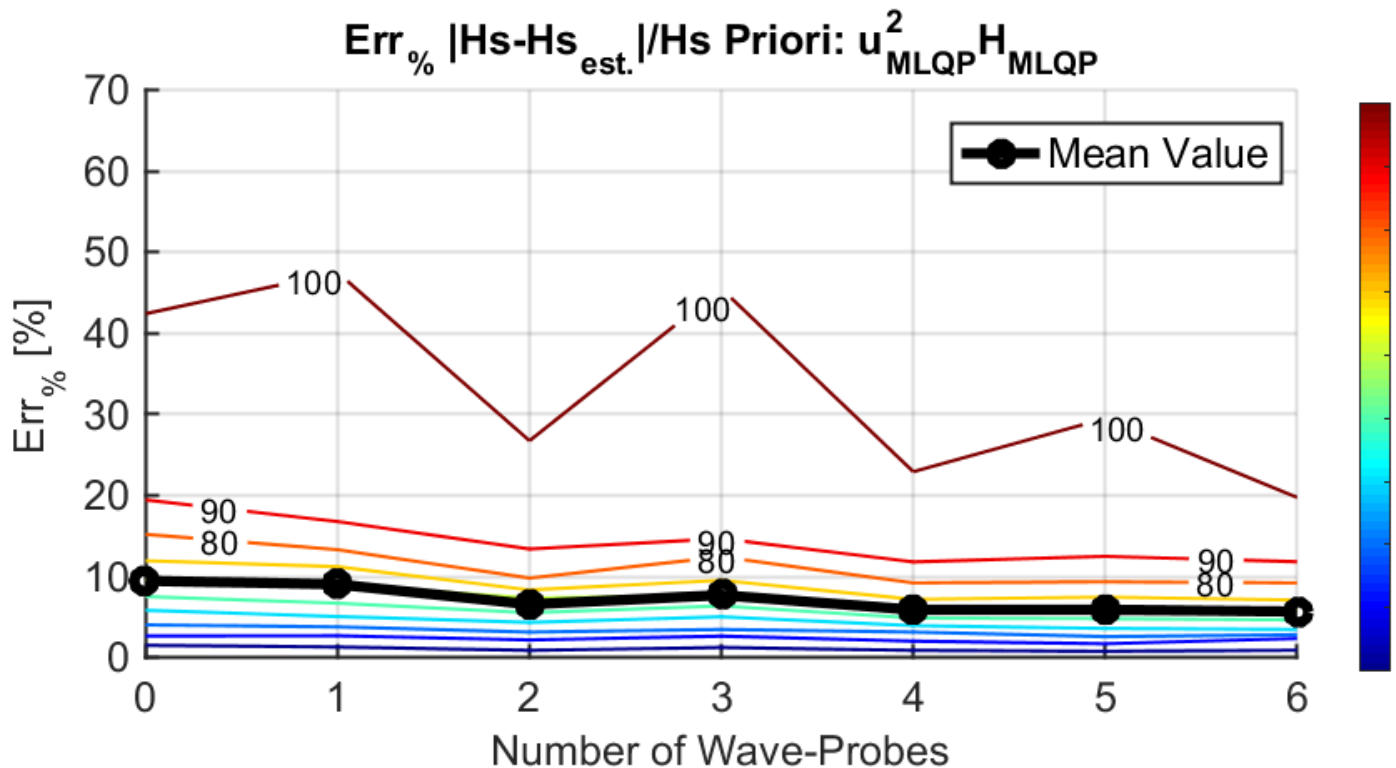

$100 \%$ $90 \%$ $80 \%$ $70 \%$ $60 \%$ $50 \%$ $40 \%$ $30 \%$ $20 \%$ $10 \%$

Err $_{\text {abs. }} \mid \mathrm{Tp}$-Tp $\mathrm{est.}_{\text {. }} \mid$ Priori: $\mathrm{u}_{1}^{2} \mathrm{H}_{1}+\mathrm{u}_{2}^{2} \mathrm{H}_{2}+\mathrm{u}_{3}^{2} \mathrm{H}_{3}$

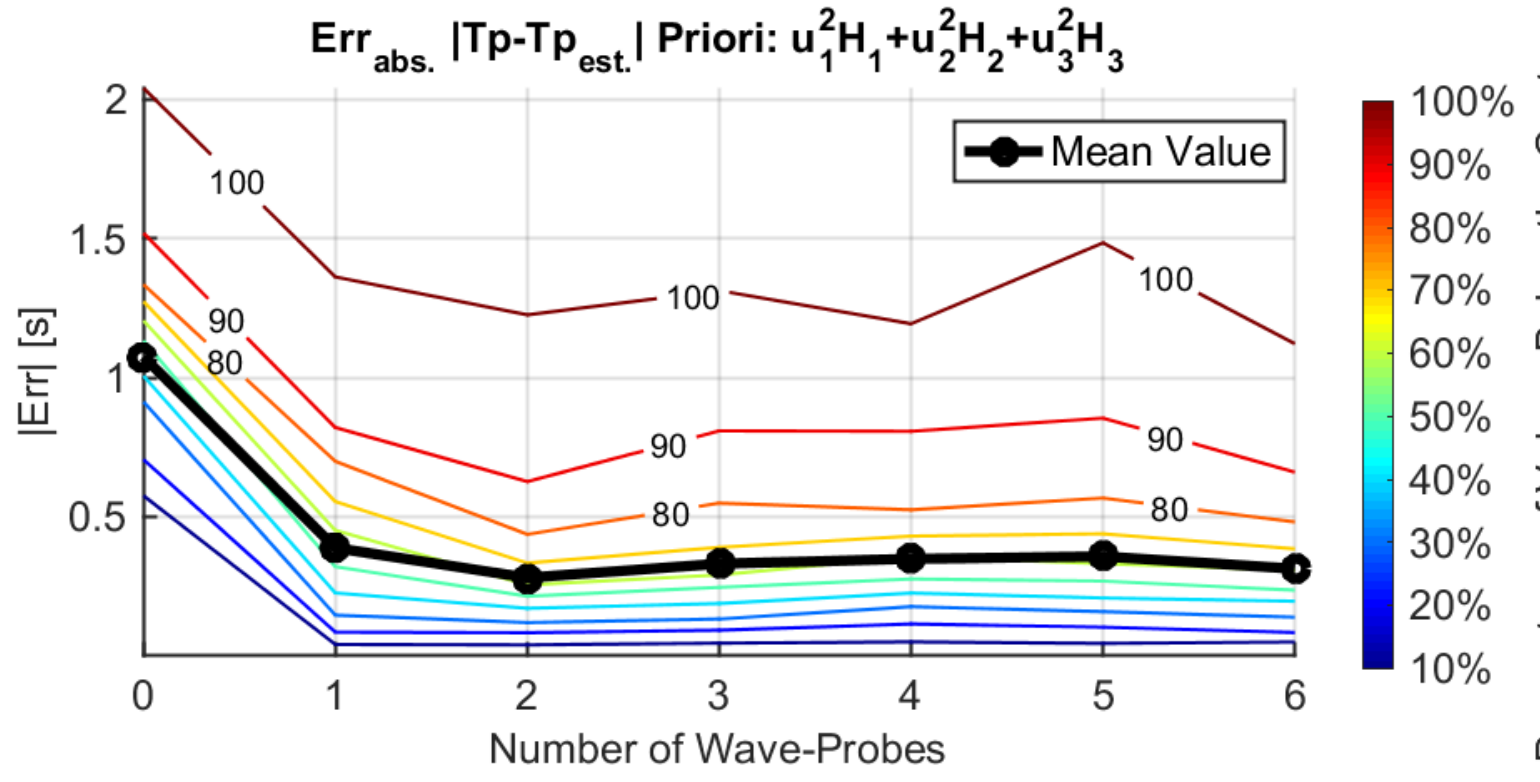



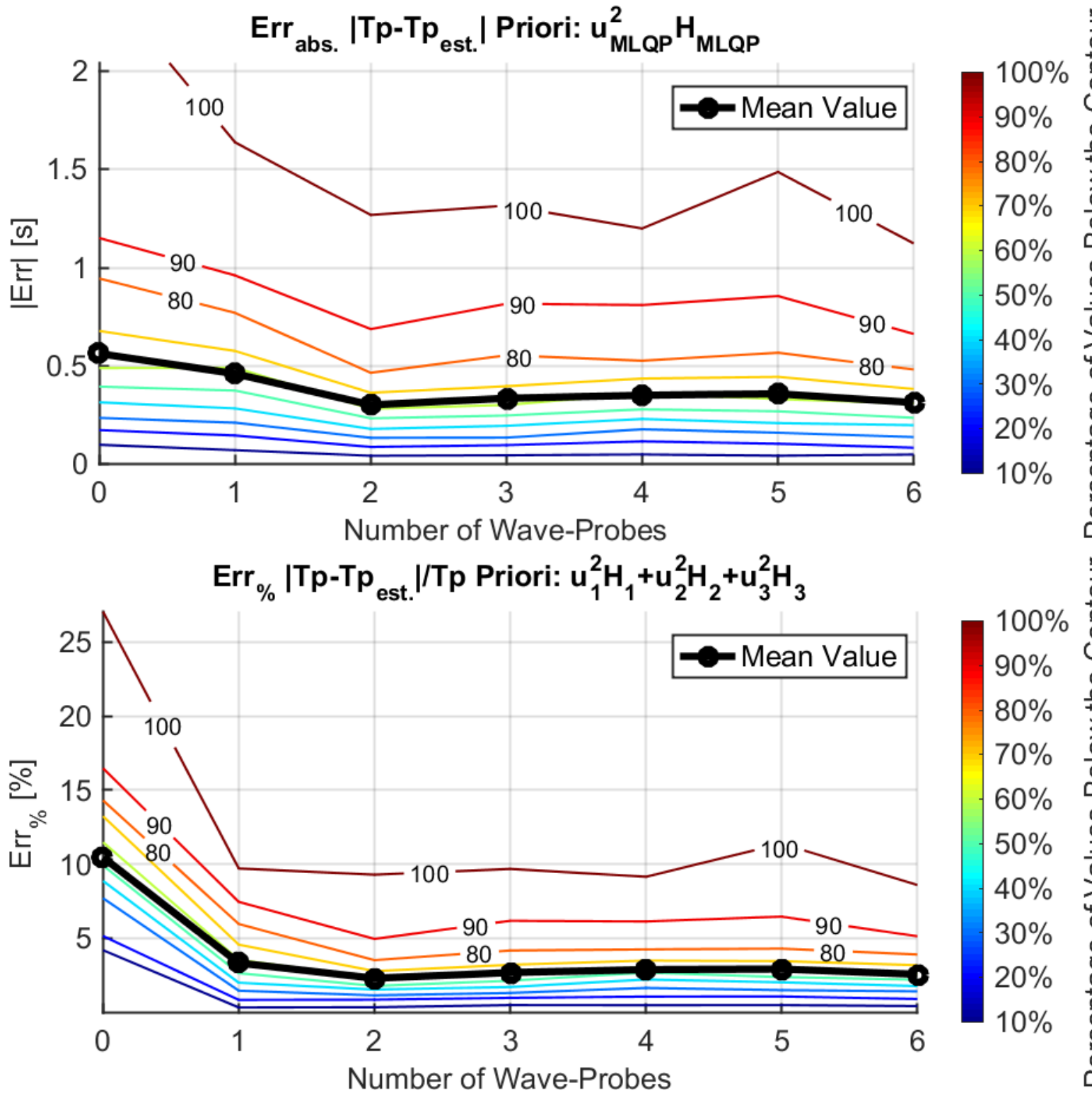

$\mathrm{Err}_{\%} \mid \mathrm{Tp}-\mathrm{Tp} \mathrm{est.}_{\text {. }} / / \mathrm{Tp}$ Priori: $\mathrm{u}_{\mathrm{MLQP}}^{2} \mathrm{H}_{\mathrm{MLQP}}$

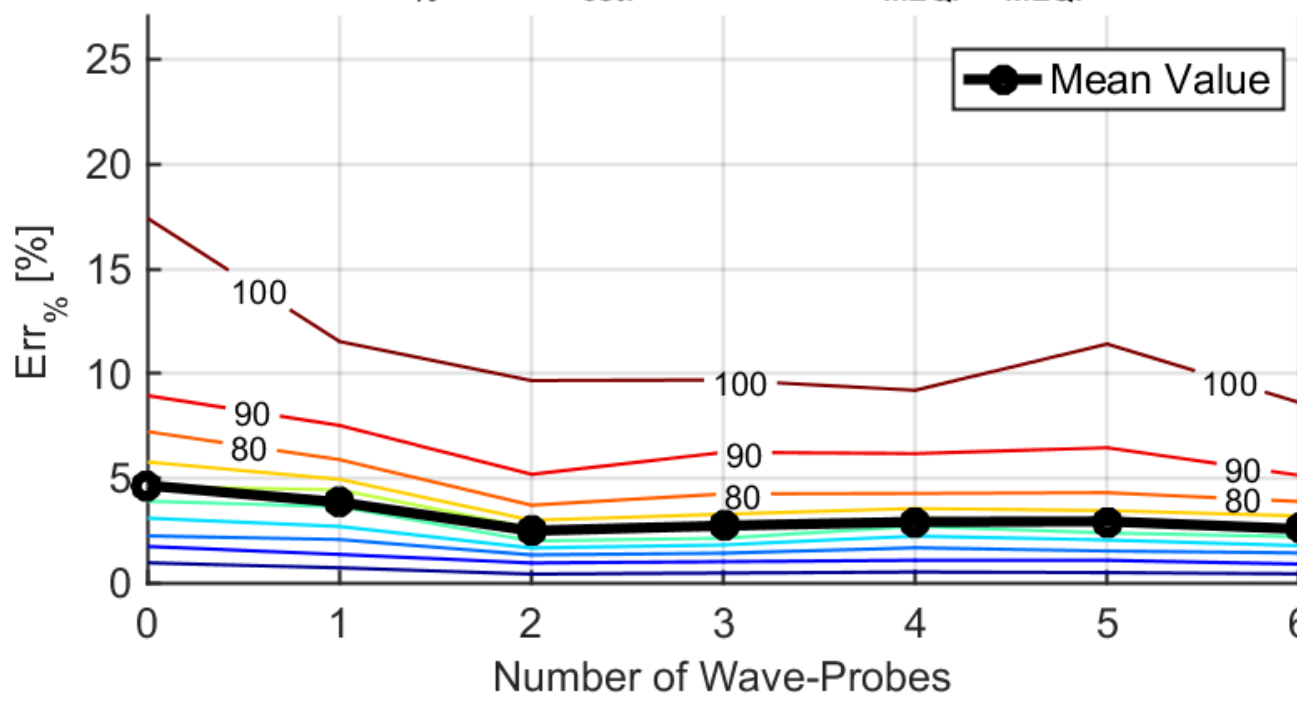



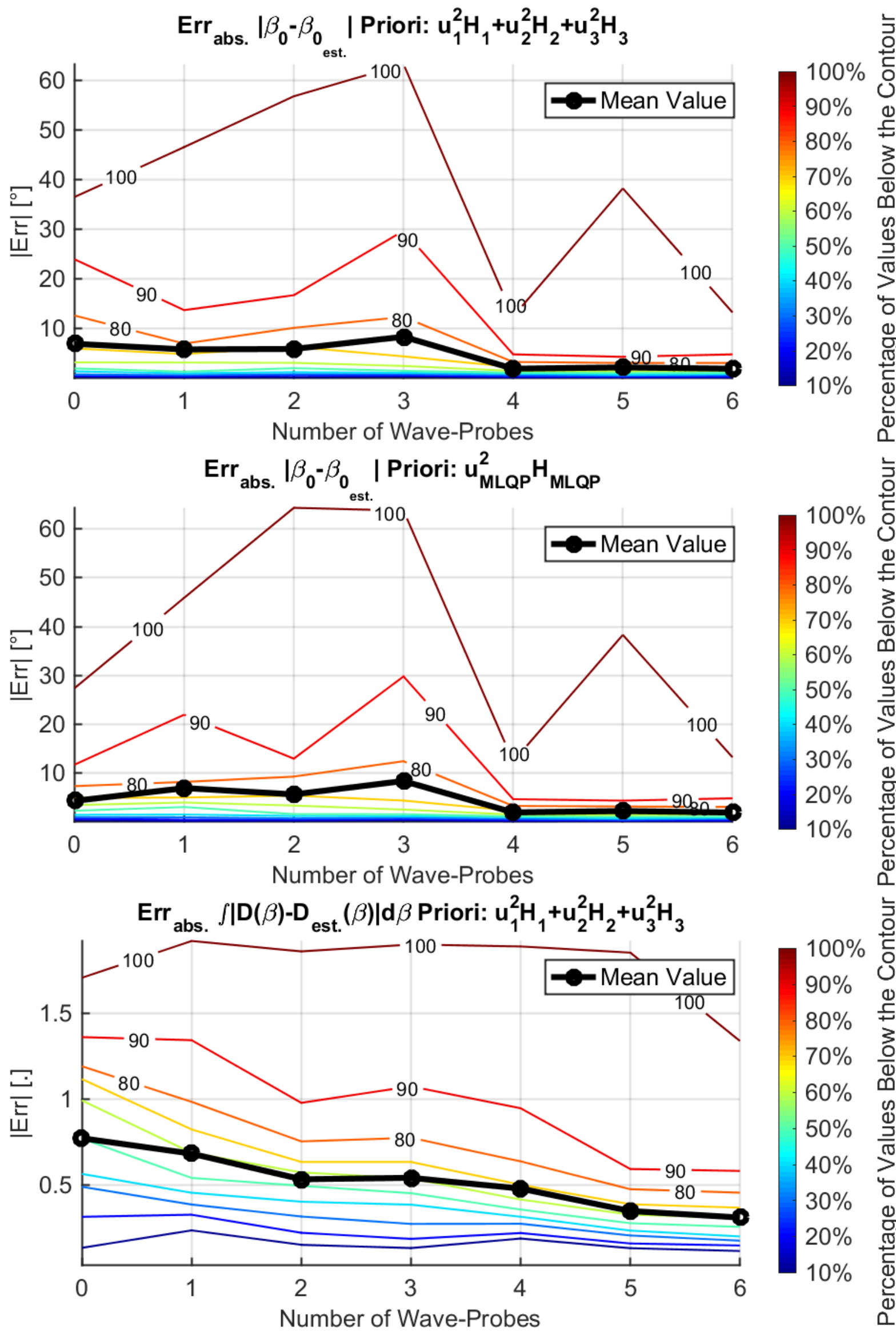


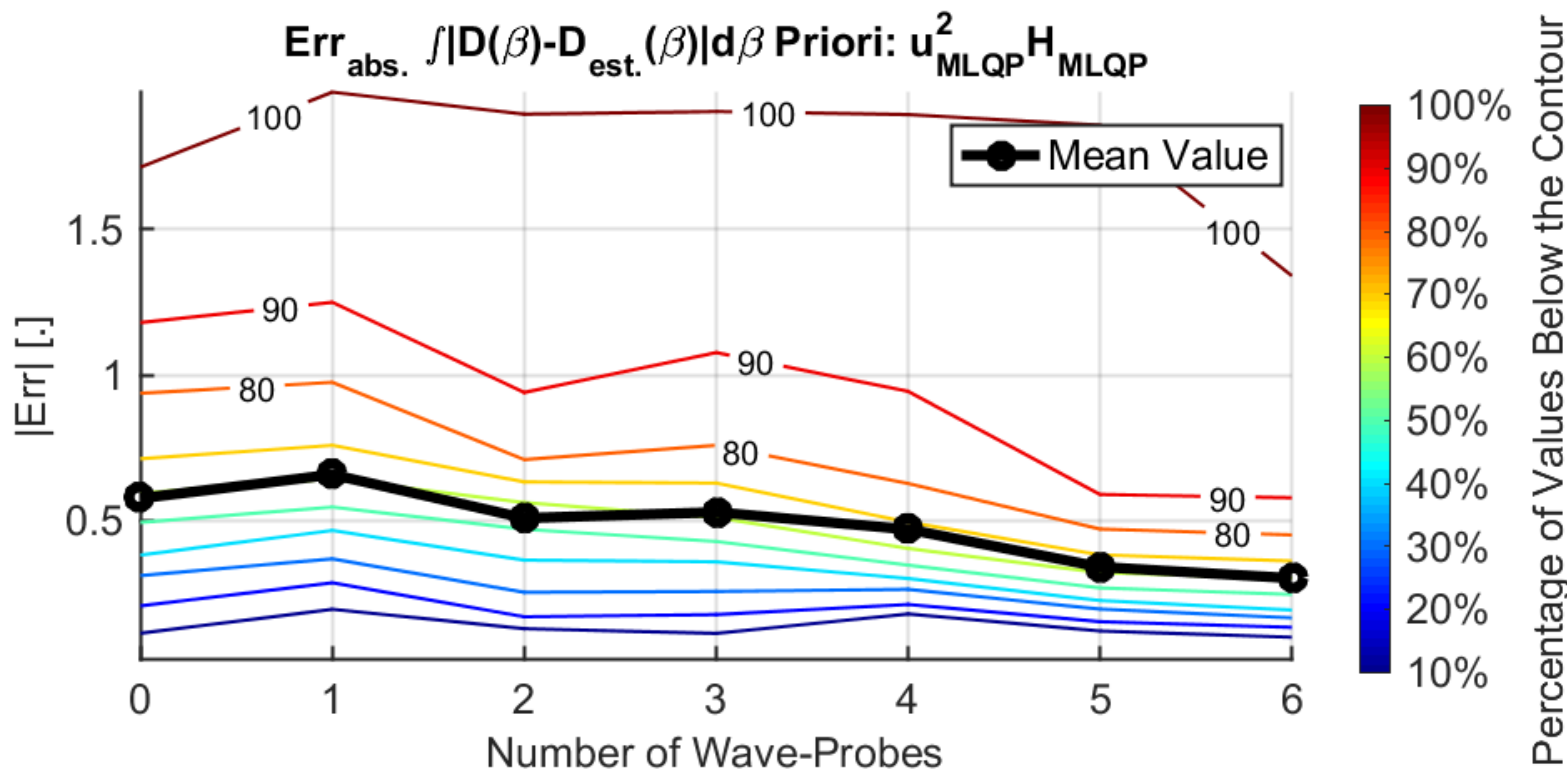

Source: Elaborated by the author.

It is possible to notice that most of the impact of the new prior happens for the estimations with only vessel movements or with only one wave-probe, with the cases with two probes changing slightly and the cases with three or more without any changes in the response.

Firstly, the cases with only vessel DoFs present a significantly improvement. In the $H_{S}$ metrics, the error of $90 \%$ of the waves drops from $1.1 \mathrm{~m}$ to $0.63 \mathrm{~m}$, with the percent error dropping from $36 \%$ to $19.5 \%$. In the $T_{P}$ metrics, the error of $90 \%$ of the waves drops from $1.52 \mathrm{~s}$ to $1.15 \mathrm{~s}$, with the percent error dropping from $16.5 \%$ to $9 \%$. In the $\beta_{0}$ metric, the error of $90 \%$ of the waves drops from $24^{\circ}$ to $12^{\circ}$; and in the $D(\theta)$ metric, dropping from 1.36 to 1.18. Examples can be seen in Figure 149 to Figure 154.

Figure 149 - Directional spectrum at $135^{\circ}$ estimated with conventional prior.
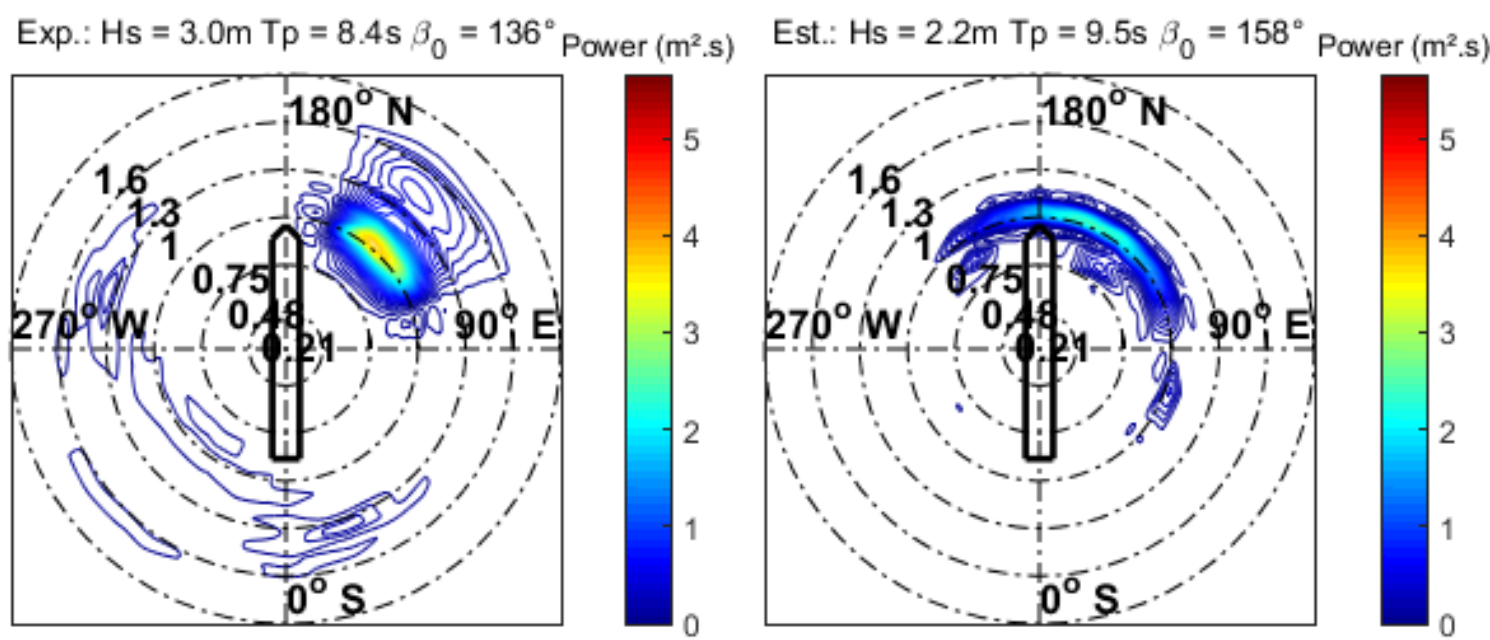

Source: Elaborated by the author. 
Figure 150 - Directional spectrum at $135^{\circ}$ estimated with MLQP.

Exp.: $\mathrm{Hs}=3.0 \mathrm{~m} \mathrm{Tp}=8.4 \mathrm{~s} \beta_{0}=136^{\circ}$ Power $\left(\mathrm{m}^{2} . \mathrm{s}\right)$

Est.: $\mathrm{Hs}=2.7 \mathrm{~m} \mathrm{Tp}=8.6 \mathrm{~s} \beta_{0}=150^{\circ}$ Power $\left(\mathrm{m}^{2} . \mathrm{s}\right)$
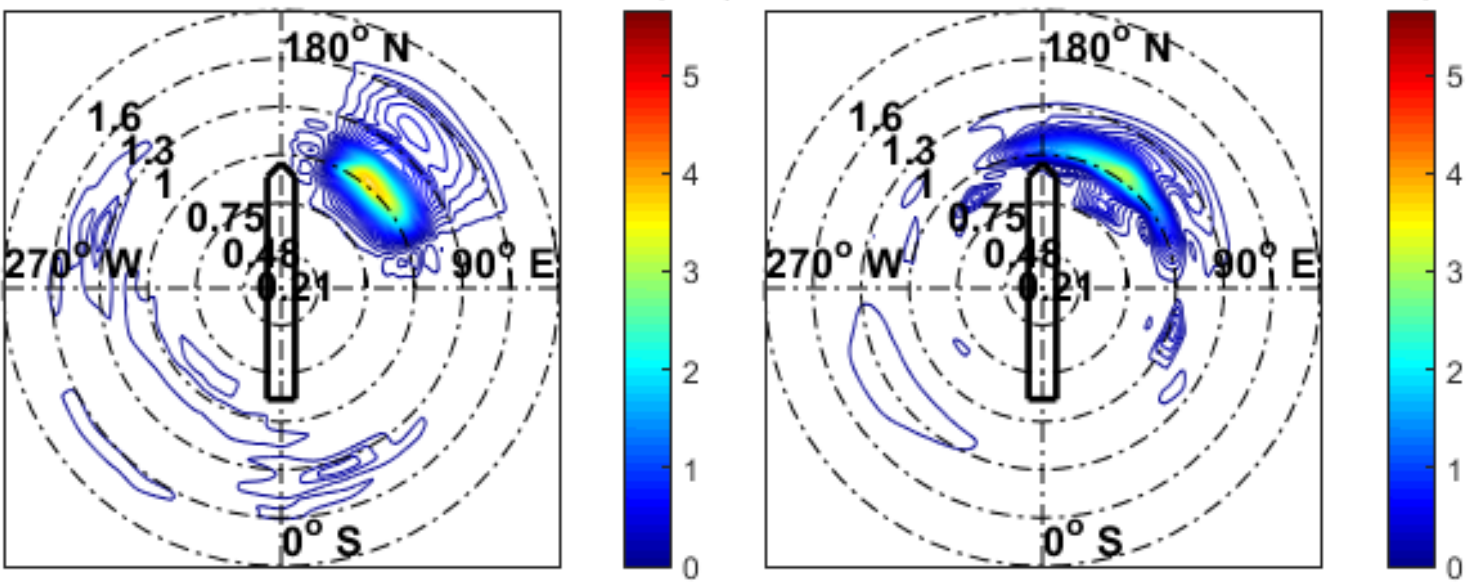

Source: Elaborated by the author.

Figure 151 - Energy spectral density at $135^{\circ}$ for different priors.

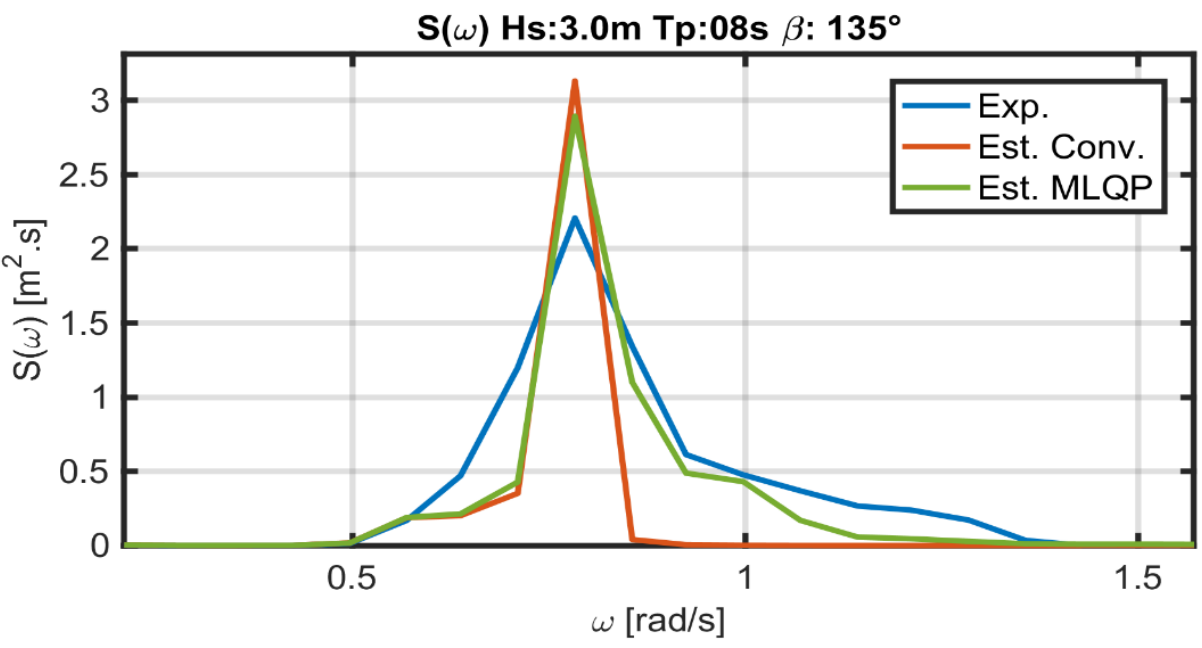

Source: Elaborated by the author.

Figure 152 - Directional spectrum at $0^{\circ}$ estimated with conventional prior.

Exp.: $\mathrm{Hs}=1.3 \mathrm{~m} \mathrm{Tp}=12.0 \mathrm{~s} \beta_{0}=000^{\circ}$ Power $\left(\mathrm{m}^{2} . \mathrm{s}\right)$
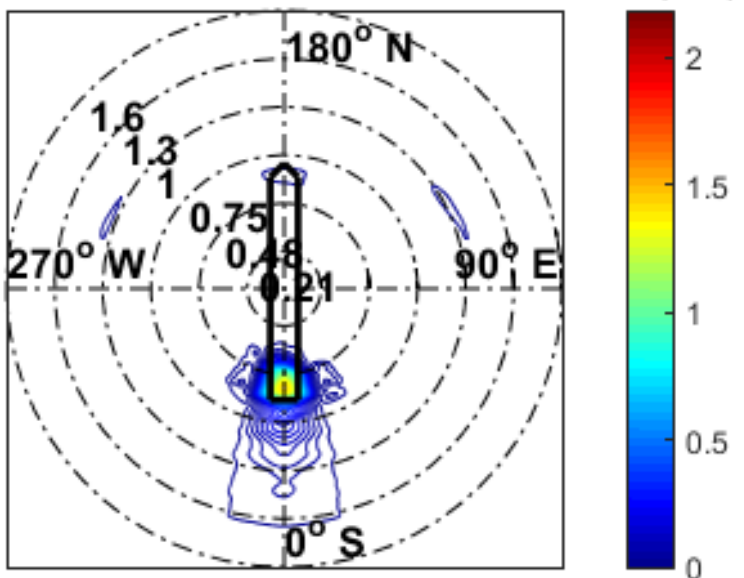

Est.: $\mathrm{Hs}=1.0 \mathrm{~m} \mathrm{Tp}=13.0 \mathrm{~s} \beta_{0}=360^{\circ}$ Power $\left(\mathrm{m}^{2} . \mathrm{s}\right)$
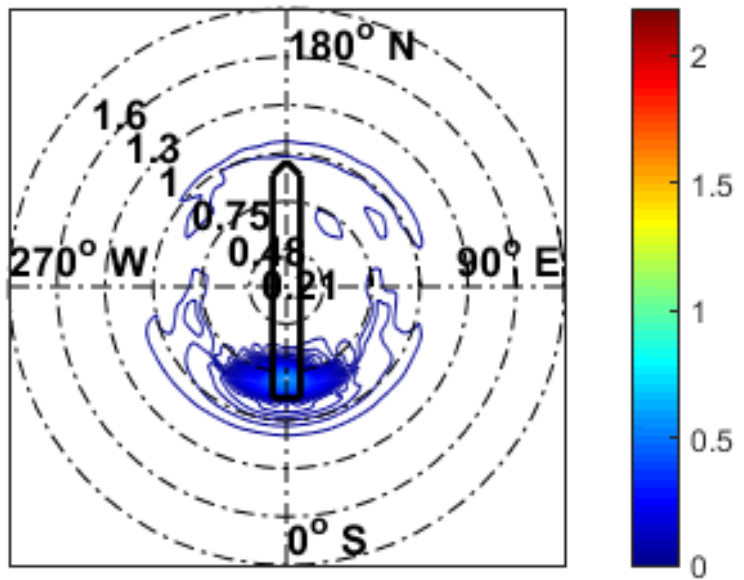

Source: Elaborated by the author. 
Figure 153 - Directional spectrum at $0^{\circ}$ estimated with MLQP.

Exp.: $\mathrm{Hs}=1.3 \mathrm{~m} \mathrm{Tp}=12.0 \mathrm{~s} \beta_{0}=000^{\circ}$ Power $\left(\mathrm{m}^{2} . \mathrm{s}\right)$
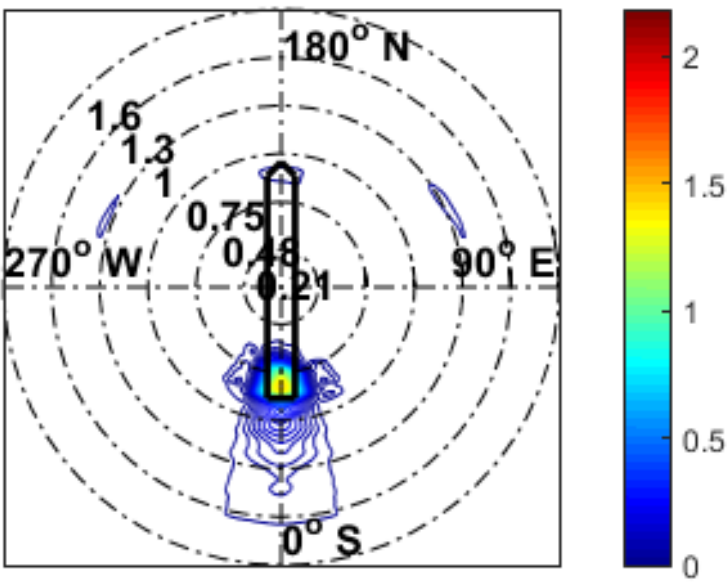

Est.: $\mathrm{Hs}=1.1 \mathrm{~m} \mathrm{Tp}=11.6 \mathrm{~s} \beta_{0}=360^{\circ}$ Power $\left(\mathrm{m}^{2} . \mathrm{s}\right)$
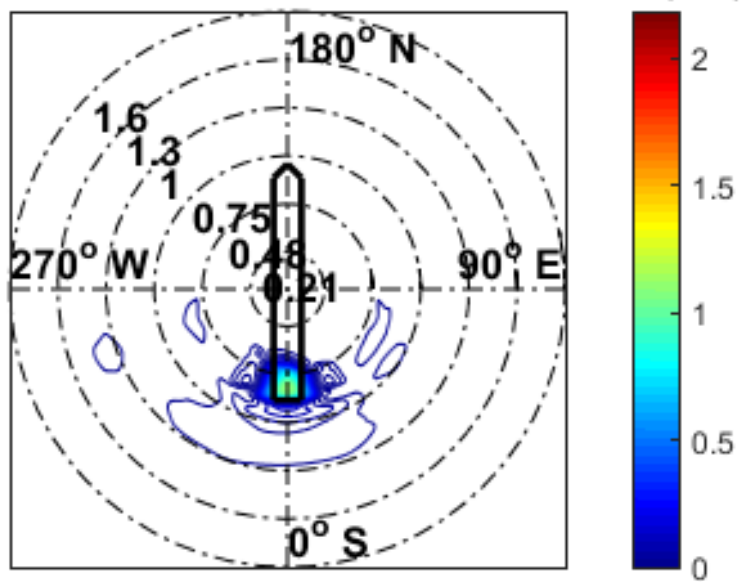

Source: Elaborated by the author.

Figure 154 - Energy spectral density at $0^{\circ}$ for different priors.

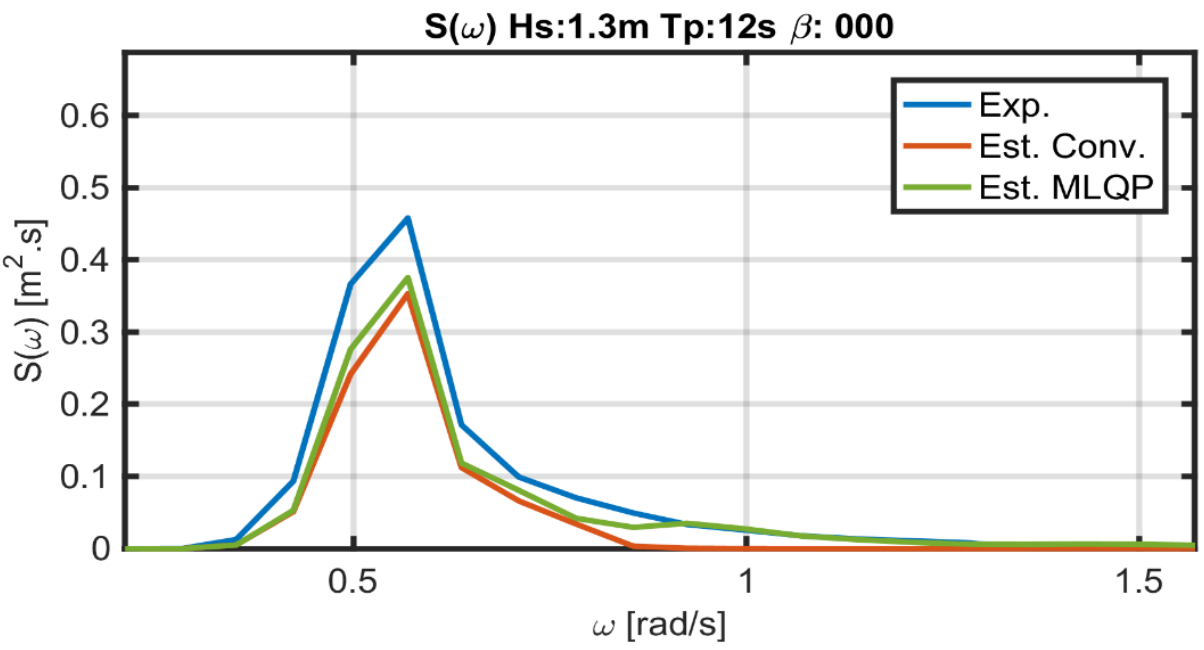

Source: Elaborated by the author.

Accordingly, to the results, the improvement seems to come from a better recovering of the energy in high frequencies/low periods using the optimal prior. This is important because the usage of this prior, by itself, can potentially improve the estimation of energies causing drift forces, the main goal of the thesis, without the installation of the wave-probes.

For extreme drafts there is no significantly change in the conclusions, so they will not be detailed in the text.

For the bimodal set, however, the results are important, since only unimodal seas were used to calibrate the optimal prior and a possible drawback of this approach would be not being able to estimate properly multi-modal seas. The histograms for the 
only vessel movements cases are shown in Figure 155; the other cases, with waveprobes, were omitted, since it was already concluded that the addition of the waveprobes diminish the influence of the change in priors.

Figure 155 - Histograms for the bimodal set comparing the priors.
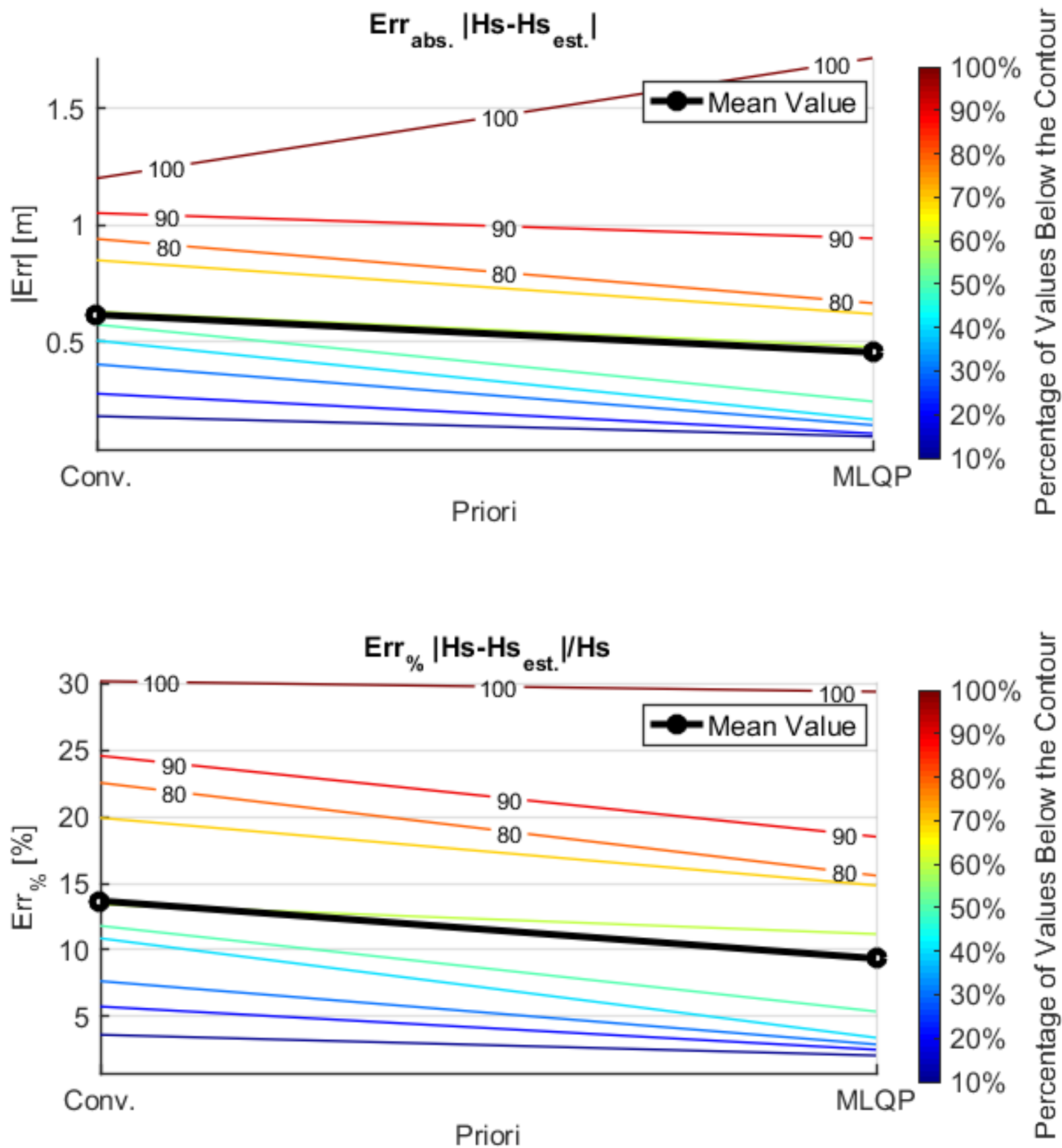


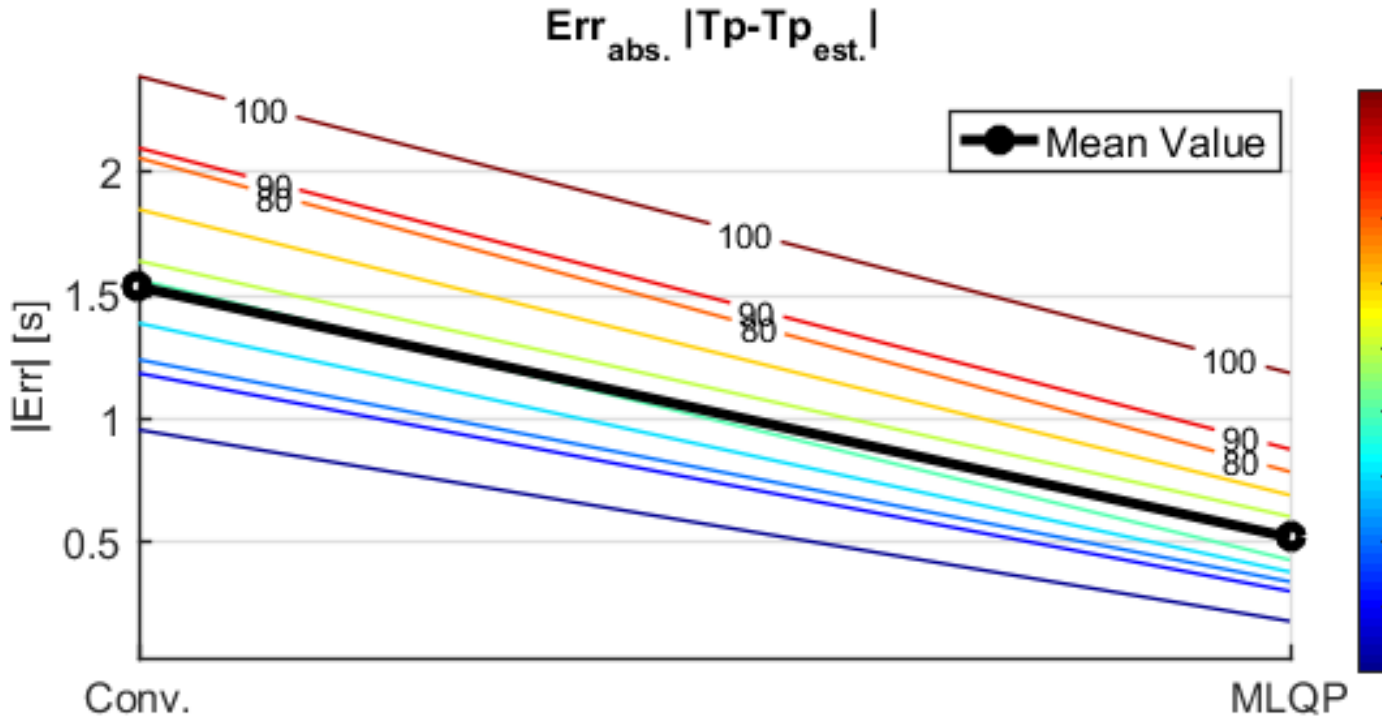

$100 \%$
$-90 \%$
$-80 \%$
$-70 \%$
$-60 \%$
$-50 \%$
$-40 \%$
$30 \%$
$20 \%$
$10 \%$
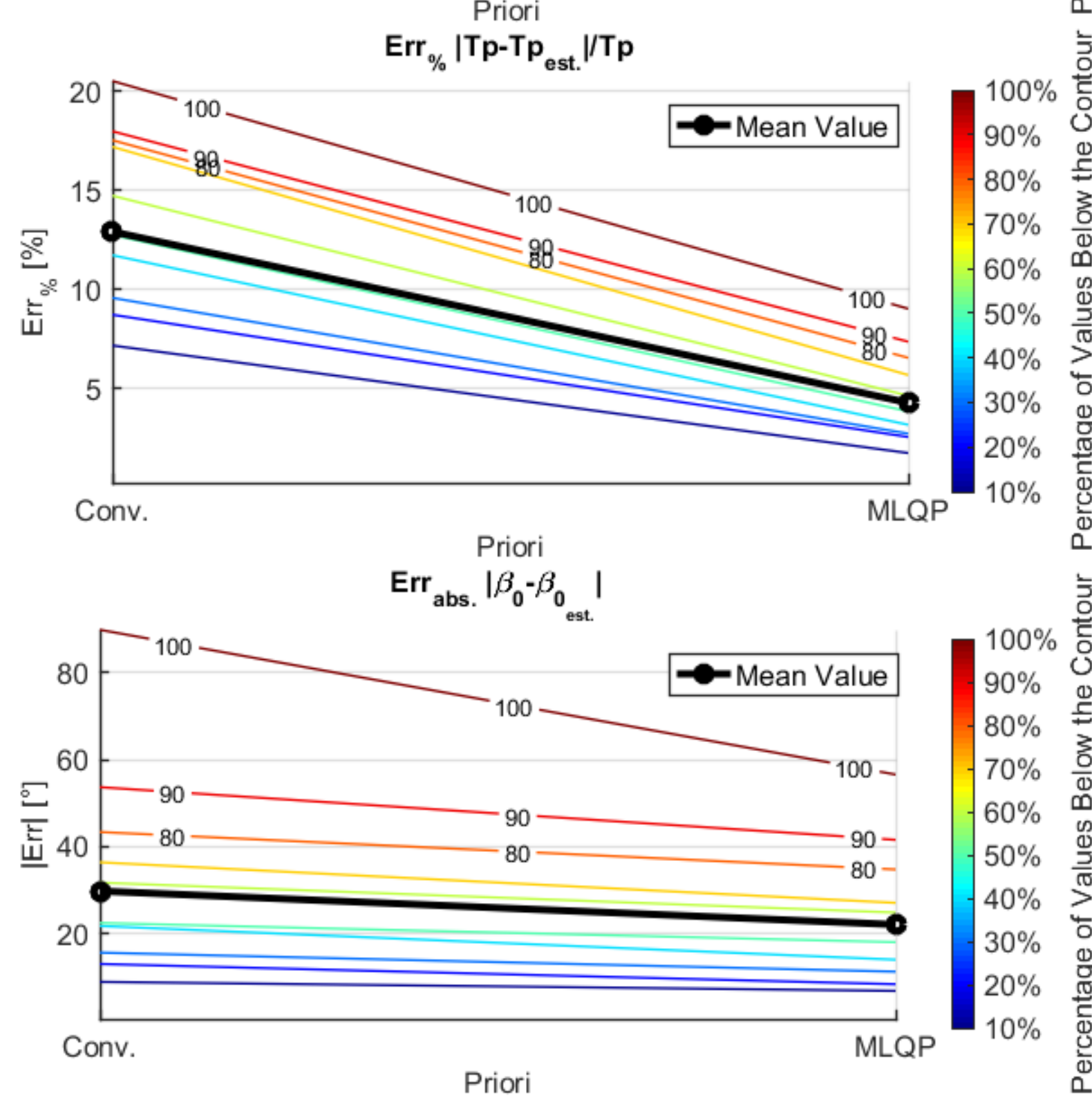

Source: Elaborated by the author. 
The results are consistent with the conclusions obtained for the unimodal set, i.e., the MLQP training with only unimodal seas is still adequate for the bimodal seas tested. This probably happens because the bimodal seas are the summation of independent unimodal seas with a reasonable distance, in the $\boldsymbol{x}$ vector indices sense, i.e., seas that are almost uncorrelated, in the covariance of the training set sense.

Some particular cases are shown in Figure 156 to Figure 159.

Figure 156 - Quartering-bow spectrum, conventional prior.

Exp.: $\mathrm{Hs}=4.1 \mathrm{~m} \mathrm{Tp}=13.9 \mathrm{~s} \beta_{0}=096^{\circ}$ Power $\left(\mathrm{m}^{2} . \mathrm{s}\right)$
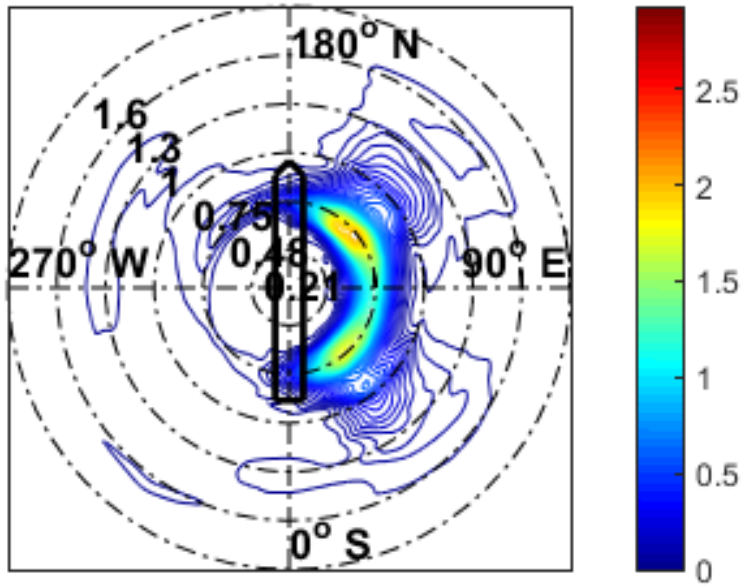

Source: Elaborated by the author.

Figure 157 - Quartering-bow spectrum, MLQP.

Exp.: $\mathrm{Hs}=4.1 \mathrm{~m} \mathrm{Tp}=13.9 \mathrm{~s} \beta_{0}=096^{\circ}$ Power $\left(\mathrm{m}^{2} . \mathrm{s}\right)$
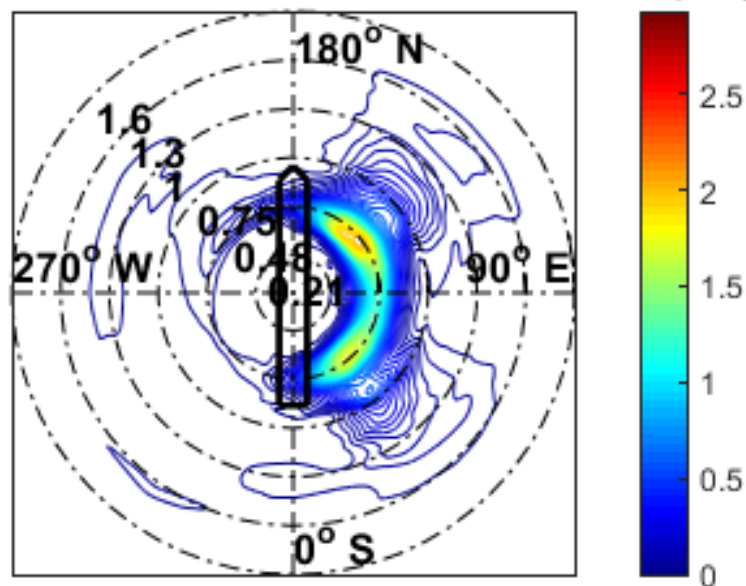

Est.: $\mathrm{Hs}=4.0 \mathrm{~m} \mathrm{Tp}=13.2 \mathrm{~s} \beta_{0}=089^{\circ}$ Power $\left(\mathrm{m}^{2} . \mathrm{s}\right)$
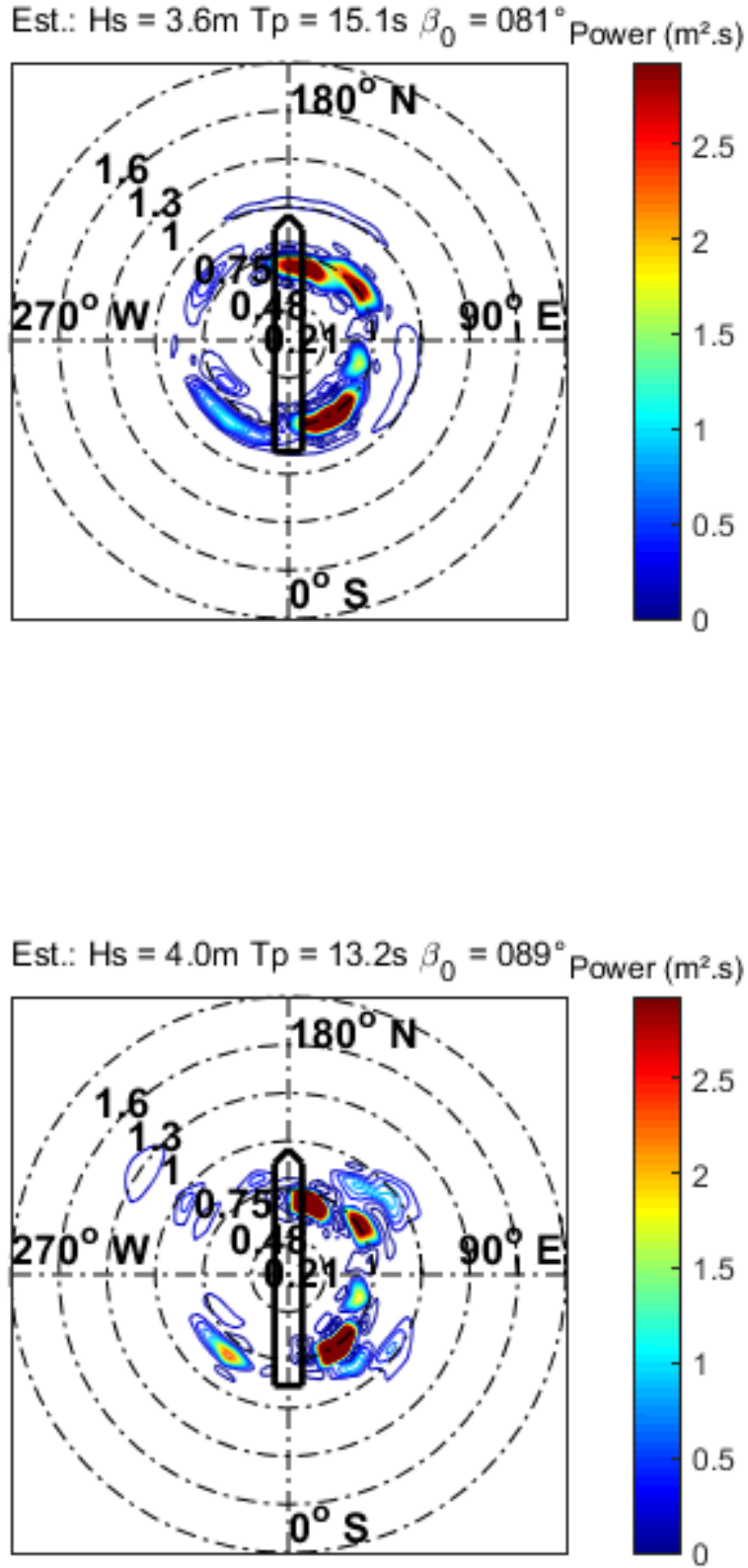

Source: Elaborated by the author. 
Figure 158 - Head-beam spectrum, conventional prior.

Exp.: $\mathrm{Hs}=5.1 \mathrm{~m} \mathrm{Tp}=13.2 \mathrm{~s} \beta_{0}=165^{\circ}$ Power $\left(\mathrm{m}^{2} . \mathrm{s}\right)$
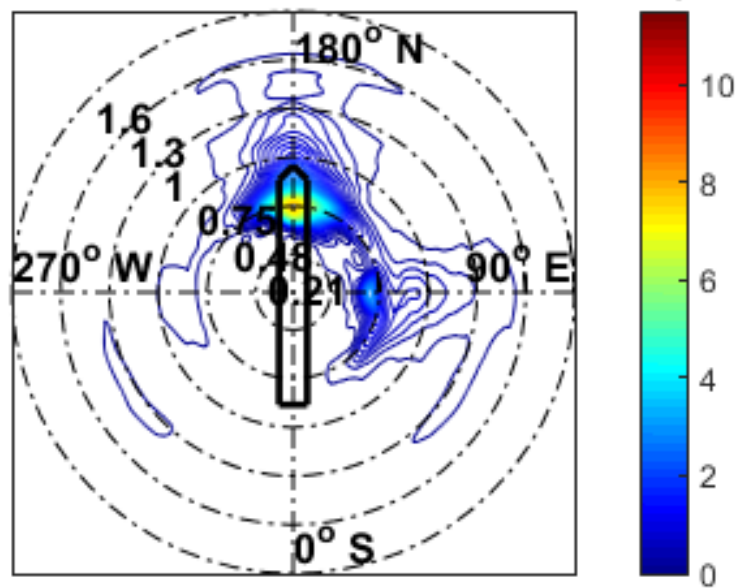

Est.: $\mathrm{Hs}=4.6 \mathrm{~m} \mathrm{Tp}=14.4 \mathrm{~s} \beta_{0}=154^{\circ}$ Power $\left(\mathrm{m}^{2} . \mathrm{s}\right)$

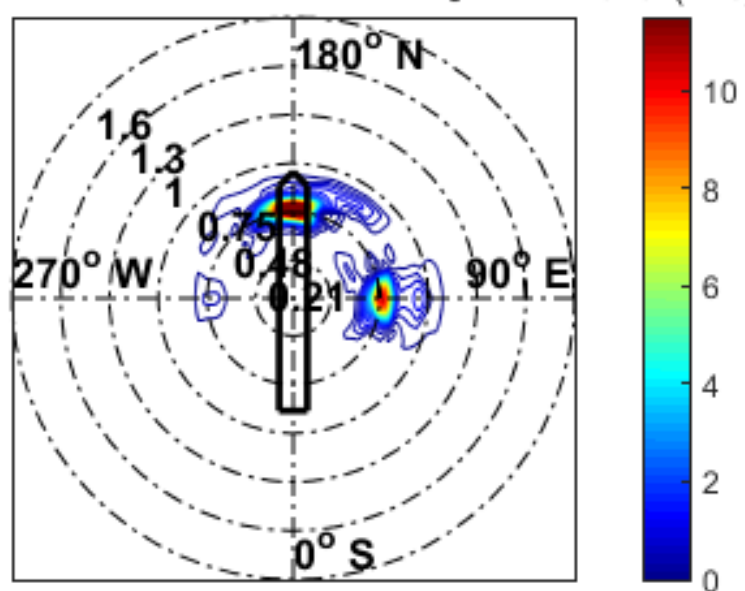

Source: Elaborated by the author.

Figure 159 - Head-beam spectrum, MLQP.

Exp.: $\mathrm{Hs}=5.1 \mathrm{~m} \mathrm{Tp}=13.2 \mathrm{~s} \beta_{0}=165^{\circ}$ Power $\left(\mathrm{m}^{2} . \mathrm{s}\right)$

Est.: $\mathrm{Hs}=5.2 \mathrm{~m} \mathrm{Tp}=13.5 \mathrm{~s} \beta_{0}=154^{\circ}$ Power $\left(\mathrm{m}^{2} . \mathrm{s}\right)$
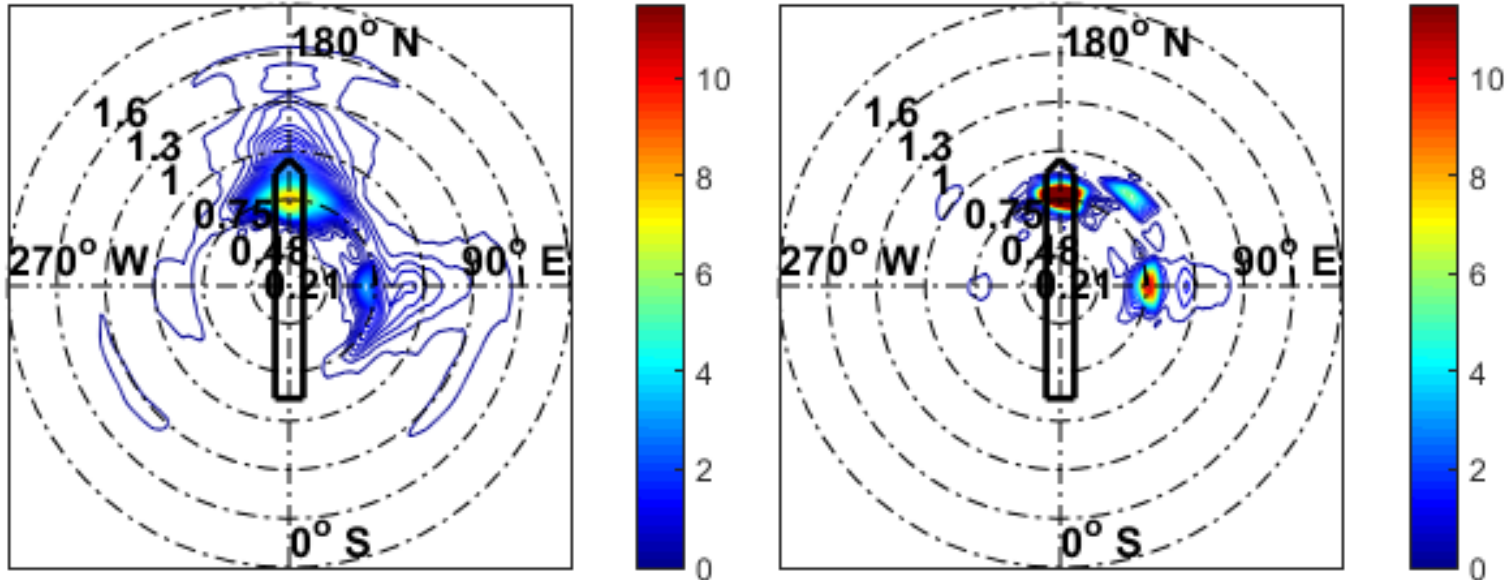

Source: Elaborated by the author. 


\section{CONCLUSIONS}

In order to overcome limitations in the current methods in directional spectrum estimation, mainly in the context of wave-buoy analogy based estimations, this thesis evaluated the usage of wave-probes as a complementary measurement strategy for enlarging the estimation period range for low periods, below $8 \mathrm{~s}$. There were already studies exploring this possibility, but a complete treatment was not yet available.

The thesis aimed to answer three main questions: how to incorporate the waveprobes in the chosen estimation method? How to define the best position of those wave-probes? How to define the best number of the wave-probes?

The incorporation of the wave-probes was solved by the extended linear model formulation, allowing the usage of the probes in a simple manner with simple linear corrections for accounting the vertical vessel movements. Starting from this model, a Bayesian Estimation approach, selected due to good results already shown in different works from different authors, was readily derived.

With the proposed method, it was possible to deduce a criterion to define the best position of the wave-probes, based on the A-optimal design of experiments or, more specifically, the utility Bayesian optimal design of experiments. Furthermore, it was proven mathematically and numerically that this simple method would provide the minimization of the upper bound of different error criteria, given support to the strategy.

After defining the best position for a given number of wave-probes, the ideal number of gauges was defined using the Elbow Criterion, a simple approach also supported by a mathematical rationale and observed experimentally.

The answers to the original questions arisen other new important questions, motivated by the strong probabilistic interpretation of the methods described, concerning how to solve the optimal design of experiments problem and the possibility of an optimal prior.

The optimization problem was solved by a heuristic method, which was able to explore some particularities of the problem, having a great potential to be expanded to more general optimal sensor placement problems due its easy adaptability to multiobjective formulations. 
The optimal prior, by its turn, was proposed to be the covariance of a sample of the seas expected for a given region, with three rationale pointing to it: the maximum likelihood quadratic prior; the prior that results in the best linear estimator; and the prior that is the closest to the real probability function given the Gaussian functional format.

All the propositions were tested and validated experimentally with a model in the towing tank at the TPN laboratory, concluding, for unimodal seas with intermediate draft, that: the addition of just one wave-probe reached approximately a 37\%-55\% improvement in the energy parameters estimations $-H_{S}$ and $T_{P}$; the addition of two or more probes reached approximately a $62 \%-65 \%$ improvement in the parameters estimations; the addition of four probes achieved the best cost benefit for mean direction estimation; and the addition of six probes was the recommendation for the best directional estimation in the entire range of the spectrum.

The optimal prior was able to improve the estimation capabilities mainly when zero or one wave-probes were used, i.e., when the prior is more needed to complement the measurement information. Furthermore, it was shown that the usage of this prior was able to increase the trace criterion impact on the other errors, i.e., it should be used at least to define the best DoFs and the best positions of wave-probes to be used.

Summarizing what was presented, the thesis was able to contribute directly with the area, opening and giving support to new possibilities, hopefully contributing to the exploration of new frontiers in the offshore industry. 


\section{PROPOSALS FOR FUTURE RESEARCHES - AN UNIFIED FRAMEWORK FOR BAYESIAN ESTIMATION}

The contributions of this thesis, despite the improvements achieved, are far from exhausting the Bayesian estimation possibilities. This final section aims to draw a general framework exploring the method capabilities, in order to map the contributions of the thesis and some next steps that can be taken in future researches.

The general framework proposed can be seen in Figure 160.

Figure 160 - General framework for Bayesian estimation.

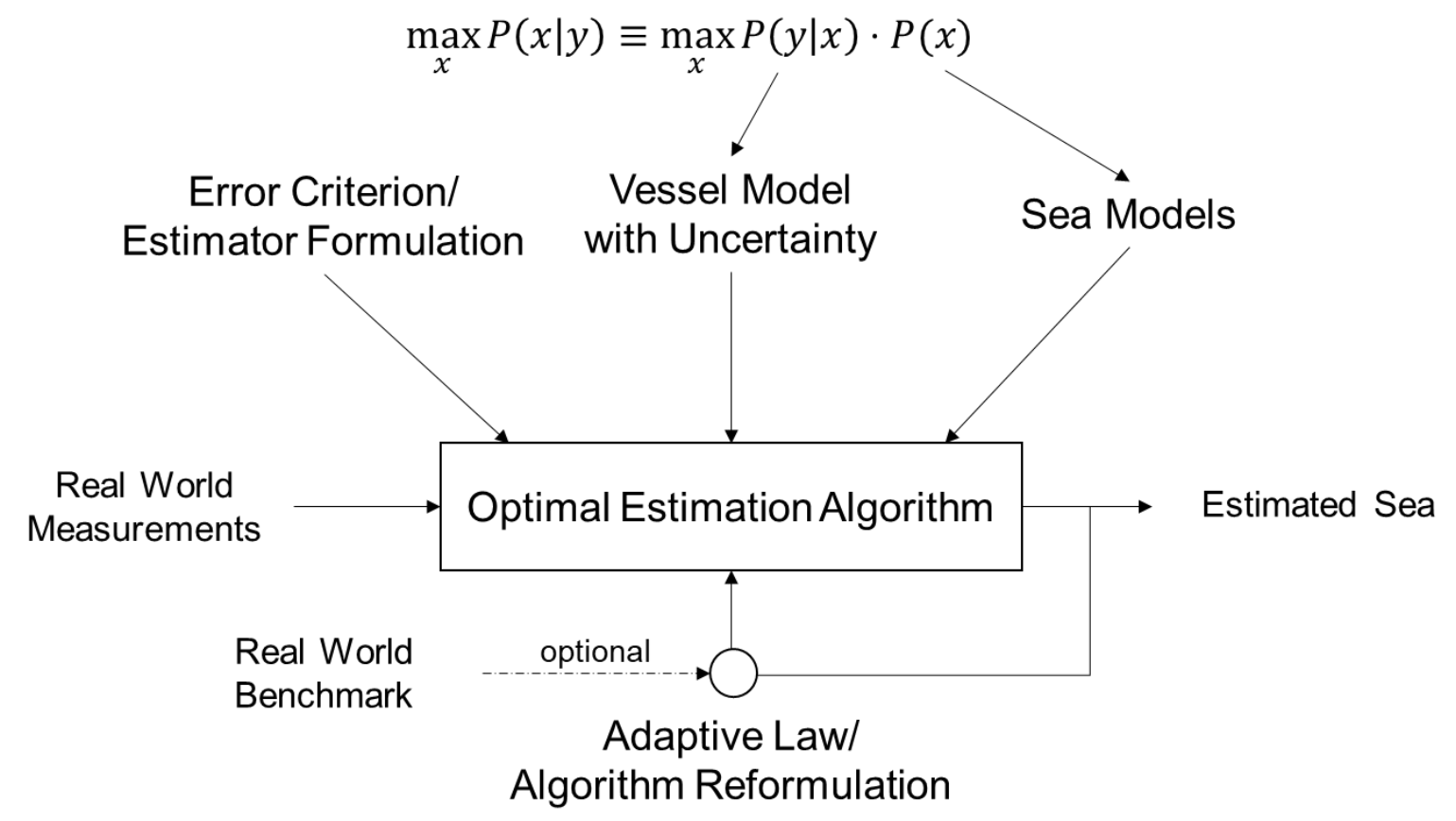

Source: Elaborated by the author.

\section{SEA MODELS}

One of the main contributions of the thesis was the idea of using model based calibrated priors instead of subjective ones. Starting from a sample of seas, it is possible, given an error criterion and estimator formulation, to derive an optimal prior.

This approach can be expanded for a different number of sea models, including bimodal models and other parametric formulations far more complex than the JONSWAP used during this text. Future researches in the area could explore those diverse models studying not only the best one in each situation, but also the robustness of each model when a set of seas different from the training sample is experienced. 


\section{VESSEL MODEL WITH UNCERTAINTIES}

The same approach used in this thesis in order to define an optimal prior could be used to define the optimal normalization matrix based on the uncertainties of the sensors employed in the estimation. Researches in this subject shall be concerned about evaluating sensors employed in real vessels, studying some possible errors, water spray, for example, and noise patterns, in order to explorer those patterns in the Bayesian estimation formulation.

A further step in this subject would be to incorporate uncertainties in the vessel model, or even non-linearities in the nominal model, which could potentially solve the roll problem, or give the algorithm an objective metric to ignore automatically this movement and its influence when estimating some frequencies or directions.

Researches in this subject can use mathematical formulations as the total least square, or more general error in variables models, which provide highly flexible tools for working with general types of uncertainties.

\section{ERROR CRITERION AND ESTIMATOR FormuLATION}

This topic complements the previous ones. During this thesis, it was shown that an optimality can be achieved for the maximum likelihood quadratic prior and for the linear estimator. The estimator formulation, however, can be expanded beyond those frameworks, using the exact probability distribution of the functions, neural networks, Bayesian nets and others models in order to increase the estimation capabilities.

Researches in this topic shall be concerned not only about the possibilities of an exact modelling of the probabilities, but also about the computational costs of the solutions.

\section{AdAPTIVE LAW AND ALGORITHM REFORMULATION}

During the thesis, one of the conclusions stated that the usage of wave-probes would be recommended only before the roll peak, avoiding the non-linearities. This is an example of an algorithm reformulation based on benchmark comparisons. This kind of conclusion, however, can be performed automatically by an online adaptive law, which makes the estimation algorithm more robust against unmodelled situations. 
Some authors have already explored this possibility. One example is (LAJIC, 2010), using fault-detection methods in onboard systems. This work is a nice example that shows that the algorithm adaptation does not need to use necessarily a benchmark, but can be based only on the onboarding measurements, comparing one measurement against the others.

A possible research in this subject could explore an algorithm based on reweighted least squares, which essentially modifies the normalization matrix of the likelihood function based on how much the noise of each sensor should be in order to explain the deviation between the measurements and the estimated sea. This is a powerful framework, with a strong probabilistic reasoning, and whose convergence was already proved in the literature.

An adaptive algorithm like the described ones could potentially have excluded the wave-probe measurements around the roll peak automatically, giving a small weight to those measurements after noticing how the discrepancies got bigger in this region.

Other possibilities could be using radars, satellite imagery or wave buoys in order to perform an online calibration of the algorithm, using the strongest part of each estimation system in the process and avoiding their weaknesses.

\section{OPTIMAL ESTIMATION ALGORITHM}

Finally, researches covering all the areas above would provide substance for the formulation of an optimal estimation algorithm. The main work of the designer of a particular estimation problem, then, would be to provide information about the models, the uncertainties and the desired computational cost, already accounting for possible adaptive laws, and an optimal algorithm could be automatically defined combining all those information, achieving a general framework for the Bayesian estimation problem. 


\section{REFERENCES}

AALBERS, A. B., TAP, R. F. and PIKSTER, J. A. 2001. An Application of Dynamic Positioning Control using Feed Forward. International Journal of Robust and Nonlinear Control. 2001.

AKAIKE, H. 1980. Likelihood and Bayes Procedure. Bayesian Statistics. Valencia : University Press, 1980.

ALPAYDIN, E. 2004. Introduction to Machine Learning. Cambridge : The MIT Press, 2004.

BERGER, J. O. 1985. Statistical Decision Theory and Bayesian Analysis. New York : Springer-Verlog, 1985.

BISHOP, C. M. 2006. Pattern Recognition and Machine Learning. New York: Springer, 2006.

BISPO, I. B. S., SIMOS, A. N., TANNURI, E. A. and CRUZ, J. J. 2012. Motion-based wave estimation by a Bayesian Inference Method: a procedure for pre-defining the Hyperparameters. 22th International Offshore and Polar Engineering Conference. 2012.

BLUEWATER. 2016. Mooring Systems. Bluewater. [Online] 2016. [Cited: Appril 01, 2016.] http://www.bluewater.com/products-technology/mooring-systems/.

CAPON, J. 1969. High-Resolution Frequency-Wavenumber Spectrum Analysis. Proc. IEEE. 1969, Vol. 57.

CARNEIRO, M. L. 2012. Desenvolvimento de Sistema de Controle para Geração e Absorção Ativa de Ondas em Tanques de Ensaios de Estruturas Oceânicas. s.l. : PhD. Thesis in Mechatronic Engineering, Escola Politécnica da Universidade de São Paulo, In Portuguese., 2012.

CHALONER \& VERDINELLI, K. 1995. Bayesian Experimental Design: A Review. 1995, Vol. 10, pp. 273-304.

COTÉ, L. J., DAVIS, J. O. and MARKS, W. 1960. The Directional Spectrum of a WindGenerated Sea as Determined from Data Obtained by the Stereo Wave Observation Project. Meteorological Papers, 2, No. 6, New York University. 1960.

DARBYSHIRE, M. 1961. A Method for Calibration of Ship-Borne Wave Recorders. J. Ocean Dynamics. 1961.

DAVIS, M. 2013. Stochastic Modelling and Control. s.I. : Springer Science \& Business Media, 2013.

DWYER, P. S. 1967. Some applications of matrix derivatives in multivariate analysis. Journal of the American Statistical Association. 1967, Vol. 62 (318), pp. 607-625.

FALTISEN \& LOKEN, O. M. 1978. Drift forces and slowly varying horizontal forces on a ship in waves. Timman Symposium on Applied Mathematics. 1978. 
FUKY MarinTech. 2016. Permanent Mooring. [Online] 2016. [Cited: October 15, 2016.] http://fukymarintech.weebly.com/mooring-types.html.

FURG. 2008. Meteorologia. [Online] Universidade Federal do Rio Grande, 2008. [Cited: October 15, 2016.] http://www.meteorologia.furg.br/.

HABER, E. 2008. Numerical Methods for Optimal Experimental Design of Large-Scale III-Posed Problems. 2008.

HASHIMOTO \& KOBUNE, N. 1988. Directional Spectrum Estimation from a Bayesian Approach. Costal Engineering Proceedings, v. 1, n. 21. 1988.

HASSELMAN, K. and BARNETT, T. P., BOUWS, E., CARLSON, H., CARTWRIGHT, D. E. 1973. Measurements of Wind-Wave Growth and Swell Decay During the Joint North Sea Wave Project (JONSWAP). Deut. Hydrogr. 1973.

HASTIE, T., TIBSHIRANI, R. and FRIEDMAN, J. 2008. The Elements of Statistical Learning: Data Mining, Inference and Prediction. Stanford : Springer, 2008.

HIMENO, Y. 1981. Prediction of Ship Roll Damping. A State of the Art. 1981. Michigan University Ann Arbor Department of Naval Architecture and Marine Engineering.

HINRICHSEN, P. F. 2014. Bifilar Suspension Measurement of Boat Inertia Parameters. Journal of Sailboat Technology. 2014.

HIRAYAMA, T. 1987. Real-time estimation of sea spectra based on motions of a running ship. Journal of the Kansai Society of Naval Architects. 1987, Vol. 204.

HOGBEN \& COBB, N. 1986. Parametric Modelling of Directional Wave Spectra. 18th Offshore Technology Conference. 1986.

HUGHES, G., et al. 2009. Close-In Precision DP using Wave Feed Forward: STLVAST Phase 2 \& 3. Dynamic Positioning Conference. 2009.

-. 2010. DP Advances in the USN Seabase: STLVAST Sea Trial and Completion. Dynamic Positioning Conference. 2010.

IBRAHIM \& GRACE, I. M. 2009. Modeling of Ship Roll Dynamics and its Coupling with Heave and Pitch. Mathematical Problems in Engineering. 2009.

ISEKI \& HIRAYAMA, T. 2012. Application of Bayesian Wave Estimation to Actual Merchant Vessels. 22th International Offshore and Polar Engineering Conference. 2012.

ISEKI \& NIELSEN, T. 2015. Study on Short-Term Variability of Ship Responses in Waves. 2015.

ISEKI \& OHTSU, T. 1999. Bayesian estimation of directional wave spectra based on ship motions. Control engineering practice. 1999, Vol. 8.

ISEKI, T., Ohtsu, K. and Fujino, M. 1992. A study on estimation of directional spectra based on ship motions. The Journal of Japan Institute of Navigation. 1992.

ISERMANN \& MUNCHOF, M. 2011. Identification of Dynamic Systems - An Introduction with Applications. s.I. : Springer, 2011. 
ISOBE, M., Kondo, K. and Horikawa, K. 1984. Extension of MLM for Estimating Directional Wave Spectrum. Proc. Symp. on Description and Modelling of Directional Seas, Lyngby, Denmark, Danish Hydraulic Institute. 1984.

JOURNÉE \& MASSIE, W. W. 2011. Offshore Hydromechanics. s.l. : Delft University of Technology, 2011.

KOBUNE \& HASHIMOTO, K. 1986. Estimation of Directional Spectrum from The Maximum Entropy Principle. Proc. 5th int. Offshore Mechanics and Artic Engineering (OMAE), Sympo., Tokyo. 1986.

KORKMAZ, S, GOKSULUK, D and ZARARSIZ, G. 2014. MVN: An R Package for Assessing Multivariate Normality. The R Journal. 2014, Vol. 6(2), pp. 151-162.

KRAUSE, A., SINGH, A. and GUESTRIN, C. 2008. Near-Optimal Sensor Placements in Gaussian Processes: Theory, Efficient Algorithms and Empirical Studies. Journal of Machine Learning Research. 2008.

LAJIC, Z. 2010. Fault-tolerant onboard monitoring and decision support systems. 2010.

LAJIC, Z., Nielsen, U. D. and Mogens, B. 2010. Fault Isolation and Quality Assessment for Shipboard Monitoring. 29th International Conference on Ocean, Offshore and Artic Engineering (OMAE). 2010.

LONGUET-HIGGINS, M. S. 1946. Measurement of sea conditions by the motion of a floating buoy. Detection of predominant groups of swell. Admiralty Research Lab, Report 103.40/N5. 1946.

LONGUET-HIGGINS, M. S., Cartwright, D. E. and Smith, N. D. 1963. Observations of the directional spectrum of sea waves using the motions of a floating buoy. Proc. Conf. Ocean Wave Spectra, Prentice-Hall. 1963.

MARTINS, J. A. A. 2003. A Análise de Ondas em Tanques de Dimensões Reduzidas com Vistas à Engenharia Oceânica. São Paulo : s.n., 2003. Phd. thesis presented to the Escola Politécnica da Universidade de São Paulo.

MELLO, C., P. 2012. Sistema de Automação e Controle para Tanques Oceânicos com Múltiplos Atuadores. São Paulo: s.n., 2012. Phd. thesis presented to the Escola Politécnica da Universidade de São Paulo..

MELLO, P. C., et al. 2013. A control and automation system for wave basins. Mechatronics. 1, 2013, Vol. 23.

MITSUYASU \& MIZUNO, H. 1976. Directional Spectra of Ocean Surface Waves. Fukuoka, Japan : Research Institute for Applied Mechanics, 1976.

MOGSTER, C. 2015. Bayesian Estimation of Non-Stacionary Ship Response Spectra. s.I. : Norwegian University of Science and Technology, 2015.

MONTAREZI, N., Jensen, J. J. and Nielsen, U. D. 2016b. Uncertainties in ship-based estimation of waves and responses. Oceans '15. 2016b. 
MONTAZERI, N., Nielsen, U. D. and Jensen, J. J. 2016a. Estimation of wind sea and swell using shipboard measurements - A refined parametric modelling approach. Applied Ocean Research. 2016a.

NEWMAN, J. N. 1999. Marine Hydrodynamics. Cambridge : The MIT Press, 1999.

NIELSEN, U. D. 2005. Estimation of Directional Wave Spectra from Measured Shipe Responses. Kongens Lyngby : s.n., 2005.

- 2006. Estimations of on-site Directional Wave Spectra from Measured Ship Responses. Marine Structures. 2006.

-. 2008a. Introducing two Hyperparameters in Bayesian Estimation of Wave Spectra. Department of Mechanical Engineering. 2008a.

-. 2011. Onboard Sea State Estimation Based on Measured Ship Motions. 12th International Ship Stability Workshop. 2011.

-. 2007. Response-based estimation of sea state parameters - influence of filtering. Ocean Engineering. 2007.

-. 2008b. The wave buoy analogy - Estimating high-frequency wave excitations. Applied Ocean Research. 2008b.

NIELSEN, U. D., Andersen, I. M. V. and Koning, J. 2013. Comparisons of Means for Estimating Sea States from an Advancing Large Container Ship. PRADS 2013. 2013.

NIELSEN, U. D., Bjerregard, M. Galezzi, R. and Fossen, T. I. 2015. New Concepts for Shipboard Sea State Estimation. 2015.

NOAA. 1996. Nondirectional and Directional Wave Data Analysis Procedures. Slidell, Louisiana : Marchall D. Earle, Neptune Sciences, Inc., 1996.

-. 2016. Satellites: Gathering data to monitor and understand our dynamic planet. [Online] 2016. [Cited: October 15, 2016.] http://www.noaanews.noaa.gov.

PASCOAL \& SOARES, R. 2006. Non-Parametric Wave Spectral Estimation using Vessel Motions. Applied Ocean Research. 2006.

PASCOAL, R., Soares, C. G. and Sorensen, A. J. 2005. Ocean Wave Spectral Estimation Using Vessel Wave Frequency Motions. Journal of Offshore Mechanics and Artic Engineering (OMAE). 2005.

PETROBRAS. 2010. Metocean Data - Campos Basin. 2010.

PIERSON \& MOSKOWITZ, H. 1964. A proposed spectral form for fully developed wind seas based on the similarity theory of S. A. Kitaigorodskii. J. Geophys. Res. 1964.

PINKSTER, J. A. 1976. Low frequency second order wave drifiting forces on vessels moored at sea. 11th Symposium on Naval Hydrodynamics, London. 1976.

-. 1978. Wave-feed-forward as a means to improve dynamic positioning. OTC3057, Houston. 1978.

PUKELSHEIM, F. 1948. Optimal Design of Experiments. 1948. 
QUALISYS. 2016. Qualysis cameras. [Online] 2016. [Cited: Appril 01, 2016.] http://www.qualisys.com/.

REMERY \& HERMANS, G. F. M. 1971. The slow drift oscillations of a moored object in random seas. Offshore Technology Conference, Houston. 1971.

RS AQUA. 2016. WaveRadar REX Datasheet. Rosemount \& RS Aqua. [Online] 2016. [Cited: October 15, 2016.] http://rsaqua.co.uk/wpcontent/uploads/2016/08/WaveRadar-Rex-v2.4.pdf.

SCRIVENS, R. 2008. The Offshore Wave Measuring King. International Ocean Systems. [Online] 2008. [Cited: October 15, 2016.] http://www.intoceansys.co.uk/articles-detail.php?iss=0000000005\&acl=0000000015.

SHIGEMURA, T., ASCE, M. and HAYASHI, K. 1988. Dynamic Behavior of a Mooring Buoy Installed in Open Shore. Coastal Engineering. 1988.

SIMOS, A. N. 2014. Hidrodinâmica - Comportamento no Mar de Sistemas Oceânicos de 1aㅡ Ordem. São Paulo : s.n., 2014. Lecture Notes in Portuguese from the Discipline PNV5200 - "First Order Hydrodynamics" at POLI-USP.

SIMOS, A. N., et al. 2010. Estimating wave spectra from the motions of moored vessels - Experimental validation. Applied Ocean Research. 2010.

SIMOS, A. N., et al. 2009. Estimating wave spectra from the motions of moored vessels: Experimental validation. Applied Ocean Research. 2009, Vol. 32.

SIMOS, A. N., Sparano, J. V. and Tannuri, E. A. 2007. Directional Wave Spectrum Estimation Based on Vessel 1st Order Motions - Field Result. 17th International Offshore and Polar Engineering Conference. 2007.

SINKE, M. 2015. Improvement of on board wave height measurement. 2015.

SLOTINE \& LI, W. 1991. Applied nonlinear control. Englewood Cliffs : prentice-Hall, 1991.

Stoica \& Moses, P. 2005. Spectral Analysis of Signals. New Jersey : Prentice Hall, 2005.

STREDULINSKY \& THORNHILL, D. C. 2011. Ship motion and wave radar data fusion for shipboard wave measurement. Journal of Ship Research. 2011, Vol. 55.

TAKEKUMA \& TAKAHASHI, K. 1972. On the Evaluation of Sea Spectra Based on Measured Ship Motions. Resistance and Propulstion Research Laboratory, Nagasaki Technical Institute. 1972.

TANNURI, E. A. and Simos, A. N., Sparano, J. V., Matos, V. L. F. 2012. Motionbased wave estimation: Small-scale tests with a crane-barge model. Marine Structures. 2012.

TANNURI, E. A. and Sparano, J. V., Simos, A. N., Cruz, J. J. 2003. Estimating directional wave spectrum based on stationary ship motion measurements. Applied Ocean Research. 2003. 
Tanque de Provas Numérico EPUSP. March 2015. Wave Measurements Aboard FPSO Unit in Peregrino Field. Technical Report 02. March 2015.

TPN. 2016. Laboratory Structure (in portuguese). Numerical Offshore Tank TPN-USP. [Online] 2016. [Cited: 02 01, 2016.] http://tpn.usp.br/category/estrutura/.

TRAENKMANN, I. 2008. Waves and currents by X-band radar. Coastal Wiki. [Online] September 21, 2008. [Cited: October 10, 2016.] http://www.coastalwiki.org/wiki/Waves_and_currents_by_X-band_radar.

TUCKER, M. J. 1956. A ship-borne wave recorder. Trans. Inst. Nav. Arch. London. 1956.

VAN OORTMERSSEN, G. 1976. The motions of a moored ship in waves. PhD Thesis, Delft University of Technology. 1976.

WAMIT, Inc. 2015. Wamit User Manual - Version 7.1. Chestnut Hill : MIT Press, 2015.

WEBSTER \& DILLINGHAM, W. 1981. Determination of Directional Seas from Ship Motions. Proceedings of Directional Wave Spectra Application, Berkeley. 1981. 


\section{APPENDIX A - POTENTIAL THEORY OF SURFACE WAVES}

This section contains a simplified deduction of the potential theory of surface waves, for the unfamiliar reader. The formulations are based on (NEWMAN, 1999) and (SIMOS, 2014).

\section{Potential Theory}

Under the hypothesis of ideal fluid, the water can be modeled as a homogenous, incompressible and frictionless continuous medium.

Using the homogenous and incompressible properties, it is possible to state that the mass, characterized by the specific mass of the fluid $\rho$, in a specified control volume $V$, will not change with time, i.e., the flux of mass through the boundary of the volume $\partial V$ will be zero. The equation can be further modified to a volume integral, using the divergence theorem, achieving the so called continuity equation, Equation (181), with $\boldsymbol{v}$ being the velocity vector of the fluid in a point, $\boldsymbol{n}$ the normal vector of the boundary in the same point and $\nabla$ the differential operator $\left[\frac{\partial}{\partial x}, \frac{\partial}{\partial y}, \frac{\partial}{\partial z}\right]$.

$$
\rho \iint_{\partial V} \boldsymbol{v} \cdot \boldsymbol{n} \partial V=\rho \iiint_{V} \nabla \cdot \boldsymbol{v} d V=0 \rightarrow \nabla \cdot \boldsymbol{v}=0
$$

The frictionless property also has a consequence, derived by the Kelvin theorem, or the circulation conservation theorem, Equation (182), which states that, under conservative forces, the circulation of a fluid does not change with time. Assuming that the fluid is in rest at an infinity previous time, the circulation will always be zero, which results, by applying the Stokes theorem, that the ideal fluid is irrotational, Equation (183). In the equations, $S$ is a surface, $\partial S$ its closed contour and $d \boldsymbol{r}$ an element over the contour.

$$
\begin{gathered}
\frac{d}{d t} \int_{\partial S} \boldsymbol{v} \cdot d \boldsymbol{r}=0 \\
\int_{\partial S} \boldsymbol{v} \cdot d \boldsymbol{r}=\iint_{S} \nabla \times \boldsymbol{v} \cdot \boldsymbol{n} d S=0 \rightarrow \nabla \times \boldsymbol{v}=0
\end{gathered}
$$


The Helmhotz theorem states that every irrotational vector field can be described as the gradient of a scalar field. Consequently, the velocity field can be described as a potential field $\varphi$, Equation (184), which gives the name potential theory.

$$
\nabla \times \boldsymbol{v}=0 \rightarrow \boldsymbol{v}=\nabla \varphi
$$

As a result, by merging Equation (181) and Equation (184), the ideal fluid problem can be stated as a second-order partial differential equation, the Laplace's equation, Equation (185), in which $\Delta$ is the Laplace's operator $\left[\frac{\partial^{2}}{\partial x^{2}}, \frac{\partial^{2}}{\partial y^{2}}, \frac{\partial^{2}}{\partial z^{2}}\right]$.

$$
\nabla \cdot \boldsymbol{v}=0 \stackrel{v=\nabla \varphi}{\longrightarrow} \nabla \cdot \nabla \varphi=\nabla^{2} \varphi=\Delta \varphi=0
$$

The relation between the velocity field and the scalar pressure field, $p$, is given by the Euler's equation, Equation (187), obtained by applying the Newton's laws to an elemental volume, Equations (186). Using the potential relation, it is possible to achieve the Bernoulli's equation, Equation (188). The gravity acceleration is $[0,0,-g]=-\nabla g z=\boldsymbol{g}$.

$$
\begin{gathered}
\boldsymbol{F}=\frac{d(\rho \boldsymbol{v})}{d t}=\rho\left(\frac{\partial \boldsymbol{v}}{\partial t}+(\nabla \cdot \boldsymbol{v}) \cdot \boldsymbol{v}\right) \text { and } \boldsymbol{F}=-\nabla p+\rho \boldsymbol{g} \\
\frac{\partial \boldsymbol{v}}{\partial t}+(\nabla \cdot \boldsymbol{v}) \cdot \boldsymbol{v}+\frac{\nabla p}{\rho}-\boldsymbol{g}=\mathbf{0} \\
\nabla\left(\frac{\partial \varphi}{\partial t}+\frac{1}{2} \nabla \varphi \cdot \nabla \varphi+\frac{p}{\rho}+g z\right)=\mathbf{0}
\end{gathered}
$$

Finally, the forces, $\boldsymbol{F}$, and moments, $\boldsymbol{M}$, acting on a floating body are obtained through pressure integration over its surface, $S_{b}$, Equations (189) and (190), with $\boldsymbol{r}_{\boldsymbol{o}}$ a vector from the moment reference to the integration point.

$$
\begin{gathered}
\boldsymbol{F}=\iint_{S_{b}} p \boldsymbol{n} d S \\
\boldsymbol{M}_{\boldsymbol{o}}=\iint_{S_{b}} p\left(\boldsymbol{r}_{\boldsymbol{o}} \times \boldsymbol{n}\right) d S
\end{gathered}
$$

Resulting in a system of equations that is enough, along with some boundary conditions, to describe the fluid behavior in a deterministic manner that is validated experimentally in a considerable number of cases, despite the strong hypothesis. 


\section{SURFACE WAVES}

Not surprisingly, waves arise as a possible solution of the Laplace's equation. Plane progressive waves, for example, are described by the potential in Equation (191), in which: $k=\frac{2 \pi}{\lambda}$ is the wave number, $\omega=\frac{2 \pi}{T}$ is the angular frequency, $z$ is the depth, $x$ the progressive direction and $C_{i}$ constants which depends on the characteristics of each particular problem.

$$
\varphi(x, z, t)=\operatorname{Re}\left[\left(C_{1} e^{k z}+C_{2} e^{-k z}\right) e^{-i k x+i \omega t}\right], \nabla^{2} \varphi=0
$$

In general, the constants are defined by some boundary conditions that are always applied for waves: the impermeability of the sea bottom, which can be ignored in some cases; and the Cauchy-Poisson condition, which establishes the kinematic and the dynamic properties of the water surface.

The first one, impermeability of the sea bottom, is stated simply as a kinematic boundary, zeroing the velocity of the fluid encountering the obstacle at depth $-h$, Equation (192). The potential, then, assumes the form in Equation (193).

$$
\begin{gathered}
v_{z}(z=-h)=\left.0 \rightarrow \frac{\partial \varphi}{\partial z}\right|_{z=-h}=0 \\
\varphi(x, z, t)=\operatorname{Re}\left[C \cosh (k(z+h)) e^{-i k x+i \omega t}\right]
\end{gathered}
$$

The second usual condition, the Cauchy-Poisson condition, states that, on the wave surface $\zeta(x, t)=A \cos (k x-\omega t)$, the pressure will be equal to the atmospheric pressure - dynamic condition, Equation (194); relating, at the same time, the velocity of the fluid with the surface variation - kinematic condition, Equation (195). The union of both statements results in Equation (196), and in the final constant defining the potential, described in Equation (197). $A$ is the wave amplitude.

Two important simplifications are made: firstly, the amplitude of the wave is assumed small in comparison with the potential order of magnitude, which means that the boundary condition can be applied in $z=0 \sim A \cos (k x-\omega t)$; secondly, the high order terms in the Bernoulli's equation are ignored, by the same argument, i.e., $\nabla \varphi(x, y, 0, t)^{2} \sim 0$. 


$$
\begin{gathered}
\left.\frac{\partial \varphi}{\partial t}\right|_{z=0}+\frac{1}{2} \nabla \varphi(x, y, 0, t)^{2}+g \zeta=-\frac{p-p_{0}}{\rho}=\left.0 \approx \frac{\partial \varphi}{\partial t}\right|_{z=0}+g \zeta \rightarrow \zeta=-\left.\frac{1}{g} \frac{\partial \varphi}{\partial t}\right|_{z=0} \\
v_{z}(z=0)=\left.\frac{\partial \zeta}{\partial t} \rightarrow \frac{\partial \varphi}{\partial z}\right|_{z=0}=\frac{\partial \zeta}{\partial t} \\
\left.\frac{\partial^{2} \varphi}{\partial t^{2}}\right|_{z=0}+\left.g \frac{\partial \varphi}{\partial z}\right|_{z=0}=0 \\
\varphi(x, z, t)=\operatorname{Re}\left[\frac{i g A}{\omega \cosh (k h)} \cosh (k(z+h)) e^{-i k x+i \omega t}\right]
\end{gathered}
$$

Applying the boundary in Equation (196) to the potential in Equation (197), it is possible to relate the angular frequency with the wave number, obtaining the dispersion relation, Equation (198). It relates the length and the frequency of the waves, accordingly with the region depth. It also gives the interesting result that the waves will travel with different velocities, depending on their frequency.

$$
k=\frac{\omega^{2}}{g \tanh (k h)}
$$

If the depth is greater than half of the wave-length, $h>\frac{1}{2} \lambda$, the potential can be further simplified to an infinite-depth potential, Equations (199) and (200).

$$
\begin{gathered}
\varphi(x, z, t)=\operatorname{Re}\left[\frac{i g A}{\omega} e^{k z} e^{-i k x+i \omega t}\right]=\frac{g A}{\omega} e^{k z} \sin (k x-\omega t) \\
k=\frac{\omega^{2}}{g}
\end{gathered}
$$

The potential obtained so far can be modified and combined to satisfy other boundary conditions. For example, if an impermeable wall is present at $x=0$, a new boundary condition appears, Equation (201), which is satisfied by the superposition of two potentials propagating in different directions, Equations (202) and (203).

$$
\begin{gathered}
v_{x}(x=0)=\left.0 \rightarrow \frac{\partial \varphi}{\partial x}\right|_{x=0}=0 \\
\varphi(x, z, t)=\frac{g A}{\omega} e^{k z} \sin (k x-\omega t)-\frac{g A}{\omega} e^{k z} \sin (k x+\omega t)=-2 \frac{g A}{\omega} e^{k z} \cos (k x) \sin (\omega t) \\
v_{x}=\frac{\partial \varphi}{\partial x}=2 k \frac{g A}{\omega} e^{k z} \overbrace{\sin (k x)}^{=\text {if } x=0} \sin (\omega t)
\end{gathered}
$$


Figure 161 - The superposition of two potentials satisfies the boundary condition.

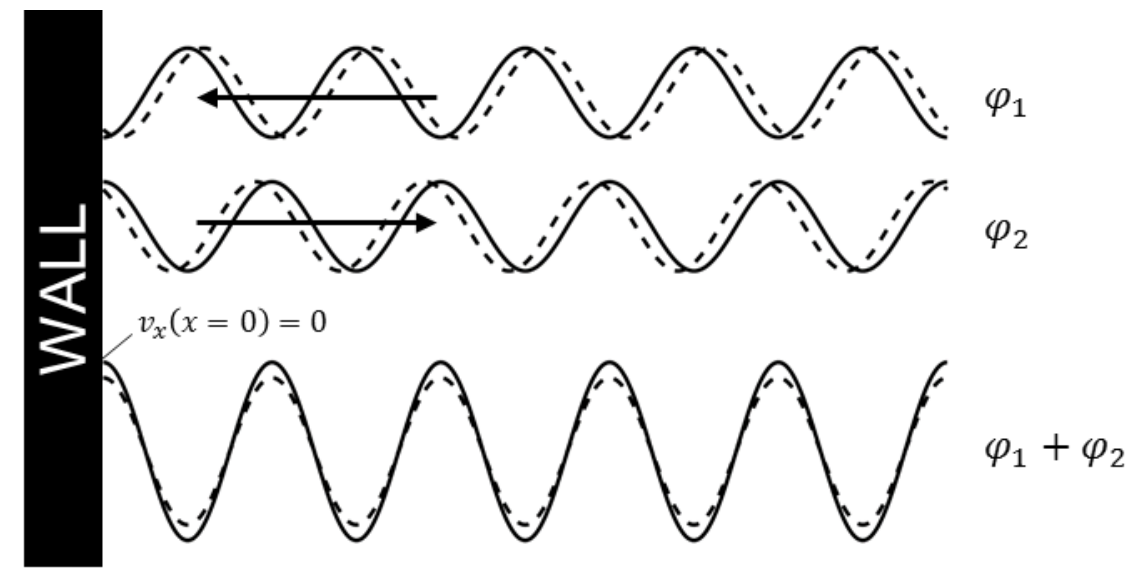

Source: Elaborated by the author.

This example, illustrated in Figure 161, is important because it justifies formally the use of wave-probes, since it also describes the asymptotical behavior of the hull of a non-moving vessel. Acting as a wall, the hull doubles the amplitude of short waves encountering it directly, in the point of contact, motivating the use of the approach proposed in this work, Equation (204).

$$
\zeta(x=0, t)=-\left.\frac{1}{g} \frac{\partial \varphi}{\partial t}\right|_{z=0}=2 A \cos (k x) \cos (\omega t) \stackrel{x=0}{=} 2 A \cos (\omega t)
$$

In this ideal case, with an infinity wall, the resulting wave is a simple stationary wave; in a more complex scenario, however, other effects happen.

\section{LINEAR RESPONSE MODEL}

When a moving floating body is inside the domain, the resultant potential can also be described as a superposition of potentials. It must account effects from:

- Radiation: the potential caused by a unitary movement of the body in each degree of freedom, $\varphi_{j}$;

- Diffraction: the unperturbed potential of the incident waves plus the scattering disturbance caused by the fixed body, $\varphi_{A}=\varphi_{0}+\varphi_{7}$.

Assuming an oscillatory body movement, with amplitude $\xi_{j}$ in each degree of freedom (DoF), the final potential will be the one presented in Equation (205). 


$$
\varphi(x, y, z, t)=\operatorname{Re}\left\{\left[\sum_{j=1}^{6} \xi_{j} \varphi_{j}(x, y, z)+A\left(\varphi_{0}(x, y, z)+\varphi_{7}(x, y, z)\right)\right] e^{i \omega t}\right\}
$$

In this case, the hypothesis made previously must be taken carefully: firstly, the frictionless hypothesis will depend on the shape of the floating body - the body must be 'smooth' - and will depend on how fast it is moving - if the body movement is too fast, non-linear effects will take control, and the potential theory is no more valid; secondly, the size of the vessel will determine the order of magnitude of the potential and, thus, the validity of the approximations made in surface wave developments - the wave amplitude must be small in comparison with the vessel.

For all potential terms, the governing equations are the previously presented continuity equation, or Laplace's equation; the Cauchy-Poisson condition; and the bottom impermeability condition. Moreover, extra equations are applied to the radiation potential and to the diffraction potential: the moving body impermeability, Equation (206); and the fixed body impermeability, Equation (207).

$$
\begin{gathered}
\left.\frac{\partial \varphi_{j}}{\partial n_{b}}\right|_{S_{b}}=i \omega n_{j}(t), j=1,2,\left.3 \quad \frac{\partial \varphi_{j}}{\partial n_{b}}\right|_{S_{b}}=i \omega(\boldsymbol{r} \times \boldsymbol{n}(t))_{j-3}, j=4,5,6 \\
\left.\frac{\partial \varphi_{A}}{\partial n_{b}}\right|_{S_{b}}=\left.0 \rightarrow \frac{\partial \varphi_{7}}{\partial n_{b}}\right|_{S_{b}}=-\left.\frac{\partial \varphi_{0}}{\partial n_{b}}\right|_{S_{b}}
\end{gathered}
$$

Again, the moving body impermeability condition, as written in the above equations, demands small movements of the body, so they can be stated as small oscillations around the same position.

And finally, by using the Bernoulli's equation, without considering high order and hydrostatic terms, it is possible to calculate the pressure in the domain and, thus, the forces and moments acting on the body; Equations (208), (209) and (210).

$$
\begin{gathered}
p(x, y, z, t)=-\rho \frac{\partial \varphi}{\partial t} \\
\boldsymbol{F}=-\rho \operatorname{Re}\left\{\sum_{j=1}^{6}\left[i \omega \xi_{j} e^{i \omega t} \iint_{S_{b}} \varphi_{j} \boldsymbol{n}_{\boldsymbol{b}} d S\right]\right\}-\rho \operatorname{Re}\left\{i \omega A e^{i \omega t} \iint_{S_{b}} \varphi_{A} \boldsymbol{n}_{\boldsymbol{b}} d S\right\} \\
\boldsymbol{M}=-\rho\left(\operatorname{Re}\left\{\sum_{j=1}^{6}\left[i \omega \xi_{j} e^{i \omega t} \iint_{S_{b}} \varphi_{j}\left(\boldsymbol{r} \times \boldsymbol{n}_{\boldsymbol{b}}\right) d S\right]\right\}-\rho \operatorname{Re}\left\{i \omega A e^{i \omega t} \iint_{S_{b}} \varphi_{A}\left(\boldsymbol{r} \times \boldsymbol{n}_{\boldsymbol{b}}\right) d S\right\}\right.
\end{gathered}
$$


Despite the complete analytical formulation, the equations presented need to be solved numerically, due to the complex geometry of the floating bodies of interest. The numerical methods often consist about discretizing the entire domain and solving the system of equations arising from it, but this particular problem can be facilitated by the third Green's identity.

The identity states that, if $\varphi$ is a harmonic function, the potential in any point $P$ of the domain can be determined by an integration over its boundary, Equation (211), with $r_{P}$ the distance between the point of integration on the surface and the point $P$ in the domain. It means the problem only needs to be discretized over the domain surface, and, with further developments, only over the floating body surface.

$$
\varphi(P)=\frac{1}{4 \pi} \iint_{\partial V}\left[\frac{1}{r_{P}} \nabla \varphi-\varphi \nabla\left(\frac{1}{r_{P}}\right)\right] \cdot \boldsymbol{n} d S
$$

This property results in a fast and accurate solving method called the panel method, which is used in the software Wamit ${ }^{\circledR}$. Essentially, the body surface is discretized in panels and a superposition of potentials is defined based on the discretization - usually, some simple potentials are positioned on the panels - with their strength being calibrated to satisfy all the boundary conditions. A complete theoretical reference can be found in (WAMIT, 2015).

After solving the potential problem, the calculated forces can be written in the matrix form, and be divided in: added mass $\boldsymbol{I}_{\boldsymbol{A}}(\omega)$ - a term proportional to the acceleration of the body, $\dot{U}_{j}$; radiation damping $\boldsymbol{B}(\omega)$ - a dissipative term proportional to the velocity of the body, $U_{j}$; and wave-exciting forces $\operatorname{Re}\left\{A e^{i \omega t} \boldsymbol{X}(\omega)\right\}-$ a term proportional to the amplitude of the incident wave; Equations (212), (213) and (214):

$$
\begin{gathered}
U_{j}(t)=\operatorname{Re}\left(i \omega \xi_{j} e^{i \omega t}\right) \\
\dot{U}_{j}(t)=-\operatorname{Re}\left(\omega^{2} \xi_{j} e^{i \omega t}\right) \\
{\left[\begin{array}{c}
\boldsymbol{F} \\
\boldsymbol{M}
\end{array}\right]_{\text {hydrodynamic }}=\boldsymbol{I}_{\boldsymbol{A}}(\omega) \dot{U}_{j}+\boldsymbol{B}(\omega) U_{j}+\operatorname{Re}\left\{A e^{i \omega t} \boldsymbol{X}(\omega)\right\}}
\end{gathered}
$$

If the body oscillates around its hydrostatic equilibrium with small movements, the hydrostatic forces can be linearized in order to be described by a simple restoration matrix $\boldsymbol{C}$, Equation (215). 


$$
\left[\begin{array}{c}
\boldsymbol{F} \\
\boldsymbol{M}
\end{array}\right]_{\text {hydrostatic }}=\operatorname{Re}\{\boldsymbol{C} \xi\}, \quad \boldsymbol{\xi}=\left\{\xi_{j} e^{i \omega t}\right\}_{j=1,2, \ldots, 6}
$$

The final dynamic system, applying all the forces on the inertia $I$ of the body, is then defined by Equation (216):

$$
\left\{-\omega^{2}\left(\boldsymbol{I}+\boldsymbol{I}_{\boldsymbol{A}}(\omega)\right)+i \omega \boldsymbol{B}(\omega)+\boldsymbol{C}\right\} \xi=A \boldsymbol{X}(\omega)
$$

Since the system is linear, it can be described by a transfer function of each possible wave - function of the angular frequency $\omega$ and the incidence angle $\beta$. This function can be discretized in a vector called Response Amplitude Operators (RAOs), which completely determine the floating body dynamics, Equation (217).

$$
\boldsymbol{R A} \boldsymbol{O}(\omega, \beta)=\frac{\boldsymbol{\xi}}{A}=\left\{-\omega^{2}\left(\boldsymbol{I}+\boldsymbol{I}_{\boldsymbol{A}}(\omega)\right)+i \omega \boldsymbol{B}(\omega)+\boldsymbol{C}\right\}^{-1} \boldsymbol{X}(\omega)
$$




\section{APPENDIX B - SUPERPOSITION OF VESSEL MOTIONS}

Usually, only the vertical motions of the vessel - heave, pitch and roll - are incorporated in the correction of the wave-probe measurements, but it is convenient to perform a sensibility analysis to justify this approach, that disregards the horizontal motions of the vessel - surge, sway and yaw.

The following analysis is made by simplifying the potential of two seas, a beam sea coming from port and a head sea, and verifying the effects of each movement in the measurements of a wave-probe located at the port of the vessel, as shown in Figure 162.

Figure 162 - Seas used in the sensibility analysis.

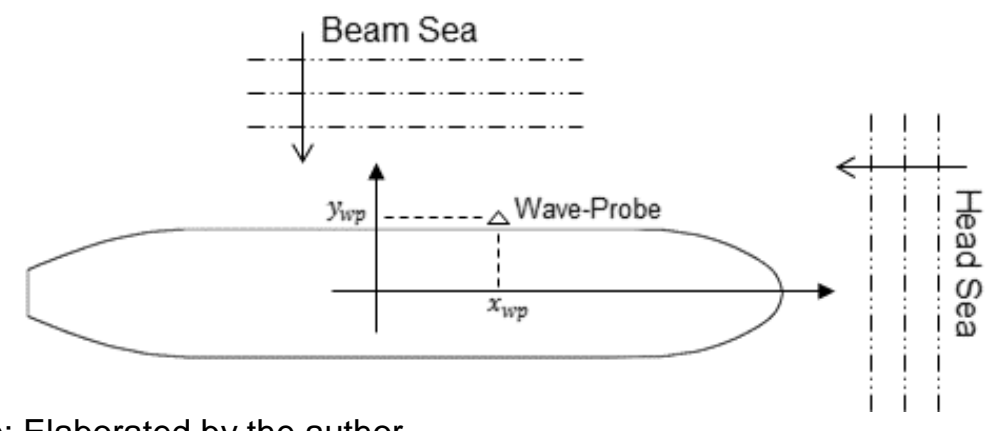

Source: Elaborated by the author.

Each movement is evaluated separately, and the angular movements are linearized, as the vessel angular displacements hardly ever surpass 0.1 radians, and, thus, the linearization error is usually much below than $0.5 \%$. The interference equations are illustrated in Figure 163 to Figure 168, in which $W P_{\text {meas. }}$ is the waveprobe measurement, i.e., the distance between the sensor and the water surface, and it is a function of the wave elevation $\zeta$ and the vessel movements $\xi$.

Figure 163 - Wave-probe measurement affected by the surge movement.

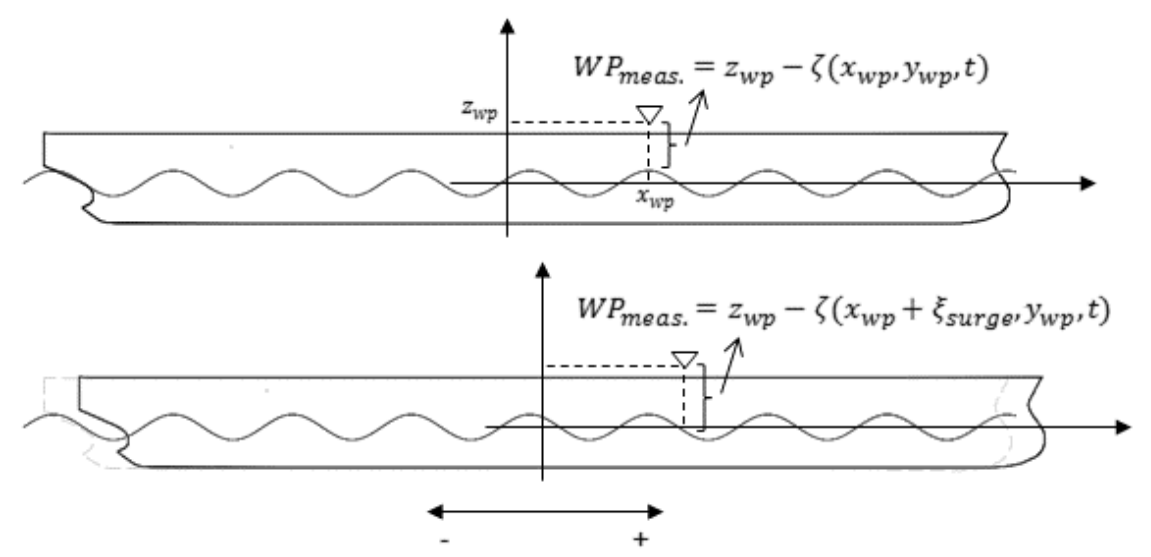

Source: Elaborated by the author. 
Figure 164 - Wave-probe measurement affected by the sway movement.
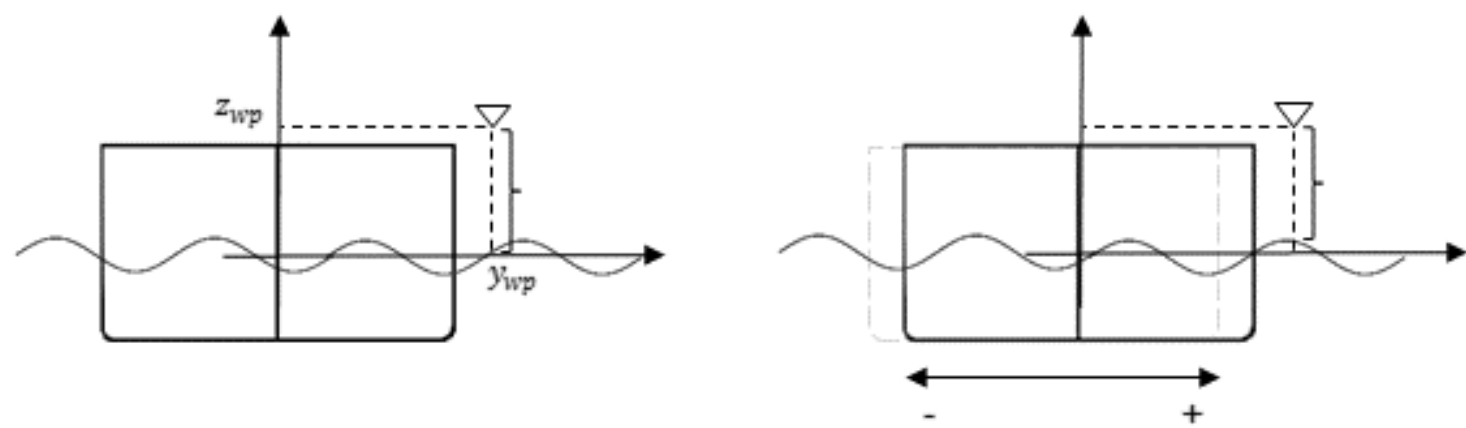

$$
W P_{\text {meas. }}=z_{w p}-\zeta\left(x_{w p}, y_{w p}, t\right)
$$$$
W P_{\text {meas. }}=z_{w p}-\zeta\left(x_{w p}, y_{w p}+\xi_{\text {sway, }}, t\right)
$$

Source: Elaborated by the author.

Figure 165 - Wave-probe measurement affected by the heave movement.
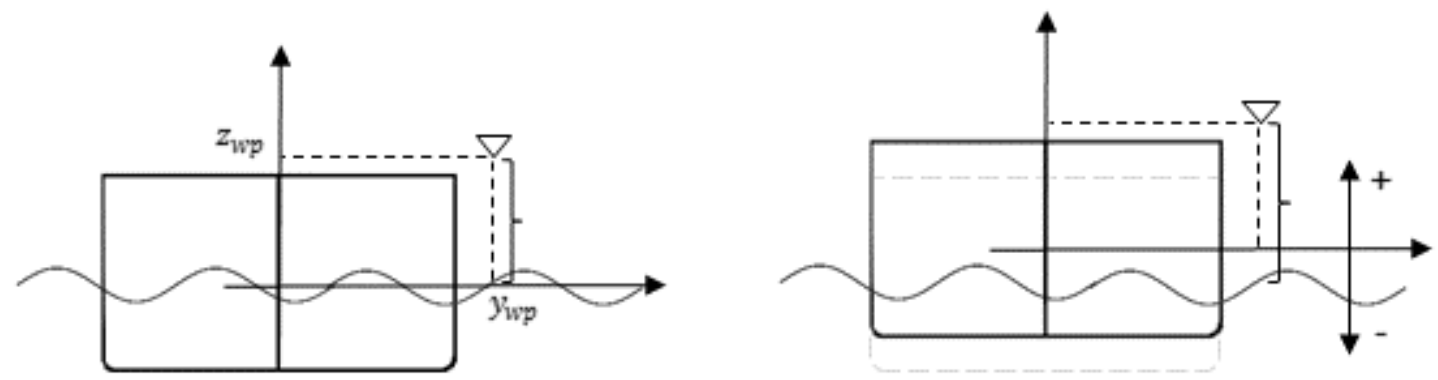

$$
W P_{\text {meas. }}=z_{w p}-\zeta\left(x_{w p}, y_{w p}, t\right)
$$

$$
W P_{\text {meas. }}=z_{w p}-\zeta\left(x_{w p}, y_{w p}, t\right)+\xi_{\text {heave }}
$$

Source: Elaborated by the author.

Figure 166 - Wave-probe measurement affected by the roll movement.
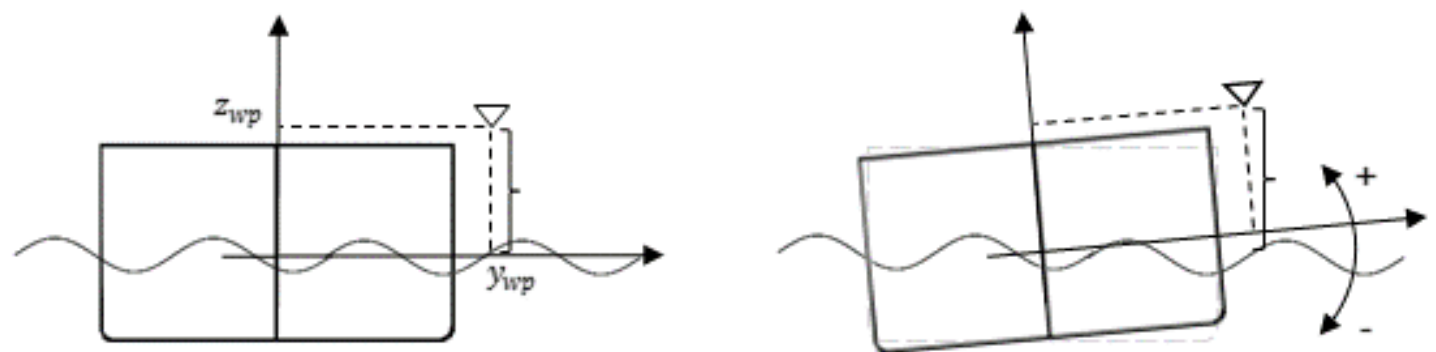

$$
W P_{\text {meas. }}=z_{w p}-\zeta\left(x_{w p}, y_{w p}, t\right)
$$

$W P_{\text {meas. }}=z_{w p}-\zeta\left(x_{w p}, y_{w p}, t\right)+\xi_{\text {roll }} \cdot y_{w p}$

Source: Elaborated by the author. 
Figure 167 - Wave-probe measurement affected by the pitch movement.

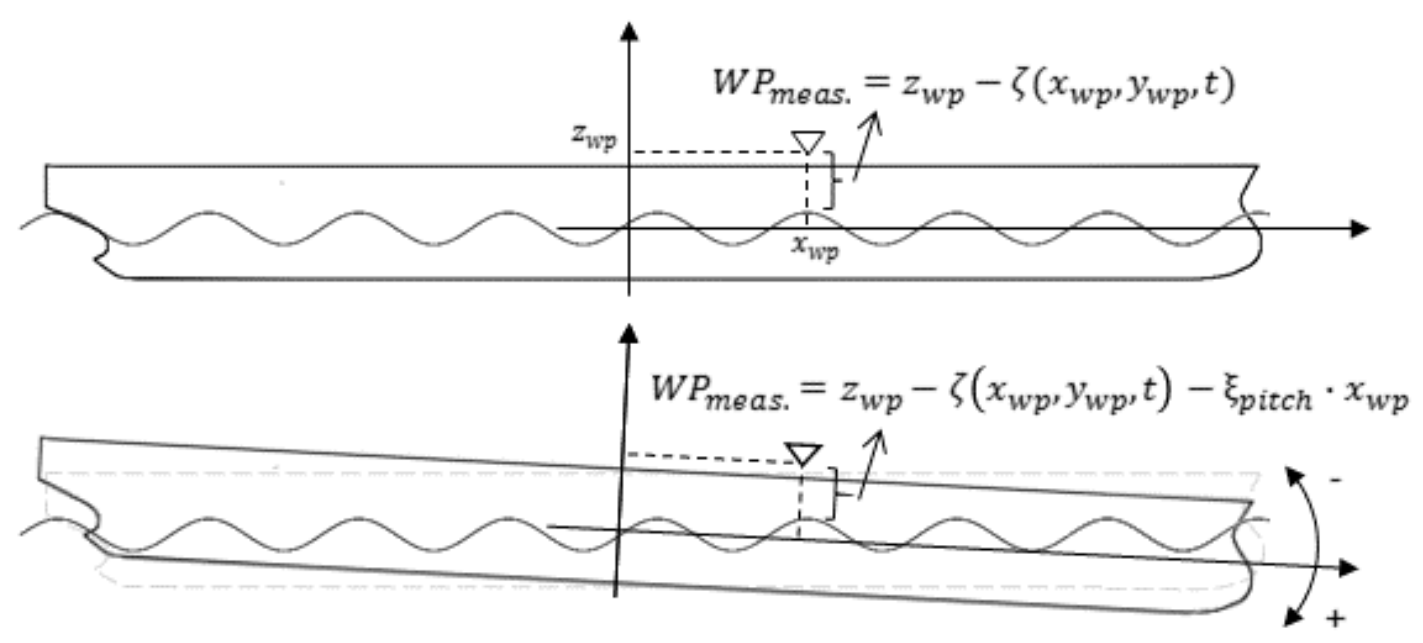

Source: Elaborated by the author.

Figure 168 - Wave-probe measurement affected by the yaw movement.

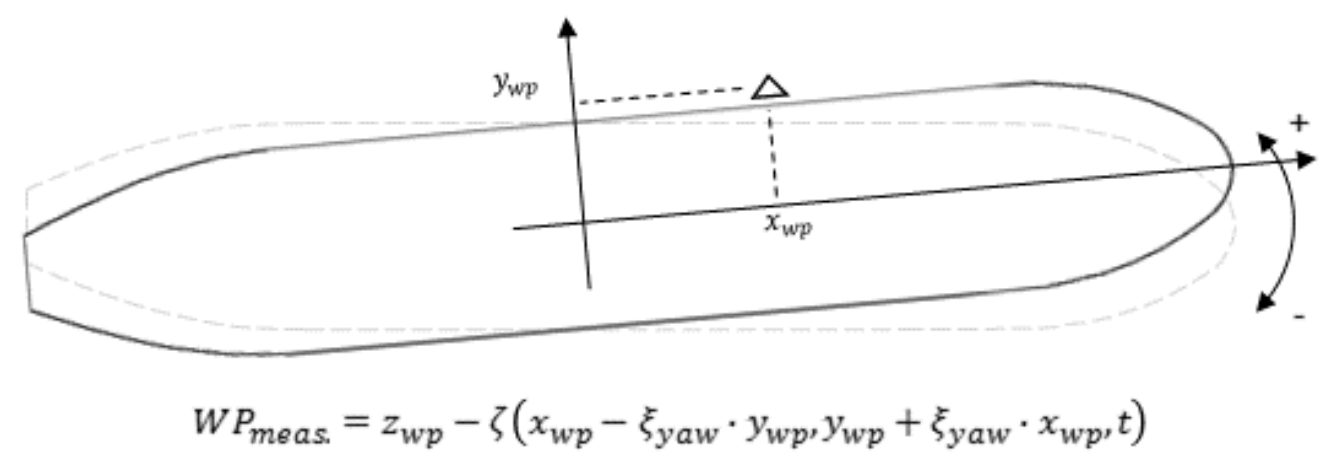

Source: Elaborated by the author.

The first sea, the beam sea, is chosen aiming at exciting the most the sway and the roll movements. The potential is simplified to an interpolation between a full reflected sea, when the period is below six seconds, and an unperturbed one, when the period is above twenty seconds. The resulting potential will be defined by the value of $C_{R}$, which is the amplification coefficient, Equation (218).

$$
\varphi(x, y, z, t)=\frac{g A}{\omega} e^{k z} \sin (k \cdot(-y)-\omega t)-C_{R} \frac{g A}{\omega} e^{k z} \sin (k \cdot(-y)+\omega t)
$$

Although the proposed potential is an approximation, it represents the qualitative behavior of the studied waves; producing, at the same time, a simple waveelevation pattern more suitable for the sensibility analysis, Equation (219).

$$
\zeta(x, y, t)=A \cos (k \cdot(-y)-\omega t)+C_{R} A \cos (k \cdot(-y)+\omega t)
$$


The vessel response is easily obtained via Wamit ${ }^{\circledR}$, and the result is shown in Figure 169 , for the floating body six degrees of freedom.

Figure 169 - Vessel response in a beam sea.
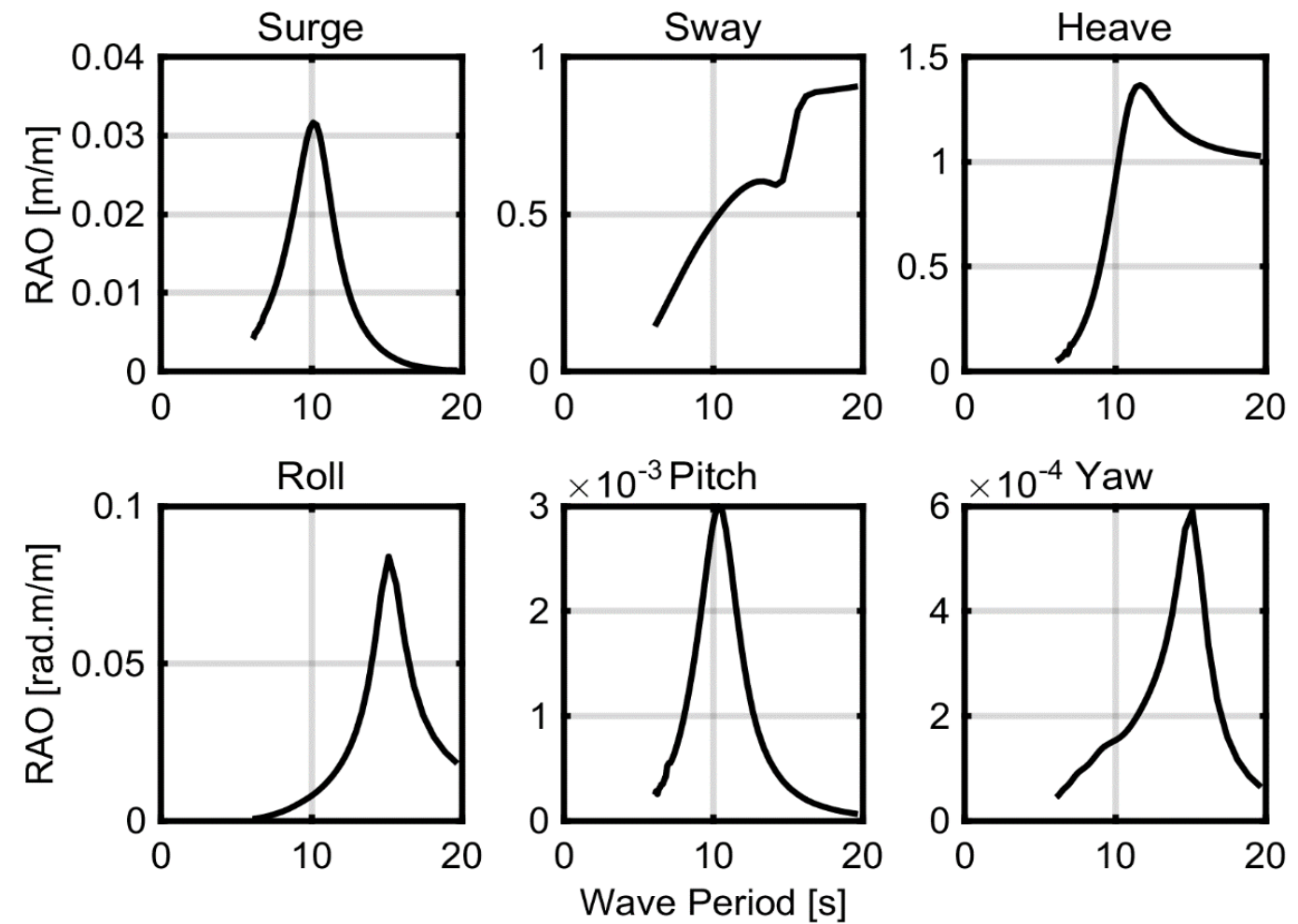

Source: Elaborated by the author.

The wave elevations in six positions along the port are shown in Figure 170.

Figure 170 - Wave-elevation in six different port positions.
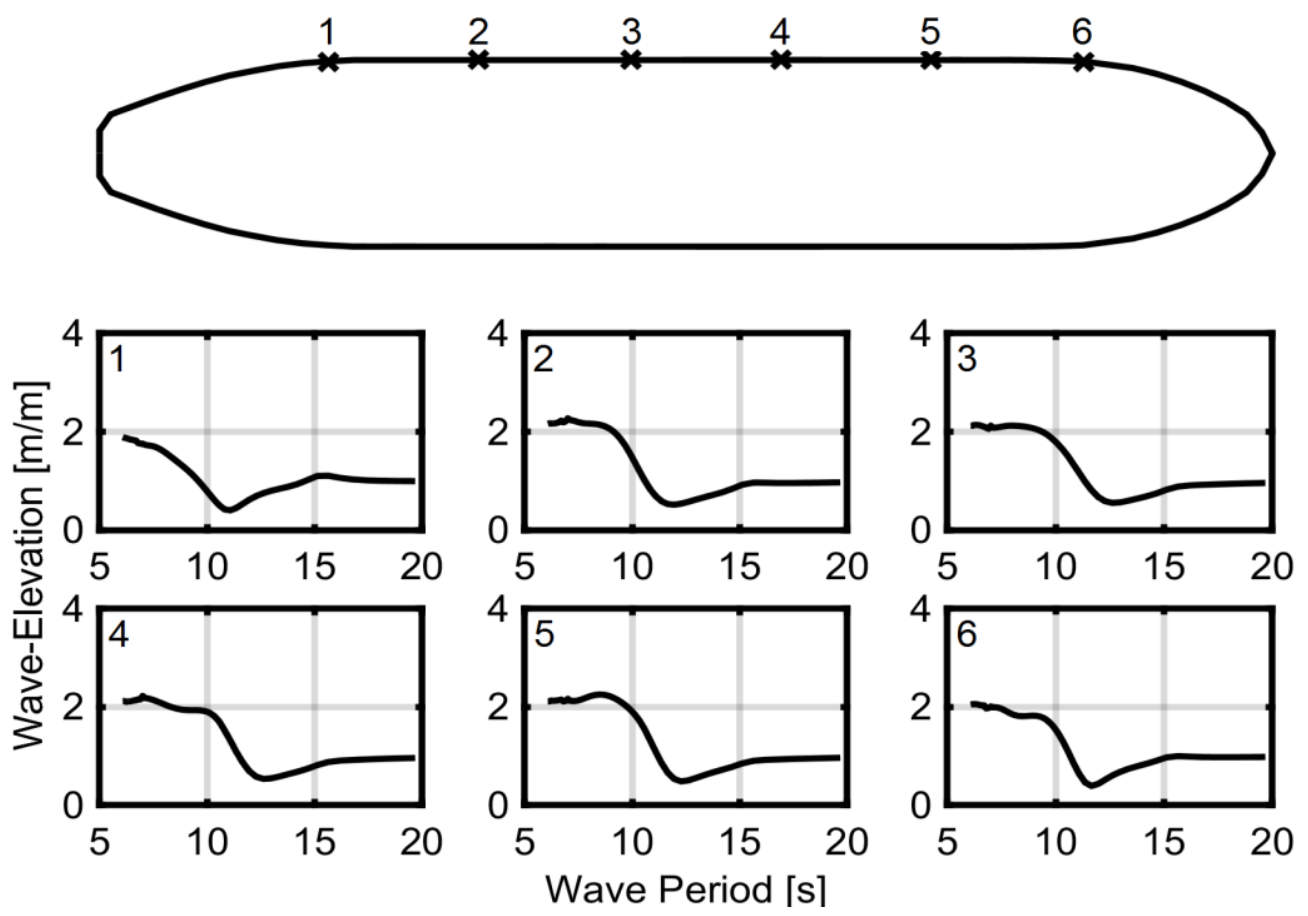

Source: Elaborated by the author. 
The responses along the starboard are shown in Figure 171.

Figure 171 - Wave-elevation in six different starboard positions.
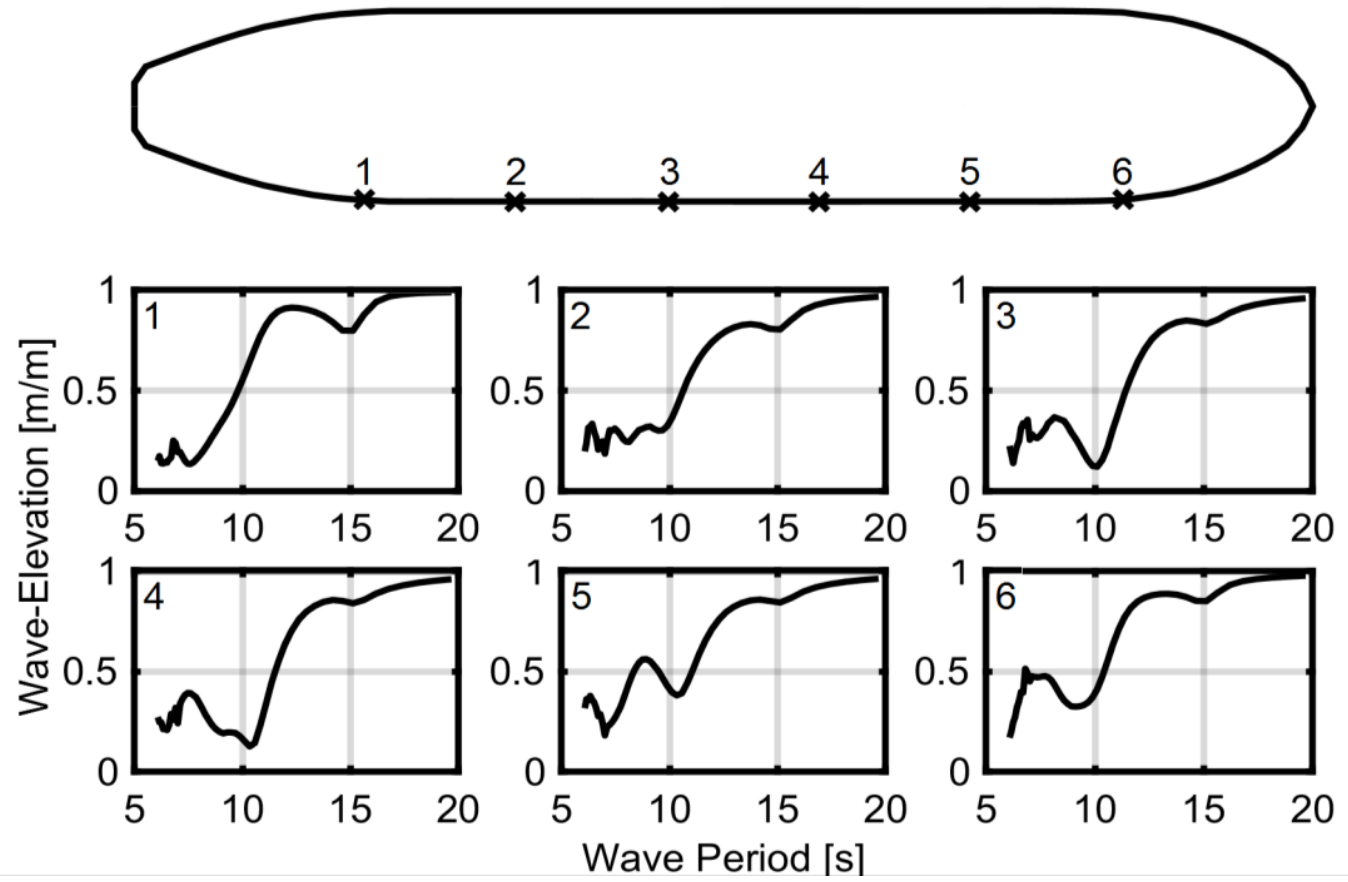

Source: Elaborated by the author.

The data is favorable to the approximations adopted. Firstly, the angular movements are always much below than 0.1 radians; and secondly, the idea of the superposition of a regular and a reflected sea is clearly seen in the port responses which starts with twice the wave amplitude and softly converges to one - and in the starboard response - which represents the potential that is able to pass through the vessel and that is not reflected.

The wave amplification coefficient can be calculated, for each period, by the average wave-elevation responses on the port, Figure 172.

Figure 172 - Amplification coefficient based on the wave-elevation on the port.

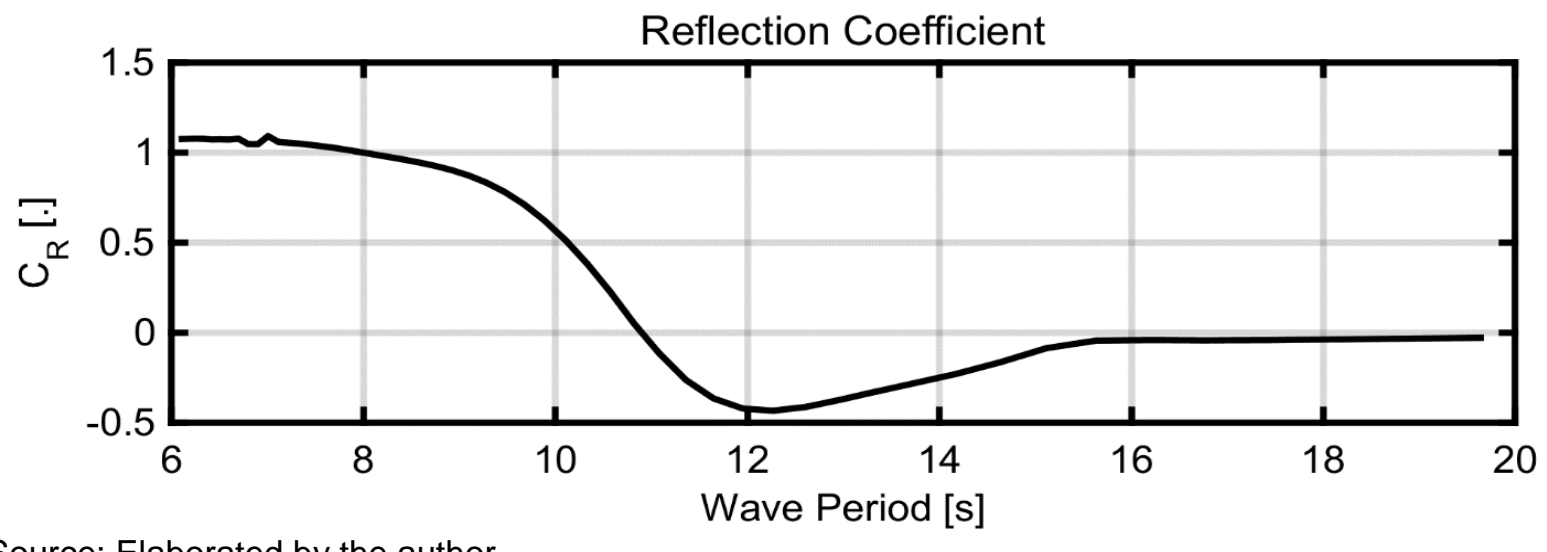

Source: Elaborated by the author. 
Finally, the maximum disturbance caused by each DoF can be calculated. For the angular movements, it is used half of the length and half of the width of the vessel as a reference for the wave-probe position; and for the horizontal movements, the phases of the responses are taken into account and the worst case is when the difference between the measured and the real wave-elevation is the greatest. The results are shown in Figure 173.

Figure 173 - Maximum disturbance cause by each DoF.

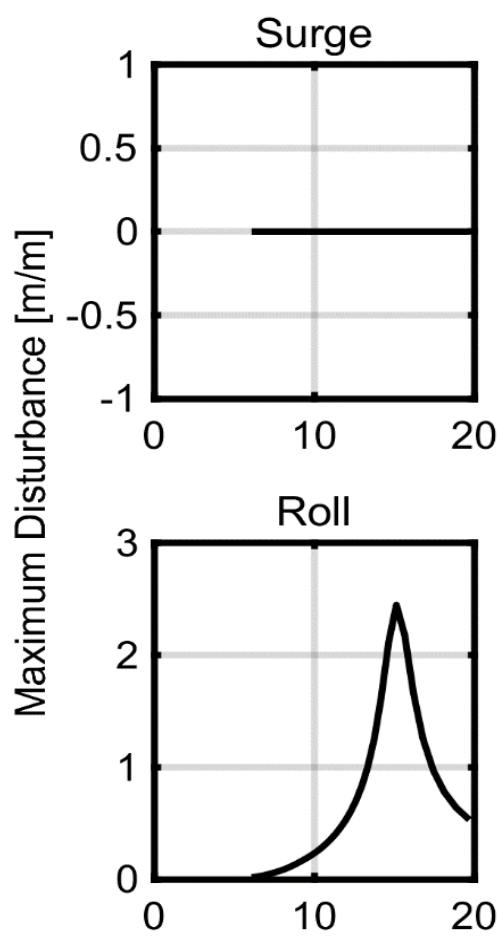

Source: Elaborated by the author.
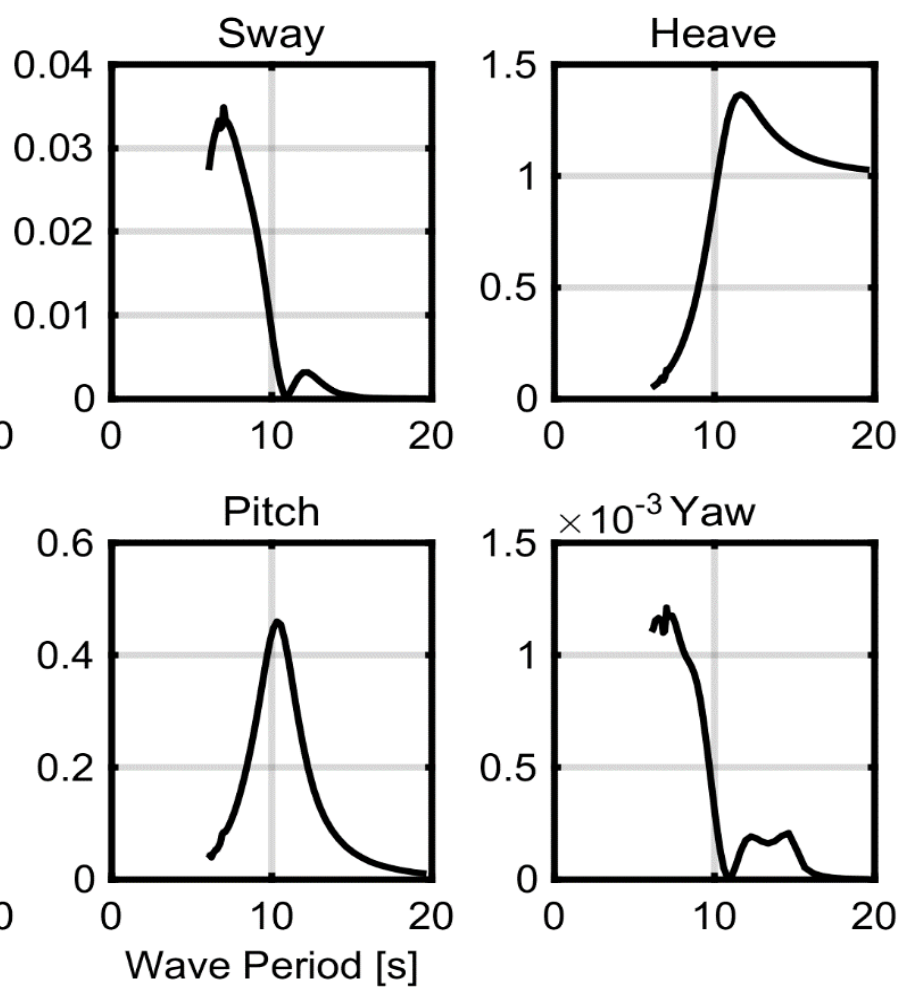

The results above suggest a clear dominance of the vertical movements. The sway horizontal movement, however, presents a non-negligible influence, around 3.5\% of the wave amplitude, or $1.75 \%$ of the wave-elevation - reminding that, below 10 seconds, the wave-elevation on the hull is twice the wave amplitude. Despite this, the effect is non-linear and it is not easily incorporated in the estimation algorithm, so it will be ignored, since the cost suppresses the possible improvements.

The second sea, the head sea, is chosen aiming at exciting the most the surge and the pitch movements. In this case, the potential is simplified to an unperturbed regular progressive wave potential, $\zeta(x, y, t)=A \cos (k \cdot(-x)-\omega t)$, a reasonable approximation if the beam, i.e., the width, of the vessel is small if compared against its length. 
The vessel response for this case is also obtained via Wamit ${ }^{\circledR}$, and the result is shown in Figure 174.

Figure 174 - Vessel response in a head sea.
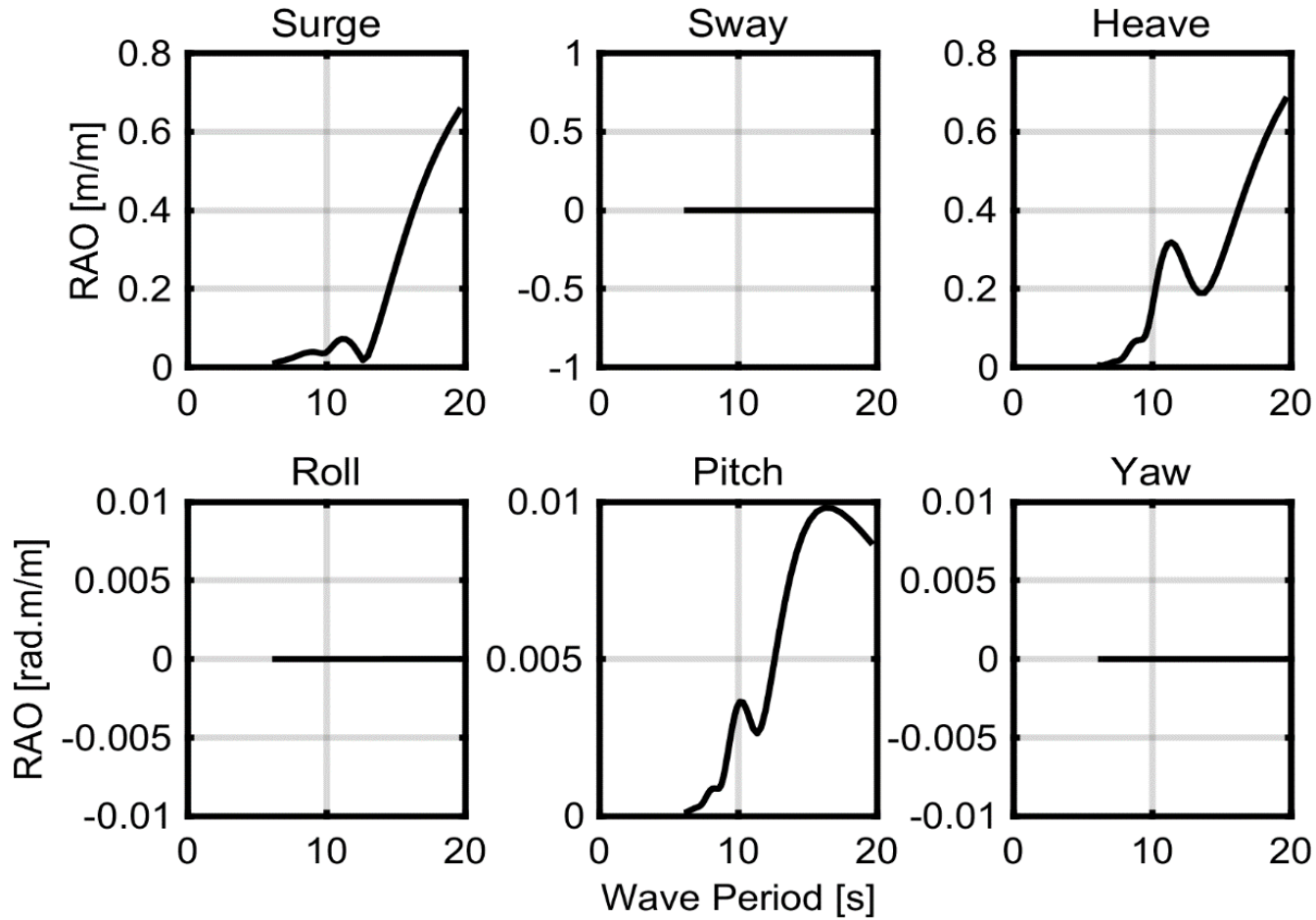

Source: Elaborated by the author.

The wave elevations in six positions along the port are shown in Figure 175.

Figure 175 - Wave-elevation in six different port positions.
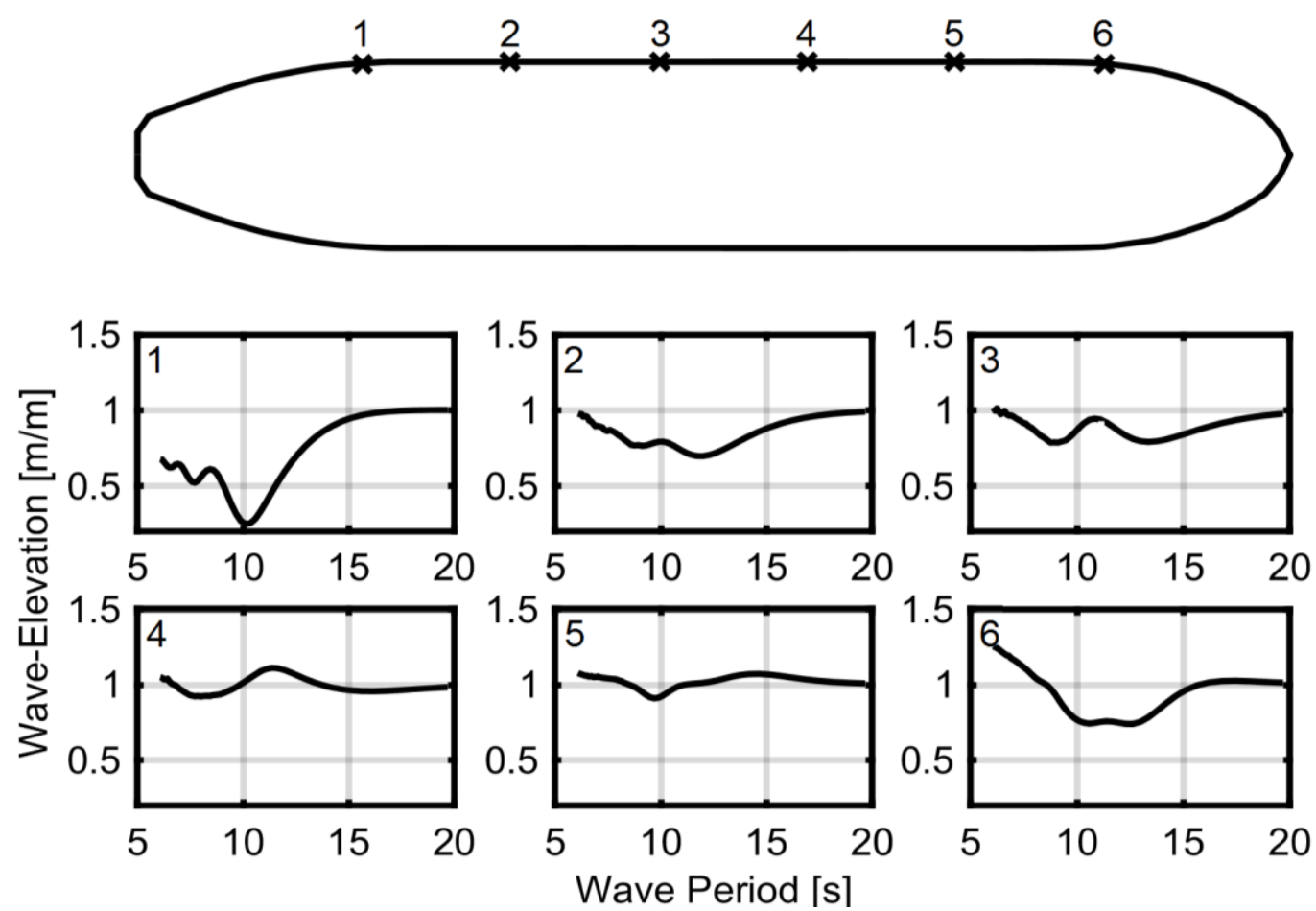

Source: Elaborated by the author. 
The responses of the probes at the starboard are not necessary, due to the symmetry of the problem.

Using the responses, the approximate potential used in the analysis is justified, mainly in the probes in the middle of the array $-2,3,4$ and 5 - which present responses almost always equal to one; the expected result in an unperturbed potential.

The final disturbances are calculated with the same considerations used in the previous example, and are shown in Figure 176.

Figure 176 - Maximum disturbance cause by each DoF.
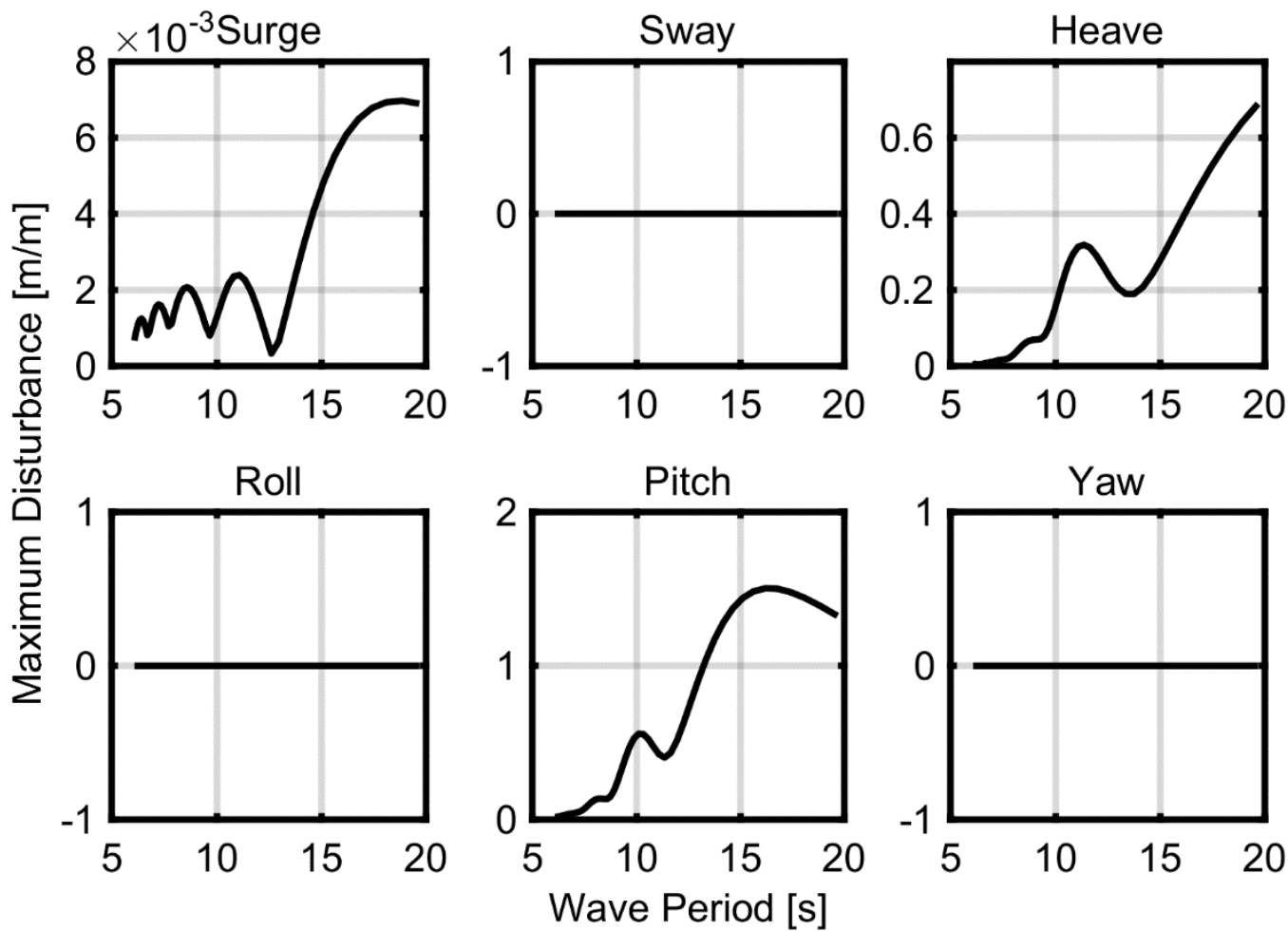

Source: Elaborated by the author.

In this case, the only horizontal motion that has influence is the surge movement. However, it only represents $0.8 \%$ of the wave amplitude and the wave elevation - the wave-elevation is equal to the wave amplitude - consequently, this movement is, indeed, negligible. The vertical movements, as in the previous case, have disturbances in the same order of magnitude that the wave-elevation, so they need to be corrected. 


\section{APPENDIX C - NUMERICAL ISSUES}

In this appendix, relevant numerical issues of the algorithms are listed. The solutions for the issues are described based on the Matlab ${ }^{\circledR}$ R2016a framework, including sample code, but can be easily expanded to any environment.

\section{INTEGRATION IN THE DIRECTIONAL DOMAIN}

Some error metrics and parametric calculations demand the integration through the direction domain, for example Equations (220) and (221):

$$
\begin{gathered}
S(\omega)=\int_{0}^{2 \pi} S(\omega, \beta) d \beta \\
D(\omega, \beta)=\frac{1}{\int_{0}^{2 \pi} \cos ^{2 s}\left(\frac{\beta-\beta_{0}}{2}\right) d \beta} \cdot \cos ^{2 s}\left(\frac{\beta-\beta_{0}}{2}\right)
\end{gathered}
$$

Suppose, for example, two discrete directional spread functions generated using the following parameters: $s=100, \Delta \beta=360^{\circ} / 20, \beta_{0_{1}}=0^{\circ}$ and $\beta_{0_{2}}=180^{\circ}$. It would be expected that the integration of those two functions would result in the same value, since they are just a translation for each other - in the first one the wave is coming from $0^{\circ}$, in the other one the wave is coming from $180^{\circ}$ - however, this is not the case:

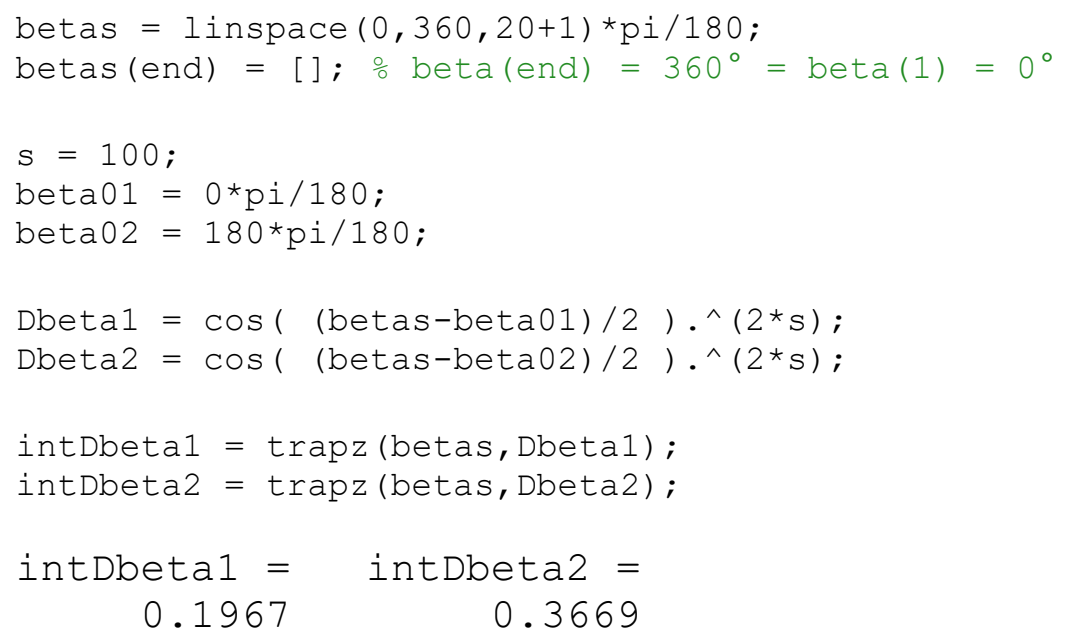

The difference source becomes clear when the resulting functions are plotted against the generating discrete directions, Figure 177. 
Figure 177 - Directional spread functions.
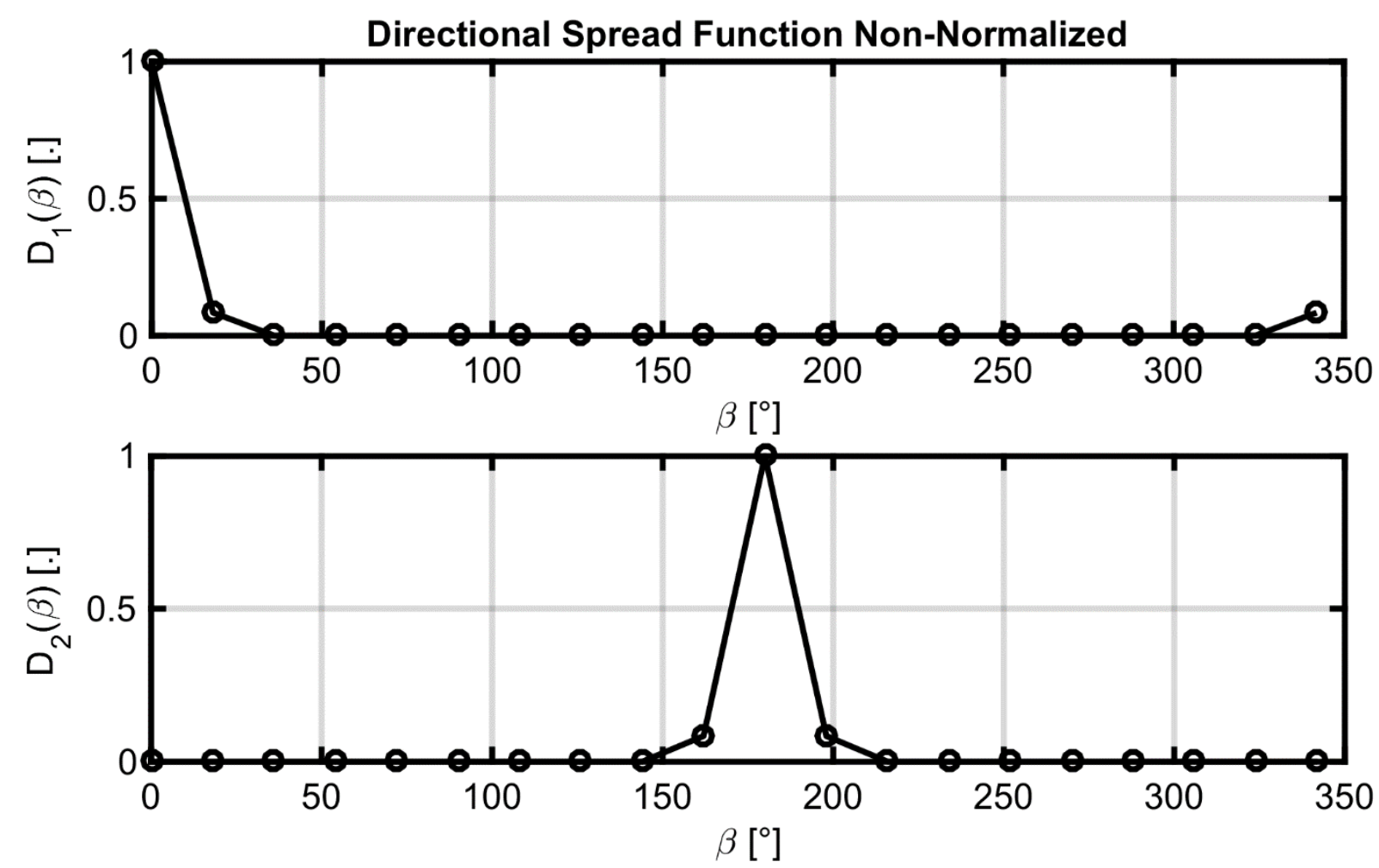

Source: Elaborated by the author.

It is possible to see that, due to the discretization, the final trapezium in $D_{1}(\beta)$, which is used in the numerical integration, is not present, causing the observed difference.

The solution for this issue is simple: to add the final trapezium. This is easily done by the function listed below:

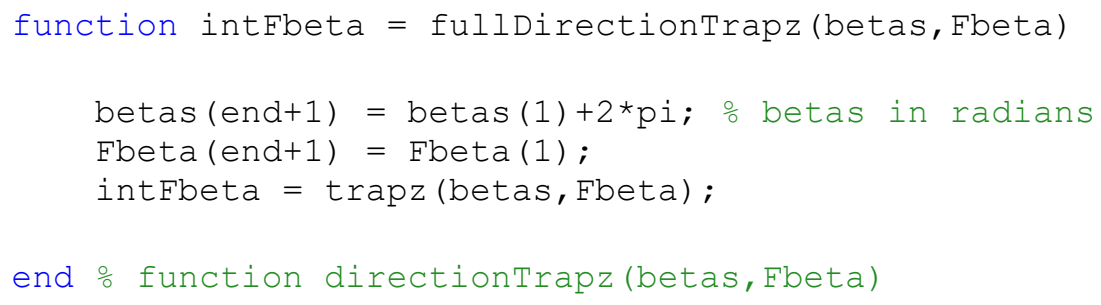

Essentially, the function adds the final integration point, completing the trapeziums before performing the numerical integration. It is important to notice that this is a simple function to illustrate the procedure, which does not verify the validity of the inputs, neither accounts for cases in which the input Fbeta is a matrix.

The effectiveness of the solution can be attested by the following code, that generates 100000 different functions, with random parameters, and compare them: 


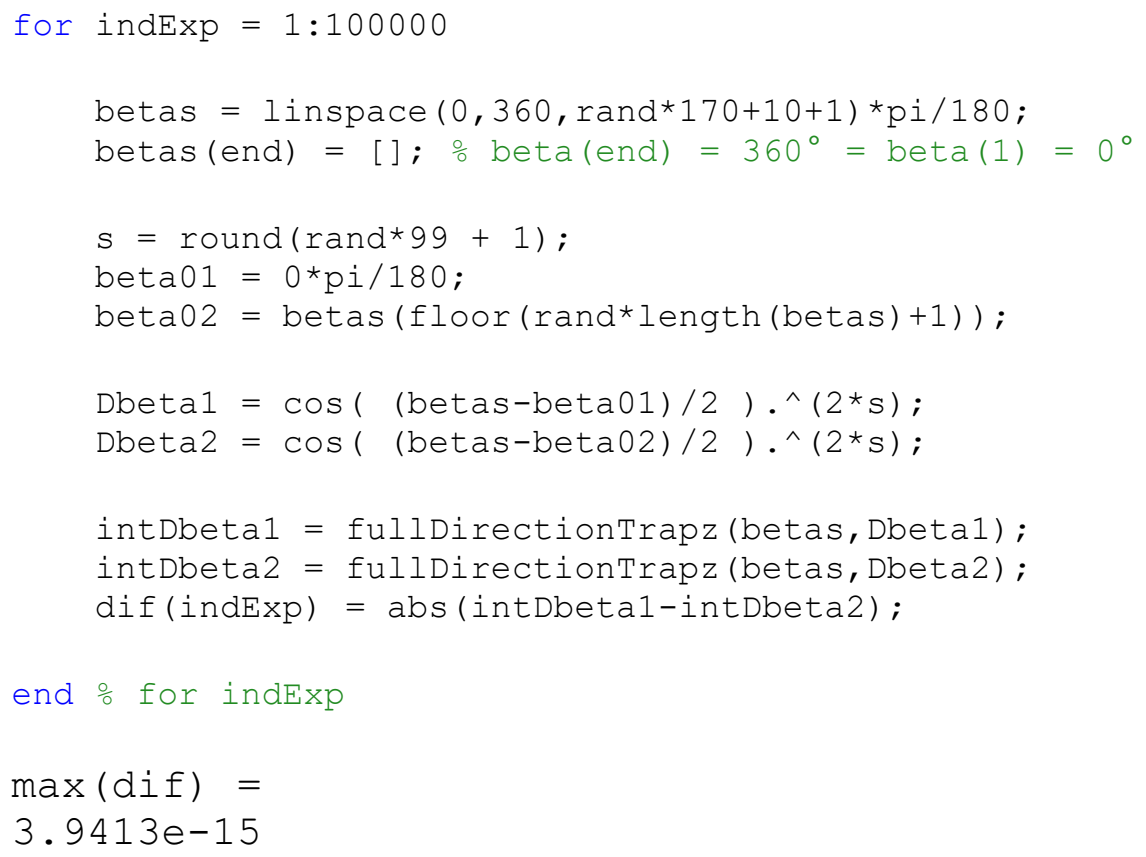

After the correction, the difference happens only due to numerical truncation.

\section{Quadratic Programming DetaILS}

After all the considerations, the final estimation algorithm consists in a quadratic programming problem with boundary constraints, Equations (222) and (223).

$$
\begin{gathered}
\min _{\boldsymbol{x}, x_{i}>0} J(\boldsymbol{x}) \equiv \min _{\boldsymbol{x}, x_{i}>0} \frac{1}{2} \boldsymbol{x}^{\boldsymbol{T}}\left[\boldsymbol{A}^{\boldsymbol{T}} \boldsymbol{A}+u_{1}^{2} \boldsymbol{H}_{1}+u_{2}^{2} \boldsymbol{H}_{2}+u_{3}^{2} \boldsymbol{H}_{3}\right] \boldsymbol{x}-\left(\boldsymbol{A}^{\boldsymbol{T}} \boldsymbol{b}\right)^{\boldsymbol{T}} \boldsymbol{x} \\
\min _{\boldsymbol{x}, x_{i}>0} \frac{1}{2} \boldsymbol{x}^{\boldsymbol{T}} \boldsymbol{H} \boldsymbol{x}+\boldsymbol{f}^{\boldsymbol{T}} \boldsymbol{x}, \boldsymbol{H}
\end{gathered}
$$

The above problem is solved by the following Matlab ${ }^{\circledR}$ code, implementing the optimization problem in Equation (224), subject to the constraints in the Equation (225), and with initial guess equally to $x_{0}$ :

$$
\begin{gathered}
\mathrm{x}=\text { quadprog }(\mathrm{H}, \mathrm{f}, \mathrm{A}, \mathrm{b}, \mathrm{Aeq}, \mathrm{beq}, \mathrm{lb}, \mathrm{ub}, \mathrm{x} 0 \text {, options }) ; \\
\min _{\boldsymbol{x}} \frac{1}{2} \boldsymbol{x}^{\boldsymbol{T}} \boldsymbol{H} \boldsymbol{x}+\boldsymbol{f}^{\boldsymbol{T}} \boldsymbol{x}, \\
\text { such that }\left\{\begin{array}{r}
\boldsymbol{A} \boldsymbol{x} \leq \boldsymbol{b} \\
\boldsymbol{A}_{\boldsymbol{e q}} \boldsymbol{x}=\boldsymbol{b} \boldsymbol{e q} \\
\boldsymbol{l} \boldsymbol{b} \leq \boldsymbol{x} \leq \boldsymbol{u b}
\end{array}\right.
\end{gathered}
$$

Resulting, for the particular problem of this thesis, in: 
The "options" field controls the optimization solver in different manners. The most significant one is the selection of the solver algorithm, which can be one of the followings:

- interior-point-convex: the default algorithm, ignores the initial search vector $x_{0}$

- trust-region-reflective: allows the usage of initial search vector;

- active-set: will be removed in future releases.

Ignoring the last one, since it will be removed in future releases, the two options would be expected to perform equally well, mainly because the quadratic programming problem with only boundary constraints is a convex problem, i.e., the local minimum is also the global minimum.

However, if only vessel movements are used with low $T_{P}$ waves, or, in a more general sense, if the DoF base is not capable of fully determine the minimum-square problem, some errors can happen in the estimation with the interior-point-convex algorithm, Figure 178, and with the trust-region-reflective algorithm if the initial guess was not provided, Figure 179.

Figure 178 - Estimation with interior-point-convex algorithm.
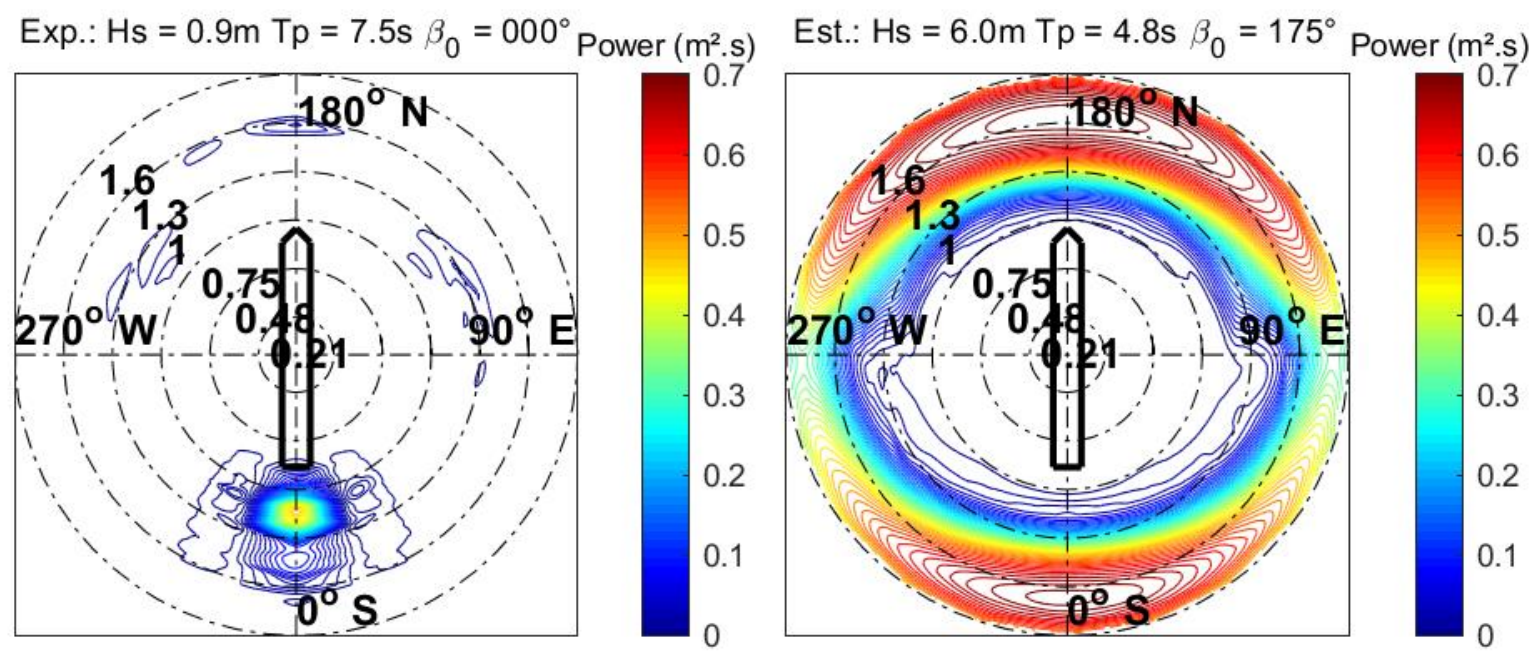

Source: Elaborated by the author. 
Figure 179 - Estimation with trust-region-reflective algorithm without initial guess.
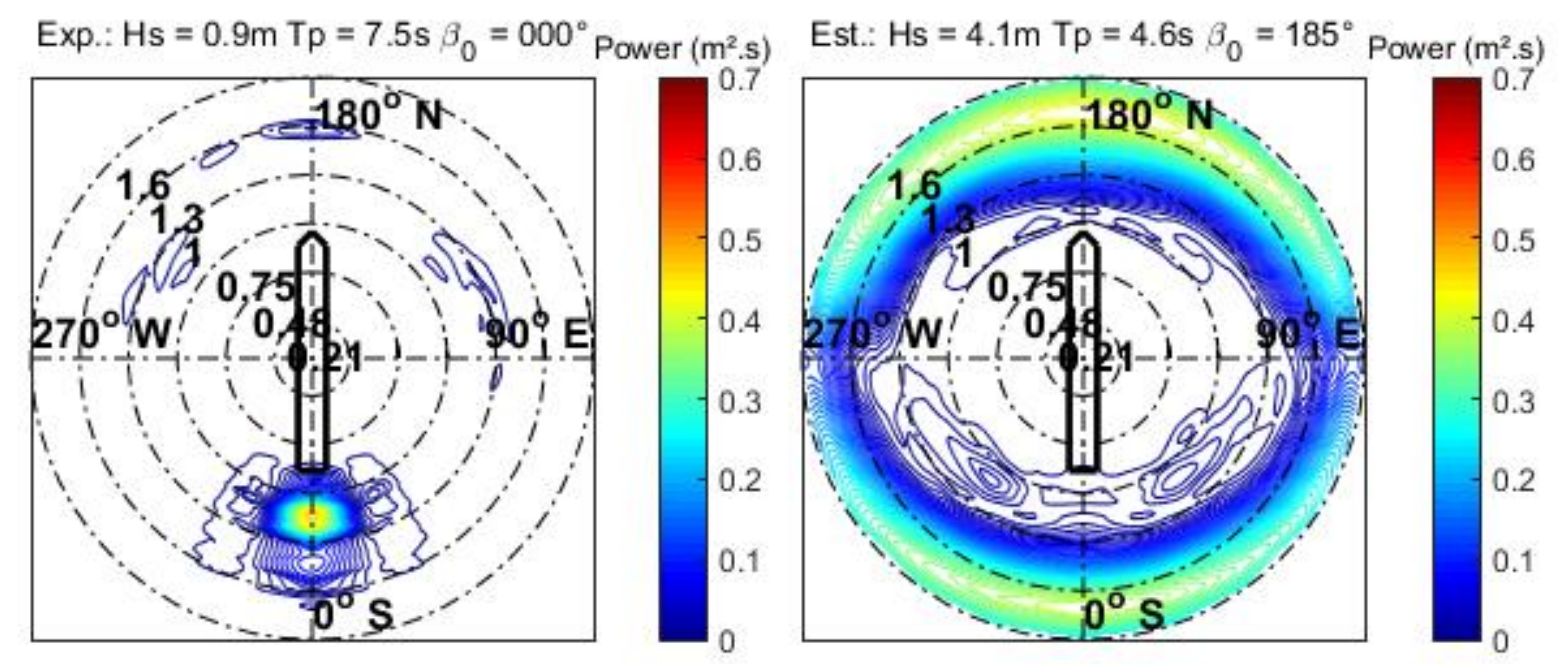

Source: Elaborated by the author.

A detailed analysis reveals that the problem happens in low periods - with frequency higher than 0.75 , i.e., periods lower than $8 \mathrm{~s}$ - exactly when the minimumsquared part of the estimation has the smallest response.

When no initial guess is provided, each algorithm has its own particular procedure, but, as a general rule, the vector $x$ will be initiated with non-zero values, usually much higher than the values expected for this particular problem. After this initial guess, the algorithms change the vector aiming at improving the cost function outcome, until some convergence criterion is reached.

Combining the results of the analysis with the knowledge about the algorithms, it is possible to explain why this kind of error occurs. After the first non-zero guess, the algorithm changes the medium and high period parts of the vector first, since they cause most of the cost function change, and the low period part starts to converge slowly, due to the low impact in the minimum-squared part of the problem, reaching the relative tolerance convergence criterion before achieving the expected zero response in those positions of the vector. Being so, non-negligible energy is "estimated" beyond the DoF base estimation capabilities.

The most straightforward solution would be increase the value of the relative tolerance criterion, but this would demand more iterations of the algorithms, increasing the time demanded to calculate the estimated response. 
A simpler strategy is to provide an initial point for the algorithm, with all position equal to zero. This strategy guarantees that the vector will change its value to nonzero only if it really causes non-negligible differences in the cost function, otherwise, it will remain equals to zero, acting as a form of regularization.

In conclusion, it is recommended that the trust-region-reflective algorithm is used, with initial guess equals to zero, correcting this kind of error, Figure 180.

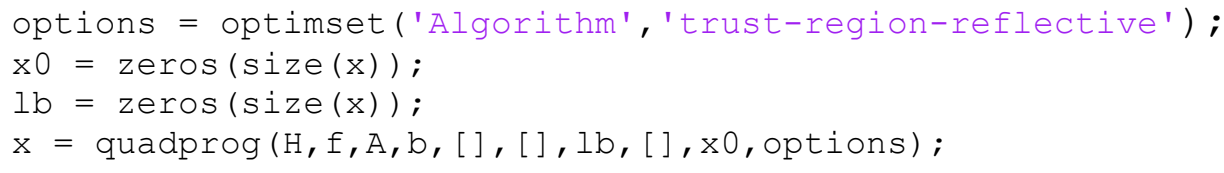

Figure 180 - Estimation with trust-region-reflective, initial guess equals to zero.
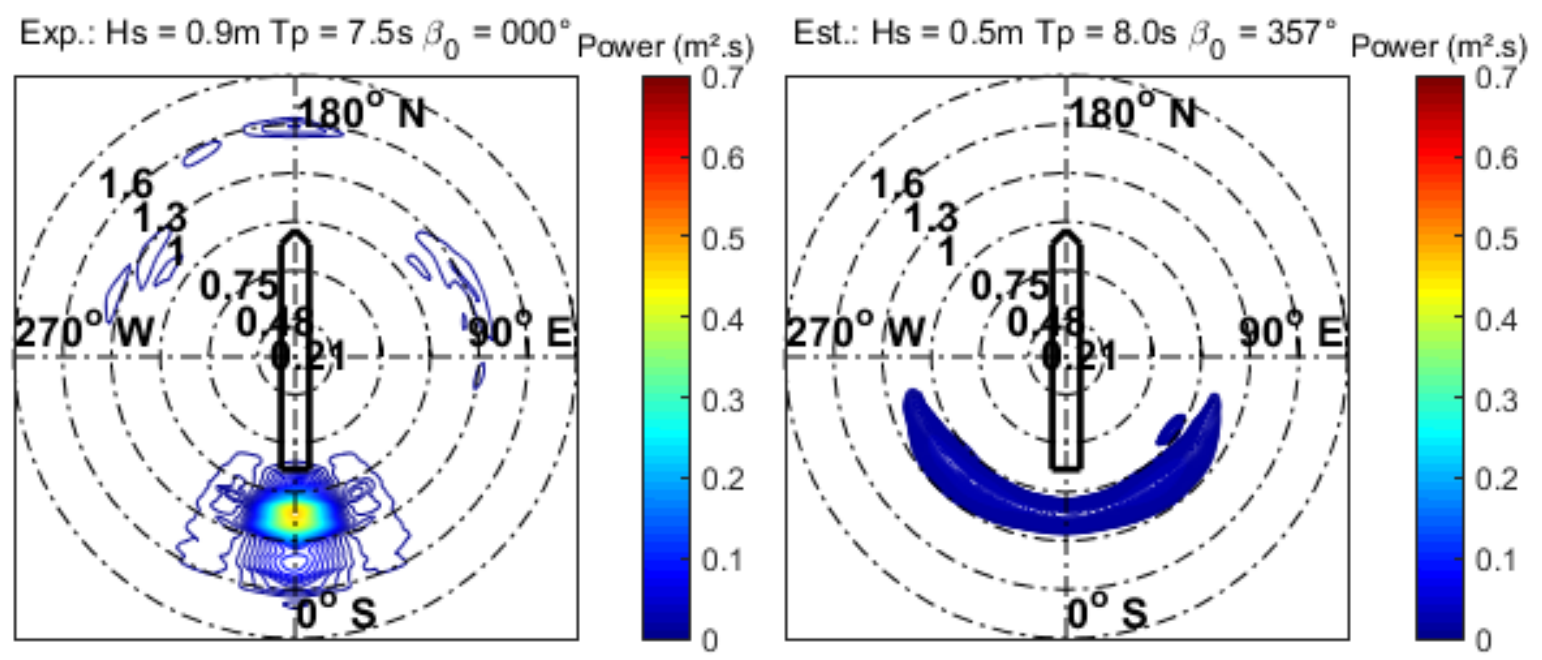

Source: Elaborated by the author.

It is important to emphasize that the response is still "bad" because a low $T_{P}$ wave is being estimated using only vessel DoFs; but the result does not "create" energy beyond the estimation capabilities anymore, correcting the issue.

\section{REDISCRETIZATION OF THE SPECTRUM}

During the error calculations based on differences between two directional spectra - experimental and estimated, for example - it is necessary the spectra to be in the same discretization, which is not always the case, mainly when the compared spectrum comes from different estimation strategies. A naïve approach is to interpolate the spectra using the new discretization in frequency and direction, but this procedure can cause non-negligible distortions that need to be taken in account. 
In order to analyze possible rediscretization strategies, the process can be divided in two: rediscretization in frequencies and rediscretization in directions.

The rediscretization in frequencies is a function that maps $S_{1}\left(\omega_{1 i}, \beta_{j}\right), \omega_{1 i}=$ $k_{i} \Delta \omega_{1}, \min (\omega) \leq \omega_{1 i} \leq \max (\omega), \beta_{j}=k_{j} \Delta \beta, 0^{\circ} \leq \beta_{j} \leq 360^{\circ}$, into $S_{2}\left(\omega_{2 i}, \beta_{j}\right), \omega_{2 i}=$ $k_{i} \Delta \omega_{2}, \min (\omega) \leq \omega_{2 i} \leq \max (\omega)$, and the linear system in Equation (226) into the linear system in Equation (227):

$$
\begin{aligned}
& b_{1}=A_{1} x_{1} \rightarrow \phi_{p k}\left(\omega_{1 i}\right)=\sum_{j=1}^{J} R A O_{p}\left(\omega_{1 i}, \beta_{j}\right) \cdot R A O_{k}^{*}\left(\omega_{1 i}, \beta_{j}\right) \cdot S\left(\omega_{1 i}, \beta_{j}\right) \Delta \beta \\
& b_{2}=A_{2} x_{2} \rightarrow \phi_{p k}\left(\omega_{2 i}\right)=\sum_{j=1}^{J} R A O_{p}\left(\omega_{2 i}, \beta_{j}\right) \cdot R A O_{k}^{*}\left(\omega_{2 i}, \beta_{j}\right) \cdot S\left(\omega_{2 i}, \beta_{j}\right) \Delta \beta
\end{aligned}
$$

It is possible to notice that the rediscretization transforms the linear system completely, changing all its rows, and essentially the problem becomes how to rediscretize the cross spectra of two signals.

Since each harmonic of a signal is independent of the other harmonics, when the signal is decomposed in a set of harmonics it is impossible to recover the amplitude of harmonics that are outside of the first set, and, in this sense, it seems that it is impossible to perform a rediscretization, unless the new $\omega_{2 i}$ set is an exact subset of the original set $\omega_{1 i}$; however, it is a premise of the problem that the energy spectral density is a continuous, smooth function, and the same with the response amplitude operators that translate it into movements and, consequently, the cross spectra. It means that an interpolation is enough to give a good estimate of an unknown harmonic that is between two other known harmonics.

The previous argument can be illustrated by the following example: given a set of 20 values $\omega_{1 i}$, a set of 30 values $\omega_{2 i}$, and a set of values $\omega_{3 i} \ni\left\{\omega_{1 i} \cup \omega_{2 i}\right\}$. In a particular position $\beta_{j}$, the theoretical directional spectrum is given by $S\left(\omega_{k i}, \beta_{j}\right)=$ $S\left(\omega_{k i}\right) \cdot D\left(\beta_{j}\right)$. Since the function $S\left(\omega_{k i}, \beta_{j}\right)$ is a smooth, continuous function, when it is plotted using different discretization procedures, the resulting values will be one above the other, which can be seen in Figure 181. 
Figure 181 - Different discretizations superimposed.

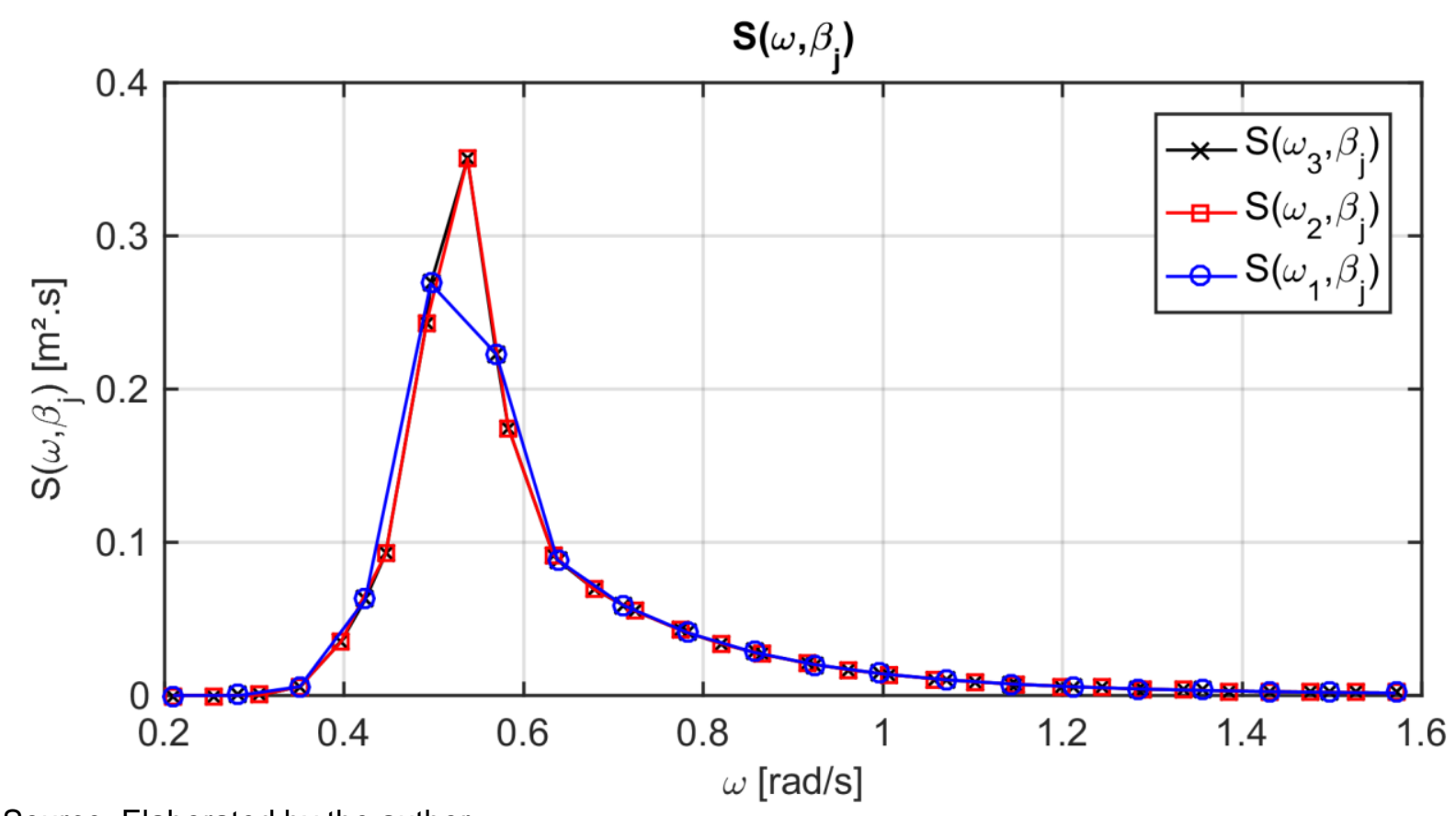

Source: Elaborated by the author.

It means that the function can be approximately converted from a higher discretization to a lower discretization by simple interpolation, Figure 182.

Figure 182 - Rediscretization using interpolation.

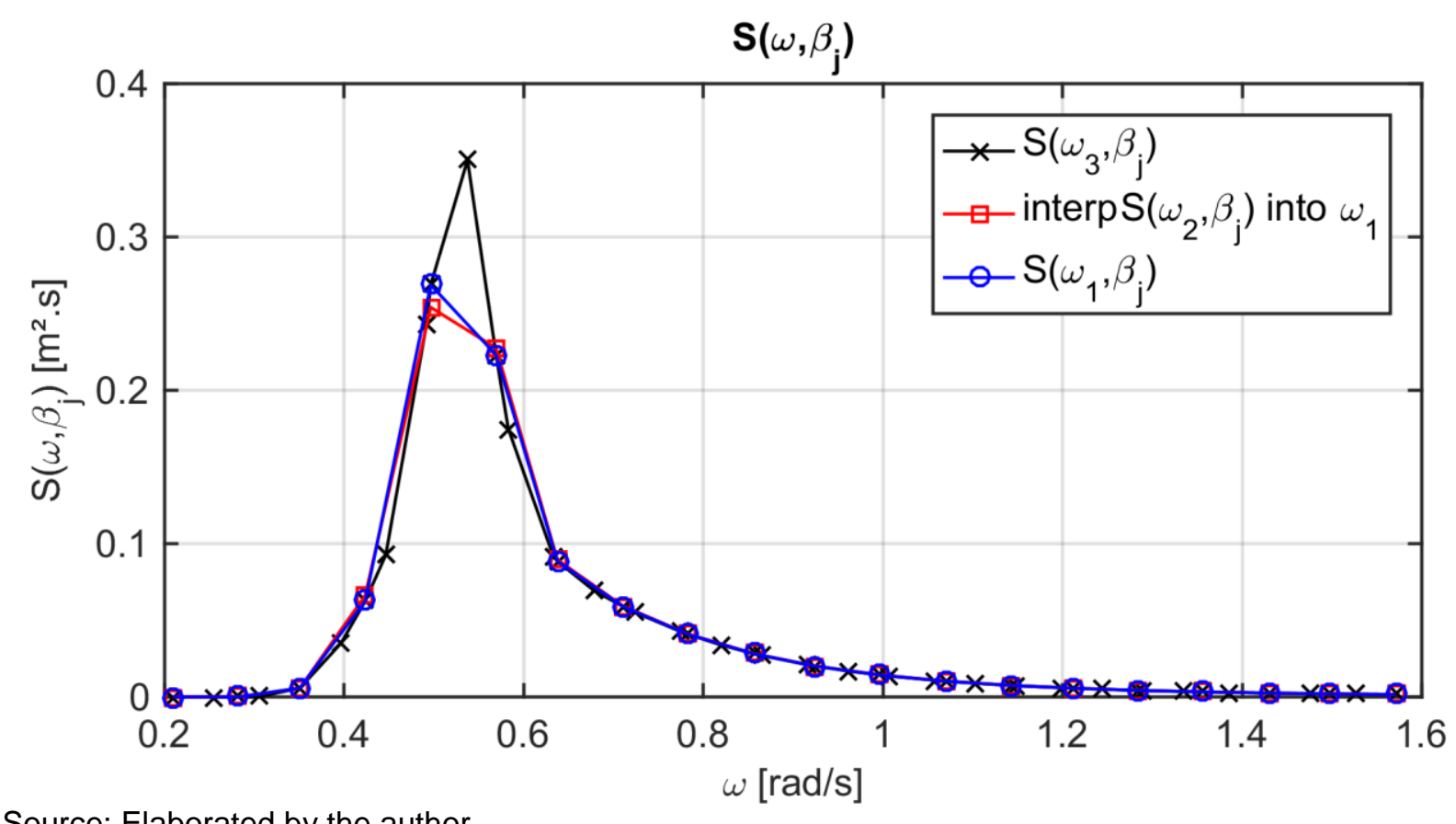

Source: Elaborated by the author.

Once more, the rediscretization from different frequencies is only possible because the function is assumed smooth and continuous, which is not true in a general spectral density function. 
The rediscretization in directions is a function that maps $S_{1}\left(\omega_{i}, \beta_{1 j}\right), \omega_{i}=$ $k_{i} \Delta \omega, \min (\omega) \leq \omega_{i} \leq \max (\omega), \beta_{1 j}=k_{1 j} \Delta \beta_{1}, 0^{\circ} \leq \beta_{1 j} \leq 360^{\circ}$, into $S_{2}\left(\omega_{i}, \beta_{2 j}\right), \beta_{2 j}=$ $k_{2 j} \Delta \beta_{2}, 0^{\circ} \leq \beta_{2 j} \leq 360^{\circ}$, and the linear system in Equation (228) into the linear system in Equation (229):

$$
\begin{aligned}
& b=A_{1} x_{1} \rightarrow \phi_{p k}\left(\omega_{i}\right)=\sum_{j_{1}=1}^{J_{1}} R A O_{p}\left(\omega_{i}, \beta_{1 j}\right) \cdot R A O_{k}^{*}\left(\omega_{i}, \beta_{1 j}\right) \cdot S\left(\omega_{i}, \beta_{1 j}\right) \Delta \beta_{1} \\
& b=A_{2} x_{2} \rightarrow \phi_{p k}\left(\omega_{i}\right)=\sum_{j_{2}=1}^{J_{2}} R A O_{p}\left(\omega_{i}, \beta_{2 j}\right) \cdot R A O_{k}^{*}\left(\omega_{i}, \beta_{2 j}\right) \cdot S\left(\omega_{i}, \beta_{2 j}\right) \Delta \beta_{2}
\end{aligned}
$$

It is possible to notice that the rediscretization does not transform the linear system completely, since the vector $b$, the cross spectra, is the same for both systems. However, the columns of the linear systems are different. The rediscretization, in this case, must guarantee that the cross spectra vector remains approximately the same, Equation (230). It means that, if $\beta_{2 j}$ is a subset of $\beta_{1 j}$, Equation (231) holds true; and, assuming RAOs almost constant in small $\Delta \beta$ intervals, Equation (232) is valid.

$$
\begin{aligned}
& \sum_{j_{1}=1}^{J_{1}} R A O_{p}\left(\omega_{i}, \beta_{1 j}\right) \cdot R A O_{k}^{*}\left(\omega_{i}, \beta_{1 j}\right) \cdot S\left(\omega_{i}, \beta_{1 j}\right) \Delta \beta_{1} \\
& =\sum_{j_{2}=1}^{J_{2}} R A O_{p}\left(\omega_{i}, \beta_{2 j}\right) \cdot R A O_{k}^{*}\left(\omega_{i}, \beta_{2 j}\right) \cdot S\left(\omega_{i}, \beta_{2 j}\right) \Delta \beta_{2} \\
& R A O_{p}\left(\omega_{i}, \beta_{2 j}\right) \cdot R A O_{k}^{*}\left(\omega_{i}, \beta_{2 j}\right) \cdot S\left(\omega_{i}, \beta_{2 j}\right) \Delta \beta_{2} \\
& =\sum_{\beta_{2 j}-\frac{\Delta \beta_{2}}{2} \leq \beta_{1 j}}^{\beta_{1 j} \leq \beta_{2 j}+\frac{\Delta \beta_{2}}{2}} R A O_{p}\left(\omega_{i}, \beta_{1 j}\right) \cdot R A O_{k}^{*}\left(\omega_{i}, \beta_{1 j}\right) \cdot S\left(\omega_{i}, \beta_{1 j}\right) \Delta \beta_{1} \\
& \quad S\left(\omega_{i}, \beta_{2 j}\right) \Delta \beta_{2}=\sum_{\beta_{2 j}-\frac{\Delta \beta_{2}}{2} \leq \beta_{1 j}}^{\beta_{2 j}} S\left(\omega_{i}, \beta_{1 j}\right) \Delta \beta_{1}
\end{aligned}
$$

The previous procedure results in the values for each $\beta_{2 j}$, Equation (233). 


$$
\left[\begin{array}{c}
\vdots \\
S\left(\omega_{i}, \beta_{2 j}\right) \\
\vdots
\end{array}\right]=\frac{1}{\Delta \beta_{2}}\left[\begin{array}{l}
\sum_{\beta_{2 j}-\frac{\Delta \beta_{2}}{2} \leq \beta_{1 j}+\frac{\Delta \beta_{2}}{2}} \\
\vdots
\end{array}\right]
$$

Until now, $\beta_{2 j}$ had to be a subset of $\beta_{1 j}$, but the result can be generalized by generating a more discretized spectrum following Equations (234) and (235).

$$
\begin{gathered}
\left\{\beta_{1 j} \cup \beta_{2 j}\right\} \in \beta_{3 j}, \Delta \beta_{3}=\text { cte. } \mid \Delta \beta_{1}=\alpha \Delta \beta_{3} \text { and } \Delta \beta_{2}=\beta \Delta \beta_{3}, \alpha \text { and } \beta \in \mathbb{Z}_{+}^{*} \\
S\left(\omega_{i}, \beta_{3 k}\right)=S\left(\omega_{i}, \beta_{1 j}\right), \quad \beta_{1 j}-\frac{\Delta \beta_{1}}{2} \leq \beta_{3 k} \leq \beta_{1 j}+\frac{\Delta \beta_{1}}{2}
\end{gathered}
$$

This spectrum with a higher discretization always satisfies Equation (236), since there will be exactly $\alpha$ values $S\left(\omega_{i}, \beta_{3 k}\right)$ inside the interval, and they will be equal to $S\left(\omega_{i}, \beta_{1 j}\right)$, Equation (237).

$$
\begin{gathered}
S\left(\omega_{i}, \beta_{1 j}\right) \Delta \beta_{1}=\sum_{\beta_{1 j}-\frac{\Delta \beta_{1}}{2} \leq \beta_{3 k}}^{\beta_{3 k} \leq \beta_{1 j}+\frac{\Delta \beta_{1}}{2}} S\left(\omega_{i}, \beta_{3 k}\right) \Delta \beta_{3} \\
S\left(\omega_{i}, \beta_{1 j}\right) \Delta \beta_{1}=\sum_{\beta_{1 j}-\frac{\Delta \beta_{1}}{2} \leq \beta_{3 k}}^{\beta_{3 k} \leq \beta_{1 j}+\frac{\Delta \beta_{1}}{2}} S\left(\omega_{i}, \beta_{1 j}\right) \Delta \beta_{3}=S\left(\omega_{i}, \beta_{1 j}\right) \alpha \Delta \beta_{3}
\end{gathered}
$$

Using this new spectrum, $S\left(\omega_{i}, \beta_{2 j}\right)$ can be calculates by Equation (233), since $\beta_{2 j}$ is a subset of $\beta_{3 j}$.

The previous argument can be illustrated by the following example: given a set of 20 values $\beta_{1 j}$, a set of 30 values $\beta_{2 j}$, and generating a set of values $\beta_{3 j} \ni\left\{\beta_{1 j} \cup \beta_{2 j}\right\}$. Assuming all sets with the first value equals $0^{\circ}$, the following code performs the rediscretization. 
o simple set that always contains betas1 and betas2

$\div$ Dbetas2/Dbetas3 $=70$, Dbetas1/Dbetas3 $=20$

betas $3=$ linspace $(0,2 *$ pi, $70 * 20+1)$ '; betas $3($ end $)=[]$;

Dbetas3 $=\operatorname{betas} 3(2)-\operatorname{betas} 3(1)$;

o generating directional spectrum example

$\mathrm{Hs}=1 ; \mathrm{Tp}=7$; beta $0=\mathrm{pi} ; \mathrm{s}=1000 ;$

$[\mathrm{x} 1, \mathrm{~S} 1]=$ genNewSea $([]$, omegas, betas1, Hs, Tp, beta $0, \mathrm{~s})$;

\% creating spectrum with higher discretization. The spectrum S1

\% is duplicated to avoid errors when beta3>max(beta1).

$\mathrm{S} 3=$ interp1 ([betas1; betas1+2*pi], [S1;S1], betas3, 'nearest');

\% pre-initializing matriz with the rediscretized spectrum

S12 = zeros (length (betas2), length (omegas));

o solving in each frequency

for indomega $=1$ : length (omegas)

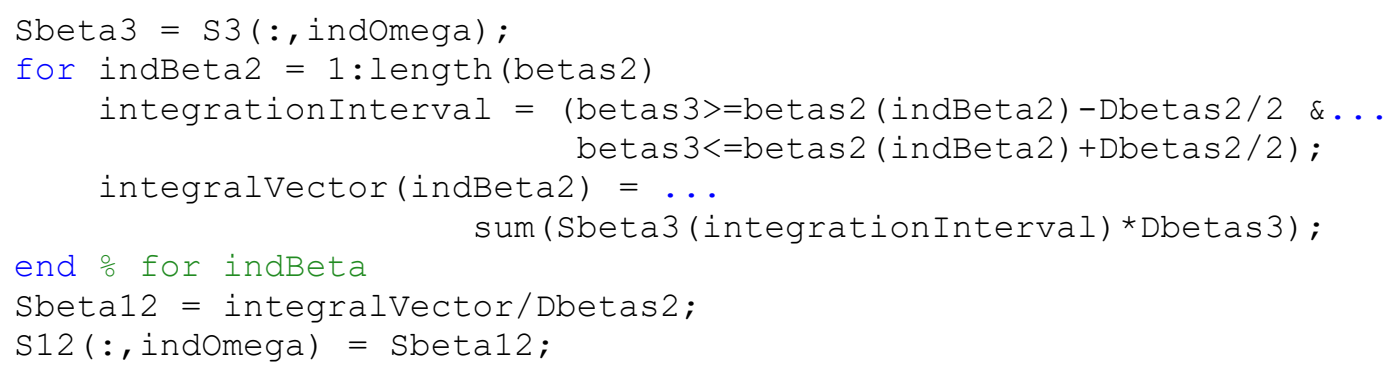

end $\frac{\circ}{\circ}$ for indomega

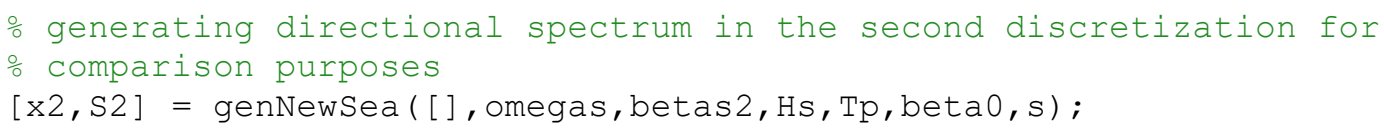

The result of the discretization can be seen in Figure 183.

Figure 183 - Original and rediscretized spectrum.
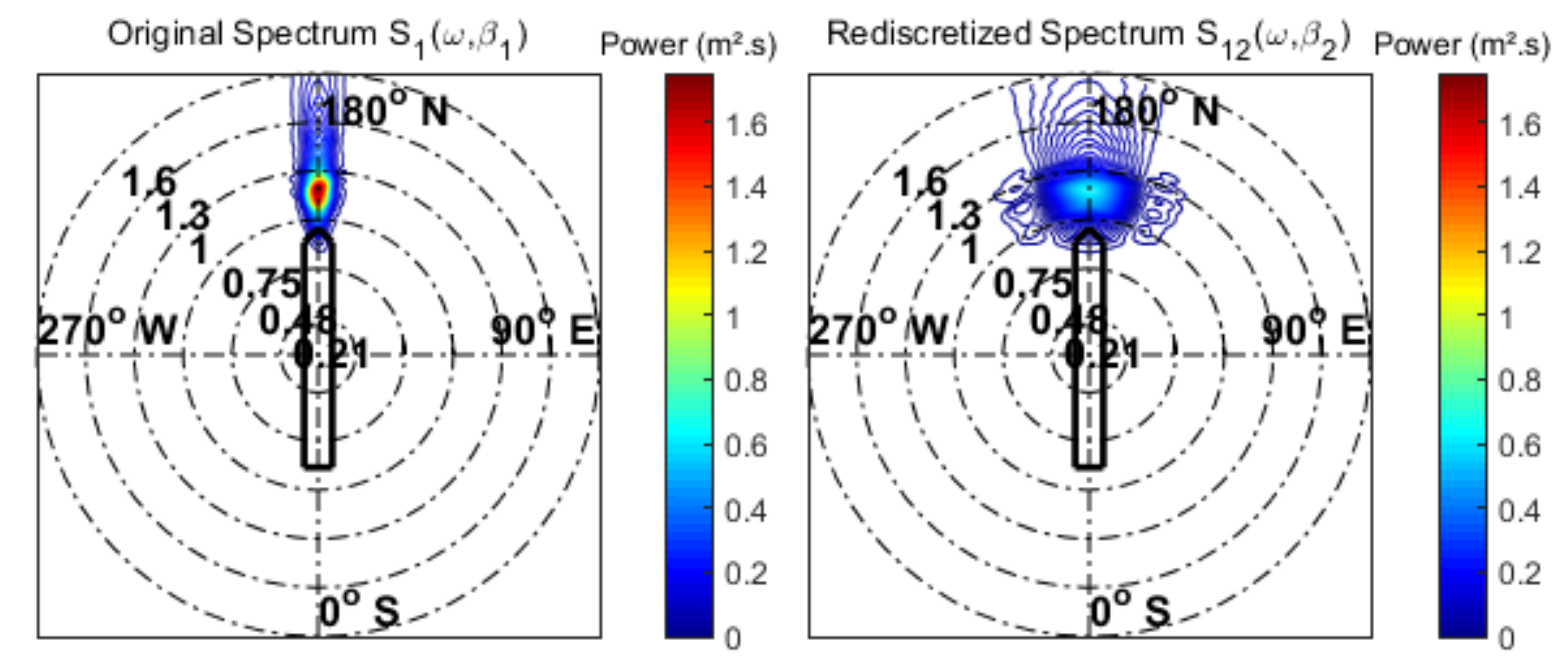

Source: Elaborated by the author. 
The previous figure illustrates how the peak of the energy becomes less pronounced in order to maintain the energy integration. This becomes clear when the spectral energy density is evaluated for each case, and both are equal, Figure 184.

Figure 184 - Comparison of the spectral energy densities.

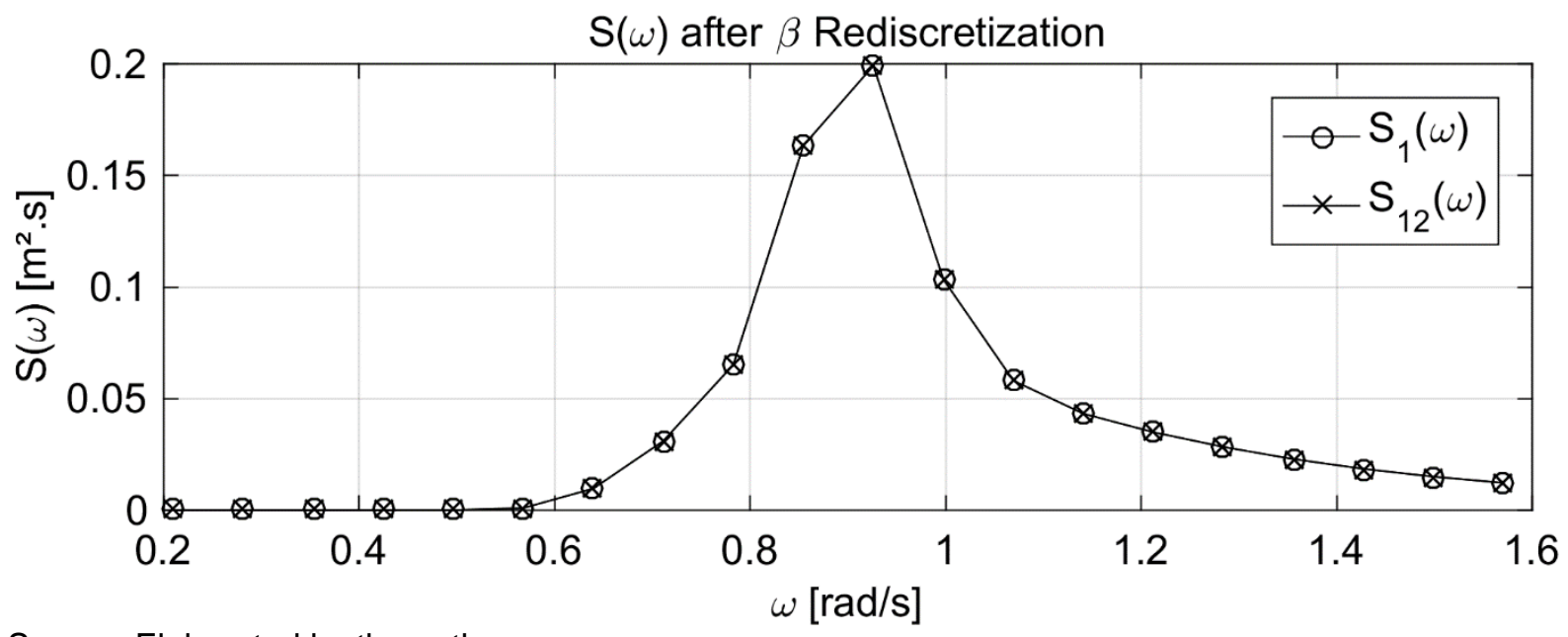

Source: Elaborated by the author.

It is also interesting to compare the rediscretized spectrum against the ideal spectrum that would be generated by the $\beta_{2 j}$ discretization, Figure 185, concluding that the rediscretized spectrum indeed recover the ideal result.

Figure 185 - Rediscretized and ideal spectrum.
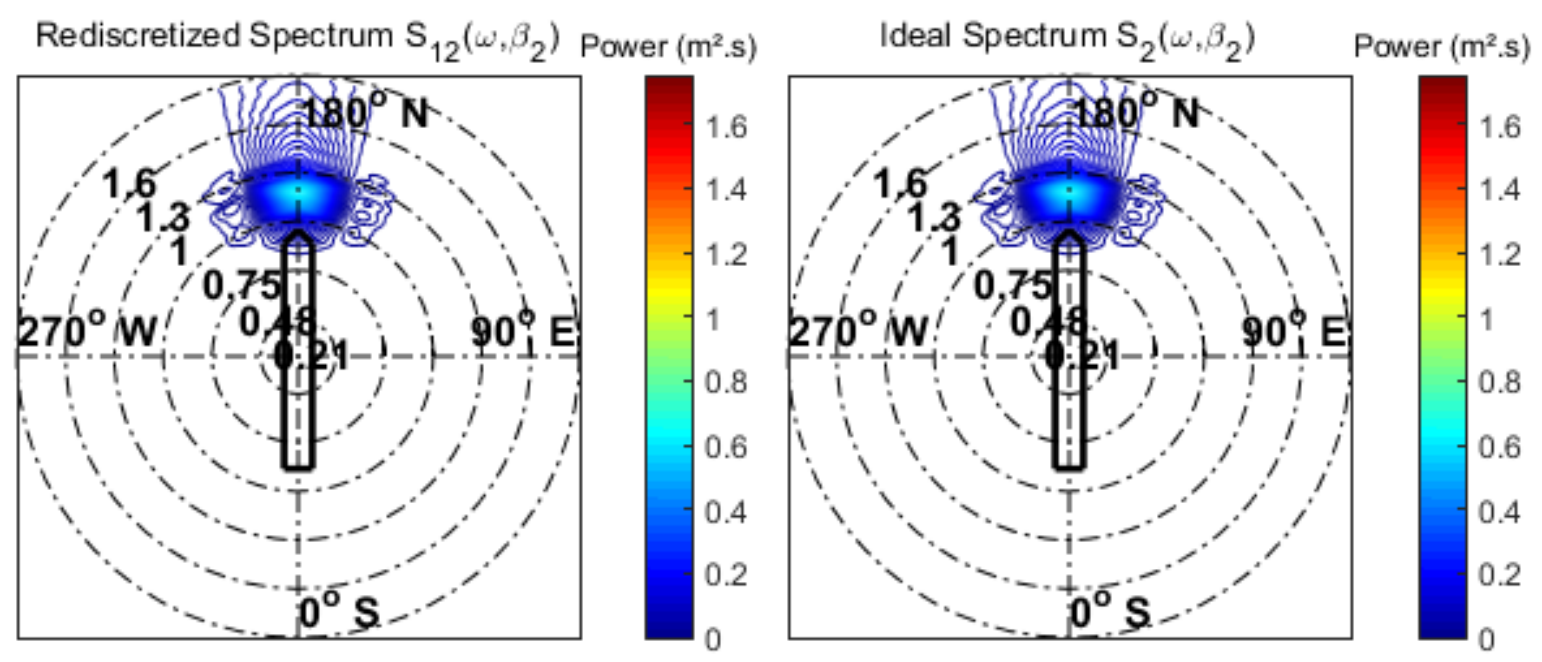

Source: Elaborated by the author.

The simple interpolation, on its turn, causes a distortion in the energy, since it takes the value of the peak, and not the value of the integral, as can be seen in Figure 186 and Figure 187. 
Figure 186 - Rediscretized spectrum by simple interpolation.
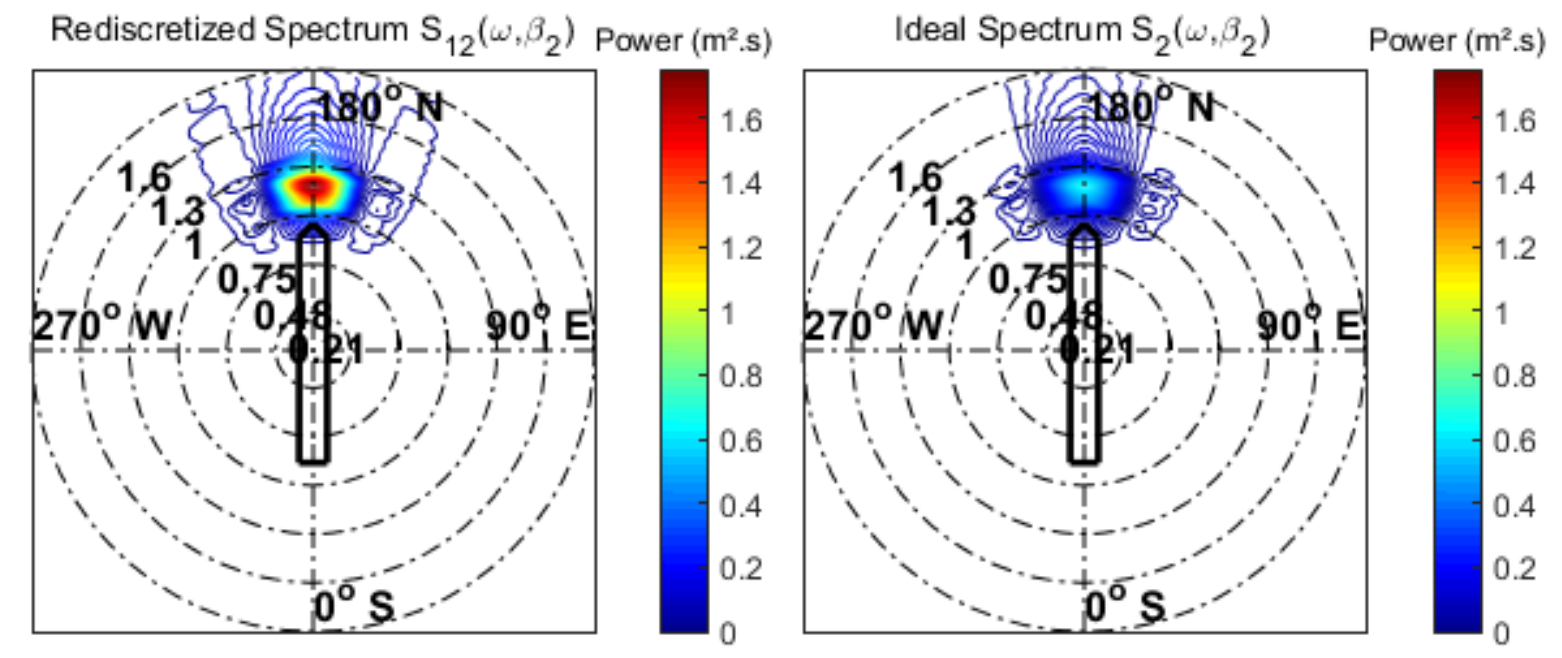

Source: Elaborated by the author.

Figure 187 - Spectral energy density of the interpolated spectrum.

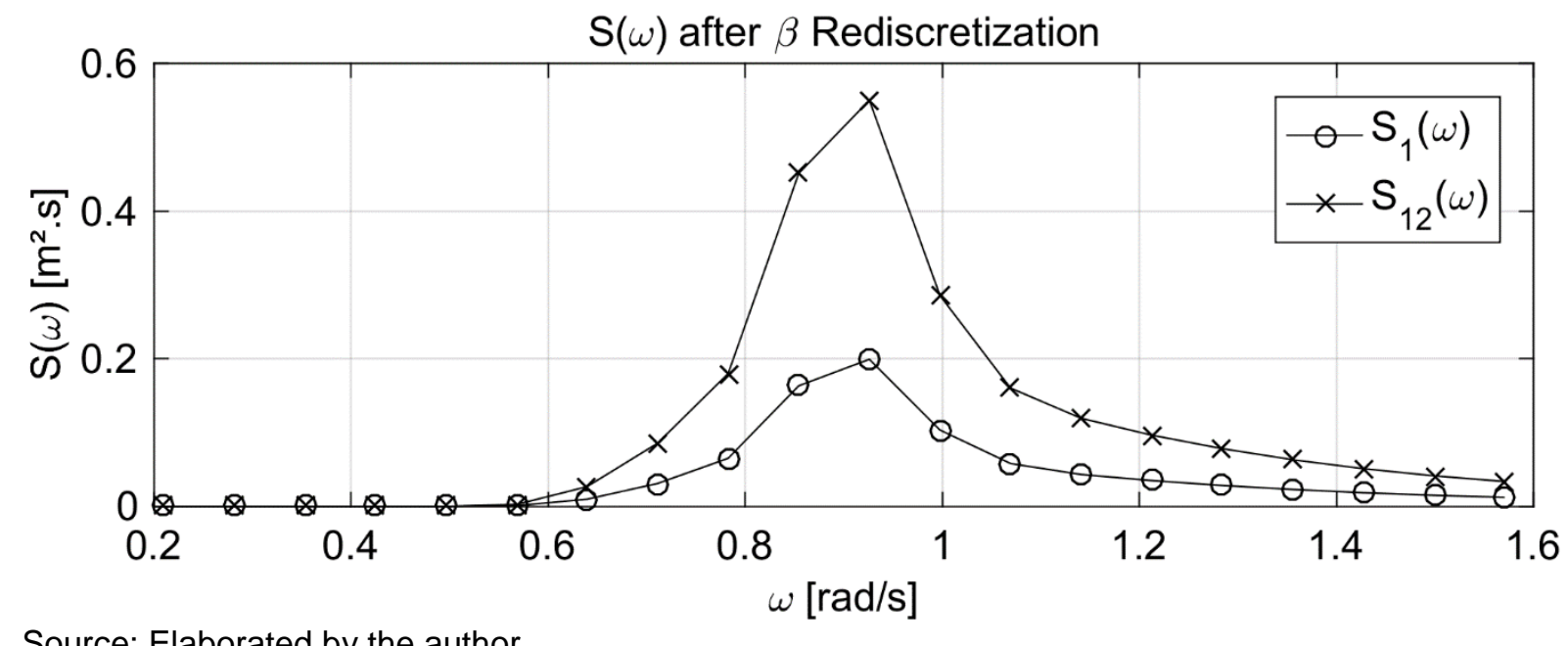

Source: Elaborated by the author.

One could argue that this is just an amplification matter, and a simple constant would correct it; however, if the peak of the spectrum with higher discretization is not exactly in a point of the new rediscretization, but slightly translated, the amplification factor changes considerably.

It happens because the interpolation takes the value that is exactly in the specific interpolated point, and the directional translation change this value. This property can be seen in Figure 188, Figure 189 and Figure 190. 
Figure 188 - Interpolation of spectrum slightly translated.

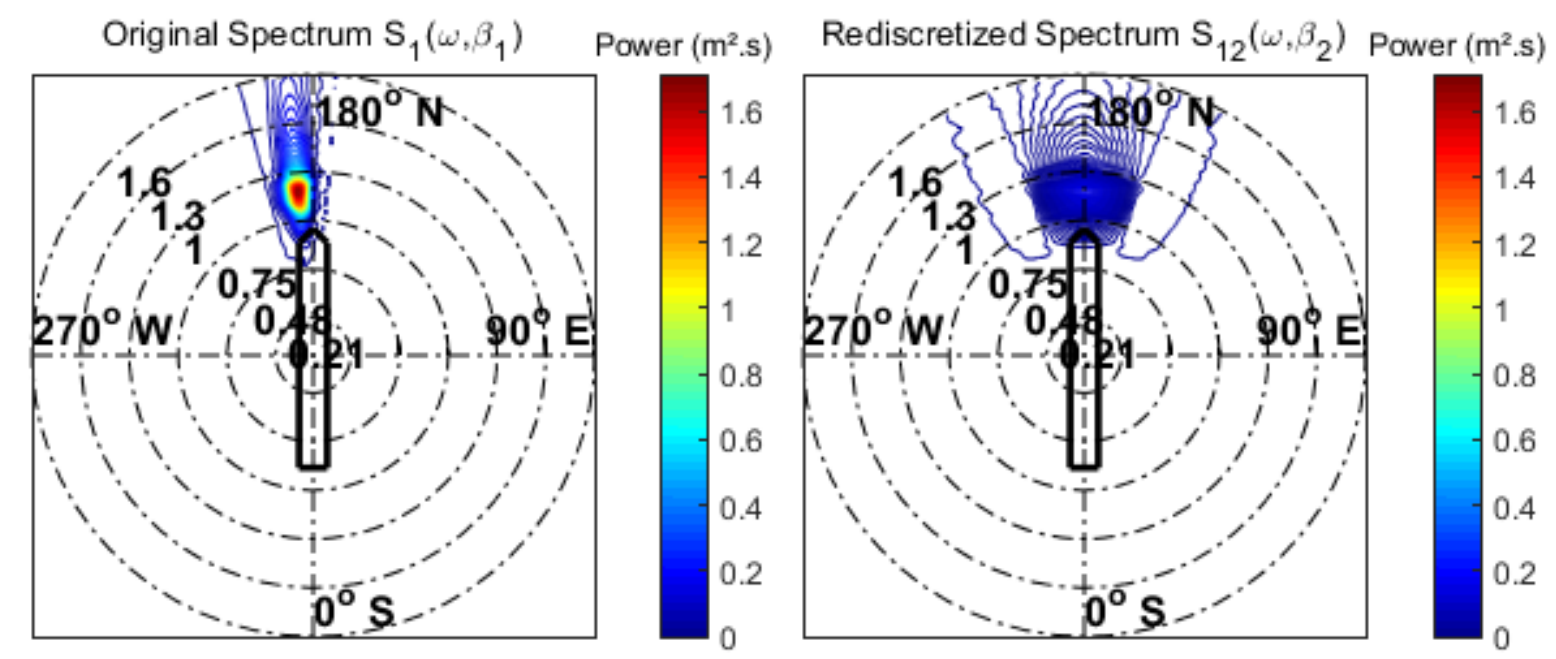

Source: Elaborated by the author.

Figure 189 - Comparison with the ideal spectrum.

Rediscretized Spectrum $\mathrm{S}_{12}\left(\omega, \beta_{2}\right)$ Power $\left(\mathrm{m}^{2} . \mathrm{s}\right)$

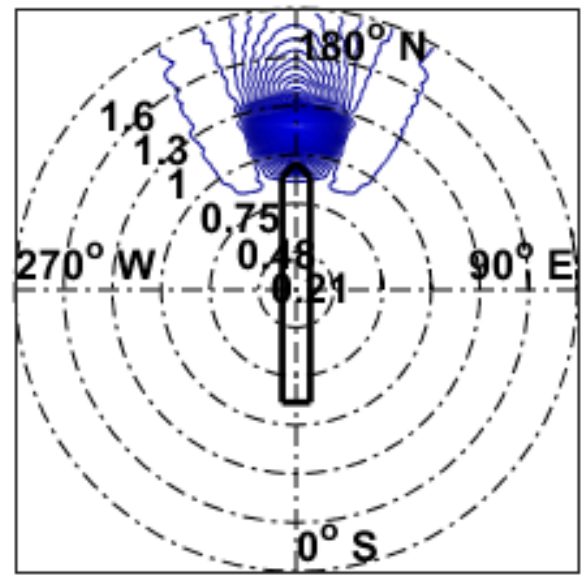

Ideal Spectrum $\mathrm{S}_{2}\left(\omega, \beta_{2}\right)$

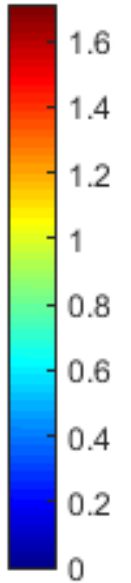

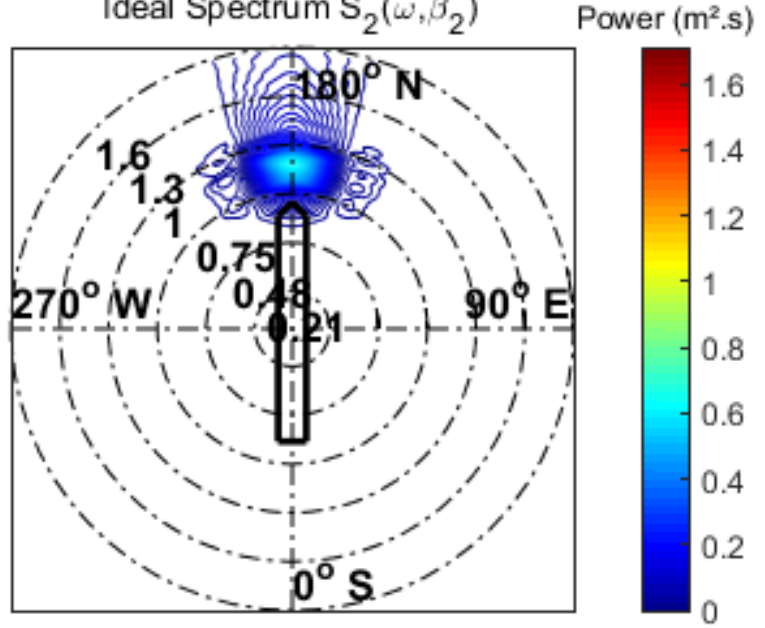

Source: Elaborated by the author.

Figure 190 - The amplification factor changes drastically.

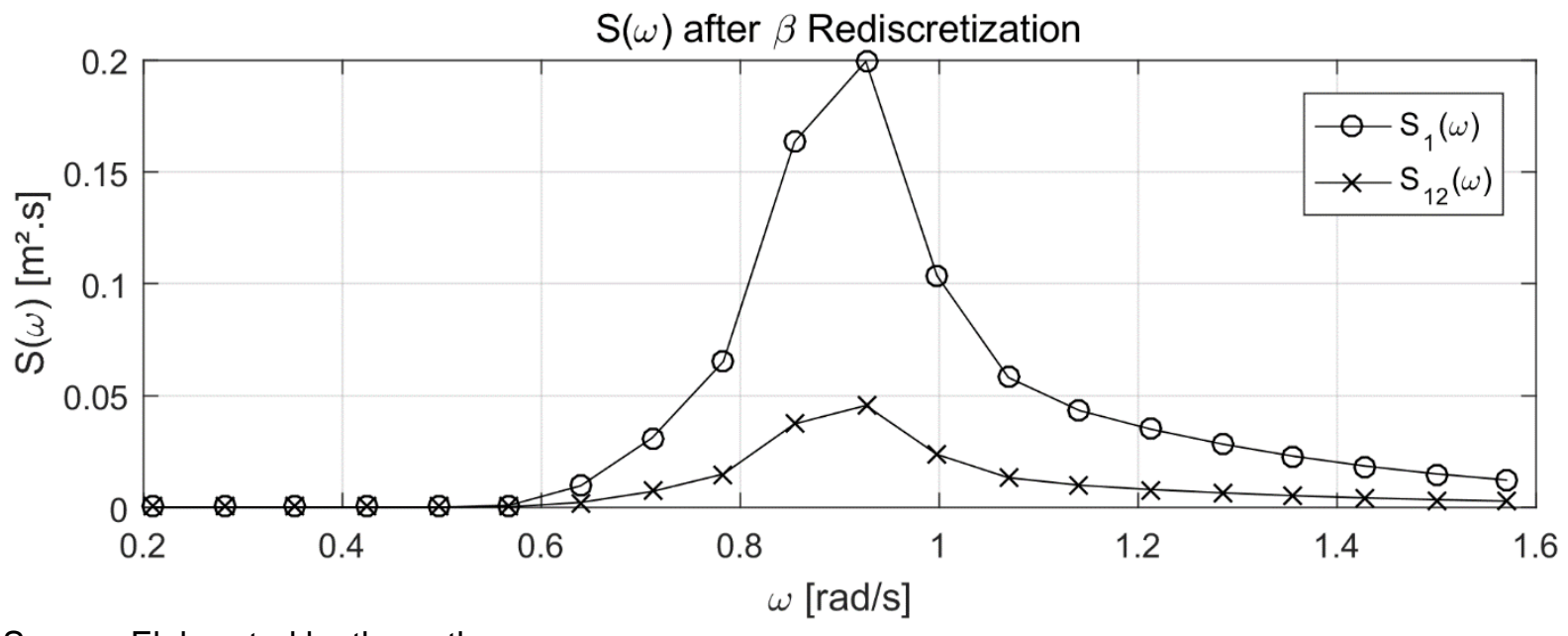

Source: Elaborated by the author. 
The proposed rediscretization procedure, however, is robust against this effect, Figure 191 and Figure 192.

Figure 191 - Rediscretization is robust against translations.
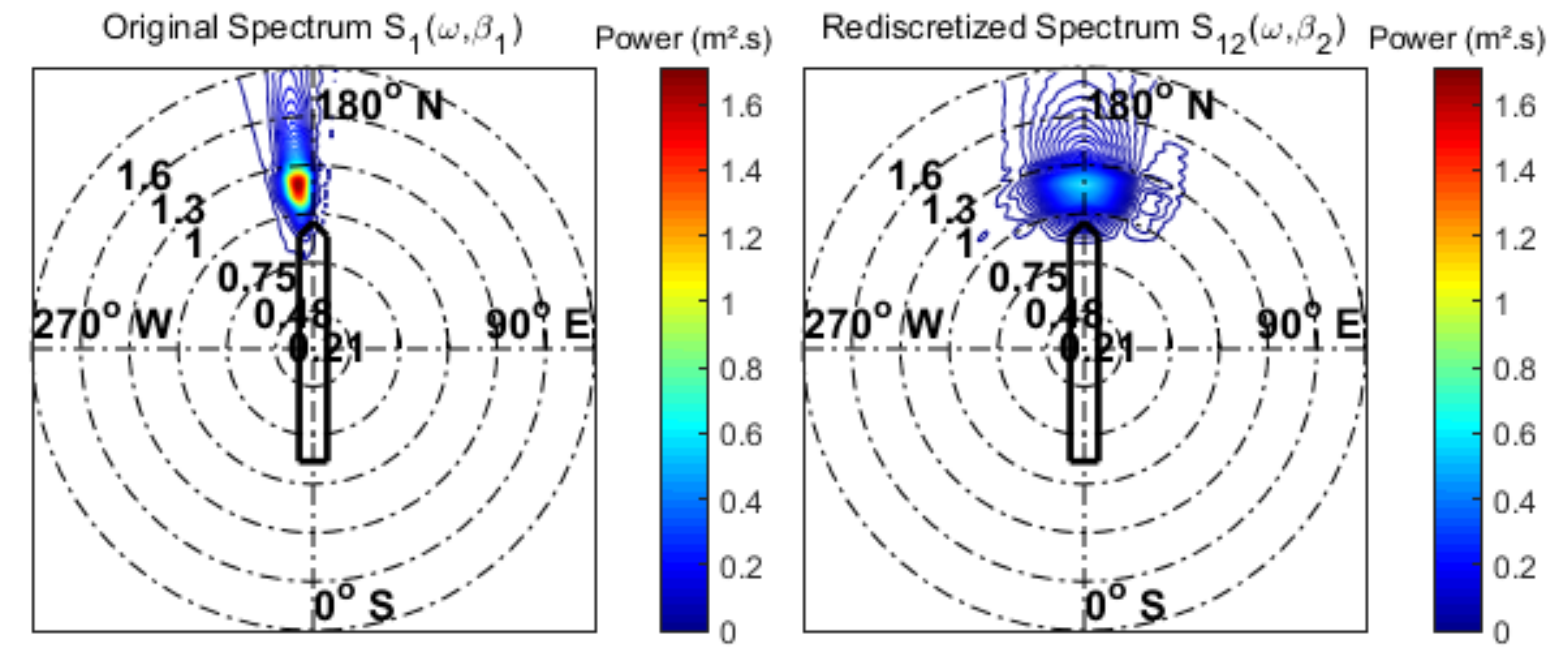

Source: Elaborated by the author.

Figure 192 - Rediscretization still compatible with the ideal spectrum.

Rediscretized Spectrum $\mathrm{S}_{12}\left(\omega, \beta_{2}\right)$ Power $\left(\mathrm{m}^{2} . \mathrm{s}\right)$
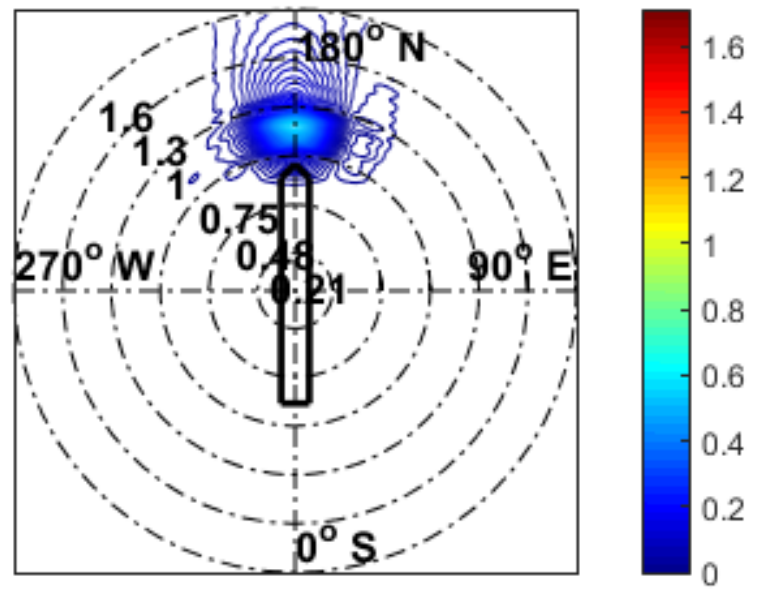

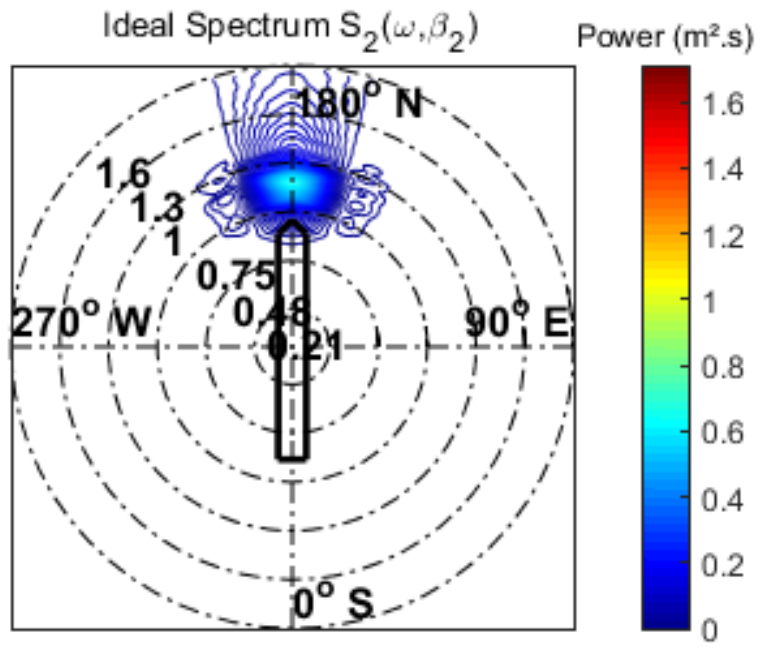

Source: Elaborated by the author.

Finally, the general discretization, in frequencies and directions, is performed one rediscretization by turn. Firstly, the spectrum is interpolated through the frequencies; secondly, the spectrum obtained by the previous procedure is rediscretized through the directions following the integral preserving method described. 